Supporting Information

\title{
Organocatalytic Atroposelective Synthesis of Isoquinolines via Dynamic Kinetic Resolution
}

\author{
Ahreum Kim,${ }^{\dagger}$ Junsoo Moon, ${ }^{\dagger}$ Chanhee Lee ${ }^{\dagger}$ Jayoung Song,${ }^{\ddagger}$ Jongchan Kim, ${ }^{\S}$ \\ Yongseok Kwon ${ }^{\dagger}$ ** \\ ${ }^{\dagger}$ School of Pharmacy, Sungkyunkwan University, Suwon 16419, Republic of Korea \\ *College of Pharmacy, Seoul National University, Seoul 08826, Republic of Korea

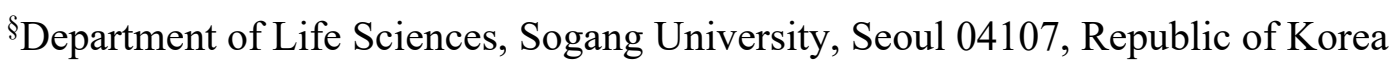

correspondence to:

y.kwon@skku.edu 


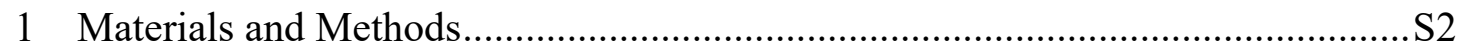

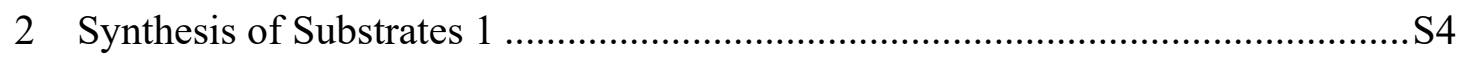

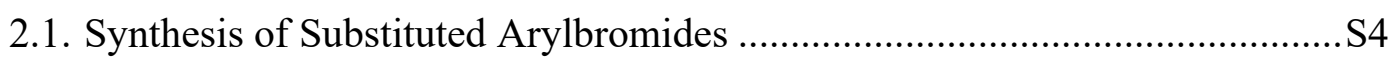

2.2. Synthesis of Substituted Biaryl Intermediates ...........................................S20

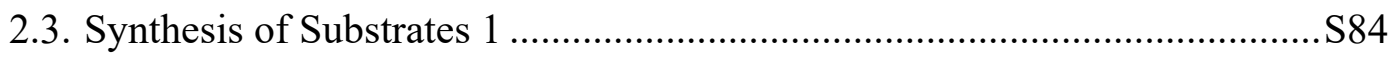

3 Reaction Optimizations and Procedures .........................................................S124

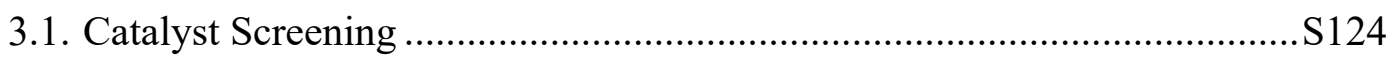

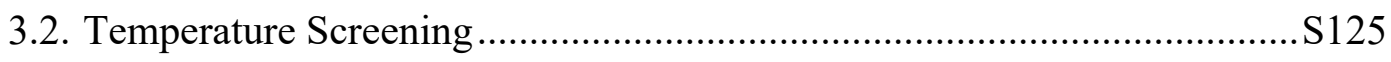

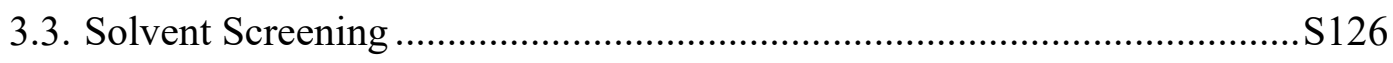

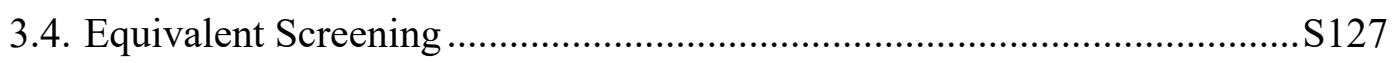

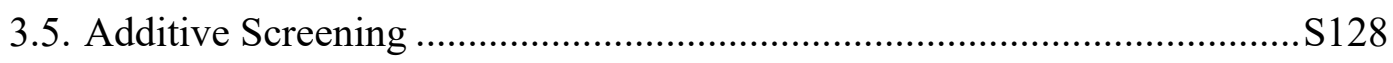

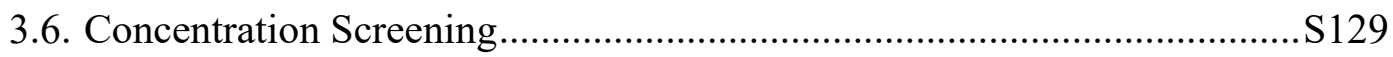

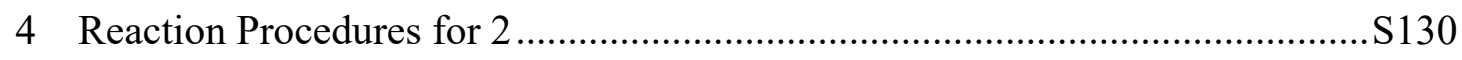

5 Characterization and Spectra of Products 2 ……………………………….......S131

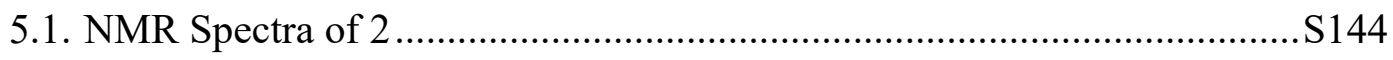

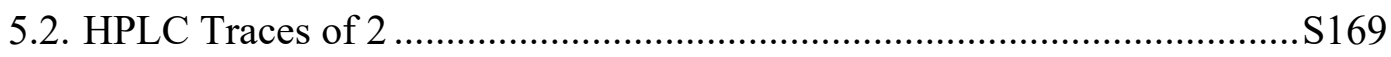

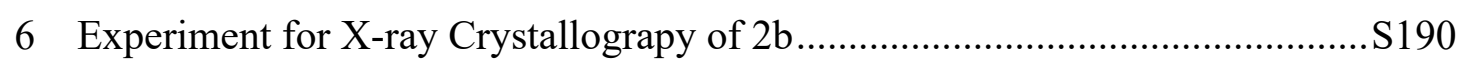

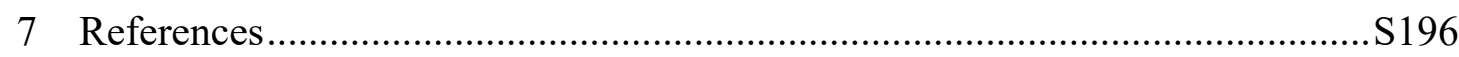




\section{Materials and Methods}

Room temperature is defined as $21-23{ }^{\circ} \mathrm{C}$. All reagents including chiral phosphoric acids were purchased from commercial suppliers and used without further purification, unless otherwise noted. All solvents were purchased from commercial suppliers and used without further purification, unless otherwise noted.

Routine ${ }^{1} \mathrm{H}$ NMR spectra were recorded on Varian $400 \mathrm{MHz}$ spectrometers at ambient temperature unless otherwise stated. All NMR solvents were purchased from Cambridge Isotope Laboratories and used without further purification. Chloroform- $d$ was stored at ambient temperature. Spectra were processed using MestReNova 14.1 using the automatic phasing and polynomial baseline correction capabilities. Splitting was determined using the automatic multiplet analysis function with manual intervention as necessary. Spectral data are reported as follows: chemical shift (multiplicity [singlet (s), broad singlet (brs), doublet (d), triplet (t), quartet (q), pentet (p), multiplet (m), doublet of doublets (dd), doublet of doublet of doublets (ddd), doublet of triplet of doublets (dtd), doublet of doublet of doublet of doublets (dddd), doublet of triplets (dt), triplet of doublets (td), etc.], coupling constant, integration). Chemical shifts are reported in ppm $(\delta)$, and coupling constants are reported in $\mathrm{Hz} .{ }^{1} \mathrm{H}$ Resonances are referenced to solvent residual peaks for $\mathrm{CDCl}_{3}(7.26 \mathrm{ppm}){ }^{1}{ }^{1}$ Routine ${ }^{13} \mathrm{C}$ NMR spectra were recorded on Varian 400 or $500 \mathrm{MHz}$ spectrometers with protons fully decoupled. ${ }^{13} \mathrm{C}$ Resonances are reported in ppm relative to solvent residual peaks for $\mathrm{CDCl}_{3}(77.16 \mathrm{ppm}) .{ }^{1}$ Note: Small deviations in chemical shifts may be observed depending on the concentration of NMR samples.

Melting points were measured using a Gallenkamp melting point apparatus without correction. Infrared spectra were recorded on a Bruker Alpha Platinum-ATR spectrometer, and $v_{\max }$ are partially reported in $\mathrm{cm}^{-1}$. Samples for high-resolution mass spectrometry were submitted to the Organic Chemistry Research Center (OCRC) at Sogang University. Data was acquired on a Thermo-Fischer Scientific LTQ Orbitrap XL instrument equipped with an ESI detector or Bruker compact instrument equipped with an ESI detector. Analytical thin-layer chromatography was performed using $60 \AA$ Silica Gel F254 pre-coated plates $(0.25 \mathrm{~mm}$ thickness $)$. TLC plates were visualized by irradiation with a UV lamp. Normal-phase column chromatography was performed using $60 \AA$ Silica Gel (32-62 micron) with an appropriate mobile phase composition and gradient. Optical rotations were recorded on a Rudolph Research Analytical Autopol I Automatic Polarimeter at the sodium D-line $(589 \mathrm{~nm})$ using a cell of $1 \mathrm{dm}$ path length. Concentration values are reported in units of g/100 mL. Normal-phase high-performance liquid chromatography was performed using an Agilent 1260 series instrument equipped with a diode array detector or multiple wavelength detector and columns (chiral supports) from Daicel Chemical Industries. 

Abbreviation
Boc tert-Butoxycarbonyl
DMAP 4-(Dimethylamino)pyridine
DME 1,2-Dimethoxyethane
DMF $\quad N, N$-Dimethylformamide
ESI Electrospray ionization
EtOAc Ethyl acetate
HFIP 1,1,1,3,3,3-Hexafluoro-2-propanol
HPLC High-performance liquid chromatography
HRMS High-resolution mass spectrometry
Hx Hexanes
MOM Methoxymethyl
rt Room temperature
THF Tetrahydrofuran
TLC Thin-layer chromatography 


\section{Synthesis of Substrates 1}

\subsection{Synthesis of Substituted Arylbromides (S1-8)}

\subsubsection{Synthesis of Substituted 2-Bromo-4-(2-nitroethyl)phenols (S1-3)}

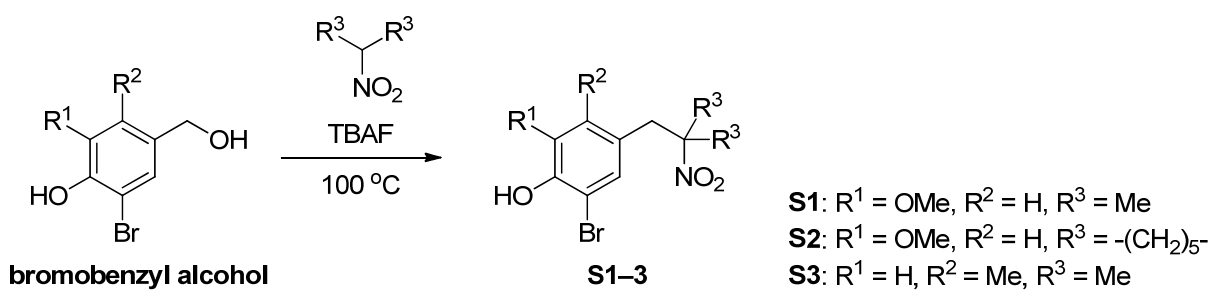

Procedure 1: To a round-bottom flask equipped with a magnetic stirring bar, a solution of bromobenzyl alcohol (1.0 equiv) in 2-nitropropane (4.0 equiv) was added. Then, tetrabutylammonium fluoride ( $1 \mathrm{M}$ solution in THF, 0.5 equiv) was added dropwise and the reaction was heated to $100^{\circ} \mathrm{C}$ in a heating block for $4.5 \mathrm{~h}$. The reaction mixture was cooled to $0{ }^{\circ} \mathrm{C}$, diluted with EtOAc and quenched with aqueous $1 \mathrm{M} \mathrm{HCl}$. The organic layer was extracted with EtOAc, dried with anhydrous $\mathrm{MgSO}_{4}$, filtered, and concentrated in vacuo. The crude material was then purified by flash chromatography to afford the desired material S1-S3. 
Characterization and Spectra of Substituted 2-Bromo-4-(2-nitroethyl)phenols (S1-3)<smiles>COc1cc(CC(C)(C)[N+](=O)[O-])cc(Br)c1O</smiles>

2-Bromo-6-methoxy-4-(2-methyl-2-nitropropyl)phenol (S1) was synthesized by following Procedure 1 using 2-bromo-4-(hydroxymethyl)-6-methoxyphenol. ${ }^{2}$ The crude material was purified by normal-phase column chromatography using an eluent of $25 \% \mathrm{EtOAc} / \mathrm{Hx}$ to provide $\mathbf{S 1}$ as a white solid (399 $\mathrm{mg}, 71 \%$ ).

m.p. $98^{\circ} \mathrm{C}$

${ }^{1} \mathrm{H}$ NMR $\left(400 \mathrm{MHz}, \mathrm{CDCl}_{3}\right) \delta 6.85(\mathrm{~s}, 1 \mathrm{H}), 6.50(\mathrm{~s}, 1 \mathrm{H}), 5.89(\mathrm{~s}, 1 \mathrm{H}), 3.85(\mathrm{~s}, 3 \mathrm{H})$, $3.08(\mathrm{~s}, 2 \mathrm{H}), 1.57(\mathrm{~s}, 6 \mathrm{H})$.

${ }^{13} \mathrm{C}$ NMR $\left(100 \mathrm{MHz}, \mathrm{CDCl}_{3}\right) \delta 147.2,142.6,127.7,126.3,111.5,108.0,88.7,56.5$, 46.2, $25.7(2 \mathrm{C})$.

IR (FT-ATR, $\mathrm{cm}^{-1}, \mathrm{CH}_{2} \mathrm{Cl}_{2}$ ) $v_{\max } 3488,2966,2939,1606,1586,1535,1498,1463$, $1422,1397,1371,1347,1279,1240,1185,1143,1045,985,957,854,824,809,790$, 733, 648, 583, 549, 521.

HRMS (ESI) $m / z$ : [M+Na] $]^{+}$Calcd for $\mathrm{C}_{11} \mathrm{H}_{14} \mathrm{BrNO}_{4}$ 326.0004; found 326.0002 .<smiles>Cc1cc(O)c(Br)cc1CC(C)(C)N=O</smiles>

2-Bromo-5-methyl-4-(2-methyl-2-nitropropyl)phenol (S2) was synthesized by following Procedure 1 using 2-bromo-4-(hydroxymethyl)-5-methylphenol. The crude material was purified by normal-phase column chromatography using an eluent of $25 \%$ EtOAc/Hx to provide $\mathbf{S} 2$ as a colorless gum (454 mg, 45\%).

${ }^{1} \mathbf{H}$ NMR $\left(400 \mathrm{MHz}, \mathrm{CDCl}_{3}\right) \delta 7.09(\mathrm{~s}, 1 \mathrm{H}), 6.84(\mathrm{~s}, 1 \mathrm{H}), 5.40(\mathrm{~s}, 1 \mathrm{H}), 3.16(\mathrm{~s}, 2 \mathrm{H})$, $2.23(\mathrm{~s}, 3 \mathrm{H}), 1.57(\mathrm{~s}, 6 \mathrm{H})$.

${ }^{13}$ C NMR $\left(100 \mathrm{MHz} \mathrm{CDCl}_{3}\right) \delta 151.4,138.8,133.6,127.5,118.0,107.4,89.2,41.9$, 25.6 (2C), 20.0 .

IR (FT-ATR, $\mathrm{cm}^{-1}, \mathrm{CH}_{2} \mathrm{Cl}_{2}$ ) $v_{\max } 3500,2987,2939,1602,1535,1489,1472,1398$, 1372, 1347, 1275, 1197, 1140, 1109, 1013, 991, 887, 857, 730, 504, 476, 452.

HRMS (ESI) $m / z$ : $[\mathrm{M}+\mathrm{Na}]^{+}$Calcd for $\mathrm{C}_{11} \mathrm{H}_{14} \mathrm{BrNO}_{3} 310.0055$; found 310.0053 . 


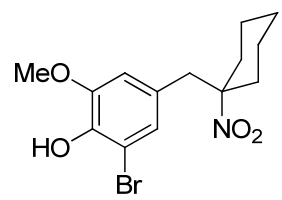

2-Bromo-6-methoxy-4-((1-nitrocyclohexyl)methyl)phenol (S3) was synthesized by following Procedure 1 using 2-bromo-4-(hydroxymethyl)-6-methoxyphenol. ${ }^{2}$ The crude material was purified by normal-phase column chromatography using an eluent of $25 \% \mathrm{EtOAc} / \mathrm{Hx}$ to provide $\mathbf{S 3}$ as a white solid (454 $\mathrm{mg}, 45 \%$ ).

m.p. $106^{\circ} \mathrm{C}$

${ }^{1} \mathrm{H}$ NMR $\left(400 \mathrm{MHz}, \mathrm{CDCl}_{3}\right) \delta 6.80(\mathrm{~s}, 1 \mathrm{H}), 6.42(\mathrm{~s}, 1 \mathrm{H}), 5.88(\mathrm{~s}, 1 \mathrm{H}), 3.83(\mathrm{~s}, 3 \mathrm{H})$, $2.97(\mathrm{~s}, 2 \mathrm{H}), 2.37(\mathrm{~d}, J=12.2 \mathrm{~Hz}, 2 \mathrm{H}), 1.71-1.52(\mathrm{~m}, 5 \mathrm{H}), 1.19-1.43(\mathrm{~m}, 3 \mathrm{H})$.

${ }^{13} \mathrm{C}$ NMR (100 MHz, $\left.\mathrm{CDCl}_{3}\right) \delta 147.1,142.6,127.0,126.0,111.4,107.9,92.00,56.5$, 46.4, 33.9 (2C), 24.8, $22.3(2 \mathrm{C})$.

IR (FT-ATR, $\mathrm{cm}^{-1}, \mathrm{CH}_{2} \mathrm{Cl}_{2}$ ) $v_{\max } 3487,2937,2862,1605,1586,1531,1498,1448$, $1422,1346,1321,1281,1241,1185,1143,1086,1044,945,925,912,860,824,788$, 735, 647, 579, 534, 455.

HRMS (ESI) $m / z$ : [M+Na] $]^{+}$Calcd for $\mathrm{C}_{14} \mathrm{H}_{18} \mathrm{BrNO}_{4}$ 366.0317; found 366.0315 . 


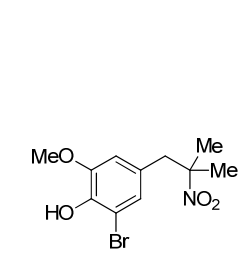

S1

$\left({ }^{1} \mathrm{H} \mathrm{NMR}, 400 \mathrm{MHz}, \mathrm{CDCl}_{3}\right)$
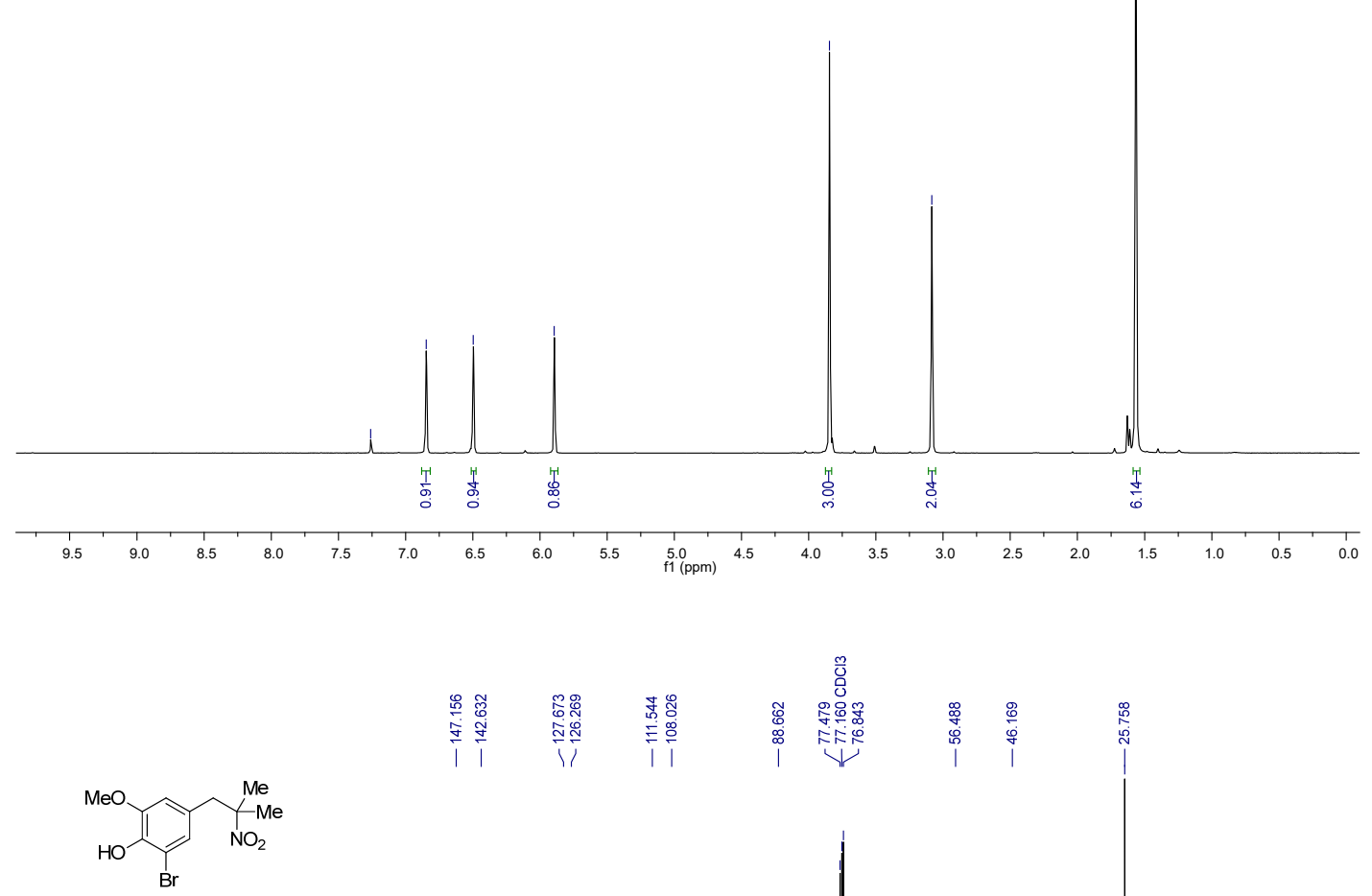

s1

$\left({ }^{13} \mathrm{C} \mathrm{NMR,}, 100 \mathrm{MHz}, \mathrm{CDCl}_{3}\right)$
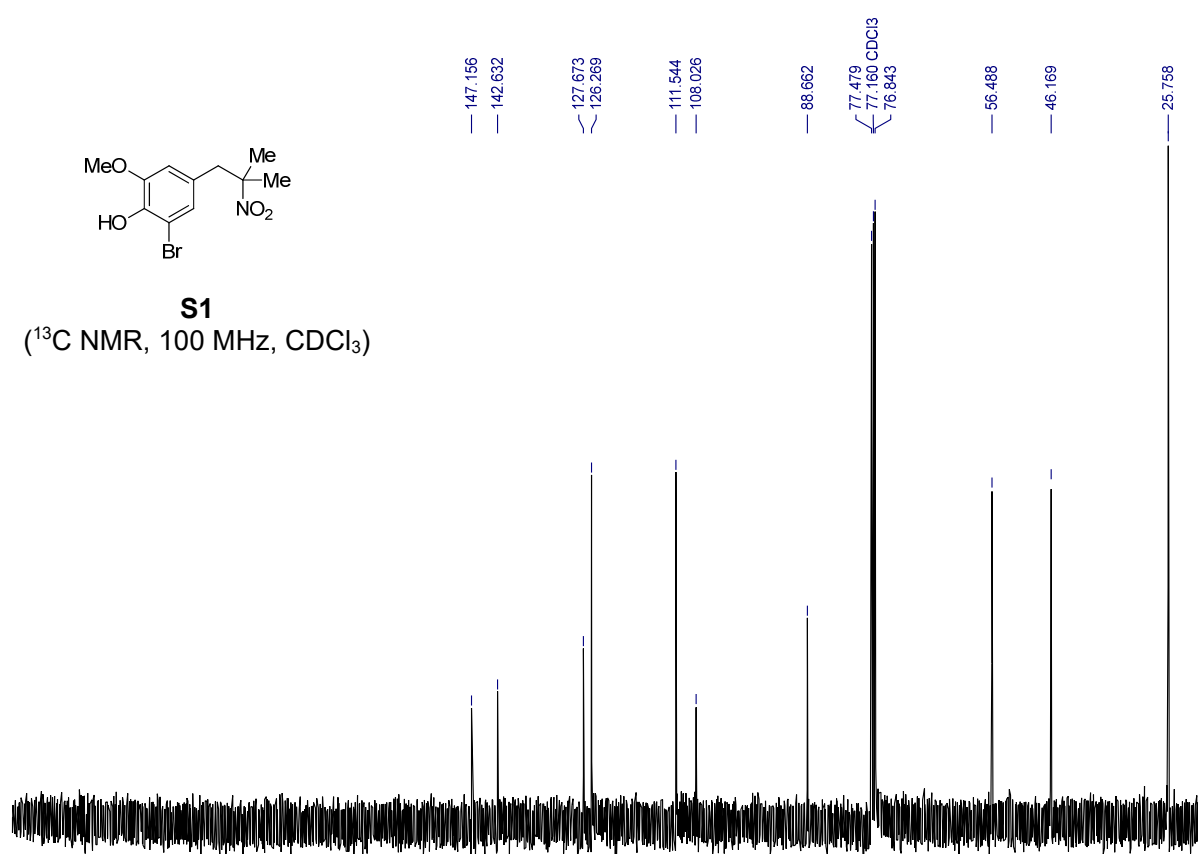

$\begin{array}{lllllllllllllllllllllllllllll}1 & 220 & 210 & 200 & 190 & 180 & 170 & 160 & 150 & 140 & 130 & 120 & \underset{\mathrm{f}}{110}(\mathrm{ppm}) & 100 & 90 & 80 & 70 & 60 & 50 & 40 & 30 & 20 & 10 & 0 & -10\end{array}$ 


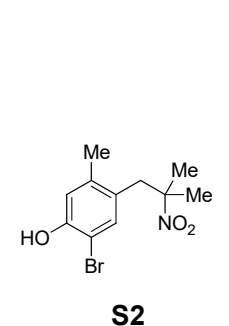

음

告

$\underset{\substack{\bar{q} \\ 0}}{1}$$$
\text { S2 }
$$

( ${ }^{1} \mathrm{H} \mathrm{NMR}, 400 \mathrm{MHz}, \mathrm{CDCl}_{3}$ )
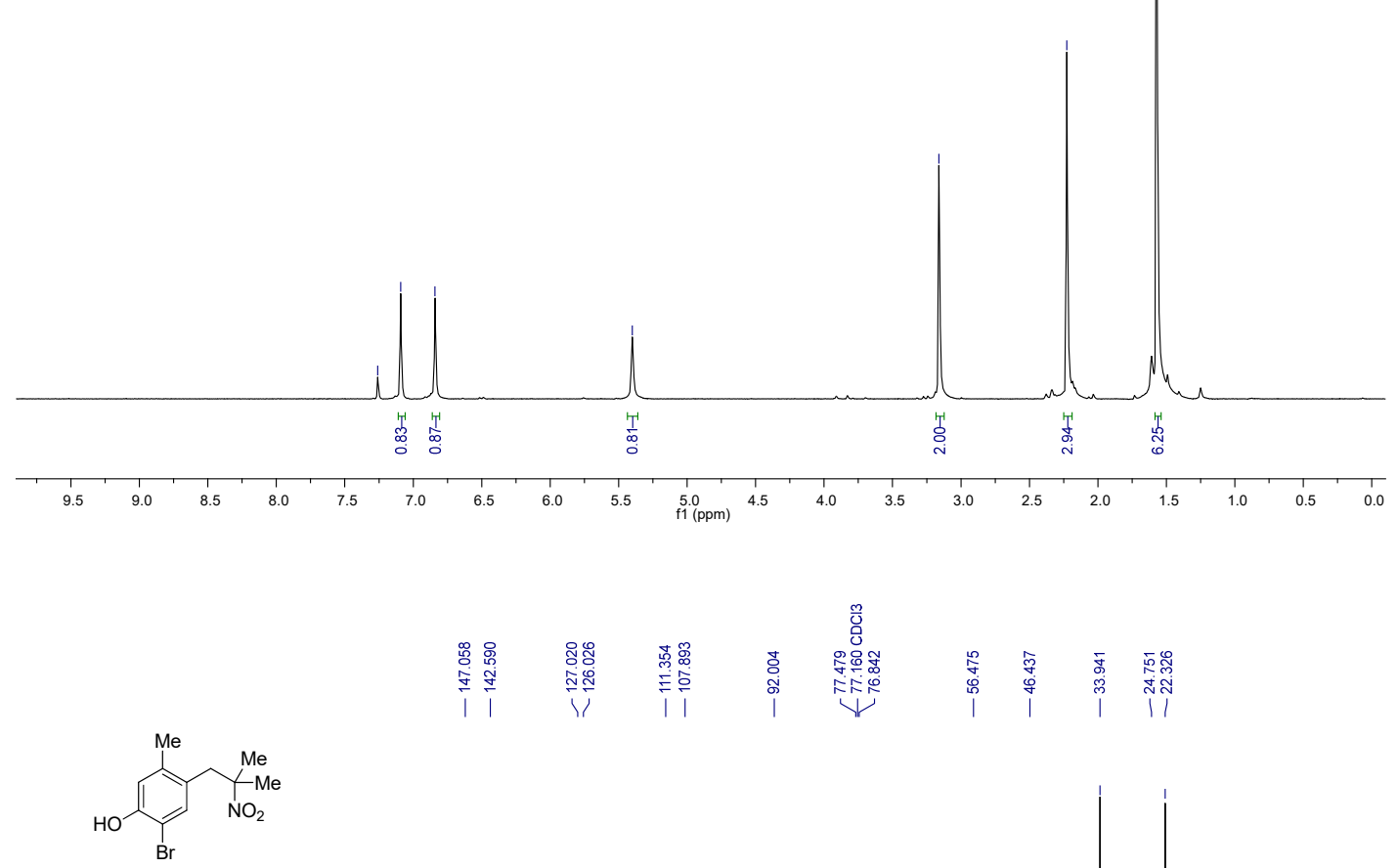

S2

$\left({ }^{13} \mathrm{C}\right.$ NMR, $\left.100 \mathrm{MHz}, \mathrm{CDCl}_{3}\right)$
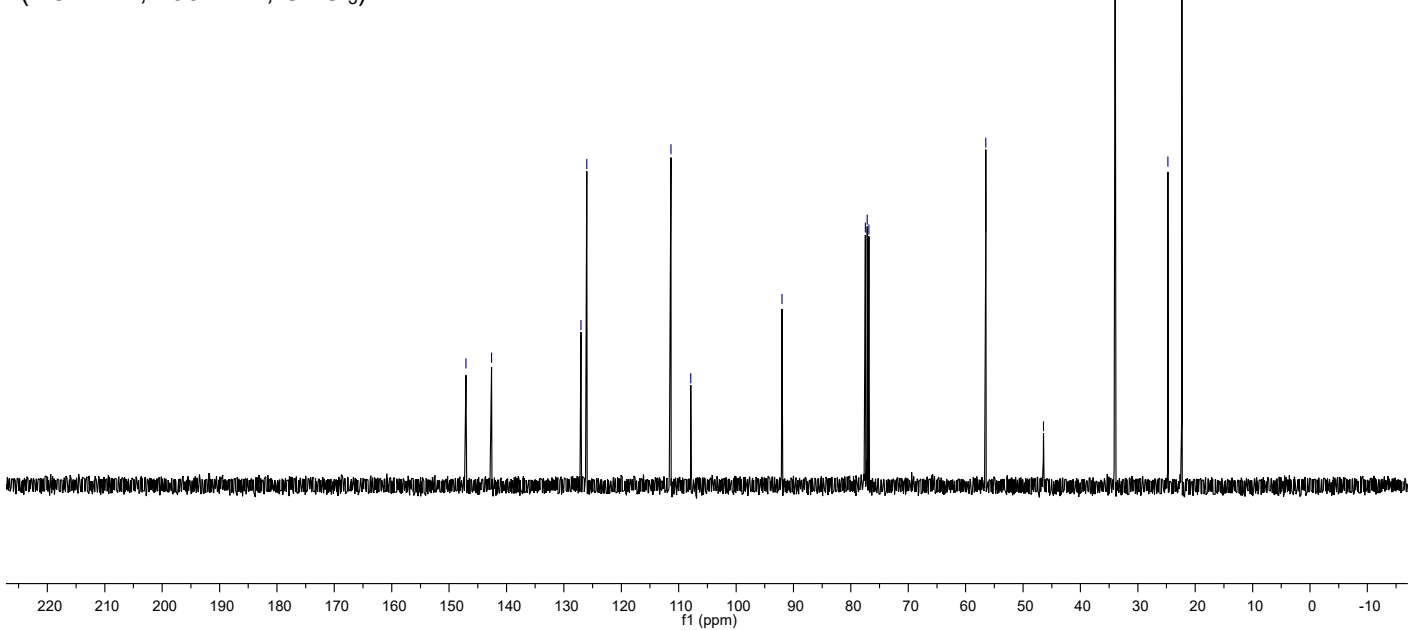

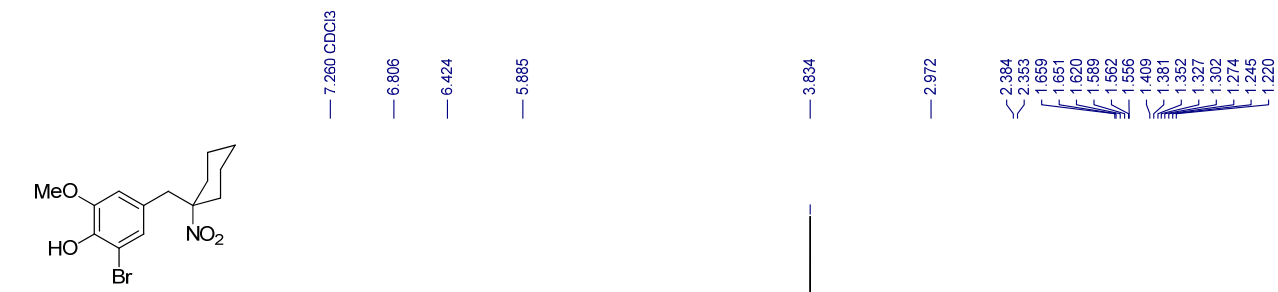

S3

$\left({ }^{1} \mathrm{H} \mathrm{NMR}, 400 \mathrm{MHz}, \mathrm{CDCl}_{3}\right)$
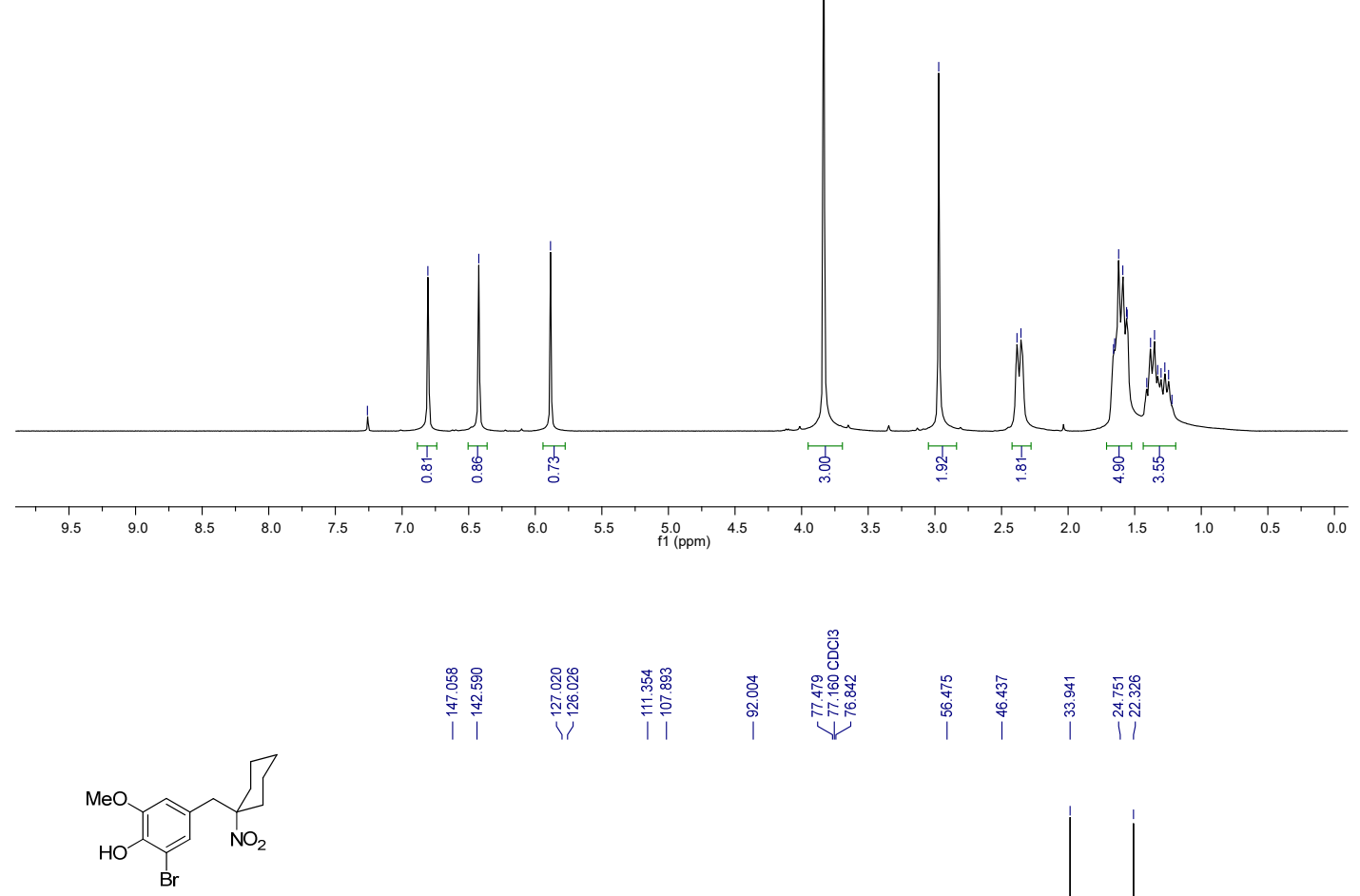

S3

$\left({ }^{13} \mathrm{C} \mathrm{NMR,}, 100 \mathrm{MHz}, \mathrm{CDCl}_{3}\right)$

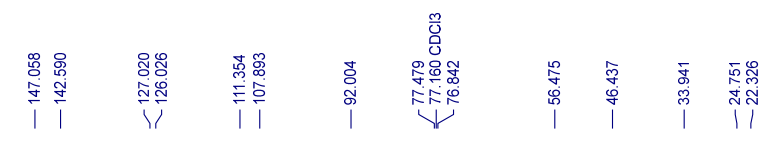

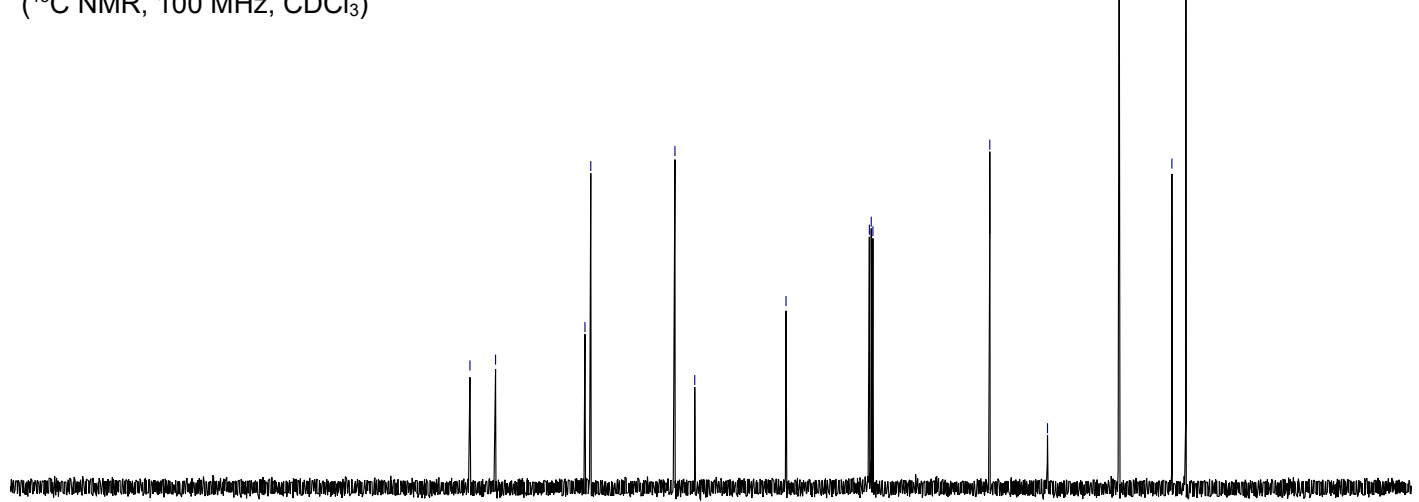

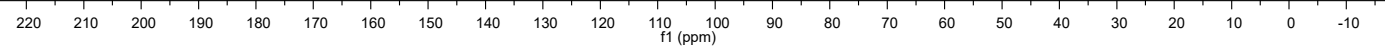




\subsubsection{Synthesis of Substituted 3-(2-Nitroethyl)arylbromides (S4-9)}
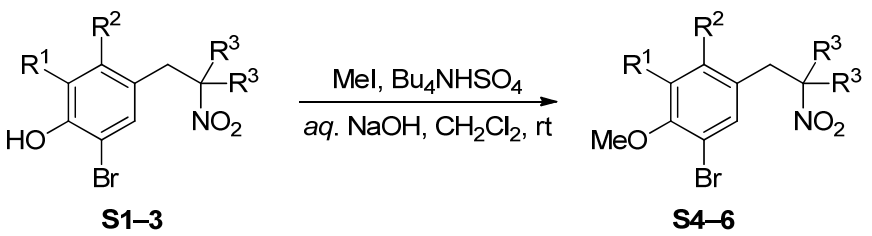

S4: $R^{1}=O M e, R^{2}=H, R^{3}=M e$
S5: $R^{1}=H, R^{2}=M e, R^{3}=M e$
S6: $R^{1}=O M e, R^{2}=H, R^{3}=-\left(C_{2}\right)_{5}^{-}$

Procedure 2: Methylation

To a round-bottom flask equipped with a magnetic stirring bar, a solution of S1-3 (1.0 equiv) in $\mathrm{CH}_{2} \mathrm{Cl}_{2}(0.2 \mathrm{M}$ ) was added. Then, aqueous $0.6 \mathrm{M} \mathrm{NaOH}$ (3.0 equiv) was added subsequently and to the reaction mixture, tetrabutylammonium hydrogen sulfate (1.0 equiv) was added. Once the mixture is dissolved, iodomethane (12.0 equiv) was added, and the reaction mixture was allowed to stir at $\mathrm{rt}$ for overnight. The reaction mixture was washed with water and extracted with $\mathrm{CH}_{2} \mathrm{Cl}_{2}$ three times. The combined organic layer was dried with anhydrous $\mathrm{MgSO}_{4}$, filtered, and concentrated in vacuo. The crude material was then purified by flash chromatography to afford the desired material S46.

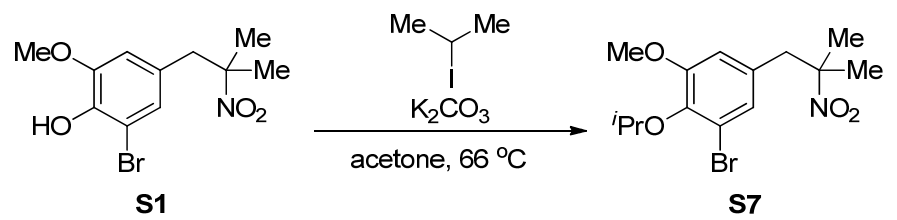

Procedure 3: Isopropylation

To a suspension of $\mathbf{S 1}$ (1.0 equiv) in acetone $(0.1 \mathrm{M})$ was added 2-iodopropane (4.0 equiv) and $\mathrm{K}_{2} \mathrm{CO}_{3}$ (2.1 equiv). The reaction mixture was heated and stirred at $66^{\circ} \mathrm{C}$ in a heating block for overnight under $\mathrm{N}_{2}$ atmosphere. Upon cooling, the suspension was concentrated under reduced pressure, diluted with EtOAc and washed with water. The organic layer was extracted with EtOAc three times, dried with anhydrous $\mathrm{MgSO}_{4}$, filtered, and concentrated in vacuo. The crude material was then purified by flash chromatography to afford the desired material S7. 

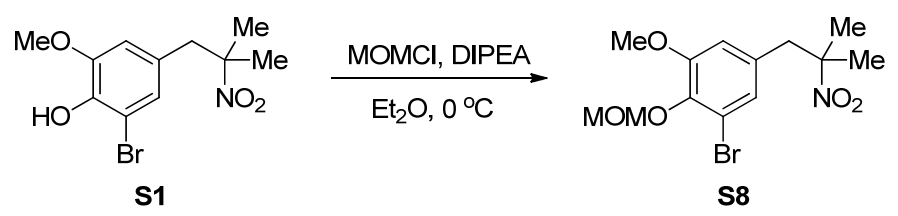

Procedure 4: MOM protection

A solution of chloromethyl methyl ether (2.0 equiv, $2.1 \mathrm{M}$ solution in $\mathrm{PhMe}$ ) was added at $\mathrm{rt}$ to a solution of $\mathbf{S 1}$ (1.0 equiv) and diethyl ether $(2.8 \mathrm{M})$. The resulting solution was cooled to $0{ }^{\circ} \mathrm{C}$ and diisopropylethylamine (1.5 equiv) was added slowly, warmed to $\mathrm{rt}$ and stirred for $14 \mathrm{~h}$. The reaction mixture was diluted with diethyl ether and quenched with saturated aqueous $\mathrm{NaHCO}_{3}$. The organic layer was extracted with diethyl ether three times, dried with $\mathrm{MgSO}_{4}$, filtered, and concentrated in vacuo. The crude product was used without purification process.

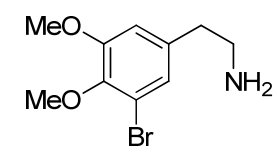

2-(3-bromo-4,5-dimethoxyphenyl) ethanamine

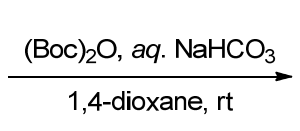<smiles>COc1cc(CCNC(=O)OCc2ccccc2)cc(Br)c1OC</smiles>

S9

Procedure 5: Boc protection

To a round-bottom flask equipped with a magnetic stirring bar, a solution of 2-(3bromo-4,5-dimethoxyphenyl)ethanamine (1.0 equiv) in 1,4-dioxane/aqueous saturated $\mathrm{NaHCO}_{3}(1: 2,0.5 \mathrm{M})$ was added. Di-tert-butyl dicarbonate (1.1 equiv) was added, and the reaction was stirred until complete consumption of starting material was indicated by TLC (about $3 \mathrm{~h}$ ). The reaction mixture was washed with brine and extracted with EtOAc three times. The combined organic layers were dried with anhydrous $\mathrm{MgSO}_{4}$, filtered, and concentrated in vacuo. The crude material was then purified by flash chromatography to afford the desired material S9. 


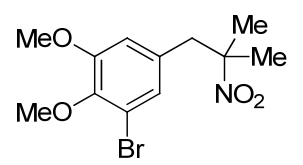

1-Bromo-2,3-dimethoxy-5-(2-methyl-2-nitropropyl)benzene (S4) was synthesized by following Procedure 2 . The crude material was purified by normal-phase column chromatography using an eluent of $13 \%$ EtOAc/Hx to provide $\mathbf{S} 4$ as a white solid (4.6 g, 99\%).

m.p. $94{ }^{\circ} \mathrm{C}$

${ }^{1} \mathbf{H}$ NMR $\left(400 \mathrm{MHz}, \mathrm{CDCl}_{3}\right) \delta 6.88(\mathrm{~s}, 1 \mathrm{H}), 6.54(\mathrm{~s}, 1 \mathrm{H}), 3.83(\mathrm{~s}, 3 \mathrm{H}), 3.81(\mathrm{~s}, 3 \mathrm{H})$, $3.11(\mathrm{~s}, 2 \mathrm{H}), 1.58(\mathrm{~s}, 6 \mathrm{H})$.

${ }^{13} \mathrm{C}$ NMR $\left(100 \mathrm{MHz}, \mathrm{CDCl}_{3}\right) \delta 153.6,146.0,132.2,126.3,117.5,113.4,88.6,60.7$, $56.2,46.2,25.8(2 \mathrm{C})$.

IR (FT-ATR, $\mathrm{cm}^{-1}, \mathrm{CH}_{2} \mathrm{Cl}_{2}$ ) $v_{\max } 3350,3062,2961,2935,2842,1672,1582,1520$, 1484, 1466, 1443, 1419, 1382, 1365, 1343, 1304, 1248, 1184, 1142, 1118, 1090, 1074, 1049, 1036, 1003, 901, 857, 820, 788, 757, 709, 692, 674, 633, 586, 531.

HRMS (ESI) $m / z$ : [M+Na] $]^{+}$Calcd for $\mathrm{C}_{12} \mathrm{H}_{16} \mathrm{BrNO}_{4} 340.0160$; found 340.0158 .<smiles>COc1cc([N+](=O)[O-])c(CC(C)(C)N=O)cc1Br</smiles>

1-Bromo-2-methoxy-4-methyl-5-(2-methyl-2-nitropropyl)benzene (S5) was synthesized by following Procedure 2. The crude material was purified by normalphase column chromatography using an eluent of 17\% EtOAc/Hx to provide $\mathbf{S 5}$ as a white solid (418 mg, 96\%).

m.p. $103^{\circ} \mathrm{C}$

${ }^{1} \mathbf{H}$ NMR $\left(400 \mathrm{MHz}, \mathrm{CDCl}_{3}\right) \delta 7.18(\mathrm{~s}, 1 \mathrm{H}), 6.69(\mathrm{~s}, 1 \mathrm{H}), 3.86(\mathrm{~s}, 3 \mathrm{H}), 3.16(\mathrm{~s}, 2 \mathrm{H})$, $2.26(\mathrm{~s}, 3 \mathrm{H}), 1.57(\mathrm{~s}, 6 \mathrm{H})$.

${ }^{13} \mathrm{C}$ NMR $\left(100 \mathrm{MHz}, \mathrm{CDCl}_{3}\right) \delta 154.9,138.0135 .1,127.2,114.2,108.6,89.0,56.3$, 42.1, 25.7 (2C), 20.3.

IR (FT-ATR, $\mathrm{cm}^{-1}, \mathrm{CH}_{2} \mathrm{Cl}_{2}$ ) v $v_{\max } 2941,1693,1598,1537,1497,1464,1396,1373$, $1347,1285,1255,1205,1147,1078,1051,888,856,717,554,506,459$.

HRMS (ESI) $m / z$ : $[\mathrm{M}+\mathrm{Na}]^{+}$Calcd for $\mathrm{C}_{12} \mathrm{H}_{16} \mathrm{BrNO}_{3} 324.0211$; found 324.0210. 
<smiles>COc1cccc(CC=CC(=O)Nc2ccc([N+](=O)[O-])c(Br)c2)c1</smiles>

\section{1-Bromo-2,3-dimethoxy-5-((1-nitrocyclohexyl)methyl)benzene} phase column chromatography using an eluent of 17\% EtOAc/Hx to provide $\mathbf{S 6}$ as a white solid (530 mg, 65\%).

m.p. $87^{\circ} \mathrm{C}$

${ }^{1} \mathrm{H}$ NMR $\left(400 \mathrm{MHz}, \mathrm{CDCl}_{3}\right) \delta 6.83(\mathrm{~s}, 1 \mathrm{H}), 6.47(\mathrm{~s}, 1 \mathrm{H}), 3.82(\mathrm{~s}, 3 \mathrm{H}), 3.80(\mathrm{~s}, 3 \mathrm{H})$, $2.99(\mathrm{~s}, 2 \mathrm{H}), 2.37$ (d, $J=11.8 \mathrm{~Hz}, 2 \mathrm{H}), 1.70-1.50(\mathrm{~m}, 5 \mathrm{H}), 1.45-1.18(\mathrm{~m}, 3 \mathrm{H})$.

${ }^{13} \mathrm{C}$ NMR $\left(100 \mathrm{MHz}, \mathrm{CDCl}_{3}\right) \delta 153.5,145.8,131.5,126.0,117.4,113.1,91.9,60.7$, 56.2, 46.4, $34.0(2 \mathrm{C}), 24.7,22.3(2 \mathrm{C})$.

IR (FT-ATR, $\mathrm{cm}^{-1}, \mathrm{CH}_{2} \mathrm{Cl}_{2}$ ) v $v_{\max } 2936,2865,1596,1565,1533,1488,1448,1428$, 1415, 1371, 1345, 1292, 1273, 1238, 1184, 1144, 1046, 1001, 947, 925, 913, 860, 816, 777, 734, 654, 611, 578, 536.

HRMS (ESI) $m / z$ : [M+Na] ${ }^{+}$Calcd for $\mathrm{C}_{15} \mathrm{H}_{20} \mathrm{BrNO}_{4} 380.0473$; found 380.0472 .

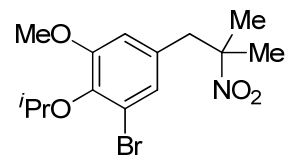

1-Bromo-2-isopropoxy-3-methoxy-5-(2-methyl-2-nitropropyl)benzene (S7) was synthesized by following Procedure 3 . The crude material was purified by normalphase column chromatography using an eluent of $25 \%$ EtOAc/Hx to provide $\mathbf{S 7}$ as a white solid (663 mg, 97\%).

m.p. $35^{\circ} \mathrm{C}$

${ }^{1} \mathbf{H}$ NMR $\left(400 \mathrm{MHz}, \mathrm{CDCl}_{3}\right) \delta 6.89$ (s, 1H), 6.53 (s, 1H), 4.51 (hept, $\left.J=6.1 \mathrm{~Hz}, 1 \mathrm{H}\right)$, $3.78(\mathrm{~s}, 3 \mathrm{H}), 3.10(\mathrm{~s}, 2 \mathrm{H}), 1.58(\mathrm{~s}, 6 \mathrm{H}), 1.31(\mathrm{~d}, J=6.2 \mathrm{~Hz}, 6 \mathrm{H})$.

${ }^{13} \mathrm{C}$ NMR $\left(100 \mathrm{MHz}, \mathrm{CDCl}_{3}\right) \delta 153.8,144.2,131.4,126.5,118.5,113.3,88.6,76.1$, $56.2,46.1,25.8(2 \mathrm{C}), 22.7(2 \mathrm{C})$.

IR (FT-ATR, $\mathrm{cm}^{-1}, \mathrm{CH}_{2} \mathrm{Cl}_{2}$ ) v $v_{\max } 2977,2934,1596,1563,1536,1482,1463,1414$, 1397, 1372, 1347, 1309, 1277, 1234, 1178, 1150, 1103, 1046, 928, 853, 815.

HRMS (ESI) $m / z$ : [M+Na] ${ }^{+}$Calcd for $\mathrm{C}_{14} \mathrm{H}_{20} \mathrm{BrNO}_{4} 368.0473$; found 368.0470 .<smiles>COc1cc(CC(C)(C)[N+](=O)[O-])cc(Br)c1OC</smiles>

1-Bromo-3-methoxy-2-(methoxymethoxy)-5-(2-methyl-2-nitropropyl)benzene (S8) was synthesized by following Procedure 4. The crude product was used without purification process to provide $\mathbf{S 8}$. 


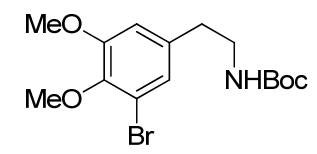

tert-Butyl 3-bromo-4,5-dimethoxyphenethylcarbamate (S9) was synthesized by following Procedure 5. The crude material was purified by normal-phase column chromatography using an eluent of $25 \% \mathrm{EtOAc} / \mathrm{Hx}$ to provide $\mathbf{S 9}$ as a white solid (917 $\mathrm{mg}, 82 \%)$.

m.p. $99^{\circ} \mathrm{C}$

${ }^{1}$ H NMR $\left(400 \mathrm{MHz}, \mathrm{CDCl}_{3}\right) \delta 6.86(\mathrm{~s}, 1 \mathrm{H}), 6.61(\mathrm{~s}, 1 \mathrm{H}), 4.84(\mathrm{~s}, 1 \mathrm{H}), 3.74(\mathrm{~d}, J=10.6$ $\mathrm{Hz}, 6 \mathrm{H}), 3.25(\mathrm{~d}, J=5.7 \mathrm{~Hz}, 2 \mathrm{H}), 2.63(\mathrm{t}, J=6.6 \mathrm{~Hz}, 2 \mathrm{H}), 1.35(\mathrm{~s}, 9 \mathrm{H})$.

${ }^{13} \mathrm{C}$ NMR $\left(100 \mathrm{MHz}, \mathrm{CDCl}_{3}\right) \delta 155.8,153.4,144.7,136.3,124.6,117.3,112.2,79.0$, $60.3,55.9,41.5,35.6,28.3(3 \mathrm{C})$.

IR (FT-ATR, $\mathrm{cm}^{-1}, \mathrm{CH}_{2} \mathrm{Cl}_{2}$ ) $v_{\max } 3382,2975,2935,2829,1691,1597,1566,1513$, $1488,1453,1429,1413,1391,1365,1341,1303,1271,1236,1164,1140,1045,1001$, $968,913,843,818,778,767,735,647,601,577,554,462$.

HRMS (ESI) $m / z$ : $[\mathrm{M}+\mathrm{Na}]^{+}$Calcd for $\mathrm{C}_{15} \mathrm{H}_{22} \mathrm{BrNO}_{4} 382.0630$; found 382.0624 . 

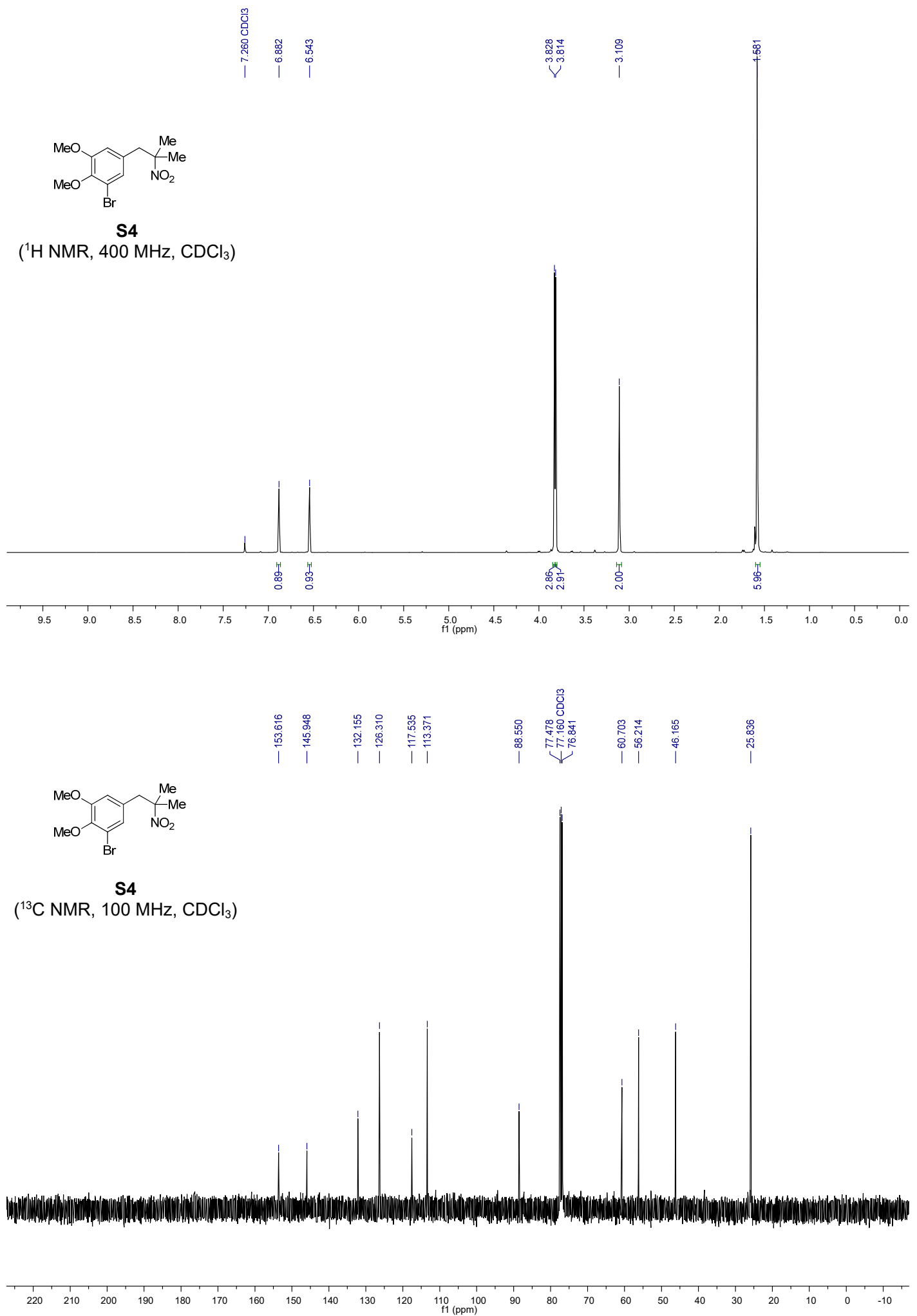

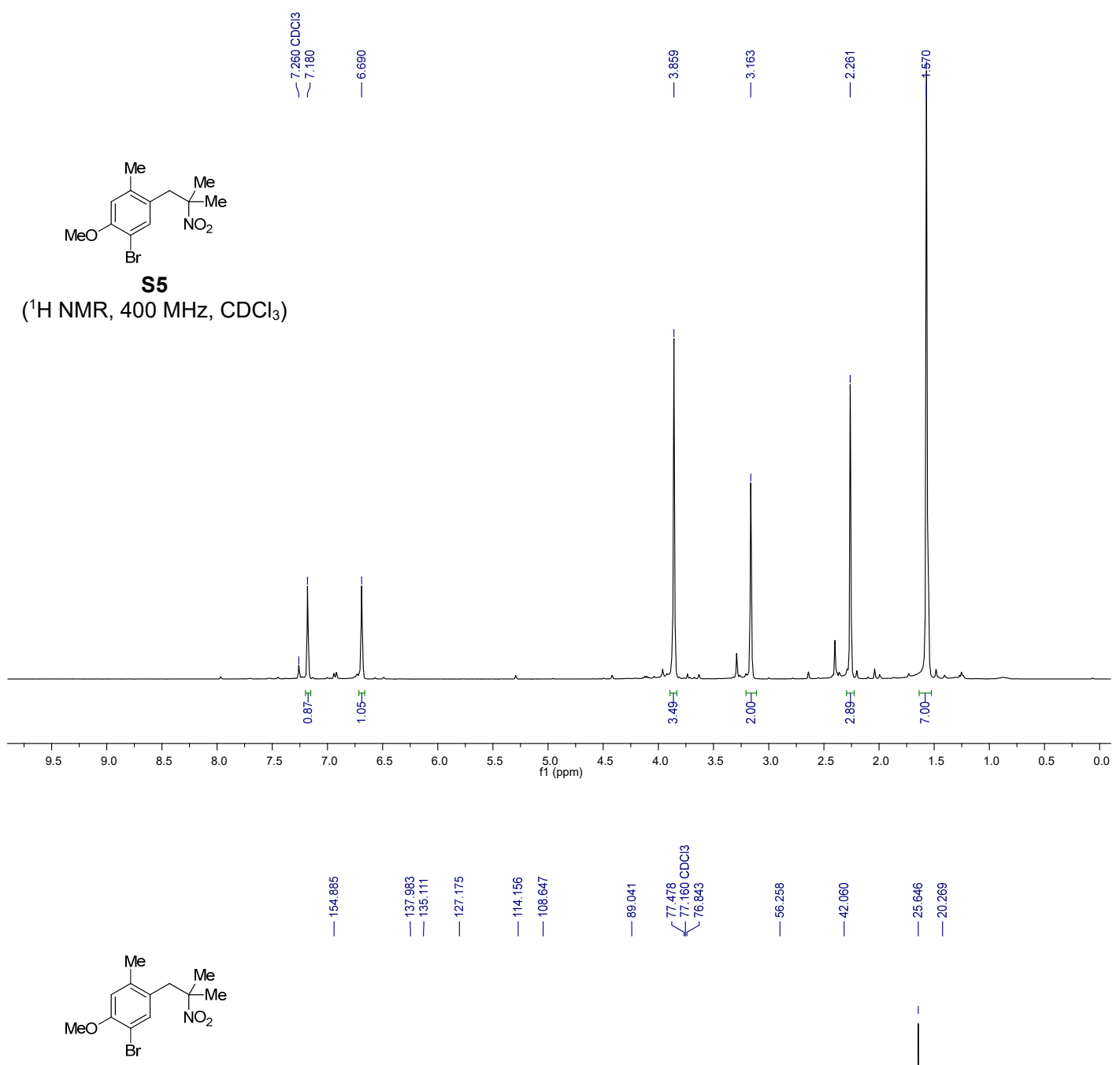

S5

$\left({ }^{13} \mathrm{C} \mathrm{NMR,}, 100 \mathrm{MHz}, \mathrm{CDCl}_{3}\right)$

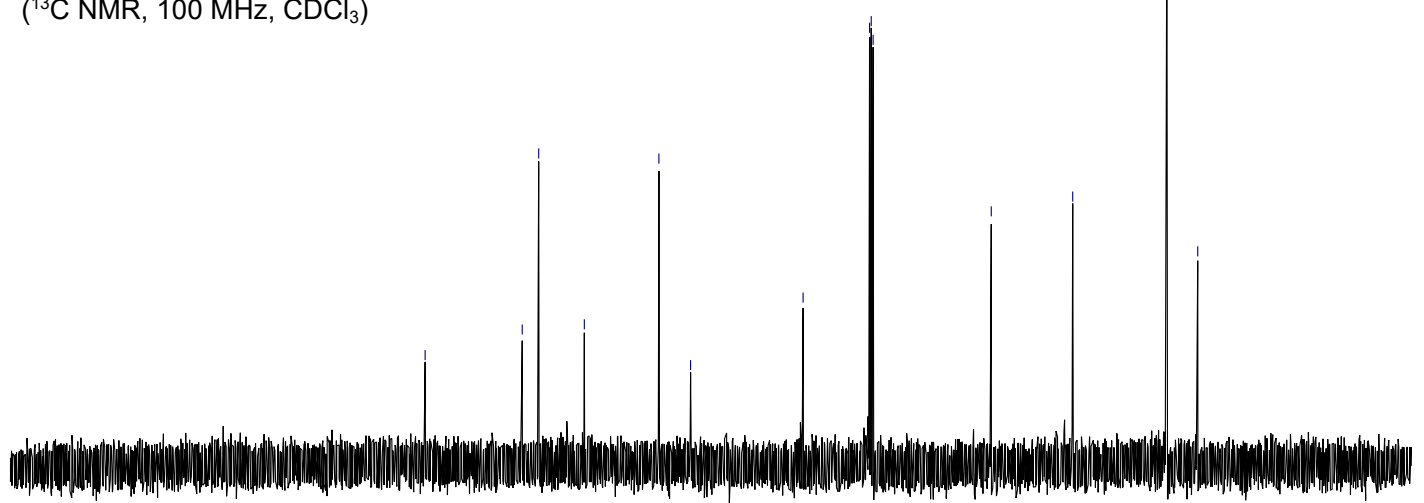

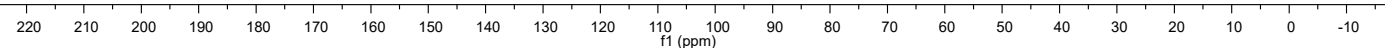




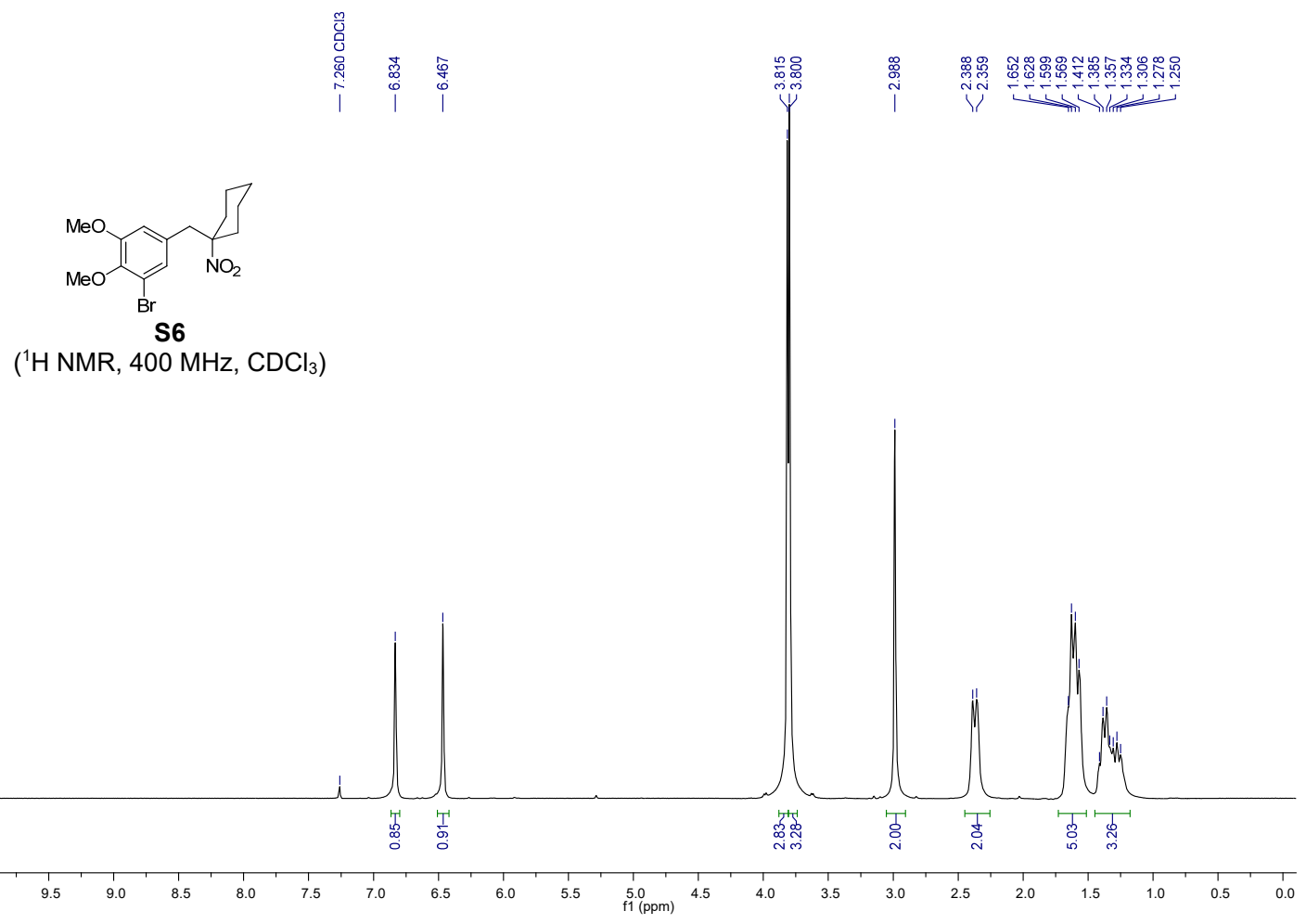

$$
\text { I }
$$

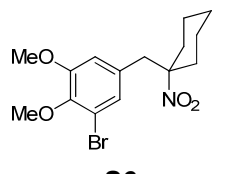

56

$\left({ }^{13} \mathrm{C}\right.$ NMR, $\left.100 \mathrm{MHz}, \mathrm{CDCl}_{3}\right)$

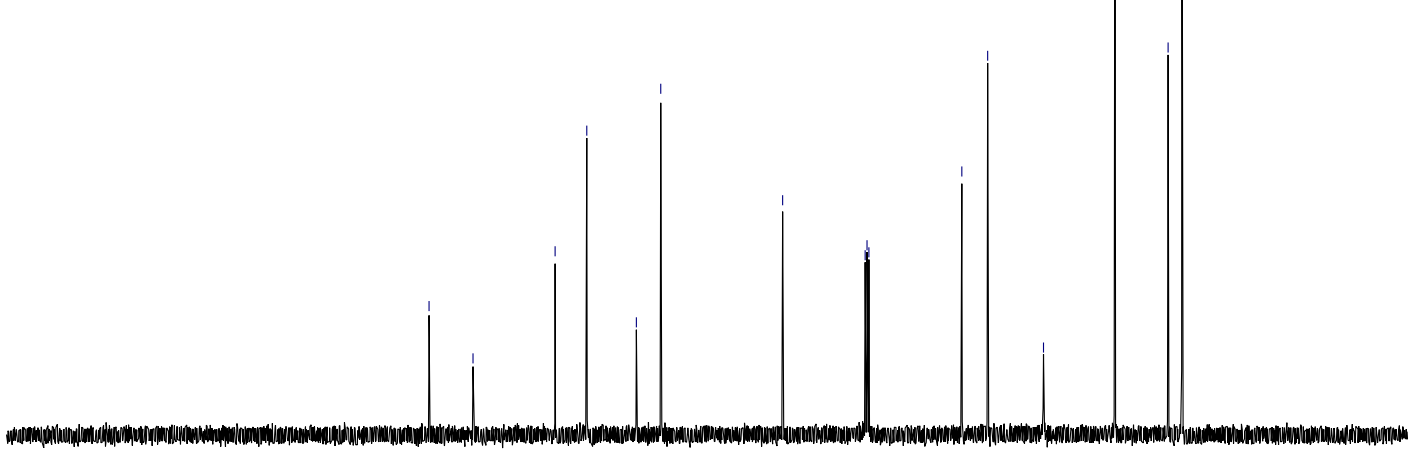

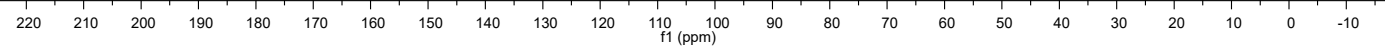




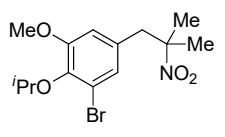

S7

( ${ }^{1} \mathrm{H}$ NMR, $400 \mathrm{MHz}, \mathrm{CDCl}_{3}$ )

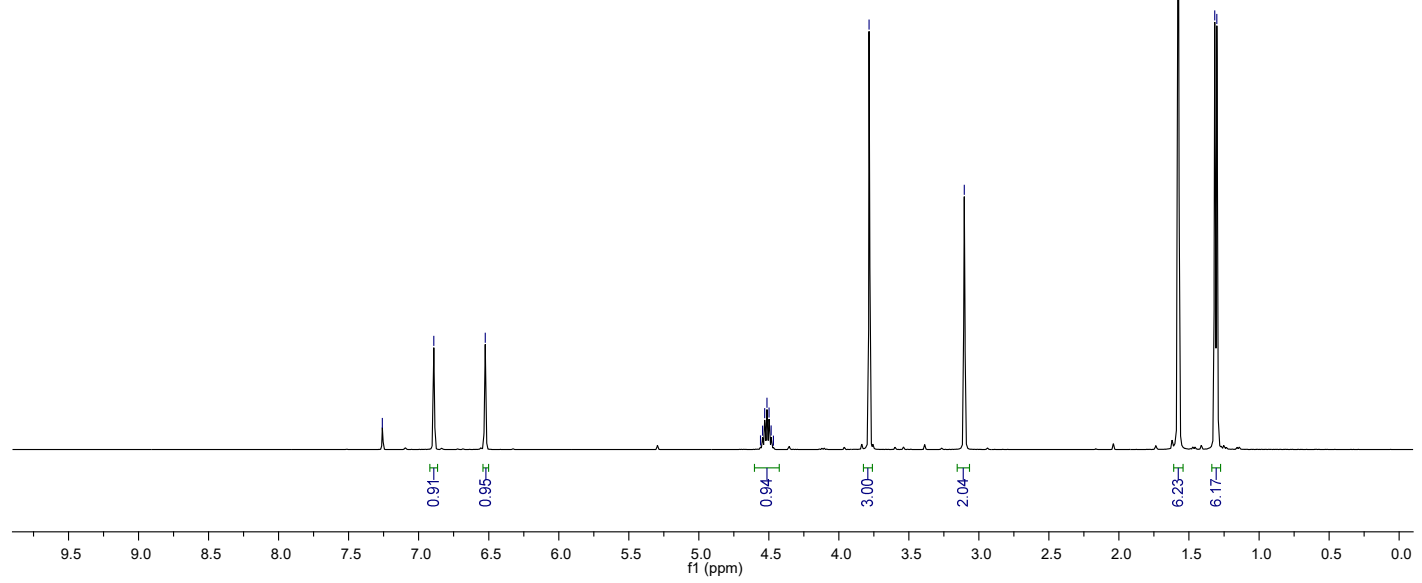<smiles>COc1cc(CC(C)(C)O)cc(Br)c1O</smiles>

S7

$\left({ }^{13} \mathrm{C}\right.$ NMR, $\left.100 \mathrm{MHz}, \mathrm{CDCl}_{3}\right)$

I

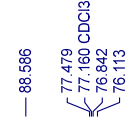

1
$\substack{0 \\ 0}$
0
0
0

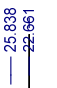

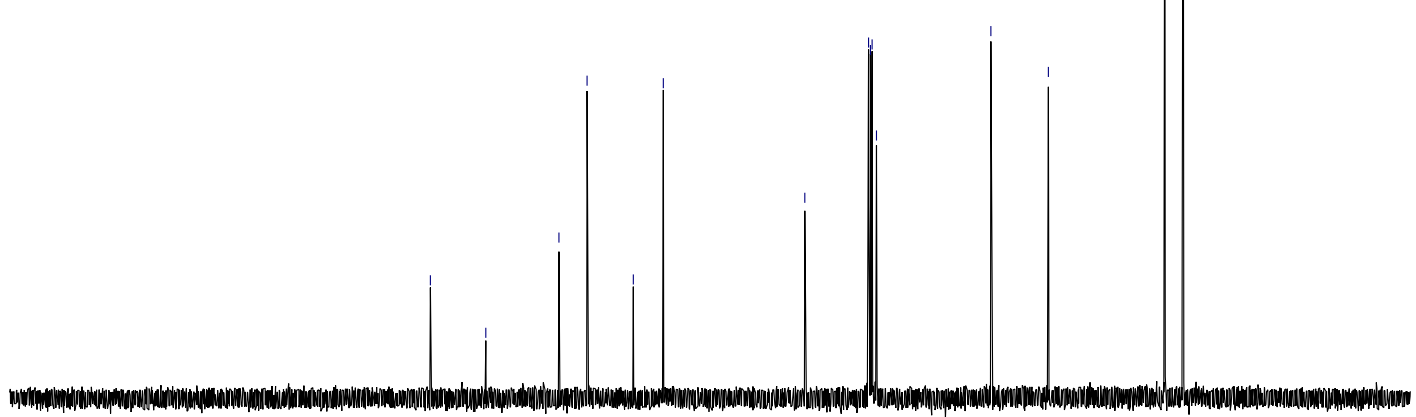

$\begin{array}{llllllllllllllllllllllllllll}1 & 1 \\ 220 & 210 & 200 & 190 & 180 & 170 & 160 & 150 & 140 & 130 & 120 & 110 & 100 & 90 & 80 & 70 & 60 & 50 & 40 & 30 & 20 & 10 & 0 & -10\end{array}$ 


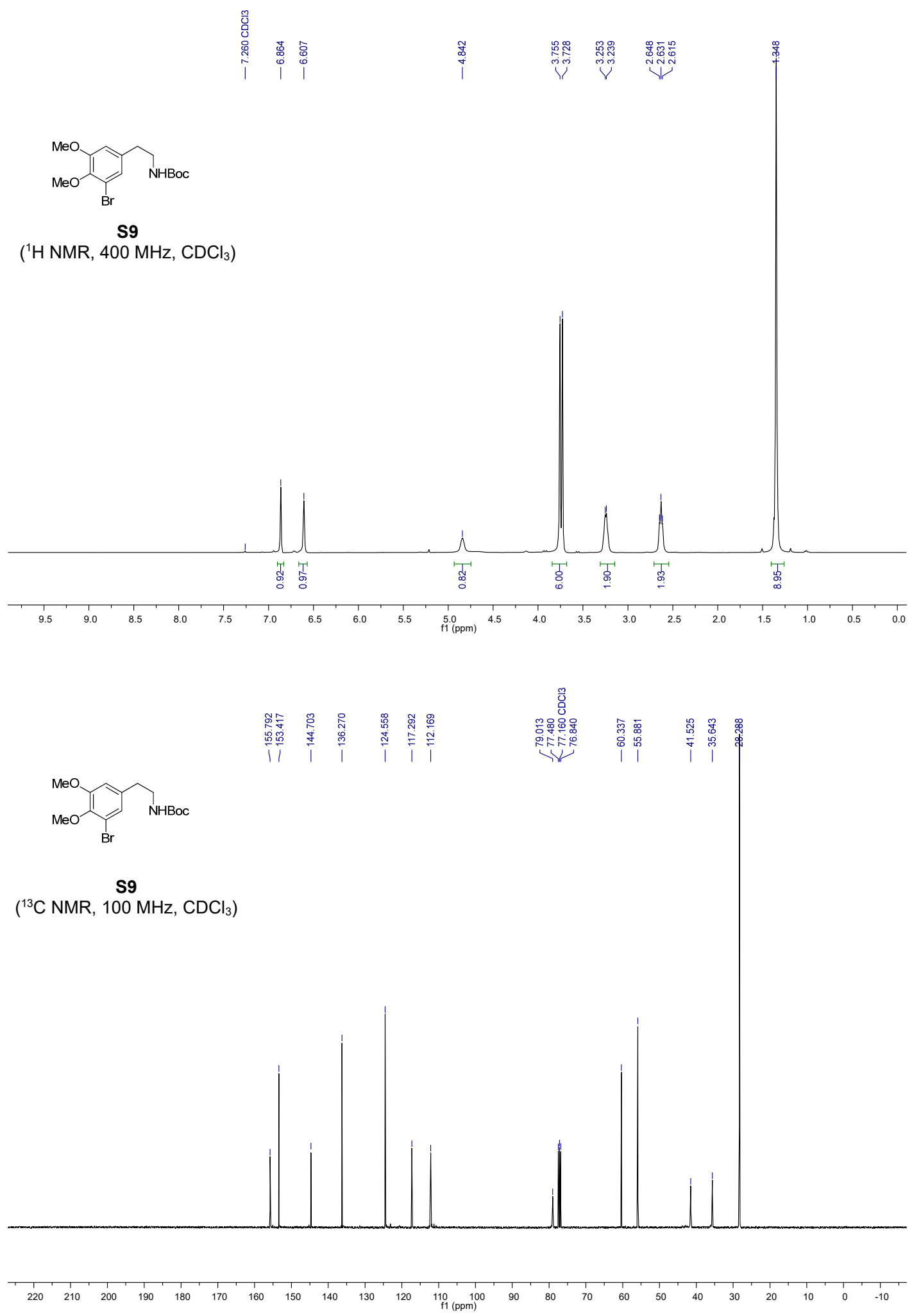




\subsection{Synthesis of Substituted Biaryl Intermediates (S10-49)}

\subsubsection{Pd-Catalyzed Cross-Coupling for Substituted Biaryl Intermediates (S10-26)}
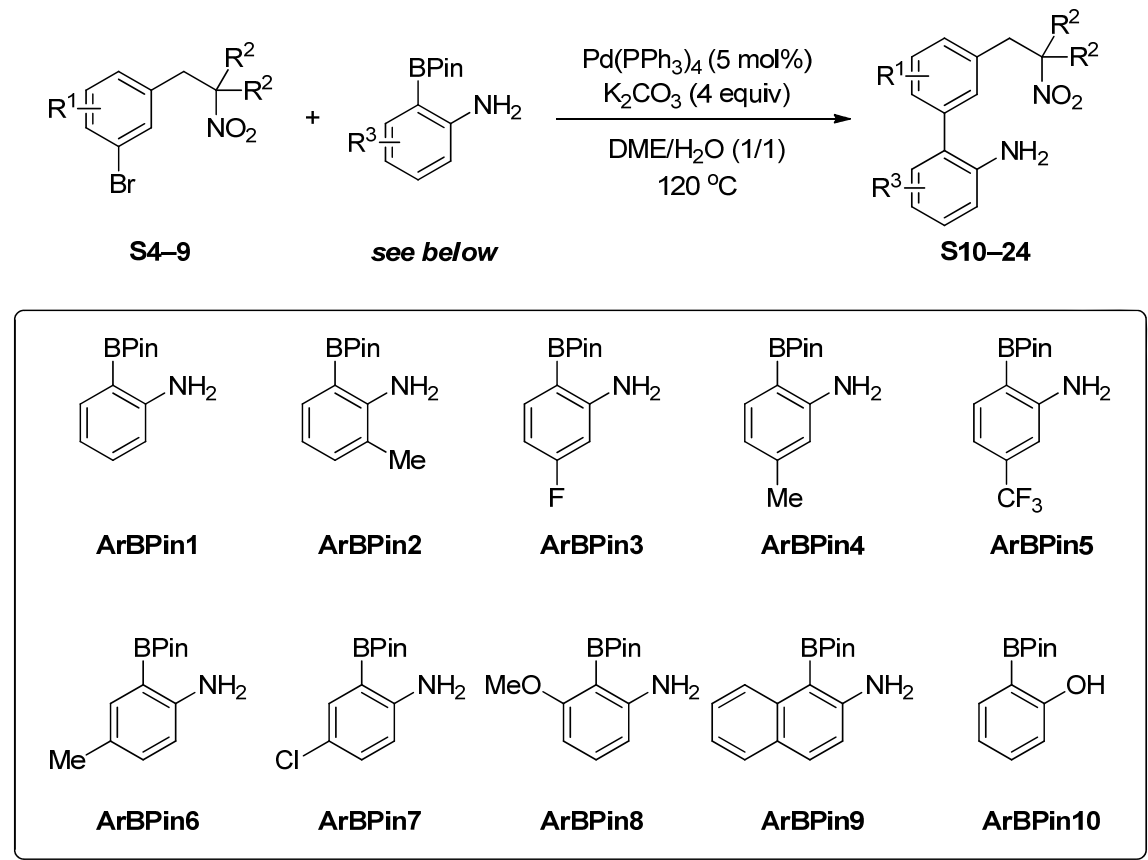

Procedure 6: An oven-dried round-bottom flask equipped with a magnetic stir bar, a solution of S4-9 (1.0 equiv) and arylboronic pinacol ester (ArBPin1-7, ${ }^{3}$ ArBPin8, ${ }^{4}$ or ArBPin9 ${ }^{5}$, ArBPin10 $\left.^{6}\right)$ (1.0 equiv) in 1,2-dimethoxyethane mixed with water $(\mathrm{DME} /$ water $=1 / 1,0.10 \mathrm{M})$ was added. Then, $\mathrm{Pd}\left(\mathrm{PPh}_{3}\right)_{4}(5 \mathrm{~mol} \%)$ and $\mathrm{K}_{2} \mathrm{CO}_{3}(4.0$ equiv) were added to flask. The reaction mixture was heated for overnight at $120^{\circ} \mathrm{C}$ in a heating block. The progress of the reaction was monitored by TLC. The reaction mixture was cooled to rt, diluted with $\mathrm{CH}_{2} \mathrm{Cl}_{2}$ and washed with brine. The organic layer was extracted with $\mathrm{CH}_{2} \mathrm{Cl}_{2}$ three times, dried with anhydrous $\mathrm{MgSO}_{4}$, filtered, and concentrated in vacuo. The crude material was then purified by flash chromatography to afford the desired material S10-24.<smiles>[R]c1ccccc1Br</smiles>

S4

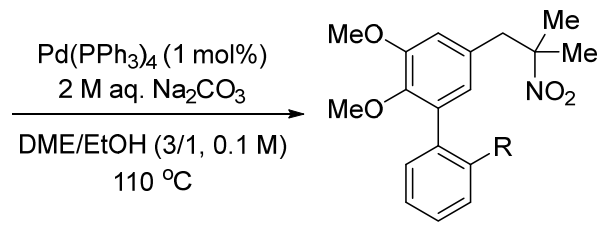

S25-26
S25: $R=M e$ S26: $\mathrm{R}=\mathrm{Ph}$

Procedure 7: An oven-dried round-bottom flask equipped with a magnetic stir bar, a solution of S4 (1.0 equiv) in 1,2-dimethoxyethane was added. Then, $\mathrm{Pd}\left(\mathrm{PPh}_{3}\right)_{4}(1 \mathrm{~mol} \%)$ was added to flask and solution of arylboronic acid (2-methylphenylboronic acid or 2phenylphenylboronic acid, 1.5 equiv) in EtOH was added. The reaction mixture was heated $4 \mathrm{~h}$ at $100^{\circ} \mathrm{C}$ in an oil bath after addition of $2.0 \mathrm{M}$ aqueous $\mathrm{Na}_{2} \mathrm{CO}_{3}$. The progress of the reaction was monitored by TLC. The reaction mixture was cooled to rt, diluted with $\mathrm{CH}_{2} \mathrm{Cl}_{2}$ and washed with brine. The organic layer was extracted with $\mathrm{CH}_{2} \mathrm{Cl}_{2}$ three times, dried with anhydrous $\mathrm{MgSO}_{4}$, filtered, and concentrated in vacuo. The crude material was then purified by flash chromatography to afford the desired material $\mathbf{S 2 5}$ and S26. 


\section{Characterization and Spectra of Biaryl Intermediates (S10-26)}

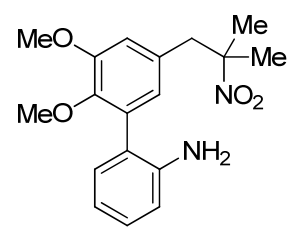

2',3'-Dimethoxy-5'-(2-methyl-2-nitropropyl)-[1,1'-biphenyl]-2-amine (S10) was synthesized by following Procedure 6 . The crude material was purified by normalphase column chromatography using an eluent of $25 \%$ EtOAc/Hx to provide $\mathbf{S 1 0}$ as a brown solid (332 mg, 95\%).

m.p. $116^{\circ} \mathrm{C}$

${ }^{1} \mathbf{H}$ NMR $\left(400 \mathrm{MHz}, \mathrm{CDCl}_{3}\right) \delta 7.17(\mathrm{t}, J=7.6 \mathrm{~Hz}, 1 \mathrm{H}), 7.09(\mathrm{~d}, J=7.5 \mathrm{~Hz}, 1 \mathrm{H}), 6.81$ $(\mathrm{t}, J=7.4 \mathrm{~Hz}, 1 \mathrm{H}), 6.76(\mathrm{~d}, J=8.0 \mathrm{~Hz}, 1 \mathrm{H}), 6.64(\mathrm{~s}, 1 \mathrm{H}), 6.62(\mathrm{~s}, 1 \mathrm{H}), 3.87(\mathrm{~s}, 3 \mathrm{H})$, 3.79 (brs, 2H), 3.57 (s, 3H), $3.16(\mathrm{~s}, 2 \mathrm{H}), 1.61(\mathrm{~s}, 6 \mathrm{H})$.

${ }^{13} \mathrm{C} \mathrm{NMR}\left(100 \mathrm{MHz}, \mathrm{CDCl}_{3}\right) \delta 152.8,146.0,144.4,133.5,131.2,130.9,128.8,125.3$, $124.3,118.4,116.0,113.2,88.8,61.1,56.1,46.7,25.85$ (2C).

IR (FT-ATR, $\mathrm{cm}^{-1}, \mathrm{CH}_{2} \mathrm{Cl}_{2}$ ) v $v_{\max } 3464,3376,2936,2836,1617,1583,1533,1497$, 1480, 1451, 1421, 1396, 1372, 1343, 1300, 1260, 1230, 1184, 1169, 1139, 1048, 1031, $1005,964,854,788,750,694,670,635,585,533$.

HRMS (ESI) $m / z$ : $[\mathrm{M}+\mathrm{H}]^{+}$Calcd for $\mathrm{C}_{18} \mathrm{H}_{22} \mathrm{~N}_{2} \mathrm{O}_{4} 331.1658$; found 331.1656.

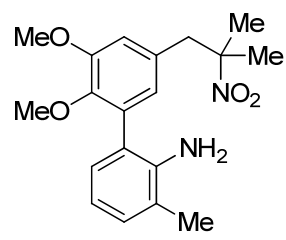

2',3'-Dimethoxy-3-methyl-5'-(2-methyl-2-nitropropyl)-[1,1'-biphenyl]-2-amine

(S11) was synthesized by following Procedure 6. The crude material was purified by normal-phase column chromatography using an eluent of $25 \% \mathrm{EtOAc} / \mathrm{Hx}$ to provide S11 as a yellow solid (426 mg, 98\%).

m.p. $98{ }^{\circ} \mathrm{C}$

${ }^{1}$ H NMR $\left(400 \mathrm{MHz}, \mathrm{CDCl}_{3}\right) \delta 7.08(\mathrm{~d}, J=7.3 \mathrm{~Hz}, 1 \mathrm{H}), 6.96(\mathrm{~d}, J=7.5 \mathrm{~Hz}, 1 \mathrm{H}), 6.74$ $(\mathrm{t}, J=7.5 \mathrm{~Hz}, 1 \mathrm{H}), 6.63(\mathrm{~s}, 1 \mathrm{H}), 6.61(\mathrm{~s}, 1 \mathrm{H}), 3.87(\mathrm{~s}, 3 \mathrm{H}), 3.68(\mathrm{~s}, 2 \mathrm{H}), 3.57(\mathrm{~s}, 3 \mathrm{H})$, $3.16(\mathrm{~d}, J=2.6 \mathrm{~Hz}, 2 \mathrm{H}), 2.23(\mathrm{~s}, 3 \mathrm{H}), 1.61(\mathrm{~s}, 6 \mathrm{H})$.

${ }^{13}$ C NMR $\left(100 \mathrm{MHz}, \mathrm{CDCl}_{3}\right) \delta 152.9,146.1,142.5,133.7,131.1,129.9,128.6,125.3$, $123.8,122.5,117.8,113.1,88.7,61.1,56.0,46.7,26.0,25.7,18.1$.

IR (FT-ATR, $\mathrm{cm}^{-1}, \mathrm{CH}_{2} \mathrm{Cl}_{2}$ ) $v_{\max } 3470,3385,2935,1671,1581,1535,1487,1461$, 1420, 1397, 1372, 1345, 1261, 1232, 1184, 1146, 1082, 1049, 1007, 855, 768, 745, 694, $576,520$.

HRMS (ESI) $m / z$ : [M+H] $]^{+}$Calcd for $\mathrm{C}_{19} \mathrm{H}_{24} \mathrm{~N}_{2} \mathrm{O}_{4} 345.1814$; found 345.1808 . 


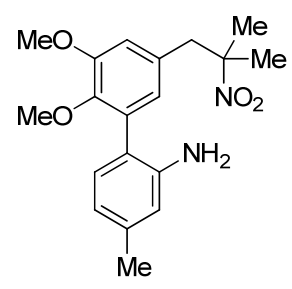

2',3'-Dimethoxy-4-methyl-5'-(2-methyl-2-nitropropyl)-[1,1'-biphenyl]-2-amine (S12) was synthesized by following Procedure 6. The crude material was purified by normal-phase column chromatography using an eluent of $25 \% \mathrm{EtOAc} / \mathrm{Hx}$ to provide S12 as a brown solid (616 mg, 79\%).

m.p. $99{ }^{\circ} \mathrm{C}$

${ }^{1} \mathbf{H}$ NMR $\left(400 \mathrm{MHz}, \mathrm{CDCl}_{3}\right) \delta 6.99(\mathrm{~d}, J=7.5 \mathrm{~Hz}, 1 \mathrm{H}), 6.70-6.56(\mathrm{~m}, 4 \mathrm{H}), 3.87(\mathrm{~s}$, $3 \mathrm{H}), 3.77$ (brs, 2H), $3.58(\mathrm{~s}, 3 \mathrm{H}), 3.16(\mathrm{~s}, 2 \mathrm{H}), 2.31(\mathrm{~s}, 3 \mathrm{H}), 1.61(\mathrm{~s}, 6 \mathrm{H})$.

${ }^{13}$ C NMR $\left(100 \mathrm{MHz}, \mathrm{CDCl}_{3}\right) \delta 152.8,146.0,144.3,138.7,133.5,131.2,130.8,125.5$, 121.6, 119.4, 116.8, 113.0, 88.8, 61.1, 56.0, 46.8, 25.9 (2C), 21.4.

IR (FT-ATR, $\mathrm{cm}^{-1}, \mathrm{CH}_{2} \mathrm{Cl}_{2}$ ) $v_{\max } 3449,3371,2997,2935,1621,1583,1535,1513$, 1481, 1462, 1418, 1397, 1372, 1344, 1299, 1261, 1231, 1184, 1162, 1137, 1076, 1042, 1007, 948, 855, 804, 732, 694, 662, 622, 598, 545, 521, 452.

HRMS (ESI) $m / z$ : $[\mathrm{M}+\mathrm{H}]^{+}$Calcd for $\mathrm{C}_{19} \mathrm{H}_{24} \mathrm{~N}_{2} \mathrm{O}_{4}$ 345.1814; found 345.1809.

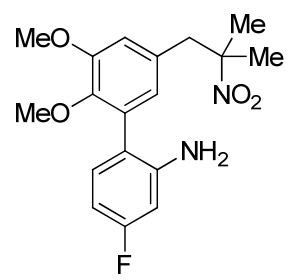

4-Fluoro-2',3'-dimethoxy-5'-(2-methyl-2-nitropropyl)-[1,1'-biphenyl]-2-amine

(S13) was synthesized by following Procedure 6. The crude material was purified by normal-phase column chromatography using an eluent of $25 \%$ to $33 \% \mathrm{EtOAc} / \mathrm{Hx}$ to provide $\mathbf{S 1 3}$ as a yellow solid (517 $\mathrm{mg}, 74 \%$ ).

m.p. $135^{\circ} \mathrm{C}$

${ }^{1} \mathbf{H}$ NMR $\left(400 \mathrm{MHz}, \mathrm{CDCl}_{3}\right) \delta 7.47(\mathrm{dd}, J=12.1,6.2 \mathrm{~Hz}, 1 \mathrm{H}), 7.35-7.21(\mathrm{~m}, 1 \mathrm{H}), 7.01$ (t, $J=7.4 \mathrm{~Hz}, 1 \mathrm{H}), 6.64(\mathrm{~s}, 1 \mathrm{H}), 6.56(\mathrm{~s}, 1 \mathrm{H}), 6.51-6.40$ (m, 2H), 3.89 (brs, 2H), 3.86 (s, 3H), $3.56(\mathrm{~s}, 3 \mathrm{H}), 3.15(\mathrm{~s}, 2 \mathrm{H}), 1.60(\mathrm{~s}, 6 \mathrm{H})$.

${ }^{13}$ C NMR $\left(100 \mathrm{MHz}, \mathrm{CDCl}_{3}\right) \delta 163.5(\mathrm{~d}, J=242.7 \mathrm{~Hz}, 1 \mathrm{C}), 152.9,146.2,146.1(\mathrm{~d}, J$ $=6.1 \mathrm{~Hz}, 1 \mathrm{C}), 132.7,132.1(\mathrm{~d}, J=9.8 \mathrm{~Hz}, 1 \mathrm{C}), 131.4,125.3,120.1(\mathrm{~d}, J=2.6 \mathrm{~Hz}, 1 \mathrm{C})$, 113.4, 105.1 (d, $J=21.4 \mathrm{~Hz}, 1 \mathrm{C}), 102.5$ (d, $J=24.4 \mathrm{~Hz}, 1 \mathrm{C}), 88.8,61.1,56.1,46.8$, $25.9(2 \mathrm{C})$.

IR (FT-ATR, $\mathrm{cm}^{-1}, \mathrm{CH}_{2} \mathrm{Cl}_{2}$ ) $v_{\max } 3477,3380,2936,1620,2584,1535,1508,1483$, $1465,1420,1397,1381,1345,1284,1263,1232,1171,1138,1067,1042$, 1005, 976, 854, 769, 740, 663, 626, 591, 549, 461.

HRMS (ESI) $m / z$ : $[\mathrm{M}+\mathrm{H}]^{+}$Calcd for $\mathrm{C}_{18} \mathrm{H}_{21} \mathrm{FN}_{2} \mathrm{O}_{4} 349.1564$; found 349.1558 . 


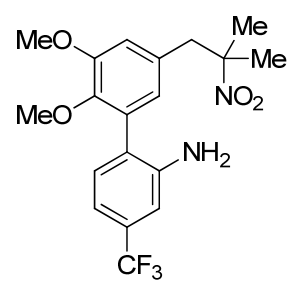

2',3'-Dimethoxy-5'-(2-methyl-2-nitropropyl)-4-(trifluoromethyl)-[1,1'-biphenyl]2-amine (S14) was synthesized by following Procedure 6. The crude material was purified by normal-phase column chromatography using an eluent of $25 \% \mathrm{EtOAc} / \mathrm{Hx}$ to provide $\mathbf{S 1 4}$ as a white solid (781 $\mathrm{mg}, 83 \%$ ).

m.p. $111^{\circ} \mathrm{C}$

${ }^{1} \mathbf{H}$ NMR $\left(400 \mathrm{MHz}, \mathrm{CDCl}_{3}\right) \delta 7.17(\mathrm{~d}, J=7.8 \mathrm{~Hz}, 1 \mathrm{H}), 7.03(\mathrm{~d}, J=7.7 \mathrm{~Hz}, 1 \mathrm{H}), 6.97$ (s, 1H), 6.67 (s, 1H), 6.57 (s, 1H), 3.95 (brs, 2H), 3.88 (s, 3H), 3.59 (s, 3H), 3.17 (s, $2 \mathrm{H}), 1.62(\mathrm{~s}, 6 \mathrm{H})$.

${ }^{13}$ C NMR $\left(100 \mathrm{MHz}, \mathrm{CDCl}_{3}\right) \delta 153.0,146.0,144.9,132.3,131.6,131.3,130.9$ (q, $J=$ $32.0 \mathrm{~Hz}, 1 \mathrm{C}), 127.3,124.7,124.3$ (q, $J=271.0 \mathrm{~Hz}, 1 \mathrm{C}), 114.7(\mathrm{q}, J=3.9 \mathrm{~Hz}, 1 \mathrm{C})$, $113.8,112.3$ (q, $J=3.8 \mathrm{~Hz}, 1 \mathrm{C}), 88.7,61.3,56.1,46.7,25.9$ (2C).

IR (FT-ATR, $\mathrm{cm}^{-1}, \mathrm{CH}_{2} \mathrm{Cl}_{2}$ ) $v_{\max } 3476,3380,2936,1625,1585,1536,1483,1466$, 1436, 1421, 1397, 1373, 1333, 1253, 1163, 1116, 1089, 1057, 1005, 926, 856, 818, 788, 746, 733, 670, 650, 590, 545, 522.

HRMS (ESI) $m / z$ : $[\mathrm{M}+\mathrm{H}]^{+}$Calcd for $\mathrm{C}_{19} \mathrm{H}_{21} \mathrm{~F}_{3} \mathrm{~N}_{2} \mathrm{O}_{4}$ 399.1532; found 399.1526.

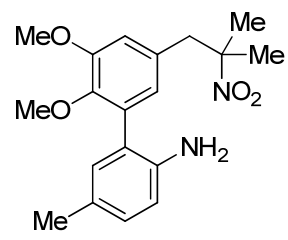

2',3'-Dimethoxy-5-methyl-5'-(2-methyl-2-nitropropyl)-[1,1'-biphenyl]-2-amine

(S15) was synthesized by following Procedure 6. The crude material was purified by normal-phase column chromatography using an eluent of $25 \% \mathrm{EtOAc} / \mathrm{Hx}$ to provide S15 as a yellow gum (540 mg, 78\%).

${ }^{1} \mathbf{H}$ NMR $\left(400 \mathrm{MHz}, \mathrm{CDCl}_{3}\right) \delta 6.98(\mathrm{~d}, J=8.0 \mathrm{~Hz}, 1 \mathrm{H}), 6.90(\mathrm{~s}, 1 \mathrm{H}), 6.68(\mathrm{~d}, J=8.0$ $\mathrm{Hz}, 1 \mathrm{H}), 6.62(\mathrm{~d}, J=5.0 \mathrm{~Hz}, 2 \mathrm{H}), 3.86(\mathrm{~s}, 3 \mathrm{H}), 3.69$ (brs, 2H), 3.57 (s, 3H), 3.16 (s, $2 \mathrm{H}), 2.27(\mathrm{~s}, 3 \mathrm{H}), 1.61(\mathrm{~s}, 6 \mathrm{H})$.

${ }^{13} \mathbf{C}$ NMR $\left(100 \mathrm{MHz}, \mathrm{CDCl}_{3}\right) \delta 152.7,145.9,141.9,133.6,131.3,131.1,129.4,127.5$, 125.3, 124.5, 116.2, 113.0, 88.7, 61.1, 56.0, 46.7, 25.8 (2C), 20.6.

IR (FT-ATR, $\mathrm{cm}^{-1}, \mathrm{CH}_{2} \mathrm{Cl}_{2}$ ) $v_{\max } 3446,3370,2935,1738,1623,1582,1534,1505$, 1481, 1463, 1423, 1397, 1372, 1343, 1289, 1262, 1231, 1184, 1151, 1132, 1046, 1005, $928,854,812,790,770,733,695,677,643,584,544,520,467$.

HRMS (ESI) $m / z$ : $[\mathrm{M}+\mathrm{H}]^{+}$Calcd for $\mathrm{C}_{19} \mathrm{H}_{24} \mathrm{~N}_{2} \mathrm{O}_{4}$ 345.1814; found 345.1809. 


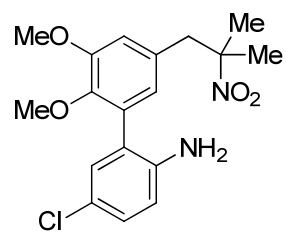

5-Chloro-2',3'-dimethoxy-5'-(2-methyl-2-nitropropyl)-[1,1'-biphenyl]-2-amine

(S16) was synthesized by following Procedure 6. The crude material was purified by normal-phase column chromatography using an eluent of 33\% EtOAc/Hx to provide S16 as a yellow gum (609 mg, 97\%).

${ }^{1} \mathbf{H}$ NMR $\left(400 \mathrm{MHz}, \mathrm{CDCl}_{3}\right) \delta 7.12(\mathrm{dd}, J=8.5,2.4 \mathrm{~Hz}, 1 \mathrm{H}), 7.06(\mathrm{~d}, J=2.4 \mathrm{~Hz}, 1 \mathrm{H})$, $6.68(\mathrm{~d}, J=8.5 \mathrm{~Hz}, 1 \mathrm{H}), 6.64(\mathrm{~d}, J=1.9 \mathrm{~Hz}, 1 \mathrm{H}), 6.58(\mathrm{~d}, J=1.9 \mathrm{~Hz}, 1 \mathrm{H}), 3.87(\mathrm{~s}, 3 \mathrm{H})$, $3.79(\mathrm{~s}, 2 \mathrm{H}), 3.59(\mathrm{~s}, 3 \mathrm{H}), 3.16(\mathrm{~s}, 2 \mathrm{H}), 1.61(\mathrm{~s}, 6 \mathrm{H})$.

${ }^{13}$ C NMR $\left(100 \mathrm{MHz}, \mathrm{CDCl}_{3}\right) \delta 152.9,145.9,143.2,132.3,131.5,130.3,128.5,125.7$, $124.9,122.8,117.1,113.6,88.7,61.2,56.0,46.7,25.7$ (2C).

IR (FT-ATR, $\mathrm{cm}^{-1}, \mathrm{CH}_{2} \mathrm{Cl}_{2}$ ) $v_{\max } 3472,3376,2936,2837,1619,1583,1534,1493$, $1481,1465,1423,1399,1372,1341,1283,1252,1233,1185,1171,1139,1094,1044$, $1004,878,855,814,788,735,693,657,640,603,583,541,521$.

HRMS (ESI) $m / z$ : [M+H] $]^{+}$Calcd for $\mathrm{C}_{18} \mathrm{H}_{21} \mathrm{ClN}_{2} \mathrm{O}_{4} 365.1268$; found 365.1263 .

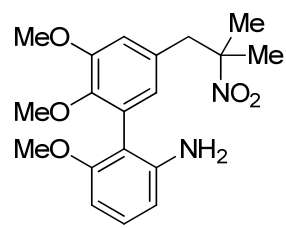

2',3',6-Trimethoxy-5'-(2-methyl-2-nitropropyl)-[1,1'-biphenyl]-2-amine (S17) was synthesized by following Procedure 6 . The crude material was purified by normalphase column chromatography using an eluent of 25\% EtOAc/Hx to provide $\mathbf{S 1 7}$ as a brown gum (205 mg, 19\%).

${ }^{1} \mathbf{H}$ NMR $\left(400 \mathrm{MHz}, \mathrm{CDCl}_{3}\right) \delta 7.12(\mathrm{t}, J=8.0 \mathrm{~Hz}, 1 \mathrm{H}), 6.63(\mathrm{~s}, 1 \mathrm{H}), 6.55(\mathrm{~s}, 1 \mathrm{H}), 6.41$ $(\mathrm{t}, J=7.8 \mathrm{~Hz}, 2 \mathrm{H}), 3.86(\mathrm{~s}, 3 \mathrm{H}), 3.69(\mathrm{~s}, 3 \mathrm{H}), 3.60(\mathrm{~s}, 3 \mathrm{H}), 3.55(\mathrm{brs}, 2 \mathrm{H}), 3.15(\mathrm{t}, J=$ $16.7 \mathrm{~Hz}, 2 \mathrm{H}), 1.60(\mathrm{~s}, 6 \mathrm{H})$.

${ }^{13}$ C NMR $\left(100 \mathrm{MHz}, \mathrm{CDCl}_{3}\right) \delta 157.9,152.9,146.8,145.6,130.8,129.1,129.0,125.8$, 113.3, 112.7, 108.8, 101.0, 88.9, 77.5, 76.8, 60.8, 55.9, 55.7, 46.8, 26.0, 25.7.

IR (FT-ATR, $\mathrm{cm}^{-1}, \mathrm{CH}_{2} \mathrm{Cl}_{2}$ ) $v_{\max } 3471,3376,2995,2937,2836,1614,1584,1534$, 1496, 1466, 1422, 1396, 1372, 1342, 1320, 1287, 1257, 1232, 1184, 1134, 1090, 1040, 1006, 944, 855, 781, 730, 696, 663, 603, 574, 528.

HRMS (ESI) $m / z$ : $[\mathrm{M}+\mathrm{H}]^{+}$Calcd for $\mathrm{C}_{19} \mathrm{H}_{24} \mathrm{~N}_{2} \mathrm{O}_{5}$ 361.1764; found 361.1760 . 


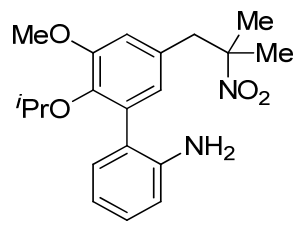

2'-Isopropoxy-3'-methoxy-5'-(2-methyl-2-nitropropyl)-[1,1'-biphenyl]-2-amine (S18) was synthesized by following Procedure 6. The crude material was purified by normal-phase column chromatography using an eluent of $25 \% \mathrm{EtOAc} / \mathrm{Hx}$ to provide $\mathbf{S 1 8}$ as a yellow gum (170 mg, 95\%).

${ }^{1} \mathbf{H}$ NMR $\left(400 \mathrm{MHz}, \mathrm{CDCl}_{3}\right) \delta 7.15(\mathrm{t}, J=7.6 \mathrm{~Hz}, 1 \mathrm{H}), 7.10(\mathrm{~d}, J=7.0 \mathrm{~Hz}, 1 \mathrm{H}), 6.80$ $(\mathrm{t}, J=7.3 \mathrm{~Hz}, 1 \mathrm{H}), 6.74(\mathrm{~d}, J=7.8 \mathrm{~Hz}, 1 \mathrm{H}), 6.63(\mathrm{~s}, 1 \mathrm{H}), 6.62(\mathrm{~s}, 1 \mathrm{H}), 4.02-3.93(\mathrm{~m}$, 1H), 3.91 (brs, 2H), 3.84 (s, 3H), 3.17 (s, 2H), 1.61 (s, 6H), 1.07 (d, $J=4.1 \mathrm{~Hz}, 3 \mathrm{H})$, $0.92(\mathrm{~d}, J=4.0 \mathrm{~Hz}, 3 \mathrm{H})$.

${ }^{13} \mathrm{C} \mathrm{NMR}\left(100 \mathrm{MHz}, \mathrm{CDCl}_{3}\right) \delta 153.3,144.6,143.9,134.7,131.3,131.1,128.6,125.4$, $125.4,118.5,116.2,112.8,88.8,76.5,56.0,46.8,26.0,25.8$ (2C), 22.4 (2C).

IR (FT-ATR, $\mathrm{cm}^{-1}, \mathrm{CH}_{2} \mathrm{Cl}_{2}$ ) v $v_{\max } 3444,3369,2975,2933,1618,1582,1536,1497$, 1447, 1422, 1396, 1371, 1343, 1301, 1259, 1225, 1173, 1142, 1104, 1049, 1033, 930, $853,801,749,671,625,586,531,485$.

HRMS (ESI) $m / z$ : [M+H] $]^{+}$Calcd for $\mathrm{C}_{20} \mathrm{H}_{26} \mathrm{~N}_{2} \mathrm{O}_{4}$ 359.1971; found 359.1965.

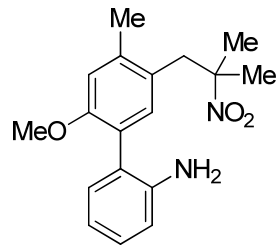

2'-Methoxy-4'-methyl-5'-(2-methyl-2-nitropropyl)-[1,1'-biphenyl]-2-amine (S19) was synthesized by following Procedure 6 . The crude material was purified by normalphase column chromatography using an eluent of $25 \% \mathrm{EtOAc} / \mathrm{Hx}$ to provide $\mathbf{S 1 9}$ as a yellow solid (332 mg, 86\%).

m.p. $55^{\circ} \mathrm{C}$

${ }^{1} \mathbf{H}$ NMR $\left(400 \mathrm{MHz}, \mathrm{CDCl}_{3}\right) \delta 7.16(\mathrm{t}, J=7.3 \mathrm{~Hz}, 1 \mathrm{H}), 7.06(\mathrm{~d}, J=7.1 \mathrm{~Hz}, 1 \mathrm{H}), 6.92$ (s, 1H), 6.85-6.79 (m, 2H), 6.76 (d, $J=7.9 \mathrm{~Hz}, 1 \mathrm{H}), 3.79$ (s, 3H), 3.69 (brs, 2H), 3.23 $(\mathrm{s}, 2 \mathrm{H}), 2.36(\mathrm{~s}, 3 \mathrm{H}), 1.61(\mathrm{~s}, 6 \mathrm{H})$.

${ }^{13}$ C NMR $\left(100 \mathrm{MHz}, \mathrm{CDCl}_{3}\right) \delta 155.8,144.6,138.0,134.0,131.2,128.6,126.0,125.9$, $124.3,118.5,115.8,113.5,89.3,55.8,42.6,25.7,20.4(2 \mathrm{C})$.

IR (FT-ATR, $\mathrm{cm}^{-1}, \mathrm{CH}_{2} \mathrm{Cl}_{2}$ ) $v_{\max } 3461,3377,2935,1614,1578,1532,1512,1489$, $1450,1395,1371,1347,1291,1227,1205,1157,1132,1045,1031,978,915,855,750$, $681,651,604,512,453$.

HRMS (ESI) $m / z$ : [M+H] $]^{+}$Calcd for $\mathrm{C}_{18} \mathrm{H}_{22} \mathrm{~N}_{2} \mathrm{O}_{3} 315.1709$; found 315.1706 . 


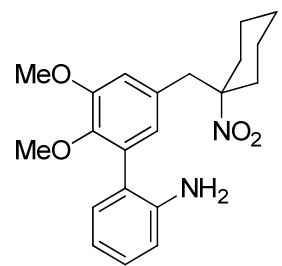

2',3'-Dimethoxy-5'-((1-nitrocyclohexyl)methyl)-[1,1'-biphenyl]-2-amine (S20) was synthesized by following Procedure 6 . The crude material was purified by normalphase column chromatography using an eluent of $25 \% \mathrm{EtOAc} / \mathrm{Hx}$ to provide $\mathbf{S 2 0}$ as a white solid (4.58 g, 90\%).

m.p. $55^{\circ} \mathrm{C}$

${ }^{1} \mathbf{H}$ NMR $\left(400 \mathrm{MHz}, \mathrm{CDCl}_{3}\right) \delta 7.16(\mathrm{t}, J=7.5 \mathrm{~Hz}, 1 \mathrm{H}), 7.09(\mathrm{~d}, J=7.5 \mathrm{~Hz}, 1 \mathrm{H}), 6.80$ $(\mathrm{t}, J=7.4 \mathrm{~Hz}, 1 \mathrm{H}), 6.75(\mathrm{~d}, J=7.9 \mathrm{~Hz}, 1 \mathrm{H}), 6.57(\mathrm{~s}, 2 \mathrm{H}), 3.86(\mathrm{~s}, 3 \mathrm{H}), 3.80$ (brs, $2 \mathrm{H})$, $3.56(\mathrm{~s}, 3 \mathrm{H}), 3.05(\mathrm{~s}, 2 \mathrm{H}), 2.42(\mathrm{~d}, J=13.2 \mathrm{~Hz}, 2 \mathrm{H}), 1.70-1.55(\mathrm{~m}, 5 \mathrm{H}), 1.43-1.22(\mathrm{~m}$, $3 \mathrm{H})$.

${ }^{13}$ C NMR $\left(100 \mathrm{MHz}, \mathrm{CDCl}_{3}\right) \delta 152.7,146.0,144.5,133.4,130.90130 .6,128.7,125.0$, $124.3,118.4,116.0,113.0,92.1,61.1,56.1,47.0,34.2,34.0,24.8,22.4(2 \mathrm{C})$.

IR (FT-ATR, $\mathrm{cm}^{-1}, \mathrm{CH}_{2} \mathrm{Cl}_{2}$ ) $v_{\max } 3465,3375,2935,2864,1671,1583,1531,1498$, 1480, 1449, 1421, 1344, 1300, 1261, 1230, 1185, 1170, 1139, 1048, 1031, 1006, 947, 926, 913, 849, 784, 749, 695, 641, 582, 534, 521, 464.

HRMS (ESI) $m / z$ : $[\mathrm{M}+\mathrm{H}]^{+}$Calcd for $\mathrm{C}_{21} \mathrm{H}_{24} \mathrm{~N}_{2} \mathrm{O}_{4} 371.1971$; found 371.1966 .

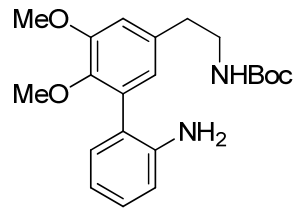

tert-Butyl (2-(2'-amino-5,6-dimethoxy-[1,1'-biphenyl]-3-yl)ethyl)carbamate (S21) was synthesized by following Procedure 6 . The crude material was purified by normal-phase column chromatography using an eluent of $25 \% \mathrm{EtOAc} / \mathrm{Hx}$ to provide S21 as a brown gum (537 mg, 96\%).

${ }^{1} \mathbf{H}$ NMR $\left(400 \mathrm{MHz}, \mathrm{CDCl}_{3}\right) \delta 7.16(\mathrm{t}, J=7.7 \mathrm{~Hz}, 1 \mathrm{H}), 7.12(\mathrm{~d}, J=7.6 \mathrm{~Hz}, 1 \mathrm{H}), 6.81$ $(\mathrm{t}, J=7.4 \mathrm{~Hz}, 1 \mathrm{H}), 6.76(\mathrm{~d}, J=5.2 \mathrm{~Hz}, 2 \mathrm{H}), 6.68(\mathrm{~s}, 1 \mathrm{H}), 4.66(\mathrm{brs}, 1 \mathrm{H}), 4.01-3.74(\mathrm{~m}$, $5 \mathrm{H}), 3.56(\mathrm{~s}, 3 \mathrm{H}), 3.38(\mathrm{~d}, J=6.0 \mathrm{~Hz}, 2 \mathrm{H}), 2.76(\mathrm{t}, J=6.6 \mathrm{~Hz}, 2 \mathrm{H}), 1.43(\mathrm{~s}, 9 \mathrm{H})$.

${ }^{13}$ C NMR (100 MHz, $\left.\mathrm{CDCl}_{3}\right) \delta 156.0,152.9,145.1,144.4,135.4,133.7,130.9,128.7$, $124.8,123.7,118.5,116.1,112.2,79.4,61.1,56.0,41.8,36.2,28.5(3 \mathrm{C})$.

IR (FT-ATR, $\mathrm{cm}^{-1}, \mathrm{CH}_{2} \mathrm{Cl}_{2}$ ) $v_{\max } 3365,2975,2934,1693,1618,1584,1503,1480$, 1453, 1421, 1392, 1366, 1343, 1251, 1230, 1167, 1138, 1049, 1032, 1007, 969, 888, 781, 750, 638, 534 .

HRMS (ESI) $m / z$ : $[\mathrm{M}+\mathrm{H}]^{+}$Calcd for $\mathrm{C}_{21} \mathrm{H}_{28} \mathrm{~N}_{2} \mathrm{O}_{4}$ 373.2127; found 373.2121 . 


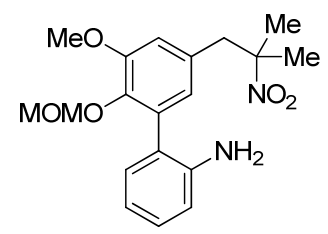

3'-Methoxy-2'-(methoxymethoxy)-5'-(2-methyl-2-nitropropyl)-[1,1'-biphenyl]-2amine (S22) was synthesized by following Procedure 6. The crude material was purified by normal-phase column chromatography using an eluent of $25 \% \mathrm{EtOAc} / \mathrm{Hx}$ to provide $\mathbf{S 2 2}$ as a brown oil (329 $\mathrm{mg}, 92 \%$ ).

${ }^{1} \mathbf{H}$ NMR $\left(400 \mathrm{MHz}, \mathrm{CDCl}_{3}\right) \delta 7.14(\mathrm{t}, J=7.7 \mathrm{~Hz}, 1 \mathrm{H}), 7.10(\mathrm{~d}, J=7.6 \mathrm{~Hz}, 1 \mathrm{H}), 6.79$ (t, $J=7.3 \mathrm{~Hz}, 1 \mathrm{H}), 6.74(\mathrm{~d}, J=7.9 \mathrm{~Hz}, 1 \mathrm{H}), 6.64(\mathrm{~d}, J=1.9 \mathrm{~Hz}, 2 \mathrm{H}), 4.84(\mathrm{~s}, 2 \mathrm{H}), 3.85$ (s, 3H), 3.76 (brs, 2H), 3.17 (s, 2H), 2.99 (s, 3H), 1.60 (s, 6H).

${ }^{13}$ C NMR $\left(100 \mathrm{MHz}, \mathrm{CDCl}_{3}\right) \delta 152.7,144.5,142.6,134.3,131.7,131.3,128.8,125.2$, 124.6, 118.4, 115.8, 113.1, 98.5, 88.7, 56.7, 56.0, 46.7, 25.9, 25.8.

IR (FT-ATR, $\mathrm{cm}^{-1}, \mathrm{CH}_{2} \mathrm{Cl}_{2}$ ) $v_{\max } 3454,3374,2936,2840,1618,1584,1535,1498$, $1481,1450,1450,1422,1397,1372,1343,1301,1261,1228,1201,1155,1139,1100$, $1075,1048,1031,957,854,807,748,734,701,672,574,532,460$.

HRMS (ESI) $m / z$ : [M+H] $]^{+}$Calcd for $\mathrm{C}_{19} \mathrm{H}_{24} \mathrm{~N}_{2} \mathrm{O}_{5}$ 361.1764; found 361.1758.

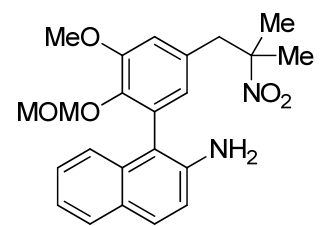

1-(3-Methoxy-2-(methoxymethoxy)-5-(2-methyl-2-

nitropropyl)phenyl)naphthalen-2-amine (S23) was synthesized by following Procedure 6 . The crude material was purified by normal-phase column chromatography using an eluent of $25 \% \mathrm{EtOAc} / \mathrm{Hx}$ to provide $\mathbf{S 2 3}$ as a red solid (780 $\mathrm{mg}, 39 \%)$.

m.p. $122^{\circ} \mathrm{C}$

${ }^{1} \mathbf{H}$ NMR $\left(400 \mathrm{MHz}, \mathrm{CDCl}_{3}\right) \delta 7.69(\mathrm{t}, J=8.5 \mathrm{~Hz}, 2 \mathrm{H}), 7.36-7.18(\mathrm{~m}, 3 \mathrm{H}), 7.02(\mathrm{~d}, J$ $=8.7 \mathrm{~Hz}, 1 \mathrm{H}), 6.73(\mathrm{~s}, 1 \mathrm{H}), 6.64(\mathrm{~s}, 1 \mathrm{H}), 4.75(\mathrm{~s}, 2 \mathrm{H}), 3.89(\mathrm{~s}, 3 \mathrm{H}), 3.77(\mathrm{brs}, 2 \mathrm{H}), 3.19$ (dd, $J=34.5,13.8 \mathrm{~Hz}, 2 \mathrm{H}), 2.72(\mathrm{~s}, 3 \mathrm{H}), 1.61(\mathrm{~d}, J=5.5 \mathrm{~Hz}, 6 \mathrm{H})$.

${ }^{13}$ C NMR $\left(100 \mathrm{MHz}, \mathrm{CDCl}_{3}\right) \delta 153.4,143.9,141.8,133.8,132.1,131.6,129.1,128.0$, $127.9,126.5,126.1,124.4,122.3,118.2,116.1,113.4,98.3,88.8,56.4,56.0,46.8,26.2$, 25.6 .

IR (FT-ATR, $\left.\mathrm{cm}^{-1}, \mathrm{CH}_{2} \mathrm{Cl}_{2}\right) v_{\max } 3471,3376,2928,1671,1582,1536,1513,1482$, $1461,1435,1426,1395,1373,1348,1335,1285,1263,1229,1202,1155,1074,1019$, $965,855,816,749,685,665,640,577,534,425$.

HRMS (ESI) $m / z$ : [M+H] $]^{+}$Calcd for $\mathrm{C}_{23} \mathrm{H}_{26} \mathrm{~N}_{2} \mathrm{O}_{5} 411.1920$; found 411.1918 . 


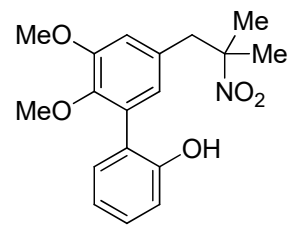

2',3'-Dimethoxy-5'-(2-methyl-2-nitropropyl)-[1,1'-biphenyl]-2-ol (S24) was synthesized by following Procedure 6 . The crude material was purified by normalphase column chromatography using an eluent of $25 \% \mathrm{EtOAc} / \mathrm{Hx}$ to provide $\mathbf{S 2 5}$ as a yellow solid (1.0 g, 98\%).

m.p. $124^{\circ} \mathrm{C}$

${ }^{1} \mathbf{H}$ NMR $\left(400 \mathrm{MHz}, \mathrm{CDCl}_{3}\right) \delta 7.32(\mathrm{t}, J=7.7 \mathrm{~Hz}, 1 \mathrm{H}), 7.27(\mathrm{~d}, J=7.6 \mathrm{~Hz}, 1 \mathrm{H}), 7.11$ $(\mathrm{s}, 1 \mathrm{H}), 7.06(\mathrm{~d}, J=8.1 \mathrm{~Hz}, 1 \mathrm{H}), 7.02(\mathrm{t}, J=7.5 \mathrm{~Hz}, 1 \mathrm{H}), 6.70(\mathrm{~s}, 1 \mathrm{H}), 6.67(\mathrm{~s}, 1 \mathrm{H})$, $3.89(\mathrm{~s}, 3 \mathrm{H}), 3.66(\mathrm{~s}, 3 \mathrm{H}), 3.20(\mathrm{~s}, 2 \mathrm{H}), 1.62(\mathrm{~s}, 6 \mathrm{H})$.

${ }^{13}$ C NMR $\left(100 \mathrm{MHz}, \mathrm{CDCl}_{3}\right) \delta 153.9,152.7,144.4,132.5,132.4,131.2,129.8,125.68$, $125.67,121.2,118.6,113.2,88.7,61.7,56.1,46.8,25.9(2 \mathrm{C})$.

IR (FT-ATR, $\mathrm{cm}^{-1}, \mathrm{CH}_{2} \mathrm{Cl}_{2}$ ) $v_{\max } 2937,2873,2360,1587,1537,1489,1454,1421$, 1396, 1371, 1346, 1261, 1225, 1186, 1140, 1095, 1051, 1034, 999, 856, 806, 787, 758, 623, 584, 540, 478, 453, 418, 415, 401, 449, 434, 422, 415, 401, 411.

HRMS (EI) $m / z$ : [M] $]^{+}$Calcd for $\mathrm{C}_{18} \mathrm{H}_{21} \mathrm{NO}_{5} 331.1420$; found 331.1420 .

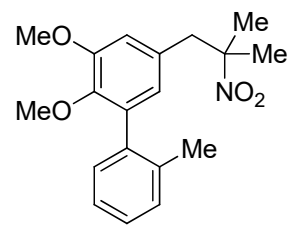

2,3-Dimethoxy-2'-methyl-5-(2-methyl-2-nitropropyl)-1,1'-biphenyl (S25) was synthesized by following Procedure 7 . The crude material was purified by normalphase column chromatography using an eluent of 14\% EtOAc/Hx to provide $\mathbf{S 2 5}$ as a colorless oil (487 mg, 98\%).

${ }^{1} \mathbf{H}$ NMR $\left(400 \mathrm{MHz}, \mathrm{CDCl}_{3}\right) \delta$ 7.30-7.25 (m, 2H), 7.25-7.20 (m, 1H), $7.18(\mathrm{t}, J=7.3$ $\mathrm{Hz}, 1 \mathrm{H}), 6.64(\mathrm{~d}, J=1.5 \mathrm{~Hz}, 1 \mathrm{H}), 6.52(\mathrm{~d}, J=1.6 \mathrm{~Hz}, 1 \mathrm{H}), 3.88(\mathrm{~s}, 3 \mathrm{H}), 3.49(\mathrm{~s}, 3 \mathrm{H})$, $3.17(\mathrm{~s}, 2 \mathrm{H}), 2.16(\mathrm{~s}, 3 \mathrm{H}), 1.61(\mathrm{~s}, 6 \mathrm{H})$.

${ }^{13} \mathbf{C ~ N M R}\left(100 \mathrm{MHz}, \mathrm{CDCl}_{3}\right) \delta 152.7,146.0,138.0,136.7,136.0,130.5,129.9,129.8$, 127.6, 125.4, 124.8, 113.0, 88.8, 60.7, 56.0, 46.8, 25.9, $20.2(2 \mathrm{C})$.

IR (FT-ATR, $\mathrm{cm}^{-1}, \mathrm{CH}_{2} \mathrm{Cl}_{2}$ ) $v_{\max } 2933,2873,2360,2339,1585,1537,1460,1423$, 1396, 1373, 1344, 1282, 1255, 1232, 1184, 1140, 1109, 1041, 1009, 854, 760, 731, 673, 634, 584, 501, 467, 449, 432, 415, 403, 407, 401, 401, 445, 434, 422, 411.

HRMS (EI) $m / z$ : [M] $]^{+}$Calcd for $\mathrm{C}_{19} \mathrm{H}_{23} \mathrm{NO}_{4} 329.1627$; found 329.1623 . 


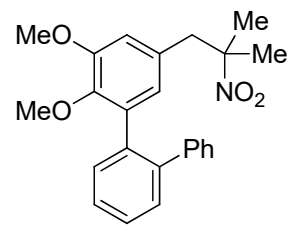

2,3-Dimethoxy-5-(2-methyl-2-nitropropyl)-1,1':2',1"'-terphenyl (S26) was synthesized by following Procedure 7 . The crude material was purified by normalphase column chromatography using an eluent of 14\% EtOAc/Hx to provide S26 as a white solid (580 mg, 99\%).

m.p. $95{ }^{\circ} \mathrm{C}$

${ }^{1} \mathbf{H}$ NMR $\left(400 \mathrm{MHz}, \mathrm{CDCl}_{3}\right) \delta$ 7.37-7.27 (m, 2H), $7.26(\mathrm{~d}, J=3.2 \mathrm{~Hz}, 2 \mathrm{H}), 7.13-7.00$ $(\mathrm{m}, 5 \mathrm{H}), 6.38(\mathrm{~d}, J=1.6 \mathrm{~Hz}, 1 \mathrm{H}), 6.20(\mathrm{~d}, J=1.7 \mathrm{~Hz}, 1 \mathrm{H}), 3.67(\mathrm{~s}, 3 \mathrm{H}), 3.46(\mathrm{~s}, 3 \mathrm{H})$, 2.86 (brs, 2H), 1.16 (s, 6H).

${ }^{13}$ C NMR $\left(100 \mathrm{MHz}, \mathrm{CDCl}_{3}\right) \delta 152.6,146.0,141.6,141.1,136.5,135.4,131.0,130.3$, $129.9,129.4$ (2C), 128.0 (2C), 127.8, 126.9, 126.6, 125.9, 112.9, 88.6, 60.5, 55.9, 46.4, $25.3(2 \mathrm{C})$.

IR (FT-ATR, $\mathrm{cm}^{-1}, \mathrm{CH}_{2} \mathrm{Cl}_{2}$ ) $v_{\max } 3680,3539,3057,2989,2937,2833,2372,2353$, 2320, 1583, 1535, 1466, 1421, 1396, 1371, 1342, 1263, 1234, 1182, 1140, 1057, 1038, 1007, 854, 739, 700, 636, 553, 519, 476, 453, 432, 422, 418, 413, 405, 411.

HRMS (EI) $m / z$ : [M] $]^{+}$Calcd for $\mathrm{C}_{24} \mathrm{H}_{25} \mathrm{NO}_{4} 391.1784$; found 391.1785 . 


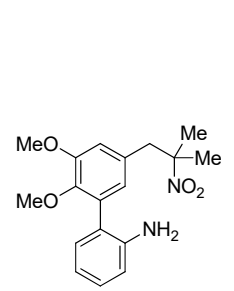

兽

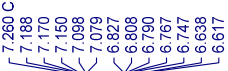

$\mathrm{S} 10$

( ${ }^{1} \mathrm{H} \mathrm{NMR}, 400 \mathrm{MHz}, \mathrm{CDCl}_{3}$ )
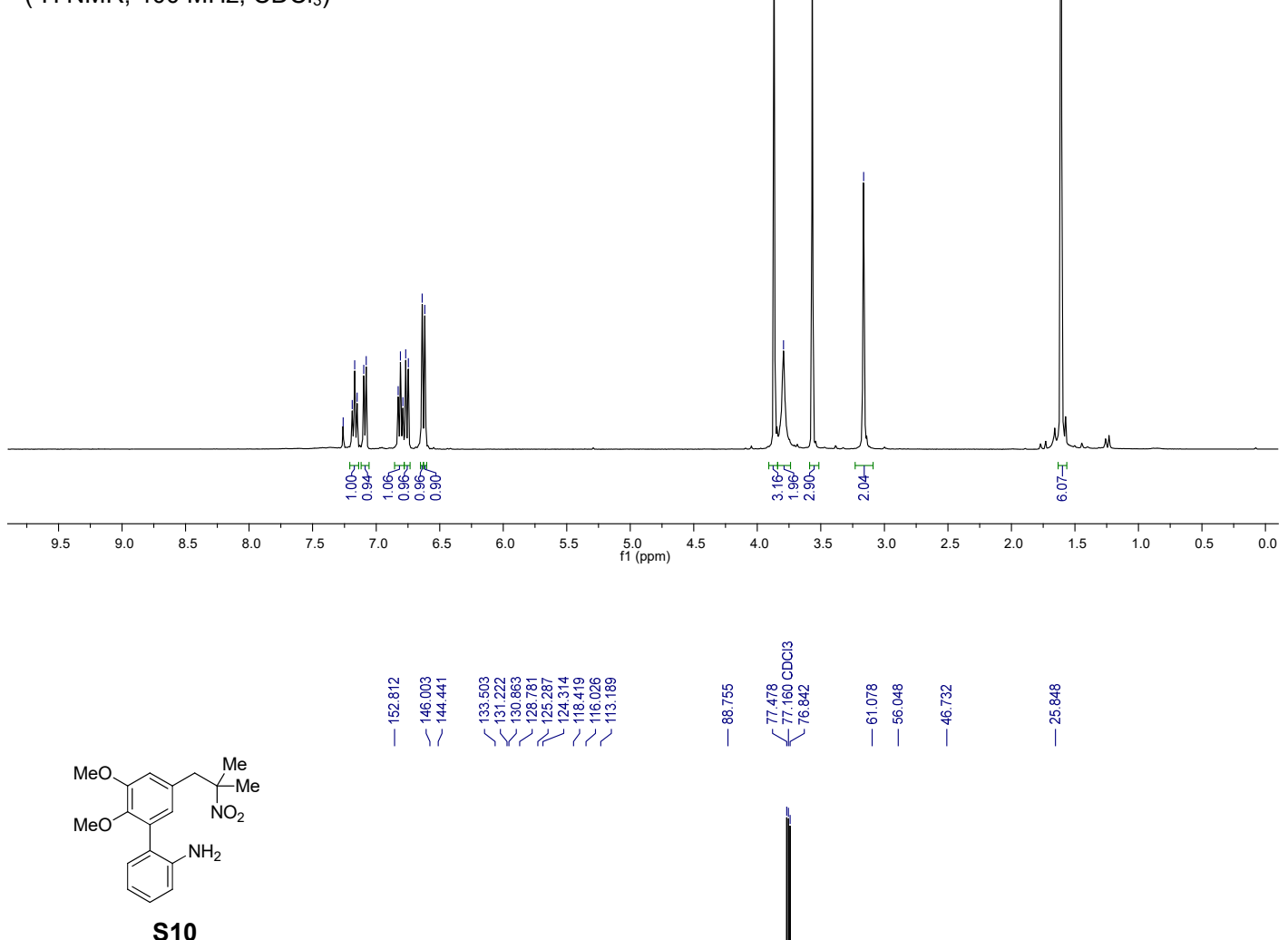

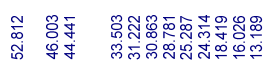

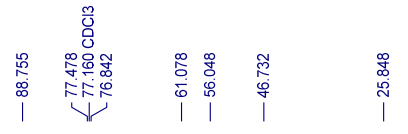

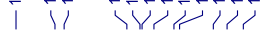

$\left({ }^{13} \mathrm{C} \mathrm{NMR}, 100 \mathrm{MHz}, \mathrm{CDCl}_{3}\right)$

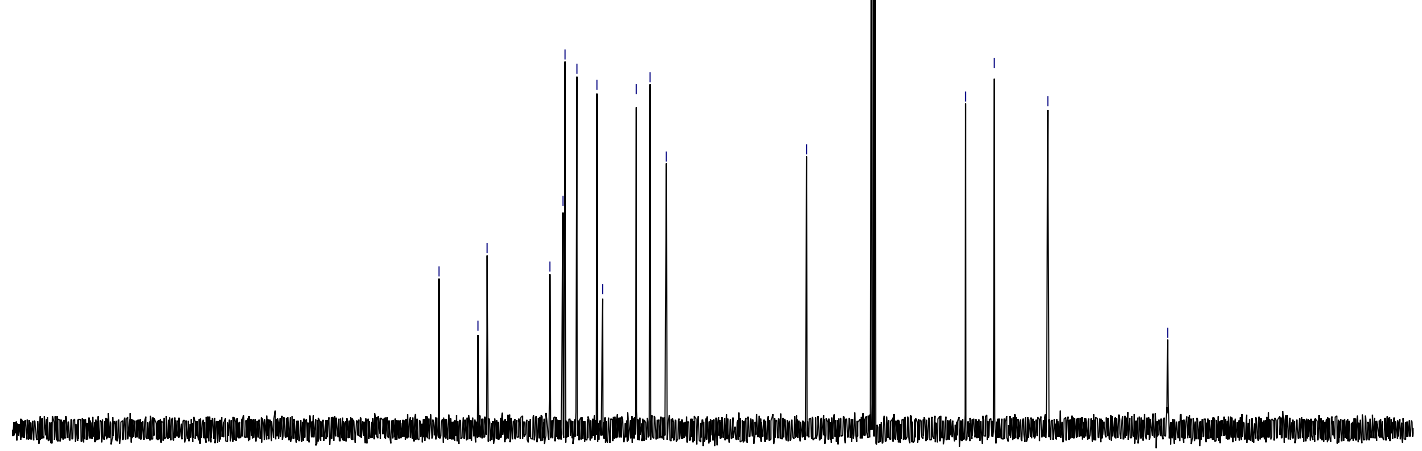

$\begin{array}{llllllllllll}220 & 210 & 200 & 190 & 180 & 170 & 160 & 150 & 140 & 130 & 120 & \begin{array}{c}110 \\ \mathrm{f} 1(\mathrm{ppm})\end{array}\end{array}$ 


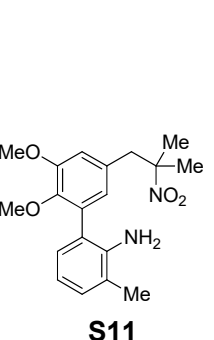

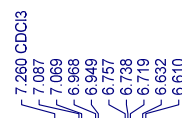

( ${ }^{1} \mathrm{H} \mathrm{NMR}, 400 \mathrm{MHz}, \mathrm{CDCl}_{3}$ )
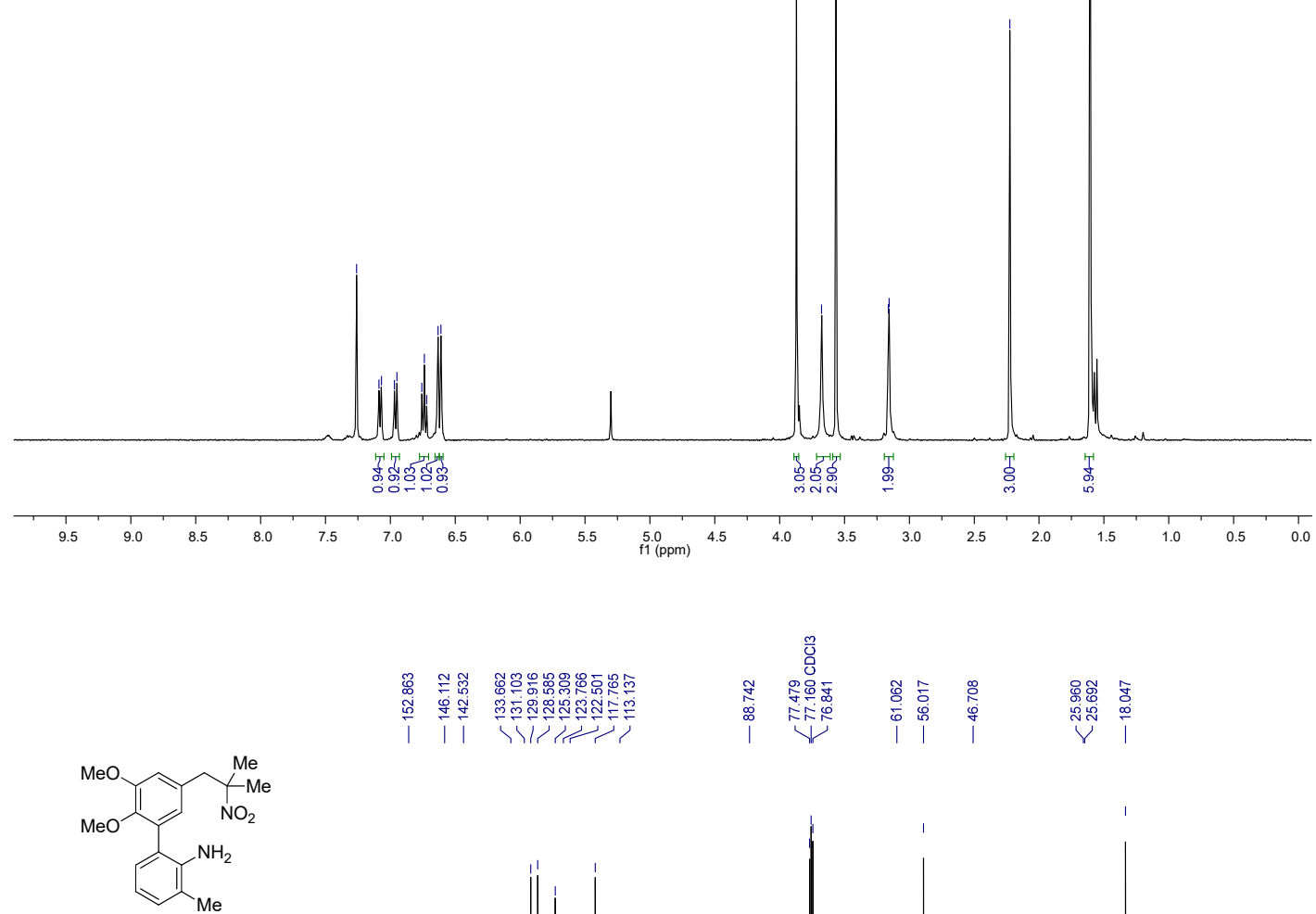

S11

$\left({ }^{13} \mathrm{C}\right.$ NMR, $\left.100 \mathrm{MHz}, \mathrm{CDCl}_{3}\right)$

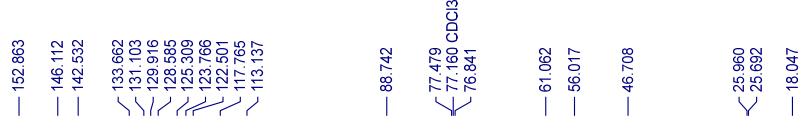

管

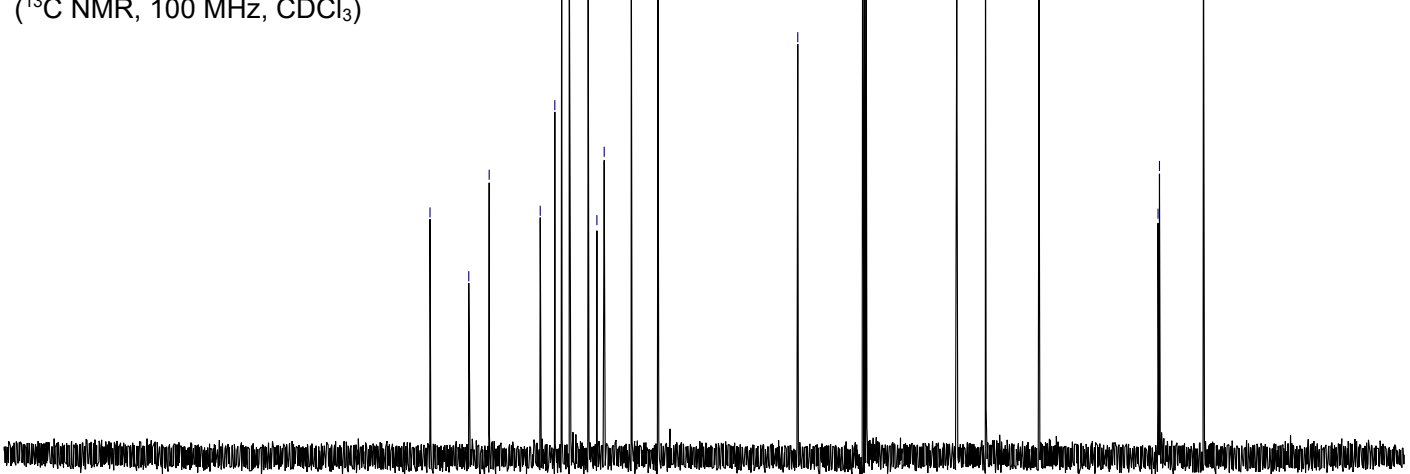

$\begin{array}{lllllllllllllllllllllllllllll}220 & 210 & 200 & 190 & 180 & 170 & 160 & 150 & 140 & 130 & 120 & \underset{f 1}{11}(\mathrm{ppm}) & 100 & 90 & 80 & 70 & 60 & 50 & 40 & 30 & 20 & 10 & 0 & -10\end{array}$ 

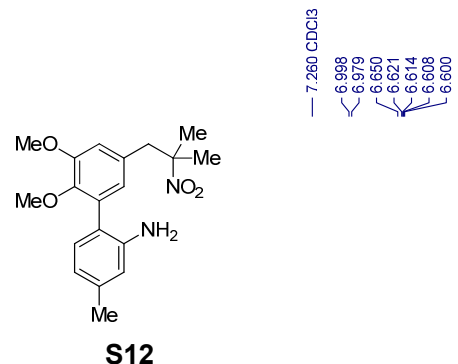

$\left({ }^{1} \mathrm{H} \mathrm{NMR}, 400 \mathrm{MHz}, \mathrm{CDCl}_{3}\right)$
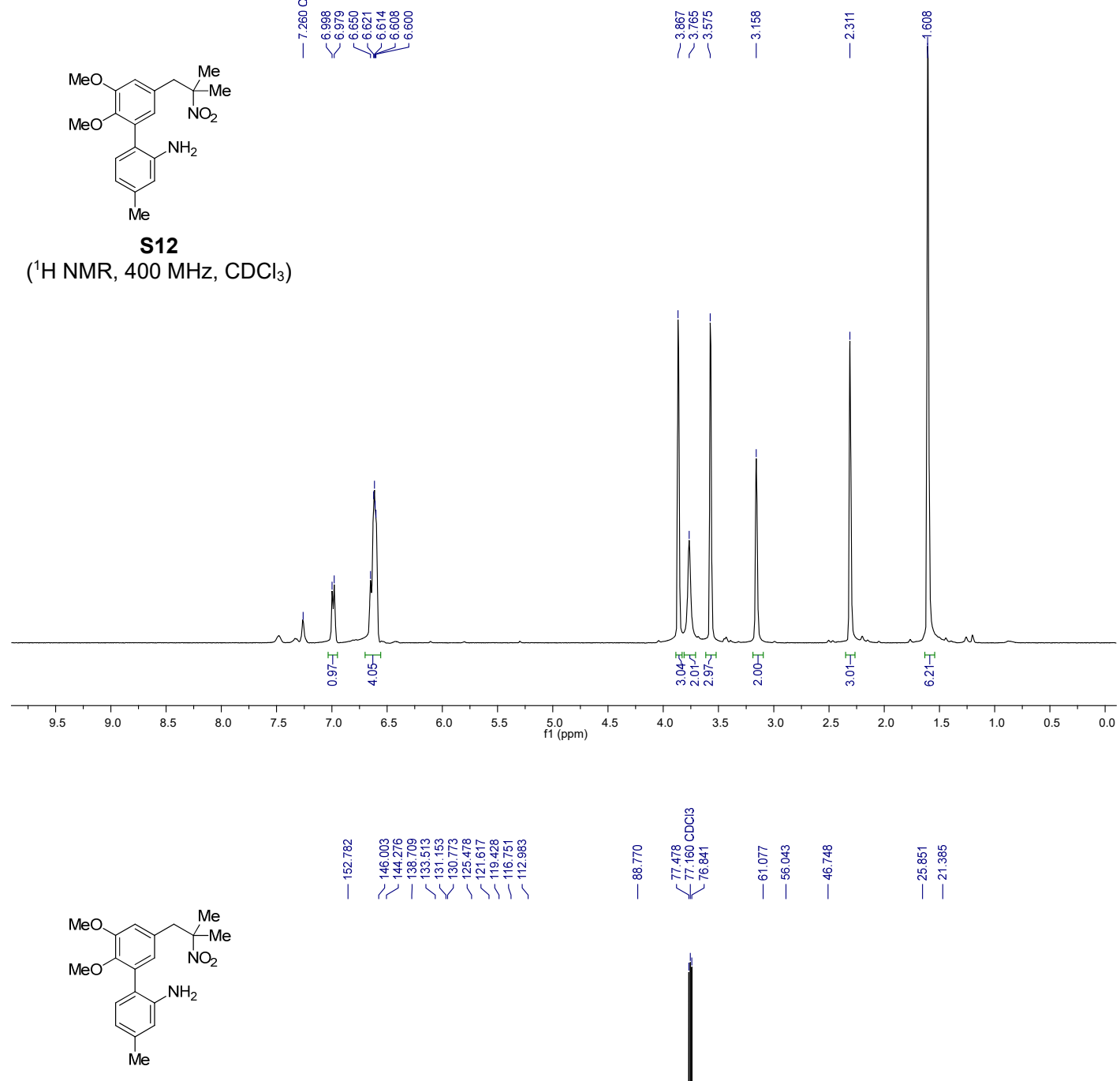

S12

$\left({ }^{13} \mathrm{C} \mathrm{NMR}, 100 \mathrm{MHz}, \mathrm{CDCl}_{3}\right)$

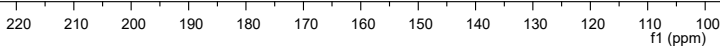

(C)

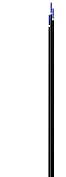



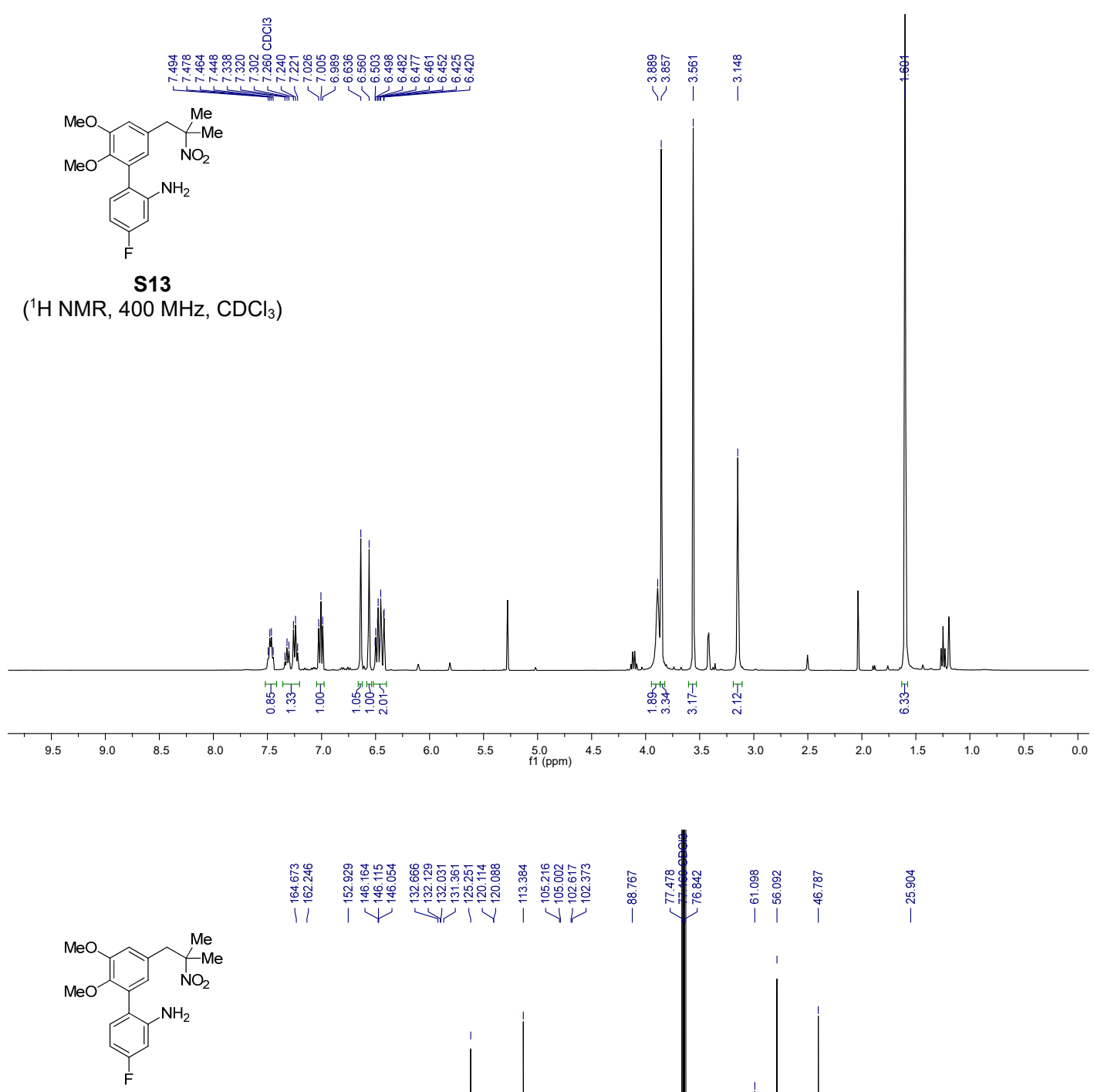

S13

$\left({ }^{13} \mathrm{C}\right.$ NMR, $\left.100 \mathrm{MHz}, \mathrm{CDCl}_{3}\right)$

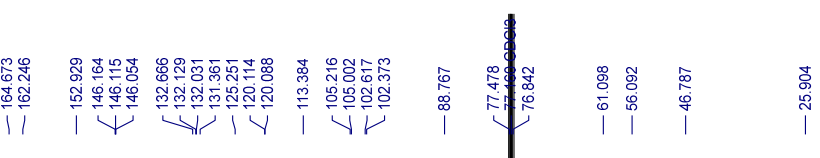

,

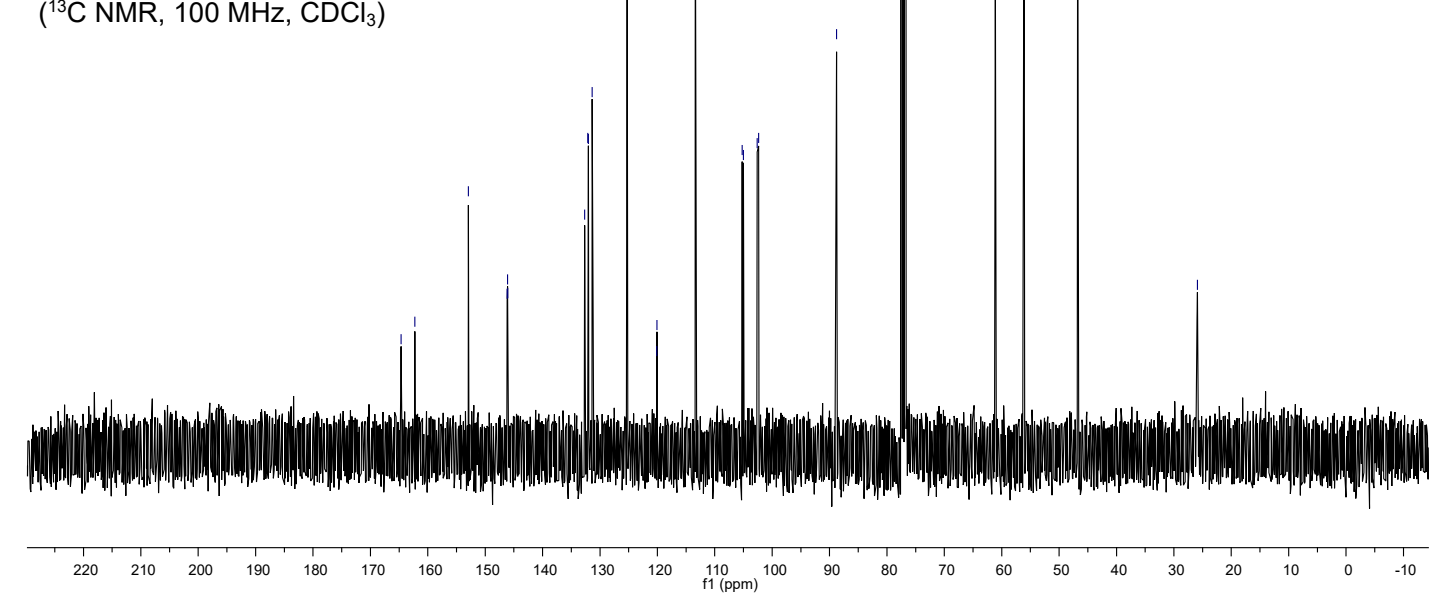




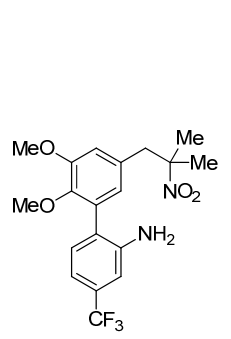

응

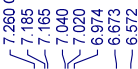

$\mathrm{S} 14$

$\left({ }^{1} \mathrm{H} \mathrm{NMR}, 400 \mathrm{MHz}, \mathrm{CDCl}_{3}\right)$
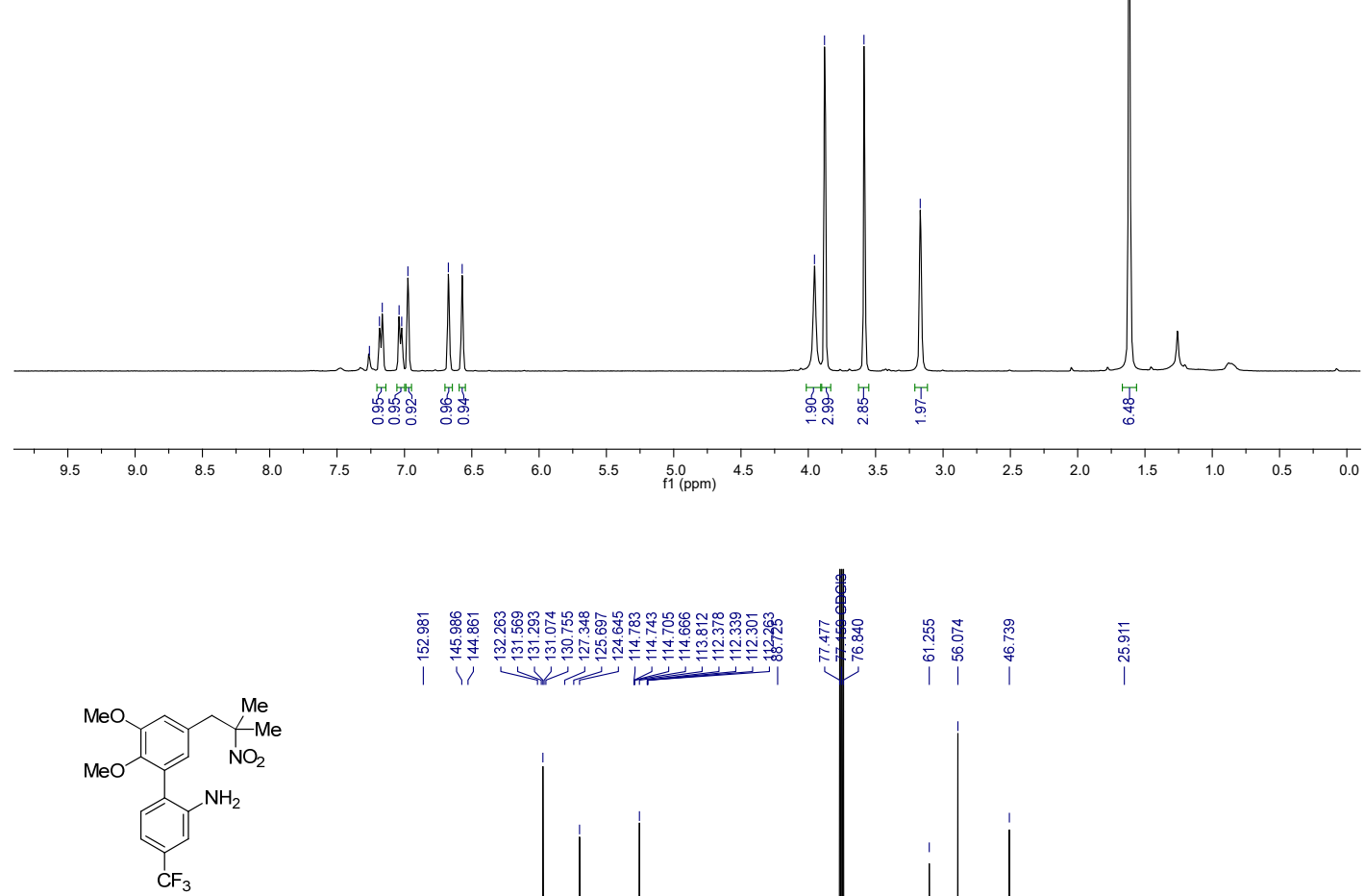

S14

$\left({ }^{13} \mathrm{C}\right.$ NMR, $\left.100 \mathrm{MHz}, \mathrm{CDCl}_{3}\right)$

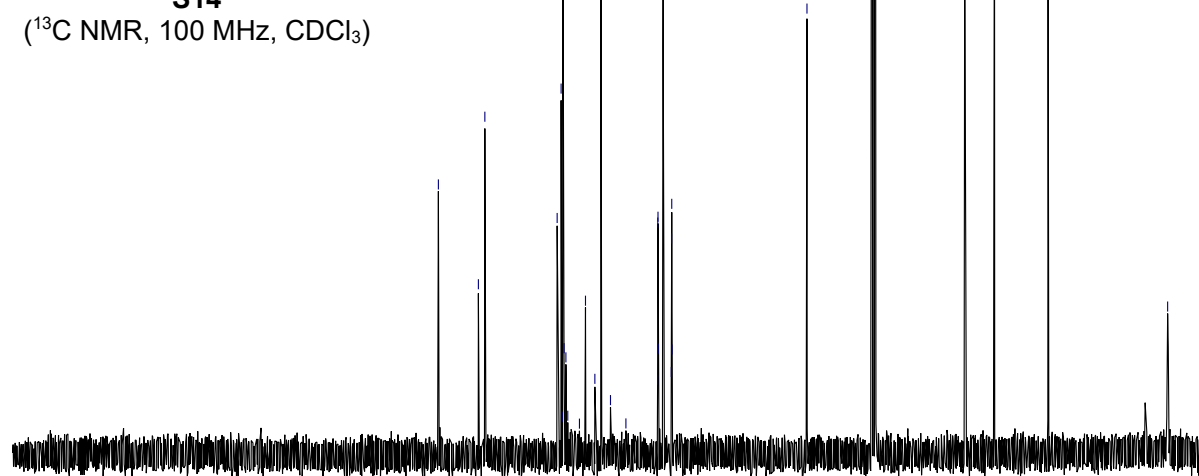

$\begin{array}{llllllllllll}220 & 210 & 200 & 190 & 180 & 170 & 160 & 150 & 140 & 130 & 120 & \begin{array}{c}110 \\ \mathrm{f} 1(\mathrm{ppm})\end{array}\end{array}$ 


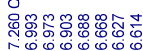

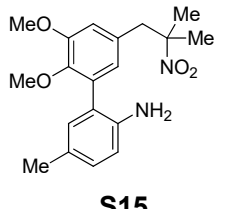

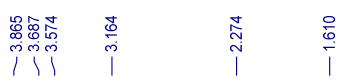

('H NMR, $400 \mathrm{MHz}, \mathrm{CDCl}_{3}$ )
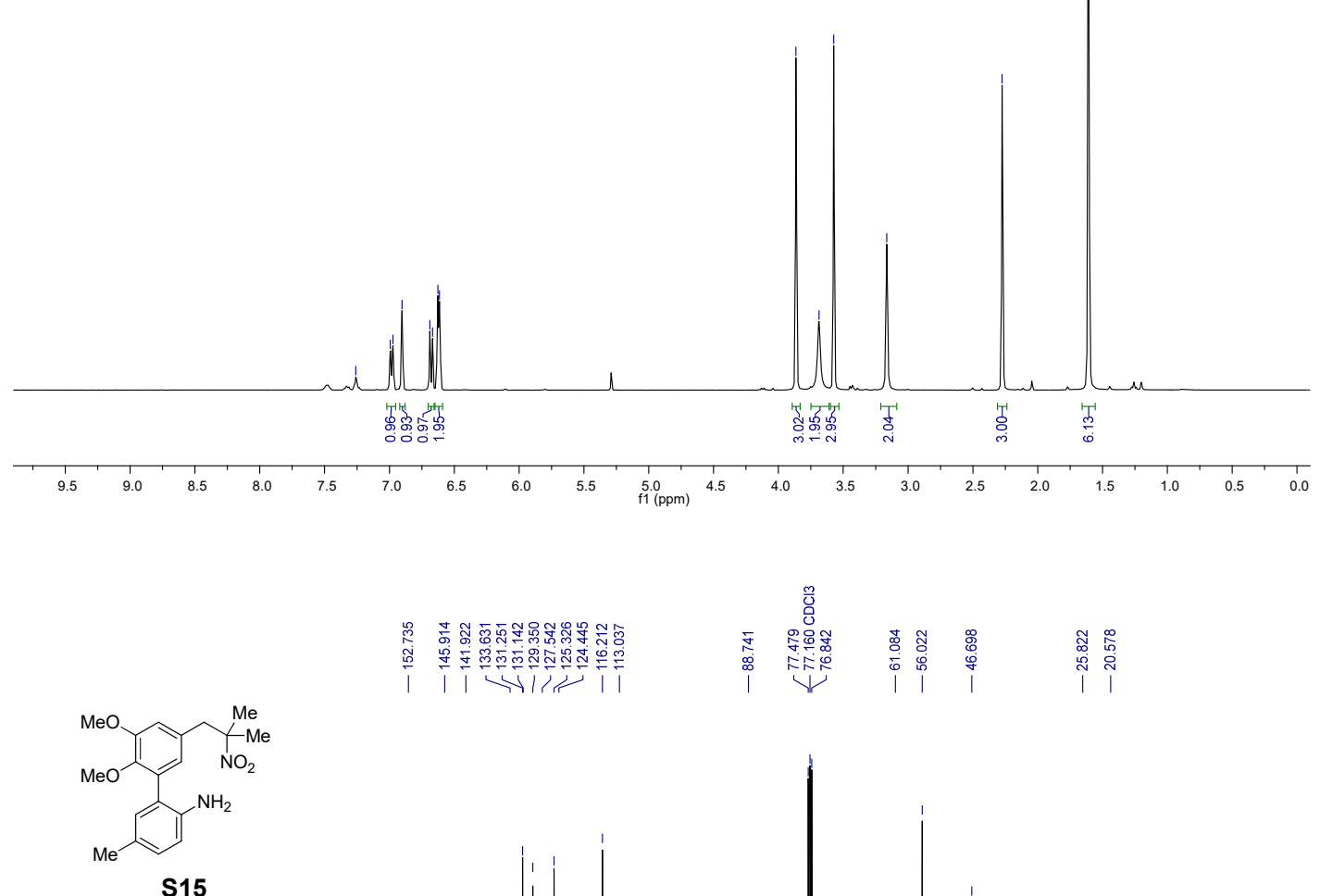

$\left({ }^{13} \mathrm{C} \mathrm{NMR}, 100 \mathrm{MHz}, \mathrm{CDCl}_{3}\right)$

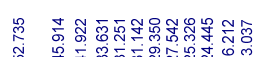

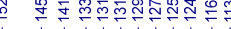

| | जाiा।

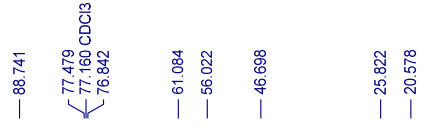

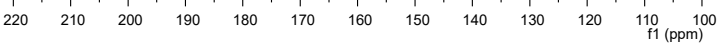




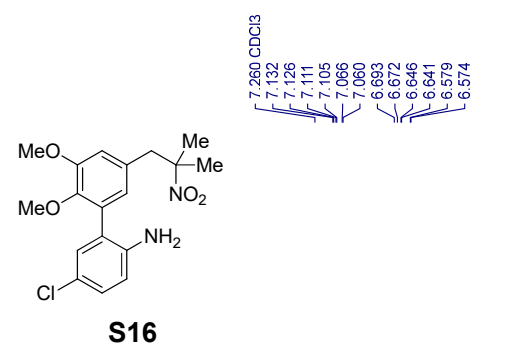

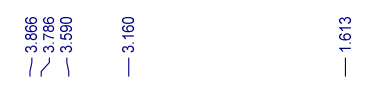

$\left({ }^{1} \mathrm{H} \mathrm{NMR}, 400 \mathrm{MHz}, \mathrm{CDCl}_{3}\right)$
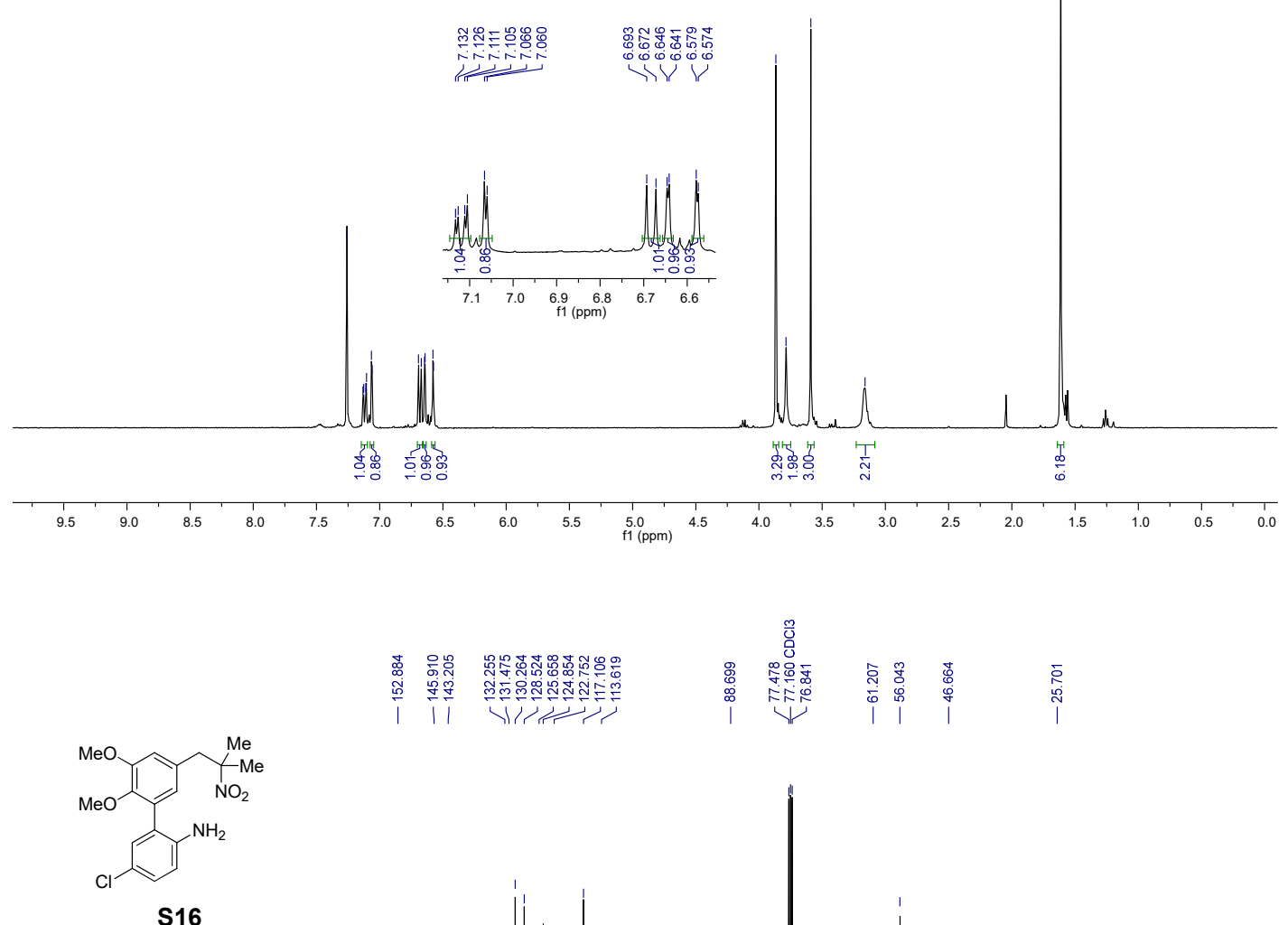

$\left({ }^{13} \mathrm{C} \mathrm{NMR}, 100 \mathrm{MHz}, \mathrm{CDCl}_{3}\right)$

\begin{tabular}{|c|c|c|c|}
\hline 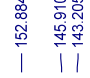 & ") & $\begin{array}{l}.8 \\
\infty \\
\infty \\
\mid \\
\mid\end{array}$ & 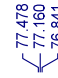 \\
\hline
\end{tabular}

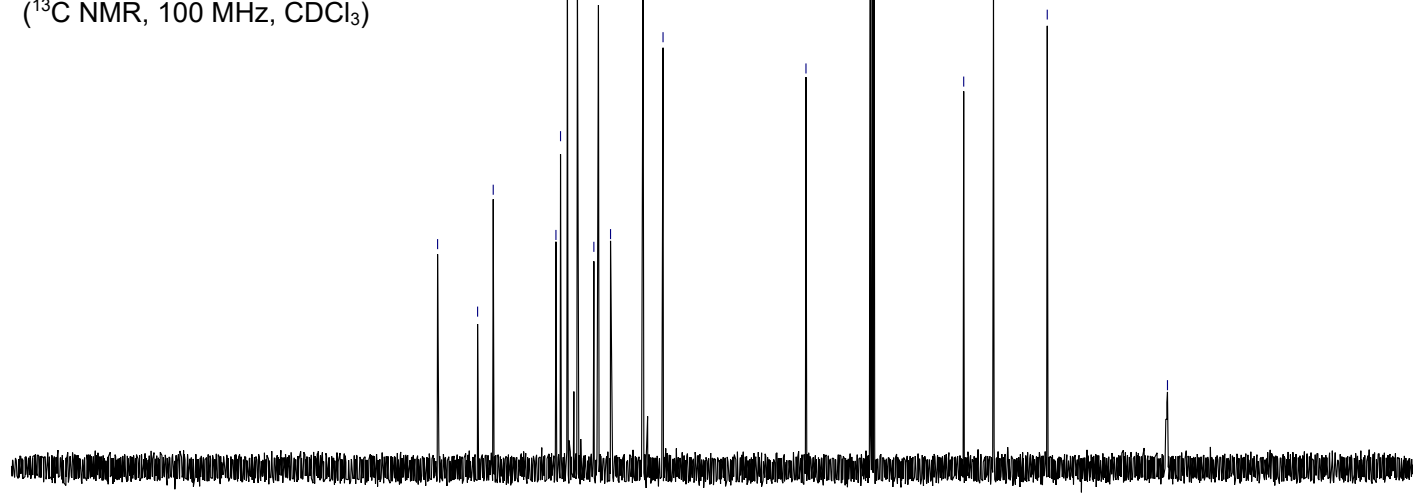

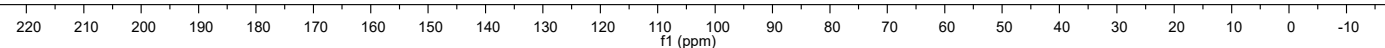




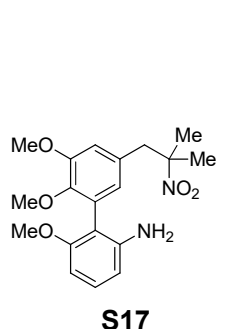

$\frac{m}{0}$

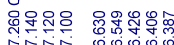

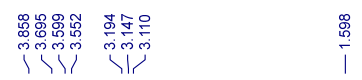

( ${ }^{1} \mathrm{H} \mathrm{NMR}, 400 \mathrm{MHz}, \mathrm{CDCl}_{3}$ )
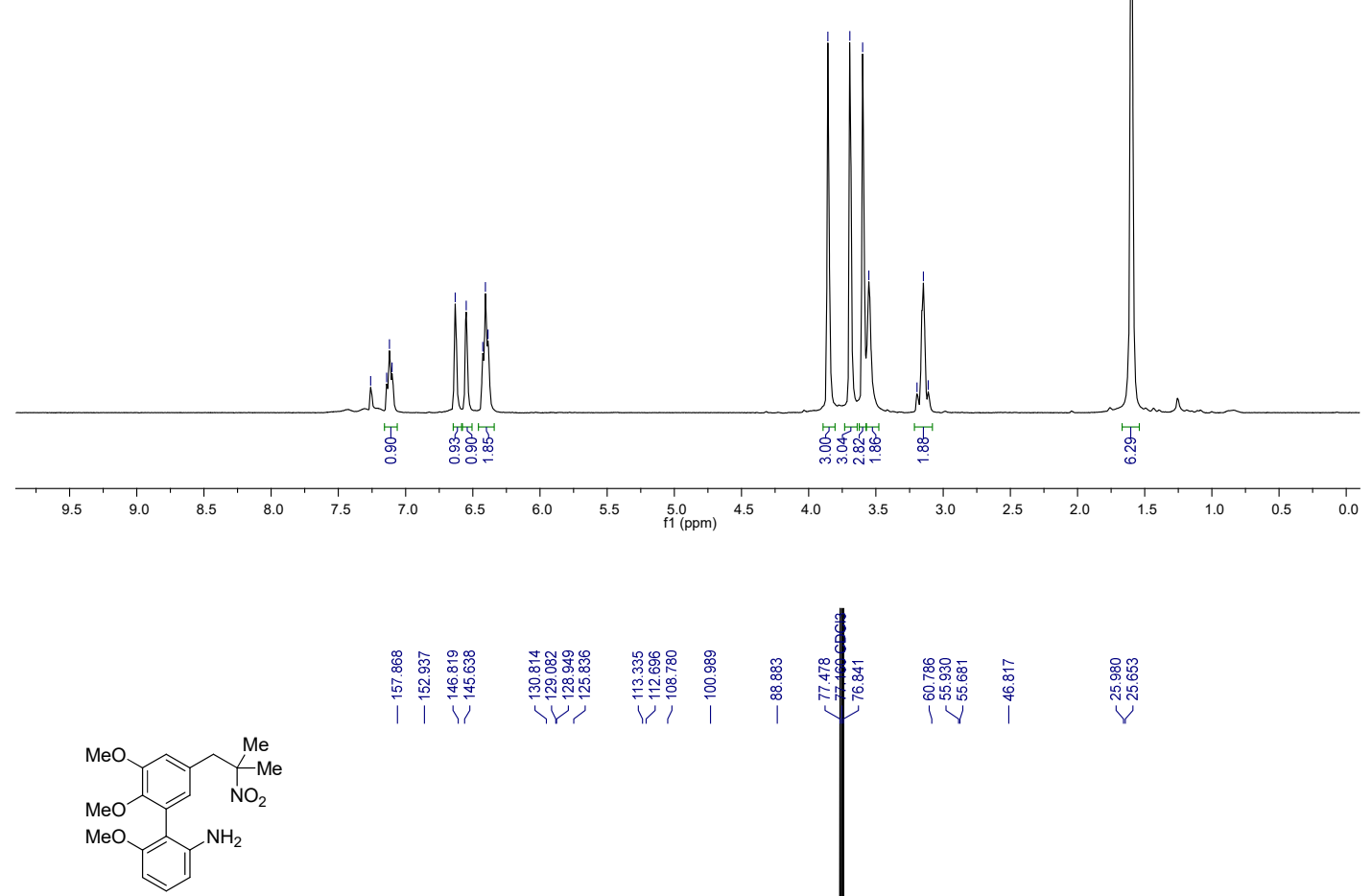

S17

$\left({ }^{13} \mathrm{C}\right.$ NMR, $\left.100 \mathrm{MHz}, \mathrm{CDCl}_{3}\right)$
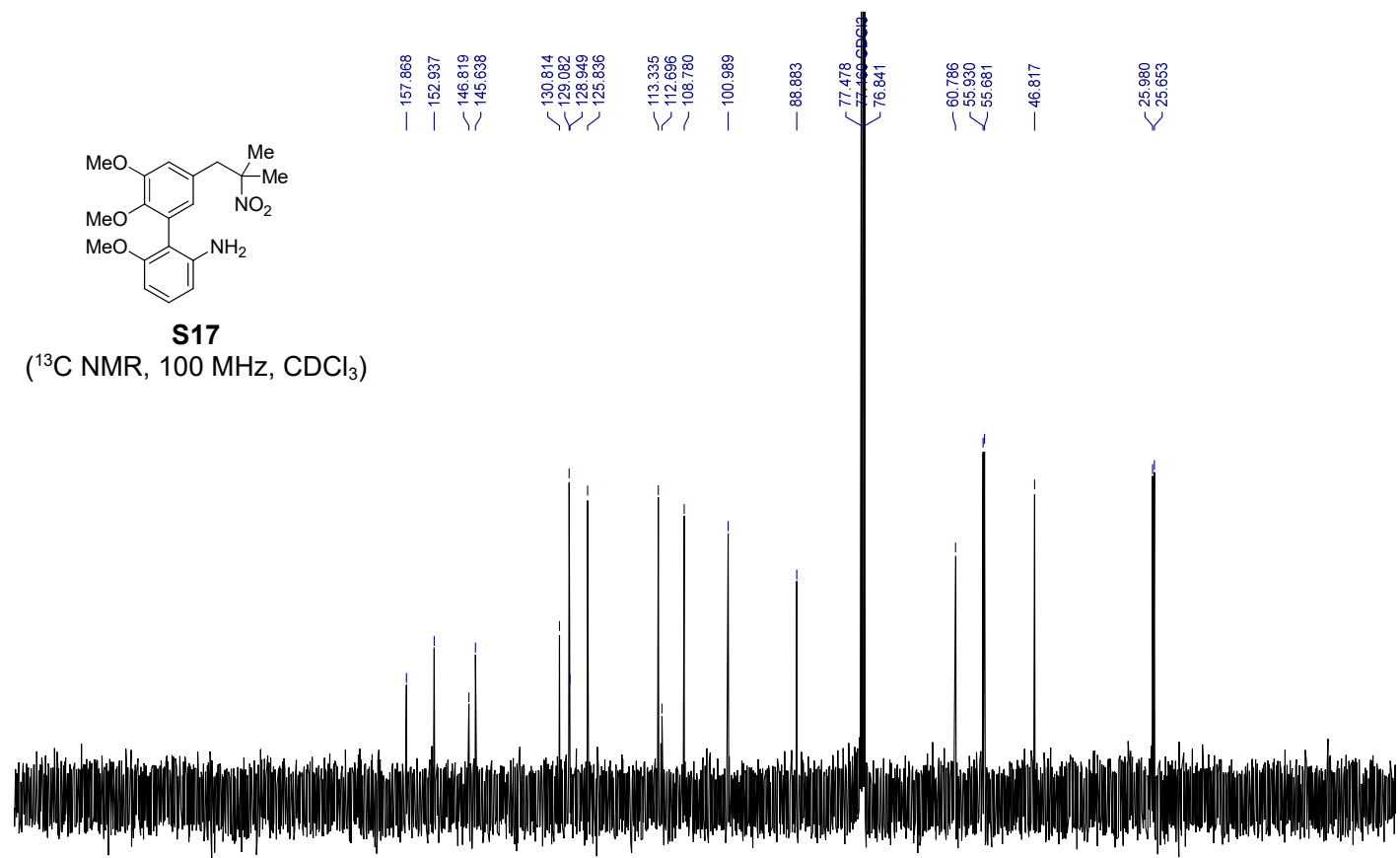

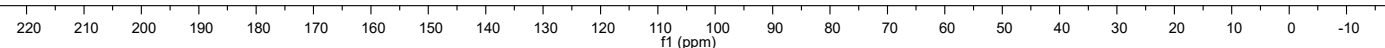



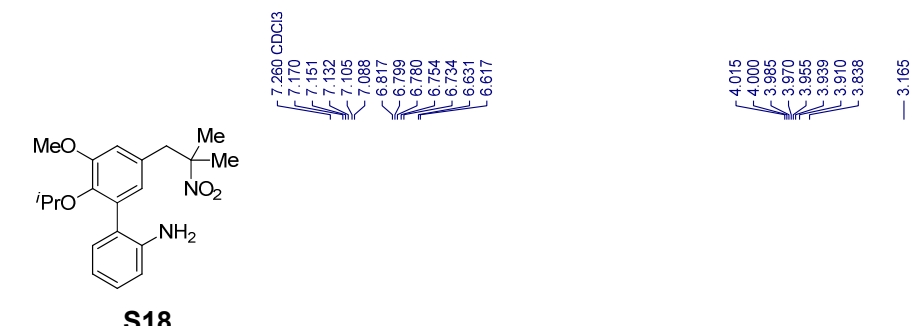

i

( $\left.{ }^{1} \mathrm{H} \mathrm{NMR}, 400 \mathrm{MHz}, \mathrm{CDCl}_{3}\right)$
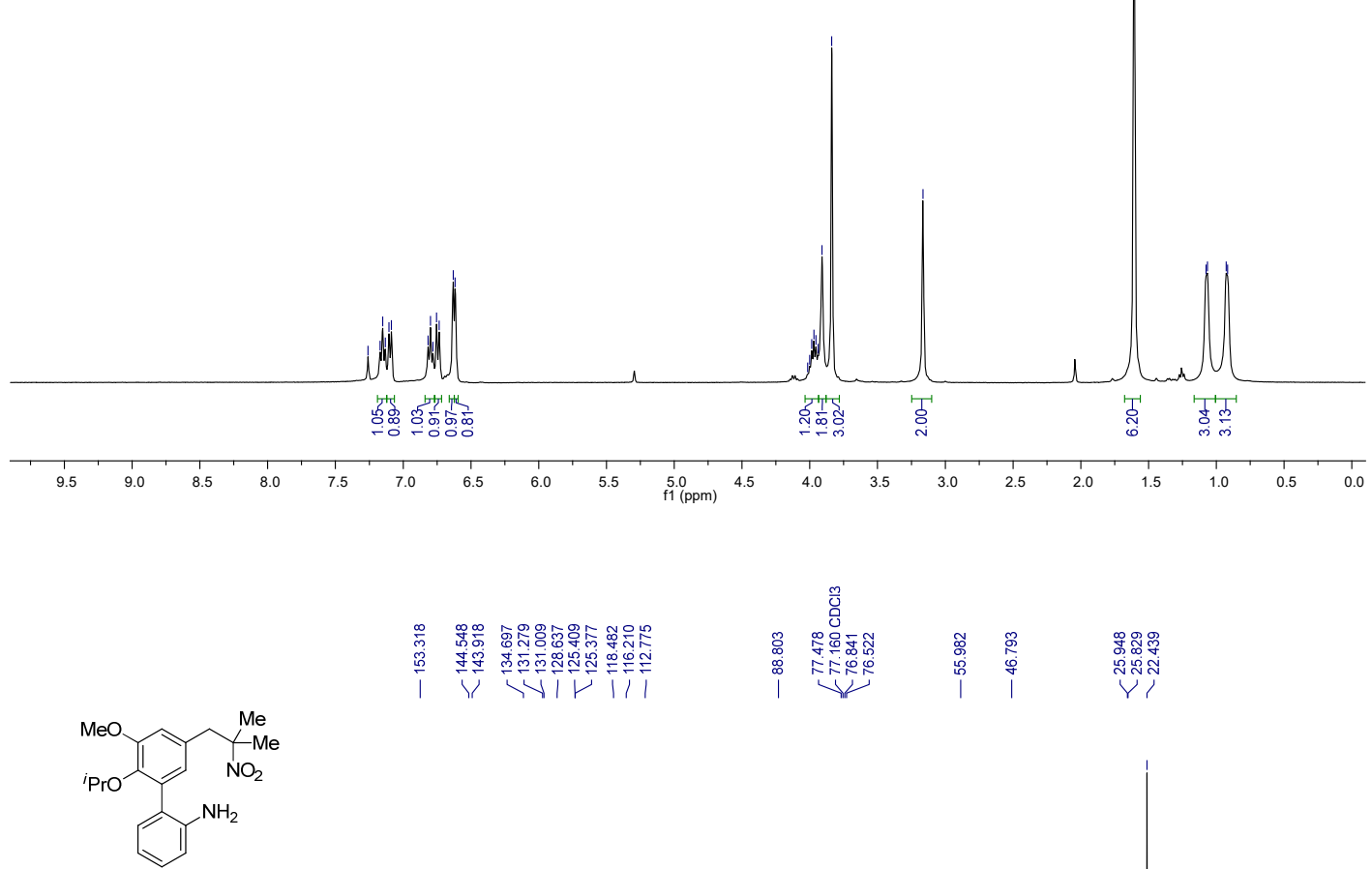

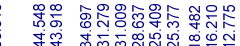

-

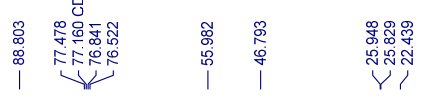

S18

$\left({ }^{13} \mathrm{C}\right.$ NMR, $\left.100 \mathrm{MHz}, \mathrm{CDCl}_{3}\right)$

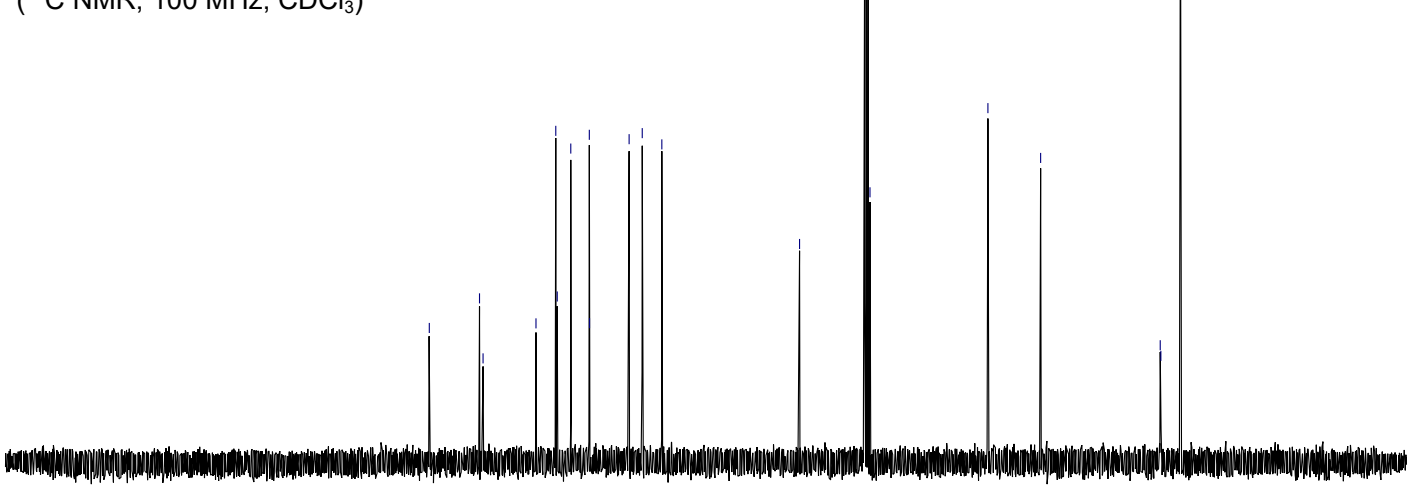

$\begin{array}{llllllllllll}220 & 210 & 200 & 190 & 180 & 170 & 160 & 150 & 140 & 130 & 120 & \begin{array}{l}110 \\ \mathrm{f} 1(\mathrm{ppm})\end{array} \\ 100\end{array}$ 

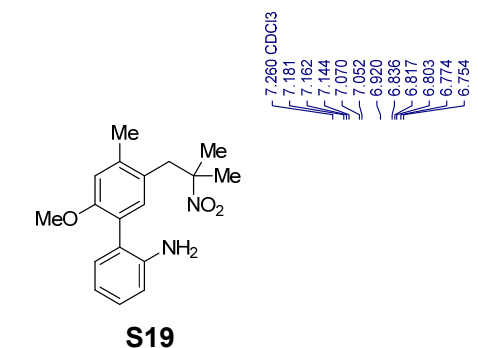

$\left({ }^{1} \mathrm{H} \mathrm{NMR}, 400 \mathrm{MHz}, \mathrm{CDCl}_{3}\right.$ )
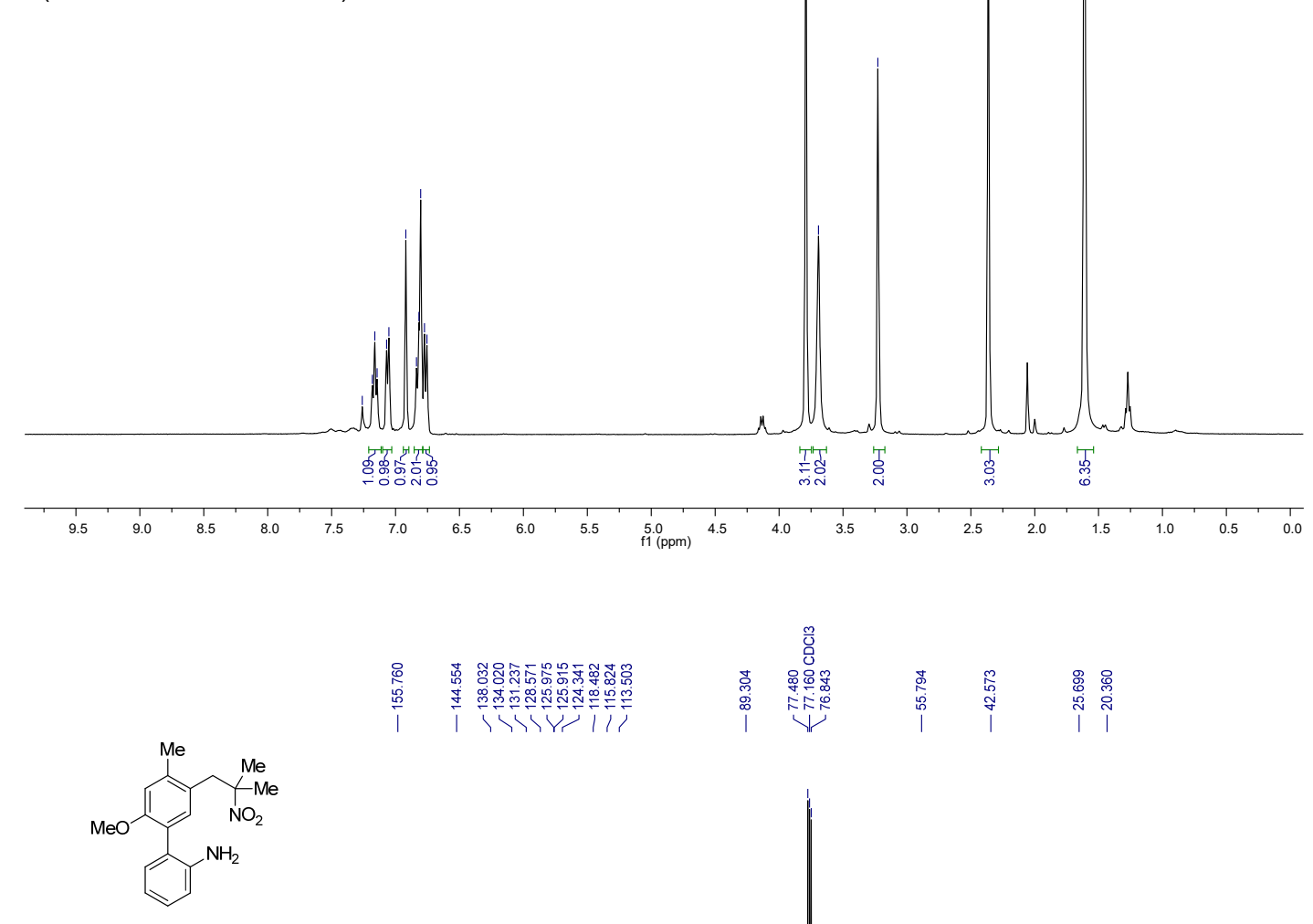

$\left({ }^{13} \mathrm{C}\right.$ NMR, $\left.100 \mathrm{MHz}, \mathrm{CDCl}_{3}\right)$

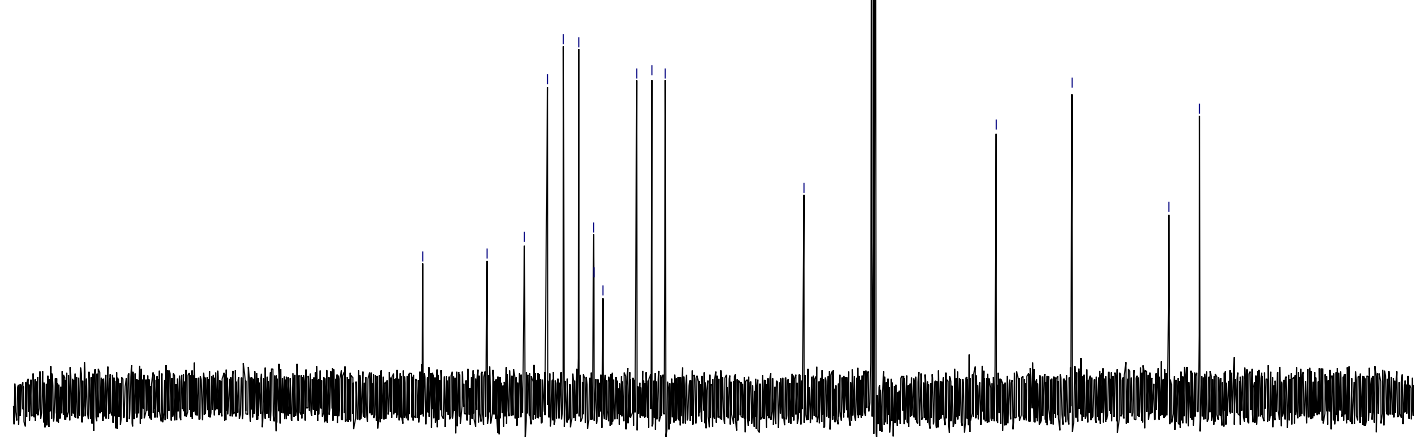

$\begin{array}{llllllllllll}220 & 210 & 200 & 190 & 180 & 170 & 160 & 150 & 140 & 130 & 120 & \begin{array}{c}110 \\ \mathrm{f} 1(\mathrm{ppm})\end{array}\end{array}$ 

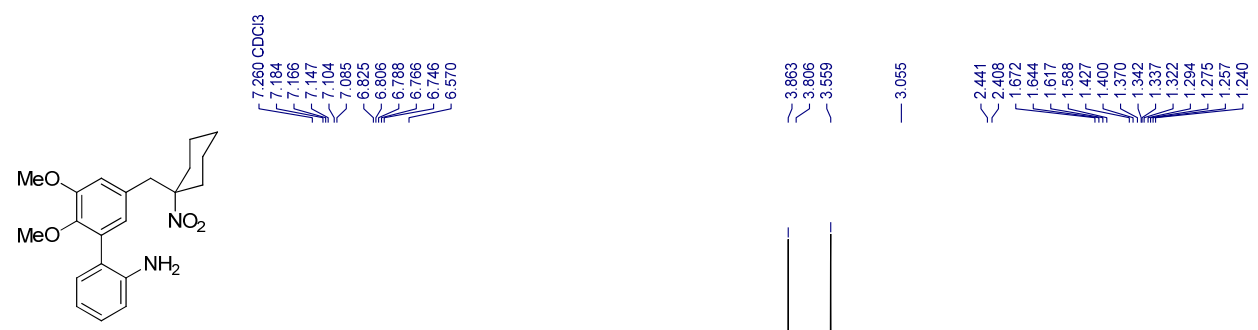

$\left({ }^{1} \mathrm{H} \mathrm{NMR}, 400 \mathrm{MHz}, \mathrm{CDCl}_{3}\right)$
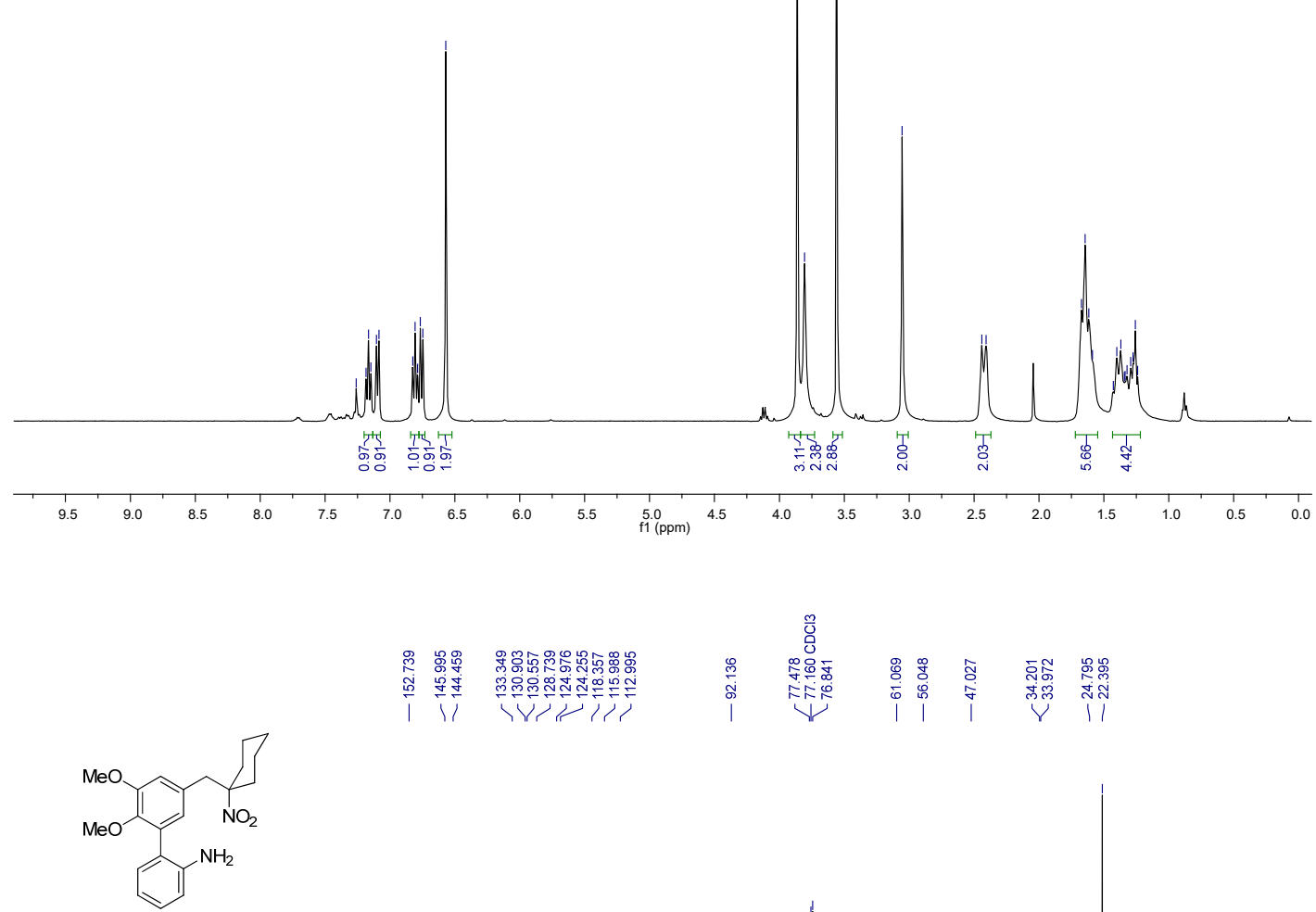

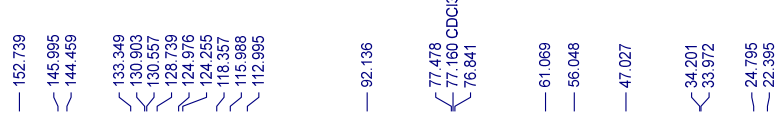

S20

$\left({ }^{13} \mathrm{C}\right.$ NMR, $\left.100 \mathrm{MHz}, \mathrm{CDCl}_{3}\right)$

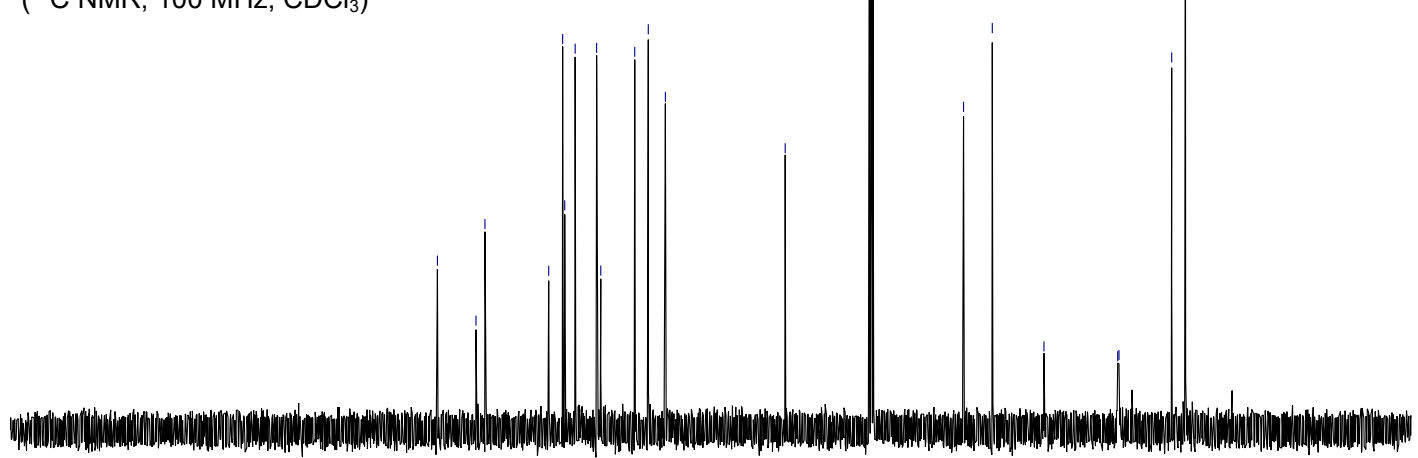

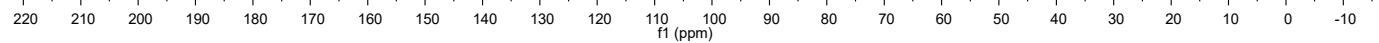




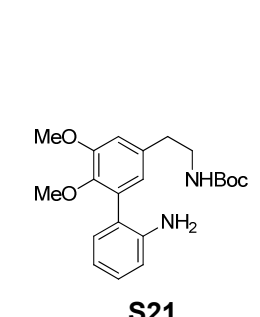

$\frac{m}{0}$

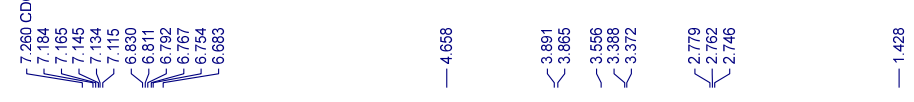

$\left({ }^{1} \mathrm{H} \mathrm{NMR}, 400 \mathrm{MHz}, \mathrm{CDCl}_{3}\right)$
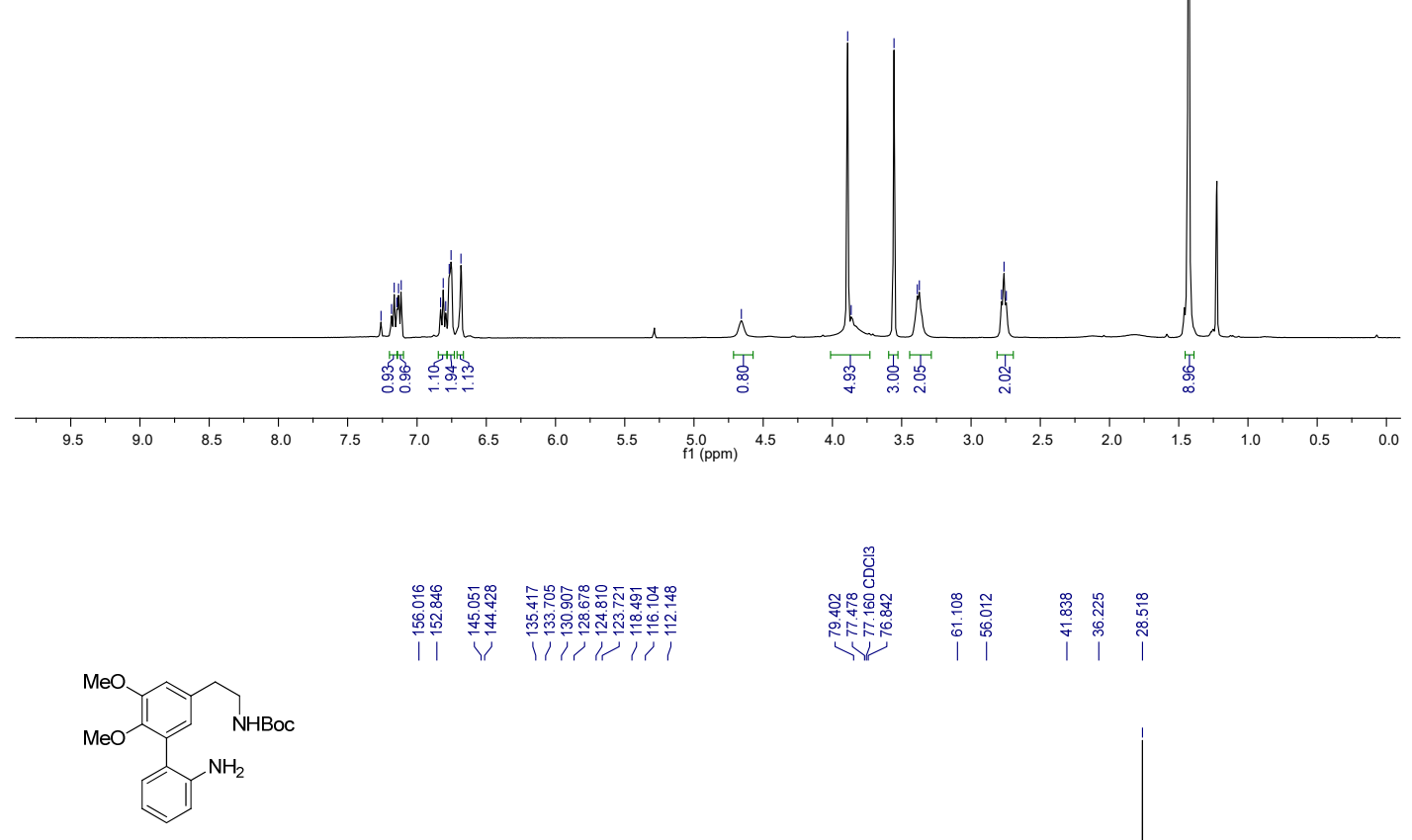

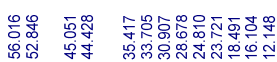

। प गारा

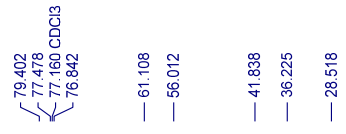

S21

$\left({ }^{13} \mathrm{C} \mathrm{NMR,}, 100 \mathrm{MHz}, \mathrm{CDCl}_{3}\right)$

(13 $\left.\mathrm{CNR}, 100 \mathrm{MHz}, \mathrm{CDCl}_{3}\right)$

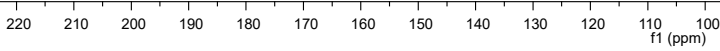




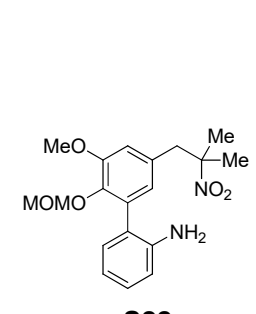

$\frac{m}{0}$

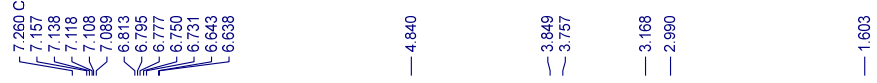

S22

('H NMR, $400 \mathrm{MHz}, \mathrm{CDCl}_{3}$ )
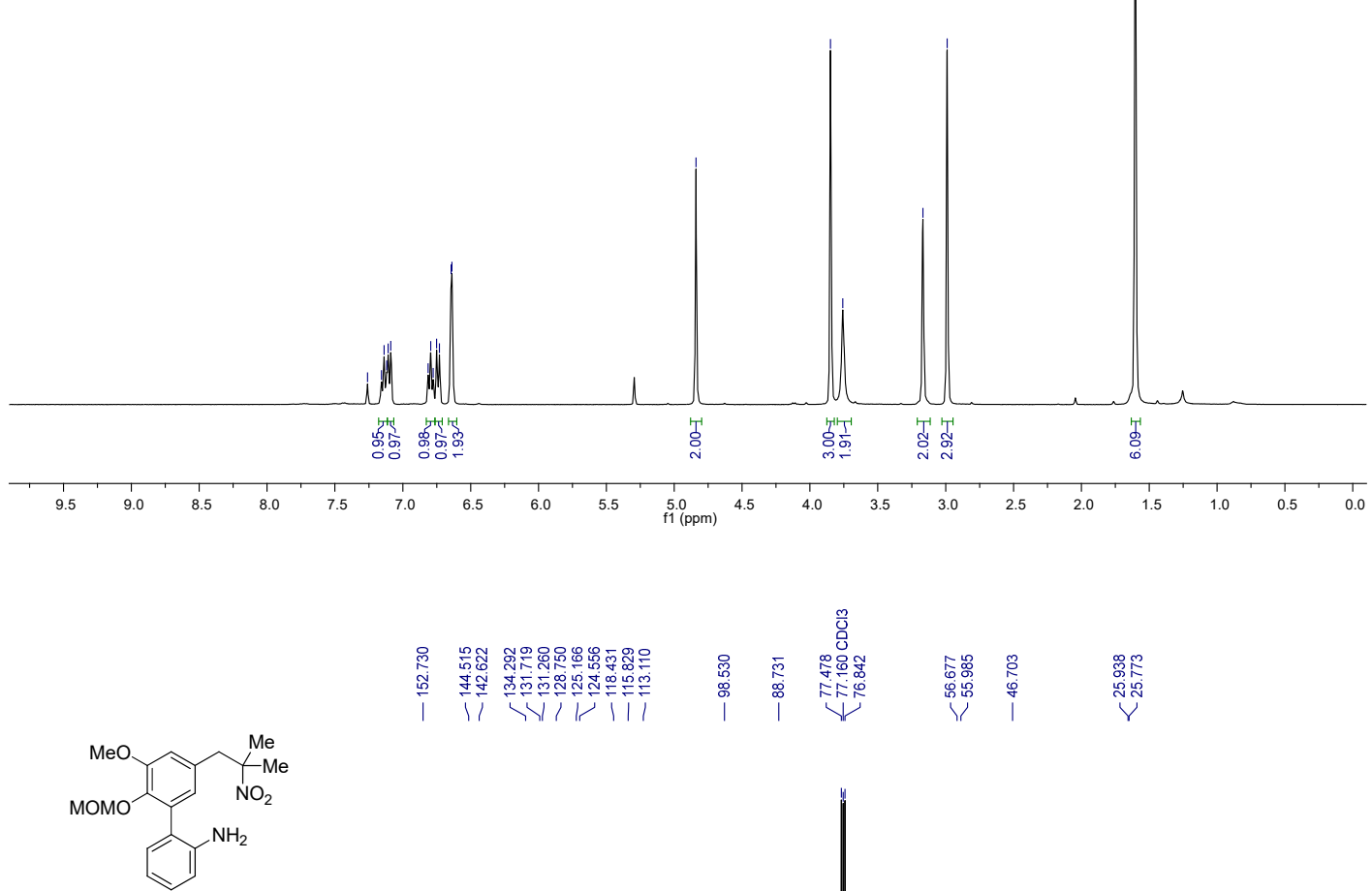

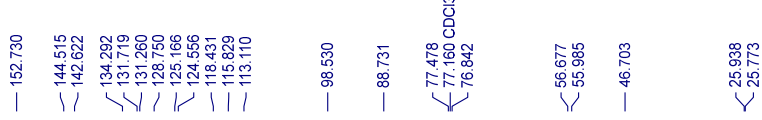

S22

$\left({ }^{13} \mathrm{C}\right.$ NMR, $\left.100 \mathrm{MHz}, \mathrm{CDCl}_{3}\right)$

$\begin{array}{llllllllllll}220 & 210 & 200 & 190 & 180 & 170 & 160 & 150 & 140 & 130 & 120 & \begin{array}{c}110 \\ \mathrm{f} 1(\mathrm{ppm})\end{array}\end{array}$ 


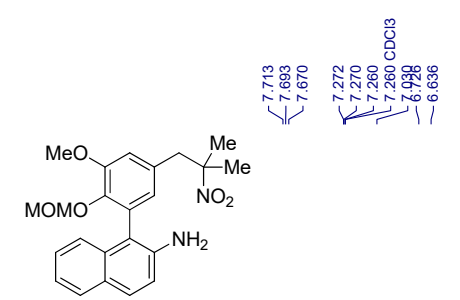

523

$\left({ }^{1} \mathrm{H} \mathrm{NMR}, 400 \mathrm{MHz}, \mathrm{CDCl}_{3}\right)$
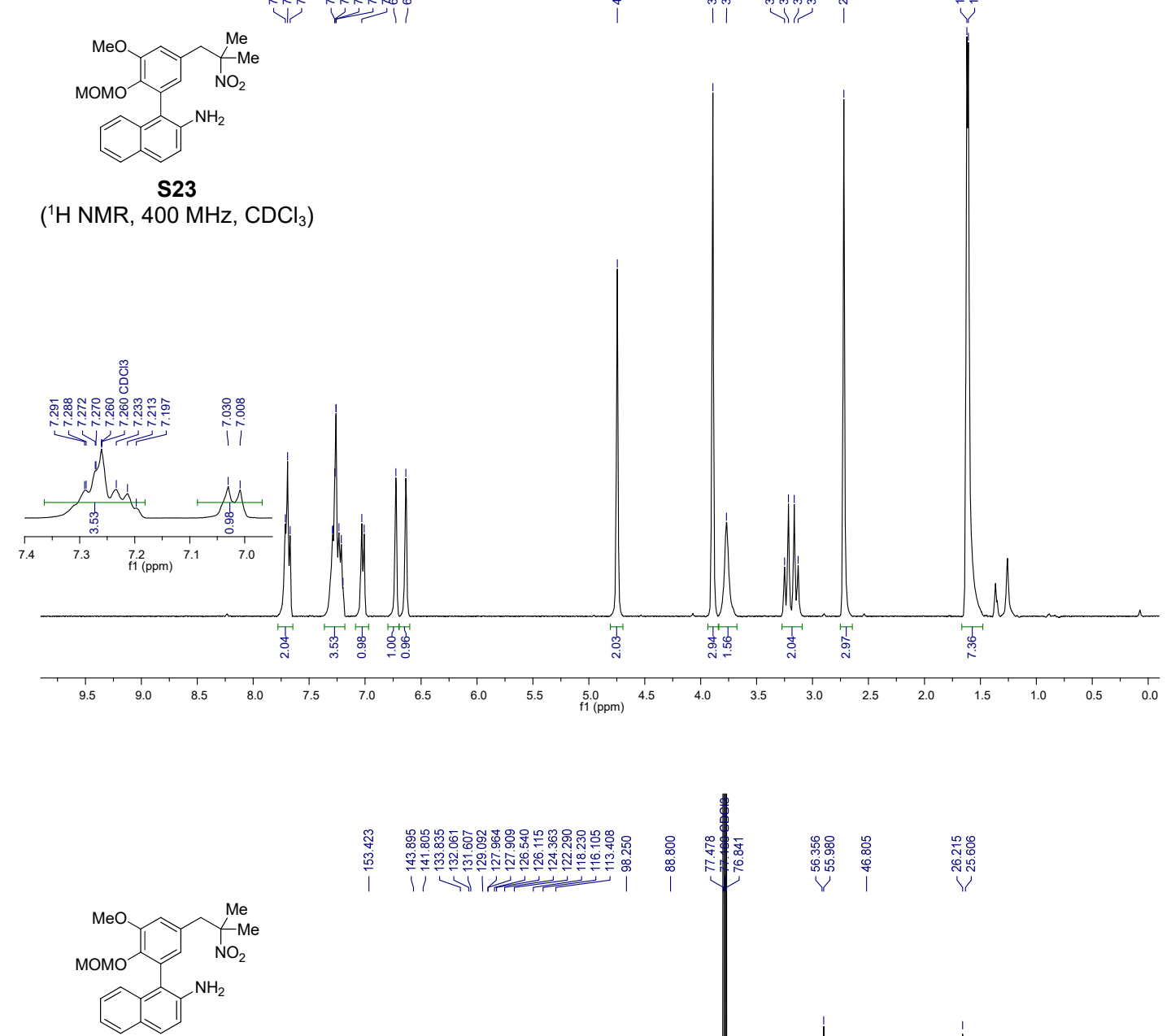

$\mathbf{S 2 3}$

$\left({ }^{13} \mathrm{C} \mathrm{NMR}, 100 \mathrm{MHz}, \mathrm{CDCl}_{3}\right)$

I

|

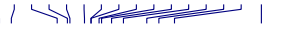

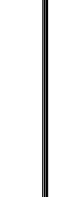

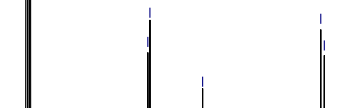

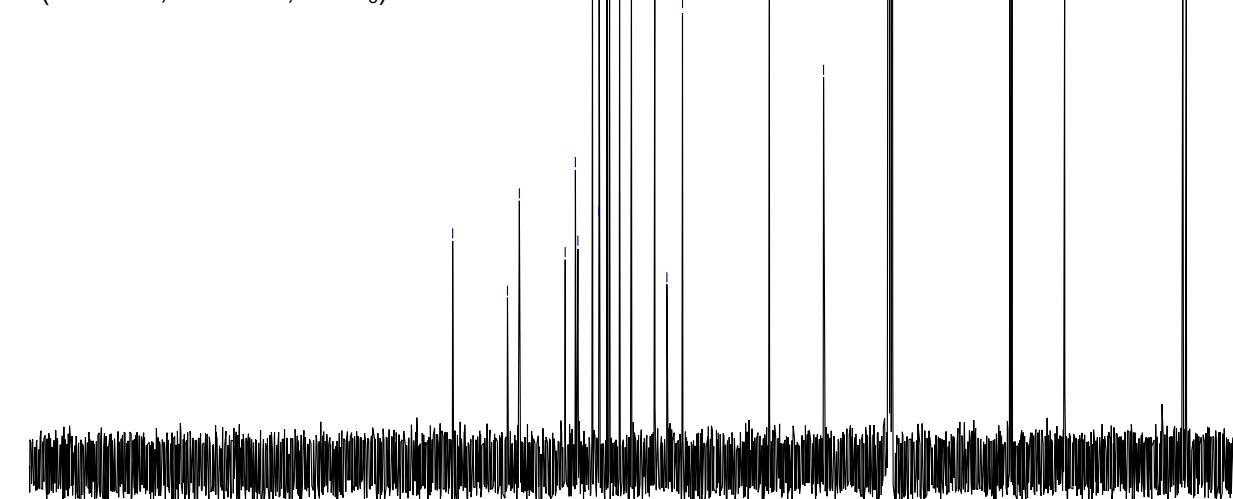

$\begin{array}{llllllllllllll}220 & 210 & 200 & 190 & 180 & 170 & 160 & 150 & 140 & 130 & 120 & \underset{f 10}{\mathrm{f} 1(\mathrm{ppm})} & 100\end{array}$ 


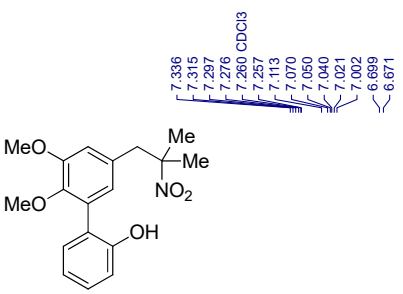

|

$\stackrel{\mathscr{0}}{1}$

S24

( ${ }^{1} \mathrm{H} \mathrm{NMR}, 400 \mathrm{MHz}, \mathrm{CDCl}_{3}$ )
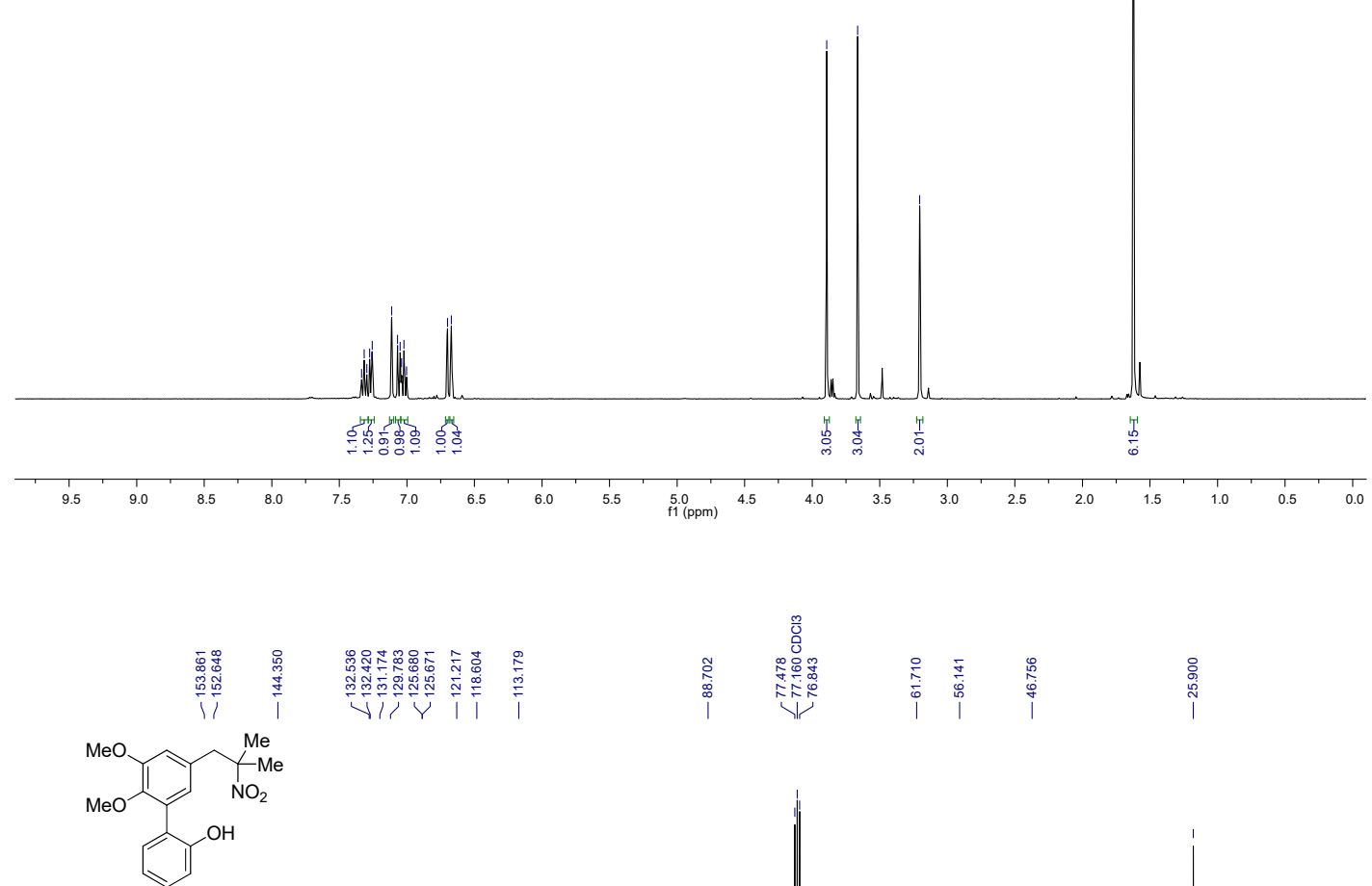

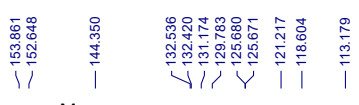

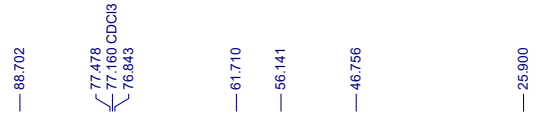

S24

$\left({ }^{13} \mathrm{C} \mathrm{NMR}, 100 \mathrm{MHz}, \mathrm{CDCl}_{3}\right)$

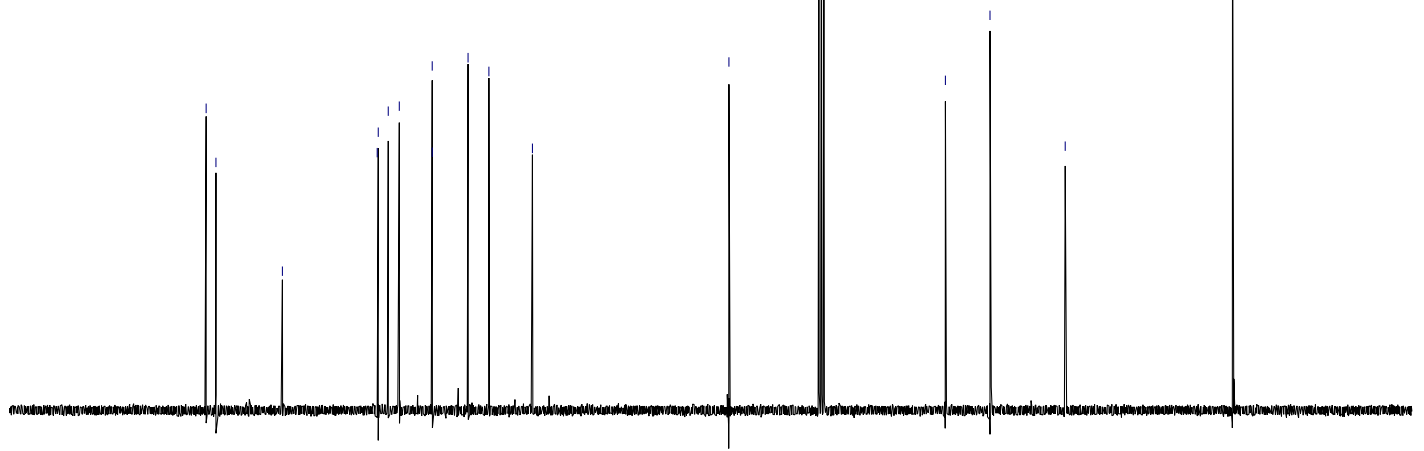




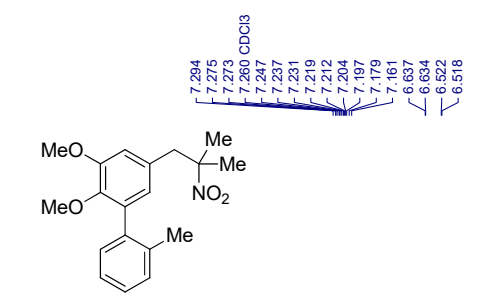

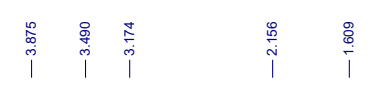

$\left({ }^{1} \mathrm{H}\right.$ NMR, $\left.400 \mathrm{MHz}, \mathrm{CDCl}_{3}\right)$
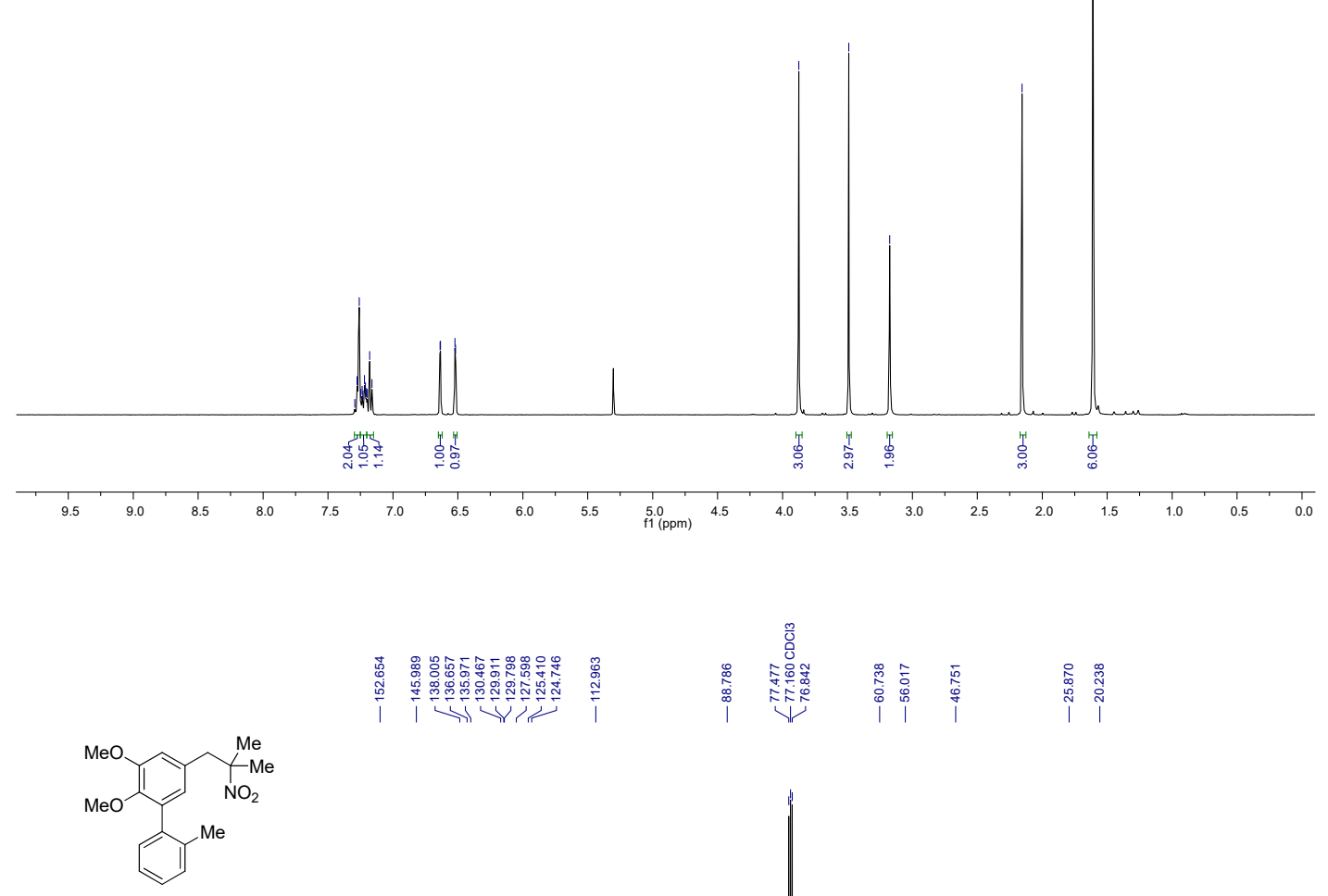

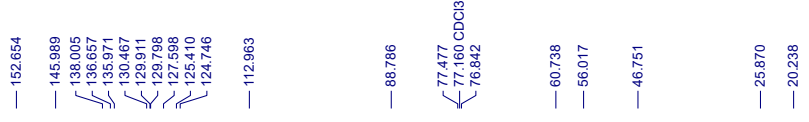

S25

$\left({ }^{13} \mathrm{C} \mathrm{NMR}, 100 \mathrm{MHz}, \mathrm{CDCl}_{3}\right)$

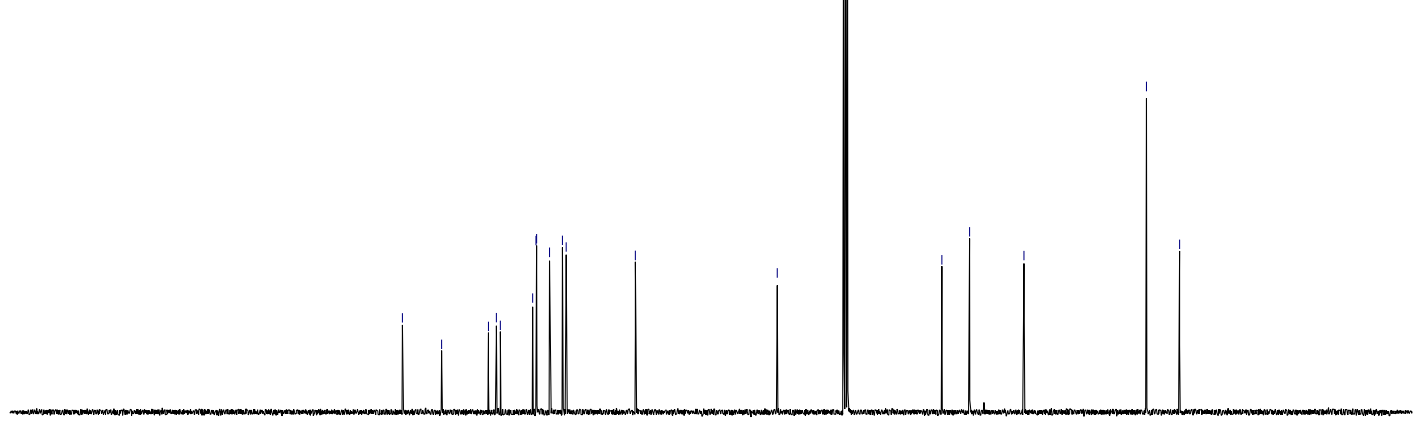

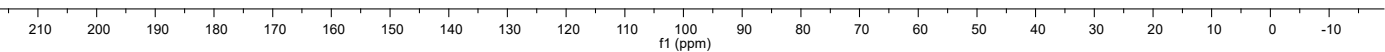




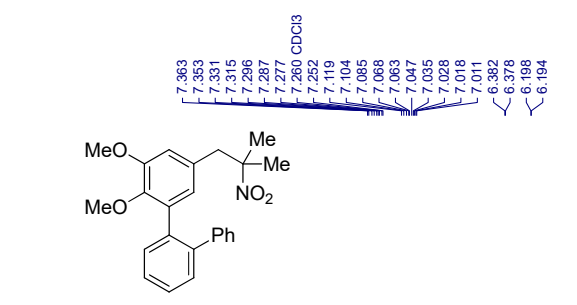

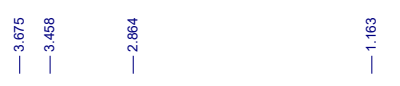

S26

('H NMR, $400 \mathrm{MHz}, \mathrm{CDCl}_{3}$ )
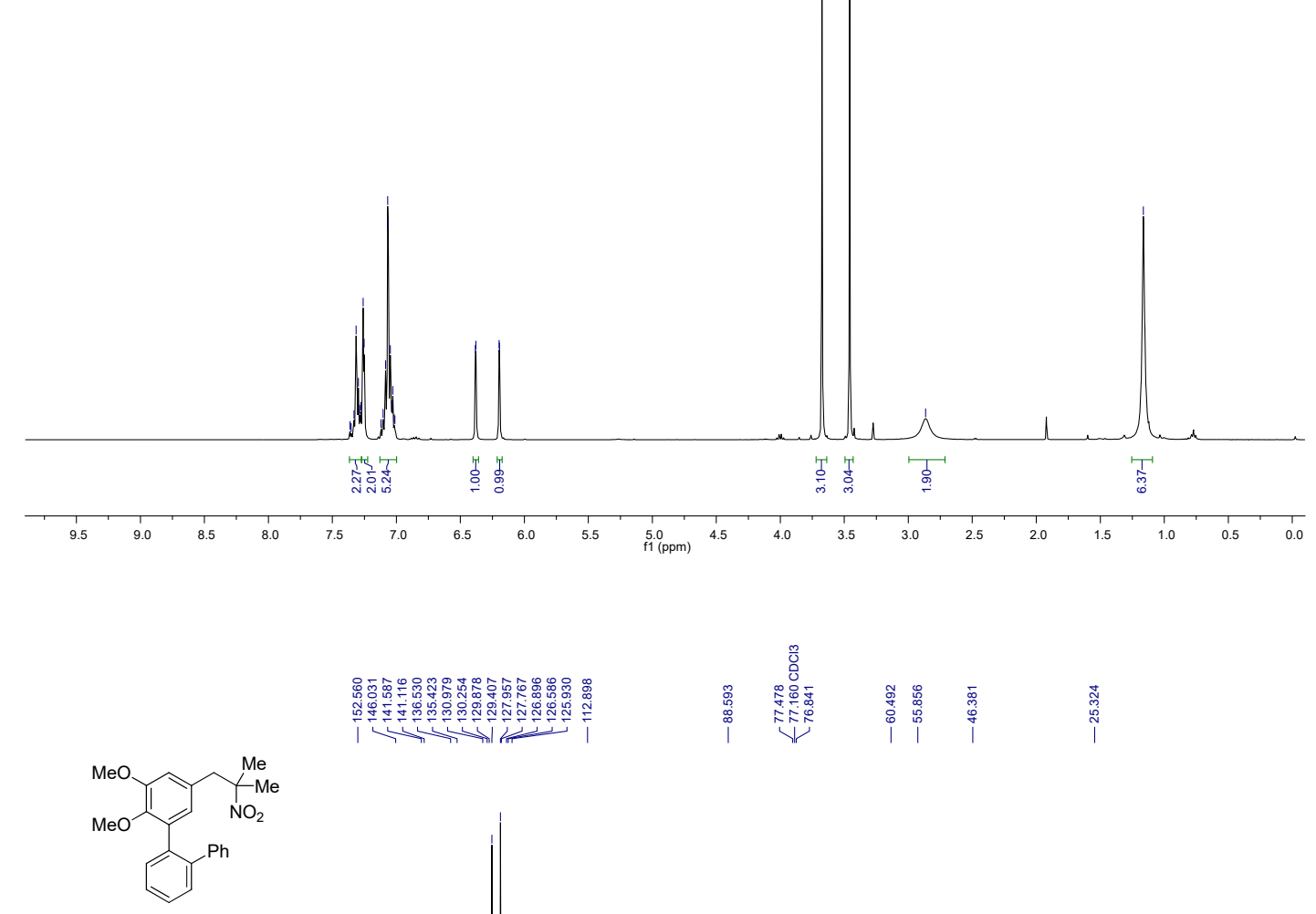

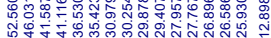

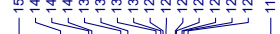

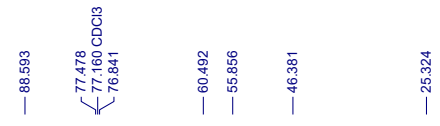

S26

$\left({ }^{13} \mathrm{C} \mathrm{NMR}, 100 \mathrm{MHz}, \mathrm{CDCl}_{3}\right)$

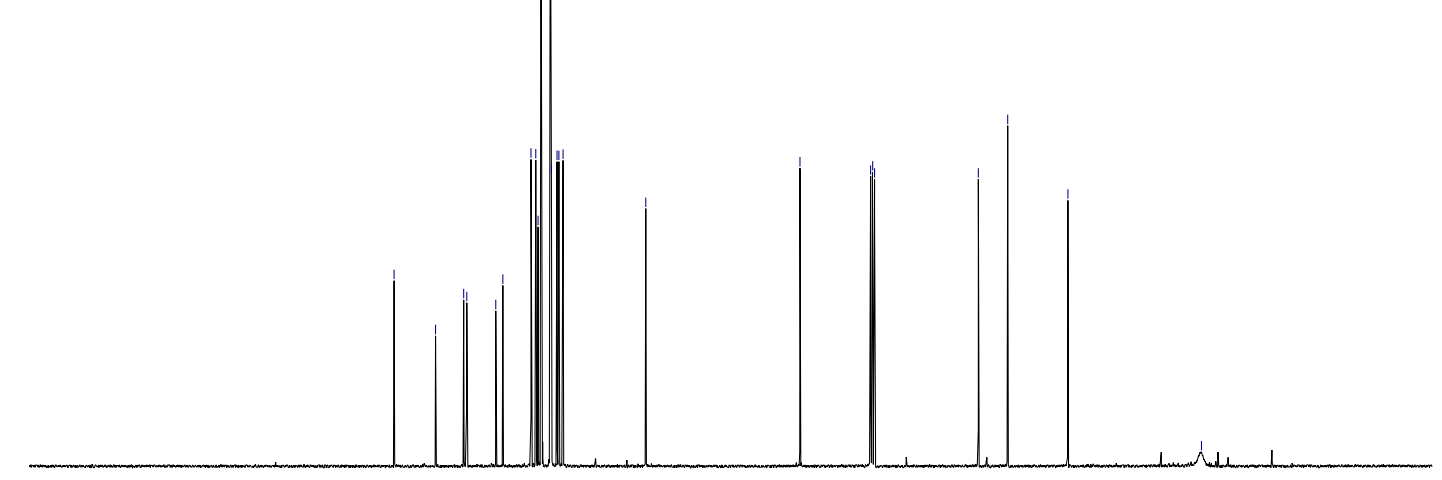

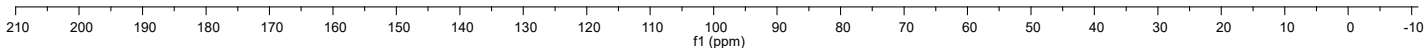




\subsubsection{MOM Deprotection for Substituted Biaryl Intermediates (S27 and S28)}

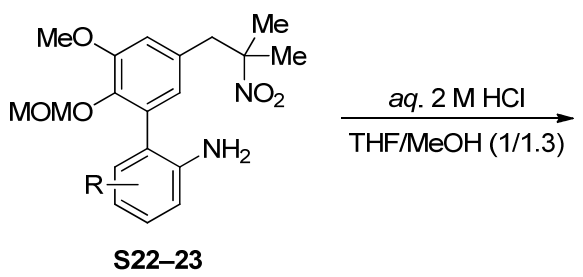<smiles>COc1cc(CC(C)(C)[N+](=O)[O-])cc(-c2c(N)cccc2N)c1O</smiles>

Procedure 8: An oven-dried round-bottom flask equipped with a magnetic stir bar, a solution of $\mathbf{S 2 2}$ or $\mathbf{S 2 3}$ in THF/MeOH $(1 / 1.3,0.10 \mathrm{M})$ was added. Then, aqueous $2 \mathrm{M}$ $\mathrm{HCl}$ (4.8 equiv) was added at $0{ }^{\circ} \mathrm{C}$. The reaction mixture was allowed to stir for overnight at $50{ }^{\circ} \mathrm{C}$ in a heating block. The reaction was quenched with saturated aqueous $\mathrm{NaHCO}_{3}$ at $0{ }^{\circ} \mathrm{C}$, extracted with EtOAc three times. The combined organic phase was washed with brine, dried with anhydrous $\mathrm{MgSO}_{4}$, filtered with EtOAc, and concentrated in vacuo. The crude product was used without purification process.<smiles>COc1cc(CC(C)(C)[N+](=O)[O-])cc(-c2ccccc2N)c1O</smiles>

2'-Amino-3-methoxy-5-(2-methyl-2-nitropropyl)-[1,1'-biphenyl]-2-ol (S27) was synthesized by following Procedure 8 . The crude product was used without purification process.<smiles>COc1cc(CC(C)(C)[N+](=O)[O-])cc(-c2c(N)ccc3ccccc23)c1O</smiles>

2-(2-Aminonaphthalen-1-yl)-6-methoxy-4-(2-methyl-2-nitropropyl)phenol (S28) was synthesized by following Procedure 8 . The crude product was used without purification process. 


\subsubsection{Fuctionalizations of Aniline for Substituted Biaryl Intermediates (S29-49)}
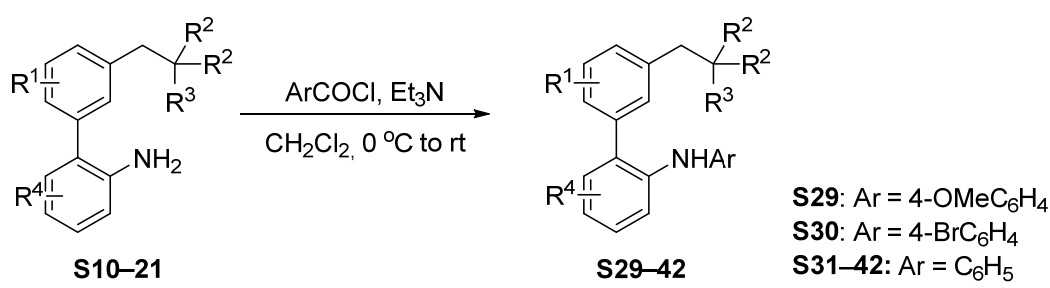

Procedure 9: Benzoylation

To a round-bottom flask equipped with a magnetic stirring bar, S10-21 (1.0 equiv), and triethylamine (2.0 equiv) were added and subsequently dissolved in $\mathrm{CH}_{2} \mathrm{Cl}_{2}(0.4 \mathrm{M})$. Then, benzoyl chloride (1.2 equiv) was added dropwise and allowed to stir for overnight at $\mathrm{rt}$. The reaction was diluted with $\mathrm{CH}_{2} \mathrm{Cl}_{2}$, transferred to a separatory funnel, and quenched with a saturated aqueous $\mathrm{NH}_{4} \mathrm{Cl}$. The organic layer was separated, and the aqueous layer was extracted an additional two times with $\mathrm{CH}_{2} \mathrm{Cl}_{2}$. The combined organic layers were then rinsed with water, dried with anhydrous $\mathrm{MgSO}_{4}$, and concentrated in vacuo. The crude material was then purified by flash chromatography to afford the desired material S29-42.

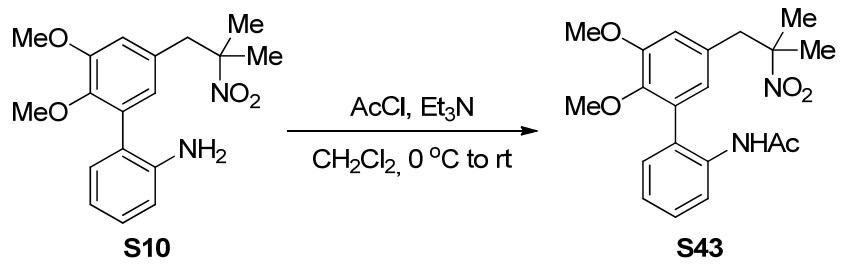

Procedure 10: Acetylation

To a round-bottom flask equipped with a magnetic stirring bar, a solution of S10 (1.0 equiv), triethylamine (1.1 equiv) in anhydrous $\mathrm{CH}_{2} \mathrm{Cl}_{2}(0.060 \mathrm{M})$ was added. Then, acetyl chloride (1.1 equiv) was added dropwise at $0{ }^{\circ} \mathrm{C}$ and the allowed to stir for overnight at $\mathrm{rt}$. The solvent was evaporated under reduced pressure, The residue was dissolved in diethyl ether and then rinsed with water. The organic layer was dried with anhydrous $\mathrm{MgSO}_{4}$, filtered with $\mathrm{CH}_{2} \mathrm{Cl}_{2}$, and concentrated in vacuo. The crude material was then purified by flash chromatography to afford the desired material $\mathbf{S 4 3}$. 

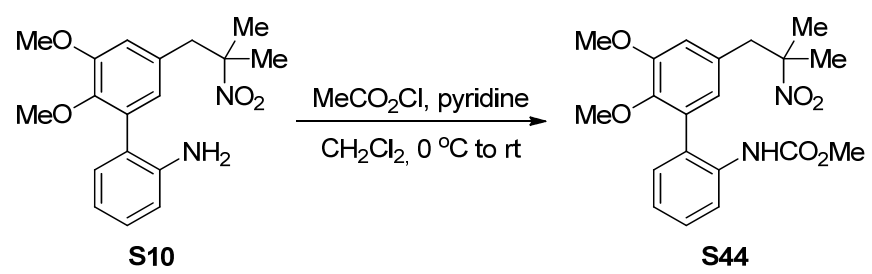

Procedure 11: Carbamoylation

To a round-bottom flask equipped with a magnetic stirring bar, $\mathbf{S 1 0}$ (1.0 equiv), and pyridine (1.3 equiv) were added and subsequently dissolved in $\mathrm{CH}_{2} \mathrm{Cl}_{2}(0.090 \mathrm{M})$. Then, methyl chloroformate (1.2 equiv) was added dropwise and allowed to stir at rt. The reaction was diluted with $\mathrm{CH}_{2} \mathrm{Cl}_{2}$, transferred to a separatory funnel, and quenched with a saturated aqueous $\mathrm{NH}_{4} \mathrm{Cl}$. The organic layer was separated, and the aqueous layer was extracted an additional two times with $\mathrm{CH}_{2} \mathrm{Cl}_{2}$. The combined organic layers were then rinsed with water, dried with anhydrous $\mathrm{MgSO}_{4}$, filtered with $\mathrm{CH}_{2} \mathrm{Cl}_{2}$, and concentrated in vacuo. The crude material was then purified by flash chromatography to afford the desired material S44.
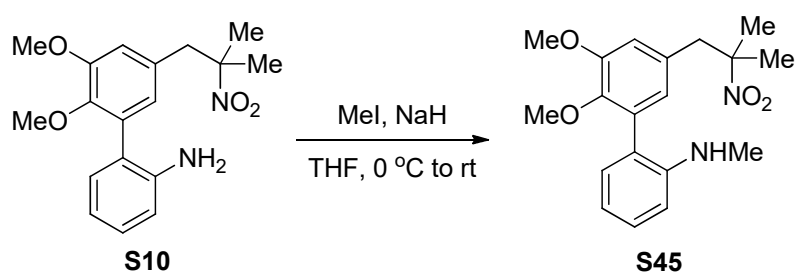

Procedure 12: Methylation

To a round-bottom flask equipped with a magnetic stirring bar, $\mathrm{NaH}$ (1.0 equiv) and THF $(0.1 \mathrm{M})$ were added. S10 (1.0 equiv) was added at $0{ }^{\circ} \mathrm{C}$ and the mixture was stirred for $30 \mathrm{~min}$. Iodomethane ( 1.5 equiv) was added and the reaction mixture was warmed to room temperature and allowed to stir for three days. Then, the reaction mixture was quenched with water and was extracted three times with EtOAc. The combined organic layers were washed with brine, dried with anhydrous $\mathrm{MgSO}_{4}$, filtered with EtOAc, and concentrated in vacuo. The crude material was then purified by flash chromatography to afford the desired material $\mathbf{S 4 5}$. 

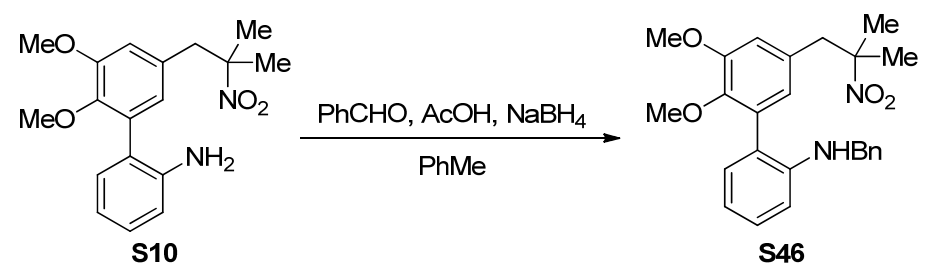

Procedure 13: Benzylation

To a round-bottom flask equipped with a magnetic stirring bar, a solution of S10 (1.0 equiv) in PhMe $(0.50 \mathrm{M})$ was added. To this solution, benzaldehyde (1.5 equiv) and acetic acid (10.5 equiv) were subsequently added. After the reaction was refluxed in a heating block for $16 \mathrm{~h}$, the reaction was cooled to $0{ }^{\circ} \mathrm{C}$. Then, sodium borohydride $(2.0$ equiv) was added portionwise. The reaction mixture was allowed to stir at $\mathrm{rt}$ for $5 \mathrm{~h}$. The reaction was cooled to $0{ }^{\circ} \mathrm{C}$ and quenched with water. The resulting mixture was extracted three times with $\mathrm{CH}_{2} \mathrm{Cl}_{2}$, and washed with brine. The combined organic layers were dried with anhydrous $\mathrm{MgSO}_{4}$, filtered with $\mathrm{CH}_{2} \mathrm{Cl}_{2}$, and concentrated in vacuo. The crude material was then purified by flash chromatography to afford the desired material S46.
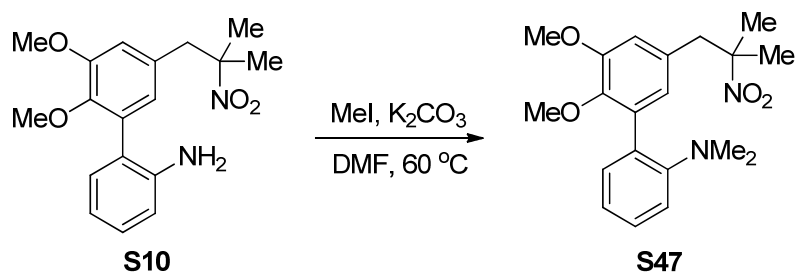

Procedure 14: Dimethylation

To a round-bottom flask equipped with a magnetic stirring bar, iodomethane (4.0 equiv) was added dropwise to stirred suspension of $\mathbf{S 1 0}$ ( 1.0 equiv) and $\mathrm{K}_{2} \mathrm{CO}_{3}$ ( 1.5 equiv) in $\operatorname{DMF}(0.50 \mathrm{M})$ at $60^{\circ} \mathrm{C}$ in a heating block for overnight. The progress of the reaction was monitored by TLC. The reaction mixture was cooled to rt and diluted with water. The aqueous phase was extracted with $\mathrm{CH}_{2} \mathrm{Cl}_{2}$ three times. The organic layer was dried with anhydrous $\mathrm{MgSO}_{4}$, filtered with $\mathrm{CH}_{2} \mathrm{Cl}_{2}$, and concentrated in vacuo. The crude material was then purified by flash chromatography to afford the desired material $\mathbf{S 4 7}$. 

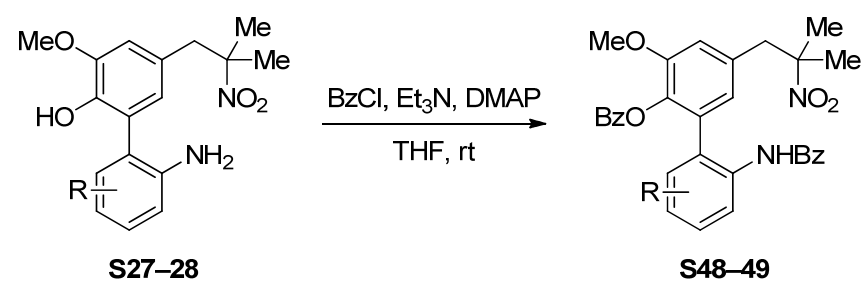

Procedure 15: $N, O$-Bisbenzoylation

To a round-bottom flask equipped with a magnetic stirring bar, a solution of $\mathbf{S 2 7}$ or $\mathbf{S 2 8}$ (1.0 equiv), DMAP (2 mol\%), and triethylamine (2.5 equiv) in THF $(0.30 \mathrm{M})$ was added. Then, the solution of benzoyl chloride (2.1 equiv) in THF $(1.4 \mathrm{M})$ was added to flask and the reaction mixture was allowed to stir for $1 \mathrm{~h}$ at $\mathrm{rt}$. The reaction mixture was concentrated in vacuo, and the crude material was then purified by flash chromatography to afford the desired material S48 or S49. 


\section{Characterization and Spectra of S29-49}

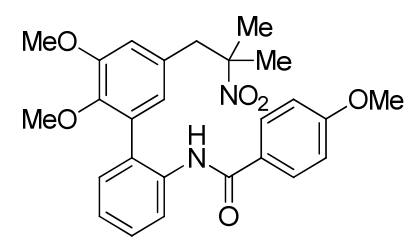

N-(2',3'-Dimethoxy-5'-(2-methyl-2-nitropropyl)-[1,1'-biphenyl]-2-yl)-4-

methoxybenzamide (S29) was synthesized by following Procedure 9. The crude material was purified by normal-phase column chromatography using an eluent of $25 \%$ EtOAc/Hx to provide $\mathbf{S 2 9}$ as a white solid (436 mg, 78\%).

m.p. $62{ }^{\circ} \mathrm{C}$

${ }^{1} \mathbf{H}$ NMR $\left(400 \mathrm{MHz}, \mathrm{CDCl}_{3}\right) \delta 8.71(\mathrm{~s}, 1 \mathrm{H}), 8.16(\mathrm{~d}, J=7.6 \mathrm{~Hz}, 1 \mathrm{H}), 7.72(\mathrm{~d}, J=8.3$ $\mathrm{Hz}, 2 \mathrm{H}), 7.43(\mathrm{t}, J=6.4 \mathrm{~Hz}, 1 \mathrm{H}), 7.30-7.18(\mathrm{~m}, 2 \mathrm{H}), 6.91(\mathrm{~d}, J=8.3 \mathrm{~Hz}, 2 \mathrm{H}), 6.67(\mathrm{~s}$, $1 \mathrm{H}), 6.62(\mathrm{~s}, 1 \mathrm{H}), 3.89(\mathrm{~s}, 3 \mathrm{H}), 3.83(\mathrm{~s}, 3 \mathrm{H}), 3.61(\mathrm{~s}, 3 \mathrm{H}), 3.26-3.08(\mathrm{~m}, 2 \mathrm{H}), 1.58(\mathrm{~d}$, $J=19.5 \mathrm{~Hz}, 6 \mathrm{H})$.

${ }^{13}$ C NMR $\left(100 \mathrm{MHz}, \mathrm{CDCl}_{3}\right) \delta 164.9,162.4,152.8,144.9,135.6,133.0,132.0,130.8$, 130.4, 129.0 (2C), 128.7, 127.2, 125.7, 124.8, 123.8, 114.0 (2C), 113.8, 88.7, 61.5, $56.2,55.5,46.6,26.1,25.7$.

IR (FT-ATR, $\mathrm{cm}^{-1}, \mathrm{CH}_{2} \mathrm{Cl}_{2}$ ) $v_{\max } 3366,2935,2839,1669,1606,1583,1535,1504$, $1485,1465,1442,1421,1396,1372,1344,1299,1247,1174,1142,1118,1089,1032$, $1002,946,899,844,789,762,734,698,675,626,572,529$.

HRMS (ESI) $m / z$ : $[\mathrm{M}+\mathrm{H}]^{+}$Calcd for $\mathrm{C}_{26} \mathrm{H}_{28} \mathrm{~N}_{2} \mathrm{O}_{6}$ 465.2026; found 465.2020.

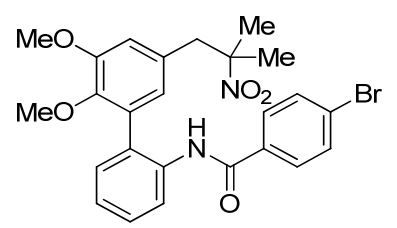

4-Bromo- $N$-(2',3'-dimethoxy-5'-(2-methyl-2-nitropropyl)-[1,1'-biphenyl]-2-

yl)benzamide (S30) was synthesized by following Procedure 9. The crude material was purified by normal-phase column chromatography using an eluent of $25 \% \mathrm{EtOAc} / \mathrm{Hx}$ to provide $\mathbf{S 3 0}$ as a white solid (405 $\mathrm{mg}, 65 \%$ ).

m.p. $137^{\circ} \mathrm{C}$

${ }^{1} \mathbf{H}$ NMR $\left(400 \mathrm{MHz}, \mathrm{CDCl}_{3}\right) \delta 8.84(\mathrm{~s}, 1 \mathrm{H}), 8.12(\mathrm{~d}, J=7.1 \mathrm{~Hz}, 1 \mathrm{H}), 7.59(\mathrm{dd}, J=25.8$, $8.3 \mathrm{~Hz}, 4 \mathrm{H}), 7.47(\mathrm{~d}, J=6.6 \mathrm{~Hz}, 1 \mathrm{H}), 7.35-7.21(\mathrm{~m}, 2 \mathrm{H}), 6.68(\mathrm{~s}, 1 \mathrm{H}), 6.62(\mathrm{~s}, 1 \mathrm{H})$, $3.89(\mathrm{~d}, J=2.4 \mathrm{~Hz}, 3 \mathrm{H}), 3.61(\mathrm{~d}, J=2.6 \mathrm{~Hz}, 3 \mathrm{H}), 3.24-3.08(\mathrm{~m}, 2 \mathrm{H}), 1.65-1.51(\mathrm{~m}$, $\mathrm{H})$.

${ }^{13}$ C NMR (100 MHz, $\left.\mathrm{CDCl}_{3}\right) \delta 164.5,152.8,144.7,135.1,133.8,132.9,132.2,132.0$ (2C), 130.9, 130.7, 128.9 (2C), 128.8, 126.5, 125.6, 125.3, 124.0, 113.8, 88.7, 61.6, $56.2,46.7,26.1,25.8$.

IR (FT-ATR, $\mathrm{cm}^{-1}, \mathrm{CH}_{2} \mathrm{Cl}_{2}$ ) $v_{\max } 3351,2931,1674,1587,1535,1485,1467,1444$, 1421, 1396, 1372, 1344, 1310, 1263, 1248, 1232, 1183, 1142, 1117, 1089, 1070, 1050, 1036, 1009, 947, 897, 754, 844, 817, 789, 756, 735, 709, 673, 625, 592, 580, 527, 456. HRMS (ESI) $m / z$ : $[\mathrm{M}+\mathrm{H}]^{+}$Calcd for $\mathrm{C}_{25} \mathrm{H}_{25} \mathrm{BrN}_{2} \mathrm{O}_{5}$ 513.1025; found 513.1019. 


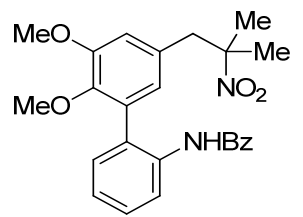

$N$-(2',3'-Dimethoxy-5'-(2-methyl-2-nitropropyl)-[1,1'-biphenyl]-2-yl)benzamide (S31) was synthesized by following Procedure 9. The crude material was purified by normal-phase column chromatography using an eluent of $25 \% \mathrm{EtOAc} / \mathrm{Hx}$ to provide S31 as a yellow gum (393 $\mathrm{mg}, 91 \%)$.

${ }^{1} \mathbf{H}$ NMR $\left(400 \mathrm{MHz}, \mathrm{CDCl}_{3}\right) \delta 8.81(\mathrm{~s}, 1 \mathrm{H}), 8.17(\mathrm{~d}, J=8.1 \mathrm{~Hz}, 1 \mathrm{H}), 7.75(\mathrm{~d}, J=7.8$ $\mathrm{Hz}, 2 \mathrm{H}), 7.52-7.38(\mathrm{~m}, 4 \mathrm{H}), 7.32-7.21(\mathrm{~m}, 1 \mathrm{H}), 6.68(\mathrm{~s}, 1 \mathrm{H}), 6.63(\mathrm{~s}, 1 \mathrm{H}), 3.88(\mathrm{~s}, 3 \mathrm{H})$, $3.62(\mathrm{~s}, 3 \mathrm{H}), 3.22-3.08(\mathrm{~m}, 2 \mathrm{H}), 1.58(\mathrm{~d}, J=18.8 \mathrm{~Hz}, 6 \mathrm{H})$.

${ }^{13} \mathrm{C} \mathrm{NMR}\left(100 \mathrm{MHz}, \mathrm{CDCl}_{3}\right) \delta 165.4,152.7,144.9,135.3,134.8,132.9,132.1,131.7$, $130.8,130.5,128.8$ (2C), 128.7, 127.2 (2C), 125.6, 125.0, 123.8, 113.9, 88.6, 61.5, 56.2, 46.6, 26.0, 25.7.

IR (FT-ATR, $\mathrm{cm}^{-1}, \mathrm{CH}_{2} \mathrm{Cl}_{2}$ ) v $v_{\max } 3352,2998,2938,2835,1673,1581,1534,1484$, $1467,1444,1421,1396,1372,1344,1303,1246,1184,1142,1119,1073,1050,1036$, 1002, 946, 906, 855, 789, 757, 734, 708, 671, 636, 588, 530 .

HRMS (ESI) $m / z$ : $[\mathrm{M}+\mathrm{Na}]^{+}$Calcd for $\mathrm{C}_{25} \mathrm{H}_{26} \mathrm{~N}_{2} \mathrm{O}_{5} 457.1739$; found 457.1736.

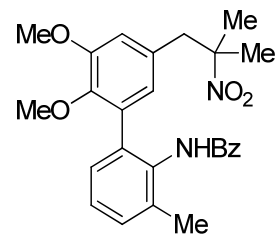

$N$-(2',3'-Dimethoxy-3-methyl-5'-(2-methyl-2-nitropropyl)-[1,1'-biphenyl]-2-

yl)benzamide (S32) was synthesized by following Procedure 9. The crude material was purified by normal-phase column chromatography using an eluent of $33 \% \mathrm{EtOAc} / \mathrm{Hx}$ to provide $\mathbf{S 3 2}$ as a white solid (377 $\mathrm{mg}, 84 \%$ ).

m.p. $163^{\circ} \mathrm{C}$

${ }^{1} \mathbf{H}$ NMR $\left(400 \mathrm{MHz}, \mathrm{CDCl}_{3}\right) \delta 8.68(\mathrm{~s}, 1 \mathrm{H}), 7.74(\mathrm{~d}, J=7.4 \mathrm{~Hz}, 2 \mathrm{H}), 7.48(\mathrm{t}, J=7.2$ $\mathrm{Hz} 1 \mathrm{H}), 7.41(\mathrm{t}, J=7.3 \mathrm{~Hz}, 2 \mathrm{H}), 7.36(\mathrm{~d}, J=7.5 \mathrm{~Hz}, 1 \mathrm{H}), 7.31(\mathrm{t}, J=7.6 \mathrm{~Hz}, 1 \mathrm{H}), 7.20$ $(\mathrm{d}, J=7.3 \mathrm{~Hz}, 1 \mathrm{H}), 6.66(\mathrm{~s}, 1 \mathrm{H}), 6.61(\mathrm{~s}, 1 \mathrm{H}), 3.85(\mathrm{~s}, 3 \mathrm{H}), 3.61(\mathrm{~s}, 3 \mathrm{H}), 3.12(\mathrm{~s}, 2 \mathrm{H})$, $2.41(\mathrm{~s}, 3 \mathrm{H}), 1.56(\mathrm{~d}, J=25.1 \mathrm{~Hz}, 6 \mathrm{H})$.

${ }^{13} \mathrm{C}$ NMR $\left(100 \mathrm{MHz}, \mathrm{CDCl}_{3}\right) \delta 165.3,152.4,144.5,136.7,135.3,134.5,134.0,133.8$, $131.7,131.5,130.7,128.6$ (2C), 128.1, 127.2 (2C), 127.1, 125.4, 113.5, 88.7, 61.5, 56.1, 46.6, 25.9, 25.7, 19.0.

IR (FT-ATR, $\mathrm{cm}^{-1}, \mathrm{CH}_{2} \mathrm{Cl}_{2}$ ) v $v_{\max } 3351,2936,1666,1580,1536,1508,1484,1455$, 1418, 1397, 1372, 1346, 1286, 1264, 1229, 1184, 1144, 1060, 1004, 932, 855, 795, 778, 740, 710, 694, 661, 610, 580, 550, 522.

HRMS (ESI) $m / z$ : $[\mathrm{M}+\mathrm{H}]^{+}$Calcd for $\mathrm{C}_{26} \mathrm{H}_{28} \mathrm{~N}_{2} \mathrm{O}_{5}$ 449.2077; found 449.2072. 


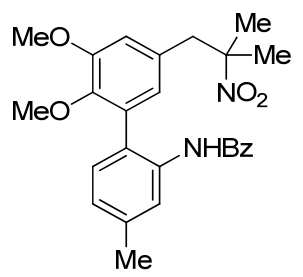

$N$-(2',3'-Dimethoxy-4-methyl-5'-(2-methyl-2-nitropropyl)-[1,1'-biphenyl]-2-

yl)benzamide (S33) was synthesized by following Procedure 9. The crude material was purified by normal-phase column chromatography using an eluent of $25 \% \mathrm{EtOAc} / \mathrm{Hx}$ to provide $\mathbf{S 3 3}$ as a white solid (730 $\mathrm{mg}, 91 \%)$.

m.p. $119^{\circ} \mathrm{C}$

${ }^{1}$ H NMR $\left(400 \mathrm{MHz}, \mathrm{CDCl}_{3}\right) \delta 8.84(\mathrm{~s}, 1 \mathrm{H}), 7.99(\mathrm{~s}, 1 \mathrm{H}), 7.80-7.68(\mathrm{~m}, 2 \mathrm{H}), 7.56-7.35$ $(\mathrm{m}, 3 \mathrm{H}), 7.19-7.13(\mathrm{~m}, 1 \mathrm{H}), 7.10-7.02(\mathrm{~m}, 1 \mathrm{H}), 6.65(\mathrm{~d}, J=2.3 \mathrm{~Hz}, 1 \mathrm{H}), 6.60(\mathrm{~d}, J=$ $2.1 \mathrm{~Hz}, 1 \mathrm{H}), 3.87(\mathrm{~d}, J=2.8 \mathrm{~Hz}, 3 \mathrm{H}), 3.61(\mathrm{~d}, J=2.9 \mathrm{~Hz}, 3 \mathrm{H}), 3.15(\mathrm{t}, J=16.2 \mathrm{~Hz}$, $2 \mathrm{H}), 2.44(\mathrm{~d}, J=2.6 \mathrm{~Hz}, 3 \mathrm{H}), 1.57(\mathrm{~d}, J=17.4 \mathrm{~Hz}, 6 \mathrm{H})$.

${ }^{13} \mathrm{C} \mathrm{NMR}\left(100 \mathrm{MHz}, \mathrm{CDCl}_{3}\right) \delta 165.3,152.7,144.8,138.7,135.0,134.9,133.0,132.0$, $131.7,130.6,128.8$ (2C), 127.7, 127.2 (2C), 126.0, 125.7, 124.4, 113.6, 88.7, 61.5, 56.2, 46.6, 26.0, 25.7, 21.5.

IR (FT-ATR, $\mathrm{cm}^{-1}, \mathrm{CH}_{2} \mathrm{Cl}_{2}$ ) $v_{\max } 3357,2937,1674,1618,1572,1534,1467,1415$, 1372, 1344, 1294, 1264, 1230, 1183, 1145, 1126, 1092, 1073, 1043, 1002, 855, 821, 798, 734, 707, 596, 547, 520 .

HRMS (ESI) $m / z$ : $[\mathrm{M}+\mathrm{H}]^{+}$Calcd for $\mathrm{C}_{26} \mathrm{H}_{28} \mathrm{~N}_{2} \mathrm{O}_{5}$ 449.2077; found 449.2072.

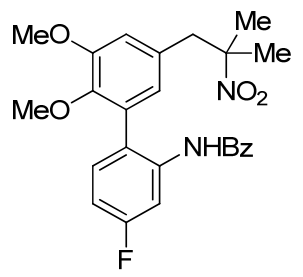

$N$-(4-Fluoro-2',3'-dimethoxy-5'-(2-methyl-2-nitropropyl)-[1,1'-biphenyl]-2-

yl)benzamide (S34) was synthesized by following Procedure 9. The crude material was purified by normal-phase column chromatography using an eluent of $25 \% \mathrm{EtOAc} / \mathrm{Hx}$ to provide $\mathbf{S 3 4}$ as a white solid (546 $\mathrm{mg}, 87 \%$ ).

m.p. $127-130{ }^{\circ} \mathrm{C}$

${ }^{1} \mathbf{H}$ NMR $\left(400 \mathrm{MHz}, \mathrm{CDCl}_{3}\right) \delta 8.76(\mathrm{~s}, 1 \mathrm{H}), 8.07(\mathrm{~d}, J=10.9 \mathrm{~Hz}, 1 \mathrm{H}), 7.73(\mathrm{~d}, J=7.7$ $\mathrm{Hz}, 2 \mathrm{H}), 7.50(\mathrm{t}, J=7.1 \mathrm{~Hz}, 1 \mathrm{H}), 7.43(\mathrm{t}, J=7.6 \mathrm{~Hz}, 2 \mathrm{H}), 7.22(\mathrm{t}, J=7.4 \mathrm{~Hz}, 1 \mathrm{H}), 6.94$ $(\mathrm{t}, J=8.2 \mathrm{~Hz}, 1 \mathrm{H}), 6.69(\mathrm{~s}, 1 \mathrm{H}), 6.58(\mathrm{~s}, 1 \mathrm{H}), 3.89(\mathrm{~s}, 3 \mathrm{H}), 3.63(\mathrm{~s}, 3 \mathrm{H}), 3.23-3.08(\mathrm{~m}$, $2 \mathrm{H}), 1.59$ (d, $J=19.8 \mathrm{~Hz}, 6 \mathrm{H})$.

${ }^{13}$ C NMR $\left(100 \mathrm{MHz}, \mathrm{CDCl}_{3}\right) \delta 165.3,162.7(\mathrm{~d}, J=244.7 \mathrm{~Hz}, 1 \mathrm{C}), 152.9,144.9,136.8$ $(\mathrm{d}, J=11.5 \mathrm{~Hz}, 1 \mathrm{C}), 134.5,132.2,132.0,131.8(\mathrm{~d}, J=9.3 \mathrm{~Hz}, 1 \mathrm{C}), 128.86(2 \mathrm{C}), 127.16$ (2C), 125.7 (d, $J=3.2 \mathrm{~Hz}, 1 \mathrm{C}), 125.5,114.1,111.7$ (d, $J=21.7 \mathrm{~Hz}, 1 \mathrm{C}), 110.4$ (d, $J=$ $26.3 \mathrm{~Hz}, 1 \mathrm{C}), 88.6,61.5,56.2,46.6,26.1,25.7$.

IR (FT-ATR, $\mathrm{cm}^{-1}, \mathrm{CH}_{2} \mathrm{Cl}_{2}$ ) $v_{\max } 3418,2937,1679,1598,1582,1525,1484,1465$, 1419, 1397, 1372, 1345, 1286, 1253, 1184, 1163, 1138, 1117, 1077, 1043, 1028, 1002, 978, 898, 856, 798, 758, 735, 707, 680, 582, 556.

HRMS (ESI) $m / z$ : $[\mathrm{M}+\mathrm{H}]^{+}$Calcd for $\mathrm{C}_{25} \mathrm{H}_{25} \mathrm{FN}_{2} \mathrm{O}_{5} 453.1826$; found 453.1820 . 


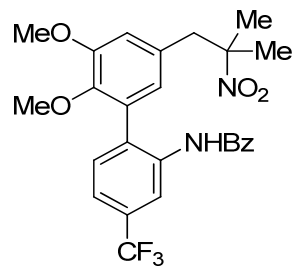

$N$-(2',3'-Dimethoxy-5'-(2-methyl-2-nitropropyl)-4-(trifluoromethyl)-[1,1'-

biphenyl]-2-yl)benzamide (S35) was synthesized by following Procedure 9. The crude material was purified by normal-phase column chromatography using an eluent of $25 \%$ EtOAc/Hx to provide $\mathbf{S 3 5}$ as a white solid (607 mg, 60\%).

m.p. $133^{\circ} \mathrm{C}$

${ }^{1} \mathbf{H}$ NMR $\left(400 \mathrm{MHz}, \mathrm{CDCl}_{3}\right) \delta 8.80(\mathrm{~s}, 1 \mathrm{H}), 8.57(\mathrm{~s}, 1 \mathrm{H}), 7.74(\mathrm{~d}, J=7.5 \mathrm{~Hz}, 2 \mathrm{H})$, 7.54-7.37 (m, 5H), $6.73(\mathrm{~s}, 1 \mathrm{H}), 6.59(\mathrm{~s}, 1 \mathrm{H}), 3.90(\mathrm{~d}, J=1.1 \mathrm{~Hz}, 3 \mathrm{H}), 3.65(\mathrm{~d}, J=1.2$ $\mathrm{Hz}, 3 \mathrm{H}), 3.25-3.09(\mathrm{~m}, 2 \mathrm{H}), 1.59(\mathrm{~d}, J=21.6 \mathrm{~Hz}, 6 \mathrm{H})$.

${ }^{13}$ C NMR $\left(100 \mathrm{MHz}, \mathrm{CDCl}_{3}\right) \delta 165.4,152.9,144.9,136.0,134.3,133.6,132.4,132.1$, 131.6, 131.3, 130.9 (q, $J=32.5 \mathrm{~Hz}, 1 \mathrm{C}), 128.9(2 \mathrm{C}), 127.2(2 \mathrm{C}), 125.1,124.0$ (q, $J=$ $271.2 \mathrm{~Hz}, 1 \mathrm{C}), 121.4$ (q, $J=3.7 \mathrm{~Hz}, 1 \mathrm{C}), 120.4$ (q, $J=4.0 \mathrm{~Hz}, 1 \mathrm{C}), 114.6,88.6,61.7$, 56.2, 46.6, 26.1, 25.7.

IR (FT-ATR, $\mathrm{cm}^{-1}, \mathrm{CH}_{2} \mathrm{Cl}_{2}$ ) $v_{\max } 3420,2939,1679,1582,1535,1484,1467,1419$, 1398, 1372, 1345, 1330, 1289, 1268, 1232, 1166, 1121, 1075, 1041, 1001, 922, 901, 880, 856, 832, 797, 707, 693, 674, 663, 585, 521.

HRMS (ESI) $m / z$ : [M+H] $]^{+}$Calcd for $\mathrm{C}_{26} \mathrm{H}_{25} \mathrm{~F}_{3} \mathrm{~N}_{2} \mathrm{O}_{5}$ 503.1794; found 503.1788.

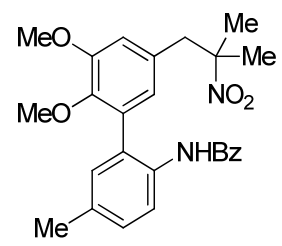

$N$-(2',3'-Dimethoxy-5-methyl-5'-(2-methyl-2-nitropropyl)-[1,1'-biphenyl]-2-

yl)benzamide (S36) was synthesized by following Procedure 9. The crude material was purified by normal-phase column chromatography using an eluent of $25 \% \mathrm{EtOAc} / \mathrm{Hx}$ to provide $\mathbf{S 3 6}$ as a white solid (572 $\mathrm{mg}, 88 \%$ ).

m.p. $66{ }^{\circ} \mathrm{C}$

${ }^{1} \mathbf{H}$ NMR $\left(400 \mathrm{MHz}, \mathrm{CDCl}_{3}\right) \delta 8.79(\mathrm{~s}, 1 \mathrm{H}), 8.02(\mathrm{~d}, J=8.1 \mathrm{~Hz}, 1 \mathrm{H}), 7.77(\mathrm{~d}, J=7.7$ $\mathrm{Hz}, 2 \mathrm{H}), 7.46$ (dt, $J=14.5,6.8 \mathrm{~Hz}, 3 \mathrm{H}), 7.28(\mathrm{~d}, J=8.1 \mathrm{~Hz}, 1 \mathrm{H}), 7.11(\mathrm{~s}, 1 \mathrm{H}), 6.68$ (s, $1 \mathrm{H}), 6.65(\mathrm{~s}, 1 \mathrm{H}), 3.89(\mathrm{~s}, 3 \mathrm{H}), 3.64(\mathrm{~s}, 3 \mathrm{H}), 3.25-3.11(\mathrm{~m}, 2 \mathrm{H}), 2.41(\mathrm{~s}, 3 \mathrm{H}), 1.60(\mathrm{~d}$, $J=19.3 \mathrm{~Hz}, 6 \mathrm{H})$.

${ }^{13}$ C NMR $\left(100 \mathrm{MHz}, \mathrm{CDCl}_{3}\right) \delta 165.3,152.7,144.8,134.9,134.7,133.1,132.7,132.0$, 131.6, 131.2, 130.6, 129.3, 128.7, 127.1, 125.6, 124.0, 113.7, 88.6, 61.5, 56.1, 46.6, 26.0, 25.7, 21.1.

IR (FT-ATR, $\mathrm{cm}^{-1}, \mathrm{CH}_{2} \mathrm{Cl}_{2}$ ) $v_{\max } 3365,2937,1672,1582,1535,1514,1466,1421$, 1397, 1372, 1343, 1301, 1266, 1229, 1184, 1144, 1123, 1095, 1073, 1049, 1027, 1001, 930, 855, 818, 794, 773, 734, 706, 673, 647, 617, 584, 545.

HRMS (ESI) $m / z$ : $[\mathrm{M}+\mathrm{H}]^{+}$Calcd for $\mathrm{C}_{26} \mathrm{H}_{28} \mathrm{~N}_{2} \mathrm{O}_{5} 449.2077$; found 449.2072. 


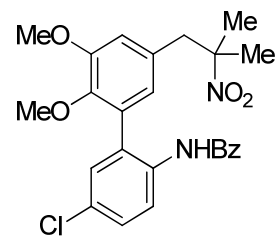

$N$-(5-Chloro-2',3'-dimethoxy-5'-(2-methyl-2-nitropropyl)-[1,1'-biphenyl]-2-

yl)benzamide (S37) was synthesized by following Procedure 9. The crude material was purified by normal-phase column chromatography using an eluent of $25 \% \mathrm{EtOAc} / \mathrm{Hx}$ to provide $\mathbf{S 3 7}$ as a white solid (480 $\mathrm{mg}, 68 \%$ ).

m.p. $69^{\circ} \mathrm{C}$

${ }^{1} \mathbf{H}$ NMR $\left(400 \mathrm{MHz}, \mathrm{CDCl}_{3}\right) \delta 8.76(\mathrm{~s}, 1 \mathrm{H}), 8.13(\mathrm{~d}, J=8.7 \mathrm{~Hz}, 1 \mathrm{H}), 7.73(\mathrm{~d}, J=8.1$ $\mathrm{Hz}, 2 \mathrm{H}), 7.49$ (t, $J=7.3 \mathrm{~Hz}, 1 \mathrm{H}), 7.42(\mathrm{t}, J=7.9 \mathrm{~Hz}, 3 \mathrm{H}), 7.27(\mathrm{~d}, J=2.2 \mathrm{~Hz}, 1 \mathrm{H}), 6.69$ $(\mathrm{s}, 1 \mathrm{H}), 6.60(\mathrm{~s}, 1 \mathrm{H}), 3.88(\mathrm{~s}, 3 \mathrm{H}), 3.65(\mathrm{~s}, 3 \mathrm{H}), 3.17(\mathrm{~s}, 2 \mathrm{H}), 1.58(\mathrm{~d}, J=18.5 \mathrm{~Hz}, 6 \mathrm{H})$. ${ }^{13}$ C NMR $\left(100 \mathrm{MHz}, \mathrm{CDCl}_{3}\right) \delta 165.4,152.8,144.7,134.5,134.1,132.3,132.1,131.9$, 131.6, 130.4, 129.9, 128.8 (2C), 128.6, 127.2 (2C), 125.2, 125.1, 114.2, 88.6, 61.7, $56.2,46.5,26.0,25.8$.

IR (FT-ATR, $\mathrm{cm}^{-1}, \mathrm{CH}_{2} \mathrm{Cl}_{2}$ ) $v_{\max } 3417,2938,1675,1602,1582,1536,1509,1486$, 1465, 1421, 1394, 1372, 1340, 1295, 1265, 1233, 1184, 1169, 1144, 1121, 1073, 1046, $1027,1000,897,875,855,820,789,705,657,640,614,583,544,419$.

HRMS (ESI) $m / z$ : $[\mathrm{M}+\mathrm{H}]^{+}$Calcd for $\mathrm{C}_{25} \mathrm{H}_{25} \mathrm{ClN}_{2} \mathrm{O}_{5} 469.1530$; found 469.1525 .

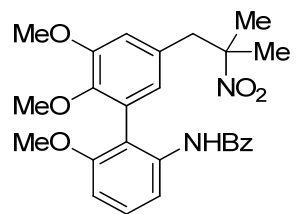

$N$-(2',3',6-Trimethoxy-5'-(2-methyl-2-nitropropyl)-[1,1'-biphenyl]-2-

yl)benzamide (S38) was synthesized by following Procedure 9. The crude material was purified by normal-phase column chromatography using an eluent of $25 \% \mathrm{EtOAc} / \mathrm{Hx}$ to provide $\mathbf{S 3 8}$ as a colorless gum (200 $\mathrm{mg}, 81 \%)$.

${ }^{1} \mathbf{H}$ NMR $\left(400 \mathrm{MHz}, \mathrm{CDCl}_{3}\right) \delta 8.04(\mathrm{~s}, 1 \mathrm{H}), 7.98(\mathrm{~d}, J=8.2 \mathrm{~Hz}, 1 \mathrm{H}), 7.58(\mathrm{~d}, J=8.2$ $\mathrm{Hz}, 2 \mathrm{H}), 7.49-7.34(\mathrm{~m}, 4 \mathrm{H}), 6.82(\mathrm{~d}, J=8.3 \mathrm{~Hz}, 1 \mathrm{H}), 6.67(\mathrm{~s}, 1 \mathrm{H}), 6.58(\mathrm{~s}, 1 \mathrm{H}), 3.88$ $(\mathrm{s}, 3 \mathrm{H}), 3.76(\mathrm{~s}, 3 \mathrm{H}), 3.60(\mathrm{~d}, J=1.3 \mathrm{~Hz}, 3 \mathrm{H}), 3.14(\mathrm{~s}, 2 \mathrm{H}), 1.57(\mathrm{~s}, 3 \mathrm{H}), 1.51(\mathrm{~s}, 3 \mathrm{H})$.

${ }^{13}$ C NMR $\left(100 \mathrm{MHz}, \mathrm{CDCl}_{3}\right) \delta 165.3,157.2,153.1,146.1,136.8,134.9,131.7,131.3$, $129.4,128.8(2 \mathrm{C}), 127.9,127.0$ (2C), 126.2, 118.2, 115.0, 114.0, 107.3, 88.7, 61.2, 56.1, 55.9, 46.7, 25.9, 25.7.

IR (FT-ATR, $\mathrm{cm}^{-1}, \mathrm{CH}_{2} \mathrm{Cl}_{2}$ ) $v_{\max } 3417,2937,2837,1717,1678,1587,1536,1484$, 1468, 1439, 1417, 1397, 1372, 1340, 1287, 1260, 1183, 1137, 1070, 1041, 1004, 935, 856, 790, 739, 709, 664, 613, 568, 416.

HRMS (ESI) $m / z$ : $[\mathrm{M}+\mathrm{H}]^{+}$Calcd for $\mathrm{C}_{26} \mathrm{H}_{28} \mathrm{~N}_{2} \mathrm{O}_{6} 465.2026$; found 465.2022. 


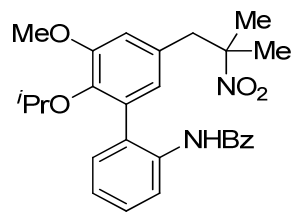

$N$-(2'-Isopropoxy-3'-methoxy-5'-(2-methyl-2-nitropropyl)-[1,1'-biphenyl]-2-

yl)benzamide (S39) was synthesized by following Procedure 9. The crude material was purified by normal-phase column chromatography using an eluent of $25 \% \mathrm{EtOAc} / \mathrm{Hx}$ to provide $\mathbf{S 3 9}$ as a white solid (164 $\mathrm{mg}, 84 \%$ ).

m.p. $78^{\circ} \mathrm{C}$

${ }^{1} \mathbf{H}$ NMR $\left(400 \mathrm{MHz}, \mathrm{CDCl}_{3}\right) \delta 9.21(\mathrm{~s}, 1 \mathrm{H}), 8.11(\mathrm{~d}, J=7.7 \mathrm{~Hz}, 1 \mathrm{H}), 7.85(\mathrm{~d}, J=7.1$ $\mathrm{Hz}, 2 \mathrm{H}), 7.53-7.40(\mathrm{~m}, 4 \mathrm{H}), 7.32-7.20(\mathrm{~m}, 2 \mathrm{H}), 6.66(\mathrm{~s}, 2 \mathrm{H}), 4.28-4.16(\mathrm{~m}, 1 \mathrm{H}), 3.86$ $(\mathrm{s}, 3 \mathrm{H}), 3.17(\mathrm{q}, J=13.9 \mathrm{~Hz}, 2 \mathrm{H}), 1.60(\mathrm{~d}, J=19.4 \mathrm{~Hz}, 6 \mathrm{H}), 1.25(\mathrm{~d}, J=5.9 \mathrm{~Hz}, 3 \mathrm{H})$, $0.84(\mathrm{~d}, J=5.9 \mathrm{~Hz}, 3 \mathrm{H})$.

${ }^{13} \mathrm{C}$ NMR $\left(100 \mathrm{MHz}, \mathrm{CDCl}_{3}\right) \delta 165.30,153.20,142.63,135.25,134.92,134.18$, $131.85,131.67,131.62,131.10,128.70$ (2C), 128.46, 127.30 (2C), 125.80, 125.11, $124.23,113.74,88.71,77.16,76.96,56.17,46.64,26.10,25.68,22.70,22.06$.

IR (FT-ATR, $\mathrm{cm}^{-1}, \mathrm{CH}_{2} \mathrm{Cl}_{2}$ ) $v_{\max } 3343,3061,2976,2933,2873,2151,1674,1582$, 1537, 1521, 1496, 1469, 1440, 1421, 1397, 1372, 1345, 1303, 1247, 1223, 1174, 1148, 1103, 1052, 1037, 928, 853, 799, 759, 709, 625, 583, 466, 413.

HRMS (ESI) $m / z$ : $[\mathrm{M}+\mathrm{H}]^{+}$Calcd for $\mathrm{C}_{27} \mathrm{H}_{30} \mathrm{~N}_{2} \mathrm{O}_{5} 463.2233$; found 463.2227.

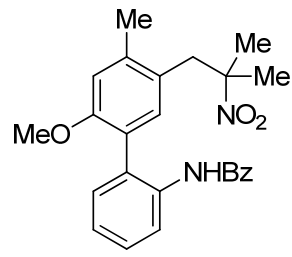

$N$-(2'-Methoxy-4'-methyl-5'-(2-methyl-2-nitropropyl)-[1,1'-biphenyl]-2-

yl)benzamide (S40) was synthesized by following Procedure 9. The crude material was purified by normal-phase column chromatography using an eluent of $25 \% \mathrm{EtOAc} / \mathrm{Hx}$ to provide $\mathbf{S 4 0}$ as a colorless gum (391 $\mathrm{mg}, 88 \%$ ).

${ }^{1} \mathbf{H}$ NMR $\left(400 \mathrm{MHz}, \mathrm{CDCl}_{3}\right) \delta 8.38(\mathrm{~s}, 1 \mathrm{H}), 8.24(\mathrm{~d}, J=7.3 \mathrm{~Hz}, 1 \mathrm{H}), 7.66(\mathrm{~d}, J=7.5$ $\mathrm{Hz}, 2 \mathrm{H}), 7.54-7.36(\mathrm{~m}, 4 \mathrm{H}), 7.23(\mathrm{~s}, 2 \mathrm{H}), 6.94(\mathrm{~s}, 1 \mathrm{H}), 6.84(\mathrm{~s}, 1 \mathrm{H}), 3.82(\mathrm{~s}, 3 \mathrm{H}), 3.22$ (s, 2H), $2.37(\mathrm{~s}, 3 \mathrm{H}), 1.58(\mathrm{~d}, J=16.1 \mathrm{~Hz}, 6 \mathrm{H})$.

${ }^{13}$ C NMR $\left(100 \mathrm{MHz}, \mathrm{CDCl}_{3}\right) \delta 154.8,139.2,135.7,135.3,134.7,131.7,131.1,130.2$, 128.8 (3C), 128.5, 126.9 (3C), 125.2, 124.9, 122.7, 113.6, 89.1, 56.2, 42.5, 26.1, 25.3, 20.4 .

IR (FT-ATR, $\mathrm{cm}^{-1}, \mathrm{CH}_{2} \mathrm{Cl}_{2}$ ) vmax 3418, 2936, 1673, 1603, 1580, 1534, 1493, 1467, 1443, 1395, 1372, 1347, 1304, 1239, 1205, 1183, 1135, 1092, 1073, 1048, 1031, 979, $900,855,796,762,735,708,673,650,592,572,512,458$.

HRMS (ESI) $m / z$ : [M+Na] $]^{+}$Calcd for $\mathrm{C}_{25} \mathrm{H}_{26} \mathrm{~N}_{2} \mathrm{O}_{4} 441.1790$; found 441.1788. 


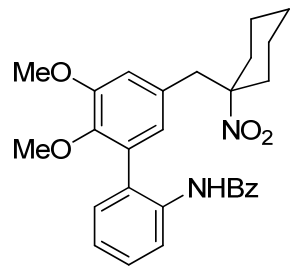

$N$-(2',3'-Dimethoxy-5'-((1-nitrocyclohexyl)methyl)-[1,1'-biphenyl]-2-

yl)benzamide (S41) was synthesized by following Procedure 9. The crude material was purified by normal-phase column chromatography using an eluent of $25 \% \mathrm{EtOAc} / \mathrm{Hx}$ to provide $\mathbf{S 4 1}$ as a colorless gum (498 $\mathrm{mg}$, 95\%).

${ }^{1} \mathbf{H}$ NMR $\left(400 \mathrm{MHz}, \mathrm{CDCl}_{3}\right) \delta 8.79(\mathrm{~s}, 1 \mathrm{H}), 8.19(\mathrm{~d}, J=7.9 \mathrm{~Hz}, 1 \mathrm{H}), 7.75(\mathrm{~d}, J=7.1$ $\mathrm{Hz}, 2 \mathrm{H}), 7.51-7.38(\mathrm{~m}, 4 \mathrm{H}), 7.31-7.20(\mathrm{~m}, 2 \mathrm{H}), 6.60(\mathrm{~d}, J=9.1 \mathrm{~Hz}, 2 \mathrm{H}), 3.88(\mathrm{~s}, 3 \mathrm{H})$, $3.61(\mathrm{~s}, 3 \mathrm{H}), 3.10-2.99(\mathrm{~m}, 2 \mathrm{H}), 2.39(\mathrm{dd}, J=35.3,12.5 \mathrm{~Hz}, 2 \mathrm{H}), 1.72-1.49(\mathrm{~m}, 4 \mathrm{H})$, $1.45-1.16(\mathrm{~m}, 4 \mathrm{H})$.

${ }^{13} \mathrm{C}$ NMR $\left(100 \mathrm{MHz}, \mathrm{CDCl}_{3}\right) \delta 165.3,152.7,144.9,135.3,134.8,132.7,131.7,131.4$, $130.8,130.4,128.8$ (2C), 128.6, 127.2 (2C), 125.3, 124.9, 123.6, 113.7, 92.0, 61.5, 56.2, 46.9, 34.3, 33.8, 24.7, 22.4, 22.3.

IR (FT-ATR, $\mathrm{cm}^{-1}, \mathrm{CH}_{2} \mathrm{Cl}_{2}$ ) $v_{\max } 3418,3343,2937,2864,1676,1584,1534,1484$, 1448, 1422, 1345, 1303, 1263, 1185, 1142, 1118, 1089, 1073, 1050, 1035, 1003, 947, $865,787,757,709,693,639,585,521$.

HRMS (ESI) $m / z$ : $[\mathrm{M}+\mathrm{H}]^{+}$Calcd for $\mathrm{C}_{28} \mathrm{H}_{30} \mathrm{~N}_{2} \mathrm{O}_{5}$ 475.2233; found 475.2231.

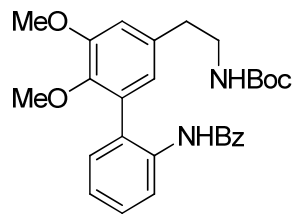

tert-Butyl (2-(2'-Benzamido-5,6-dimethoxy-[1,1'-biphenyl]-3-yl)ethyl)carbamate (S42) was synthesized by following Procedure 9. The crude material was purified by normal-phase column chromatography using an eluent of $25 \% \mathrm{EtOAc} / \mathrm{Hx}$ to provide S42 as a white solid (611 mg, 89\%).

m.p. $69^{\circ} \mathrm{C}$

${ }^{1} \mathbf{H}$ NMR $\left(400 \mathrm{MHz}, \mathrm{CDCl}_{3}\right) \delta 8.97(\mathrm{~s}, 1 \mathrm{H}), 8.17(\mathrm{~d}, J=7.7 \mathrm{~Hz}, 1 \mathrm{H}), 7.80(\mathrm{~d}, J=7.1$ $\mathrm{Hz}, 2 \mathrm{H}), 7.56-7.40(\mathrm{~m}, 4 \mathrm{H}), 7.39-7.32(\mathrm{~m}, 1 \mathrm{H}), 7.30-7.20(\mathrm{~m}, 1 \mathrm{H}), 6.83(\mathrm{~s}, 1 \mathrm{H}), 6.70$ $(\mathrm{s}, 1 \mathrm{H}), 4.77(\mathrm{~s}, 1 \mathrm{H}), 3.92(\mathrm{~s}, 3 \mathrm{H}), 3.62(\mathrm{~s}, 3 \mathrm{H}), 3.37(\mathrm{~s}, 2 \mathrm{H}), 2.78(\mathrm{~s}, 2 \mathrm{H}), 1.42(\mathrm{~s}, 9 \mathrm{H})$. ${ }^{13}$ C NMR $\left(100 \mathrm{MHz}, \mathrm{CDCl}_{3}\right) \delta 165.3,155.9,152.7,143.8,136.3,135.2,134.8,133.0$, 131.6, 130.9, 130.8, 128.7 (2C), 128.5, 127.1 (2C), 124.9, 124.0, 123.9, 112.8, 79.3, $61.5,56.1,41.8,36.2,28.4(3 \mathrm{C})$.

IR (FT-ATR, $\mathrm{cm}^{-1}, \mathrm{CH}_{2} \mathrm{Cl}_{2}$ ) v $v_{\max } 3349,3058,2975,2934,1703,1670,1579,1516$, 1484, 1468, 1442, 1420, 1391, 1365, 1342, 1303, 1246, 1165, 1139, 1119, 1050, 1036, $1001,969,905,850,819,783,756,734,705,626,583,530$.

HRMS (ESI) $m / z$ : [M+Na] $]^{+}$Calcd for $\mathrm{C}_{28} \mathrm{H}_{32} \mathrm{~N}_{2} \mathrm{O}_{5} 499.2209$; found 499.2203. 


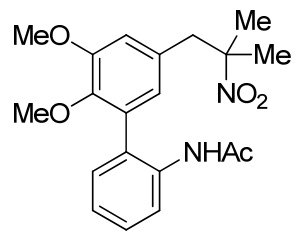

$N$-(2',3'-Dimethoxy-5'-(2-methyl-2-nitropropyl)-[1,1'-biphenyl]-2-yl)acetamide

(S43) was synthesized by following Procedure 10. The crude material was purified by normal-phase column chromatography using an eluent of $25 \% \mathrm{EtOAc} / \mathrm{Hx}$ to provide $\mathbf{S 4 3}$ as a yellow gum (476 mg, 70\%).

${ }^{1} \mathbf{H}$ NMR $\left(400 \mathrm{MHz}, \mathrm{CDCl}_{3}\right) \delta 7.96(\mathrm{~d}, J=8.1 \mathrm{~Hz}, 1 \mathrm{H}), 7.84(\mathrm{~s}, 1 \mathrm{H}), 7.34(\mathrm{t}, J=7.5$ $\mathrm{Hz}, 1 \mathrm{H}), 7.21-7.11(\mathrm{~m}, 2 \mathrm{H}), 6.66(\mathrm{~s}, 1 \mathrm{H}), 6.55(\mathrm{~s}, 1 \mathrm{H}), 3.86(\mathrm{~s}, 3 \mathrm{H}), 3.51(\mathrm{~s}, 3 \mathrm{H}), 3.14$ (s, 2H), $1.99(\mathrm{~s}, 3 \mathrm{H}), 1.59(\mathrm{~s}, 6 \mathrm{H})$.

${ }^{13}$ C NMR $\left(100 \mathrm{MHz}, \mathrm{CDCl}_{3}\right) \delta 168.6,152.5,144.9,135.1,132.7,131.7,130.7,129.9$, $128.4,125.1,124.7,123.7,113.5,88.6,61.2,55.9,46.5,25.8(2 \mathrm{C}), 24.5$.

IR (FT-ATR, $\left.\mathrm{cm}^{-1}, \mathrm{CH}_{2} \mathrm{Cl}_{2}\right) v_{\max } 3412,2993,2938,2834,1687,1585,1536,1486$, 1447, 1422, 1397, 1345, 1298, 1264, 1234, 1185, 1142, 1102, 1037, 1005, 855, 759, $733,684,627,581,540$.

HRMS (ESI) $m / z$ : $[\mathrm{M}+\mathrm{H}]^{+}$Calcd for $\mathrm{C}_{20} \mathrm{H}_{24} \mathrm{~N}_{2} \mathrm{O}_{5} 373.1764$; found 373.1758 .<smiles>COc1cc(CC(C)(C)[N+](=O)[O-])cc(-c2ccccc2NC(C)=O)c1OC</smiles>

\section{Methyl} (2',3'-dimethoxy-5'-(2-methyl-2-nitropropyl)-[1,1'-biphenyl]-2yl)carbamate (S44) was synthesized by following Procedure 11. The crude material was purified by normal-phase column chromatography using an eluent of $25 \%$ EtOAc/Hx to provide $\mathbf{S 4 4}$ as a colorless gum (472 $\mathrm{mg}$, 81\%).

${ }^{1} \mathbf{H}$ NMR $\left(400 \mathrm{MHz}, \mathrm{CDCl}_{3}\right) \delta 7.93(\mathrm{~s}, 1 \mathrm{H}), 7.38(\mathrm{t}, J=7.6 \mathrm{~Hz}, 1 \mathrm{H}), 7.21-7.10(\mathrm{~m}$, $3 \mathrm{H}), 6.67(\mathrm{~s}, 1 \mathrm{H}), 6.57(\mathrm{~s}, 1 \mathrm{H}), 3.88(\mathrm{~s}, 3 \mathrm{H}), 3.67(\mathrm{~s}, 3 \mathrm{H}), 3.53(\mathrm{~s}, 3 \mathrm{H}), 3.22-3.12(\mathrm{~m}$, $2 \mathrm{H}), 1.61(\mathrm{~d}, J=4.1 \mathrm{~Hz}, 6 \mathrm{H})$.

${ }^{13}$ C NMR $\left(100 \mathrm{MHz}, \mathrm{CDCl}_{3}\right) \delta 154.6,152.8,145.3,135.5,132.6,131.7,130.7(2 \mathrm{C})$, $128.7(2 \mathrm{C}), 125.3,123.9,113.6,88.7,61.2,56.0,52.2,46.6,26.0,25.8$.

IR (FT-ATR, $\mathrm{cm}^{-1}, \mathrm{CH}_{2} \mathrm{Cl}_{2}$ ) $v_{\max } 3420,2941,2837,1735,1586,1536,1523,1485$, 1448, 1421, 1397, 1372, 1344, 1301, 1263, 1216, 1189, 1142, 1107, 1067, 1048, 1037, $1004,954,855,833,767,734,676,625,581$.

HRMS (ESI) $m / z$ : $[\mathrm{M}+\mathrm{H}]^{+}$Calcd for $\mathrm{C}_{20} \mathrm{H}_{24} \mathrm{~N}_{2} \mathrm{O}_{6} 389.1713$; found 389.1708. 


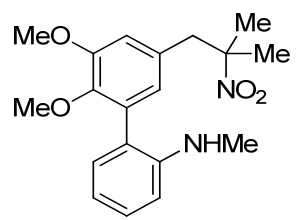

2',3'-Dimethoxy- $N$-methyl-5'-(2-methyl-2-nitropropyl)-[1,1'-biphenyl]-2-amine (S45) was synthesized by following Procedure 12. The crude material was purified by normal-phase column chromatography using an eluent of $25 \% \mathrm{EtOAc} / \mathrm{Hx}$ to provide S45 as a yellow oil (176 mg, 26\%).

${ }^{1} \mathbf{H}$ NMR $\left(400 \mathrm{MHz}, \mathrm{CDCl}_{3}\right) \delta 7.29(\mathrm{t}, J=7.8 \mathrm{~Hz}, 1 \mathrm{H}), 7.06(\mathrm{~d}, J=7.4 \mathrm{~Hz}, 1 \mathrm{H}), 6.76$ $(\mathrm{t}, J=7.4 \mathrm{~Hz}, 1 \mathrm{H}), 6.71(\mathrm{~d}, J=8.2 \mathrm{~Hz}, 1 \mathrm{H}), 6.64(\mathrm{~s}, 1 \mathrm{H}), 6.59(\mathrm{~s}, 1 \mathrm{H}), 3.88(\mathrm{~s}, 4 \mathrm{H})$, $3.55(\mathrm{~s}, 3 \mathrm{H}), 3.24-3.09(\mathrm{~m}, 2 \mathrm{H}), 2.81(\mathrm{~s}, 3 \mathrm{H}), 1.61(\mathrm{~s}, 6 \mathrm{H})$.

${ }^{13}$ C NMR $\left(100 \mathrm{MHz}, \mathrm{CDCl}_{3}\right) \delta 152.9,146.9,146.2,133.3,131.2,130.4,129.0,125.4$, $124.0,116.6,113.1,110.1,88.8,61.1,56.0,46.8,30.9,26.1,25.7$.

IR (FT-ATR, $\mathrm{cm}^{-1}, \mathrm{CH}_{2} \mathrm{Cl}_{2}$ ) $v_{\max } 3430,2987,2934,2815,1600,1580,1535,1514$, $1482,1458,1418,1396,1371,1342,1314,1287,1262,1231,1167,1137,1094,1066$, $1035,1006,964,854,790,746,701,673,629,585,545,520$.

HRMS (ESI) $m / z$ : [M+H] $]^{+}$Calcd for $\mathrm{C}_{19} \mathrm{H}_{24} \mathrm{~N}_{2} \mathrm{O}_{4}$ 345.1814; found 345.1809.

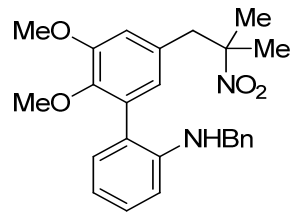

$N$-Benzyl-2',3'-dimethoxy-5'-(2-methyl-2-nitropropyl)-[1,1'-biphenyl]-2-amine (S46) was synthesized by following Procedure 13. The crude material was purified by normal-phase column chromatography using an eluent of $25 \% \mathrm{EtOAc} / \mathrm{Hx}$ to provide S46 as a yellow gum (499 mg, 83\%).

${ }^{1} \mathbf{H}$ NMR $\left(400 \mathrm{MHz}, \mathrm{CDCl}_{3}\right) \delta 7.35-7.26(\mathrm{~m}, 4 \mathrm{H}), 7.23(\mathrm{~d}, J=6.8 \mathrm{~Hz}, 1 \mathrm{H}), 7.18(\mathrm{t}, J$ $=7.9 \mathrm{~Hz}, 1 \mathrm{H}), 7.08(\mathrm{~d}, J=7.3 \mathrm{~Hz}, 1 \mathrm{H}), 6.75(\mathrm{t}, J=7.3 \mathrm{~Hz}, 1 \mathrm{H}), 6.67-6.60(\mathrm{~s}, 3 \mathrm{H})$, 4.40-4.26 (m, 3H), 3.87 (s, 3H), 3.60 (s, 3H), $3.16(\mathrm{~s}, 2 \mathrm{H}), 1.59(\mathrm{~s}, 6 \mathrm{H})$.

${ }^{13}$ C NMR $\left(100 \mathrm{MHz}, \mathrm{CDCl}_{3}\right) \delta 152.9,146.3,145.4,139.7,133.2,131.2,130.6,128.9$, 128.5 (2C), 127.1 (2C), 126.9, 125.4, 123.9, 116.8, 113.3, 111.0, 88.7, 61.1, 56.0, 48.0, 46.7, 25.9, 25.7.

IR (FT-ATR, $\mathrm{cm}^{-1}, \mathrm{CH}_{2} \mathrm{Cl}_{2}$ ) $v_{\max } 3435,2927,2852,1734,1599,1581,1537,1514$, 1483, 1454, 1421, 1396, 1372, 1344, 1322, 1295, 1263, 1233, 1184, 1163, 1142, 1064, $1039,1008,855,790,749,699,632,585,461,428$.

HRMS (ESI) $m / z$ : [M+H] $]^{+}$Calcd for $\mathrm{C}_{25} \mathrm{H}_{28} \mathrm{~N}_{2} \mathrm{O}_{4} 421.2127$; found 421.2123. 


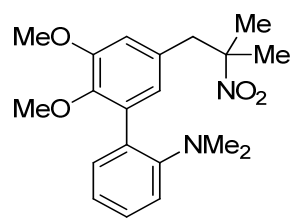

2',3'-Dimethoxy- $N, N$-dimethyl-5'-(2-methyl-2-nitropropyl)-[1,1'-biphenyl]-2amine (S47) was synthesized by following Procedure 14. The crude material was purified by normal-phase column chromatography using an eluent of $25 \% \mathrm{EtOAc} / \mathrm{Hx}$ to provide $\mathbf{S 4 7}$ as a yellow gum (292 $\mathrm{mg}, 68 \%$ ).

${ }^{1} \mathbf{H}$ NMR $\left(400 \mathrm{MHz}, \mathrm{CDCl}_{3}\right) \delta 7.29(\mathrm{t}, J=7.7 \mathrm{~Hz}, 1 \mathrm{H}), 7.20(\mathrm{~d}, J=7.4 \mathrm{~Hz}, 1 \mathrm{H}), 7.05$ $(\mathrm{d}, J=8.0 \mathrm{~Hz}, 1 \mathrm{H}), 6.99$ (t, $J=7.4 \mathrm{~Hz}, 1 \mathrm{H}), 6.77(\mathrm{~s}, 1 \mathrm{H}), 6.60(\mathrm{~s}, 1 \mathrm{H}), 3.87(\mathrm{~s}, 3 \mathrm{H})$, $3.69(\mathrm{~s}, 3 \mathrm{H}), 3.17(\mathrm{~s}, 2 \mathrm{H}), 2.55(\mathrm{~s}, 6 \mathrm{H}), 1.61(\mathrm{~s}, 6 \mathrm{H})$.

${ }^{13} \mathrm{C}$ NMR $\left(100 \mathrm{MHz}, \mathrm{CDCl}_{3}\right) \delta 152.9,151.7,145.5,135.4,131.9,130.6,130.1,128.3$, $125.1,121.0,117.8,112.6,88.9,60.4,55.9,46.7,43.4(2 \mathrm{C}), 25.8(2 \mathrm{C})$.

IR (FT-ATR, $\mathrm{cm}^{-1}, \mathrm{CH}_{2} \mathrm{Cl}_{2}$ ) $v_{\max } 2937,2869,2828,2779,1583,1536,1497,1451$, 1419, 1396, 1371, 1342, 1321, 1251, 1233, 1186, 1158, 1138, 1100, 1052, 1039, 1009, 948, 854, 808, 787, 759, 669, 634, 583, 553.

HRMS (ESI) $m / z$ : [M+H] $]^{+}$Calcd for $\mathrm{C}_{20} \mathrm{H}_{26} \mathrm{~N}_{2} \mathrm{O}_{4} 359.1971$; found 359.1965.

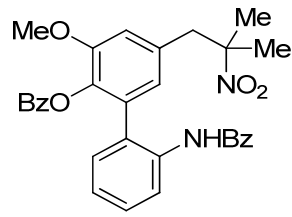

2'-Benzamido-3-methoxy-5-(2-methyl-2-nitropropyl)-[1,1'-biphenyl]-2-yl

Benzoate (S48) was synthesized by following Procedure 15. The crude material was purified by normal-phase column chromatography using an eluent of $25 \% \mathrm{EtOAc} / \mathrm{Hx}$ to provide $\mathbf{S 4 8}$ as a white solid (223 $\mathrm{mg}, 73 \%$ ).

m.p. $138^{\circ} \mathrm{C}$

${ }^{1} \mathbf{H}$ NMR $\left(400 \mathrm{MHz}, \mathrm{CDCl}_{3}\right) \delta 8.24-7.99(\mathrm{~m}, 1 \mathrm{H}), 7.94(\mathrm{~d}, J=7.7 \mathrm{~Hz}, 2 \mathrm{H}), 7.75(\mathrm{~d}, J$ $=7.3 \mathrm{~Hz}, 2 \mathrm{H}), 7.54(\mathrm{t}, J=7.5 \mathrm{~Hz}, 1 \mathrm{H}), 7.49(\mathrm{~d}, J=7.3 \mathrm{~Hz}, 1 \mathrm{H}), 7.46-7.27(\mathrm{~m}, 6 \mathrm{H})$, $7.24(\mathrm{~d}, J=7.4 \mathrm{~Hz}, 1 \mathrm{H}), 7.12(\mathrm{t}, J=7.5 \mathrm{~Hz}, 1 \mathrm{H}), 6.74(\mathrm{~s}, 1 \mathrm{H}), 6.68(\mathrm{~s}, 1 \mathrm{H}), 3.83(\mathrm{~s}$, $3 \mathrm{H}), 3.23(\mathrm{~d}, J=13.8 \mathrm{~Hz}, 1 \mathrm{H}), 3.08(\mathrm{~d}, J=13.9 \mathrm{~Hz}, 1 \mathrm{H}), 1.42(\mathrm{~d}, J=60.4 \mathrm{~Hz}, 6 \mathrm{H})$.

${ }^{13}$ C NMR $\left(100 \mathrm{MHz}, \mathrm{CDCl}_{3}\right) \delta 165.3,152.0,137.5,135.6,134.5,134.3,133.8,131.8$, $130.5,130.2$ (2C), 129.9, 129.1, 128.8 (2C), 128.6 (3C), 128.5, 128.4, 128.3, 127.2 (2C), 124.5, 124.4, 113.5, 88.5, 56.3, 46.6, 26.2, 25.1.

IR (FT-ATR, $\mathrm{cm}^{-1}, \mathrm{CH}_{2} \mathrm{Cl}_{2}$ ) v $v_{\max } 3416,3062,2924,2853,1730,1675,1592,1583$, 1536, 1494, 1480, 1451, 1422, 1397, 1372, 1346, 1305, 1262, 1204, 1176, 1141, 1079, $1061,1034,1024,942,855,798,761,707,618,587,481,435,419$.

HRMS (ESI) $m / z$ : $[\mathrm{M}+\mathrm{H}]^{+}$Calcd for $\mathrm{C}_{31} \mathrm{H}_{28} \mathrm{~N}_{2} \mathrm{O}_{6}$ 525.2026; found 525.2021. 


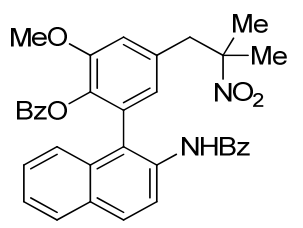

2-(2-Benzamidonaphthalen-1-yl)-6-methoxy-4-(2-methyl-2-nitropropyl)phenyl benzoate (S49) was synthesized by following Procedure 15. The crude material was purified by normal-phase column chromatography using an eluent of 33\% EtOAc/ $\mathrm{Hx}$ to provide $\mathbf{S 4 9}$ as a white solid (310 $\mathrm{mg}, 88 \%$ ).

m.p. $76^{\circ} \mathrm{C}$

${ }^{1} \mathbf{H}$ NMR $\left(400 \mathrm{MHz}, \mathrm{CDCl}_{3}\right) \delta 8.37(\mathrm{~s}, 1 \mathrm{H}), 8.18(\mathrm{~d}, J=7.0 \mathrm{~Hz}, 1 \mathrm{H}), 7.91-7.76(\mathrm{~m}$, $4 \mathrm{H}), 7.71(\mathrm{~d}, J=7.3 \mathrm{~Hz}, 2 \mathrm{H}), 7.55-7.37(\mathrm{~m}, 8 \mathrm{H}), 7.24(\mathrm{t}, J=7.1 \mathrm{~Hz}, 1 \mathrm{H}), 6.82(\mathrm{~s}, 1 \mathrm{H})$, $6.70(\mathrm{~s}, 1 \mathrm{H}), 3.87(\mathrm{~s}, 3 \mathrm{H}), 3.25(\mathrm{~d}, J=13.9 \mathrm{~Hz}, 1 \mathrm{H}), 3.10(\mathrm{~d}, J=13.8 \mathrm{~Hz}, 1 \mathrm{H}), 1.47(\mathrm{~s}$, $3 \mathrm{H}), 1.31(\mathrm{~s}, 3 \mathrm{H})$.

${ }^{13}$ C NMR $\left(100 \mathrm{MHz}, \mathrm{CDCl}_{3}\right) \delta 165.5,152.1,138.4,134.9,134.3,133.9,133.7,132.4$, 131.9, 131.194, 131.188, 131.0, 130.1 (2C), 129.0, 128.8 (3C), 128.4 (3C), 128.3, 128.1, 127.3 (2C), 126.7, 125.4, 125.3, 125.0, 113.6, 88.5, 56.3, 46.6, 26.1, 25.0.

IR (FT-ATR, $\mathrm{cm}^{-1}, \mathrm{CH}_{2} \mathrm{Cl}_{2}$ ) $v_{\max } 3402,3063,2939,1725,1677,1621,1597,1537$, $1503,1488,1461,1426,1383,1346,1331,1316,1268,1204,1178,1142,1082,1062$, 1024, 1002, 964, 855, 818, 797, 750, 708, 688, 619, 537, 427.

HRMS (ESI) $m / z$ : $[\mathrm{M}+\mathrm{H}]^{+}$Calcd for $\mathrm{C}_{35} \mathrm{H}_{30} \mathrm{~N}_{2} \mathrm{O}_{6}$ 575.2182; found 575.2180. 


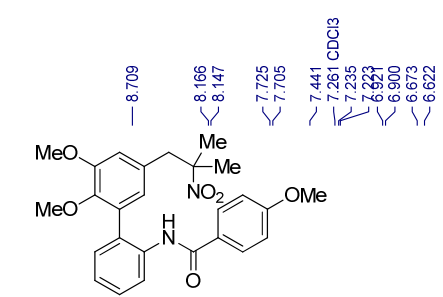

$\mathbf{S 2 9}$

$\left({ }^{1} \mathrm{H} \mathrm{NMR}, 400 \mathrm{MHz}, \mathrm{CDCl}_{3}\right)$

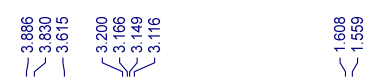

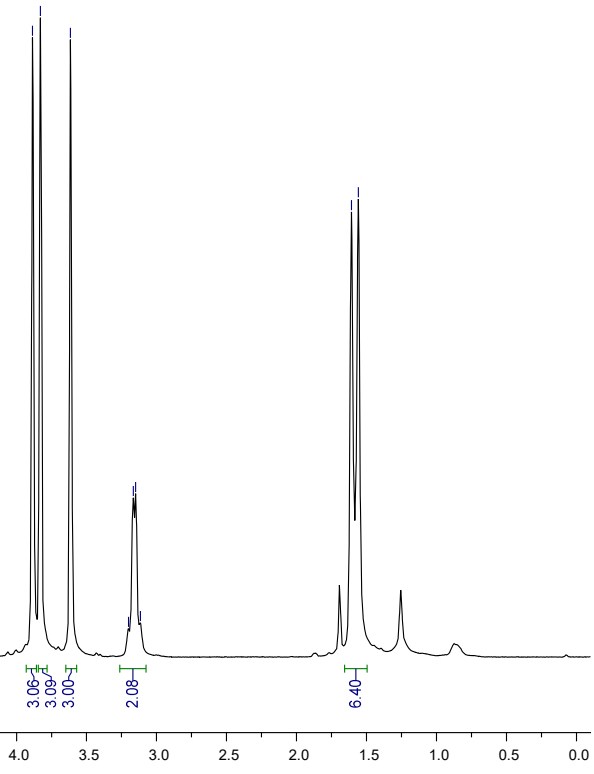

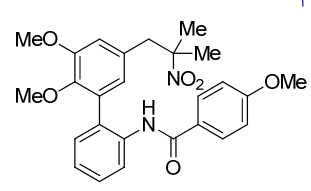

S29

$\left({ }^{13} \mathrm{C}\right.$ NMR, $\left.100 \mathrm{MHz}, \mathrm{CDCl}_{3}\right)$

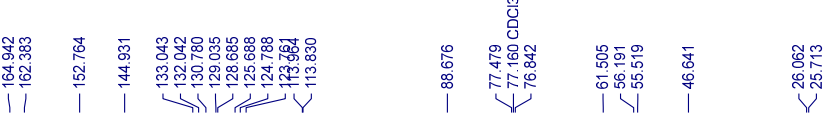

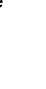



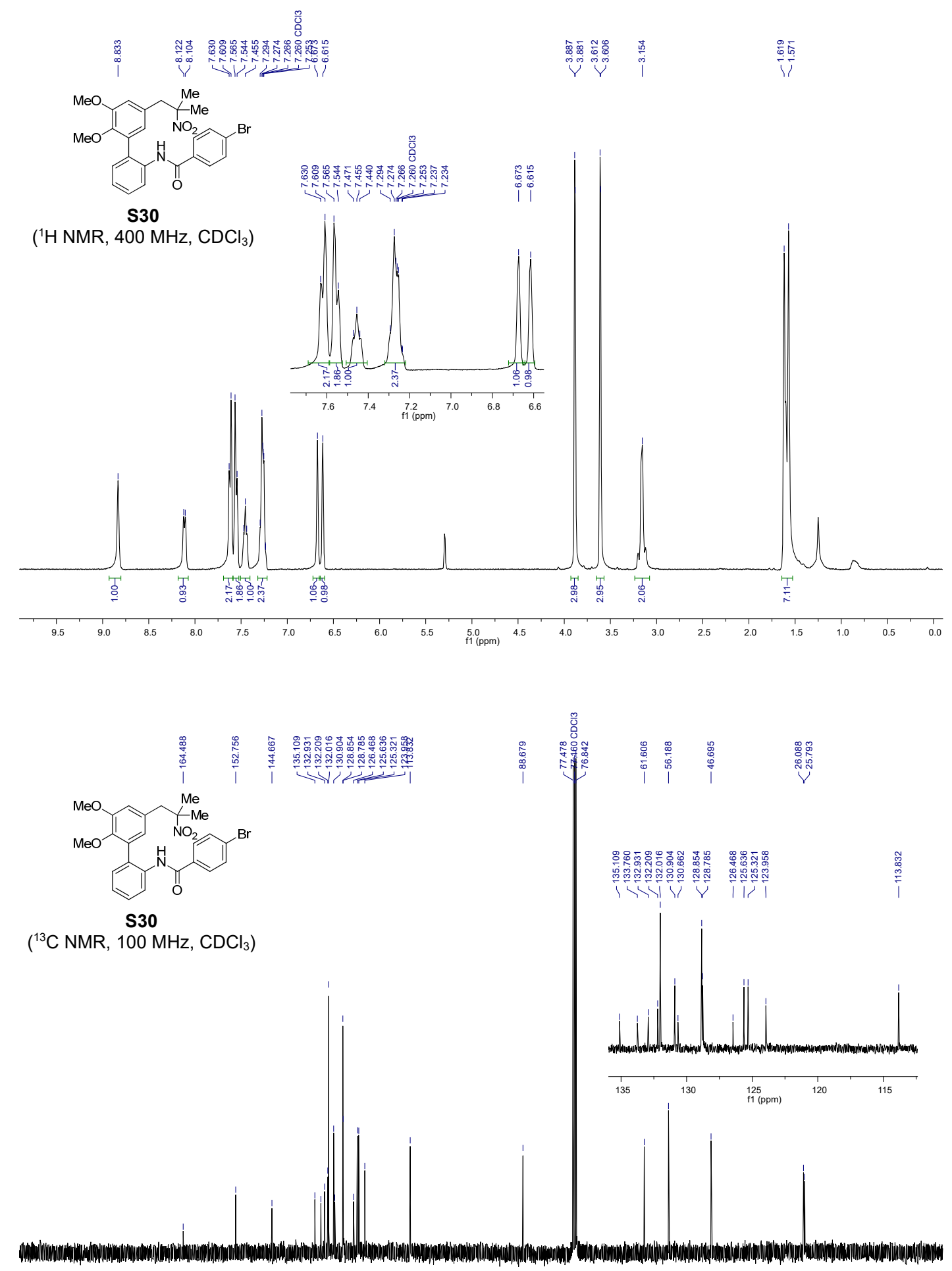


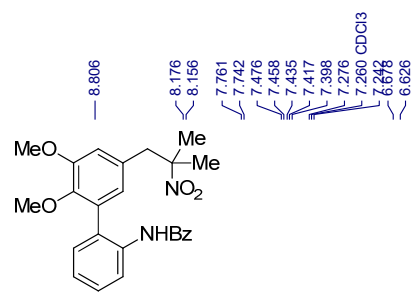

要

S31

$\left({ }^{1} \mathrm{H} \mathrm{NMR}, 400 \mathrm{MHz}, \mathrm{CDCl}_{3}\right)$
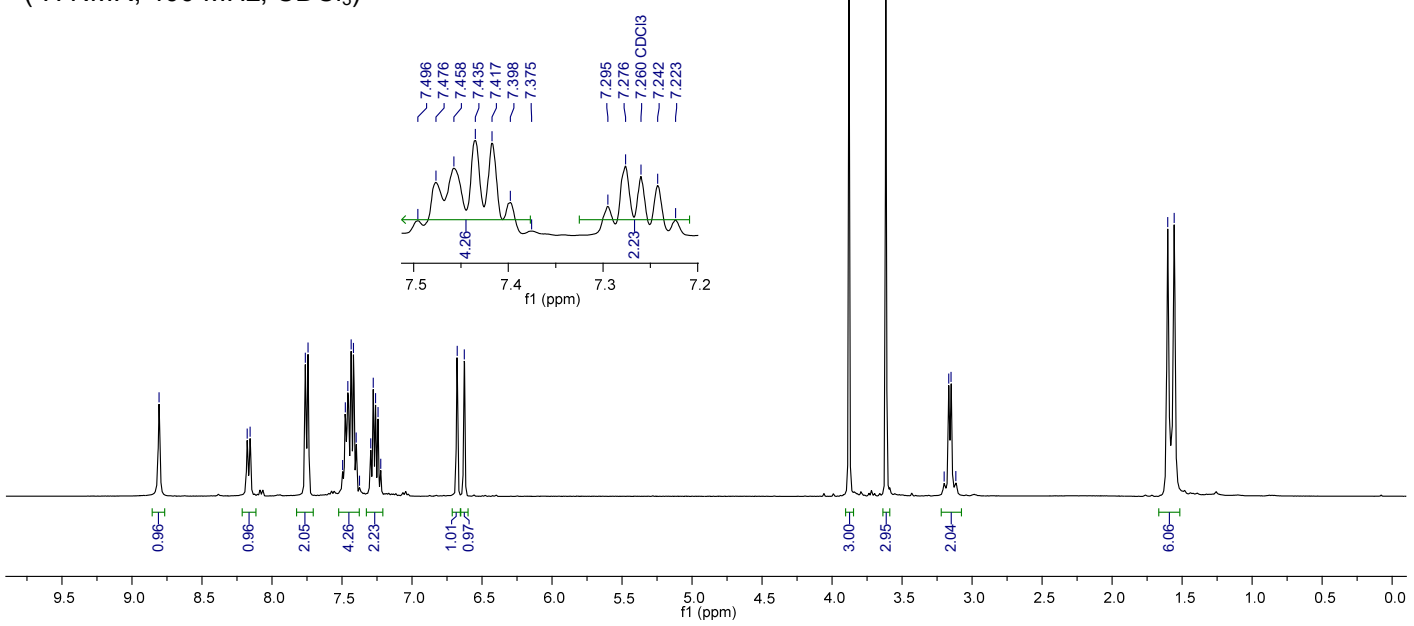

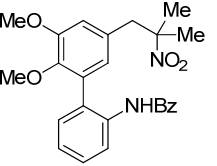

S31

$\left({ }^{13} \mathrm{C}\right.$ NMR, $\left.100 \mathrm{MHz}, \mathrm{CDCl}_{3}\right)$

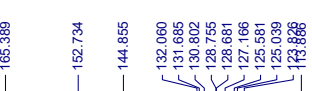

Me

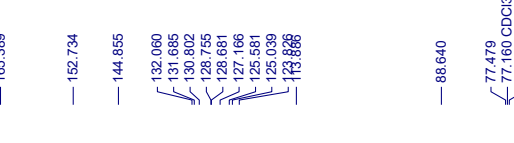

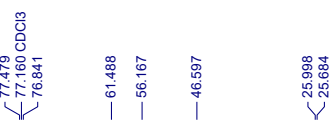

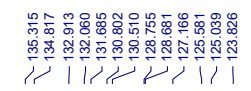

Vi T)

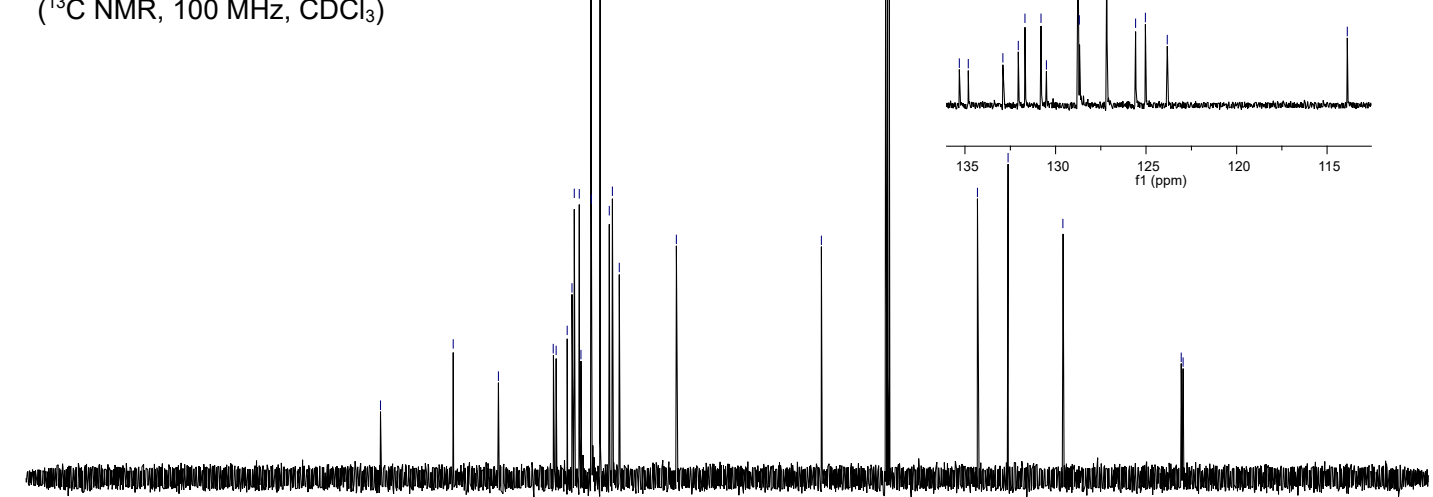

$\begin{array}{llllllllllllll}220 & 210 & 200 & 190 & 180 & 170 & 160 & 150 & 140 & 130 & 120 & 110 \\ \mathrm{f} 1(\mathrm{ppm}) & 100\end{array}$ 


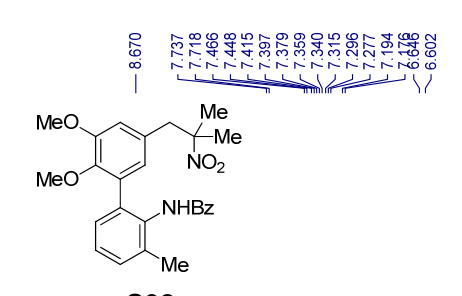

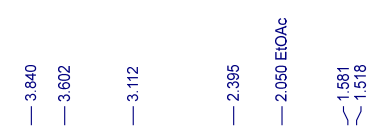

S32

( ${ }^{1} \mathrm{H}$ NMR, $400 \mathrm{MHz}, \mathrm{CDCl}_{3}$ )

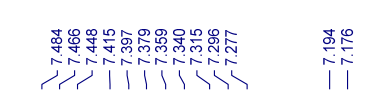
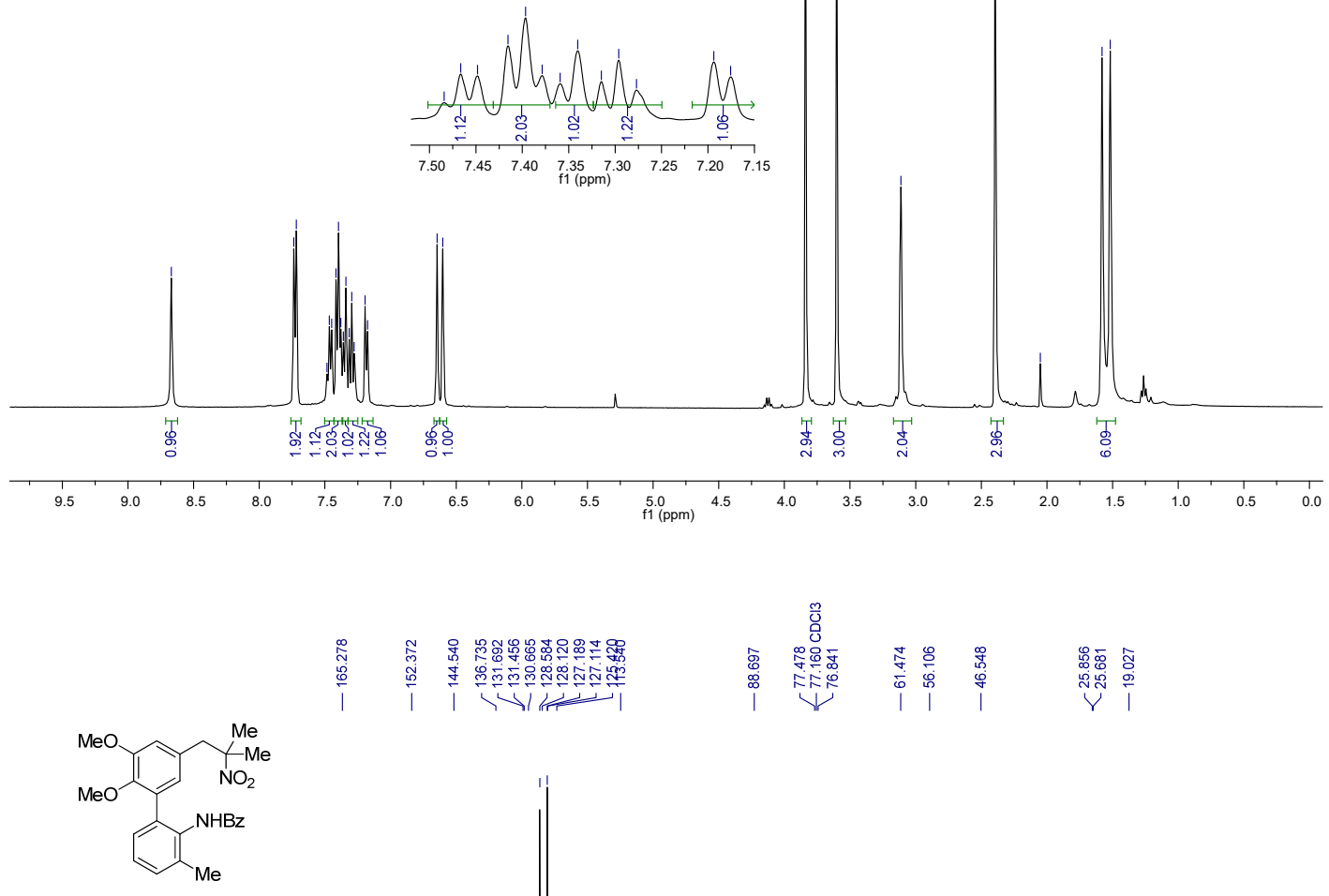

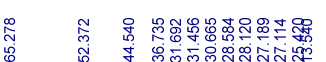

।

1
0
0
0
0
0

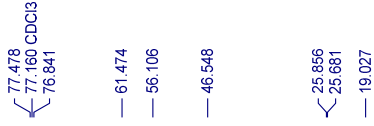

S32

$\left({ }^{13} \mathrm{C} \mathrm{NMR,} 100 \mathrm{MHz}, \mathrm{CDCl}_{3}\right)$

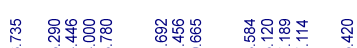

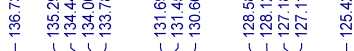

I 11 1/ II ।

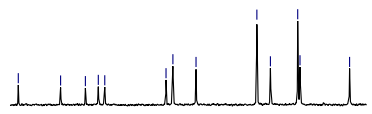

\begin{tabular}{lllllll}
\hline 136 & 134 & $\underset{132}{\mathrm{f} 1(\mathrm{ppm})}$ & 130 & 128 & 126 \\
\hline
\end{tabular}

$\begin{array}{lllllllllllll}220 & 210 & 200 & 190 & 180 & 170 & 160 & 150 & 140 & 130 & 120 & 110 & 100 \\ \mathrm{f} 1(\mathrm{ppm})\end{array}$ 


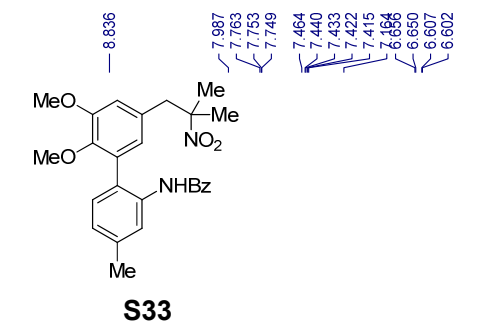

ソソ \}

( ${ }^{\mathrm{H}} \mathrm{NMR}, 400 \mathrm{MHz}, \mathrm{CDCl}_{3}$ )
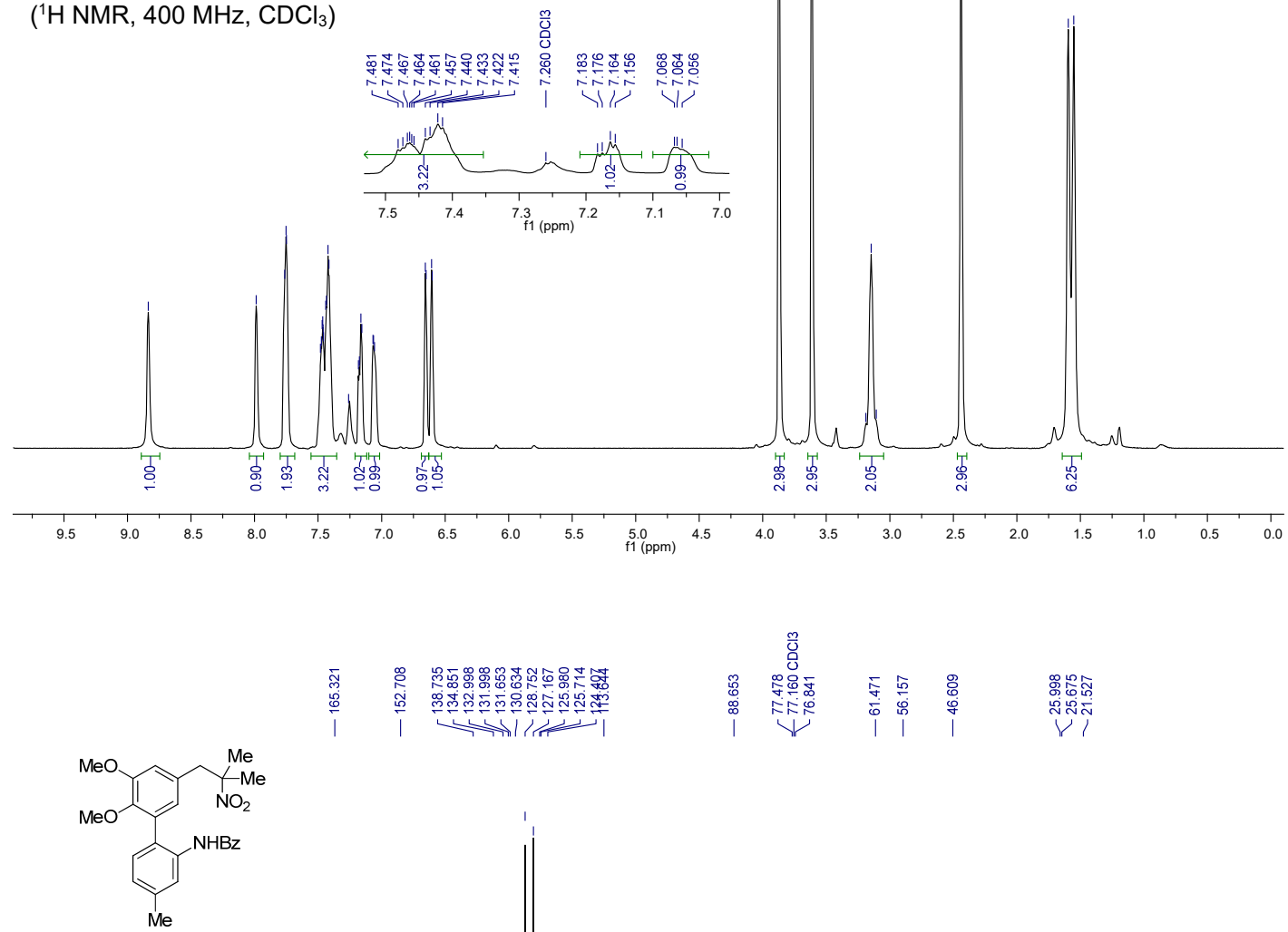

S33

$\left({ }^{13} \mathrm{C}\right.$ NMR, $\left.100 \mathrm{MHz}, \mathrm{CDCl}_{3}\right)$

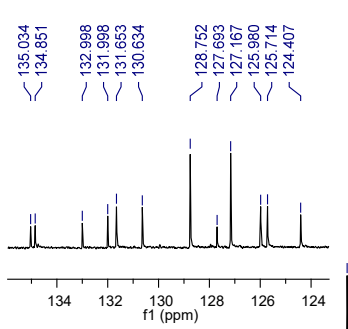




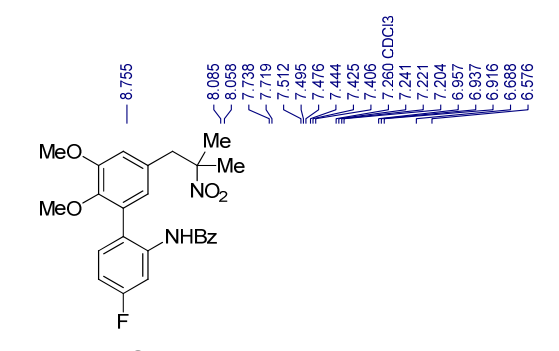

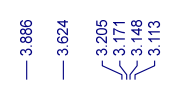

$\frac{1}{10}$

('H NMR, $400 \mathrm{MHz}, \mathrm{CDCl}_{3}$ )
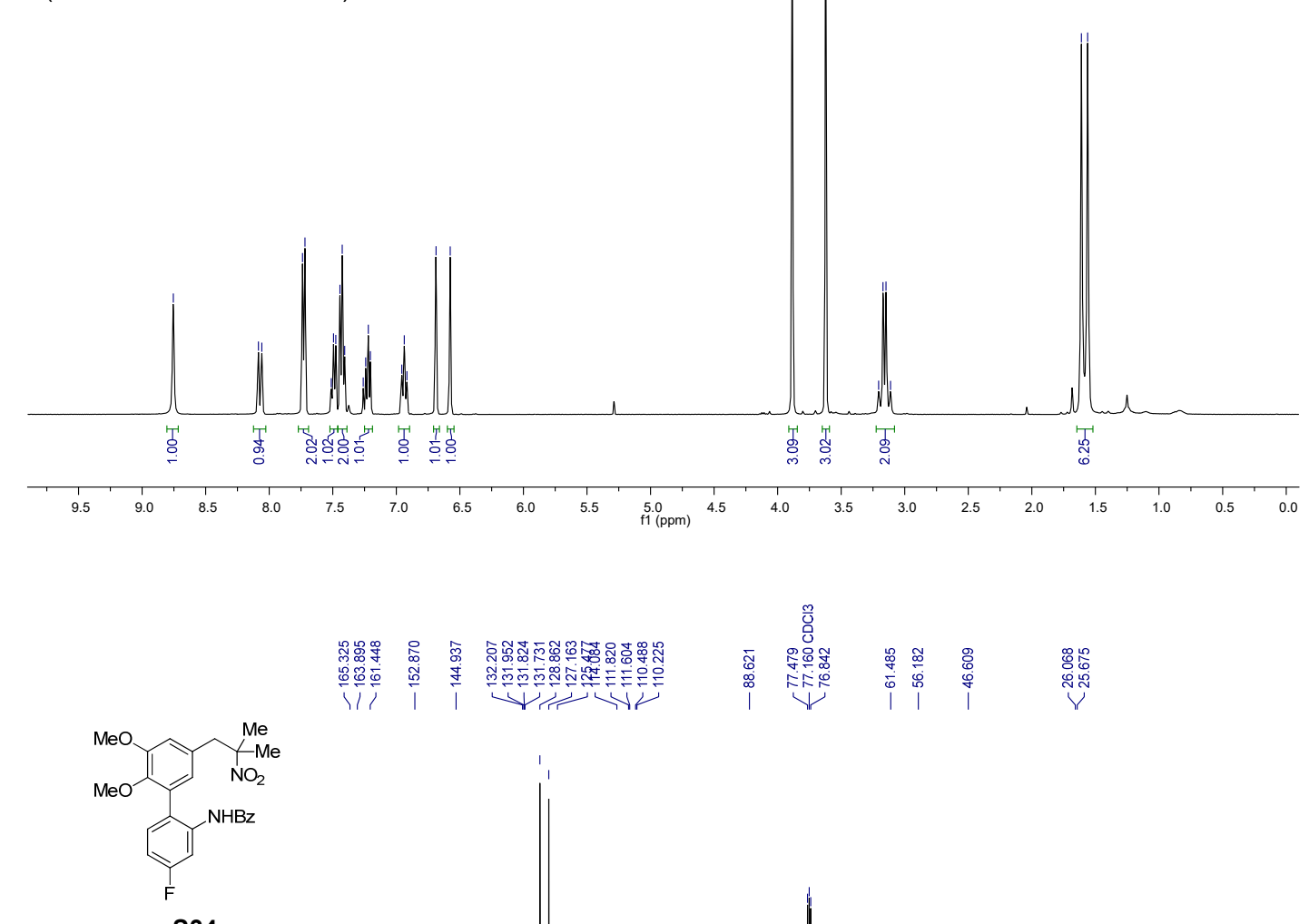

S34

$\left({ }^{13} \mathrm{C} \mathrm{NMR,}, 100 \mathrm{MHz}, \mathrm{CDCl}_{3}\right)$

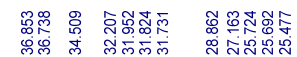

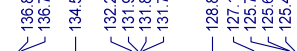

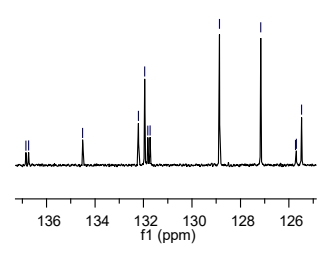

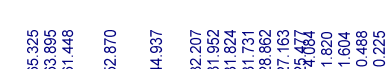

11। I I

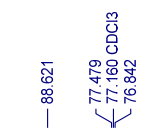

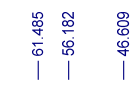

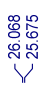
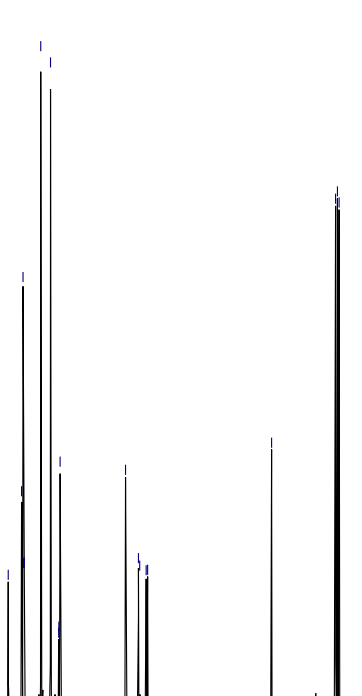

$\begin{array}{lllll}220 & 210 & 200 & 190 & 180\end{array}$

$160 \quad 150 \quad 140$

$\underset{\mathrm{f} 1(\mathrm{ppm})}{110} 100$ 
응

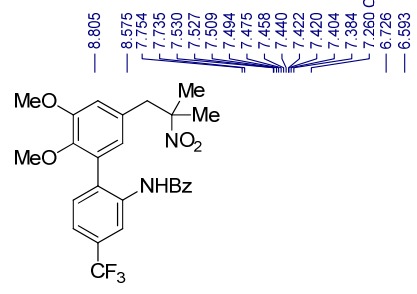

S35

( ${ }^{1} \mathrm{H}$ NMR, $400 \mathrm{MHz}, \mathrm{CDCl}_{3}$ )
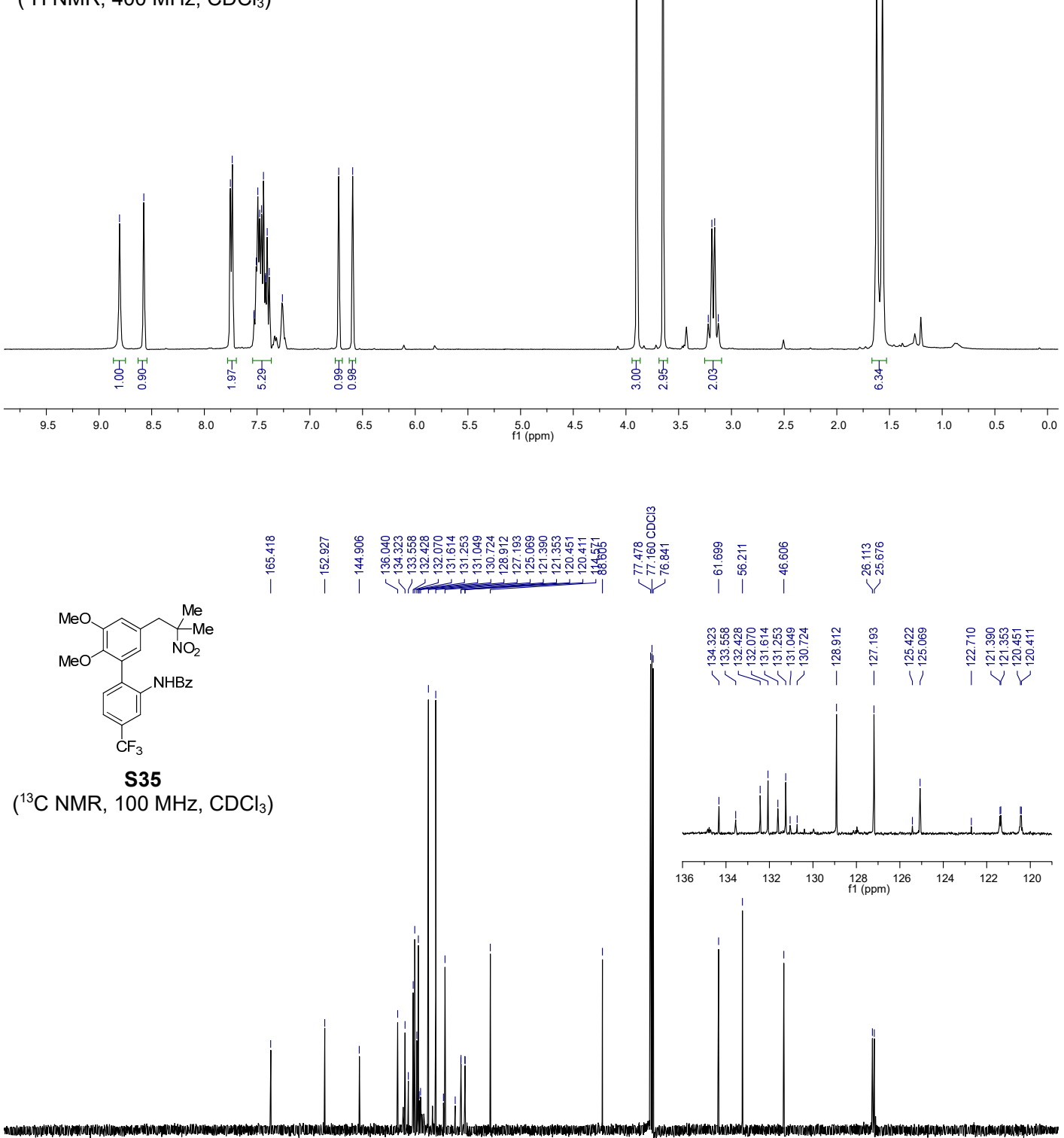

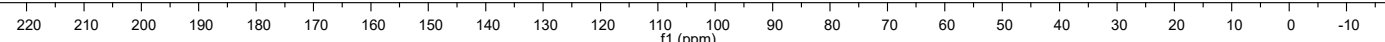



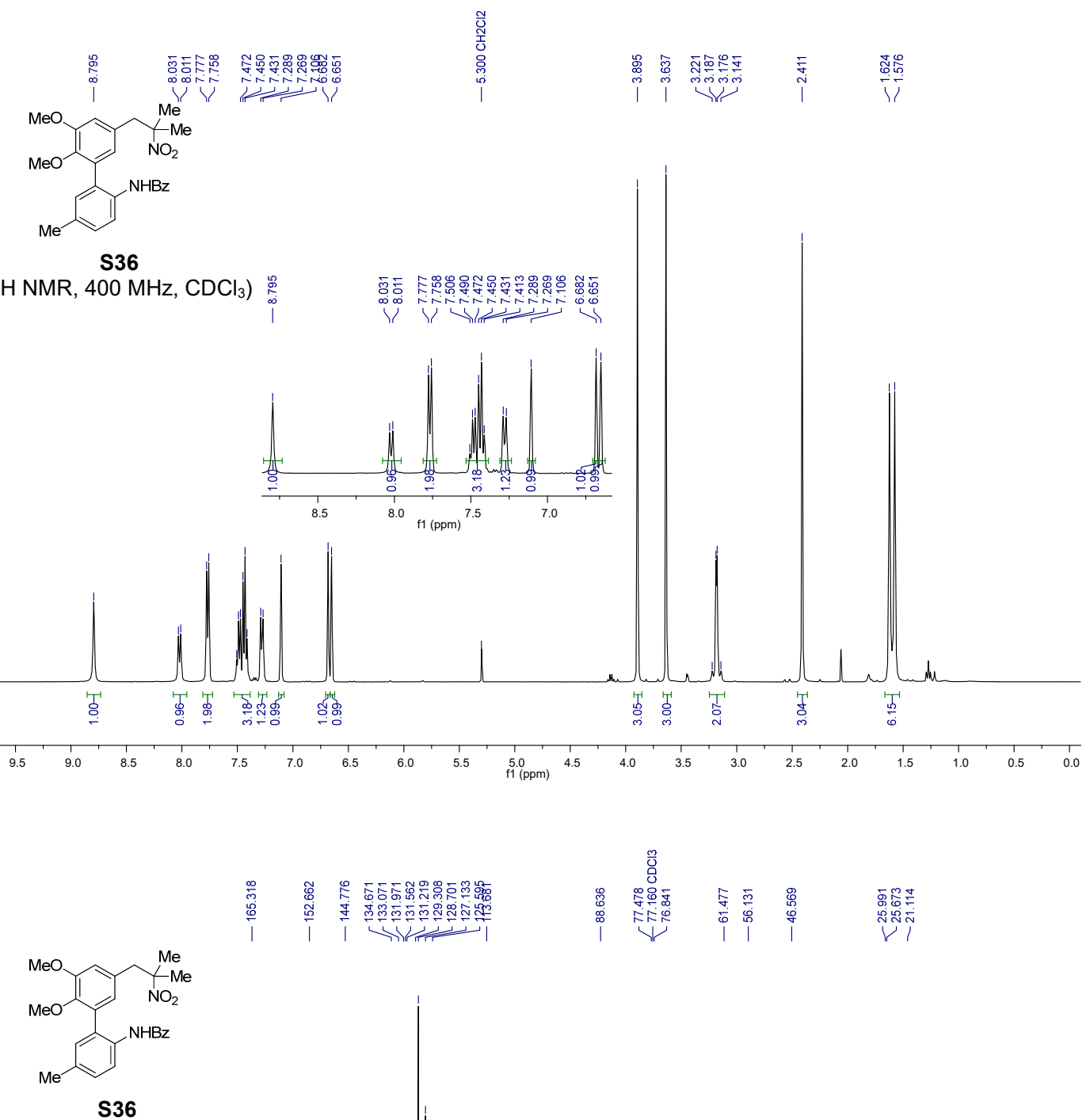

$\left({ }^{13} \mathrm{C}\right.$ NMR, $\left.100 \mathrm{MHz}, \mathrm{CDCl}_{3}\right)$

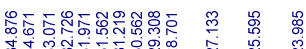
|i
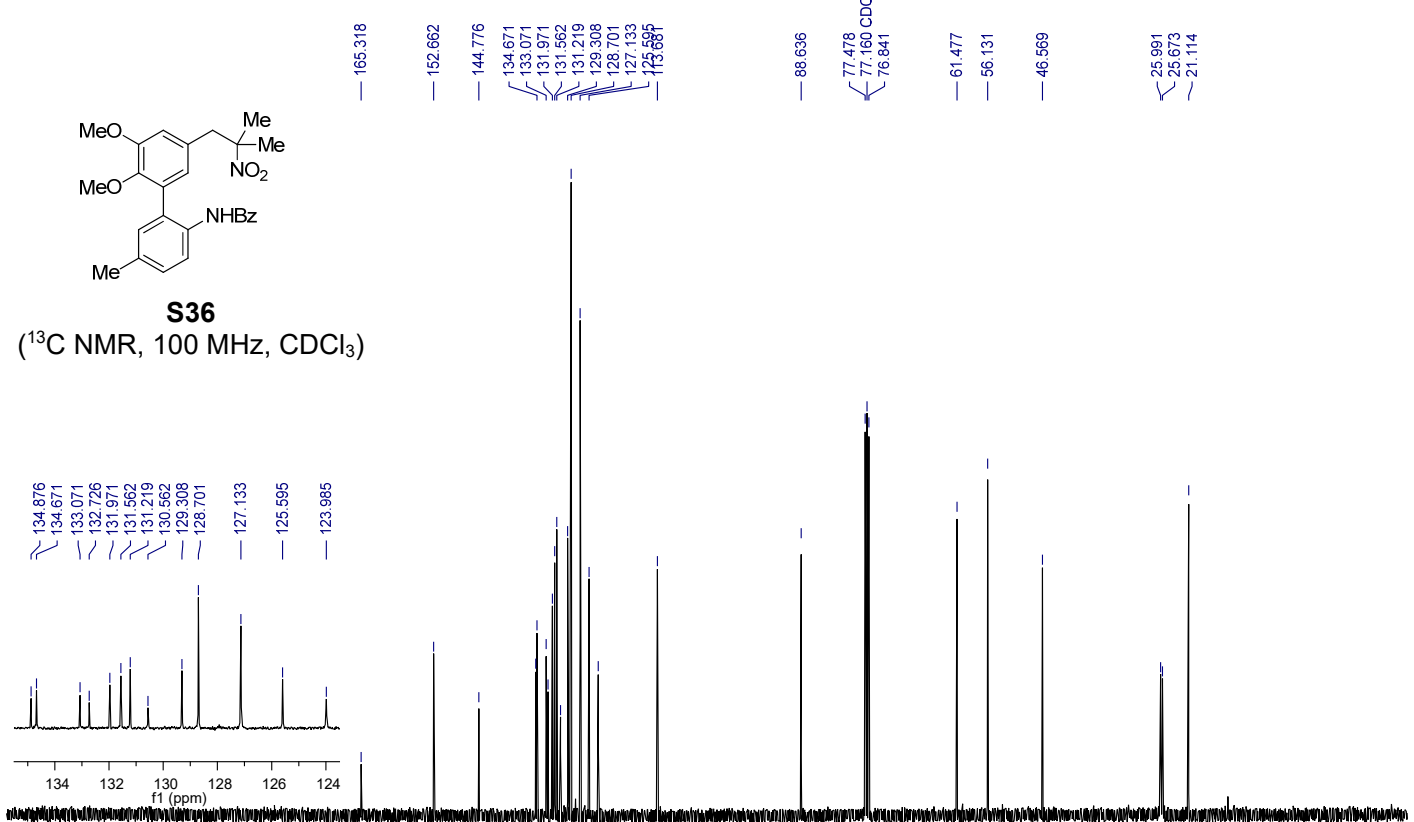

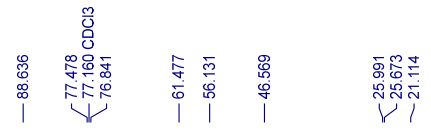

$\begin{array}{llllllllllll}120 & 210 & 200 & 190 & 180 & 170 & 160 & 150 & 140 & 130 & 120 & \begin{array}{c}110 \\ \mathrm{f} 1(\mathrm{pmm})\end{array} \\ 100 & & & & & & & & & & & \end{array}$ 


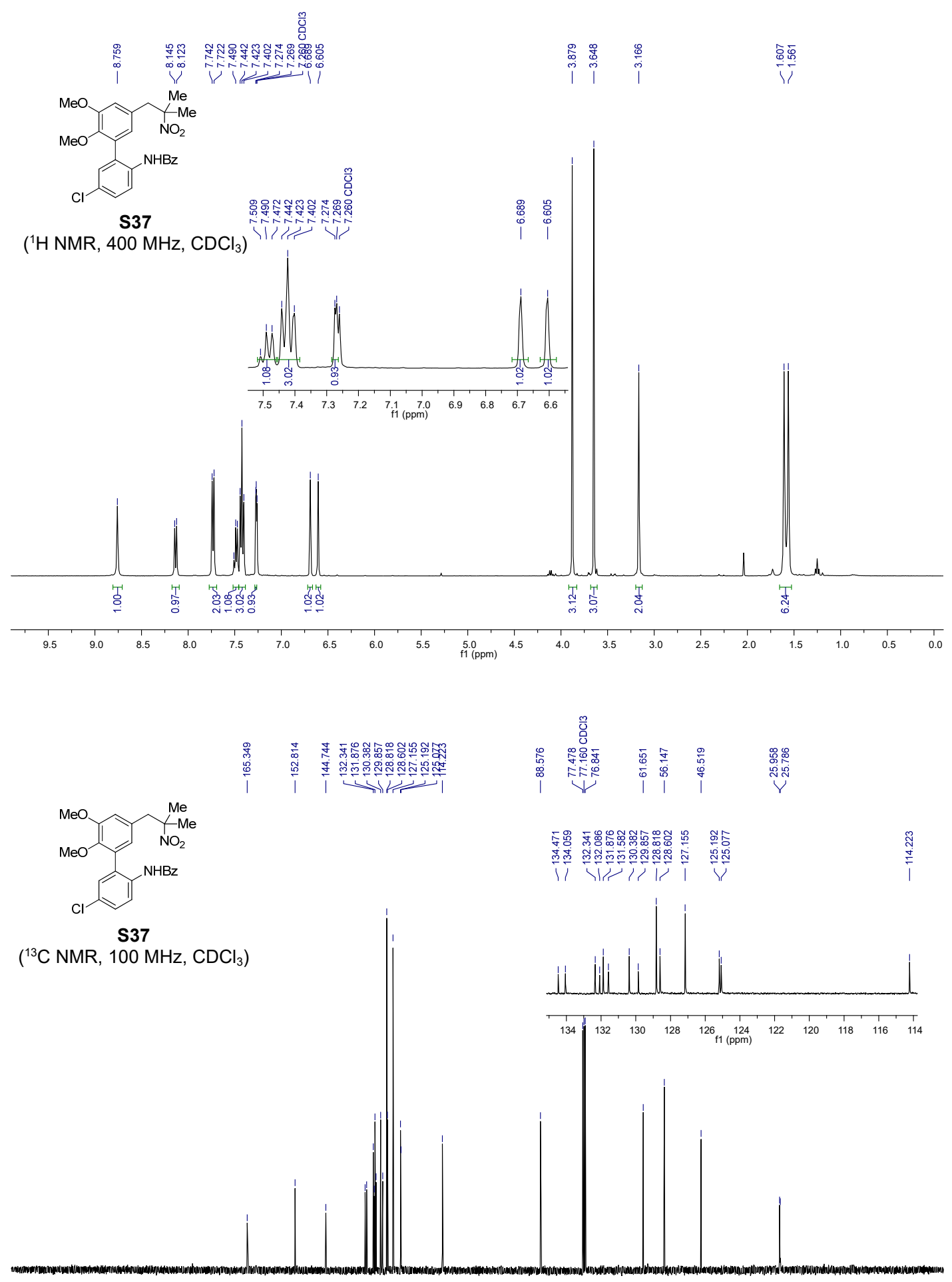

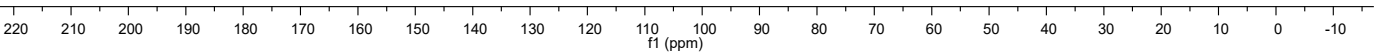




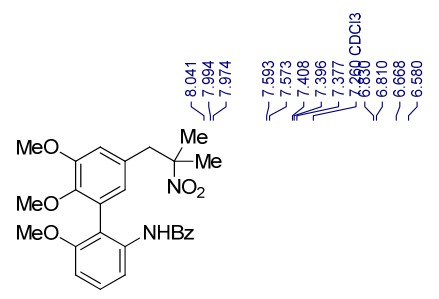

( ${ }^{1} \mathrm{H}$ NMR, $400 \mathrm{MHz}, \mathrm{CDCl}_{3}$ )
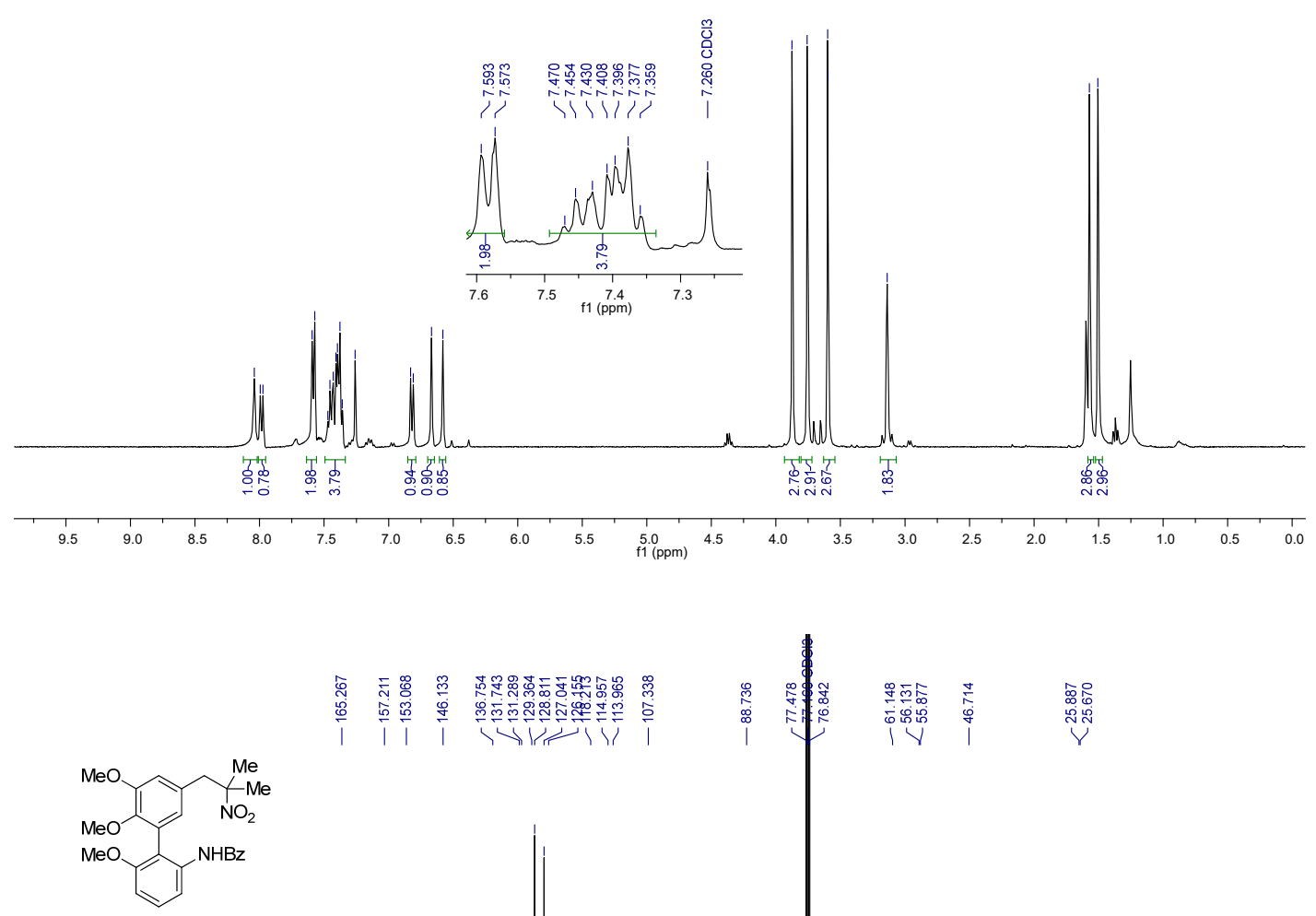

S38

$\left({ }^{13} \mathrm{C} \mathrm{NMR}, 100 \mathrm{MHz}, \mathrm{CDCl}_{3}\right)$

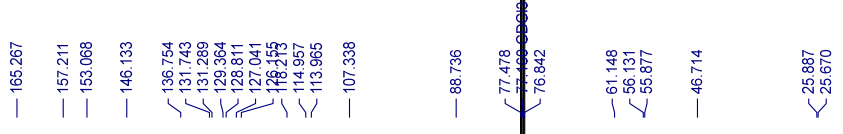

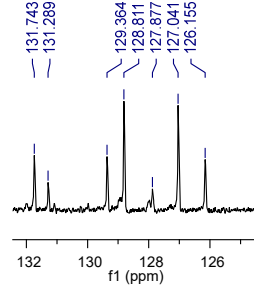

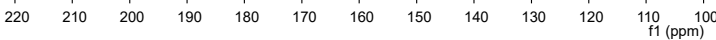




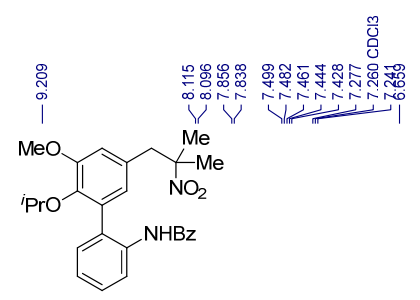

( ${ }^{1} \mathrm{H} \mathrm{NMR}, 400 \mathrm{MHz}, \mathrm{CDCl}_{3}$ )
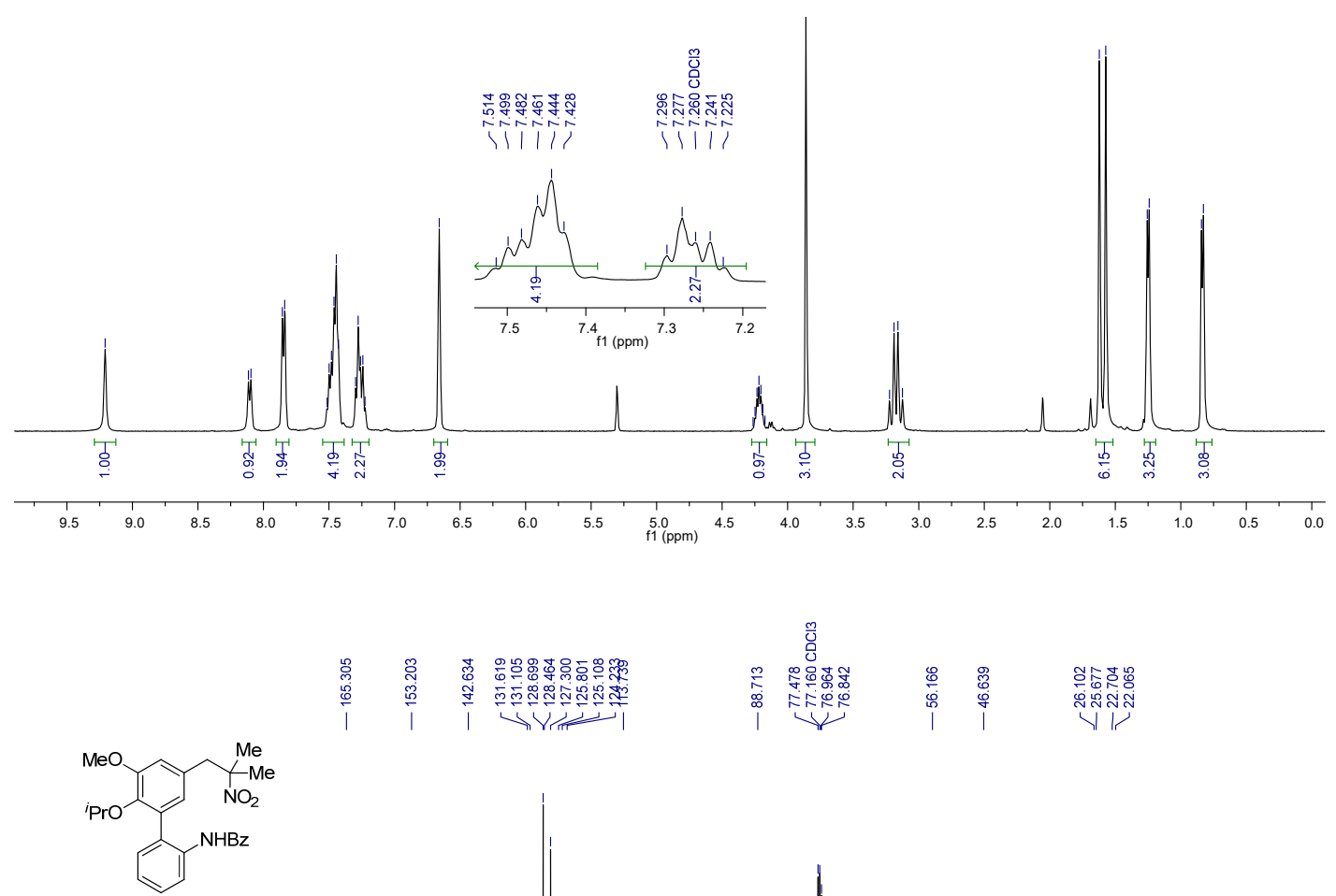

S39

$\left({ }^{13} \mathrm{C}\right.$ NMR, $\left.100 \mathrm{MHz}, \mathrm{CDCl}_{3}\right)$

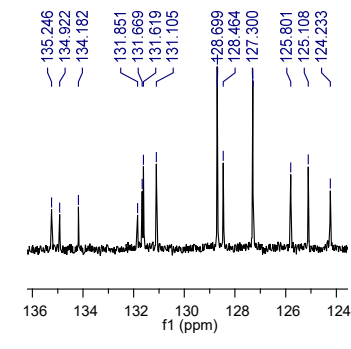

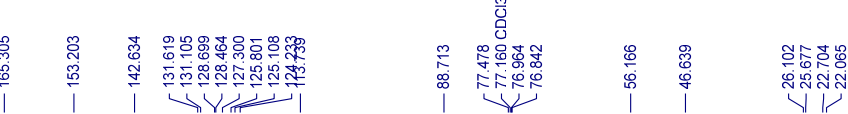

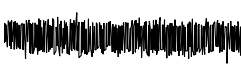

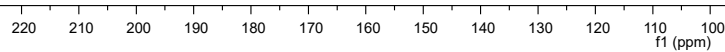




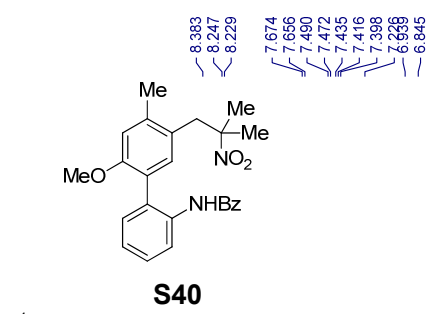

$\left({ }^{1} \mathrm{H}\right.$ NMR, $400 \mathrm{MHz}, \mathrm{CDCl}_{3}$ )
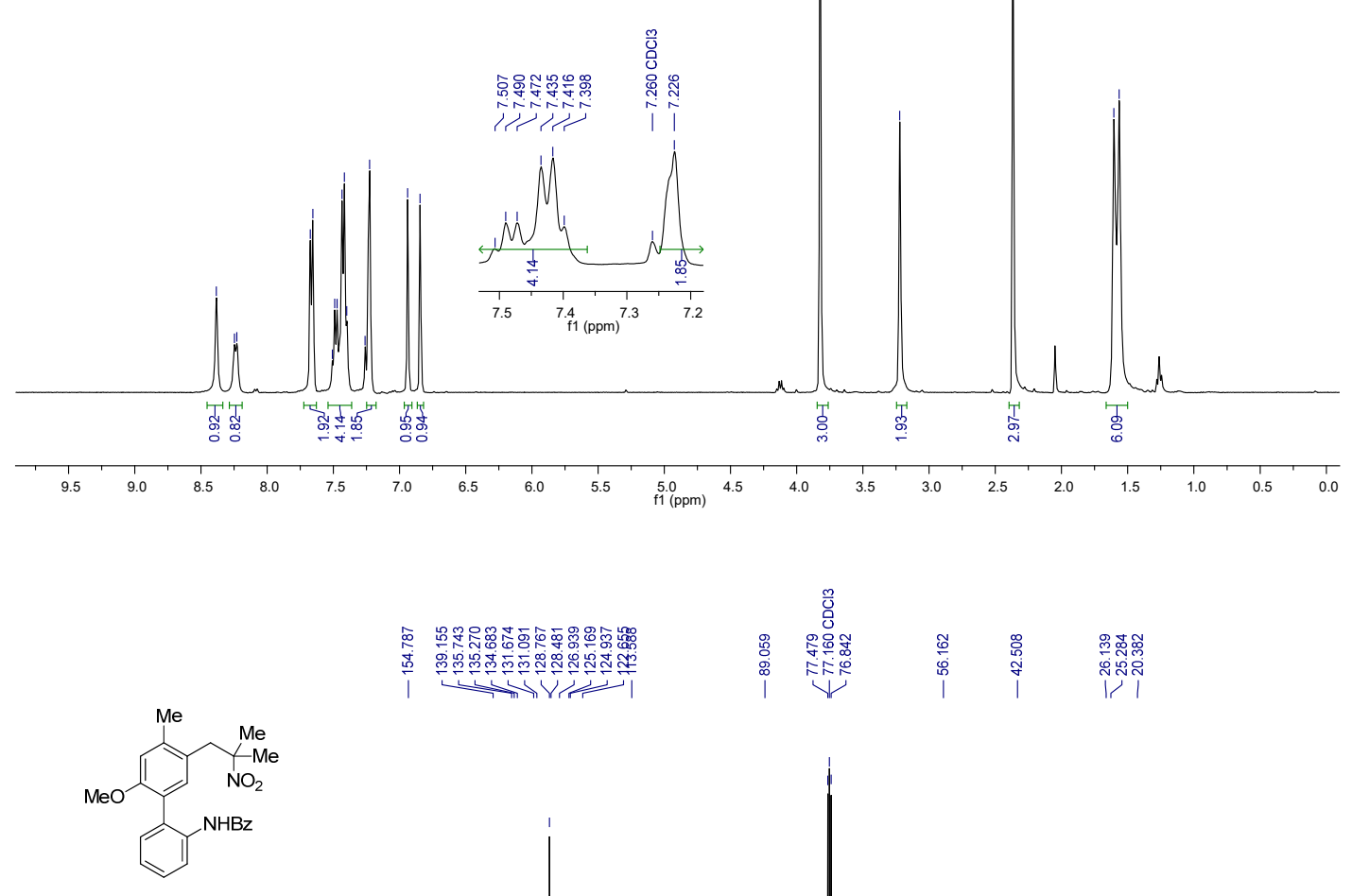

0
0
0

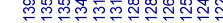

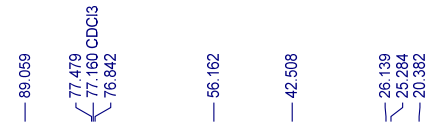

S40

$\left({ }^{13} \mathrm{C}\right.$ NMR, $\left.100 \mathrm{MHz}, \mathrm{CDCl}_{3}\right)$

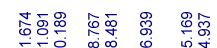

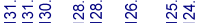

$111 \mathrm{Y}+\mathrm{Y}$

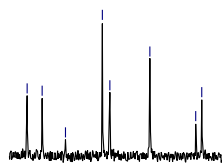

${ }_{132} \quad \begin{array}{lll}130 \\ \mathrm{fl}(\mathrm{ppm})\end{array}$

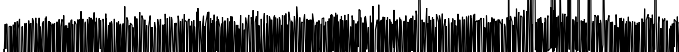

Whim

$\begin{array}{lllllll}220 & 210 & 200 & 190 & 180 & 170 & 160\end{array}$

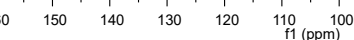




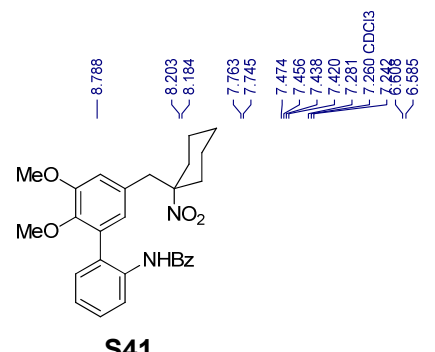

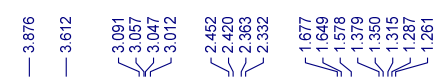

$\left({ }^{1} \mathrm{H} \mathrm{NMR}, 400 \mathrm{MHz}, \mathrm{CDCl}_{3}\right)$
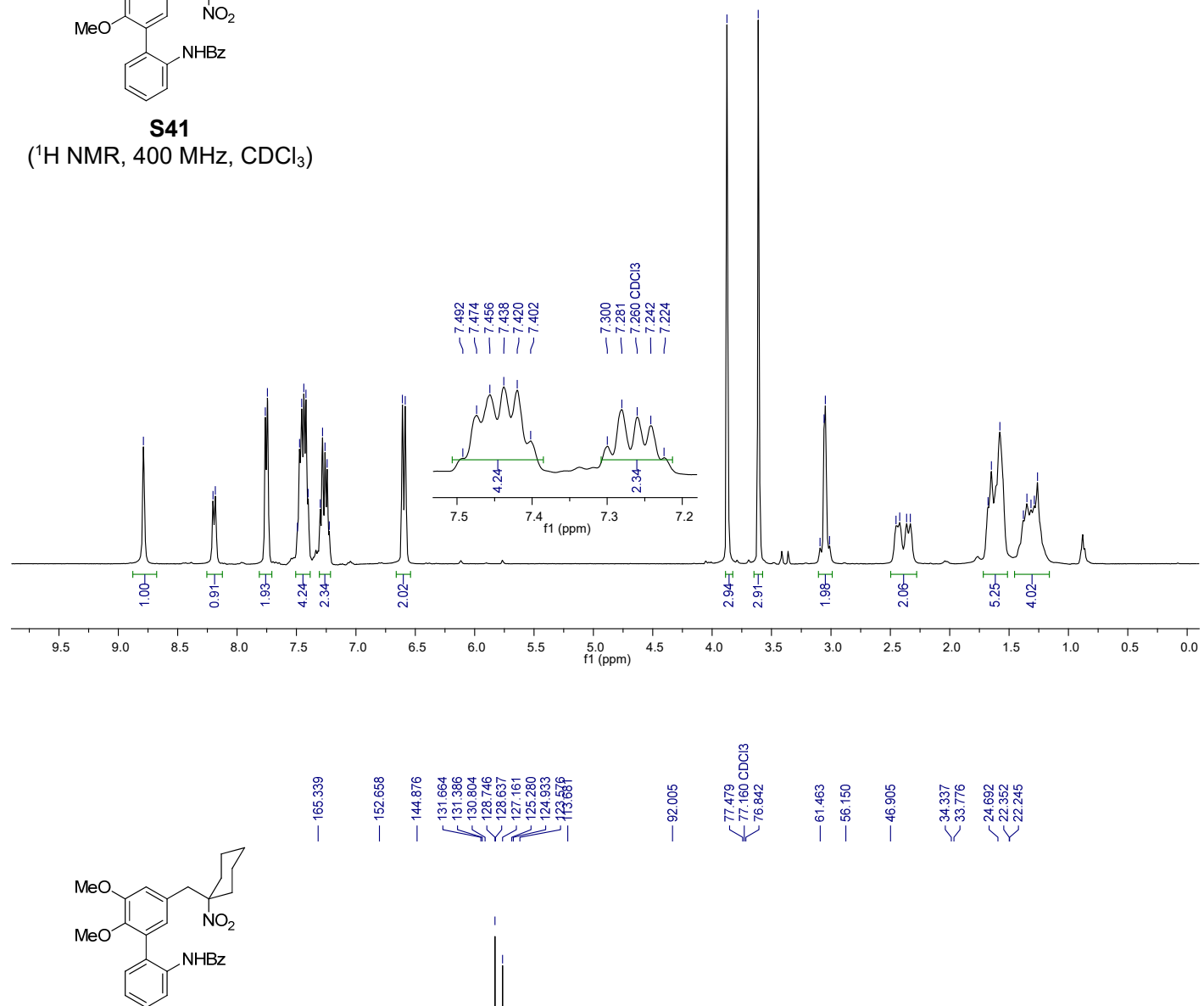

S41

$\left({ }^{13} \mathrm{C}\right.$ NMR, $\left.100 \mathrm{MHz}, \mathrm{CDCl}_{3}\right)$

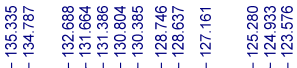

ार रोण ए।
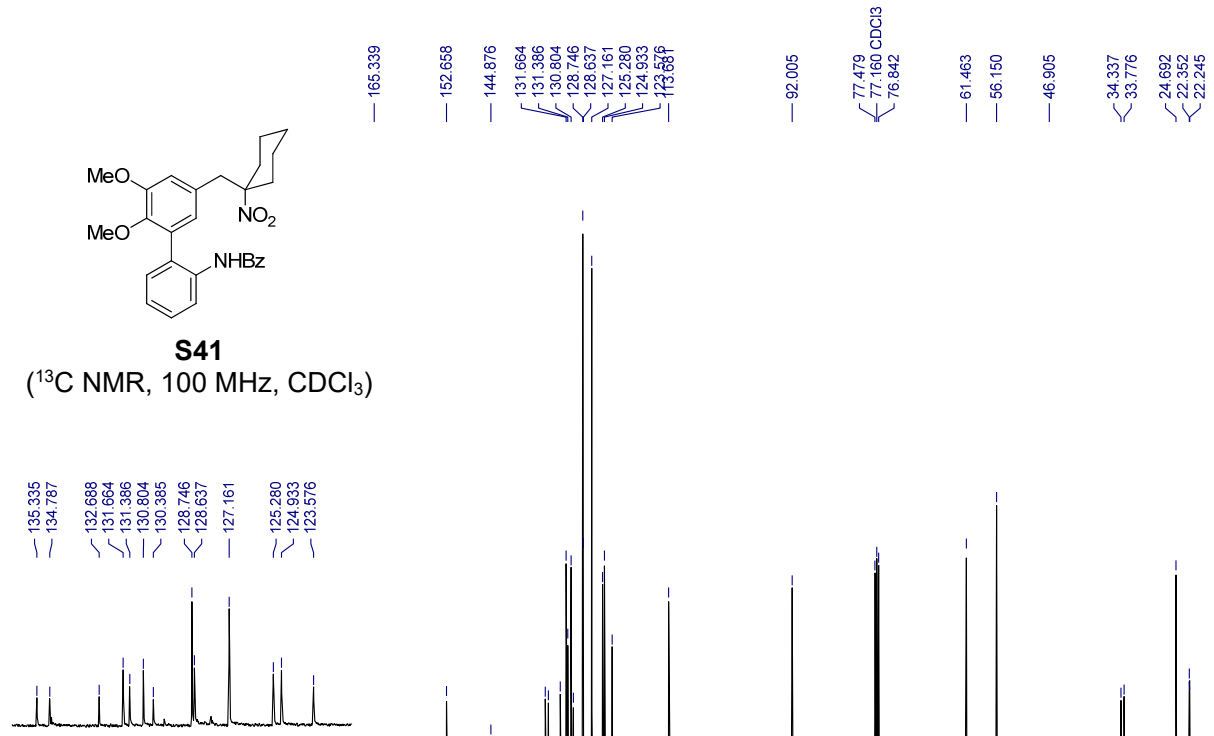

$\begin{array}{lllllllllll}T 136 & 134 & 132 & \begin{array}{l}130 \\ \mathrm{f} 1(\mathrm{ppm})\end{array} & 128 & 126 & 124 & 122\end{array}$

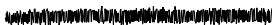




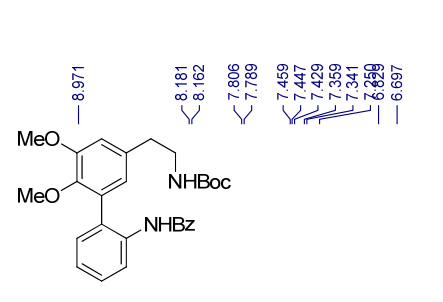

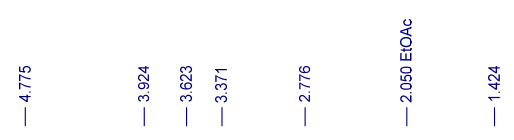

S42

$\left({ }^{1} \mathrm{H} \mathrm{NMR}, 400 \mathrm{MHz}, \mathrm{CDCl}_{3}\right)$
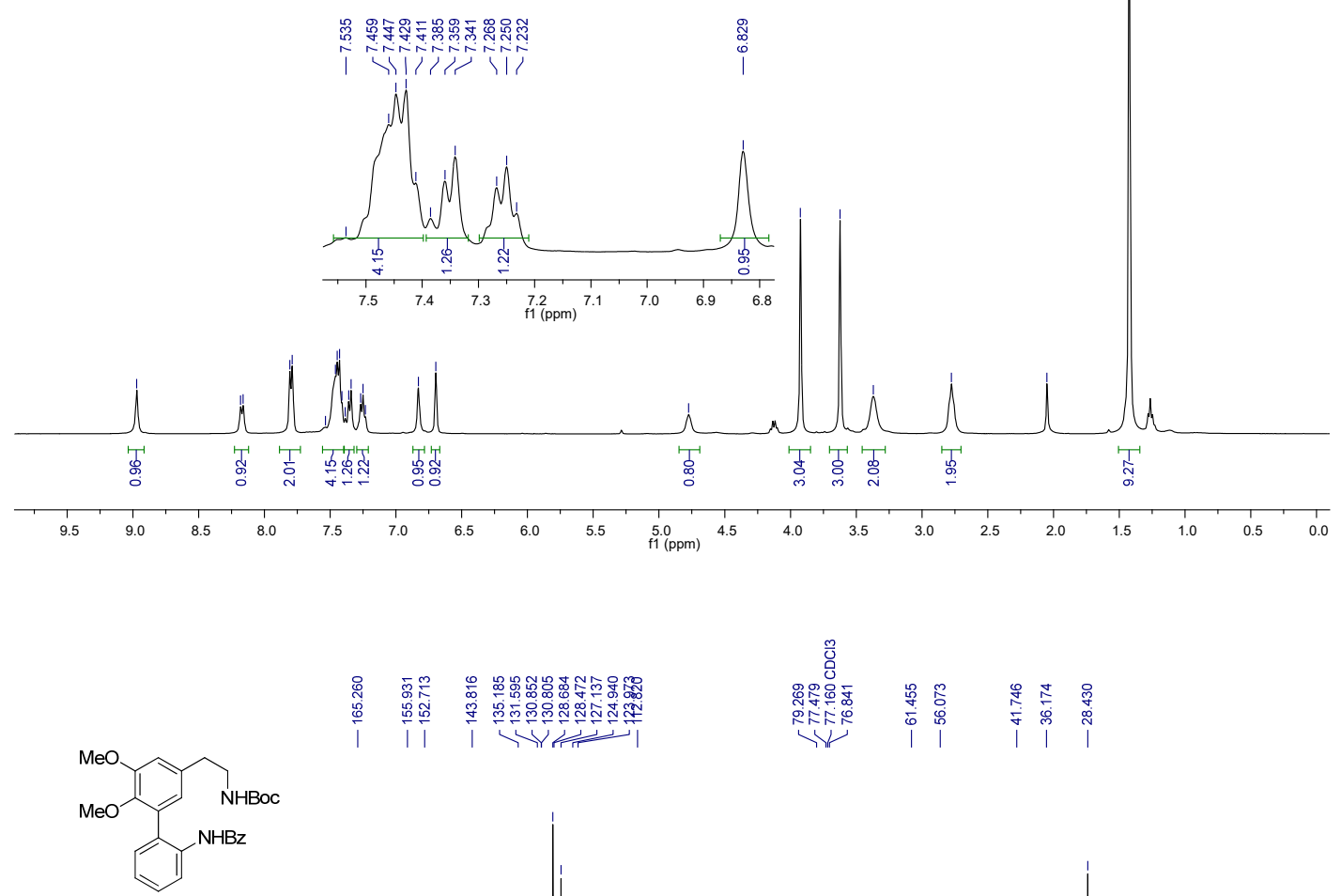

S42

$\left({ }^{13} \mathrm{C} \mathrm{NMR}, 100 \mathrm{MHz}, \mathrm{CDCl}_{3}\right)$

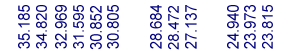

ili

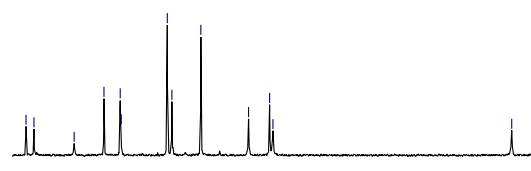

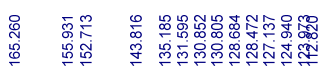

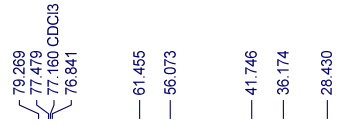

$\begin{array}{lllllllllllll}134 & 132 & 130 & 128 & 126 & 124 & 122 & 120 & 118 & 116 & 114 & 112\end{array}$

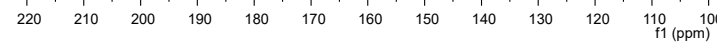




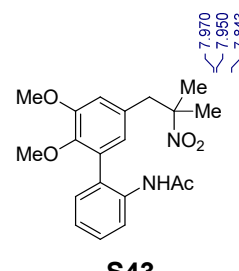

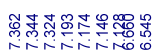

ininivio

543

( ${ }^{1} \mathrm{H} \mathrm{NMR}, 400 \mathrm{MHz}, \mathrm{CDCl}_{3}$ )
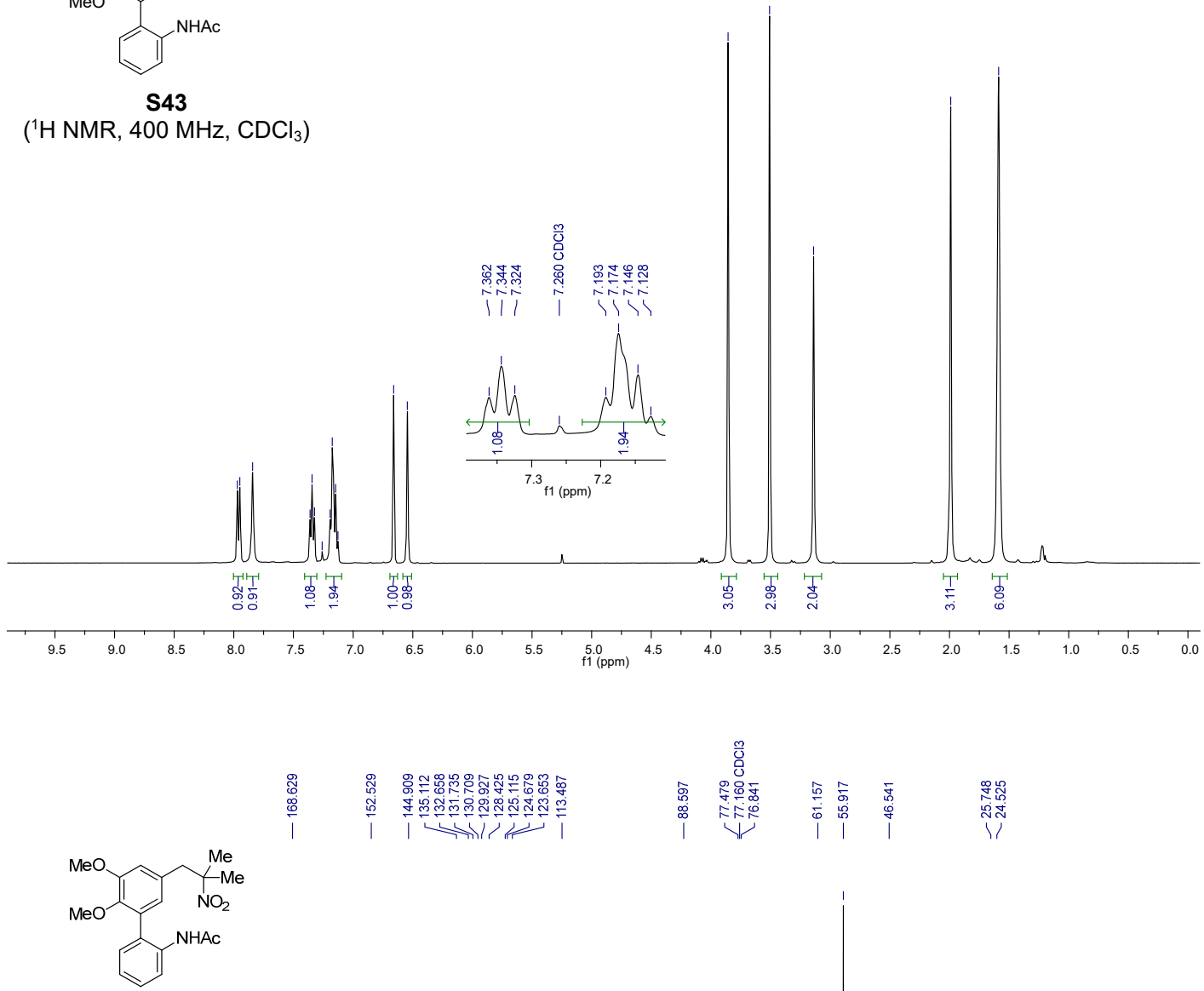

S43

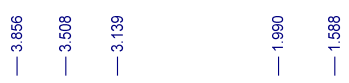

$\left({ }^{13} \mathrm{C}\right.$ NMR, $\left.100 \mathrm{MHz}, \mathrm{CDCl}_{3}\right)$

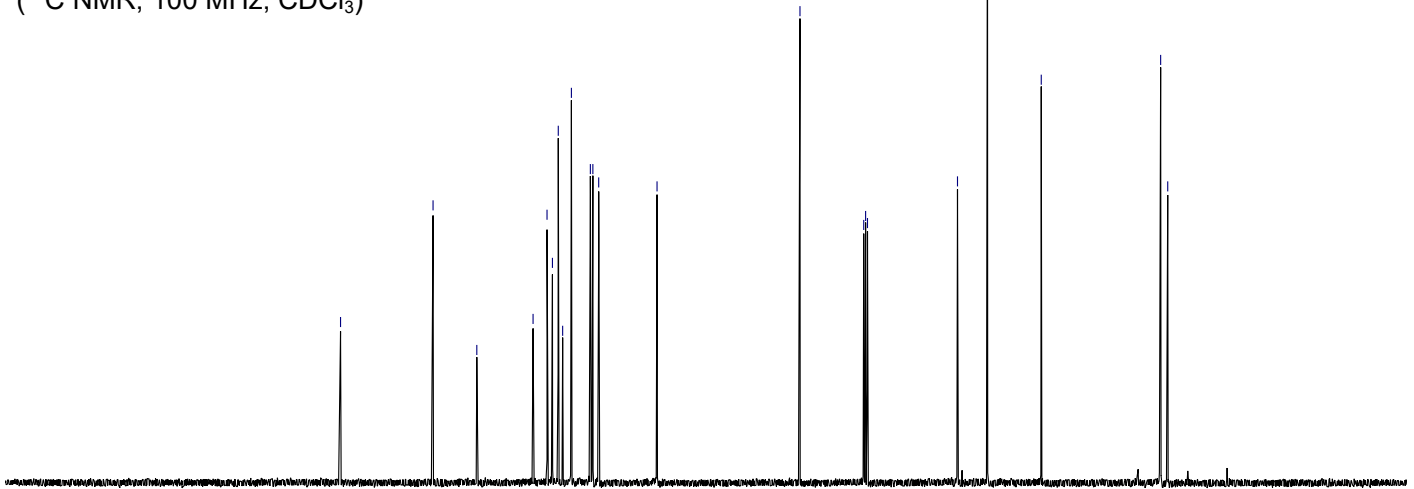

$\begin{array}{llllllllllll}220 & 210 & 200 & 190 & 180 & 170 & 160 & 150 & 140 & 130 & 120 & \begin{array}{l}110 \\ \mathrm{f} 1(\mathrm{ppm})\end{array}\end{array}$ 


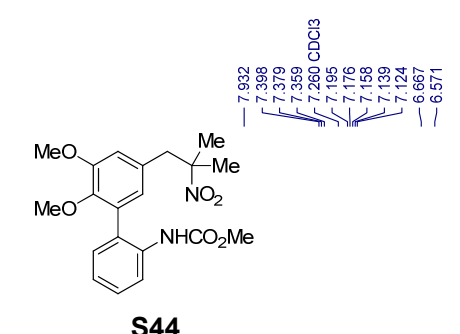

$\left({ }^{1} \mathrm{H}\right.$ NMR, $\left.400 \mathrm{MHz}, \mathrm{CDCl}_{3}\right)$
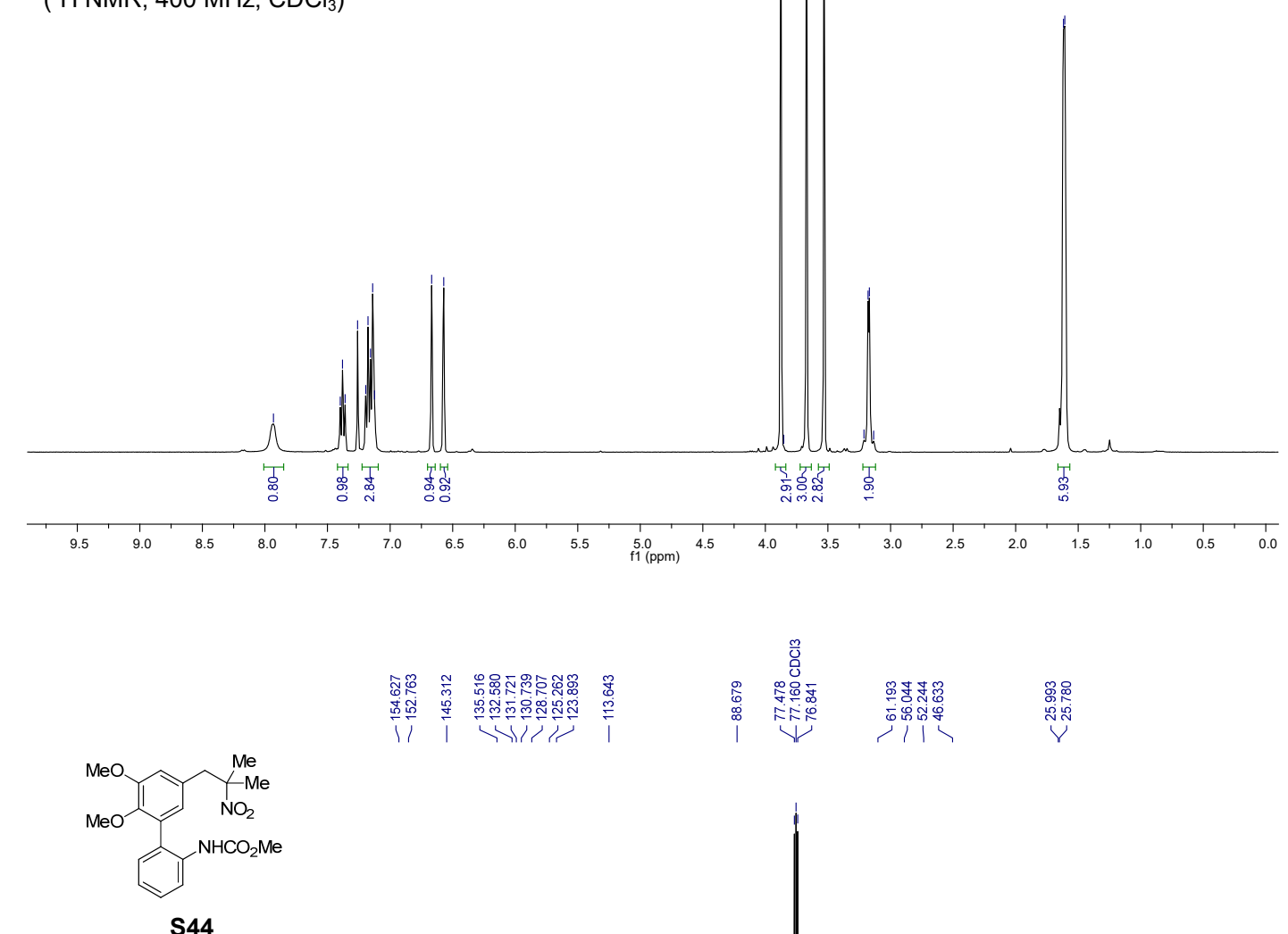

$\left({ }^{13} \mathrm{C} \mathrm{NMR}, 100 \mathrm{MHz}, \mathrm{CDCl}_{3}\right)$

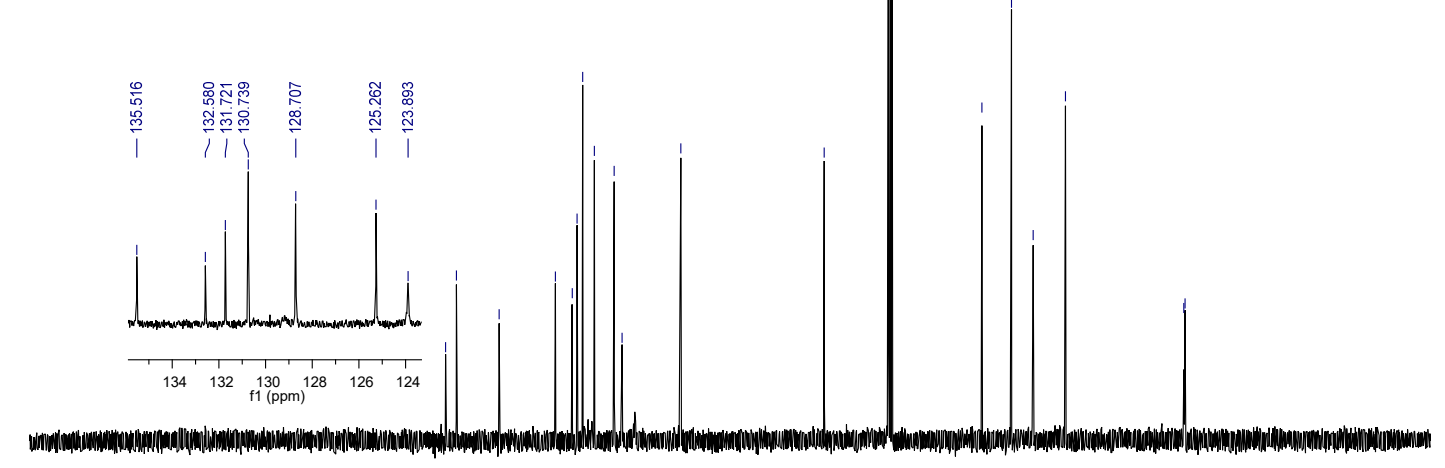

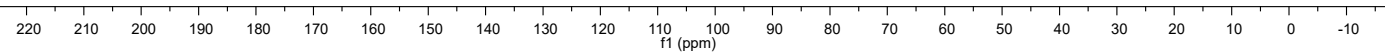




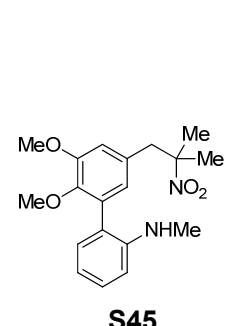

$\left({ }^{1} \mathrm{H} \mathrm{NMR}, 400 \mathrm{MHz}, \mathrm{CDCl}_{3}\right)$
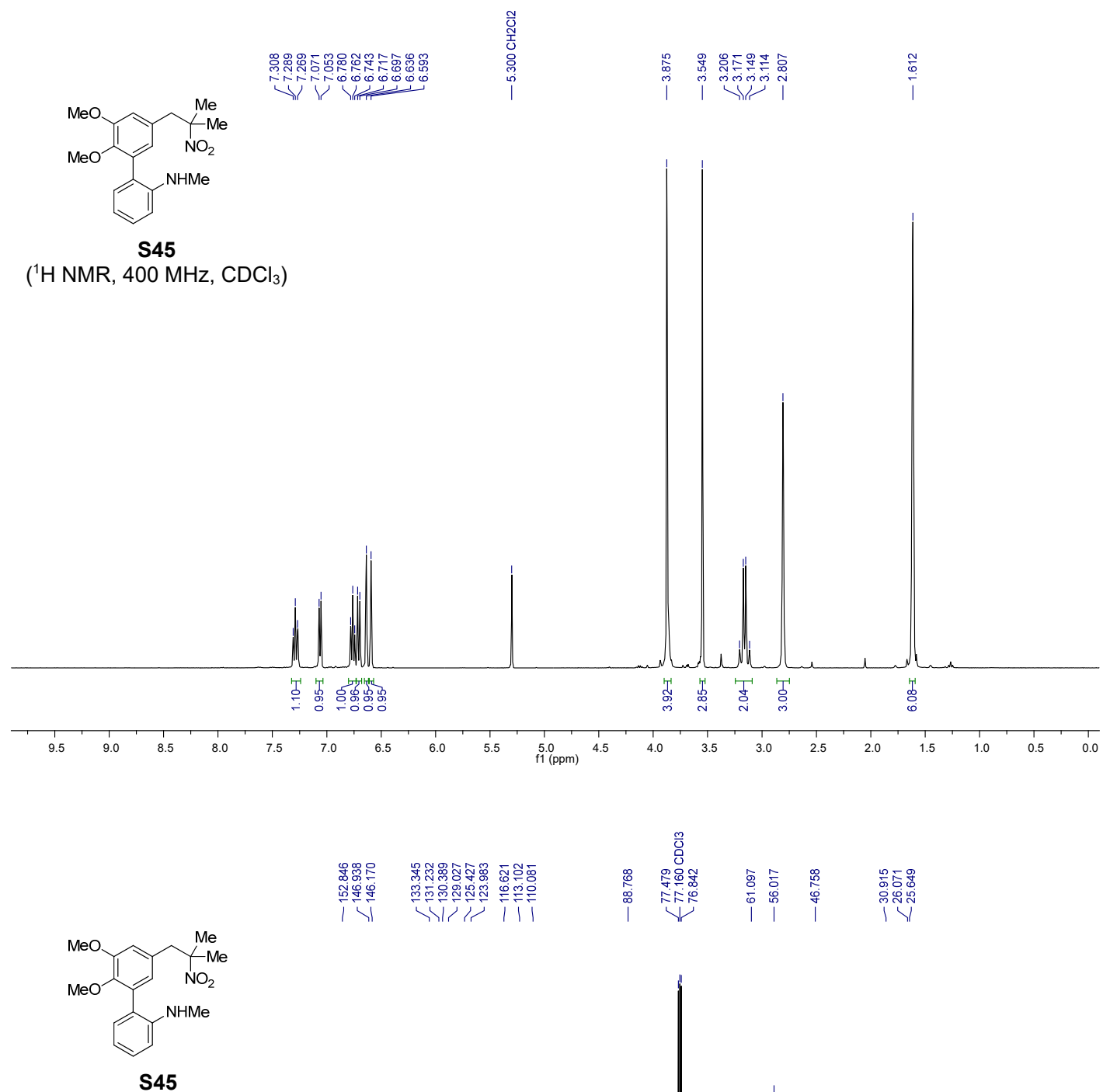

$\left({ }^{13} \mathrm{C}\right.$ NMR, $\left.100 \mathrm{MHz}, \mathrm{CDCl}_{3}\right)$
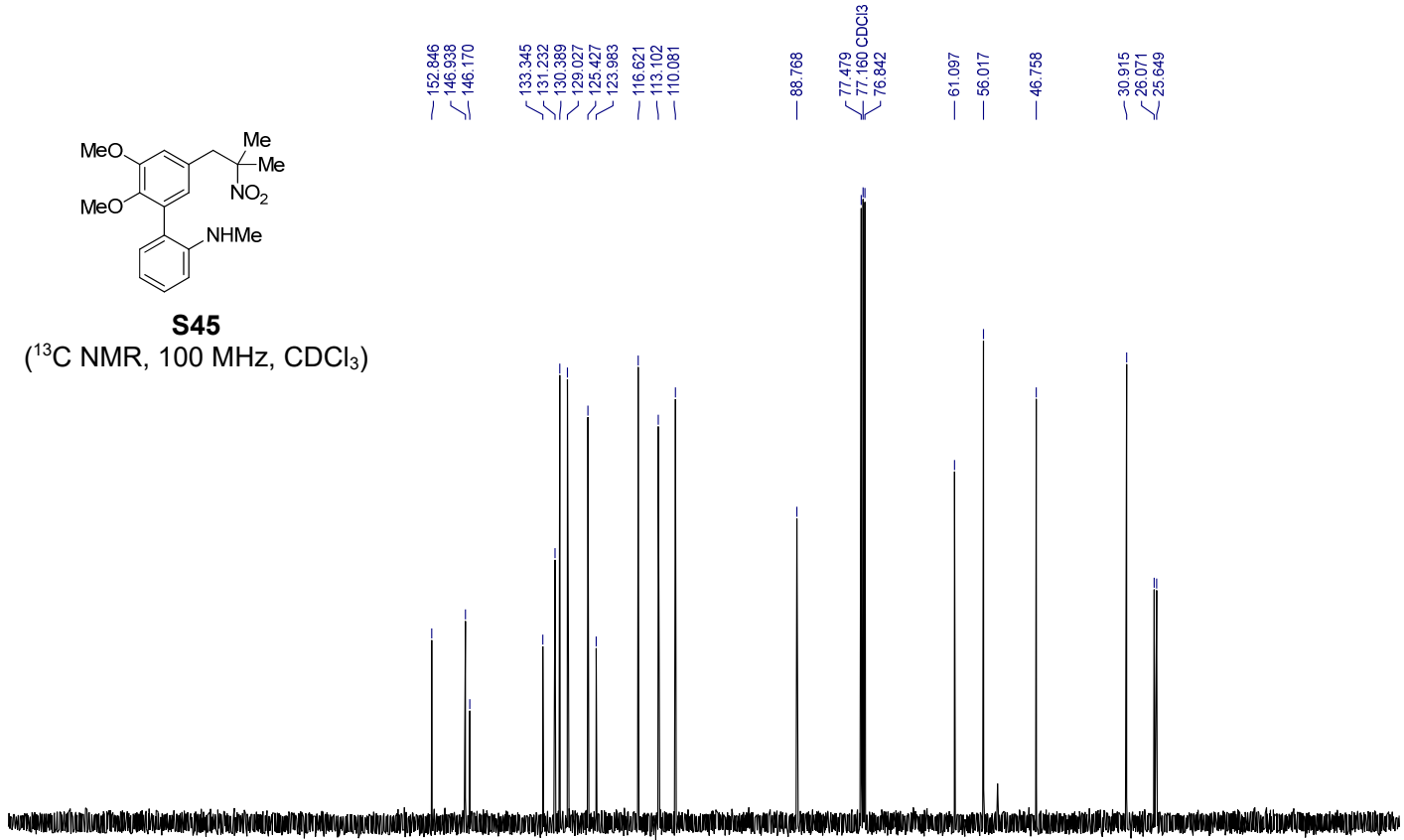

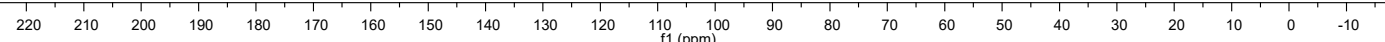



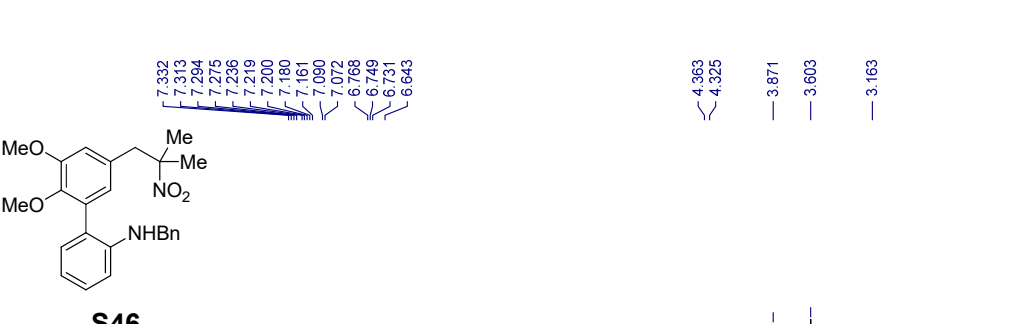

S46
$\left({ }^{1} \mathrm{H}\right.$ NMR, $\left.400 \mathrm{MHz}, \mathrm{CDCl}_{3}\right)$
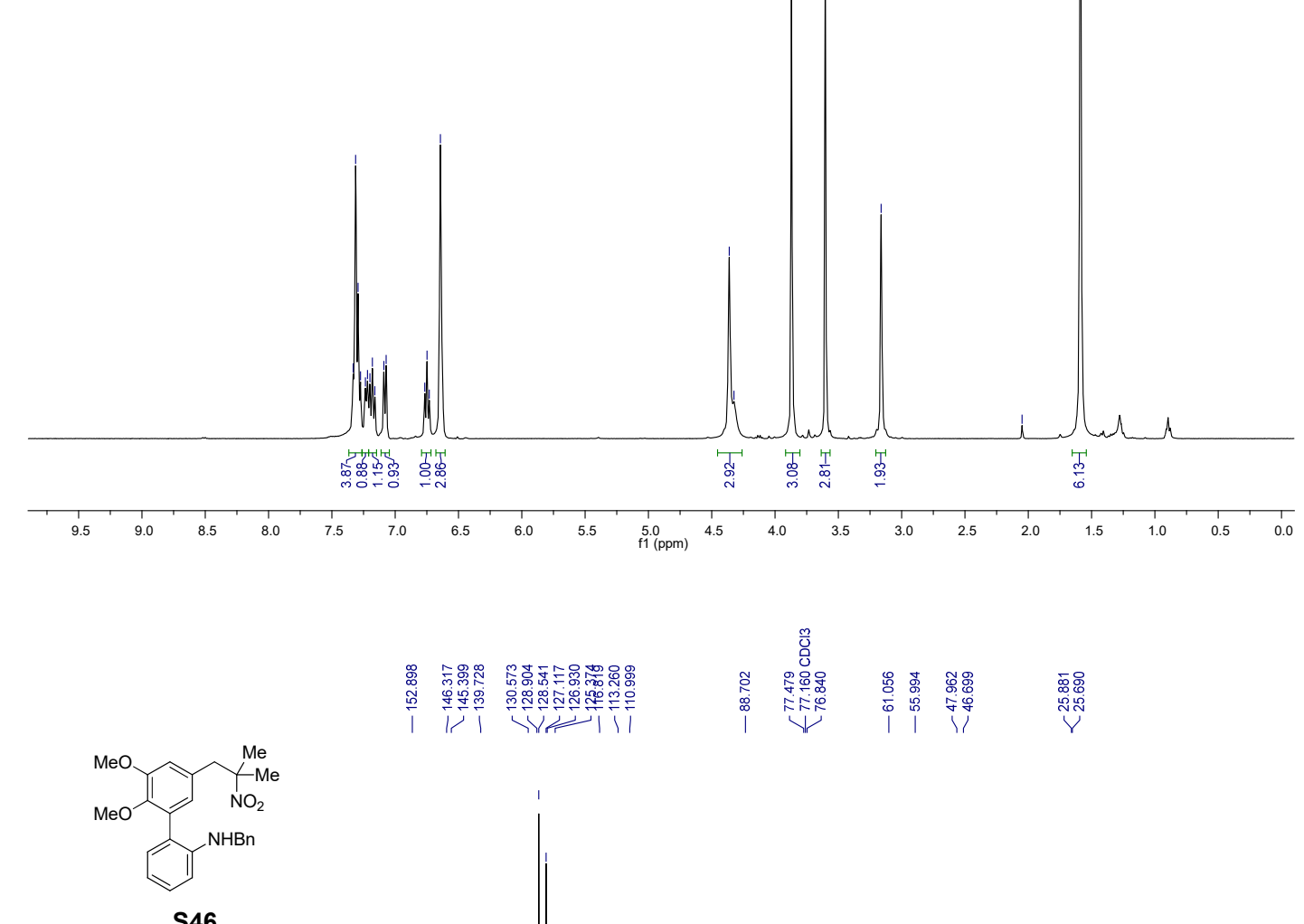

$\left({ }^{13} \mathrm{C}\right.$ NMR, $\left.100 \mathrm{MHz}, \mathrm{CDCl}_{3}\right)$

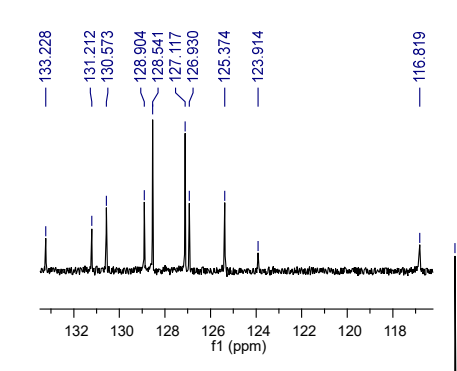

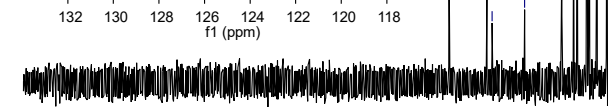

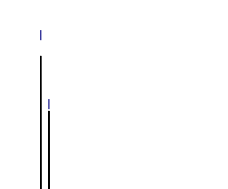



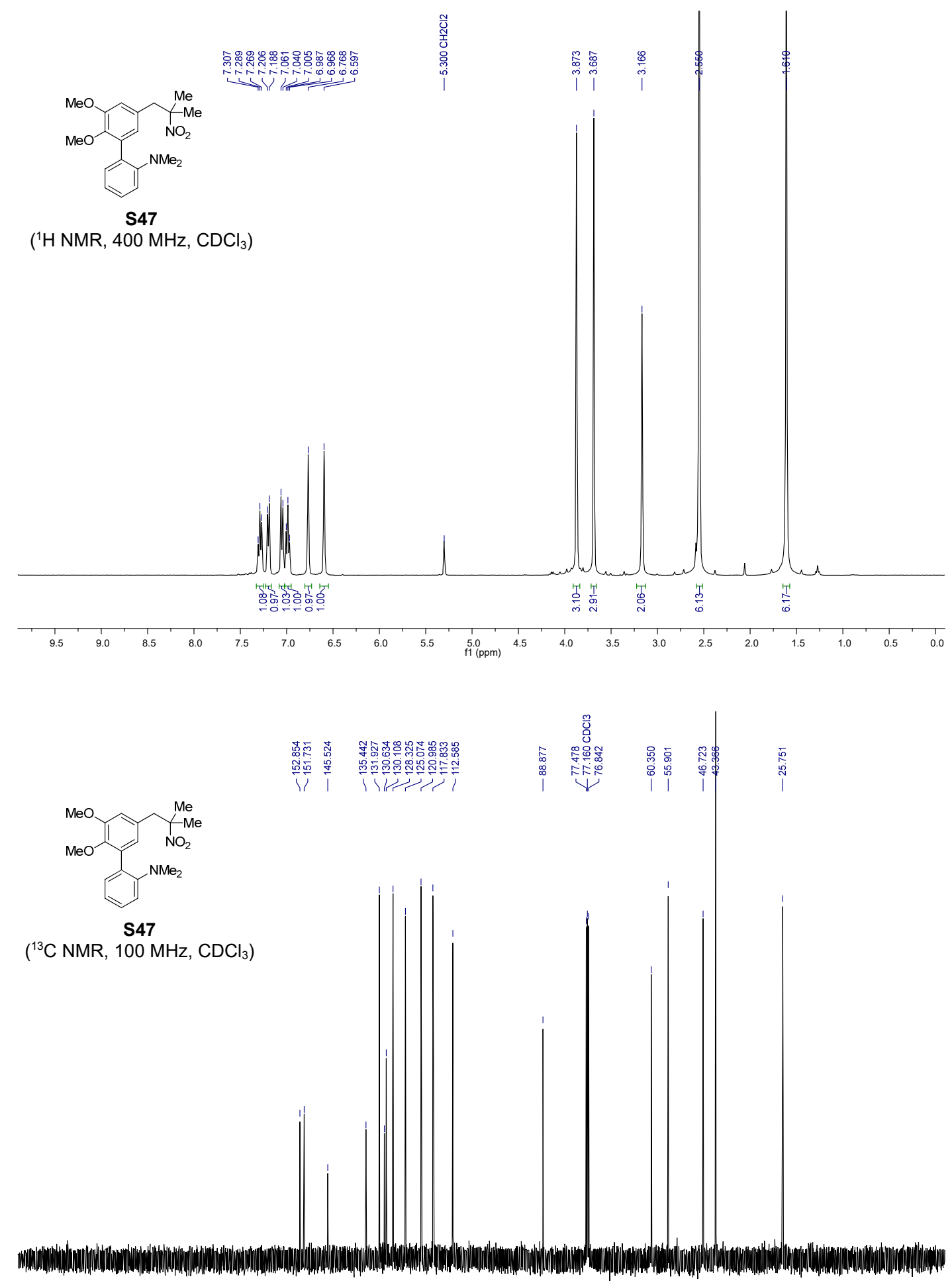

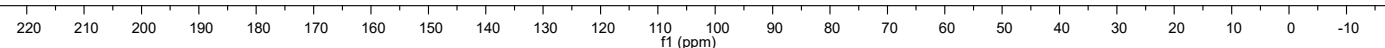



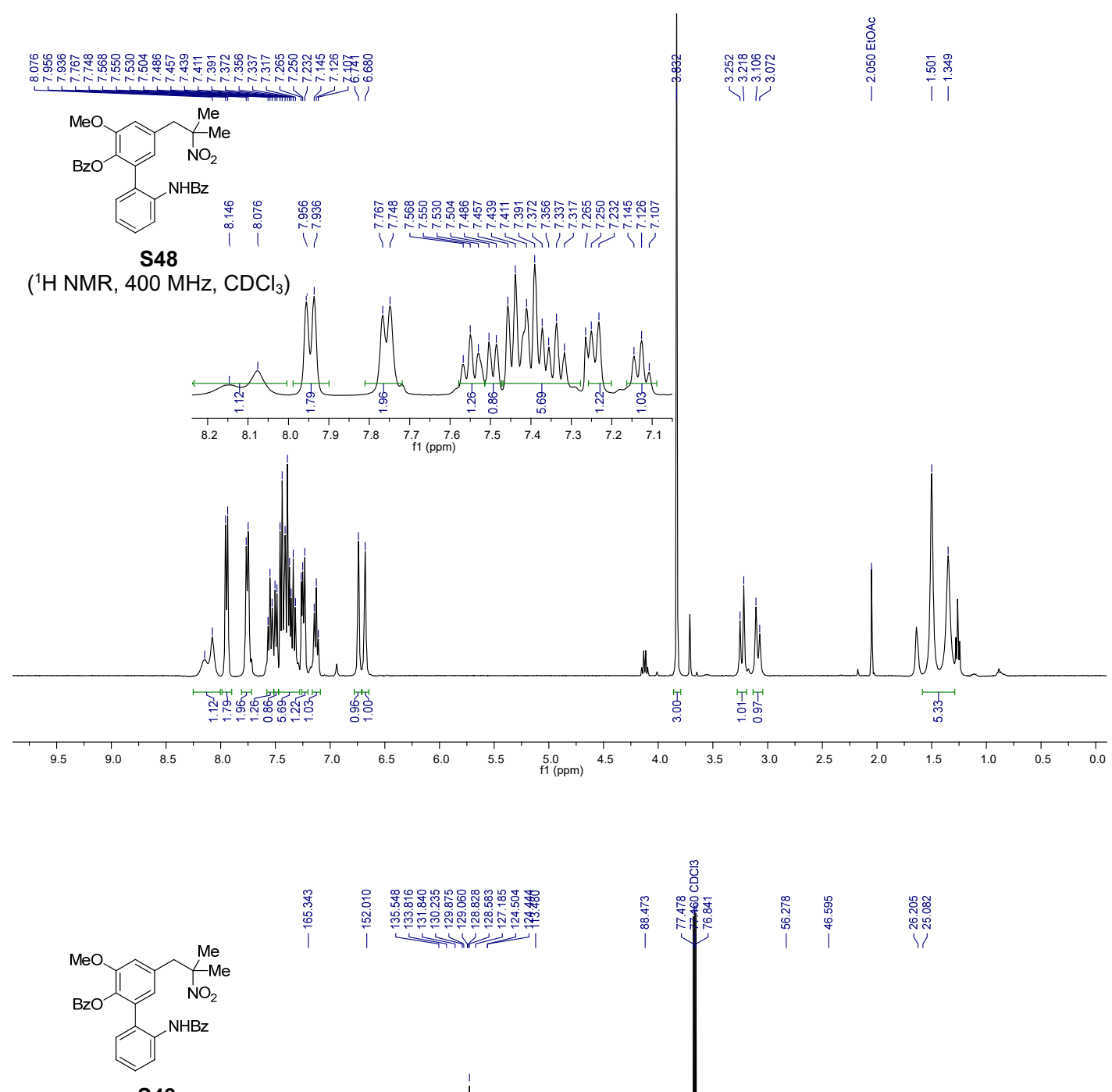

$\left({ }^{13} \mathrm{C} N M R, 100 \mathrm{MHz}, \mathrm{CDCl}_{3}\right)$

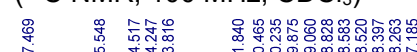

至

广 广1 广
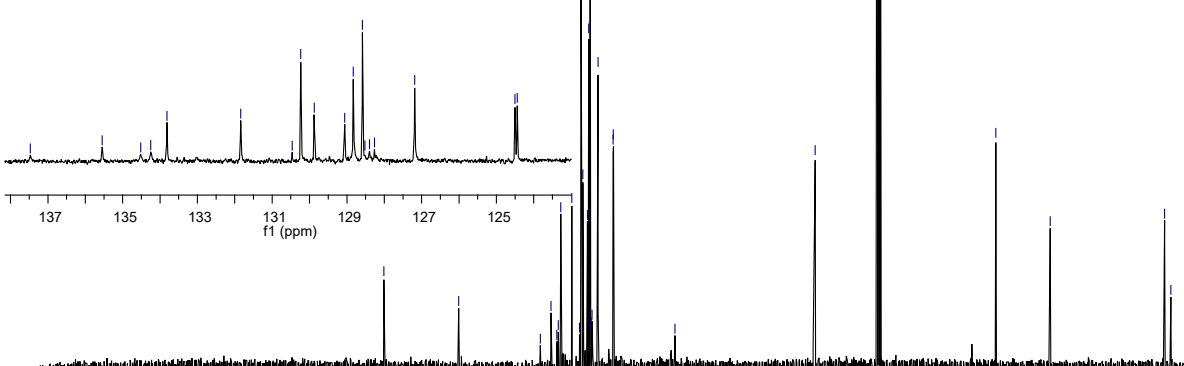

$\begin{array}{llllllllllll}220 & 210 & 200 & 190 & 180 & 170 & 160 & 150 & 140 & 130 & 120 & \begin{array}{c}110 \\ \mathrm{f} 1(\mathrm{ppm})\end{array}\end{array}$ 

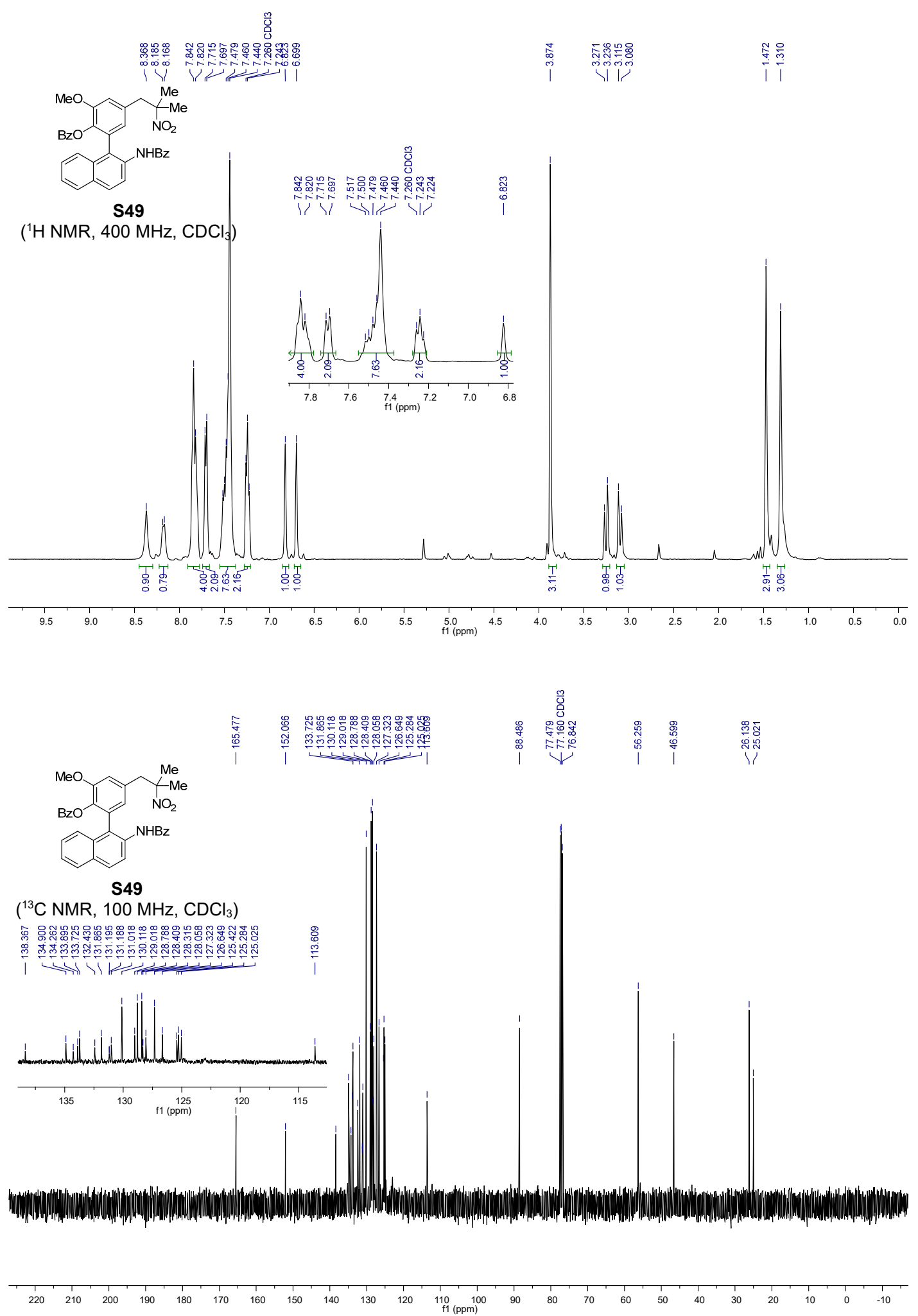


\subsection{Synthesis of Substrate 1}
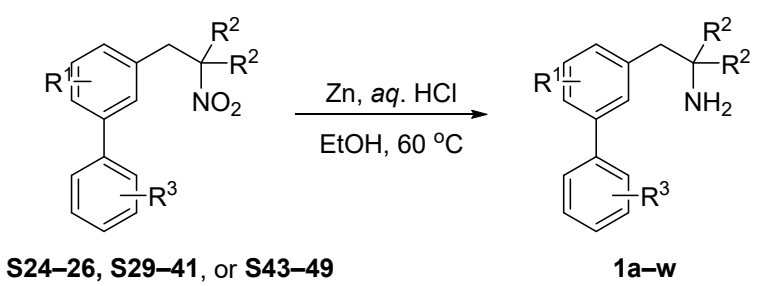

Procedure 16: To a round-bottom flask equipped with a magnetic stirring bar and a refluxing condenser, S24-26, S29-41, or $\mathbf{S 4 3 - 4 9}$ (1.0 equiv) and zinc dust (18.2 equiv) were added and subsequently dissolved in $\mathrm{EtOH}(0.10 \mathrm{M})$. To the reaction mixture, diluted aqueous $\mathrm{HCl}(2.0$ equiv, $0.40 \mathrm{M})$ was added, and the reaction was heated to $60{ }^{\circ} \mathrm{C}$ in a heating block and allowed to stir for overnight at $60^{\circ} \mathrm{C}$. The reaction mixture was cooled to rt and filtered with cotton plug. After removing the solvent in vacuo, the residue was diluted with $\mathrm{CH}_{2} \mathrm{Cl}_{2}$, transferred to a separatory funnel, and basified with aqueous $1 \mathrm{M} \mathrm{NaOH}$. The organic layer was separated, and the aqueous layer was extracted an additional two times with $\mathrm{CH}_{2} \mathrm{Cl}_{2}$. The combined organic layers were then rinsed with water, dried with anhydrous $\mathrm{MgSO}_{4}$, and concentrated in vacuo. The crude material was then purified by flash chromatography to afford the desired material 1aw.
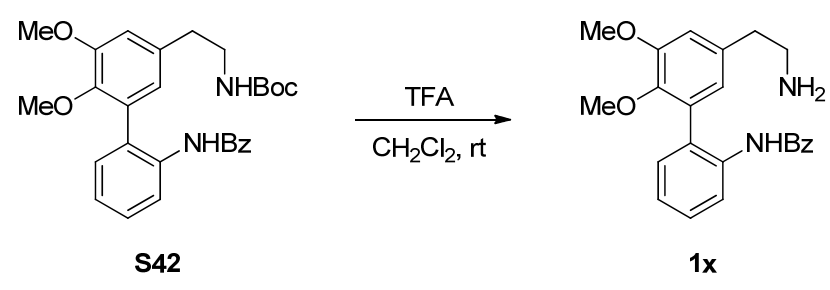

Procedure 17: Boc deprotection

To a round-bottom flask equipped with a magnetic stirring bar, $\mathbf{S 4 2}$ (1.0 equiv) was added and subsequently dissolved in $\mathrm{CH}_{2} \mathrm{Cl}_{2}(0.20 \mathrm{M})$. Trifluoroacetic acid (13.0 equiv) was added at $\mathrm{rt}$ and the reaction mixture was allowed to stir until complete consumption of starting material which was indicated by TLC (about $1.5 \mathrm{~h}$ ). The reaction mixture was quenched with saturated aqueous $\mathrm{Na}_{2} \mathrm{CO}_{3}$ and extracted three times with $\mathrm{CH}_{2} \mathrm{Cl}_{2}$. The combined organic layers were dried by anhydrous $\mathrm{MgSO}_{4}$, filtered, and concentrated in vacuo. The crude material was then purified by flash chromatography to afford the desired material $\mathbf{1 x}$. 


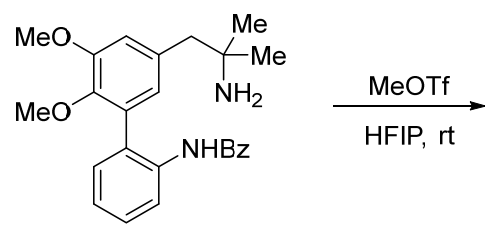

$1 a$

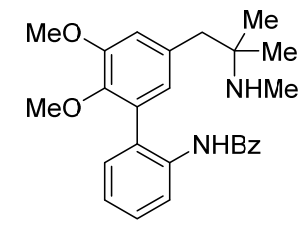

$1 y$

Procedure 18: Methylation

To a solution of HFIP (10 equiv) and 1a (1.0 equiv) was added methyl triflate (1.5 equiv). The mixture was stirred for $1 \mathrm{~h}$ at $\mathrm{rt}$ and then quenched by an aqueous $\mathrm{HCl}(2$ $\mathrm{N}, 1 \mathrm{~mL}$ ). Volatiles were evaporated under reduced pressure, and the resulting mixture was neutralized with a saturated aqueous solution of $\mathrm{NaHCO}_{3}$ and extracted three times with $\mathrm{CH}_{2} \mathrm{Cl}_{2}$. Combined organic layers were dried with $\mathrm{MgSO}_{4}$, filtered, and concentrated in vacuo. The crude material was then purified by flash chromatography to afford the desired material $\mathbf{1 y}$. 


\section{Characterization and Spectra of Substrate 1}

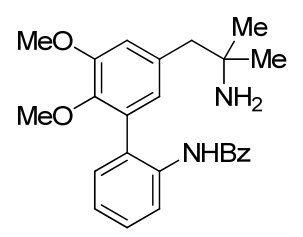

$N$-(5'-(2-Amino-2-methylpropyl)-2',3'-dimethoxy-[1,1'-biphenyl]-2-yl)benzamide (1a) was synthesized by following Procedure 16 from S31. The crude material was purified by normal-phase column chromatography using an eluent of $10 \%$ $\mathrm{MeOH} / \mathrm{EtOAc}$ with $1 \%$ aqueous $\mathrm{NH}_{3}$ to provide 1a as a white solid (348 mg, 95\%).

m.p. $118^{\circ} \mathrm{C}$

${ }^{1} \mathbf{H}$ NMR $\left(400 \mathrm{MHz}, \mathrm{CDCl}_{3}\right) \delta 8.93(\mathrm{~s}, 1 \mathrm{H}), 8.16(\mathrm{~d}, J=8.1 \mathrm{~Hz}, 1 \mathrm{H}), 7.77(\mathrm{~d}, J=7.5$ $\mathrm{Hz}, 2 \mathrm{H}), 7.48-7.37(\mathrm{~m}, 4 \mathrm{H}), 7.32(\mathrm{~d}, J=7.5 \mathrm{~Hz}, 1 \mathrm{H}), 7.23(\mathrm{t}, J=7.5 \mathrm{~Hz}, 1 \mathrm{H}), 6.81(\mathrm{~s}$, $1 \mathrm{H}), 6.69(\mathrm{~s}, 1 \mathrm{H}), 3.91(\mathrm{~s}, 3 \mathrm{H}), 3.61(\mathrm{~s}, 3 \mathrm{H}), 2.63(\mathrm{~s}, 2 \mathrm{H}), 1.50(\mathrm{brs}, 2 \mathrm{H}), 1.12(\mathrm{~s}, 6 \mathrm{H})$.

${ }^{13}$ C NMR (100 MHz, $\left.\mathrm{CDCl}_{3}\right) \delta 165.3,152.2,143.9,135.7,135.3,134.9,132.5,131.6$, $130.9,130.8,128.7$ (2C), 128.5, 127.2 (2C), 125.8, 124.9, 123.7, 114.7, 61.5, 56.2, $50.8,50.2,30.6,30.5$.

IR (FT-ATR, $\mathrm{cm}^{-1}, \mathrm{CH}_{2} \mathrm{Cl}_{2}$ ) $v_{\max } 3350,3062,2961,2935,2842,1672,1582,1529$, 1484, 1466, 1443, 1419, 1382, 1365, 1343, 1304, 1248, 1184, 1142, 1118, 1090, 1074 , 1049, 1036, 1003, 901, 857, 820, 788, 757, 709, 692, 674, 633, 586, 531.

HRMS (ESI) $m / z$ : $[\mathrm{M}+\mathrm{H}]^{+}$Calcd for $\mathrm{C}_{25} \mathrm{H}_{28} \mathrm{~N}_{2} \mathrm{O}_{3}$ 405.2178; found 405.2173.

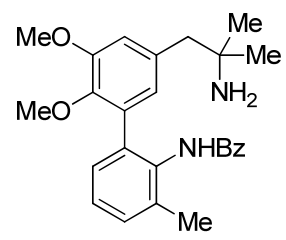

$\mathrm{N}$-(5'-(2-Amino-2-methylpropyl)-2',3'-dimethoxy-3-methyl-[1,1'-biphenyl]-2yl)benzamide (1b) was synthesized by following Procedure 16 from $\mathbf{S 3 2}$. The crude material was purified by normal-phase column chromatography using an eluent of $10 \%$ $\mathrm{MeOH} /$ EtOAc with $1 \%$ aqueous $\mathrm{NH}_{3}$ to provide $\mathbf{1 b}$ as a white solid $(273 \mathrm{mg}, 86 \%$ ).

m.p. $60{ }^{\circ} \mathrm{C}$

${ }^{1} \mathbf{H}$ NMR $\left(400 \mathrm{MHz}, \mathrm{CDCl}_{3}\right) \delta 8.78(\mathrm{~s}, 1 \mathrm{H}), 7.75(\mathrm{~d}, J=7.2 \mathrm{~Hz}, 2 \mathrm{H}), 7.46(\mathrm{t}, J=7.3$ $\mathrm{Hz}, 1 \mathrm{H}), 7.40$ (t, $J=7.3 \mathrm{~Hz}, 2 \mathrm{H}), 7.34(\mathrm{~d}, J=7.2 \mathrm{~Hz}, 1 \mathrm{H}), 7.29(\mathrm{t}, J=7.4 \mathrm{~Hz}, 1 \mathrm{H}), 7.22$ $(\mathrm{d}, J=7.0 \mathrm{~Hz}, 1 \mathrm{H}), 6.72(\mathrm{~d}, J=10.1 \mathrm{~Hz}, 2 \mathrm{H}), 3.88(\mathrm{~s}, 3 \mathrm{H}), 3.61(\mathrm{~s}, 3 \mathrm{H}), 2.59(\mathrm{~s}, 2 \mathrm{H})$, 2.40 (s, 3H), 1.44 (brs, 2H)1.10 (s, 6H).

${ }^{13}$ C NMR $\left(100 \mathrm{MHz}, \mathrm{CDCl}_{3}\right) \delta 165.2,151.9,143.7,136.6,135.6,135.3,134.5,133.8$, 133.6, 131.4, 130.5, 128.6 (2C), 128.2, 127.3 (2C), 127.0, 125.6, 114.3, 61.5, 56.1, $50.9,50.1,30.7,30.4,19.1$.

IR (FT-ATR, $\mathrm{cm}^{-1}, \mathrm{CH}_{2} \mathrm{Cl}_{2}$ ) $v_{\max } 3347,3056,2961,2933,1662,1579,1509,1484$, 1455, 1417, 1382, 1366, 1346, 1286, 1261, 1228, 1183, 1143, 1087, 1060, 1004, 931, 901, 853, 795, 776, 758, 737, 708, 694, 664, 609, 574, 549, 526.

HRMS (ESI) $m / z$ : $[\mathrm{M}+\mathrm{H}]^{+}$Calcd for $\mathrm{C}_{26} \mathrm{H}_{30} \mathrm{~N}_{2} \mathrm{O}_{3} 419.2335$; found 419.2331. 


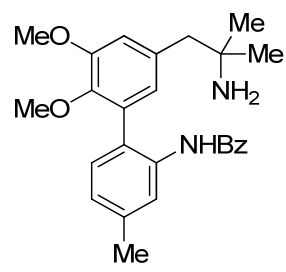

$N$-(5'-(2-Amino-2-methylpropyl)-2',3'-dimethoxy-4-methyl-[1,1'-biphenyl]-2-

yl)benzamide (1c) was synthesized by following Procedure 16 from $\mathbf{S 3 3}$. The crude material was purified by normal-phase column chromatography using an eluent of 5\% $\mathrm{MeOH} / \mathrm{CH}_{2} \mathrm{Cl}_{2}$ with $1 \%$ aqueous $\mathrm{NH}_{3}$ to provide $1 \mathrm{c}$ as a colorless gum (137 mg, $\left.42 \%\right)$.

${ }^{1} \mathbf{H}$ NMR $\left(400 \mathrm{MHz}, \mathrm{CDCl}_{3}\right) \delta 8.97(\mathrm{~s}, 1 \mathrm{H}), 7.99(\mathrm{~s}, 1 \mathrm{H}), 7.79(\mathrm{~d}, J=7.9 \mathrm{~Hz}, 2 \mathrm{H}), 7.48$ $(\mathrm{t}, J=6.7 \mathrm{~Hz}, 1 \mathrm{H}), 7.42(\mathrm{t}, J=7.5 \mathrm{~Hz}, 2 \mathrm{H}), 7.21(\mathrm{~d}, J=7.8 \mathrm{~Hz}, 1 \mathrm{H}), 7.05(\mathrm{~d}, J=7.8$ $\mathrm{Hz}, 1 \mathrm{H}), 6.80(\mathrm{~s}, 1 \mathrm{H}), 6.68(\mathrm{~s}, 1 \mathrm{H}), 3.91(\mathrm{~s}, 3 \mathrm{H}), 3.62(\mathrm{~s}, 3 \mathrm{H}), 2.63(\mathrm{~s}, 2 \mathrm{H}), 2.44(\mathrm{~s}, 3 \mathrm{H})$, 1.34 (brs, 2H) $1.12(\mathrm{~s}, 6 \mathrm{H})$.

${ }^{13}$ C NMR $\left(100 \mathrm{MHz}, \mathrm{CDCl}_{3}\right) \delta 165.3,152.3,144.0,138.6,135.7,135.0,135.0,132.6$, $131.6,130.8,128.7$ (2C), 128.1, 127.2 (2C), 125.94, 125.92, 124.4, 114.5, 61.5, 56.2, $50.9,50.2,30.7,30.6,21.5$.

IR (FT-ATR, $\mathrm{cm}^{-1}, \mathrm{CH}_{2} \mathrm{Cl}_{2}$ ) v $v_{\max } 3350,2961,2933,1674,1618,1581,1527,1467$, 1415,1382 , 1362, 1344, 1295, 1260, 1184, 1145, 1123, 1092, 1074, 1043, 1004, 951, 901, 857, 819, 799, 786, 759, 708, 592, 553, 480.

HRMS (ESI) $m / z$ : [M+H] $]^{+}$Calcd for $\mathrm{C}_{26} \mathrm{H}_{30} \mathrm{~N}_{2} \mathrm{O}_{3} 419.2335$; found 419.2330.

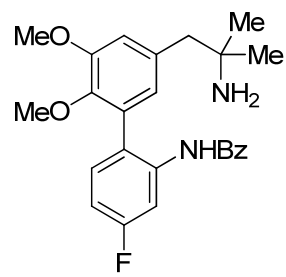

$N$-(5'-(2-Amino-2-methylpropyl)-4-fluoro-2',3'-dimethoxy-[1,1'-biphenyl]-2-

yl)benzamide (1d) was synthesized by following Procedure 16 from $\mathbf{S 3 4}$. The crude material was purified by normal-phase column chromatography using an eluent of $10 \%$ $\mathrm{MeOH} / \mathrm{CH}_{2} \mathrm{Cl}_{2}$ with $1 \%$ aqueous $\mathrm{NH}_{3}$ to provide $1 \mathbf{d}$ as a colorless gum (341 mg, $67 \%$ ).

${ }^{1} \mathbf{H}$ NMR $\left(400 \mathrm{MHz}, \mathrm{CDCl}_{3}\right) \delta 8.94(\mathrm{~s}, 1 \mathrm{H}), 8.10(\mathrm{~d}, J=10.9 \mathrm{~Hz}, 1 \mathrm{H}), 7.79(\mathrm{~d}, J=8.2$ $\mathrm{Hz}, 2 \mathrm{H}), 7.51(\mathrm{t}, J=6.8 \mathrm{~Hz}, 1 \mathrm{H}), 7.45(\mathrm{t}, J=7.3 \mathrm{~Hz}, 2 \mathrm{H}), 7.31(\mathrm{t}, J=7.4 \mathrm{~Hz}, 1 \mathrm{H}), 6.96$ $(\mathrm{td}, J=8.2,2.4 \mathrm{~Hz}, 1 \mathrm{H}), 6.87(\mathrm{~s}, 1 \mathrm{H}), 6.68(\mathrm{~s}, 1 \mathrm{H}), 3.95(\mathrm{~s}, 3 \mathrm{H}), 3.66(\mathrm{~s}, 3 \mathrm{H}), 2.67(\mathrm{~s}$, 2H), 1.45 (brs, 2H), 1.16 (s, 6H).

${ }^{13}$ C NMR $\left(100 \mathrm{MHz}, \mathrm{CDCl}_{3}\right) \delta 165.2,162.5(\mathrm{~d}, J=244.3 \mathrm{~Hz}, 1 \mathrm{C}), 152.3,143.9,136.7$ $(\mathrm{d}, J=11.3 \mathrm{~Hz}, 1 \mathrm{C}), 135.9,134.5,131.83$ (d, $J=9.2 \mathrm{~Hz}, 1 \mathrm{C}), 131.82,131.4,128.7$ (2C), 127.1 (2C), 126.1 (d, $J=3.3 \mathrm{~Hz}, 1 \mathrm{C}), 125.6,114.8,111.5$ (d, $J=21.2 \mathrm{~Hz}, 1 \mathrm{C}$ ), $110.2(\mathrm{~d}, J=26.3 \mathrm{~Hz}, 1 \mathrm{C}), 61.4,56.1,50.7,50.1,30.7,30.5$.

IR (FT-ATR, $\mathrm{cm}^{-1}, \mathrm{CH}_{2} \mathrm{Cl}_{2}$ ) $v_{\max } 3414,3350,2961,2936,1677,1597,1581,1524$, $1483,1465,1417,1383,1365,1345,1286,1253,1184,1163,1141,1117,1079,1043$, $1028,1002,978,858,816,798,785,758,732,707,680,622,584,551,476,446$.

HRMS (ESI) $m / z$ : $[\mathrm{M}+\mathrm{H}]^{+}$Calcd for $\mathrm{C}_{25} \mathrm{H}_{27} \mathrm{FN}_{2} \mathrm{O}_{3}$ 423.2084; found 423.2078. 


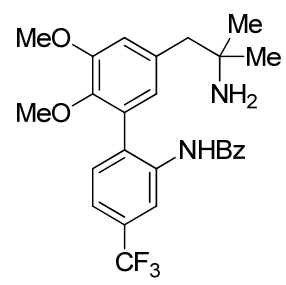

$N$-(5'-(2-Amino-2-methylpropyl)-2',3'-dimethoxy-4-(trifluoromethyl)-[1,1'-

biphenyl]-2-yl)benzamide (1e) was synthesized by following Procedure 16 from S35. The crude material was purified by normal-phase column chromatography using an eluent of $10 \% \mathrm{MeOH} / \mathrm{CH}_{2} \mathrm{Cl}_{2}$ with $1 \%$ aqueous $\mathrm{NH}_{3}$ to provide $1 \mathrm{e}$ as a white solid (215 $\mathrm{mg}, 63 \%)$.

m.p. $73{ }^{\circ} \mathrm{C}$

${ }^{1} \mathbf{H}$ NMR $\left(400 \mathrm{MHz}, \mathrm{CDCl}_{3}\right) \delta 8.97(\mathrm{~s}, 1 \mathrm{H}), 8.56(\mathrm{~s}, 1 \mathrm{H}), 7.77(\mathrm{~d}, J=7.0 \mathrm{~Hz}, 2 \mathrm{H})$, $7.54-7.38(\mathrm{~m}, 5 \mathrm{H}), 6.87(\mathrm{~s}, 1 \mathrm{H}), 6.66(\mathrm{~s}, 1 \mathrm{H}), 3.93(\mathrm{~s}, 3 \mathrm{H}), 3.65(\mathrm{~d}, J=1.5 \mathrm{~Hz}, 3 \mathrm{H})$, $2.64(\mathrm{~s}, 2 \mathrm{H}), 1.28$ (brs, 2H), $1.13(\mathrm{~s}, 6 \mathrm{H})$.

${ }^{13} \mathrm{C} \mathrm{NMR}\left(100 \mathrm{MHz}, \mathrm{CDCl}_{3}\right) \delta 165.4,152.4,143.9,136.2,135.9,134.4,134.0,132.0$, $131.4,131.2,130.6(\mathrm{q}, J=32.6 \mathrm{~Hz}, 1 \mathrm{C}), 128.8(2 \mathrm{C}), 127.2(2 \mathrm{C}), 125.3,124.1$ (q, $J=$ $271.2 \mathrm{~Hz}, 1 \mathrm{C}), 121.3$ (q, $J=3.6 \mathrm{~Hz}, 1 \mathrm{C}), 120.4$ (q, $J=3.9 \mathrm{~Hz}, 1 \mathrm{C}), 115.3,61.7,56.2$, $50.8,50.1,30.8,30.6$.

IR (FT-ATR, $\mathrm{cm}^{-1}, \mathrm{CH}_{2} \mathrm{Cl}_{2}$ ) $v_{\max } 2962,1677,1582,1534,1483,1466,1417,1383$, 1329, 1264, 1230, 1165, 1120, 1075, 1041, 1001, 923, 903, 878, 857, 829, 787, 757, 734, 706, 694, 677, 663, 583.

HRMS (ESI) $m / z$ : $[\mathrm{M}+\mathrm{H}]^{+}$Calcd for $\mathrm{C}_{26} \mathrm{H}_{27} \mathrm{~F}_{3} \mathrm{~N}_{2} \mathrm{O}_{3}$ 473.2052; found 473.2046.

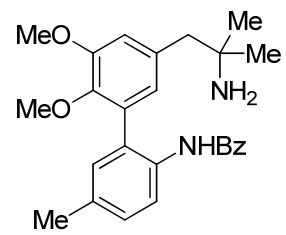

$N$-(5'-(2-Amino-2-methylpropyl)-2',3'-dimethoxy-5-methyl-[1,1'-biphenyl]-2-

yl)benzamide (1f) was synthesized by following Procedure 16 from S36. The crude material was purified by normal-phase column chromatography using an eluent of $10 \%$ $\mathrm{MeOH} / \mathrm{EtOAc}$ with $1 \%$ aqueous $\mathrm{NH}_{3}$ to provide $\mathbf{1 f}$ as a white solid (448 $\mathrm{mg}, 92 \%$ ).

m.p. $51{ }^{\circ} \mathrm{C}$

${ }^{1} \mathbf{H}$ NMR $\left(400 \mathrm{MHz}, \mathrm{CDCl}_{3}\right) \delta 8.94(\mathrm{~s}, 1 \mathrm{H}), 8.02(\mathrm{~d}, J=8.1 \mathrm{~Hz}, 1 \mathrm{H}), 7.80(\mathrm{~d}, J=7.6$ $\mathrm{Hz}, 2 \mathrm{H}), 7.49(\mathrm{t}, J=6.8 \mathrm{~Hz}, 1 \mathrm{H}), 7.43(\mathrm{t}, J=7.5 \mathrm{~Hz}, 2 \mathrm{H}), 7.27(\mathrm{~d}, J=8.6 \mathrm{~Hz}, 1 \mathrm{H}), 7.15$ $(\mathrm{s}, 1 \mathrm{H}), 6.83(\mathrm{~s}, 1 \mathrm{H}), 6.72(\mathrm{~s}, 1 \mathrm{H}), 3.94(\mathrm{~s}, 3 \mathrm{H}), 3.65(\mathrm{~s}, 3 \mathrm{H}), 2.66(\mathrm{~s}, 2 \mathrm{H}), 2.41(\mathrm{~s}, 3 \mathrm{H})$, 1.48 (brs, 2H), 1.15 (s, 6H).

${ }^{13} \mathrm{C}$ NMR $\left(100 \mathrm{MHz}, \mathrm{CDCl}_{3}\right) \delta 165.3,152.2,143.9,135.6,135.0,134.6,132.7,131.5$, $131.4,130.9,129.1,128.7$ (3C), 127.2 (2C), 125.8, 124.0, 114.5, 61.5, 56.2, 50.8, 50.1, 30.7, 30.6, 21.1.

IR (FT-ATR, $\mathrm{cm}^{-1}, \mathrm{CH}_{2} \mathrm{Cl}_{2}$ ) $v_{\max } 3351,2960,2934,1671,1581,1515,1464,1419$, 1399, 1365, 1342, 1301, 1265, 1228, 1184, 1144, 1120, 1095, 1073, 1049, 1027, 1002, $929,855,818,793,772,758,733,706,675,647,617,577,545,482$.

HRMS (ESI) $m / z$ : $[\mathrm{M}+\mathrm{H}]^{+}$Calcd for $\mathrm{C}_{26} \mathrm{H}_{30} \mathrm{~N}_{2} \mathrm{O}_{3} 419.2335$; found 419.2332. 


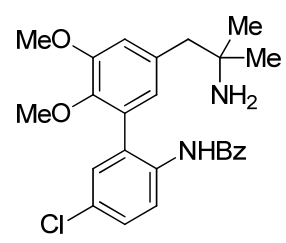

$N$-(5'-(2-Amino-2-methylpropyl)-5-chloro-2',3'-dimethoxy-[1,1'-biphenyl]-2yl)benzamide (1g) was synthesized by following Procedure 16 from S37. The crude material was purified by normal-phase column chromatography using an eluent of $10 \%$ $\mathrm{MeOH} /$ EtOAc with $1 \%$ aqueous $\mathrm{NH}_{3}$ to provide $1 \mathrm{~g}$ as a white solid (346 mg, 86\%).

m.p. $58^{\circ} \mathrm{C}$

${ }^{1} \mathbf{H}$ NMR $\left(400 \mathrm{MHz}, \mathrm{CDCl}_{3}\right) \delta 8.12(\mathrm{~d}, J=8.7 \mathrm{~Hz}, 1 \mathrm{H}), 7.76(\mathrm{~d}, J=8.0 \mathrm{~Hz}, 2 \mathrm{H}), 7.48$ (t, $J=6.9 \mathrm{~Hz}, 1 \mathrm{H}), 7.41(\mathrm{t}, J=7.9 \mathrm{~Hz}, 3 \mathrm{H}), 7.31(\mathrm{~d}, J=2.0 \mathrm{~Hz}, 1 \mathrm{H}), 6.83(\mathrm{~s}, 1 \mathrm{H}), 6.66$ (s, 1H), $3.91(\mathrm{~s}, 3 \mathrm{H}), 3.65(\mathrm{~s}, 3 \mathrm{H}), 2.63(\mathrm{~s}, 2 \mathrm{H}), 1.42$ (brs, 2H), $1.12(\mathrm{~s}, 6 \mathrm{H})$.

${ }^{13} \mathrm{C}$ NMR $\left(100 \mathrm{MHz}, \mathrm{CDCl}_{3}\right) \delta 165.3,152.3,143.8,136.0,134.6,134.0,132.5,131.8$, $131.2,130.5,129.8,128.8$ (2C), 128.4, 127.2 (2C), 125.4, 125.1, 115.1, 61.7, 56.2, $50.8,50.2,30.7,30.6$.

IR (FT-ATR, $\mathrm{cm}^{-1}, \mathrm{CH}_{2} \mathrm{Cl}_{2}$ ) $v_{\max } 2960,2936,1673,1602,1581,1510,1485,1464$, 1418, 1392, 1330, 1296, 1263, 1230, 1184, 1144, 1119, 1074, 1046, 1027, 1001, 902, 874, 856, 819, 787, 758, 734, 706, 659, 638, 613, 575, 543, 480, 418.

HRMS (ESI) $m / z$ : $[\mathrm{M}+\mathrm{H}]^{+}$Calcd for $\mathrm{C}_{25} \mathrm{H}_{27} \mathrm{ClN}_{2} \mathrm{O}_{3} 439.1789$; found 439.1786.

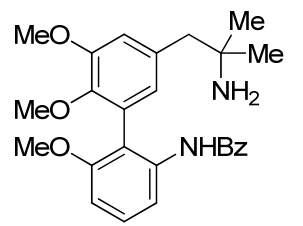

$N$-(5'-(2-Amino-2-methylpropyl)-2',3',6-trimethoxy-[1,1'-biphenyl]-2-

yl)benzamide (1h) was synthesized by following Procedure 16 from $\mathbf{S 3 8}$. The crude material was purified by normal-phase column chromatography using an eluent of $10 \%$ $\mathrm{MeOH} / \mathrm{EtOAc}$ with $1 \%$ aqueous $\mathrm{NH}_{3}$ to provide $\mathbf{1 h}$ as a white solid (150 mg, 89\%).

m.p. $67{ }^{\circ} \mathrm{C}$

${ }^{1} \mathbf{H}$ NMR $\left(400 \mathrm{MHz}, \mathrm{CDCl}_{3}\right) \delta 8.19(\mathrm{~s}, 1 \mathrm{H}), 7.96(\mathrm{~d}, J=8.1 \mathrm{~Hz}, 1 \mathrm{H}), 7.60(\mathrm{~d}, J=7.4$ $\mathrm{Hz}, 2 \mathrm{H}), 7.54-7.30(\mathrm{~m}, 4 \mathrm{H}), 6.81(\mathrm{~d}, J=8.5 \mathrm{~Hz}, 2 \mathrm{H}), 6.64(\mathrm{~s}, 1 \mathrm{H}), 3.90(\mathrm{~s}, 2 \mathrm{H}), 3.74$ (s, 3H), $3.60(\mathrm{~s}, 3 \mathrm{H}), 2.67-2.52(\mathrm{~m}, 2 \mathrm{H}), 1.87$ (brs, 2H), $1.09(\mathrm{~s}, 6 \mathrm{H})$.

${ }^{13}$ C NMR $\left(100 \mathrm{MHz}, \mathrm{CDCl}_{3}\right) \delta 165.2,157.2,152.5,145.1,136.6,134.9,134.6,131.6$, $129.1,128.7$ (2C), 127.3, 127.0 (2C), 126.3, 118.5, 114.9, 114.6, 107.3, 61.1, 56.1, 55.8, 50.6, 50.4, 30.2, 30.1.

IR (FT-ATR, $\mathrm{cm}^{-1}, \mathrm{CH}_{2} \mathrm{Cl}_{2}$ ) v $v_{\max } 3413,2960,2936,2837,1677,1586,1525,1484$, 1468, 1439, 1417, 1382, 1366, 1329, 1304, 1286, 1259, 1184, 113, 1071, 1041, 1005, 934, 893, 852, 792, 754, 738, 710, 694, 669, 609, 571.

HRMS (ESI) $m / z$ : $[\mathrm{M}+\mathrm{H}]^{+}$Calcd for $\mathrm{C}_{27} \mathrm{H}_{30} \mathrm{~N}_{2} \mathrm{O}_{4} 447.2284$; found 447.2281. 


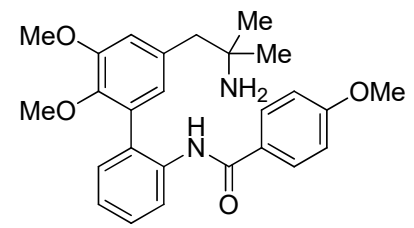

$N$-(5'-(2-Amino-2-methylpropyl)-2',3'-dimethoxy-[1,1'-biphenyl]-2-yl)-4-

methoxybenzamide (1i) was synthesized by following Procedure 16 from S29. The crude material was purified by normal-phase column chromatography using an eluent of $10 \% \mathrm{MeOH} / \mathrm{CH}_{2} \mathrm{Cl}_{2}$ with $1 \%$ aqueous $\mathrm{NH}_{3}$ to provide $1 \mathrm{i}$ as a colorless gum $(244 \mathrm{mg}$, $78 \%)$.

${ }^{1} \mathbf{H}$ NMR $\left(400 \mathrm{MHz}, \mathrm{CDCl}_{3}\right) \delta 8.87(\mathrm{~s}, 1 \mathrm{H}), 8.14(\mathrm{~d}, J=8.0 \mathrm{~Hz}, 1 \mathrm{H}), 7.75(\mathrm{~d}, J=8.6$ $\mathrm{Hz}, 2 \mathrm{H}), 7.43(\mathrm{t}, J=7.6 \mathrm{~Hz}, 1 \mathrm{H}), 7.31(\mathrm{~d}, J=7.6 \mathrm{~Hz}, 1 \mathrm{H}), 7.22(\mathrm{t}, J=7.3 \mathrm{~Hz}, 1 \mathrm{H}), 6.91$ $(\mathrm{d}, J=8.6 \mathrm{~Hz}, 2 \mathrm{H}), 6.81(\mathrm{~s}, 1 \mathrm{H}), 6.69(\mathrm{~s}, 1 \mathrm{H}), 3.92(\mathrm{~s}, 3 \mathrm{H}), 3.83(\mathrm{~s}, 3 \mathrm{H}), 3.62(\mathrm{~s}, 3 \mathrm{H})$, $2.64(\mathrm{~s}, 2 \mathrm{H}), 1.48$ (brs, 2H)1.13 (s, 6H).

${ }^{13}$ C NMR $\left(100 \mathrm{MHz}, \mathrm{CDCl}_{3}\right) \delta 165.0,162.3,152.3,144.0,135.7,135.5,132.7,131.0$, $130.8,129.1$ (2C), 128.5, 127.3, 125.9, 124.8, 123.8, 114.6, 113.9 (2C), 61.6, 56.2, $55.54,50.9,50.3,30.7,30.6$.

IR (FT-ATR, $\mathrm{cm}^{-1}, \mathrm{CH}_{2} \mathrm{Cl}_{2}$ ) $v_{\max } 3353,2960,2935,2839,1668,1606,1583,1527$, $1505,1484,1465,1442,1419,1382,1365,1344,1300,1240,1176,1142,1119,1090$, 1034, 1004, 901, 845, 788, 762, 681, 626, 573, 532, 478.

HRMS (ESI) $m / z$ : [M+H] $]^{+}$Calcd for $\mathrm{C}_{26} \mathrm{H}_{30} \mathrm{~N}_{2} \mathrm{O}_{4} 435.2284$; found 435.2278.

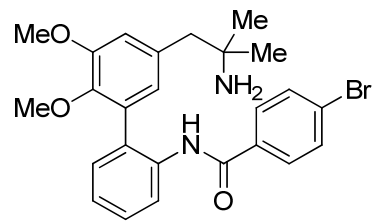

$N$-(5'-(2-Amino-2-methylpropyl)-2',3'-dimethoxy-[1,1'-biphenyl]-2-yl)-4-

bromobenzamide (1j) was synthesized by following Procedure 16 from S30. The crude material was purified by normal-phase column chromatography using an eluent of $10 \% \mathrm{MeOH} / \mathrm{CH}_{2} \mathrm{Cl}_{2}$ with $1 \%$ aqueous $\mathrm{NH}_{3}$ to provide $\mathbf{1} \mathbf{j}$ as a yellow gum (256 $\mathrm{mg}$, $71 \%)$.

${ }^{1}$ H NMR $\left(400 \mathrm{MHz}, \mathrm{CDCl}_{3}\right) \delta 8.99(\mathrm{~s}, 1 \mathrm{H}), 8.11(\mathrm{~d}, J=7.8 \mathrm{~Hz}, 1 \mathrm{H}), 7.65(\mathrm{~d}, J=8.2$ $\mathrm{Hz}, 2 \mathrm{H}), 7.55(\mathrm{~d}, J=8.1 \mathrm{~Hz}, 2 \mathrm{H}), 7.45(\mathrm{t}, J=7.6 \mathrm{~Hz}, 1 \mathrm{H}), 7.34(\mathrm{~d}, J=7.5 \mathrm{~Hz}, 1 \mathrm{H})$, $7.24(\mathrm{~d}, J=7.7 \mathrm{~Hz}, 1 \mathrm{H}), 6.82(\mathrm{~s}, 1 \mathrm{H}), 6.69(\mathrm{~s}, 1 \mathrm{H}), 3.93(\mathrm{~s}, 3 \mathrm{H}), 3.62(\mathrm{~s}, 3 \mathrm{H}), 2.64(\mathrm{~s}$, 2H), 1.42 (brs, 2H), 1.14 (s, 6H).

${ }^{13}$ C NMR (100 MHz, $\left.\mathrm{CDCl}_{3}\right) \delta 164.5,152.3,143.7,135.9,135.1,133.8,132.5,132.0$ (2C), 131.1 (2C), 128.9 (2C), 128.6, 126.4, 125.9, 125.3, 123.9, 114.6, 61.6, 56.2, 50.9, $50.2,30.8,30.6$.

IR (FT-ATR, $\mathrm{cm}^{-1}, \mathrm{CH}_{2} \mathrm{Cl}_{2}$ ) $v_{\max } 3349,3053,2961,2934,2844,1673,1586,1522$, $1484,1466,1443,1419,1382,1365,1344,1310,1261,1248,1230,1183,1142,1117$, 1089, 1070, 1050, 1036, 1009, 946, 900, 843, 817, 787, 755, 710, 679, 625, 591, 573, $528,481,448$.

HRMS (ESI) $m / z$ : $[\mathrm{M}+\mathrm{H}]^{+}$Calcd for $\mathrm{C}_{25} \mathrm{H}_{27} \mathrm{BrN}_{2} \mathrm{O}_{3} 483.1283$; found 483.1278 . 


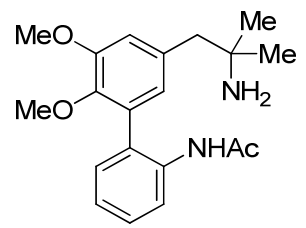

$N$-(5'-(2-Amino-2-methylpropyl)-2',3'-dimethoxy-[1,1'-biphenyl]-2-yl)acetamide (1k) was synthesized by following Procedure 16 from S43. The crude material was purified by normal-phase column chromatography using an eluent of $5 \%$ $\mathrm{MeOH} / \mathrm{CH}_{2} \mathrm{Cl}_{2}$ with $1 \%$ aqueous $\mathrm{NH}_{3}$ to provide $1 \mathbf{k}$ as a yellow oil $(297 \mathrm{mg}, 83 \%)$.

${ }^{1} \mathbf{H}$ NMR $\left(400 \mathrm{MHz}, \mathrm{CDCl}_{3}\right) \delta 8.02(\mathrm{~s}, 1 \mathrm{H}), 7.97(\mathrm{~d}, J=8.1 \mathrm{~Hz}, 1 \mathrm{H}), 7.36(\mathrm{t}, J=7.7$ $\mathrm{Hz}, 1 \mathrm{H}), 7.24(\mathrm{~d}, J=7.5 \mathrm{~Hz}, 1 \mathrm{H}), 7.16(\mathrm{t}, J=7.4 \mathrm{~Hz}, 1 \mathrm{H}), 6.80(\mathrm{~s}, 1 \mathrm{H}), 6.64(\mathrm{~s}, 1 \mathrm{H})$, $3.90(\mathrm{~s}, 3 \mathrm{H}), 3.52(\mathrm{~s}, 3 \mathrm{H}), 2.63(\mathrm{~s}, 2 \mathrm{H}), 2.00(\mathrm{~s}, 3 \mathrm{H}), 1.26(\mathrm{brs}, 2 \mathrm{H}) 1.14(\mathrm{~s}, 6 \mathrm{H})$.

${ }^{13}$ C NMR $\left(100 \mathrm{MHz}, \mathrm{CDCl}_{3}\right) \delta 168.6,152.1,144.0,135.6,135.2,132.4,130.9,130.4$, 128.4, 125.6, 124.7, 123.7, 114.2, 61.3, 56.0, 50.9, 50.1, 30.74, 30.65, 24.7.

IR (FT-ATR, $\mathrm{cm}^{-1}, \mathrm{CH}_{2} \mathrm{Cl}_{2}$ ) $v_{\max } 3345,2960,2934,2843,1676,1584,1519,1485$, 1464, 1444, 1419, 1367, 1344, 1298, 1261, 1230, 1184, 1138, 1103, 1049, 1037, 1005, $968,944,856,788,756,733,688,660,634,676,540,478$.

HRMS (ESI) $m / z$ : [M+H] $]^{+}$Calcd for $\mathrm{C}_{20} \mathrm{H}_{26} \mathrm{~N}_{2} \mathrm{O}_{3}$ 343.2022; found 343.2016.

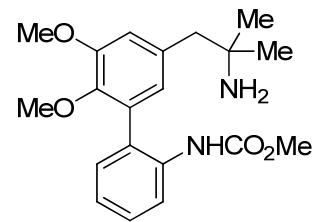

Methyl (5'-(2-Amino-2-methylpropyl)-2',3'-dimethoxy-[1,1'-biphenyl]-2yl)carbamate (11) was synthesized by following Procedure 16 from $\mathbf{S 4 4}$. The crude material was purified by normal-phase column chromatography using an eluent of $10 \%$ $\mathrm{MeOH} / \mathrm{EtOAc}$ with $1 \%$ aqueous $\mathrm{NH}_{3}$ to provide $1 \mathrm{l}$ as a white solid (294 mg, 76\%).

m.p. $125^{\circ} \mathrm{C}$

${ }^{1}$ H NMR $\left(400 \mathrm{MHz}, \mathrm{CDCl}_{3}\right) \delta 7.92$ (brs, $\left.1 \mathrm{H}\right), 7.34$ (t, $\left.J=7.7 \mathrm{~Hz}, 1 \mathrm{H}\right), 7.25-7.17$ (m, 2H), $7.10(\mathrm{t}, J=7.4 \mathrm{~Hz}, 1 \mathrm{H}), 6.79(\mathrm{~s}, 1 \mathrm{H}), 6.61(\mathrm{~s}, 1 \mathrm{H}), 3.88(\mathrm{~s}, 3 \mathrm{H}), 3.63(\mathrm{~s}, 3 \mathrm{H}), 3.51$ (s, 3H), 2.62 (s, 2H), 1.29 (brs, 2H), 1.13 (s, 6H).

${ }^{13}$ C NMR $\left(100 \mathrm{MHz}, \mathrm{CDCl}_{3}\right) \delta 154.5,152.2,144.4,135.4,135.3,132.0,130.7,129.4$, $128.4,125.3,123.7,121.4,114.3,61.1,56.0,52.1,50.8,50.1,30.6(2 \mathrm{C})$.

IR (FT-ATR, $\mathrm{cm}^{-1}, \mathrm{CH}_{2} \mathrm{Cl}_{2}$ ) $v_{\max } 3417,3351,2958,1731,1585,1521,1484,1446$, 1418,1382 , 1344, 1299, 1259, 1214, 1187, 1139, 1108, 1067, 1048, 1036, 1004, 953, $856,832,788,756,734,682,622,574,530,479$.

HRMS (ESI) $m / z$ : [M+H] $]^{+}$Calcd for $\mathrm{C}_{20} \mathrm{H}_{26} \mathrm{~N}_{2} \mathrm{O}_{4}$ 359.1971; found 359.1969. 


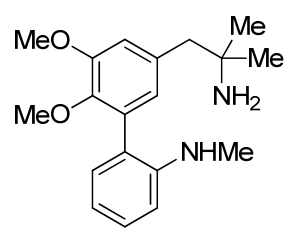

5'-(2-Amino-2-methylpropyl)-2',3'-dimethoxy- $N$-methyl-[1,1'-biphenyl]-2-amine (1m) was synthesized by following Procedure 16 from S45. The crude material was purified by normal-phase column chromatography using an eluent of $10 \%$ $\mathrm{MeOH} /$ EtOAc with $1 \%$ aqueous $\mathrm{NH}_{3}$ to provide $\mathbf{1 m}$ as a colorless oil (114 $\left.\mathrm{mg}, 83 \%\right)$.

${ }^{1} \mathbf{H}$ NMR $\left(400 \mathrm{MHz}, \mathrm{CDCl}_{3}\right) \delta$ 7.31-7.24 (m, 1H), $7.09(\mathrm{~d}, J=7.3 \mathrm{~Hz}, 1 \mathrm{H}), 6.79-6.72$ $(\mathrm{m}, 2 \mathrm{H}), 6.70(\mathrm{~d}, J=8.1 \mathrm{~Hz}, 1 \mathrm{H}), 6.65(\mathrm{~s}, 1 \mathrm{H}), 4.00-3.81(\mathrm{~m}, 4 \mathrm{H}), 3.54(\mathrm{~s}, 3 \mathrm{H}), 2.79$ (s, 3H), $2.63(\mathrm{~s}, 2 \mathrm{H}), 1.35$ (brs, 2H), $1.15(\mathrm{~s}, 6 \mathrm{H})$.

${ }^{13} \mathrm{C} \mathrm{NMR}\left(100 \mathrm{MHz}, \mathrm{CDCl}_{3}\right) \delta 152.4,147.0,145.2,135.0,133.0,130.5,128.9,125.7$, 124.6, 116.7, 113.8, 110.1, 61.2, 56.0, 51.1, 50.2, 31.0, 30.7, 30.6.

IR (FT-ATR, $\mathrm{cm}^{-1}, \mathrm{CH}_{2} \mathrm{Cl}_{2}$ ) $v_{\max } 3426,2959,2930,2814,1601,1579,1514,1481$, 1459, 1417, 1381, 1365, 1342, 1314, 1293, 1261, 1230, 1184, 1167, 1135, 1094, 1067, 1035, 1008, 965, 929, 855, 789, 748, 681, 629, 575, 540, 515.

HRMS (ESI) $m / z$ : $[\mathrm{M}+\mathrm{H}]^{+}$Calcd for $\mathrm{C}_{19} \mathrm{H}_{26} \mathrm{~N}_{2} \mathrm{O}_{2}$ 315.2073; found 315.2072.

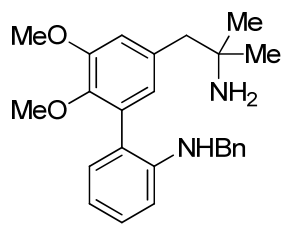

5'-(2-Amino-2-methylpropyl)- $N$-benzyl-2',3'-dimethoxy-[1,1'-biphenyl]-2-amine (1n) was synthesized by following Procedure 16 from S46. The crude material was purified by normal-phase column chromatography using an eluent of $10 \%$ $\mathrm{MeOH} / \mathrm{EtOAc}$ with $1 \%$ aqueous $\mathrm{NH}_{3}$ to provide $\mathbf{1 n}$ as a colorless gum (180 mg, 56\%).

${ }^{1} \mathbf{H}$ NMR $\left(400 \mathrm{MHz}, \mathrm{CDCl}_{3}\right) \delta$ 7.37-7.26 (m, 4H), 7.26-7.15 (m, 2H), $7.13(\mathrm{~d}, J=7.1$ $\mathrm{Hz}, 1 \mathrm{H}), 6.82-6.74(\mathrm{~m}, 2 \mathrm{H}), 6.71(\mathrm{~s}, 1 \mathrm{H}), 6.65(\mathrm{~d}, J=8.1 \mathrm{~Hz}, 1 \mathrm{H}), 4.45-4.30(\mathrm{~m}, 3 \mathrm{H})$, $3.92(\mathrm{~s}, 3 \mathrm{H}), 3.62(\mathrm{~s}, 3 \mathrm{H}), 2.67$ (s, 2H), 1.74 (brs, 2H) $1.17(\mathrm{~s}, 6 \mathrm{H})$.

${ }^{13} \mathrm{C}$ NMR $\left(100 \mathrm{MHz}, \mathrm{CDCl}_{3}\right) \delta 152.5,145.5,139.8,134.7,132.9,130.6,128.8,128.5$ (2C), 127.2 (2C), 126.9 (2C), 125.7, 124.4, 116.8, 114.0, 110.9, 61.1, 56.1, 50.7, 50.5, 48.0, $30.4(2 \mathrm{C})$.

IR (FT-ATR, $\mathrm{cm}^{-1}, \mathrm{CH}_{2} \mathrm{Cl}_{2}$ ) v $v_{\max } 3431,3061,3027,2959,2930,2870,2842,1738$, 1599, 1579, 1513, 1482, 1452, 1417, 1381, 1363, 1343, 1323, 1295, 1259, 1229, 1184 , $1163,1141,1121,1082,1065,1038,1008,966,930,854,789,747,698,681,631,578$, $538,511,458$.

HRMS (ESI) $m / z$ : [M+H $]^{+}$Calcd for $\mathrm{C}_{19} \mathrm{H}_{24} \mathrm{~N}_{2} \mathrm{O}_{4}$ 345.1814; found 345.1809. 


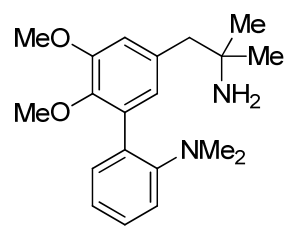

5'-(2-Amino-2-methylpropyl)-2',3'-dimethoxy- $N, N$-dimethyl-[1,1'-biphenyl]-2-

amine (10) was synthesized by following Procedure 16 from S47. The crude material was purified by normal-phase column chromatography using an eluent of $10 \%$ $\mathrm{MeOH} / \mathrm{EtOAc}$ with $1 \%$ aqueous $\mathrm{NH}_{3}$ to provide lo as a colorless oil $(214 \mathrm{mg}, 89 \%$ ).

${ }^{1} \mathbf{H}$ NMR $\left(400 \mathrm{MHz}, \mathrm{CDCl}_{3}\right) \delta 7.29(\mathrm{t}, J=7.7 \mathrm{~Hz}, 1 \mathrm{H}), 7.24(\mathrm{~d}, J=7.5 \mathrm{~Hz}, 1 \mathrm{H}), 7.06$ $(\mathrm{d}, J=8.1 \mathrm{~Hz}, 1 \mathrm{H}), 7.00(\mathrm{t}, J=7.4 \mathrm{~Hz}, 1 \mathrm{H}), 6.83(\mathrm{~s}, 1 \mathrm{H}), 6.72(\mathrm{~s}, 1 \mathrm{H}), 3.91(\mathrm{~s}, 3 \mathrm{H})$, $3.70(\mathrm{~s}, 3 \mathrm{H}), 2.64(\mathrm{~s}, 2 \mathrm{H}), 2.56(\mathrm{~s}, 6 \mathrm{H}), 1.41$ (brs, 2H) $1.15(\mathrm{~s}, 6 \mathrm{H})$.

${ }^{13}$ C NMR $\left(100 \mathrm{MHz}, \mathrm{CDCl}_{3}\right) \delta 152.4,151.7,144.6,135.0,133.7,132.0,131.0,128.2$, $125.1,121.0,117.8,113.2,60.4,55.9,51.0,50.2,43.4(2 \mathrm{C}), 30.4(2 \mathrm{C})$.

IR (FT-ATR, $\mathrm{cm}^{-1}, \mathrm{CH}_{2} \mathrm{Cl}_{2}$ ) $v_{\max } 2958,2935,2866,2828,2779,1582,1497,1475$, $1463,1451,1418,1381,1364,1342$, 1328, 1270, 1254, 1231, 1185, 1141, 1100, 1052 , 1039, 1010, 948, 849, 786, 756, 675, 627, 577, 555, 540, 498, 447.

HRMS (ESI) $m / z$ : $[\mathrm{M}+\mathrm{H}]^{+}$Calcd for $\mathrm{C}_{20} \mathrm{H}_{28} \mathrm{~N}_{2} \mathrm{O}_{2}$ 329.2229; found 329.2227.

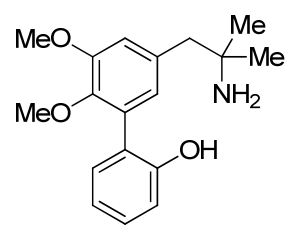

5'-(2-Amino-2-methylpropyl)-2',3'-dimethoxy-[1,1'-biphenyl]-2-ol (1p) was synthesized by following Procedure 16 from S24. The crude material was purified by normal-phase column chromatography using an eluent of $10 \% \mathrm{MeOH} / \mathrm{EtOAc}$ with $1 \%$ aqueous $\mathrm{NH}_{3}$ to provide $\mathbf{l o}$ as a white solid ( $\left.820 \mathrm{mg}, 95 \%\right)$.

m.p. $145^{\circ} \mathrm{C}$

${ }^{1} \mathbf{H}$ NMR $\left(400 \mathrm{MHz}, \mathrm{CDCl}_{3}\right) \delta 7.30(\mathrm{t}, J=6.4 \mathrm{~Hz}, 2 \mathrm{H}), 7.05(\mathrm{~d}, J=8.3 \mathrm{~Hz}, 1 \mathrm{H}), 7.00$ $(\mathrm{t}, J=7.5 \mathrm{~Hz}, 1 \mathrm{H}), 6.80(\mathrm{~s}, 1 \mathrm{H}), 6.77(\mathrm{~s}, 1 \mathrm{H}), 3.91(\mathrm{~s}, 3 \mathrm{H}), 3.66(\mathrm{~s}, 3 \mathrm{H}), 2.67(\mathrm{~s}, 2 \mathrm{H})$, $1.17(\mathrm{~s}, 6 \mathrm{H})$.

${ }^{13} \mathrm{C}$ NMR $\left(100 \mathrm{MHz}, \mathrm{CDCl}_{3}\right) \delta 154.0,152.2,143.7,135.8,132.2,131.2,129.6,126.0$, $125.9,121.0,118.5,114.0,61.7,56.1,50.9,50.3,30.6(2 \mathrm{C})$.

IR (FT-ATR, $\mathrm{cm}^{-1}, \mathrm{CH}_{2} \mathrm{Cl}_{2}$ ) $v_{\max } 3344,3276,3060,2962,2935,2592,2351,1585$, $1485,1450,1421,1344,1259,1230,1184,1140,1101,1036,1011,868,756,509,492$, 471, 453, 445, 426, 405, 418, 415, 401, 449, 434, 422, 415, 401, 411.

HRMS (EI) $m / z$ : $[\mathrm{M}]^{+}$Calcd for $\mathrm{C}_{18} \mathrm{H}_{23} \mathrm{NO}_{3} 301.1678$; found 301.1677. 


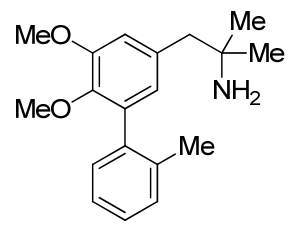

1-(5,6-Dimethoxy-2'-methyl-[1,1'-biphenyl]-3-yl)-2-methylpropan-2-amine (1q) was synthesized by following Procedure 16 from S25. The crude material was purified by normal-phase column chromatography using an eluent of $10 \% \mathrm{MeOH} / \mathrm{EtOAc}$ with $1 \%$ aqueous $\mathrm{NH}_{3}$ to provide lq as a colorless oil $(127.3 \mathrm{mg}, 85 \%)$.

${ }^{1} \mathbf{H}$ NMR $\left(400 \mathrm{MHz}, \mathrm{CDCl}_{3}\right) \delta$ 7.27-7.23 (m, 2H), 7.22-7.17 (m, 2H), $6.77(\mathrm{~s}, 1 \mathrm{H})$, $6.58(\mathrm{~s}, 1 \mathrm{H}), 3.90(\mathrm{~s}, 3 \mathrm{H}), 3.49(\mathrm{~s}, 3 \mathrm{H}), 2.65(\mathrm{~s}, 2 \mathrm{H}), 2.18(\mathrm{~s}, 3 \mathrm{H}), 1.50$ (brs, 2H), 1.16 $(\mathrm{s}, 6 \mathrm{H})$.

${ }^{13} \mathrm{C}$ NMR $\left(100 \mathrm{MHz}, \mathrm{CDCl}_{3}\right) \delta 152.2,145.1,138.4,136.7,135.6,134.0,129.87$, $129.85,127.5,125.4,125.0,113.6,60.8,56.0,51.0,50.3,30.5,20.3$ (2C).

IR (FT-ATR, $\mathrm{cm}^{-1}, \mathrm{CH}_{2} \mathrm{Cl}_{2}$ ) $v_{\max } 3367,2958,2931,2871,2372,2351,2320,1726$, $1583,1537,1479,1462,1419,1379,1342,1281,1254,1230,1184,1140,1109,1041$, 1011, 852, 756, 729, 679, 631, 579, 501, 480, 449, 434, 422, 415, 401, 411.

HRMS (EI) $m / z$ : [M] $]^{+}$Calcd for $\mathrm{C}_{19} \mathrm{H}_{25} \mathrm{NO}_{2} 299.1885$; found 299.1886.

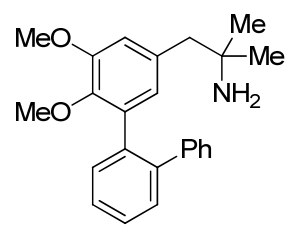

1-(5,6-dimethoxy-[1,1':2',1'-terphenyl]-3-yl)-2-methylpropan-2-amine (1r) was synthesized by following Procedure 16 from S26. The crude material was purified by normal-phase column chromatography using an eluent of $10 \% \mathrm{MeOH} / \mathrm{EtOAc}$ with $1 \%$ aqueous $\mathrm{NH}_{3}$ to provide $\mathbf{~ I r}$ as a white solid (416 mg, 83\%).

m.p. $70{ }^{\circ} \mathrm{C}$

${ }^{1} \mathbf{H}$ NMR $\left(400 \mathrm{MHz}, \mathrm{CDCl}_{3}\right) \delta$ 7.46-7.41 (m, 3H), 7.41-7.35 (m, 1H), 7.21-7.12 (m, $5 \mathrm{H}), 6.59(\mathrm{~s}, 1 \mathrm{H}), 6.31(\mathrm{~s}, 1 \mathrm{H}), 3.85(\mathrm{~s}, 3 \mathrm{H}), 3.65(\mathrm{~s}, 3 \mathrm{H}), 2.44(\mathrm{~s}, 2 \mathrm{H}), 0.91(\mathrm{~s}, 6 \mathrm{H})$.

${ }^{13}$ C NMR $\left(100 \mathrm{MHz}, \mathrm{CDCl}_{3}\right) \delta 152.3,145.4,141.8,141.2,136.9,135.1,133.4,131.1$, $130.3,129.5(2 \mathrm{C}), 127.9(2 \mathrm{C}), 127.7,126.9,126.7,126.0,113.7,60.7,56.0,50.8,49.9$, $30.2(2 \mathrm{C})$.

IR (FT-ATR, $\mathrm{cm}^{-1}, \mathrm{CH}_{2} \mathrm{Cl}_{2}$ ) $v_{\max } 3369,3057,2958,2931,2871,2839,2372,2353$, 1581, 1466, 1417, 1381, 1363, 1342, 1261, 1230, 1184, 1138, 1109, 1059, 1039, 1009, 849, 742, 700, 677, 636, 575, 553, 494, 465, 455, 451, 445, 434, 422, 411.

HRMS (EI) $m / z$ : [M] $]^{+}$Calcd for $\mathrm{C}_{24} \mathrm{H}_{27} \mathrm{NO}_{2} 361.2042$; found 361.2040. 


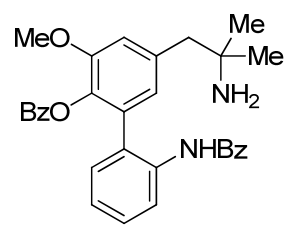

5-(2-Amino-2-methylpropyl)-2'-benzamido-3-methoxy-[1,1'-biphenyl]-2-yl

benzoate (1s) was synthesized by following Procedure 16 from S48. The crude material was purified by normal-phase column chromatography using an eluent of $5 \%$ $\mathrm{MeOH} / \mathrm{EtOAc}$ with $1 \%$ aqueous $\mathrm{NH}_{3}$ to provide $1 \mathrm{~s}$ as a colorless oil (128 mg, 60\%).

${ }^{1} \mathbf{H}$ NMR $\left(400 \mathrm{MHz}, \mathrm{CDCl}_{3}\right) 8.18$ (brs, 1H), 8.12 (s, 1H), 7.95 (d, J=7.6 Hz, 2H), 7.74 $(\mathrm{d}, J=7.4 \mathrm{~Hz}, 2 \mathrm{H}), 7.53(\mathrm{t}, J=7.4 \mathrm{~Hz}, 1 \mathrm{H}), 7.47(\mathrm{t}, J=7.3 \mathrm{~Hz}, 1 \mathrm{H}), 7.39(\mathrm{dd}, J=14.0$, $7.1 \mathrm{~Hz}, 4 \mathrm{H}), 7.32$ (t, $J=7.8 \mathrm{~Hz}, 1 \mathrm{H}), 7.29-7.24(\mathrm{~m}, 1 \mathrm{H}), 7.11(\mathrm{t}, J=7.4 \mathrm{~Hz}, 1 \mathrm{H}), 6.91$ (s, 1H), $6.74(\mathrm{~s}, 1 \mathrm{H}), 3.86(\mathrm{~s}, 3 \mathrm{H}), 2.64(\mathrm{~s}, 2 \mathrm{H}), 1.51$ (brs, 2H), 1.00 (d, J=16.8 Hz, $6 \mathrm{H})$.

${ }^{13}$ C NMR $\left(100 \mathrm{MHz}, \mathrm{CDCl}_{3}\right) \delta 165.4,160.2,151.5,137.9,136.6,135.6,134.8,133.7$, $132.4,131.7,130.5,130.2$ (2C), 123.0, 128.9, 128.81, 128.8 (2C), 128.6 (2C), 128.2, $127.2(2 \mathrm{C}), 124.6,124.4,114.5,56.3,50.9,50.1,30.5,30.4$.

IR (FT-ATR, $\mathrm{cm}^{-1}, \mathrm{CH}_{2} \mathrm{Cl}_{2}$ ) $v_{\max } 3409,3062,2960,2926,2851,1726,1673,1583$, 1523, 1480, 1450, 1420, 1381, 1346, 1306, 1264, 1202, 1177, 1139, 1080, 1061, 1034, 1024, 968, 898, 857, 795, 759, 735, 707, 617, 586, 532, 485, 422.

HRMS (ESI) $m / z$ : $[\mathrm{M}+\mathrm{H}]^{+}$Calcd for $\mathrm{C}_{31} \mathrm{H}_{30} \mathrm{~N}_{2} \mathrm{O}_{4}$ 495.2284; found 495.2278.

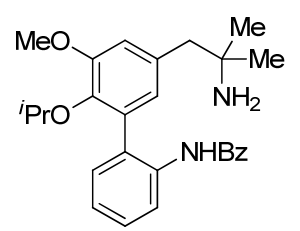

$N$-(5'-(2-Amino-2-methylpropyl)-2'-isopropoxy-3'-methoxy-[1,1'-biphenyl]-2-

yl)benzamide (1t) was synthesized by following Procedure 16 from S39. The crude material was purified by normal-phase column chromatography using an eluent of $10 \%$ $\mathrm{MeOH} / \mathrm{EtOAc}$ with $1 \%$ aqueous $\mathrm{NH}_{3}$ to provide $\mathbf{1 t}$ as a white solid (105 $\left.\mathrm{mg}, 17 \%\right)$.

m.p. $88^{\circ} \mathrm{C}$

${ }^{1} \mathbf{H}$ NMR $\left(400 \mathrm{MHz}, \mathrm{CDCl}_{3}\right) \delta 9.32(\mathrm{~s}, 1 \mathrm{H}), 8.09(\mathrm{~d}, J=7.9 \mathrm{~Hz}, 1 \mathrm{H}), 7.86(\mathrm{~d}, J=7.2$ $\mathrm{Hz}, 2 \mathrm{H}), 7.51-7.37(\mathrm{~m}, 4 \mathrm{H}), 7.31(\mathrm{~d}, J=7.5 \mathrm{~Hz}, 1 \mathrm{H}), 7.21(\mathrm{t}, J=7.4 \mathrm{~Hz}, 1 \mathrm{H}), 6.79$ (s, 1H), $6.70(\mathrm{~s}, 1 \mathrm{H}), 4.19$ (p, $J=6.0 \mathrm{~Hz}, 1 \mathrm{H}), 3.87$ (s, 3H), $2.63(\mathrm{~s}, 2 \mathrm{H}), 1.74$ (brs, 2H), $1.24(\mathrm{~d}, J=5.8 \mathrm{~Hz}, 3 \mathrm{H}), 1.13(\mathrm{~s}, 6 \mathrm{H}), 0.83(\mathrm{~d}, J=5.8 \mathrm{~Hz}, 3 \mathrm{H})$.

${ }^{13}$ C NMR $\left(100 \mathrm{MHz}, \mathrm{CDCl}_{3}\right) \delta 165.2,152.7,141.7,135.2,135.1,134.9,133.7,132.2$, $131.5,131.1,128.6(2 \mathrm{C}), 128.2,127.3$ (2C), 125.9, 125.0, 124.1, 114.6, 76.8, 56.2, $50.7,50.3,30.5,30.3,22.6,22.0$.

IR (FT-ATR, $\mathrm{cm}^{-1}, \mathrm{CH}_{2} \mathrm{Cl}_{2}$ ) $v_{\max } 3350,3063,2971,2931,2870,1673,1582,1520$, 1495, 1468, 1439, 1419, 1382, 1343, 1303, 1247, 1220, 1186, 1148, 1104, 1051, 1037 , 929, 904, 850, 819, 800, 757, 708, 692, 675, 625, 585, 528, 490, 443.

HRMS (ESI) $m / z$ : $[\mathrm{M}+\mathrm{H}]^{+}$Calcd for $\mathrm{C}_{27} \mathrm{H}_{32} \mathrm{~N}_{2} \mathrm{O}_{3} 433.2491$; found 433.2489. 


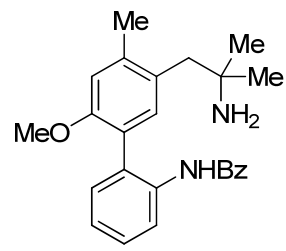

$N$-(5'-(2-Amino-2-methylpropyl)-2'-methoxy-4'-methyl-[1,1'-biphenyl]-2-

yl)benzamide (1u) was synthesized by following Procedure 16 from $\mathbf{S 4 0}$. The crude material was purified by normal-phase column chromatography using an eluent of $10 \%$ $\mathrm{MeOH} / \mathrm{EtOAc}$ with $1 \%$ aqueous $\mathrm{NH}_{3}$ to provide $1 \mathbf{u}$ as a colorless gum (323 mg, 97\%).

${ }^{1} \mathbf{H}$ NMR $\left(400 \mathrm{MHz}, \mathrm{CDCl}_{3}\right) \delta 8.64(\mathrm{~s}, 1 \mathrm{H}), 8.29(\mathrm{~d}, J=7.3 \mathrm{~Hz}, 1 \mathrm{H}), 7.72(\mathrm{~d}, J=7.2$ $\mathrm{Hz}, 2 \mathrm{H}), 7.56-7.38(\mathrm{~m}, 4 \mathrm{H}), 7.33(\mathrm{~d}, J=7.2 \mathrm{~Hz}, 1 \mathrm{H}), 7.25(\mathrm{t}, J=7.1 \mathrm{~Hz}, 1 \mathrm{H}), 7.12(\mathrm{~s}$, $1 \mathrm{H}), 6.90(\mathrm{~s}, 1 \mathrm{H}), 3.84(\mathrm{~s}, 3 \mathrm{H}), 2.71(\mathrm{~d}, J=6.9 \mathrm{~Hz}, 2 \mathrm{H}), 2.46(\mathrm{~s}, 3 \mathrm{H}), 1.47(\mathrm{~s}, 2 \mathrm{H}), 1.16$ $(\mathrm{s}, 7 \mathrm{H})$.

${ }^{13}$ C NMR $\left(100 \mathrm{MHz}, \mathrm{CDCl}_{3}\right) \delta 165.0,153.7,138.8,135.5,135.1,135.0,131.4,131.0$, $130.5,130.2,128.5(2 \mathrm{C}), 127.9,126.7$ (2C), 124.5, 124.2, 122.4, 113.3, 55.9, 51.2, 45.7, 30.7, 30.3, 20.7.

IR (FT-ATR, $\mathrm{cm}^{-1}, \mathrm{CH}_{2} \mathrm{Cl}_{2}$ ) v $v_{\max } 3358,3062,3027,2959,2864,1738,1672,1603$, 1580, 1520, 1493, 1466, 1443, 1392, 1380, 1304, 1248, 1216, 1202, 1184, 1144, 1117 , 1092, 1073, 1047, 1031, 896, 847, 810, 797, 761, 732, 707, 674, 648, 592, 570, 530, $500,457$.

HRMS (ESI) $m / z$ : [M+H] $]^{+}$Calcd for $\mathrm{C}_{25} \mathrm{H}_{28} \mathrm{~N}_{2} \mathrm{O}_{2}$ 389.2229; found 389.2227.

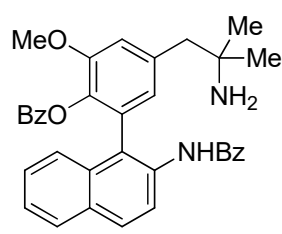

4-(2-Amino-2-methylpropyl)-2-(2-benzamidonaphthalen-1-yl)-6-methoxyphenyl benzoate (1v) was synthesized by following Procedure 16 from S49. The crude material was purified by normal-phase column chromatography using an eluent of $10 \%$ $\mathrm{MeOH} / \mathrm{EtOAc}$ with $1 \%$ aqueous $\mathrm{NH}_{3}$ to provide $1 \mathrm{v}$ as a white solid (250 mg, 80\%).

m.p. $199^{\circ} \mathrm{C}$

${ }^{1} \mathbf{H}$ NMR $\left(400 \mathrm{MHz}, \mathrm{CDCl}_{3}\right) \delta 8.39(\mathrm{~s}, 1 \mathrm{H}), 8.21(\mathrm{~d}, J=6.4 \mathrm{~Hz}, 1 \mathrm{H}), 7.88-7.54(\mathrm{~m}$, $4 \mathrm{H}), 7.71(\mathrm{~d}, J=7.3 \mathrm{~Hz}, 2 \mathrm{H}), 7.55-7.32(\mathrm{~m}, 7 \mathrm{H}), 7.23(\mathrm{t}, J=7.0 \mathrm{~Hz}, 2 \mathrm{H}), 7.00(\mathrm{~s}, 1 \mathrm{H})$, $6.75(\mathrm{~s}, 1 \mathrm{H}), 3.91(\mathrm{~s}, 3 \mathrm{H}), 2.65(\mathrm{~s}, 2 \mathrm{H}), 1.27$ (brs, 2H), $0.96(\mathrm{~d}, J=21.4 \mathrm{~Hz}, 6 \mathrm{H})$.

${ }^{13} \mathbf{C}$ NMR $\left(100 \mathrm{MHz}, \mathrm{CDCl}_{3}\right) \delta 165.4,151.5,138.6,137.4,134.5,133.9,133.6,132.5$, $131.8,131.0,130.5,130.1$ (2C), 128.8, 128.7 (2C), 128.5, 128.4 (2C), 128.0, 127.3 (2C), 126.5, 125.6, 125.2, 125.0, 122.8, 114.5, 56.3, 51.0, 50.0, 30.4, 30.3.

IR (FT-ATR, $\mathrm{cm}^{-1}, \mathrm{CH}_{2} \mathrm{Cl}_{2}$ ) $v_{\max } 3061,2961,1722,1674,1621,1598,1523,1503$, $1488,1461,1453,1424,1382,1317,1268,1202$, 1178, 1140, 1083, 1063, 1023, 964 , $900,865,817,797,752,708,689,619,571,537,430$.

HRMS (ESI) $m / z$ : $[\mathrm{M}+\mathrm{H}]^{+}$Calcd for $\mathrm{C}_{35} \mathrm{H}_{32} \mathrm{~N}_{2} \mathrm{O}_{4}$ 545.2440; found 545.2438. 


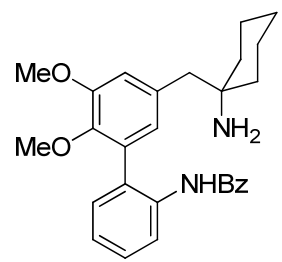

$N$-(5'-((1-Aminocyclohexyl)methyl)-2',3'-dimethoxy-[1,1'-biphenyl]-2-

yl)benzamide (1w) was synthesized by following Procedure 16 from $\mathbf{S 4 1}$. The crude material was purified by normal-phase column chromatography using an eluent of $10 \%$ $\mathrm{MeOH} / \mathrm{EtOAc}$ with $1 \%$ aqueous $\mathrm{NH}_{3}$ to provide $1 \mathbf{w}$ as a white solid (230 mg, $79 \%$ ).

m.p. $75^{\circ} \mathrm{C}$

${ }^{1}$ H NMR $\left(400 \mathrm{MHz}, \mathrm{CDCl}_{3}\right) \delta 8.98(\mathrm{~s}, 1 \mathrm{H}), 8.15(\mathrm{~d}, J=7.3 \mathrm{~Hz}, 1 \mathrm{H}), 7.78(\mathrm{~d}, J=6.8$ $\mathrm{Hz}, 2 \mathrm{H}), 7.50-7.37(\mathrm{~m}, 4 \mathrm{H}), 7.32(\mathrm{~d}, J=7.0 \mathrm{~Hz}, 1 \mathrm{H}), 7.22(\mathrm{t}, J=6.8 \mathrm{~Hz}, 1 \mathrm{H}), 6.84(\mathrm{~s}$, $1 \mathrm{H}), 6.69(\mathrm{~s}, 1 \mathrm{H}), 3.90(\mathrm{~s}, 3 \mathrm{H}), 3.61(\mathrm{~s}, 3 \mathrm{H}), 2.64(\mathrm{~s}, 2 \mathrm{H}), 1.61-1.19(\mathrm{~m}, 10 \mathrm{H})$.

${ }^{13}$ C NMR $\left(100 \mathrm{MHz}, \mathrm{CDCl}_{3}\right) \delta 165.2,152.1,143.8,135.2,134.9,134.8,132.3,131.5$, $130.9,130.8,128.6(2 \mathrm{C}), 128.4,127.1$ (2C), 125.8, 124.9, 123.7, 114.8, 61.4, 56.1, $51.3,48.8,38.5(2 \mathrm{C}), 25.8,22.1(2 \mathrm{C})$.

IR (FT-ATR, $\mathrm{cm}^{-1}, \mathrm{CH}_{2} \mathrm{Cl}_{2}$ ) $v_{\max } 3006,2970,2926,2848,1738,1675,1582,1523$, 1484, 1444, 1421, 1366, 1304, 1229, 1217, 1140, 1119, 1091, 1036, 1004, 904, 814, 788, 756, 708, 585, 539, 528.

HRMS (ESI) $m / z$ : [M+H] $]^{+}$Calcd for $\mathrm{C}_{28} \mathrm{H}_{32} \mathrm{~N}_{2} \mathrm{O}_{3}$ 445.2491; found 445.2489.<smiles>COc1cc(CCN)cc(-c2ccccc2N(C)C(=O)c2ccccc2)c1OC</smiles>

$N$-(5'-(2-Aminoethyl)-2',3'-dimethoxy-[1,1'-biphenyl]-2-yl)benzamide (1x) was synthesized by following Procedure 17 from S42. The crude material was purified by normal-phase column chromatography using an eluent of $10 \% \mathrm{MeOH} / \mathrm{EtOAc}$ with $1 \%$ aqueous $\mathrm{NH}_{3}$ to provide $1 \times$ as a yellow gum (340 mg, 79\%).

${ }^{1} \mathbf{H}$ NMR $\left(400 \mathrm{MHz}, \mathrm{CDCl}_{3}\right) \delta 8.94(\mathrm{~s}, 1 \mathrm{H}), 8.13(\mathrm{~d}, J=7.4 \mathrm{~Hz}, 1 \mathrm{H}), 7.75(\mathrm{~d}, J=6.7$ $\mathrm{Hz}, 2 \mathrm{H}), 7.57-7.28(\mathrm{~m}, 5 \mathrm{H}), 7.21(\mathrm{t}, J=6.8 \mathrm{~Hz}, 1 \mathrm{H}), 6.79(\mathrm{~s}, 1 \mathrm{H}), 6.68(\mathrm{~s}, 1 \mathrm{H}), 3.88(\mathrm{~s}$, $3 \mathrm{H}), 3.58(\mathrm{~s}, 3 \mathrm{H}), 2.91(\mathrm{t}, J=5.5 \mathrm{~Hz}, 2 \mathrm{H}), 2.68$ (t, $J=5.4 \mathrm{~Hz}, 2 \mathrm{H}), 1.81$ (brs, 2H).

${ }^{13}$ C NMR $\left(100 \mathrm{MHz}, \mathrm{CDCl}_{3}\right) \delta 165.2,152.6,143.7,136.9,135.1,134.7,132.8,131.5$, 130.8, 130.7, 128.6 (2C), 128.3, 127.0 (2C), 124.9, 123.8, 123.7, 112.9, 61.3, 56.0, 43.2, 39.6.

IR (FT-ATR, $\mathrm{cm}^{-1}, \mathrm{CH}_{2} \mathrm{Cl}_{2}$ ) $v_{\max } 3370,3060,3000,2937,2845,1671,1583,1520$, 1484, 1469, 1444, 1420, 1343, 1304, 1251, 1229, 1184, 1140, 1119, 1090, 1074, 1049, $1035,1003,903,848,783,758,709,614,586,529,485,406$.

HRMS (ESI) $m / z$ : [M+H] $]^{+}$Calcd for $\mathrm{C}_{23} \mathrm{H}_{24} \mathrm{~N}_{2} \mathrm{O}_{3} 377.1865$; found 377.1860. 


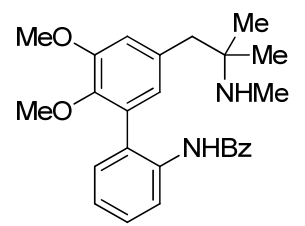

$N$-(2',3'-Dimethoxy-5'-(2-methyl-2-(methylamino)propyl)-[1,1'-biphenyl]-2yl)benzamide (1y) was synthesized by following Procedure 18 from 1a. The crude material was purified by normal-phase column chromatography using an eluent of $10 \%$ $\mathrm{MeOH} / \mathrm{EtOAc}$ with $1 \%$ aqueous $\mathrm{NH}_{3}$ to provide $1 \mathbf{y}$ as a colorless gum $(117 \mathrm{mg}, 73 \%)$.

${ }^{1} \mathbf{H}$ NMR $\left(400 \mathrm{MHz}, \mathrm{CDCl}_{3}\right) \delta 8.93(\mathrm{~s}, 1 \mathrm{H}), 8.17(\mathrm{~d}, J=7.2 \mathrm{~Hz}, 1 \mathrm{H}), 7.78(\mathrm{~d}, J=6.6$ $\mathrm{Hz}, 2 \mathrm{H}), 7.44$ (dd, $J=11.6,4.8 \mathrm{~Hz}, 4 \mathrm{H}), 7.32(\mathrm{~d}, J=6.4 \mathrm{~Hz}, 1 \mathrm{H}), 7.25(\mathrm{~d}, J=7.2 \mathrm{~Hz}$, $1 \mathrm{H}), 6.79(\mathrm{~s}, 1 \mathrm{H}), 6.67(\mathrm{~s}, 1 \mathrm{H}), 3.91(\mathrm{~s}, 3 \mathrm{H}), 3.62(\mathrm{~s}, 3 \mathrm{H}), 2.74-2.62(\mathrm{~m}, 2 \mathrm{H}), 2.38(\mathrm{~s}$, $3 \mathrm{H}), 1.77$ (brs, 2H), 1.07 (s, 6H).

${ }^{13}$ C NMR $\left(100 \mathrm{MHz}, \mathrm{CDCl}_{3}\right) \delta 165.4,152.3,143.9,135.5,135.3,134.9,132.6,131.7$, $130.9,130.9,128.7(2 \mathrm{C}), 128.5,127.2(2 \mathrm{C}), 125.8,125.0,123.8,114.7,61.5,56.2$, 53.5, 46.7, 28.9, 26.24, 26.20.

IR (FT-ATR, $\left.\mathrm{cm}^{-1}, \mathrm{CH}_{2} \mathrm{Cl}_{2}\right) v_{\max } 3335,3063,2964,2936,2799,1674,1582,1520$, 1484, 1466, 1443, 1419, 1379, 1361, 1342, 1303, 1247, 1227, 1183, 1141, 1118, 1090, 1073, 1050, 1035, 1003, 897, 853, 819, 788, 756, 708, 692, 637, 585, 574, 530.

HRMS (ESI) $m / z$ : $[\mathrm{M}+\mathrm{H}]^{+}$Calcd for $\mathrm{C}_{26} \mathrm{H}_{30} \mathrm{~N}_{2} \mathrm{O}_{3} 419.2335$; found 419.2329. 


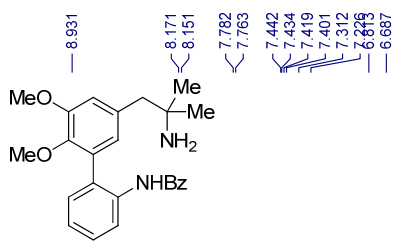

\}

$1 \mathrm{a}$

('H NMR, $\left.400 \mathrm{MHz}, \mathrm{CDCl}_{3}\right)$
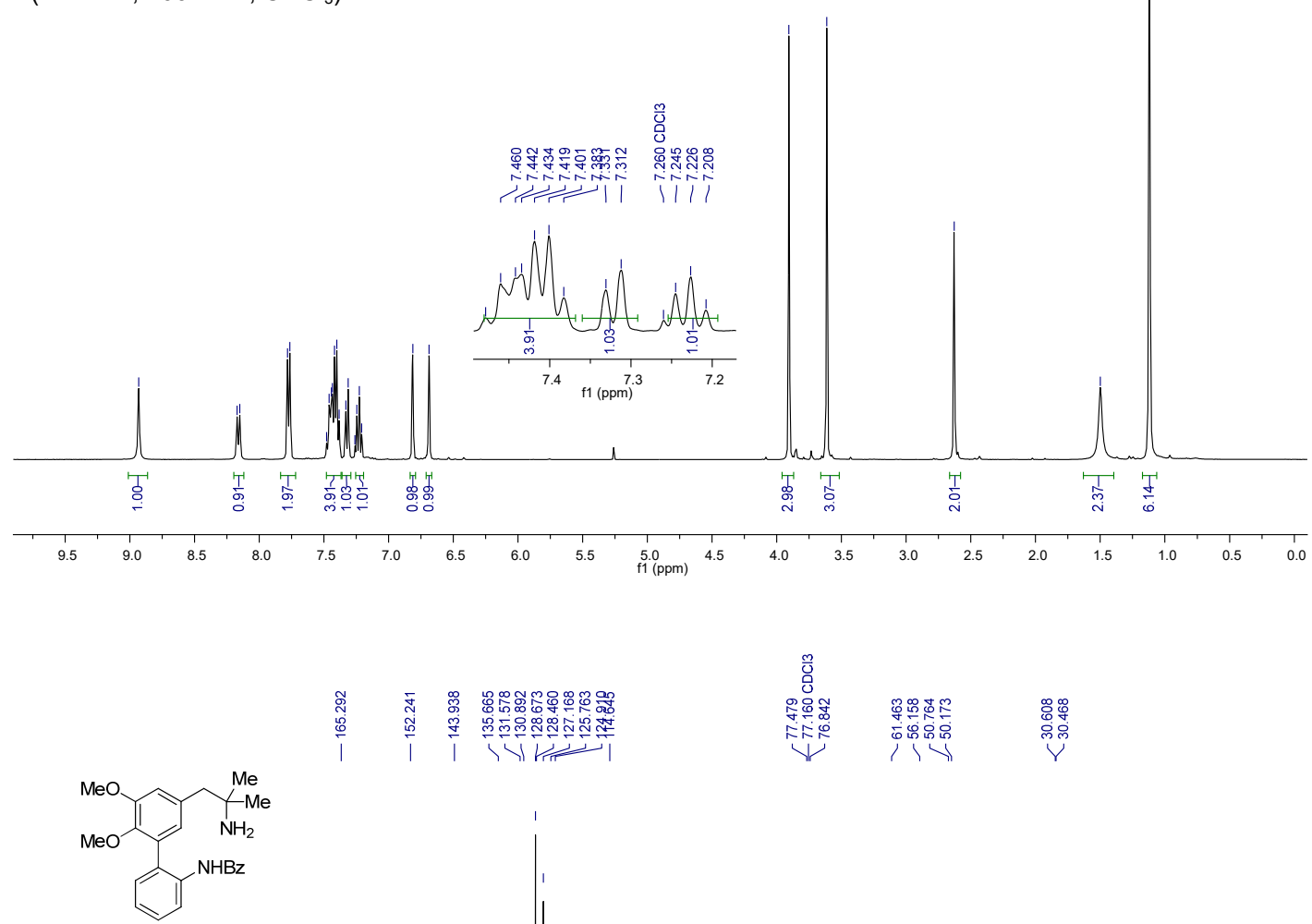

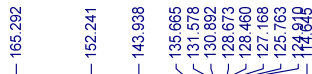

$1 \mathrm{a}$

$\left({ }^{13} \mathrm{C}\right.$ NMR, $\left.100 \mathrm{MHz}, \mathrm{CDCl}_{3}\right)$

每

$\because \underset{1}{-1}$
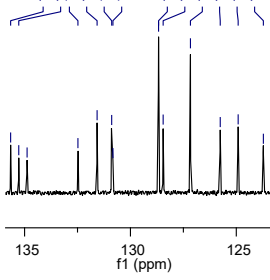

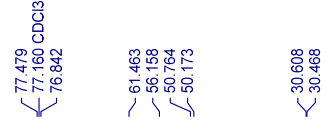

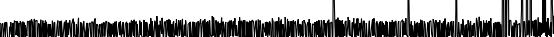

$\begin{array}{lllllllllll}220 & 210 & 200 & 190 & 180 & 170 & 160 & 150 & 140 & 130 & 120\end{array}$ 100 


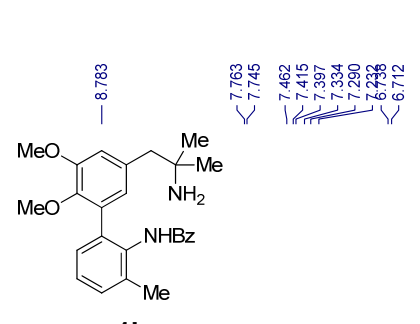

( ${ }^{1} \mathrm{H}$ NMR, $400 \mathrm{MHz}, \mathrm{CDCl}_{3}$ )

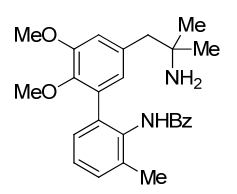

$1 b$

$\left({ }^{13} \mathrm{C}\right.$ NMR, $\left.100 \mathrm{MHz}, \mathrm{CDCl}_{3}\right)$

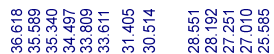

管

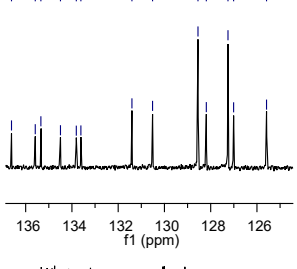

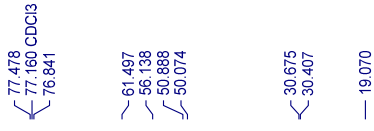

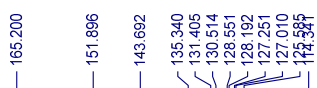

禽䓂 (n)

范

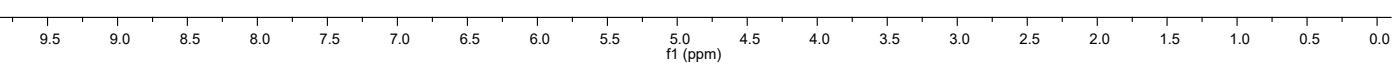



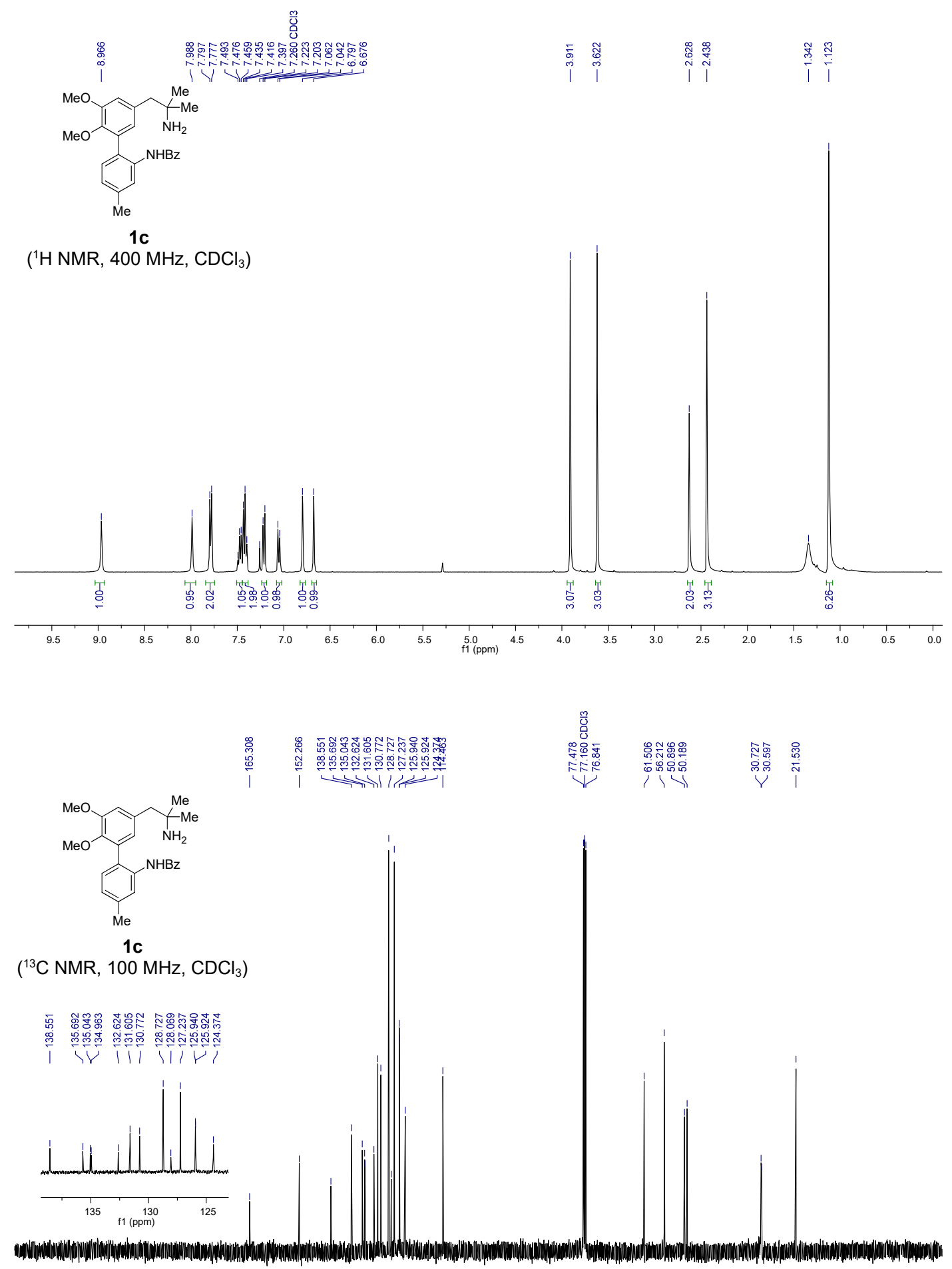

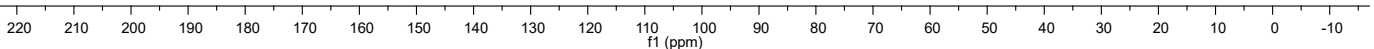



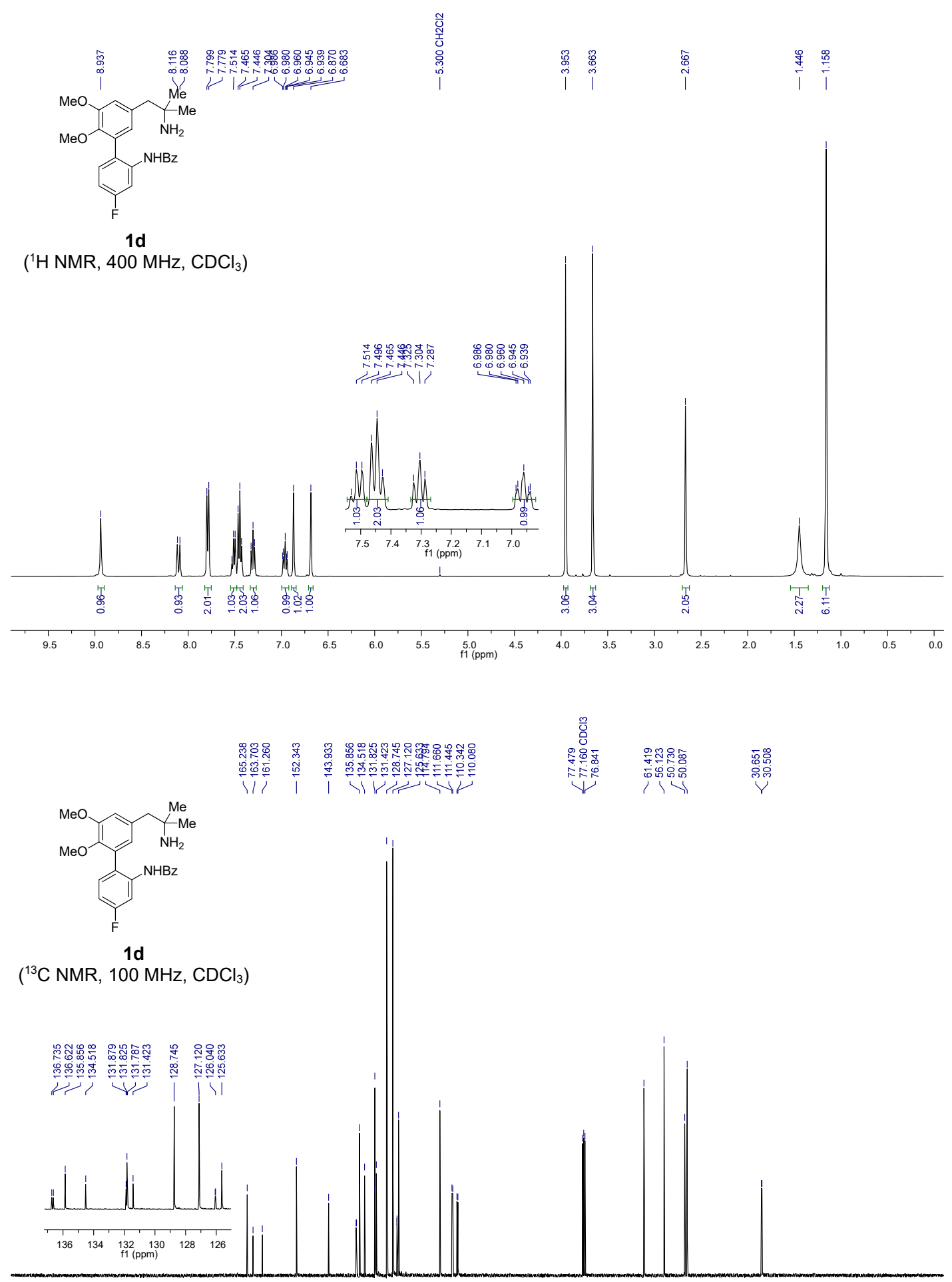

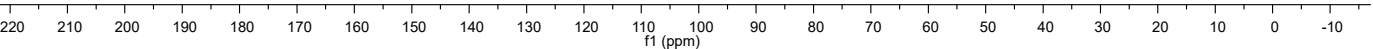




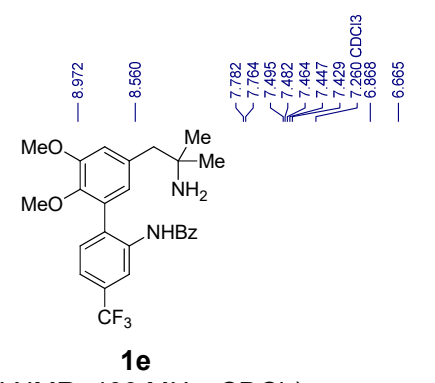

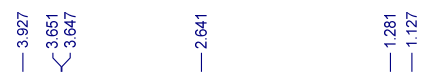

$\left({ }^{1} \mathrm{H}\right.$ NMR, $400 \mathrm{MHz}, \mathrm{CDCl}_{3}$ )
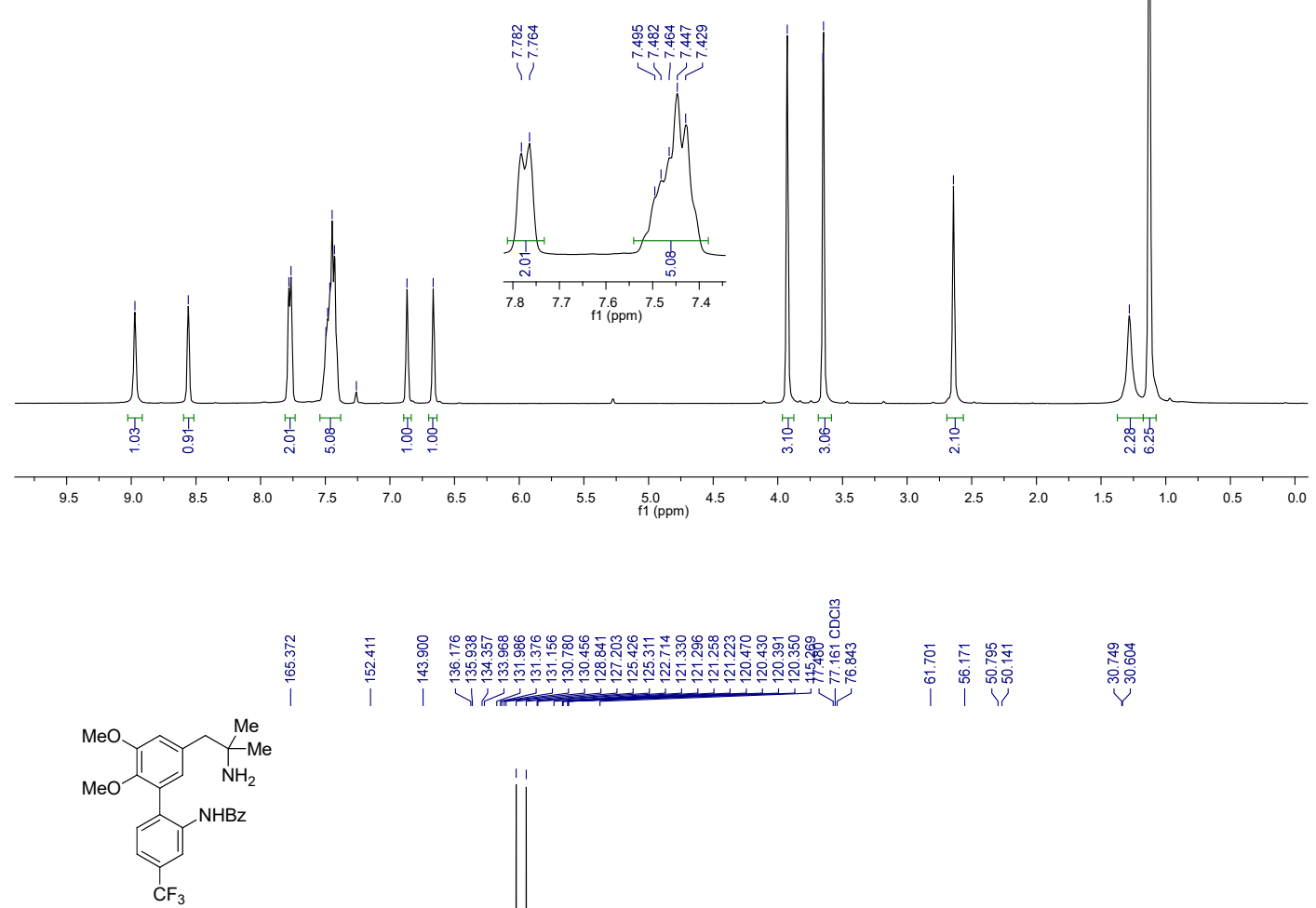

$1 \mathrm{e}$

$\left({ }^{13} \mathrm{C} \mathrm{NMR}, 100 \mathrm{MHz}, \mathrm{CDCl}_{3}\right)$

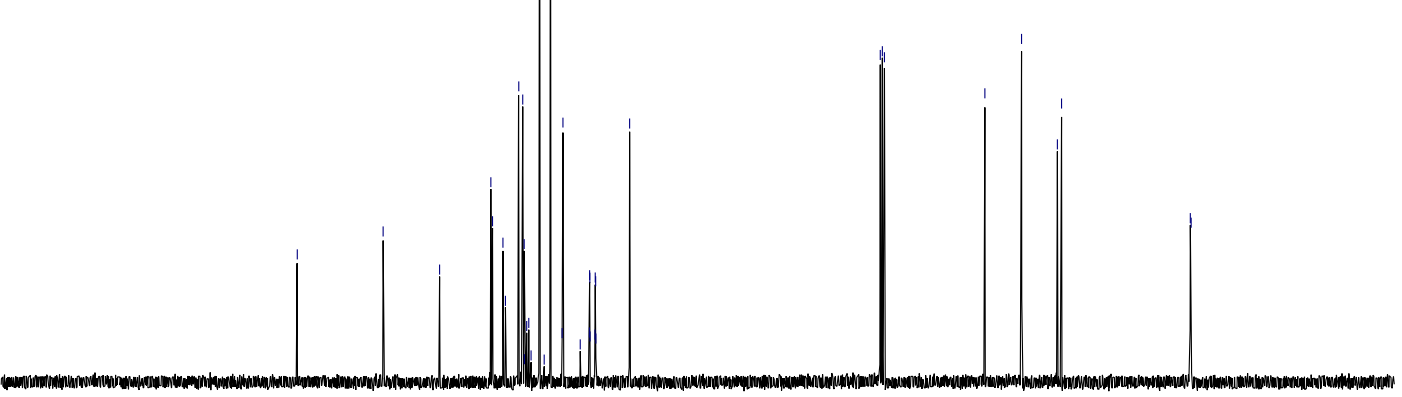

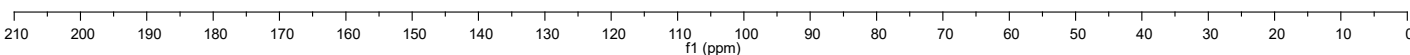



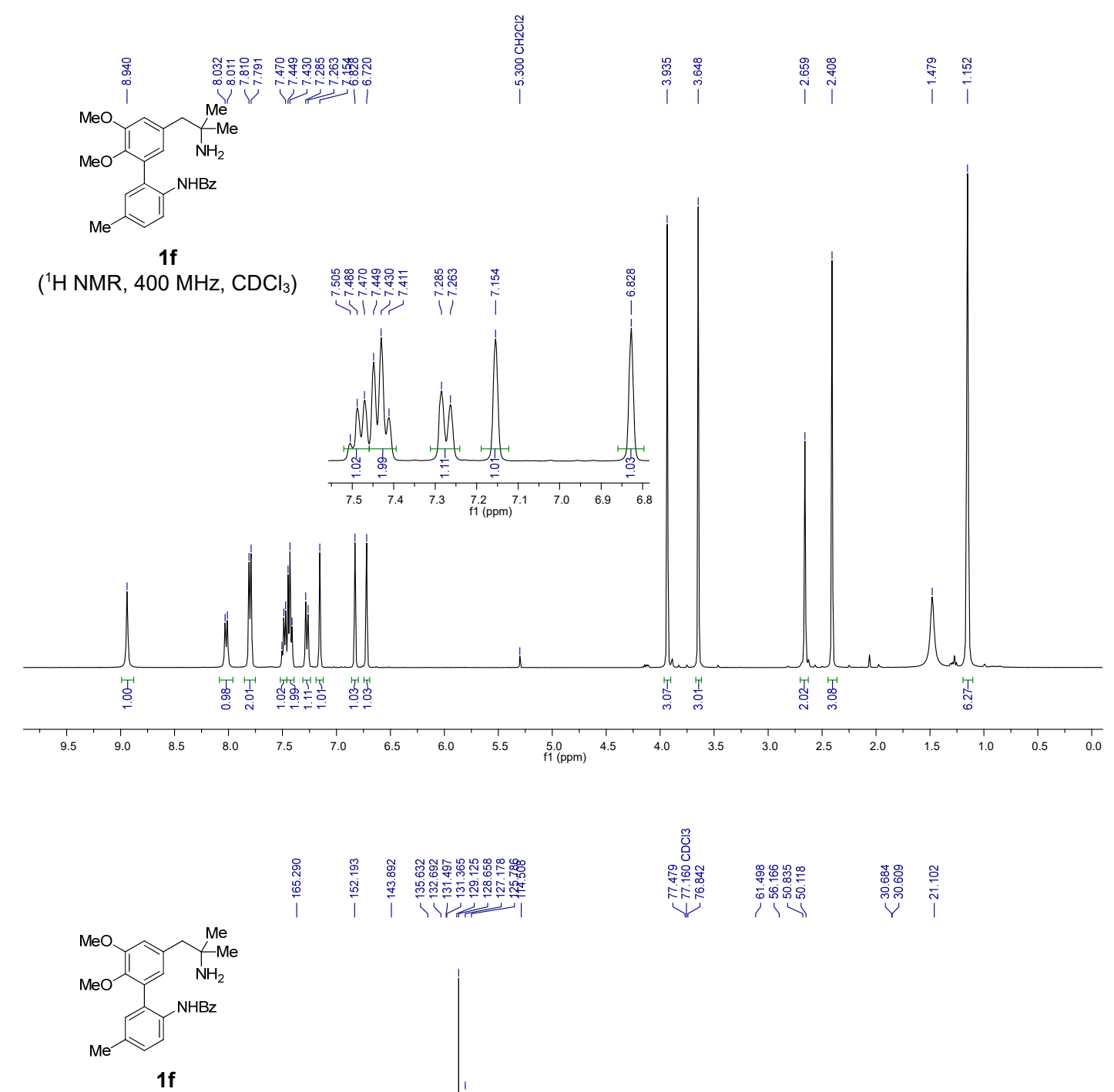

$\left({ }^{13} \mathrm{C} \mathrm{NMR}, 100 \mathrm{MHz}, \mathrm{CDCl}_{3}\right)$
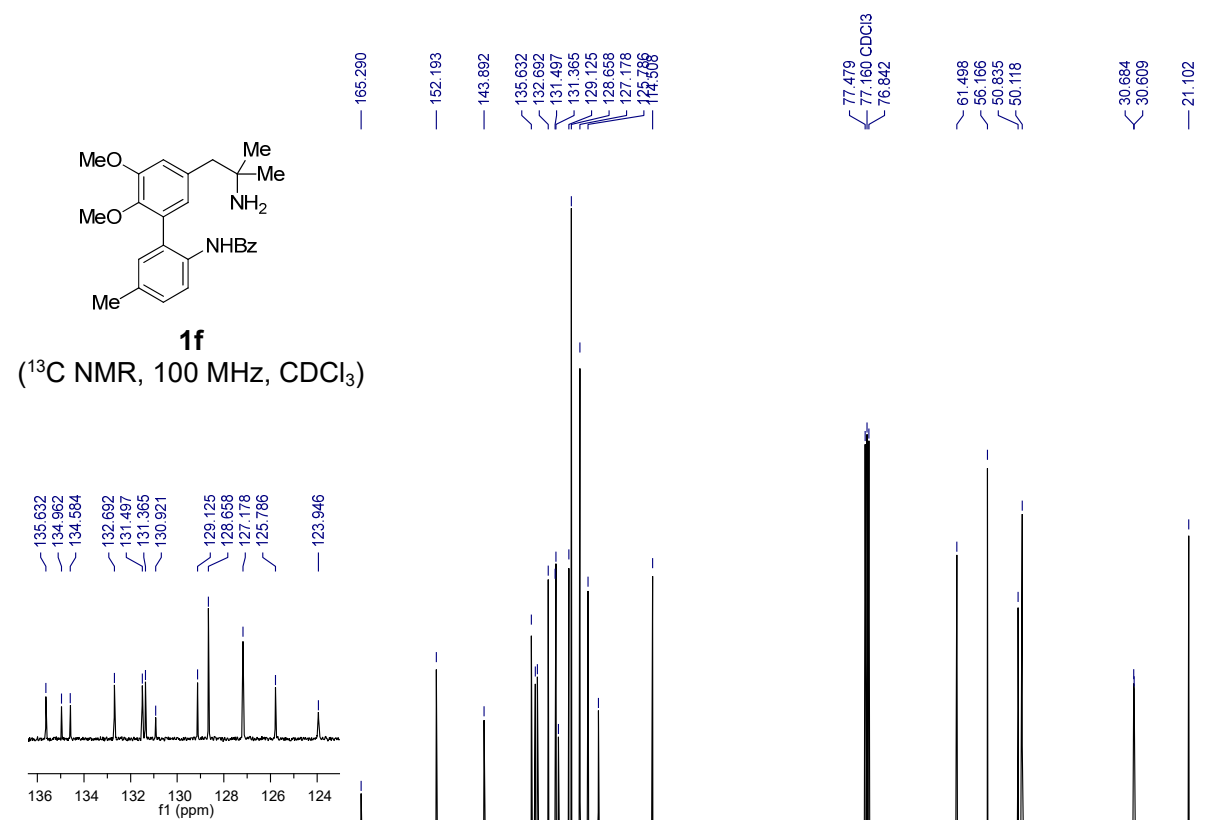

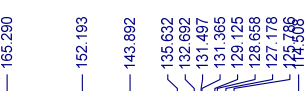

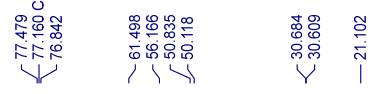

$\begin{array}{lllllll}220 & 210 & 200 & 190 & 180 & 170 & 160\end{array}$ f1 (ppm) 


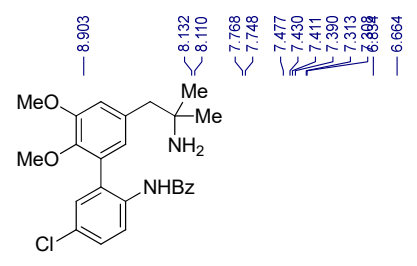

$1 \mathrm{~g}$

( ${ }^{1} \mathrm{H}$ NMR, $400 \mathrm{MHz}, \mathrm{CDCl}_{3}$ )
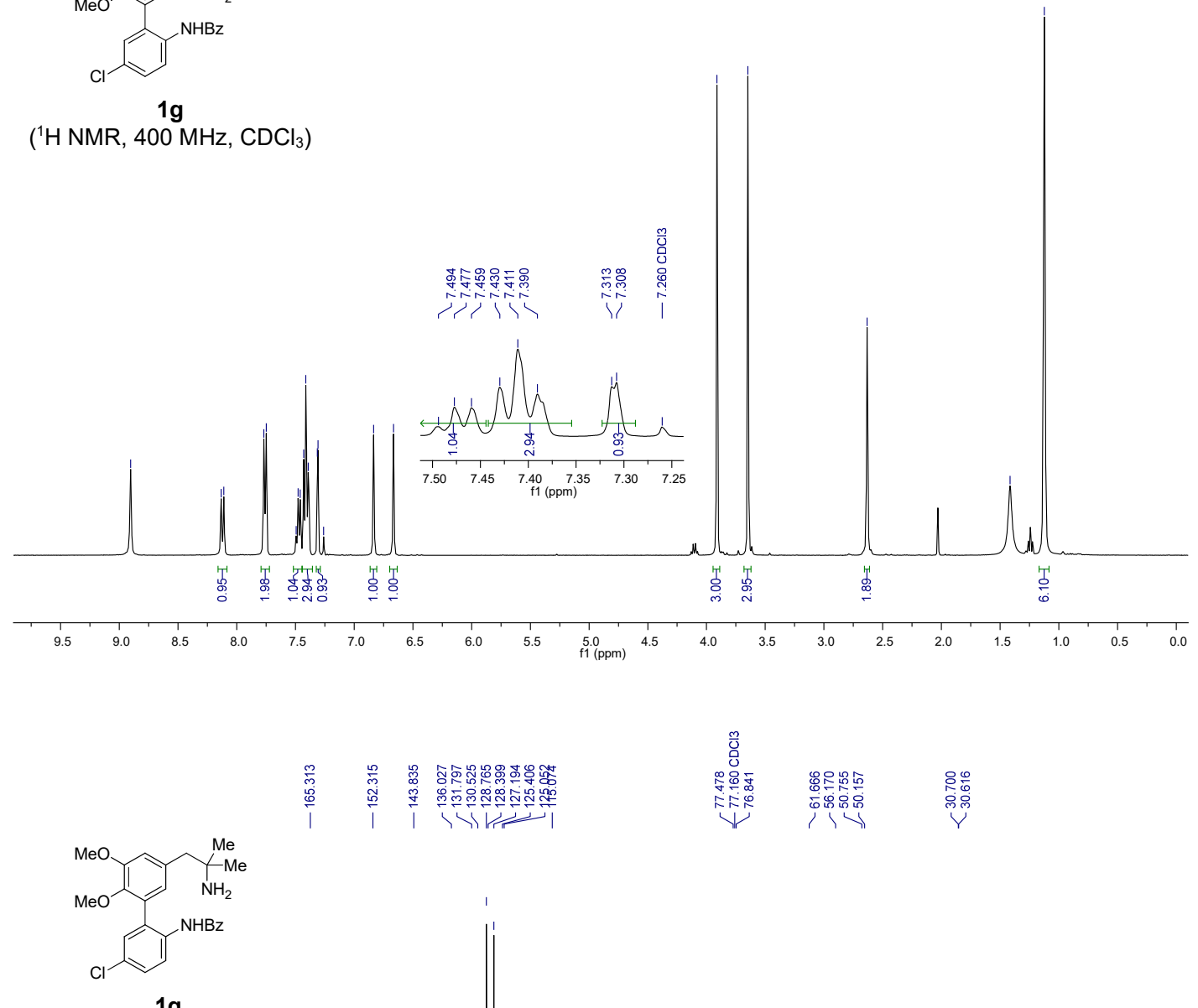

$1 \mathrm{~g}$

$\left({ }^{13} \mathrm{C}\right.$ NMR, $\left.100 \mathrm{MHz}, \mathrm{CDCl}_{3}\right)$

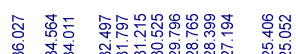

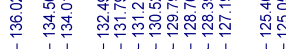

11111111111

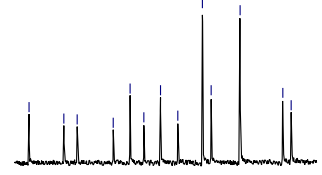

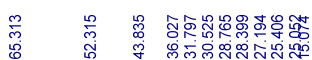

।

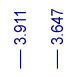

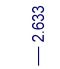

$\stackrel{i}{\frac{1}{2}}$

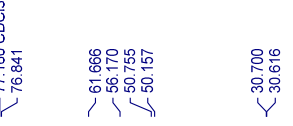

$\begin{array}{llllllllll}136 & 134 & 132 & 130 & 128 & 126 & 124 \\ & & & & 1(p p m)\end{array}$ 


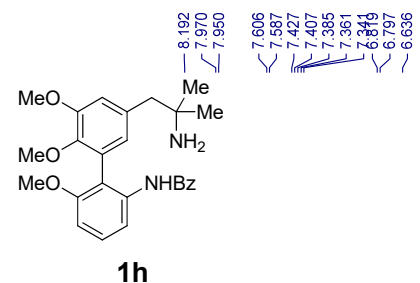

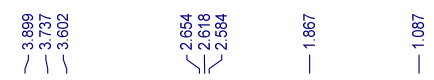

$\left({ }^{1} \mathrm{H} \mathrm{NMR}, 400 \mathrm{MHz}, \mathrm{CDCl}_{3}\right.$ )
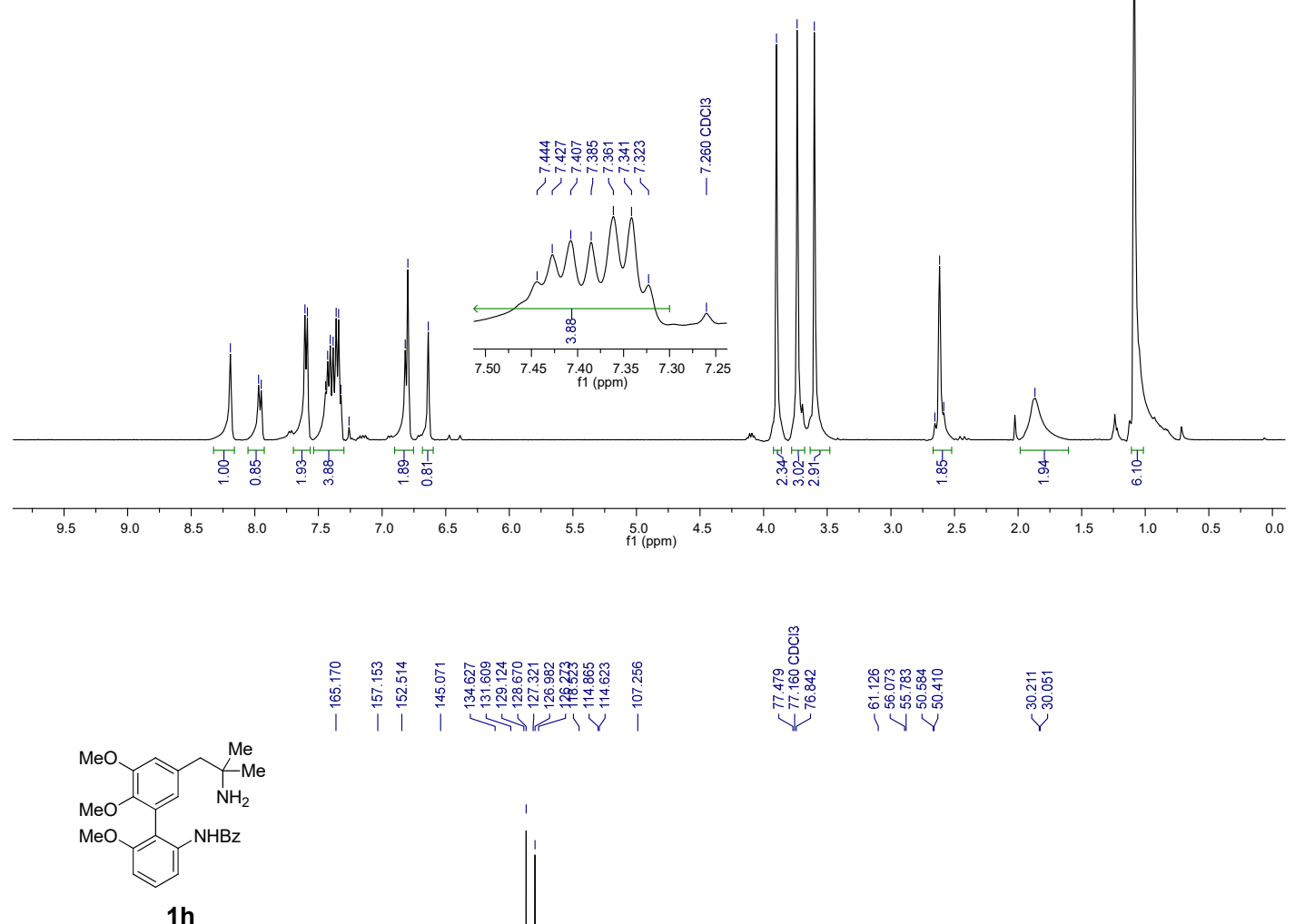

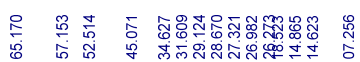

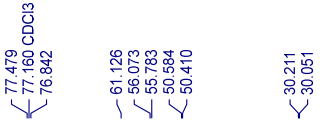

$\left({ }^{13} \mathrm{C} \mathrm{NMR}, 100 \mathrm{MHz}, \mathrm{CDCl}_{3}\right)$
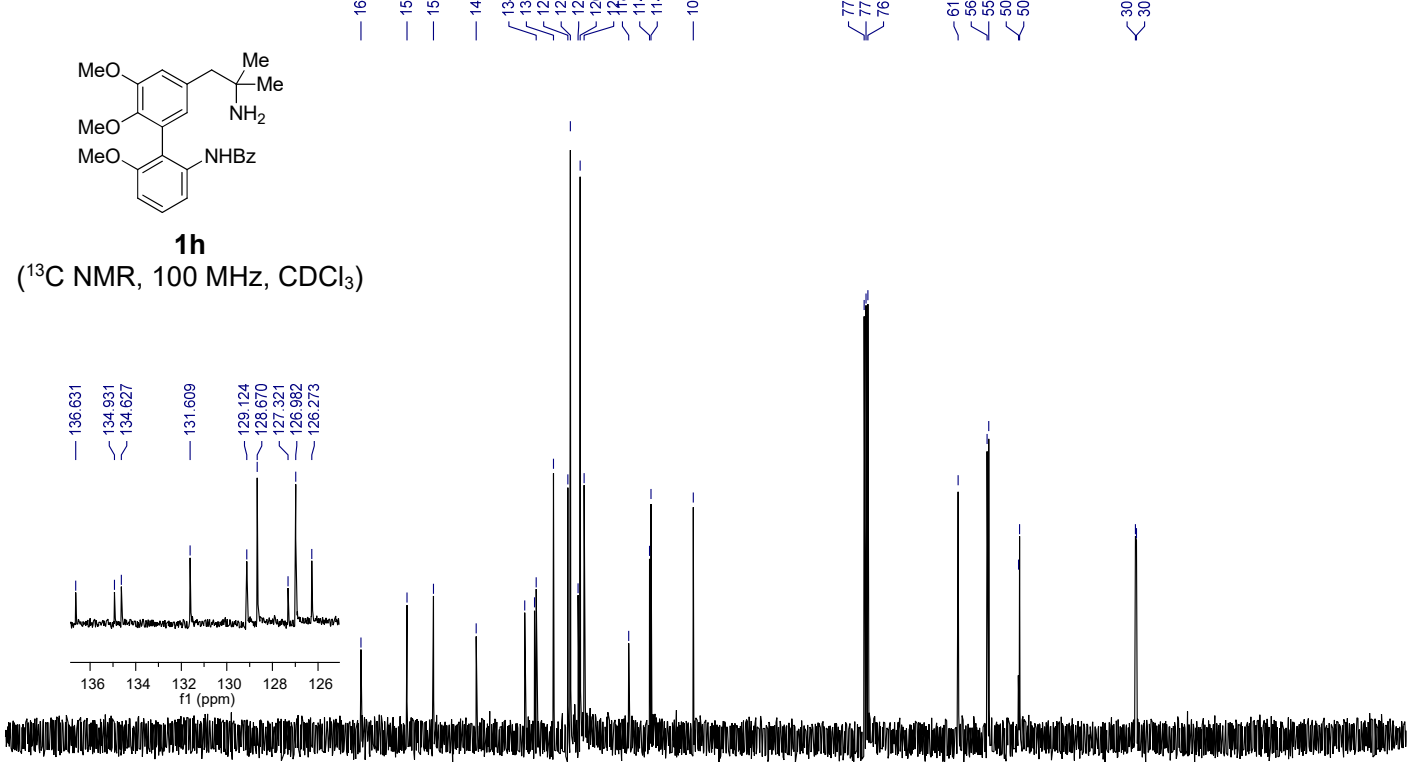

Vل

$\begin{array}{llllllllllll}220 & 210 & 200 & 190 & 180 & 170 & 160 & 150 & 140 & 130 & 120 & \begin{array}{c}110 \\ \mathrm{f} 1(\mathrm{ppm})\end{array}\end{array}$ 


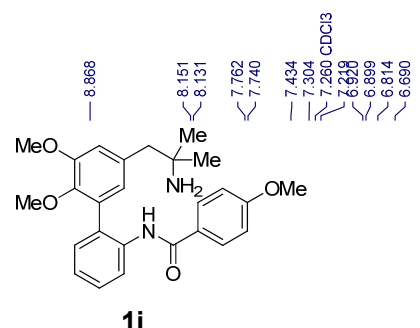

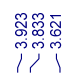

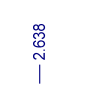

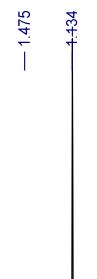

( ${ }^{1} \mathrm{H}$ NMR, $400 \mathrm{MHz}, \mathrm{CDCl}_{3}$ )
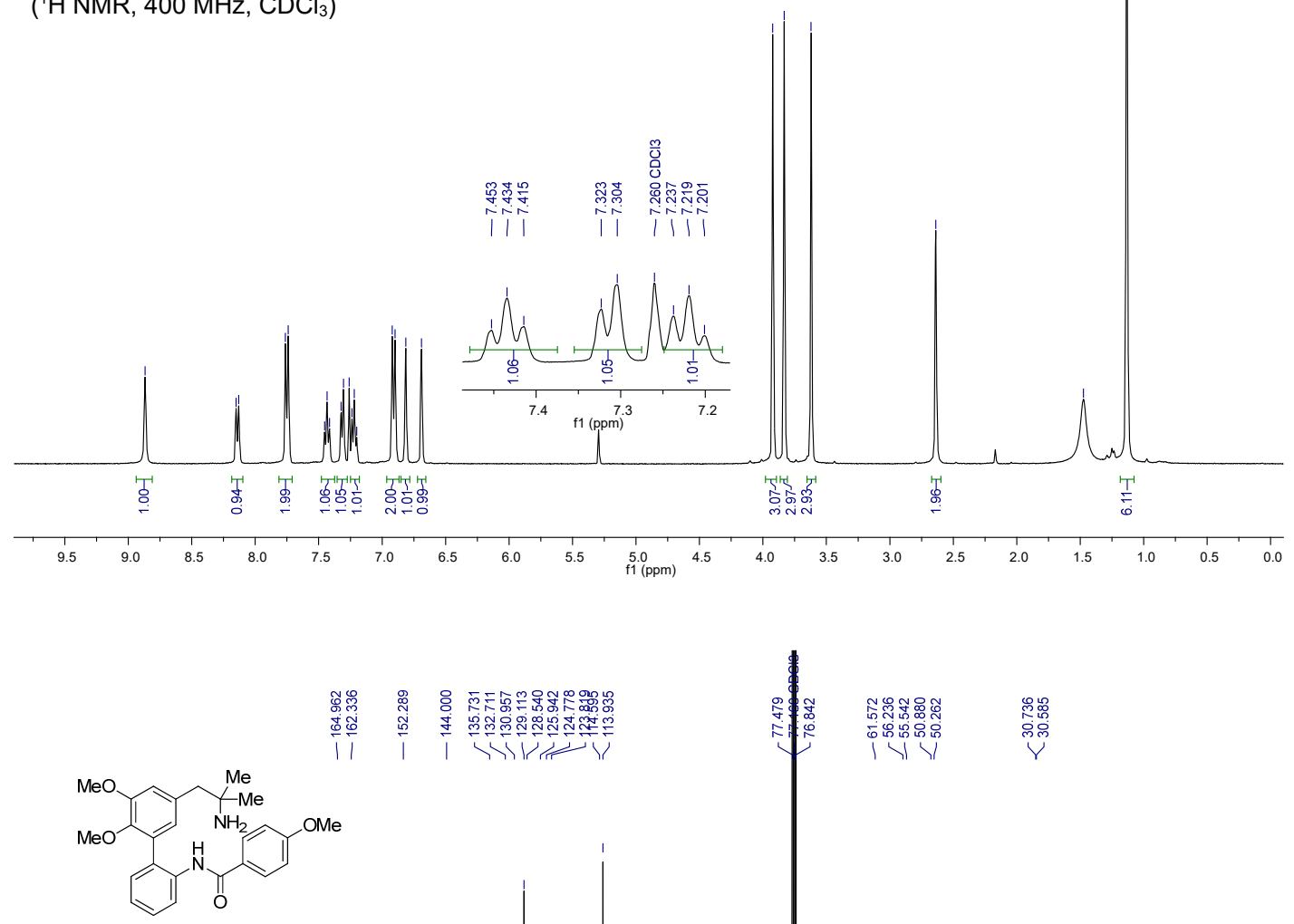

$1 \mathrm{i}$

$\left({ }^{13} \mathrm{C} \mathrm{NMR}, 100 \mathrm{MHz}, \mathrm{CDCl}_{3}\right)$

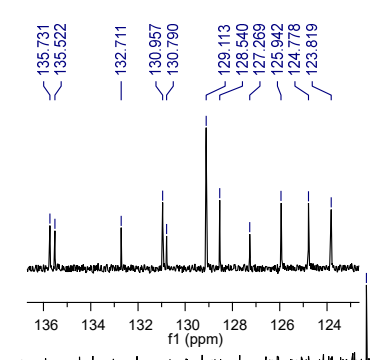

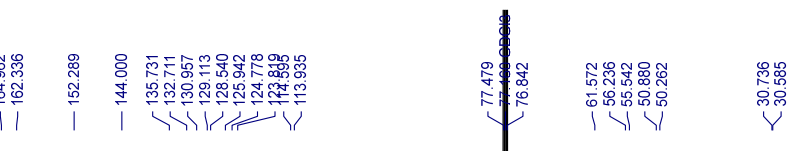

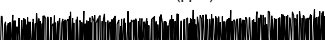

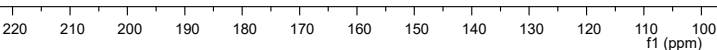




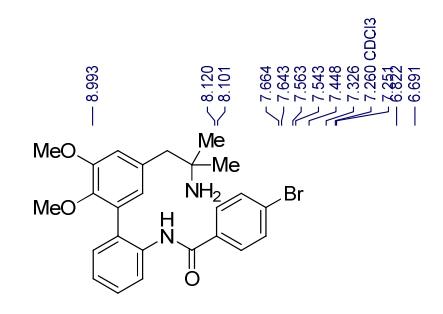

$1 \mathrm{j}$

( ${ }^{1} \mathrm{H} \mathrm{NMR}, 400 \mathrm{MHz}, \mathrm{CDCl}_{3}$ )
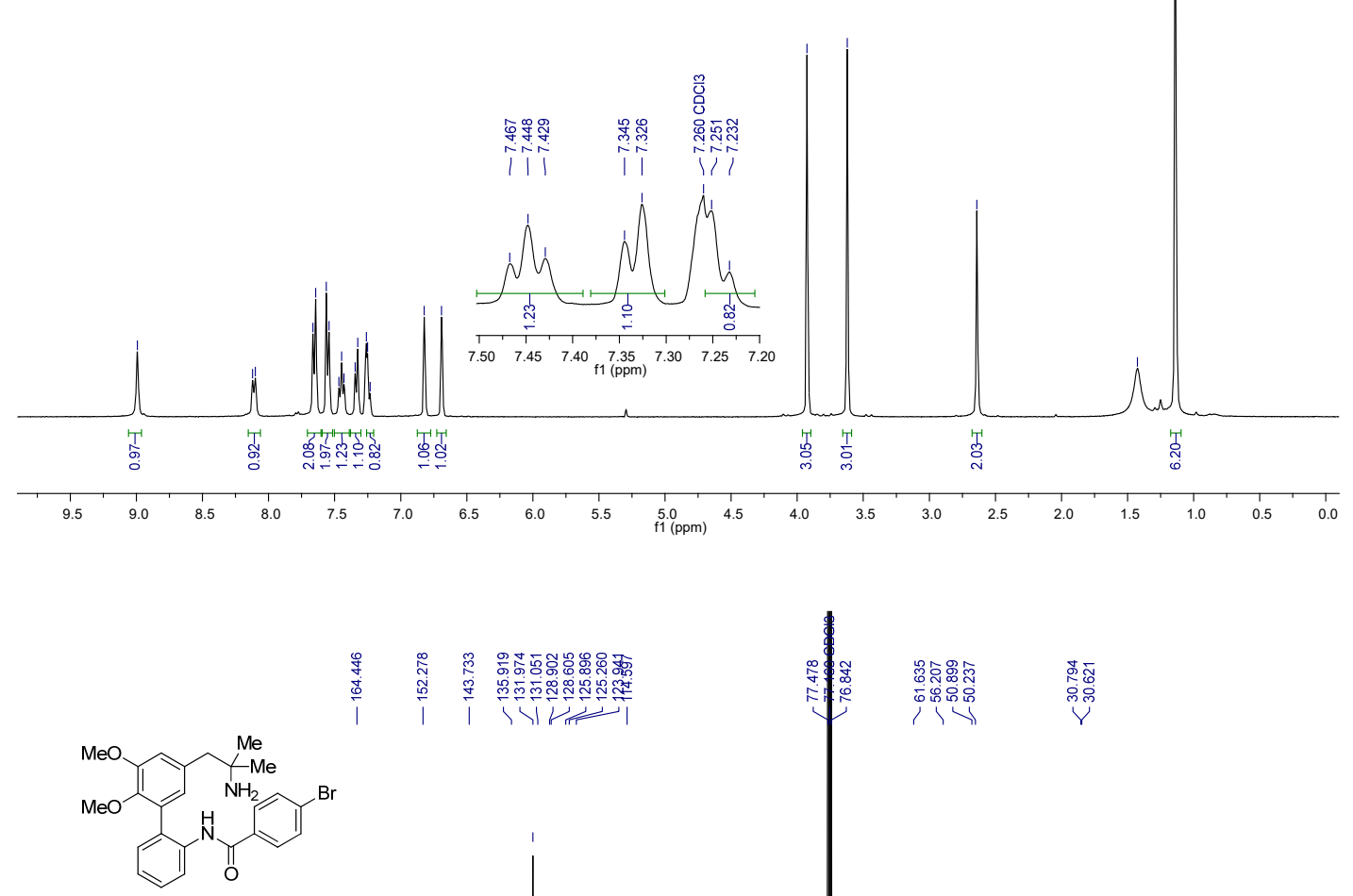

$1 \mathrm{j}$

$\left({ }^{13} \mathrm{C} \mathrm{NMR}, 100 \mathrm{MHz}, \mathrm{CDCl}_{3}\right)$

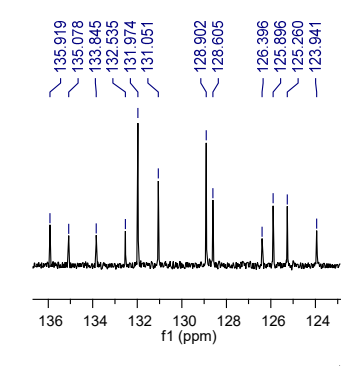

$\begin{array}{lllllllllllll}220 & 210 & 200 & 190 & 180 & 170 & 160 & 150 & 140 & 130 & 120 & \begin{array}{c}110 \\ \mathrm{f} 1(\mathrm{ppm})\end{array} & 100 \\ & & & & & \end{array}$ 


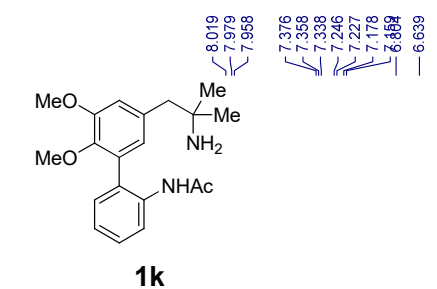

i

( ${ }^{1} \mathrm{H} \mathrm{NMR}, 400 \mathrm{MHz}, \mathrm{CDCl}_{3}$ )
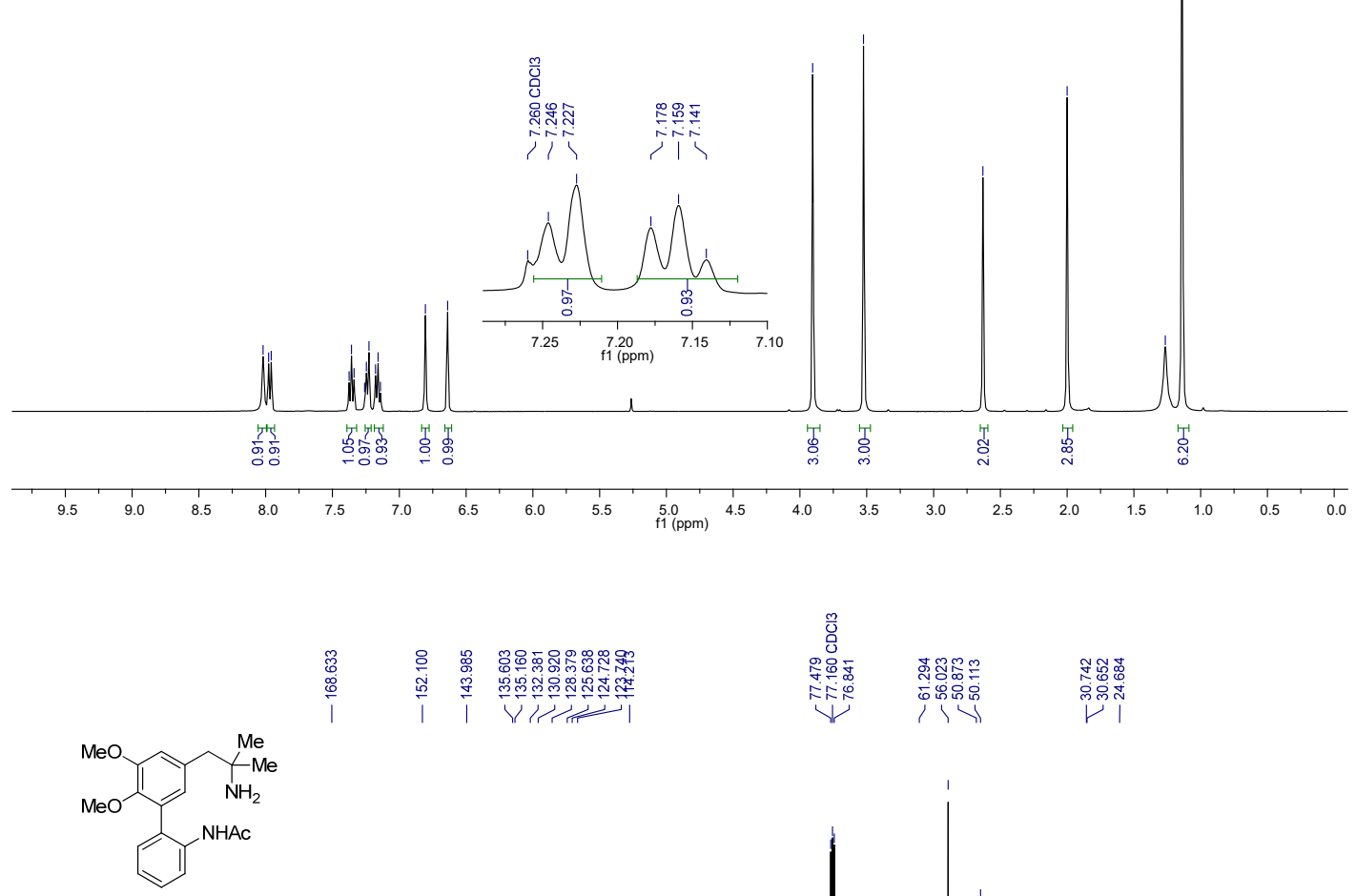

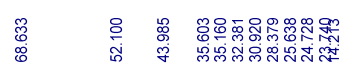

i $\quad$

$1 \mathrm{k}$

$\left({ }^{13} \mathrm{C}\right.$ NMR, $\left.100 \mathrm{MHz}, \mathrm{CDCl}_{3}\right)$
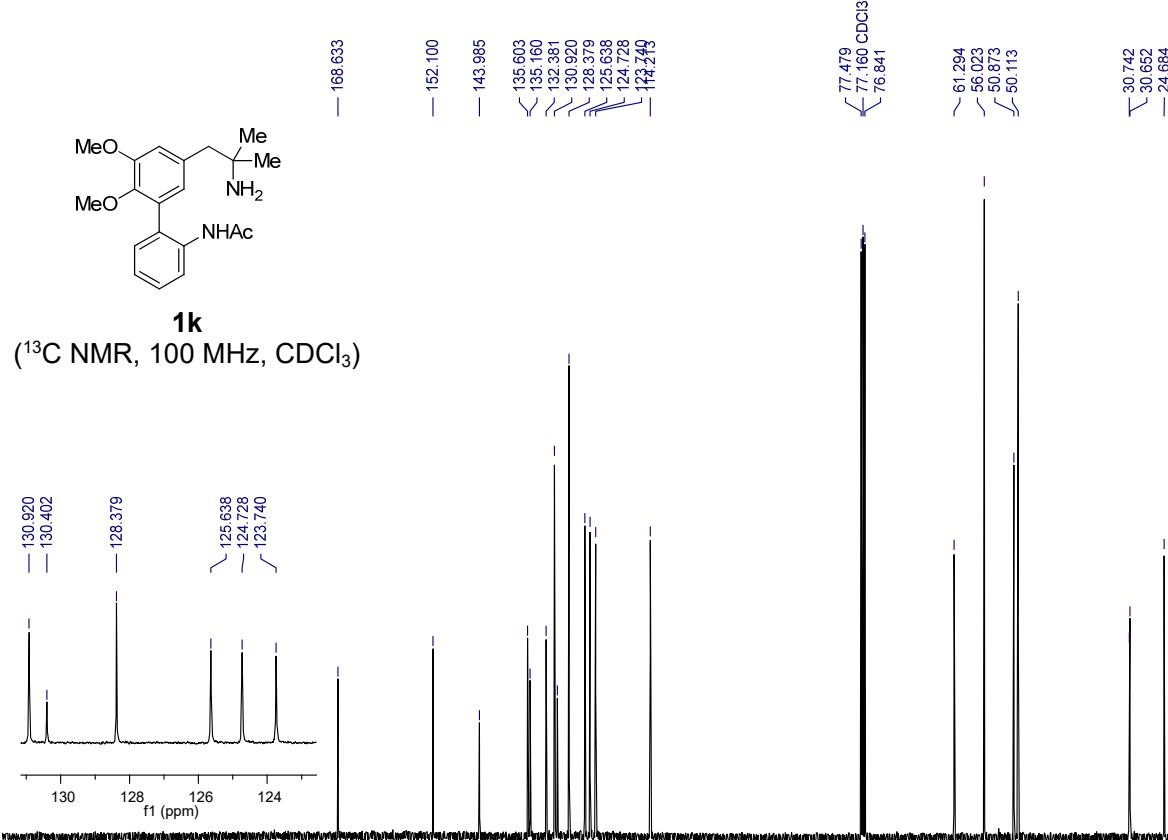

$\begin{array}{llllllllllll}220 & 210 & 200 & 190 & 180 & 170 & 160 & 150 & 140 & 130 & 120 & \begin{array}{c}110 \\ \mathrm{f} 1(\mathrm{ppm})\end{array}\end{array}$ 


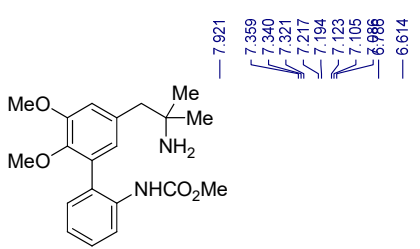

11

( ${ }^{1} \mathrm{H} \mathrm{NMR}, 400 \mathrm{MHz}, \mathrm{CDCl}_{3}$ )

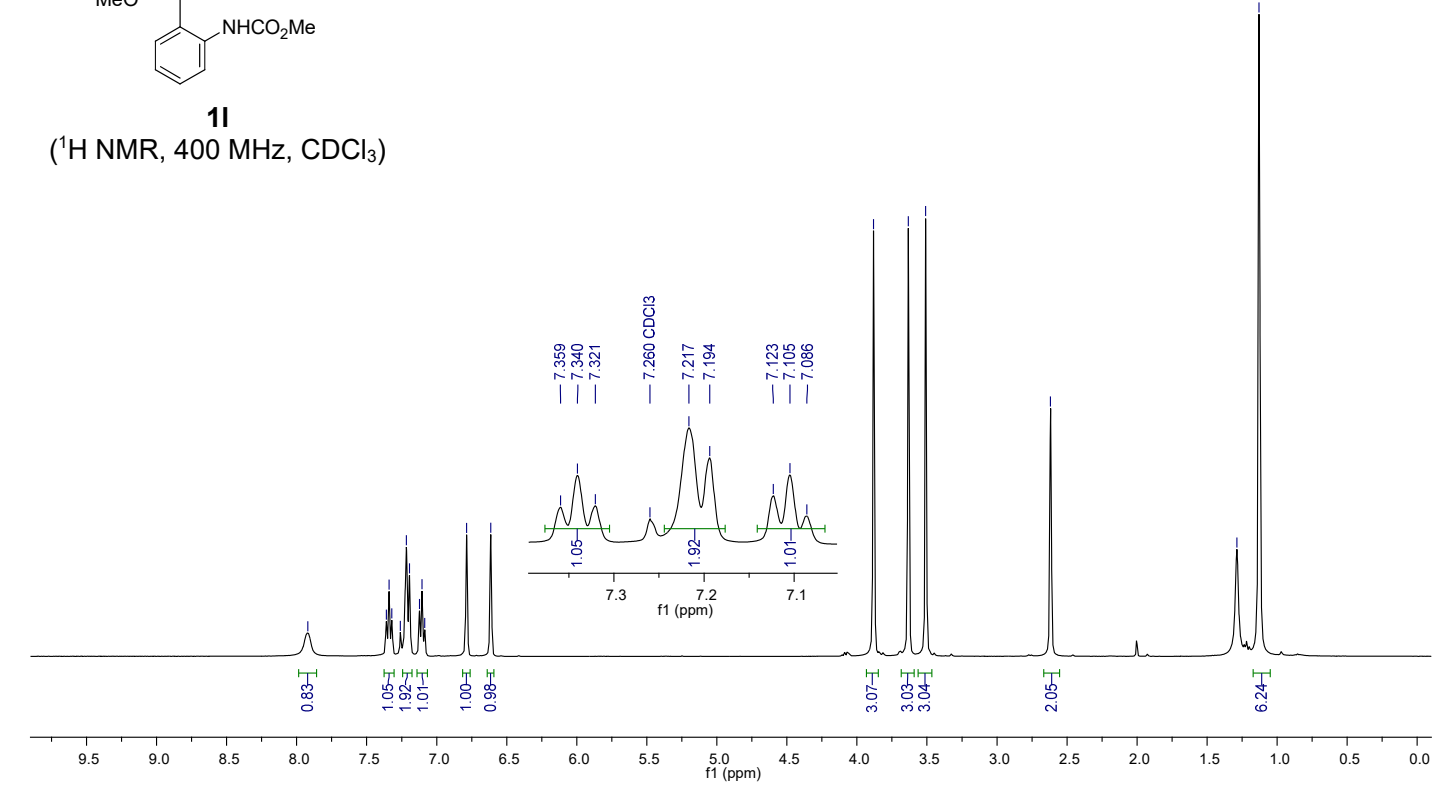

iฺ

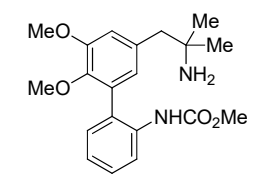

11

$\left({ }^{13} \mathrm{C} \mathrm{NMR}, 100 \mathrm{MHz}, \mathrm{CDCl}_{3}\right)$

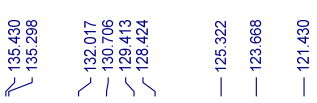

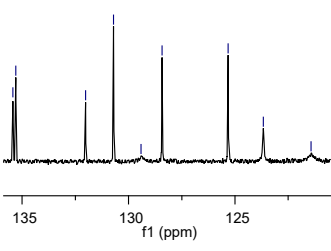

잉

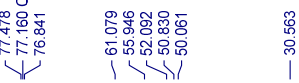

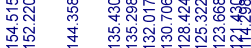

11 IIti

पititit

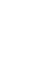

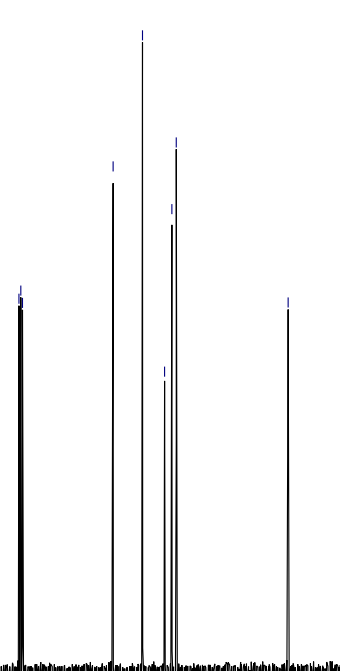



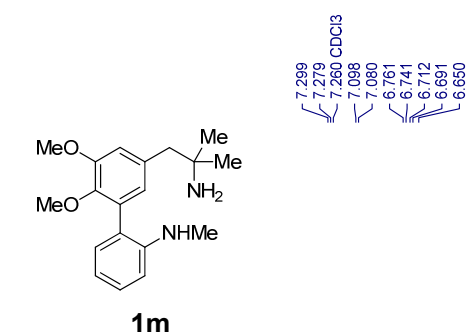

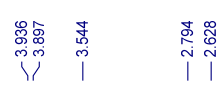

$\stackrel{i}{i}$

('H NMR, $400 \mathrm{MHz}, \mathrm{CDCl}_{3}$ )
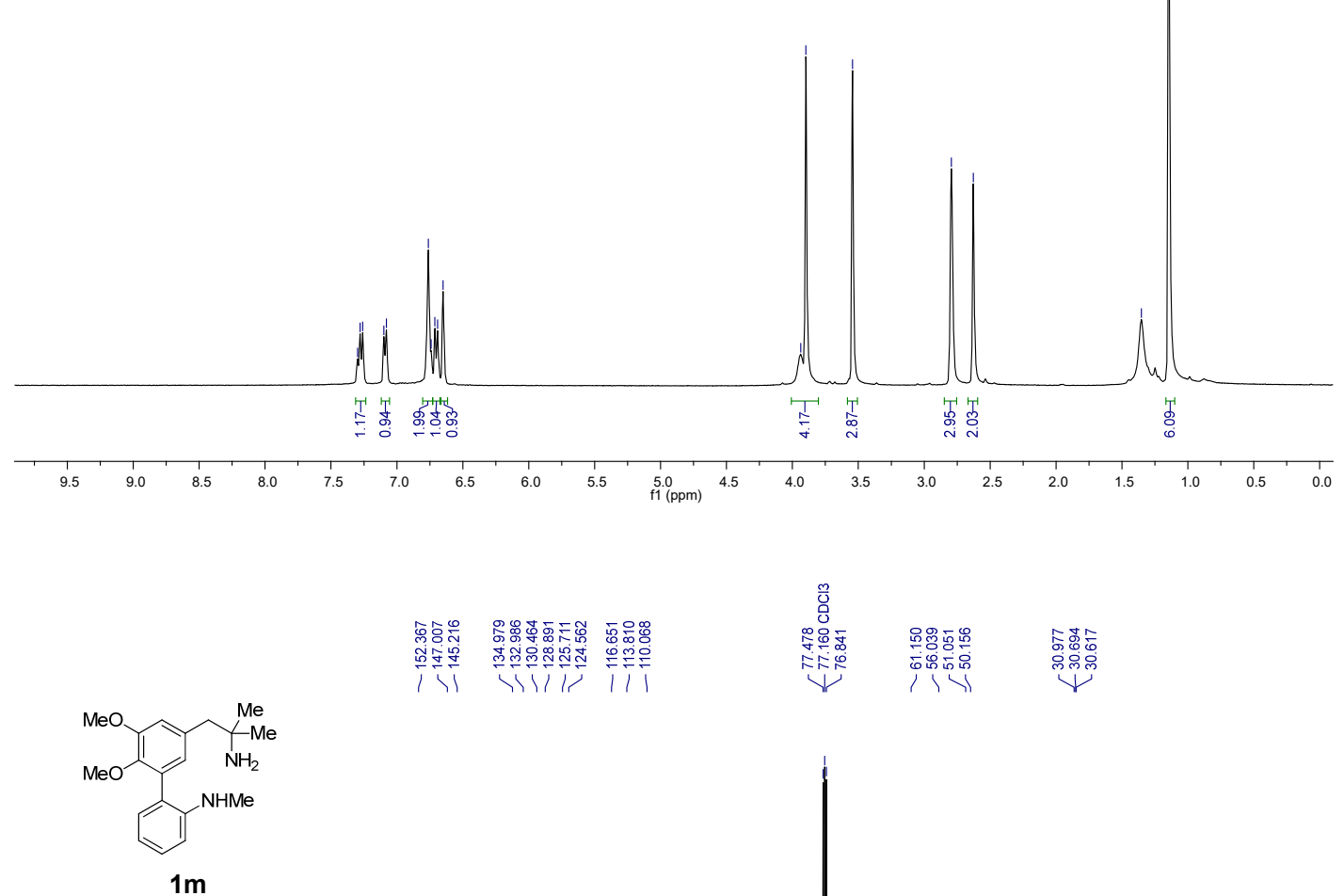

$\left({ }^{13} \mathrm{C} \mathrm{NMR}, 100 \mathrm{MHz}, \mathrm{CDCl}_{3}\right)$

$\begin{array}{lllllllllllll}220 & 210 & 200 & 190 & 180 & 170 & 160 & 150 & 140 & 130 & 120 & \underset{\mathrm{f} 1}{110}(\mathrm{ppm}) & 100\end{array}$ 

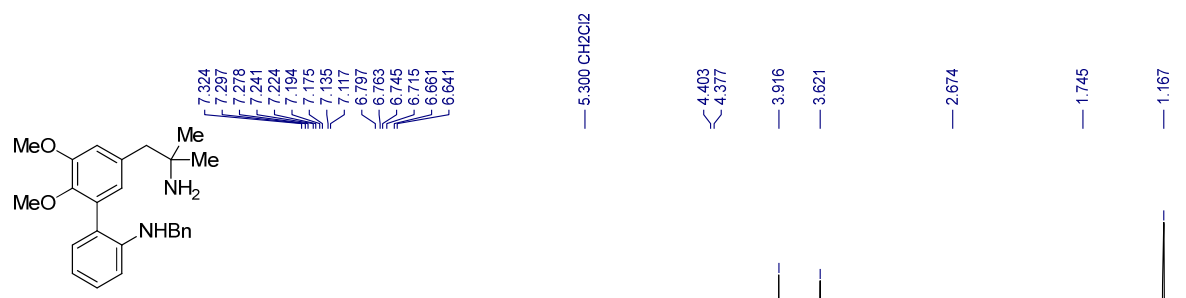

$1 n$

( ${ }^{1} \mathrm{H} \mathrm{NMR}, 400 \mathrm{MHz}, \mathrm{CDCl}_{3}$ )
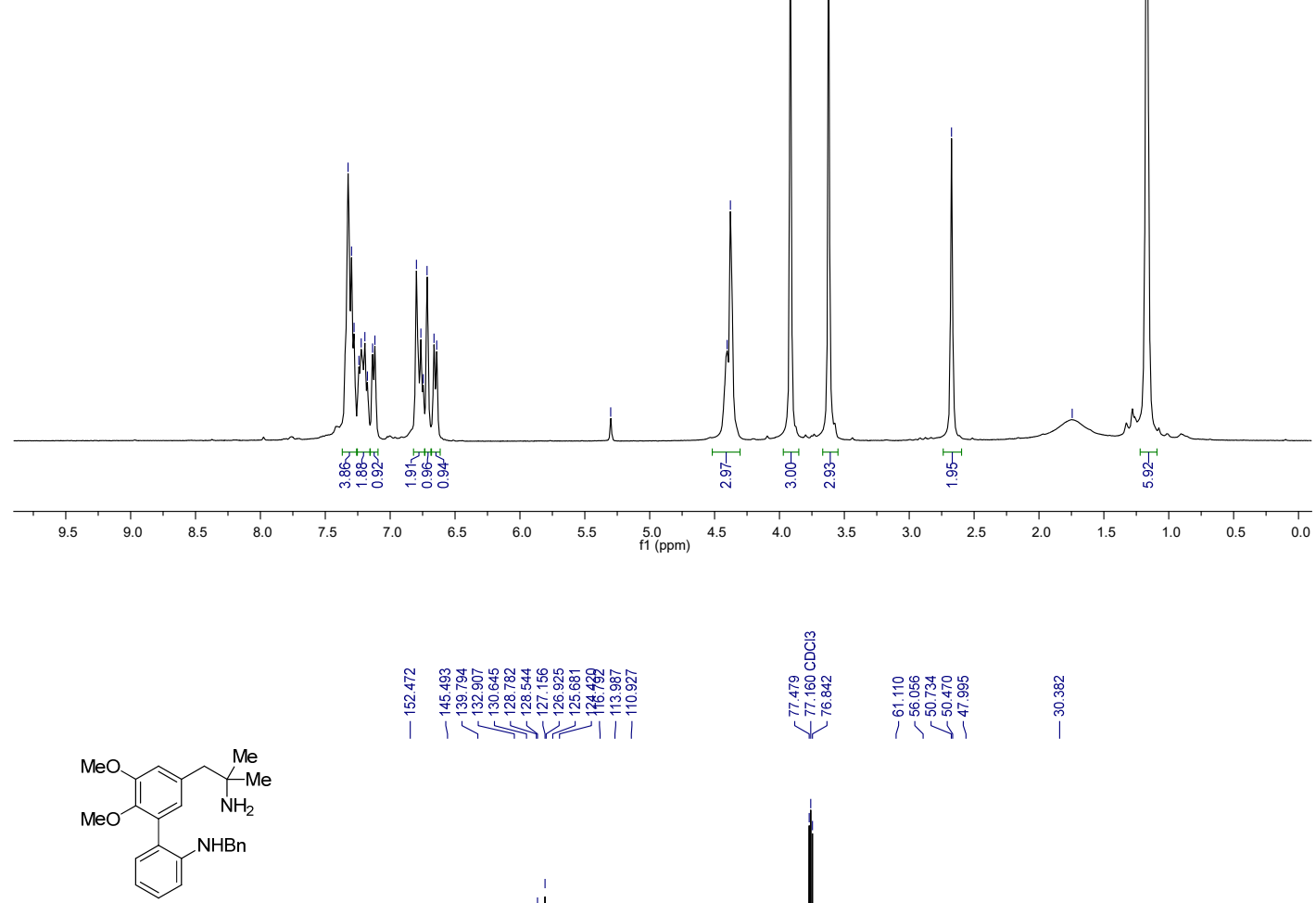

它

1 -

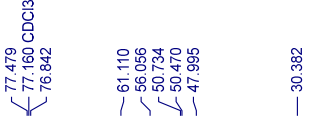

1n

$\left({ }^{13} \mathrm{C} \mathrm{NMR,}, 100 \mathrm{MHz}, \mathrm{CDCl}_{3}\right)$

i

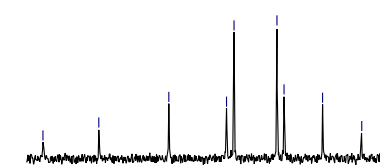

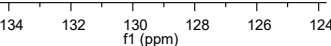
Initw

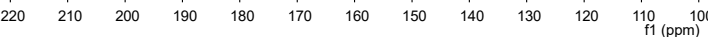



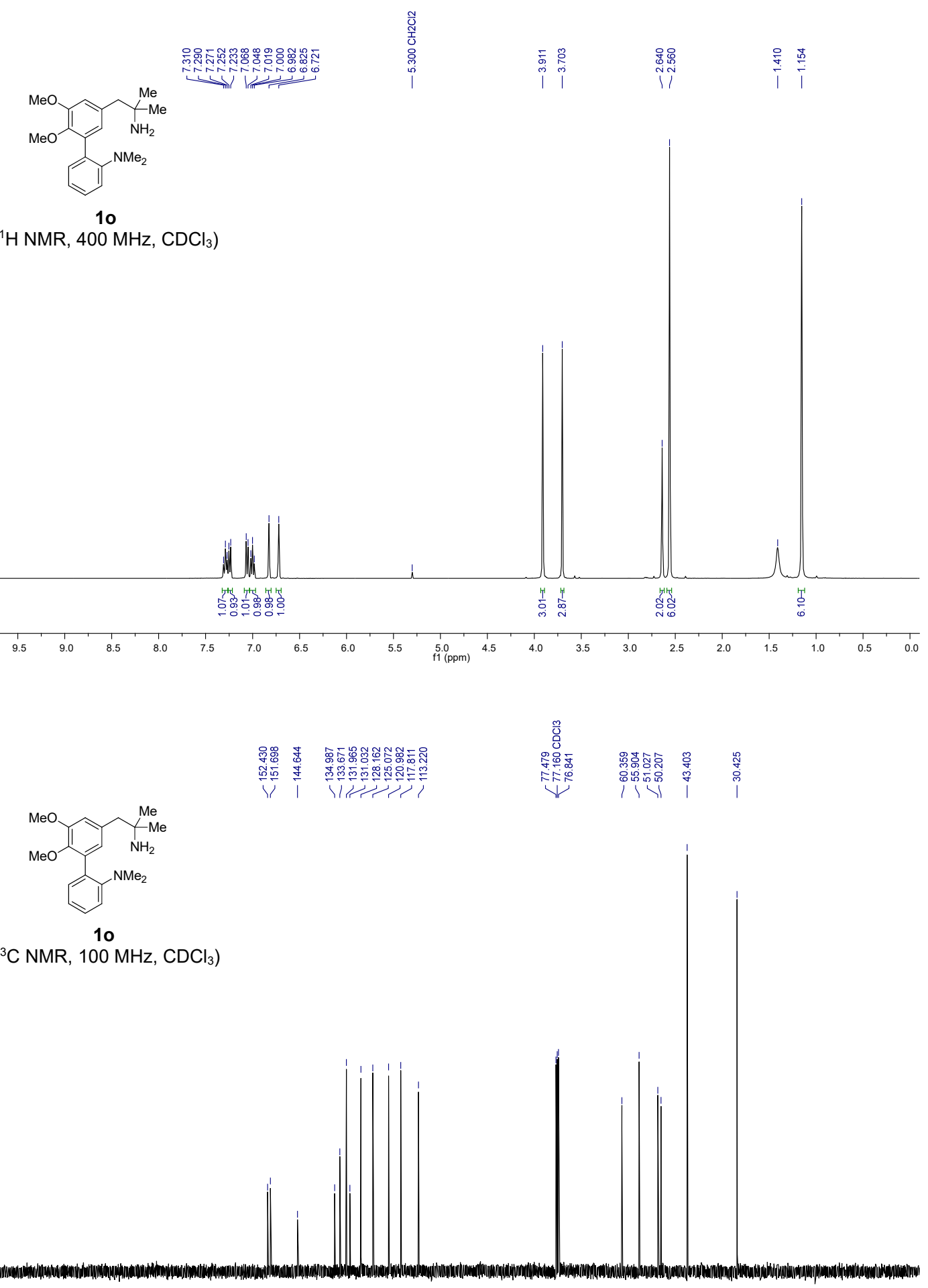

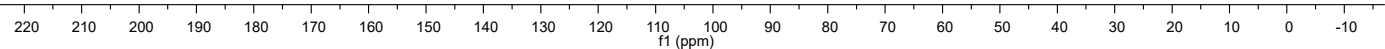




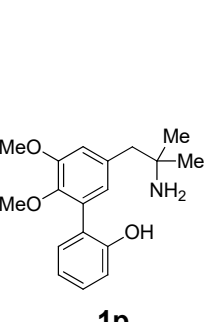

竞兽

( $\left.{ }^{1} \mathrm{H} \mathrm{NMR}, 400 \mathrm{MHz}, \mathrm{CDCl}_{3}\right)$
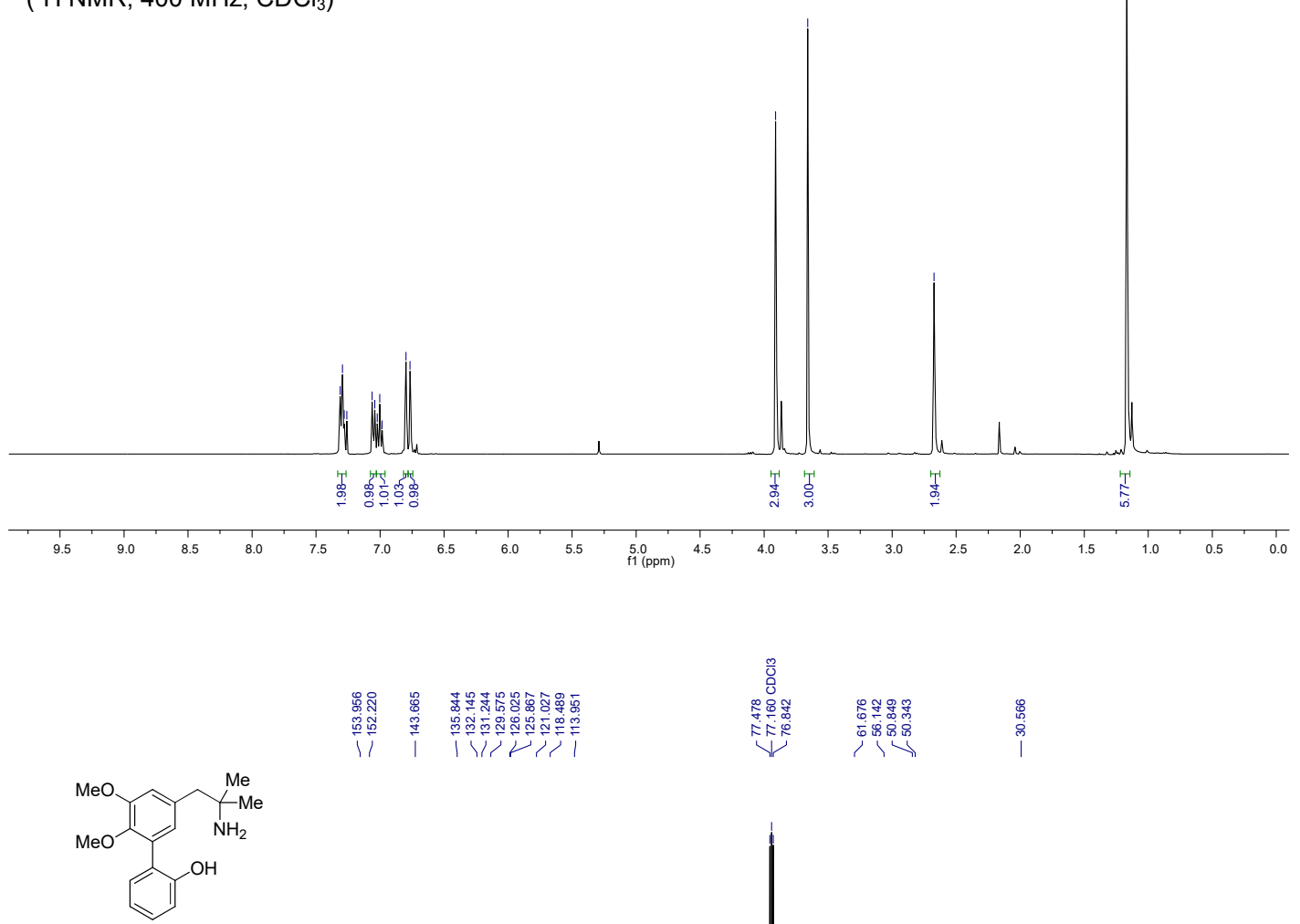

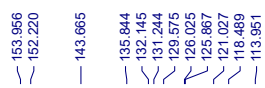

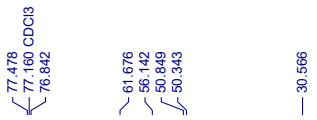

$$
1 \mathrm{p}
$$

$\left({ }^{13} \mathrm{C} \mathrm{NMR}, 100 \mathrm{MHz}, \mathrm{CDCl}_{3}\right)$

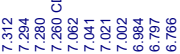

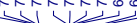

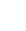
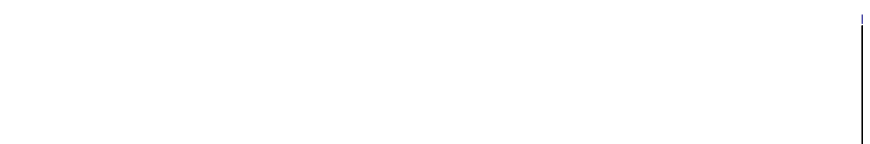


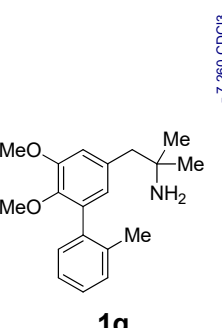

等

总

('H NMR, $400 \mathrm{MHz}, \mathrm{CDCl}_{3}$ )
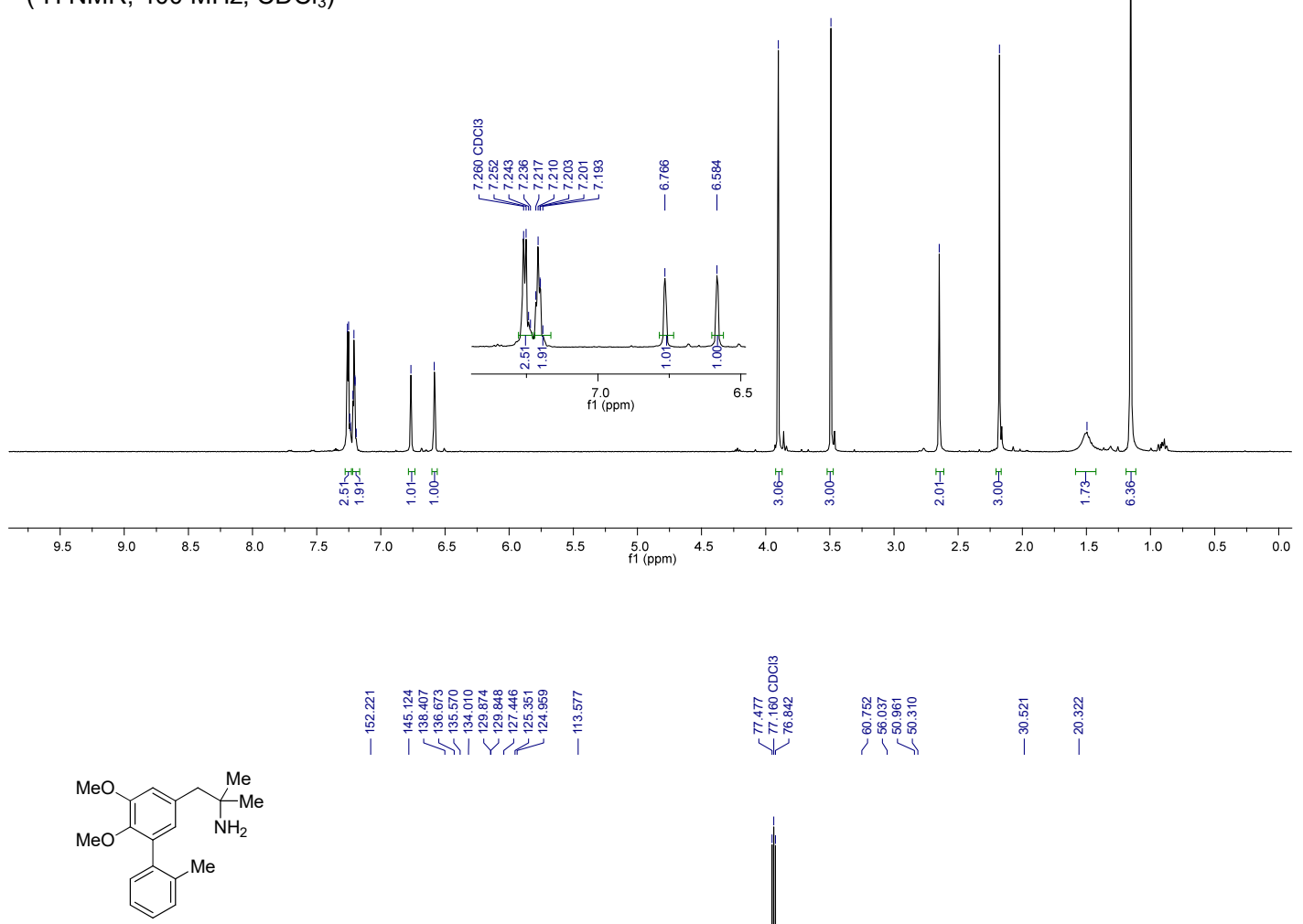

|

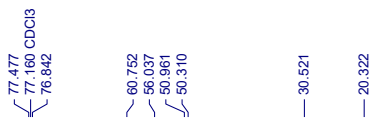

$$
1 q
$$

$\left({ }^{13} \mathrm{C} \mathrm{NMR}, 100 \mathrm{MHz}, \mathrm{CDCl}_{3}\right)$

$\begin{array}{lllllll}210 & 200 & 190 & 180 & 170 & 160 & 150\end{array}$

$110 \underset{f 1(\mathrm{ppm})}{100}$ 

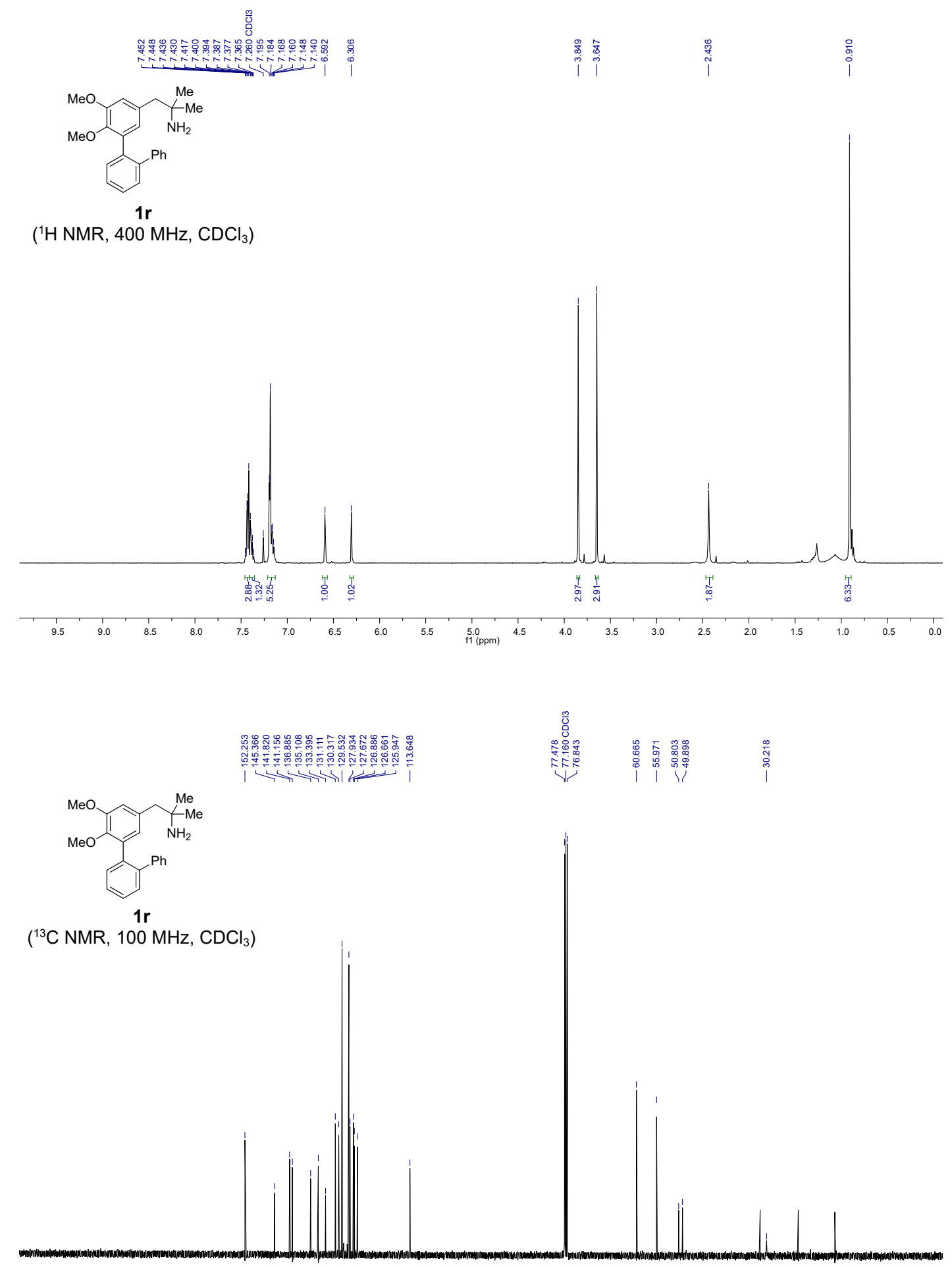


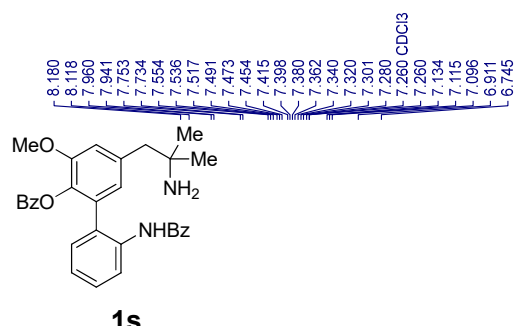

$\left({ }^{1} \mathrm{H} \mathrm{NMR}, 400 \mathrm{MHz}, \mathrm{CDCl}_{3}\right)$
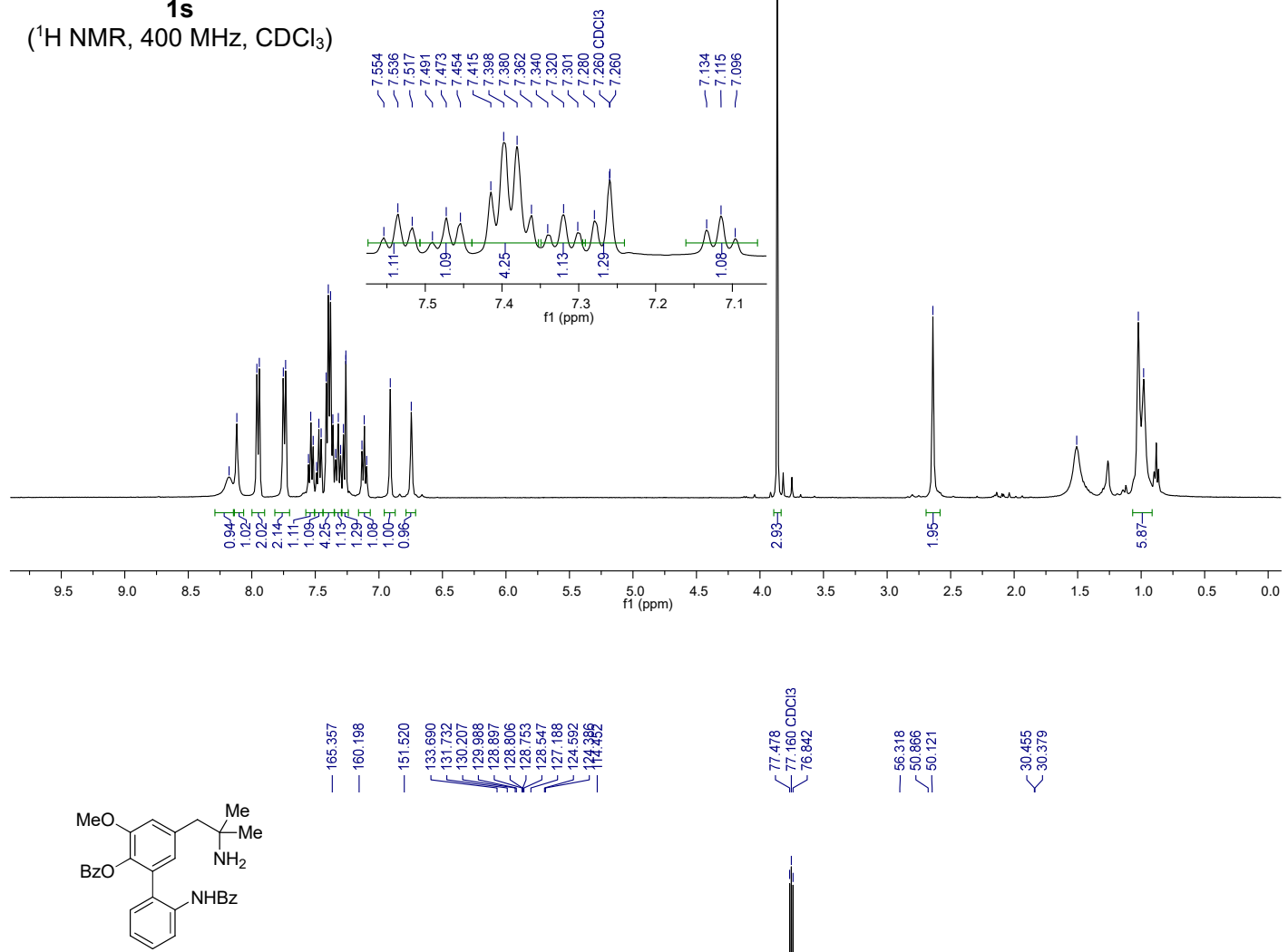

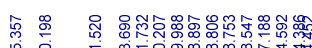

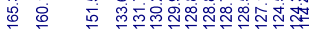

i i

$1 \mathrm{~s}$

$\left({ }^{13} \mathrm{C}\right.$ NMR, $\left.100 \mathrm{MHz}, \mathrm{CDCl}_{3}\right)$

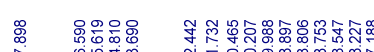

管

तार 1141 पा।

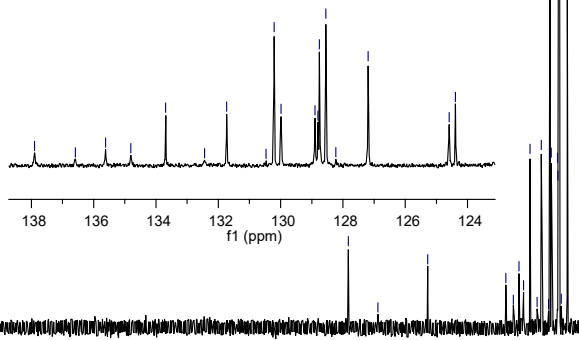

$220 \quad 210 \quad 200 \quad 190 \quad 190^{\prime}, r^{\prime} \mid$

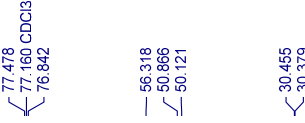




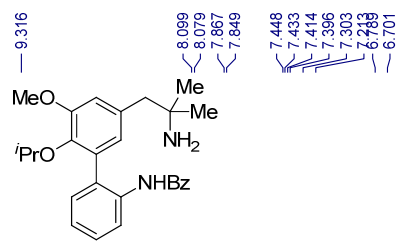

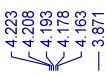

广

$1 \mathrm{t}$

('H NMR, $400 \mathrm{MHz}, \mathrm{CDCl}_{3}$ )
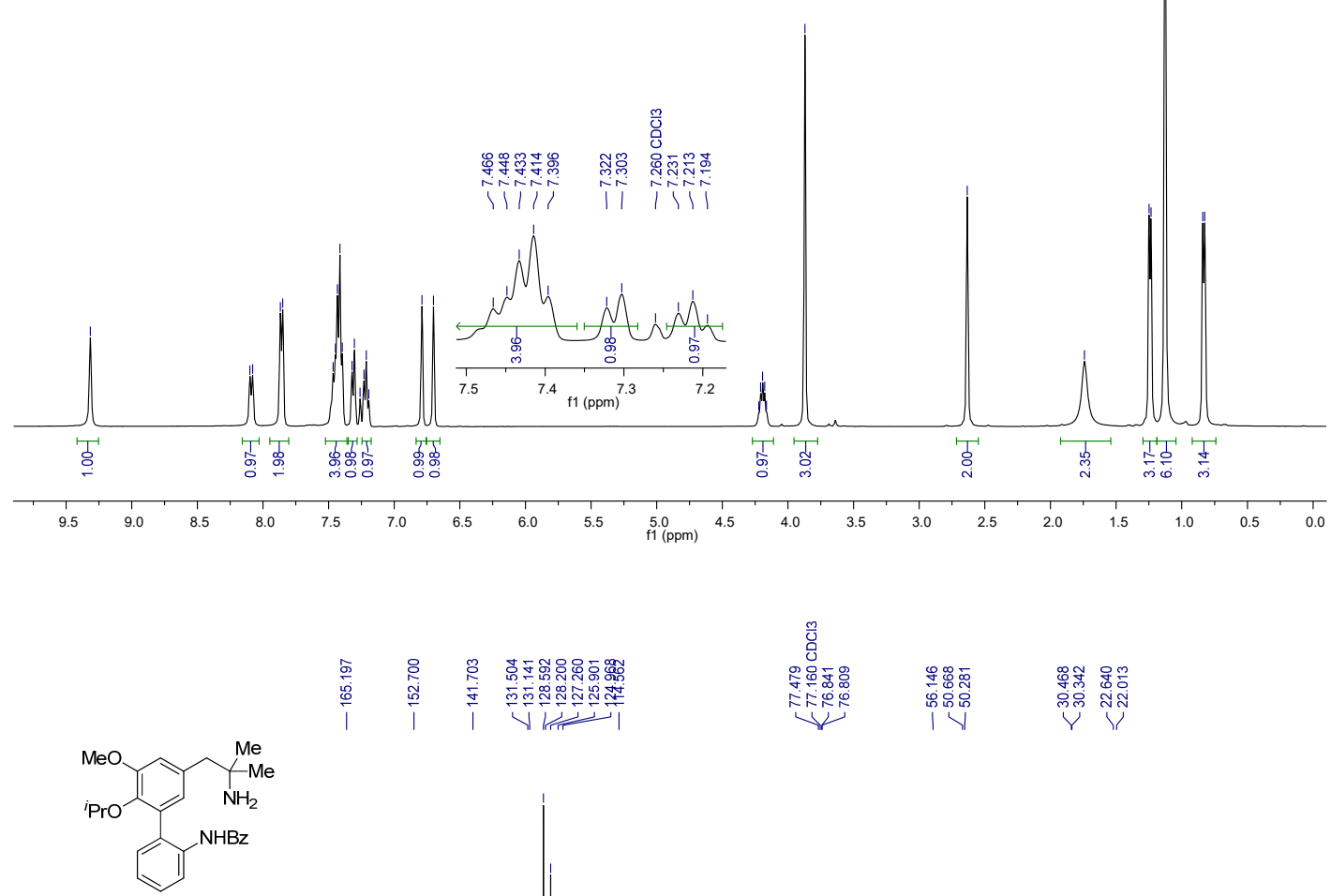

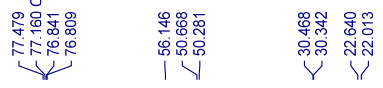

$1 \mathrm{t}$

$\left({ }^{13} \mathrm{C}\right.$ NMR, $\left.100 \mathrm{MHz}, \mathrm{CDCl}_{3}\right)$

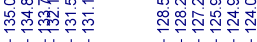

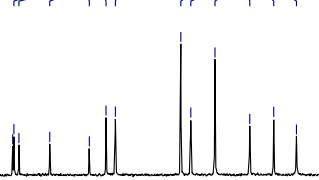

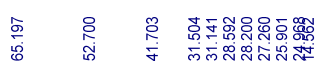

I) । 

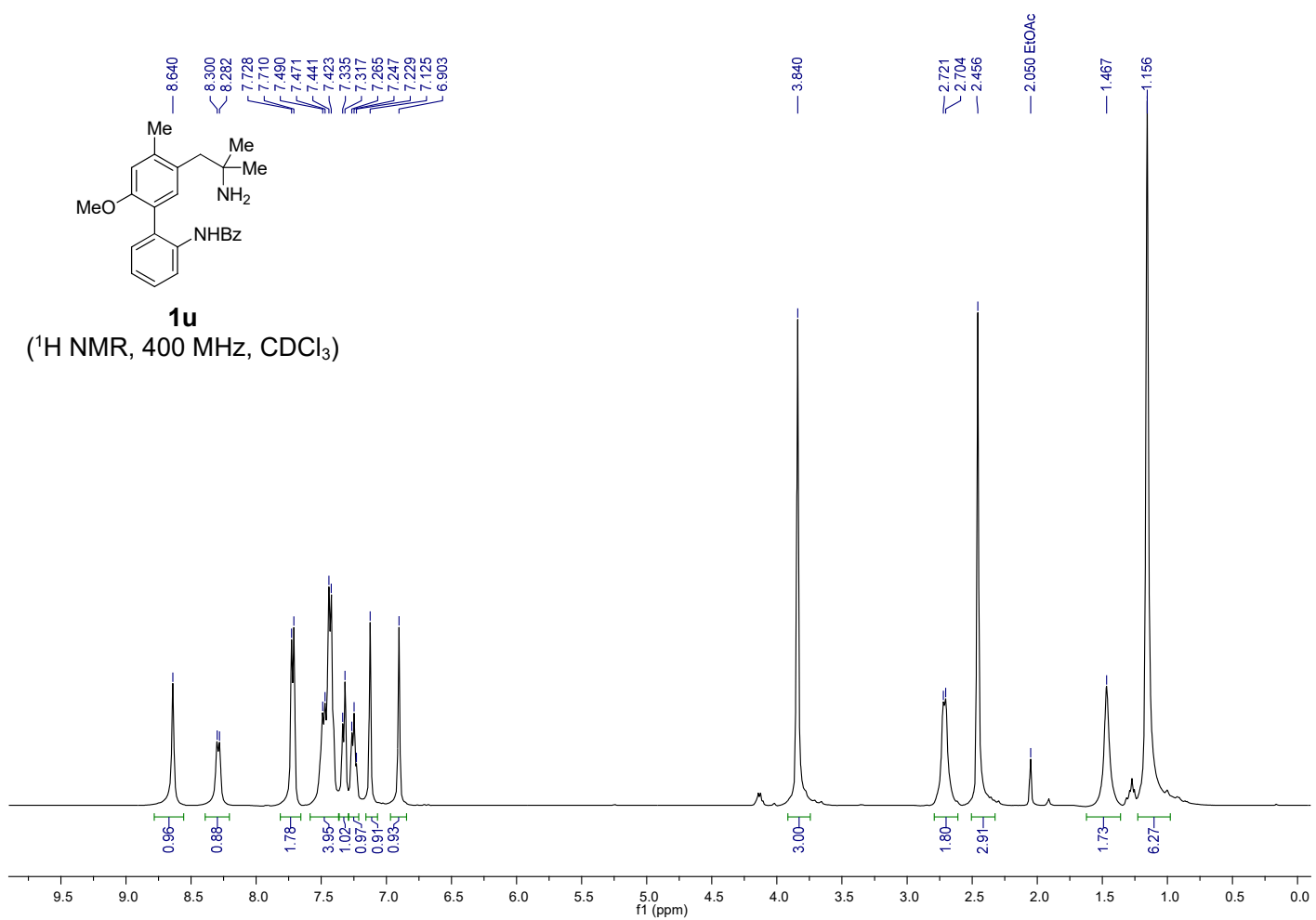

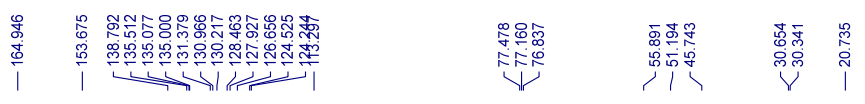

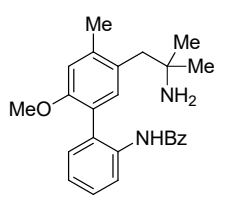

$1 \mathrm{u}$

$\left({ }^{13} \mathrm{C} \mathrm{NMR}, 100 \mathrm{MHz}, \mathrm{CDCl}_{3}\right)$

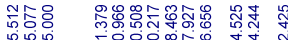

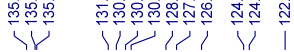

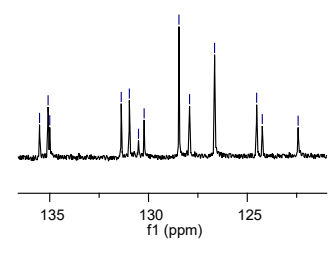

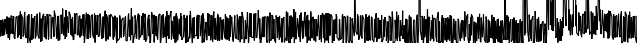

$\begin{array}{lllllllllllll}220 & 210 & 200 & 190 & 180 & 170 & 160 & 150 & 140 & 130 & 120 & 110 & 100 \\ \mathrm{f} 1(\mathrm{ppm})\end{array}$ 

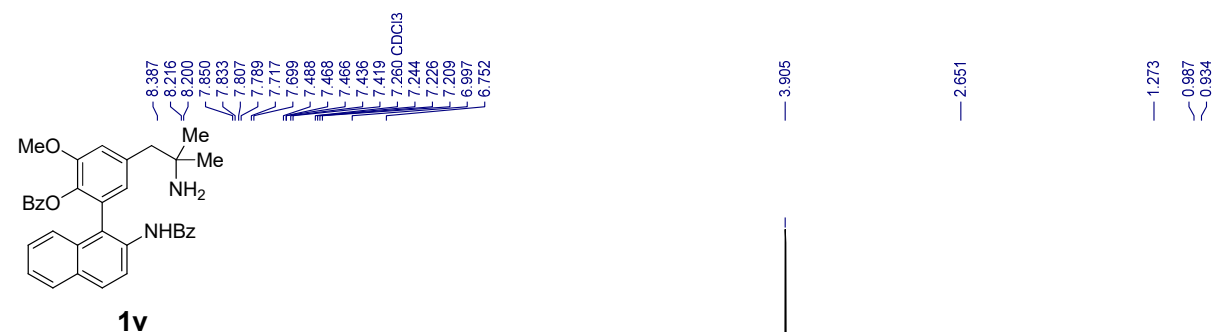

( ${ }^{1} \mathrm{H}$ NMR, $400 \mathrm{MHz}, \mathrm{CDCl}_{3}$ )
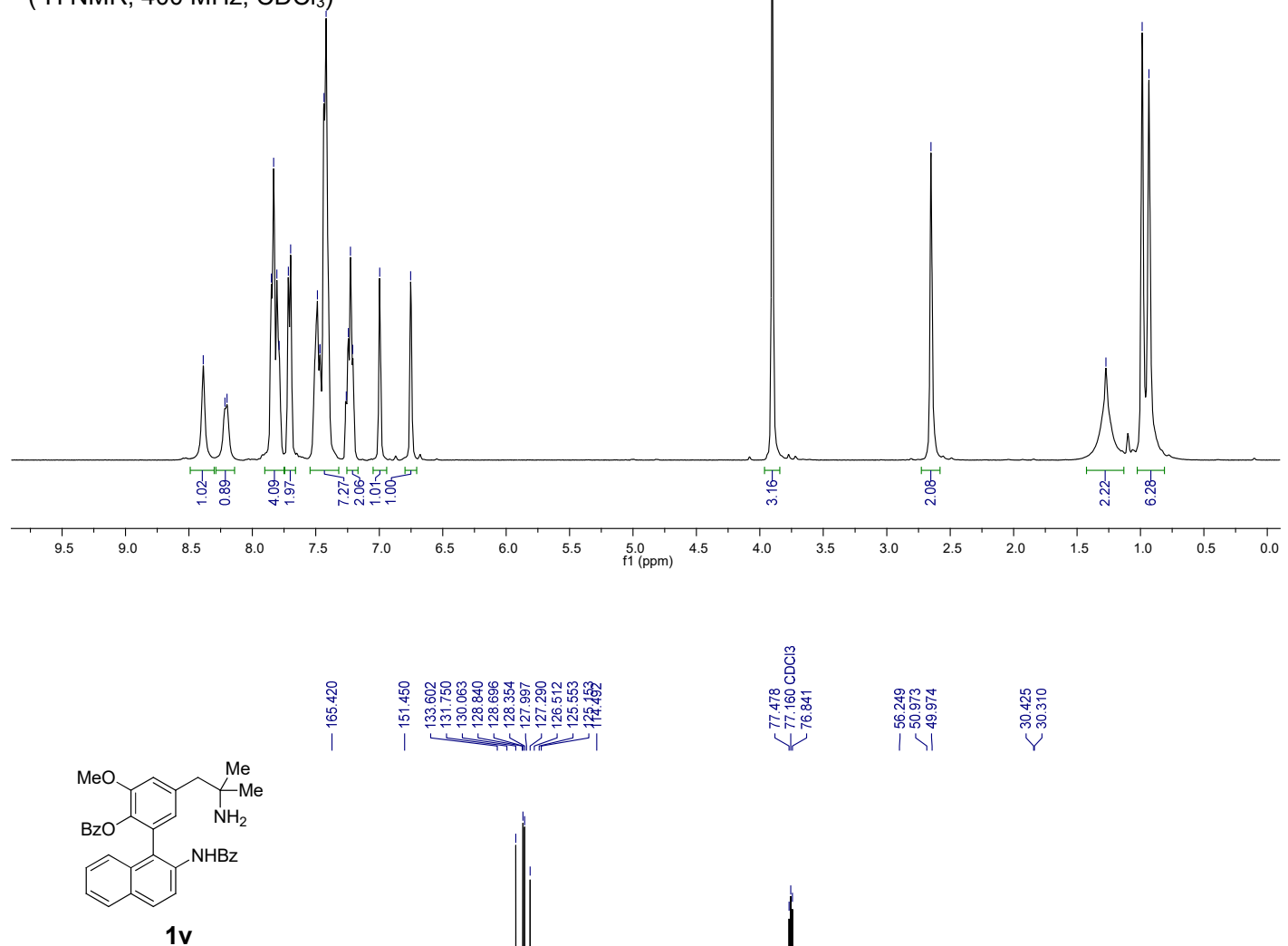

$\left({ }^{13} \mathrm{C} \mathrm{NMR}, 100 \mathrm{MHz}, \mathrm{CDCl}_{3}\right)$

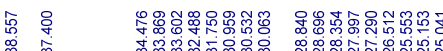

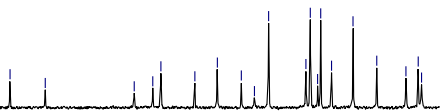

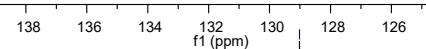

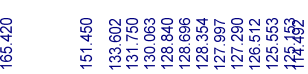

品 لvi 年
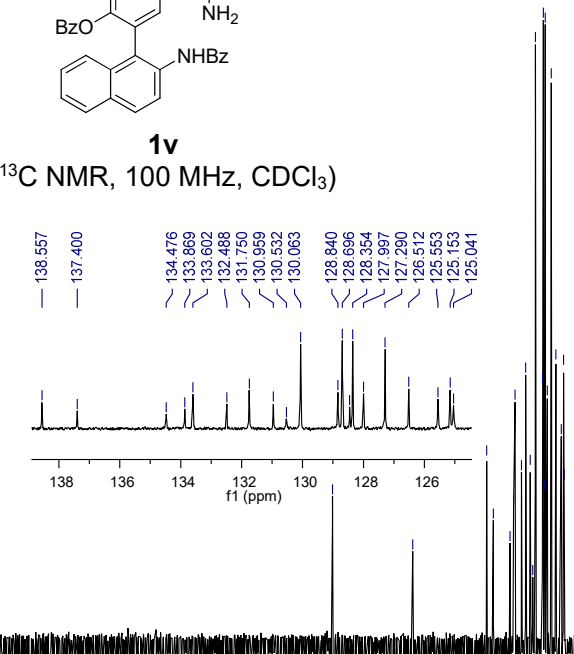

$\begin{array}{lllllll}220 & 210 & 200 & 190 & 180 & 170\end{array}$ $\underset{\mathrm{f} 1(\mathrm{ppm})}{100}$ 


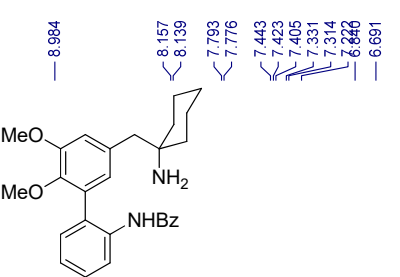

$1 w$

$\left({ }^{1} \mathrm{H}\right.$ NMR, $400 \mathrm{MHz}, \mathrm{CDCl}_{3}$ )
।

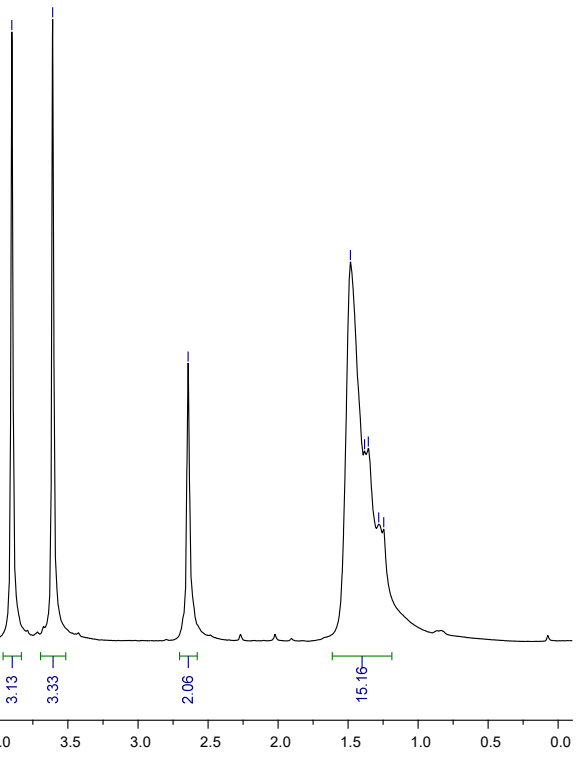

प.

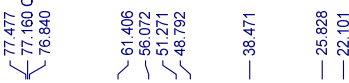

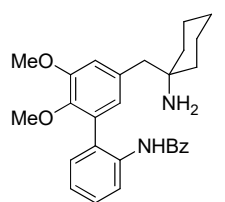

$1 w$

$\left({ }^{13} \mathrm{C}\right.$ NMR, $\left.100 \mathrm{MHz}, \mathrm{CDCl}_{3}\right)$

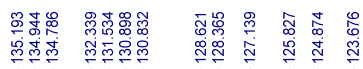

iा एर । । ।

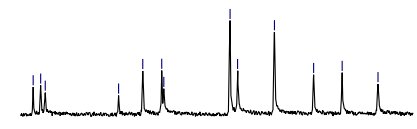

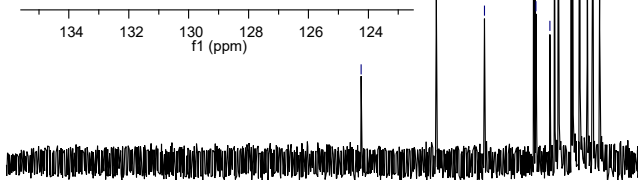

$\begin{array}{lllllllllllll}220 & 210 & 200 & 190 & 180 & 170 & 160 & 150 & 140 & 130 & 120 & 110 & 100 \\ \mathrm{f1}(\mathrm{ppm})\end{array}$ 


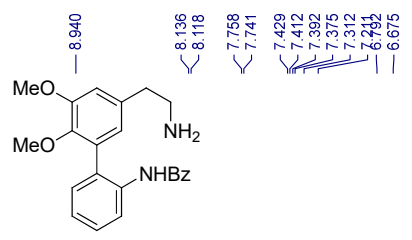

$1 \mathrm{x}$

$\left({ }^{1} \mathrm{H} \mathrm{NMR}, 400 \mathrm{MHz}, \mathrm{CDCl}_{3}\right)$

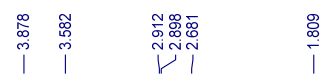

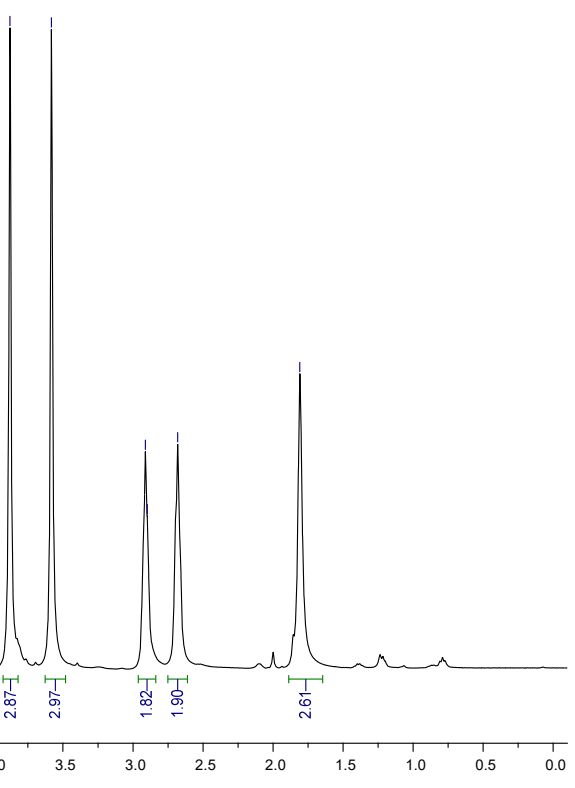

\section{0}

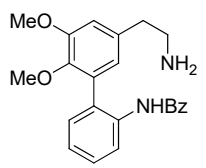

$1 \mathrm{x}$

$\left({ }^{13} \mathrm{C}\right.$ NMR, $\left.100 \mathrm{MHz}, \mathrm{CDCl}_{3}\right)$

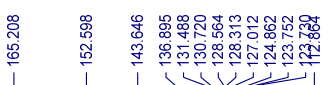

誉

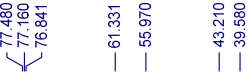

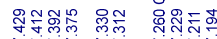

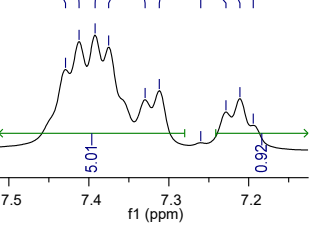

尊
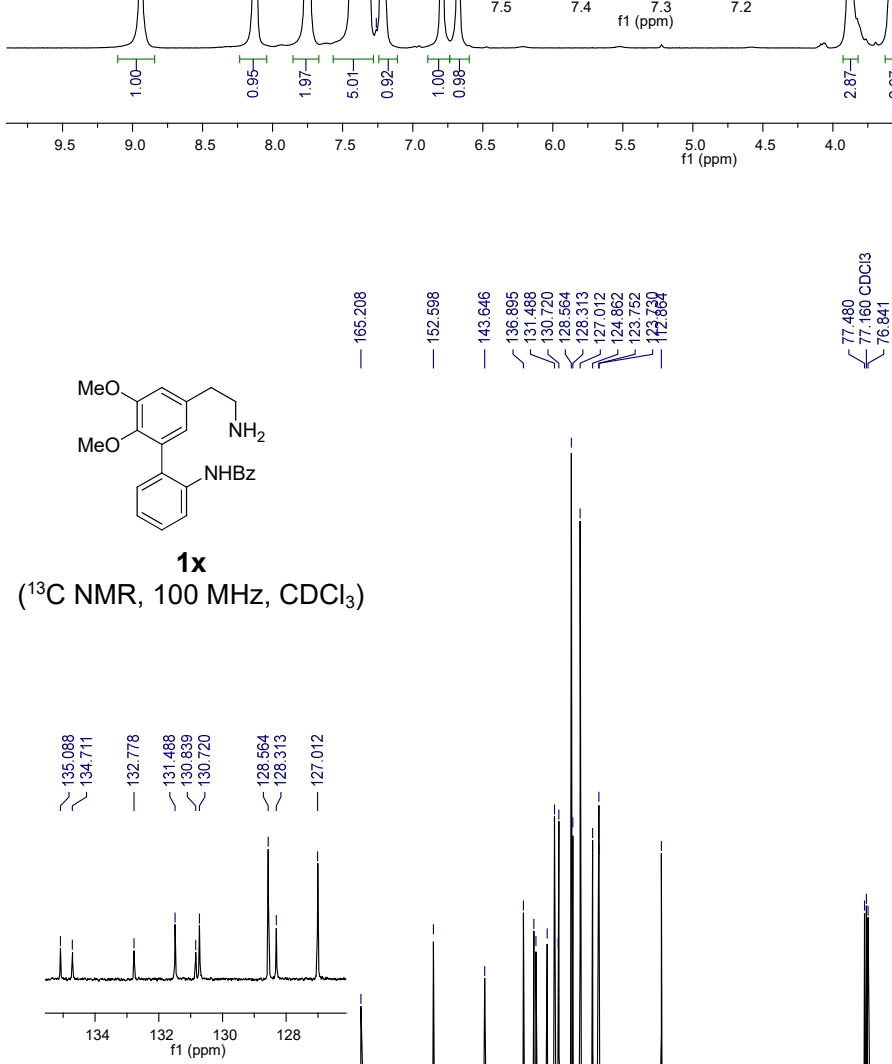

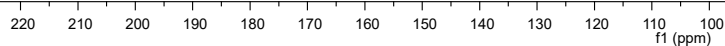




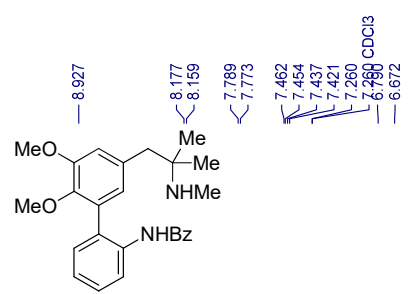

$1 y$

告

( ${ }^{1} \mathrm{H} \mathrm{NMR}, 400 \mathrm{MHz}, \mathrm{CDCl}_{3}$ )
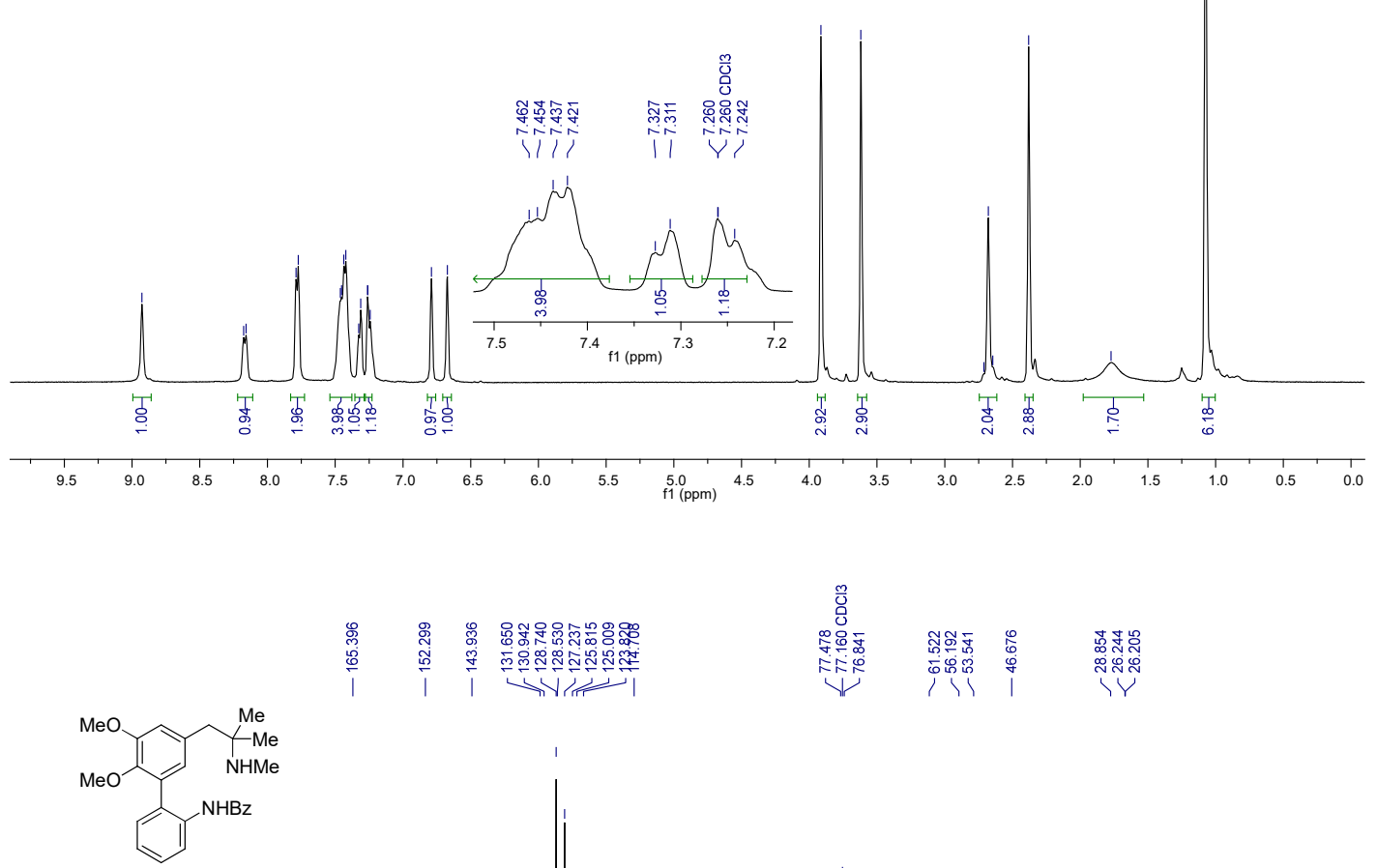

$1 y$

$\left({ }^{13} \mathrm{C} \mathrm{NMR}, 100 \mathrm{MHz}, \mathrm{CDCl}_{3}\right)$

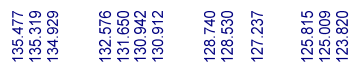

यi iर

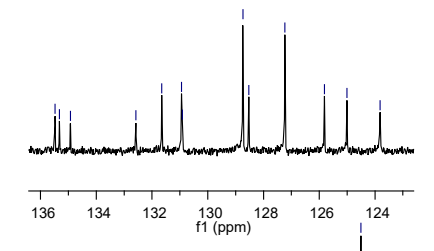

\%

|

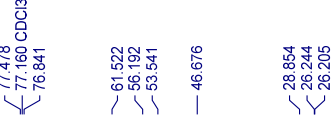

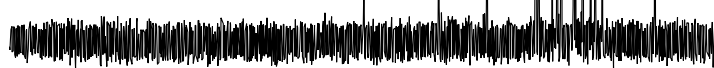

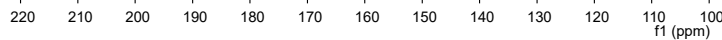




\section{Reaction Optimizations and Procedures}

\subsection{Catalyst Screening}

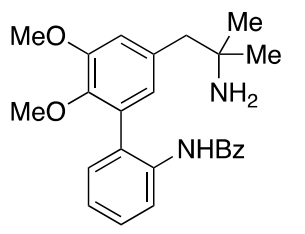

1a

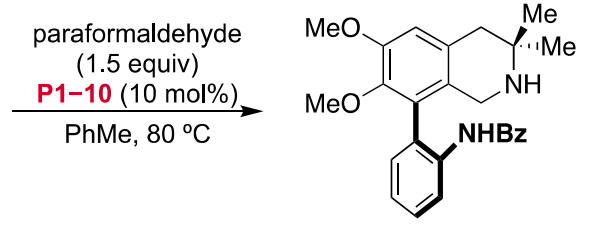

2a

To an oven dried $4 \mathrm{~mL}$ vial equipped with a magnetic stir bar was added substrate 1a (20.2 mg, $0.05 \mathrm{mmol}, 1$ equiv), paraformaldehyde ( $2.3 \mathrm{mg}, 0.075 \mathrm{mmol}, 1.5$ equiv) and P1-10 (0.005 mmol, 0.1 equiv). After dilution with toluene $(0.5 \mathrm{~mL}, 0.1 \mathrm{M})$, the vial was sealed with a Teflon cap and further secured with Parafilm $\mathrm{M}^{\circledR}$. The reaction mixture was left to stir at $80^{\circ} \mathrm{C}$, and then the solvent was removed in vacuo. The crude material was purified by flash column chromatography using an eluent of $10 \%$ $\mathrm{MeOH} /$ EtOAc with $1 \%$ aqueous $\mathrm{NH}_{3}$ as an additive. The enantioselectivity was determined by chiral HPLC.

Table S1.

\begin{tabular}{|c|c|c|c|c|}
\hline & & $\begin{array}{l}\text { P1: } \mathrm{R}^{\prime}=2,4,6-{ }^{i} \mathrm{Pr}_{3} \mathrm{C}_{6} \mathrm{H}_{2} \\
\text { P2: } \mathrm{R}^{\prime}=2,4,6-\mathrm{Cy}_{3} \mathrm{C}_{6} \mathrm{H}_{2} \\
\text { P3: R' }=\text { 9-anthracenyl } \\
\text { P4: R' = 9-phenanthryl }\end{array}$ & \multicolumn{2}{|c|}{$\begin{array}{l}\text { P5: } \mathrm{R}^{\prime}=4-{ }^{-} \mathrm{BuC}_{6} \mathrm{H}_{4} \\
\text { P6: } \mathrm{R}^{\prime}=4-\mathrm{OMeC}_{6} \mathrm{H}_{4} \\
\text { P7: } \mathrm{R}^{\prime}=4-\mathrm{CF}_{3} \mathrm{C}_{6} \mathrm{H}_{4} \\
\text { P8: } \mathrm{R}^{\prime}=3,5-\mathrm{Ph}_{2} \mathrm{C}_{6} \mathrm{H}_{3} \\
\text { P9: } \mathrm{R}^{\prime}=3,5-\left(\mathrm{CF}_{3}\right)_{2} \mathrm{C}_{6} \mathrm{H}_{3}\end{array}$} \\
\hline Entry & Catalyst & Time (h) & Yield $^{a}(\%)$ & e.e. ${ }^{b}(\%)$ \\
\hline 1 & P1 & 9 & 50 & 96 \\
\hline 2 & $\mathbf{P 2}$ & 9 & 50 & 95 \\
\hline 3 & $\mathbf{P 3}$ & 12 & 14 & 70 \\
\hline 4 & P4 & 11 & $<15$ & n.d. ${ }^{c}$ \\
\hline 5 & P5 & 12 & 18 & $31^{d}$ \\
\hline 6 & P6 & 12 & $<15$ & n.d. ${ }^{c}$ \\
\hline 7 & P7 & 12 & 15 & $54^{d}$ \\
\hline 8 & P8 & 12 & 24 & $24^{d}$ \\
\hline 9 & P9 & 12 & 26 & $14^{d}$ \\
\hline
\end{tabular}

${ }^{a}$ Isolated yields. ${ }^{b}$ Enantiomeric excesses were determined by chiral HPLC analysis. ${ }^{c}$ Not determined. ${ }^{d}$ The opposite enantiomer was obtained as a major isomer. 


\subsection{Temperature Screening}

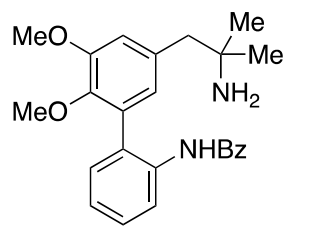

1a

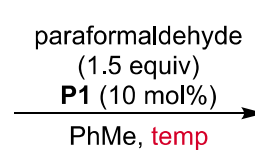

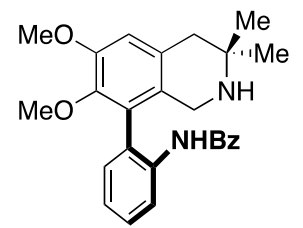

$2 a$

To an oven dried $4 \mathrm{~mL}$ vial equipped with a magnetic stir bar was added substrate 1a (20.2 mg, $0.05 \mathrm{mmol}, 1$ equiv), paraformaldehyde ( $2.3 \mathrm{mg}, 0.075 \mathrm{mmol}, 1.5$ equiv) and P1 (0.005 mmol, 0.1 equiv). After dilution with PhMe $(0.5 \mathrm{~mL}, 0.1 \mathrm{M})$, the vial was sealed with a Teflon cap and further secured with Parafilm $\mathrm{M}^{\circledR}$. The reaction mixture was heated to stir, and then the solvent was removed in vacuo. The crude material was purified by flash column chromatography using an eluent of $10 \% \mathrm{MeOH} / \mathrm{EtOAc}$ with $1 \%$ aqueous $\mathrm{NH}_{3}$ as an additive. The enantioselectivity was determined by chiral HPLC.

Table S2.

\begin{tabular}{ccccc}
\hline Entry & Temp $\left({ }^{\circ} \mathrm{C}\right)$ & Time & Yield $^{a}(\%)$ & $e e^{b}(\%)$ \\
\hline 1 & 100 & $6 \mathrm{~h}$ & 56 & 94 \\
2 & 80 & $12 \mathrm{~h}$ & 69 & 95 \\
3 & 60 & $3 \mathrm{~d}$ & 55 & 97 \\
4 & 40 & $3 \mathrm{~d}$ & 24 & 99
\end{tabular}

${ }^{a}$ Isolated yields. ${ }^{b}$ Enantiomeric excesses were determined by chiral HPLC analysis. 


\subsection{Solvent Screening}

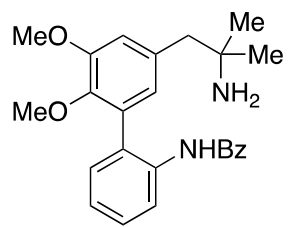

$1 \mathrm{a}$

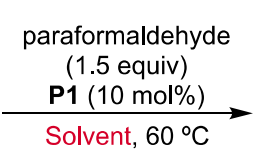

Solvent, $60^{\circ} \mathrm{C}$

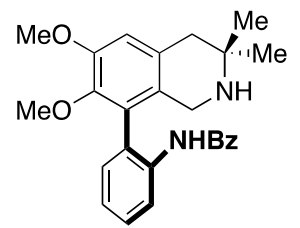

$2 \mathrm{a}$

To an oven dried $4 \mathrm{~mL}$ vial equipped with a magnetic stir bar was added substrate (20.2 $\mathrm{mg}, 0.05 \mathrm{mmol}, 1$ equiv), paraformaldehyde $(2.3 \mathrm{mg}, 0.075 \mathrm{mmol}, 1.5$ equiv) and $\mathbf{P 1}$ ( $0.005 \mathrm{mmol}, 0.1$ equiv). After dilution with solvent $(0.5 \mathrm{~mL}, 0.1 \mathrm{M})$, the vial was sealed with a Teflon cap and further secured with Parafilm $\mathrm{M}^{\circledR}$. The reaction mixture was left to stir for three days at $60{ }^{\circ} \mathrm{C}$, and then the solvent was removed in vacuo. The crude material was purified by flash column chromatography using an eluent of $10 \%$ $\mathrm{MeOH} /$ EtOAc with $1 \%$ aqueous $\mathrm{NH}_{3}$ as an additive. The enantioselectivity was determined by chiral HPLC.

Table S3.

\begin{tabular}{cccc}
\hline Entry & Solvent & Yield $^{a}(\%)$ & e.e. $^{b}(\%)$ \\
\hline 1 & PhMe & 41 & 96 \\
2 & THF & 49 & 91 \\
3 & 1,2-dichloroethane & 27 & 85 \\
4 & $\mathrm{CHCl}_{3}$ & 44 & 95 \\
5 & 1,4-dioxane & 37 & 96 \\
6 & cyclohexane & 18 & 98 \\
${ }^{a}$ Isolated yields. ${ }^{b}$ Enantiomeric excesses were determined by chiral HPLC analysis
\end{tabular}




\subsection{Equivalent Screening}<smiles>COc1cc(CC(C)(C)N)cc(-c2ccccc2NC(=O)c2ccccc2)c1OC</smiles>

1a

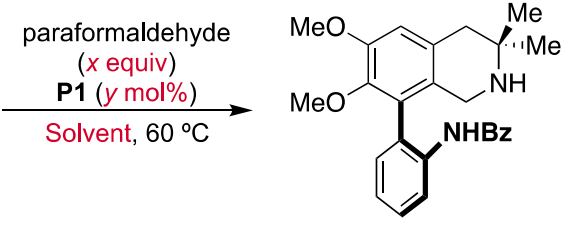

2a

To an oven dried $4 \mathrm{~mL}$ vial equipped with a magnetic stir bar was added substrate 1a (20.2 mg, $0.05 \mathrm{mmol}, 1$ equiv), paraformaldehyde ( $x$ equiv) and $\mathbf{P 1}$ ( $y$ mol\%). After dilution with solvent $(0.5 \mathrm{~mL}, 0.1 \mathrm{M})$, the vial was sealed with a Teflon cap and further secured with Parafilm $\mathrm{M}^{\circledR}$. The reaction mixture was left to stir for three days at $60{ }^{\circ} \mathrm{C}$, and then the solvent was removed in vacuo. The crude material was purified by flash column chromatography using an eluent of $10 \% \mathrm{MeOH} / \mathrm{EtOAc}$ with $1 \%$ aqueous $\mathrm{NH}_{3}$ as an additive. The enantioselectivity was determined by chiral HPLC.

Table S4.

\begin{tabular}{ccccccc}
\hline Entry & $\begin{array}{c}\text { Paraformaldehyde } \\
(x \text { equiv })\end{array}$ & $\begin{array}{c}\mathbf{P 1} \\
(y \text { mol\%) }\end{array}$ & Solvent & $\begin{array}{c}\text { Temp } \\
\left({ }^{\circ} \mathrm{C}\right)\end{array}$ & $\begin{array}{c}\text { Yield }^{a} \\
(\%)\end{array}$ & $\begin{array}{c}\text { e.e. }^{b} \\
(\%)\end{array}$ \\
\hline 1 & 1.5 & 10 & PhMe & 60 & 41 & 96 \\
2 & 1.5 & 10 & THF & 60 & 49 & 91 \\
3 & 1.5 & 10 & $\mathrm{CHCl}_{3}$ & 60 & 44 & 95 \\
4 & 1.5 & 10 & $1,4-$ dioxane & 60 & 37 & 96 \\
5 & 1.5 & 20 & PhMe & 60 & 67 & 96 \\
6 & 1.5 & 20 & $\mathrm{THF}$ & 60 & 64 & 91 \\
7 & 1.5 & 20 & $\mathrm{CHCl}$ & 60 & 58 & 95 \\
8 & 1.5 & 20 & $1,4-$ dioxane & 60 & 54 & 95 \\
9 & 1 & 20 & PhMe & 60 & 38 & 91 \\
10 & 2 & 20 & PhMe & 60 & 38 & 96 \\
11 & 3 & 20 & PhMe & 60 & 31 & 96 \\
\hline
\end{tabular}

${ }^{a}$ Isolated yields. ${ }^{b}$ Enantiomeric excesses were determined by chiral HPLC analysis. 


\subsection{Additive screening}

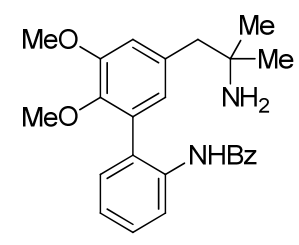

$1 \mathrm{a}$

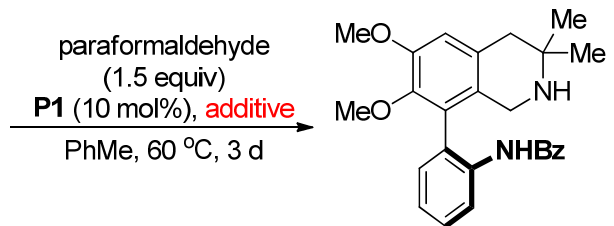

$2 \mathbf{a}$

To an oven dried $4 \mathrm{~mL}$ vial equipped with a magnetic stir bar was added substrate $(20.2$ $\mathrm{mg}, 0.05 \mathrm{mmol}, 1$ equiv), paraformaldehyde ( $2.3 \mathrm{mg}, 0.075 \mathrm{mmol}, 1.5$ equiv), P1 (10 $\mathrm{mol} \%$ ) and additive $(100 \mathrm{mg})$. After dilution with $\mathrm{PhMe}(0.5 \mathrm{~mL}, 0.1 \mathrm{M})$, the vial was sealed with a Teflon cap and further secured with Parafilm $\mathrm{M}^{\circledR}$. The reaction mixture was left to stir for three days at $60{ }^{\circ} \mathrm{C}$, and then the solvent was removed in vacuo. The crude material was purified by flash column chromatography using an eluent of $10 \%$ $\mathrm{MeOH} / \mathrm{EtOAc}$ with $1 \%$ aqueous $\mathrm{NH}_{3}$ as an additive. The enantioselectivity was determined by chiral HPLC.

Table S5

\begin{tabular}{cccc}
\hline Entry & Additive & Yield $^{a}(\%)$ & e.e. $^{b}(\%)$ \\
\hline 1 & $3 \AA$ (rod) & 18 & 97 \\
2 & $4 \AA$ (rod) & 27 & 96 \\
3 & $4 \AA$ (bead) & 27 & 95 \\
4 & $5 \AA$ (rod) & 44 & 96 \\
5 & $\mathrm{Na}_{2} \mathrm{SO}_{4}$ & 33 & 95 \\
6 & $\mathrm{MgSO}_{4}$ & 17 & 96 \\
\hline
\end{tabular}

${ }^{a}$ Isolated yields. ${ }^{b}$ Enantiomeric excesses were determined by chiral HPLC analysis. 


\subsection{Concentration screening}

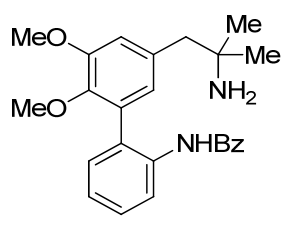

$1 \mathrm{a}$

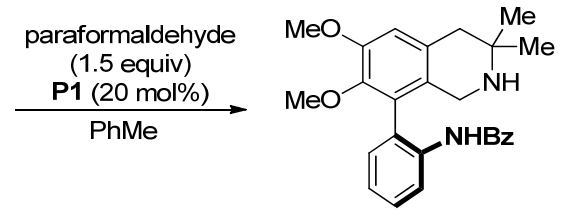

$2 a$

To an oven dried vial equipped with a magnetic stir bar was added substrate 1a $(20.2$ $\mathrm{mg}, 0.05 \mathrm{mmol}, 1$ equiv), paraformaldehyde ( $2.3 \mathrm{mg}, 0.075 \mathrm{mmol}, 1.5$ equiv) and $\mathbf{P 1}$ ( $0.01 \mathrm{mmol}, 0.2$ equiv). After dilution with $\mathrm{PhMe}$, the vial was sealed with a Teflon cap and further secured with Parafilm $\mathrm{M}^{\circledR}$. The reaction mixture was heated, and then the solvent was removed in vacuo. The crude material was purified by flash column chromatography using an eluent of $10 \% \mathrm{MeOH} /$ EtOAc with $1 \%$ aqueous $\mathrm{NH}_{3}$ as an additive. The enantioselectivity was determined by chiral HPLC.

Table S6

\begin{tabular}{cccccc}
\hline Entry & ${\text { Temp }\left({ }^{\circ} \mathrm{C}\right)} \mathrm{M}(\mathrm{mol} / \mathrm{L})$ & Time & Yield $^{a}(\%)$ & e.e. $^{b}(\%)$ \\
\hline 1 & 60 & 0.1 & $3 \mathrm{~d}$ & 67 & 96 \\
2 & 60 & 0.033 & $60 \mathrm{~h}$ & 77 & 96 \\
3 & 60 & 0.01 & $24 \mathrm{~h}$ & 83 & 96 \\
4 & 80 & 0.1 & $8 \mathrm{~h}$ & 56 & 96 \\
5 & 80 & 0.033 & $5 \mathrm{~h}$ & 70 & 95 \\
6 & 80 & 0.01 & $4.5 \mathrm{~h}$ & 79 & 94 \\
\hline
\end{tabular}

${ }^{a}$ Isolated yields. ${ }^{b}$ Enantiomeric excesses were determined by chiral HPLC analysis. 


\section{Reaction Procedure for 2}<smiles>[R][R]1ccccc1-c1c[R1]([H])cc(CC([R])(C)N)c1</smiles>

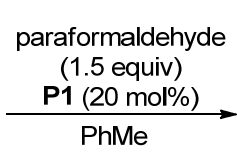

$$
\mathrm{R}^{3} \frac{r_{1}}{1}
$$

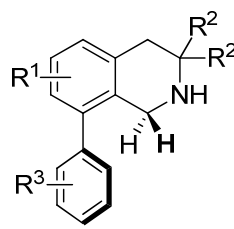

2

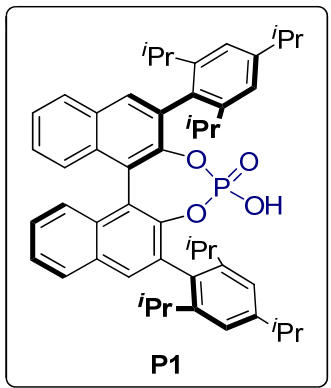

Procedure 19: To an oven dried $20 \mathrm{~mL}$ vial equipped with a magnetic stir bar was added substrate 1 ( $0.05 \mathrm{mmol}, 1$ equiv), paraformaldehyde ( $2.3 \mathrm{mg}, 0.075 \mathrm{mmol}, 1.5$ equiv) and $\mathbf{P 1}(0.01 \mathrm{mmol}, 0.2$ equiv). After dilution with PhMe $(5 \mathrm{~mL}, 0.01 \mathrm{M})$, the vial was sealed with a Teflon cap and further secured with Parafilm $\mathrm{M}^{\circledR}$. The reaction mixture was heated and stirred at $60{ }^{\circ} \mathrm{C}$ or $80{ }^{\circ} \mathrm{C}$ in an oil bath until complete consumption of 1 . Then the solvent was removed in vacuo. The crude material was purified by flash column chromatography using an eluent of $10 \% \mathrm{MeOH} / \mathrm{EtOAc}$ with $1 \%$ aqueous $\mathrm{NH}_{3}$ as an additive. The enantioselectivity was determined by chiral HPLC.

Procedure 19-1 (1.0 mmol scale): To an oven dried round-bottom flask equipped with a magnetic stirring bar was added substrate 1a (404.5 mg, $1.0 \mathrm{mmol}, 1$ equiv), paraformaldehyde (45.0 mg, $1.5 \mathrm{mmol}, 1.5$ equiv) and $\mathbf{P 1}$ (150.6 mg, $0.2 \mathrm{mmol}, 0.2$ equiv). After dilution with PhMe (100 mL, $0.01 \mathrm{M})$, the reflux condenser was connected to flask and sealed with teflon tape and Parafilm $\mathrm{M}^{\circledR}$. The reaction mixture was heated and stirred at $60{ }^{\circ} \mathrm{C}$ in an oil bath until complete consumption of 1a. Then the solvent was removed in vacuo. The crude material was purified by flash column chromatography using an eluent of $10 \% \mathrm{MeOH} / \mathrm{EtOAc}$ with $1 \%$ aqueous $\mathrm{NH}_{3}$ as an additive. The enantioselectivity was determined by chiral HPLC.

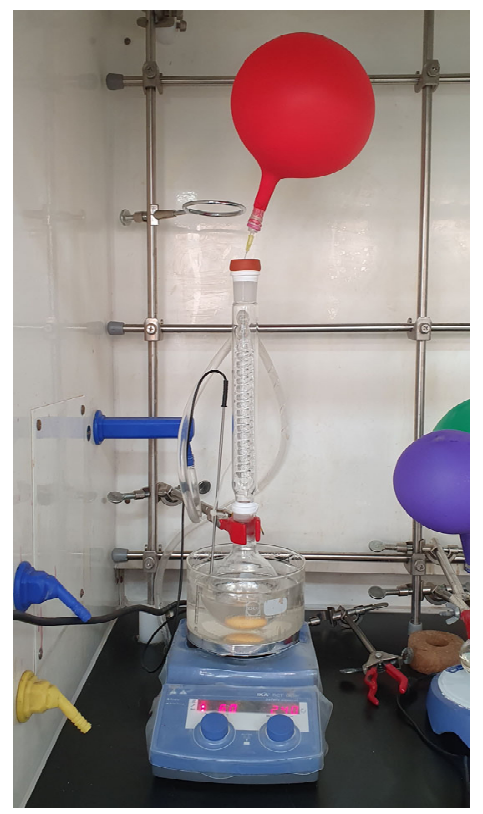




\section{Characterization and Spectra of Products 2}

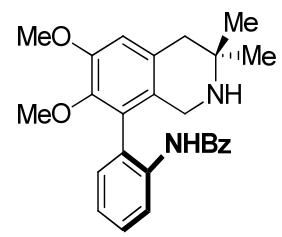

$\mathrm{N}$-(2-(6,7-Dimethoxy-3,3-dimethyl-1,2,3,4-tetrahydroisoquinolin-8-

yl)phenyl)benzamide (2a) was synthesized by following Procedure 19 from 1a. The crude material was purified by normal-phase column chromatography using an eluent of $10 \% \mathrm{MeOH} / \mathrm{EtOAc}$ with $0.5 \%$ aqueous $\mathrm{NH}_{3}$ to give $\mathbf{2 a}$ as a white solid (17.3 $\mathrm{mg}$, $83 \%$ yield, $60{ }^{\circ} \mathrm{C} ; 362 \mathrm{mg}, 86 \%$ yield, $\left.60^{\circ} \mathrm{C}, 1 \mathrm{mmol} \mathrm{scale}\right)$.

m.p. $110^{\circ} \mathrm{C}$

${ }^{1} \mathbf{H}$ NMR $\left(400 \mathrm{MHz}, \mathrm{CDCl}_{3}\right) \delta 8.40(\mathrm{~d}, J=8.2 \mathrm{~Hz}, 1 \mathrm{H}), 7.94(\mathrm{~s}, 1 \mathrm{H}), 7.57(\mathrm{~d}, J=7.9$ $\mathrm{Hz}, 2 \mathrm{H}), 7.44(\mathrm{t}, J=7.3 \mathrm{~Hz}, 2 \mathrm{H}), 7.34(\mathrm{t}, J=7.6 \mathrm{~Hz}, 2 \mathrm{H}), 7.24-7.14(\mathrm{~m}, 2 \mathrm{H}), 6.70(\mathrm{~s}$, $1 \mathrm{H}), 3.89(\mathrm{~s}, 3 \mathrm{H}), 3.72(\mathrm{~d}, J=16.9 \mathrm{~Hz}, 1 \mathrm{H}), 3.54(\mathrm{~d}, J=0.9 \mathrm{~Hz}, 3 \mathrm{H}), 3.35(\mathrm{~d}, J=17.0$ $\mathrm{Hz}, 1 \mathrm{H}), 2.62$ (s, 2H), 1.79 (brs, 1H) $1.16(\mathrm{~s}, 3 \mathrm{H}), 1.06(\mathrm{~s}, 3 \mathrm{H})$.

${ }^{13}$ C NMR $\left(100 \mathrm{MHz}, \mathrm{CDCl}_{3}\right) \delta 165.2,151.3,144.3,135.6,134.9,131.8,131.7,130.1$, 129.3, 128.8, 128.7 (2C), 127.1, 127.0 (2C), 125.7, 124.3, 122.0, 113.6, 61.1, 56.0, 48.3, 42.8, 42.0, 28.2, 26.9.

IR (FT-ATR, $\mathrm{cm}^{-1}, \mathrm{CH}_{2} \mathrm{Cl}_{2}$ ) $v_{\max } 3350,2940,2594,2188,2031,1674,1580,1523$, $1485,1469,1445,1423,1379,1306,1254,1230,1202,1179,1136,1050,1036,1002$, 909, 833, 799, 758, 720, 710, 692, 674, 587, 519, 454, 416.

HRMS (ESI) $m / z$ : $[\mathrm{M}+\mathrm{H}]^{+}$Calcd for $\mathrm{C}_{26} \mathrm{H}_{28} \mathrm{~N}_{2} \mathrm{O}_{3} 417.2178$; found 417.2176.

Optical: $[a]^{20} \mathrm{D}=+7.0^{\circ}\left(c=0.83, \mathrm{CHCl}_{3}, 96 \%\right.$ e.e. $)$

HPLC (Chiralpak AD, ${ }^{i} \mathrm{PrOH} / n$-hexane $=10 / 90$ with $0.1 \% \mathrm{Et}_{2} \mathrm{NH}$, flow rate $=1.0$ $\mathrm{mL} / \mathrm{min}, \lambda=254 \mathrm{~nm}$ ) $t_{\mathrm{R}}=58.3 \min$ (major), $31.4 \min$ (minor)

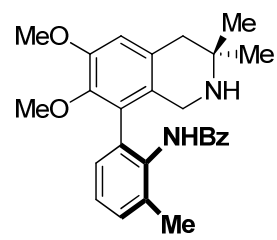

$N$-(2-(6,7-Dimethoxy-3,3-dimethyl-1,2,3,4-tetrahydroisoquinolin-8-yl)-6-

methylphenyl)benzamide (2b) was synthesized by following Procedure 19 from $\mathbf{1 b}$. The crude material was purified by normal-phase column chromatography using an eluent of $10 \% \mathrm{MeOH} / \mathrm{EtOAc}$ with $0.5 \%$ aqueous $\mathrm{NH}_{3}$ to give $\mathbf{2 b}$ as a white solid (17.9 mg, $83 \%$ yield, $60{ }^{\circ} \mathrm{C}$ ).

m.p. $183^{\circ} \mathrm{C}$

${ }^{1} \mathbf{H}$ NMR $\left(400 \mathrm{MHz}, \mathrm{CDCl}_{3}\right) \delta 7.90(\mathrm{~s}, 1 \mathrm{H}), 7.60(\mathrm{~d}, J=7.8 \mathrm{~Hz}, 2 \mathrm{H}), 7.45(\mathrm{t}, J=7.3$ $\mathrm{Hz}, 1 \mathrm{H}), 7.36(\mathrm{t}, J=7.4 \mathrm{~Hz}, 3 \mathrm{H}), 7.29(\mathrm{t}, J=7.5 \mathrm{~Hz}, 1 \mathrm{H}), 7.14(\mathrm{~d}, J=7.3 \mathrm{~Hz}, 1 \mathrm{H}), 6.60$ $(\mathrm{s}, 1 \mathrm{H}), 3.87(\mathrm{~s}, 3 \mathrm{H}), 3.82(\mathrm{~d}, J=17.1 \mathrm{~Hz}, 1 \mathrm{H}), 3.54(\mathrm{~s}, 3 \mathrm{H}), 3.46(\mathrm{~d}, J=17.0 \mathrm{~Hz}, 1 \mathrm{H})$, 2.57 (dd, $J=44.1,16.1 \mathrm{~Hz}, 2 \mathrm{H}), 2.41$ (s, 3H), 1.73 (brs, 1H), 1.18 (s, 3H), 1.04 (s, 3H). 
${ }^{13} \mathrm{C}$ NMR $\left(100 \mathrm{MHz}, \mathrm{CDCl}_{3}\right) \delta 165.6,150.7,143.5,137.1,134.6,134.1,133.5,131.6$, 131.3, 131.2, 130.4, 128.5 (2C), 127.9, 127.1 (2C), 126.8, 125.9, 112.9, 61.1, 55.9, 48.20, 42.9, 42.1, 28.0, 27.2, 19.0.

IR (FT-ATR, $\mathrm{cm}^{-1}, \mathrm{CH}_{2} \mathrm{Cl}_{2}$ ) $v_{\max } 2930,2850,2630,1667,1599,1579,1315,1484$, 1459, 1429, 1395, 1380, 1362, 1330, 1257, 1198, 1132, 1118, 1057, 1027, 1004, 965, 930, 839, 798, 736, 720, 617, 598, 519, 480, 443, 422.

HRMS (ESI) $m / z$ : $[\mathrm{M}+\mathrm{H}]^{+}$Calcd for $\mathrm{C}_{27} \mathrm{H}_{30} \mathrm{~N}_{2} \mathrm{O}_{3} 431.2335$; found 431.2332 .

Optical: $[a]^{20}{ }_{\mathrm{D}}=+3.9^{\circ}\left(c=0.96, \mathrm{CHCl}_{3}, 98 \%\right.$ e.e. $)$

HPLC (Chiralpak AD, ${ }^{i} \mathrm{PrOH} / n$-hexane $=20 / 80$ with $0.1 \% \mathrm{Et}_{2} \mathrm{NH}$, flow rate $=1.0$ $\mathrm{mL} / \mathrm{min}, \lambda=254 \mathrm{~nm}$ ) $t_{\mathrm{R}}=31.7 \mathrm{~min}$ (major), $23.9 \mathrm{~min}$ (minor)

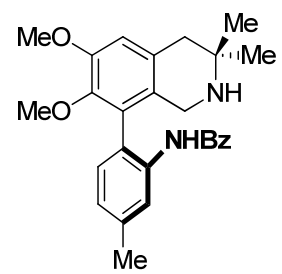

$N$-(2-(6,7-Dimethoxy-3,3-dimethyl-1,2,3,4-tetrahydroisoquinolin-8-yl)-5-

methylphenyl)benzamide (2c) was synthesized by following Procedure 19 from 1c. The crude material was purified by normal-phase column chromatography using an eluent of $1.5 \% \mathrm{MeOH} / \mathrm{EtOAc}$ with $10 \%$ aqueous $\mathrm{NH}_{3}$ to give $2 \mathbf{c}$ as a white solid (16.1 $\mathrm{mg}, 75 \%$ yield, $60{ }^{\circ} \mathrm{C}$ ).

m.p. $96{ }^{\circ} \mathrm{C}$

${ }^{1} \mathbf{H}$ NMR $\left(400 \mathrm{MHz}, \mathrm{CDCl}_{3}\right) \delta 8.19(\mathrm{~s}, 1 \mathrm{H}), 7.90(\mathrm{~s}, 1 \mathrm{H}), 7.56(\mathrm{~d}, J=7.9 \mathrm{~Hz}, 2 \mathrm{H}), 7.43$ $(\mathrm{t}, J=7.3 \mathrm{~Hz}, 1 \mathrm{H}), 7.34(\mathrm{t}, J=7.5 \mathrm{~Hz}, 2 \mathrm{H}), 7.03(\mathrm{~s}, 2 \mathrm{H}), 6.67(\mathrm{~s}, 1 \mathrm{H}), 3.88(\mathrm{~s}, 3 \mathrm{H}), 3.78$ $(\mathrm{d}, J=16.8 \mathrm{~Hz}, 1 \mathrm{H}), 3.53(\mathrm{~s}, 3 \mathrm{H}), 3.41(\mathrm{~d}, J=16.8 \mathrm{~Hz}, 1 \mathrm{H}), 2.68(\mathrm{~s}, 2 \mathrm{H}), 2.44(\mathrm{~s}, 3 \mathrm{H})$, $1.20(\mathrm{~s}, 3 \mathrm{H}), 1.11(\mathrm{~s}, 3 \mathrm{H})$.

${ }^{13}$ C NMR $\left(100 \mathrm{MHz}, \mathrm{CDCl}_{3}\right) \delta 165.4,151.6,144.6,138.9,135.3,134.9,131.7,130.9$, 129.9, 129.7, 128.7 (2C), 127.0 (2C), 125.5, 124.1, 124.1, 123.1, 113.3, 61.2, 56.0, 49.4, 42.1, 41.3, 27.6, 26.0, 21.7.

IR (FT-ATR, $\mathrm{cm}^{-1}, \mathrm{CH}_{2} \mathrm{Cl}_{2}$ ) $v_{\max } 3414,3307,2959,2925,2854,1677,1619,1575$, 1529, 1466, 1425, 1379, 1330, 1314, 1295, 1255, 1193, 1150, 1126, 1091, 1029, 963, 838, 798, 708, 599, 482.

HRMS (ESI) $m / z$ : $[\mathrm{M}+\mathrm{H}]^{+}$Calcd for $\mathrm{C}_{27} \mathrm{H}_{30} \mathrm{~N}_{2} \mathrm{O}_{3} 431.2335$; found 431.2330.

Optical: $[a]^{20}{ }_{\mathrm{D}}=+7.4^{\mathrm{o}}\left(c=0.54, \mathrm{CHCl}_{3}, 97 \%\right.$ e.e. $)$

HPLC (Chiralpak AD, ${ }^{i} \mathrm{PrOH} / n$-hexane $=20 / 80$ with $0.1 \% \mathrm{Et}_{2} \mathrm{NH}$, flow rate $=1.0$ $\mathrm{mL} / \mathrm{min}, \lambda=254 \mathrm{~nm}$ ) $t_{\mathrm{R}}=25.9 \min$ (major), $20.2 \min$ (minor)

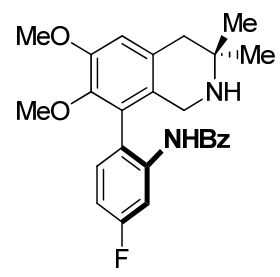

$N$-(2-(6,7-Dimethoxy-3,3-dimethyl-1,2,3,4-tetrahydroisoquinolin-8-yl)-5-

fluorophenyl)benzamide (2d) was synthesized by following Procedure 19 from 1d. 
The crude material was purified by normal-phase column chromatography using an eluent of $2 \% \mathrm{MeOH} / \mathrm{EtOAc}$ with $10 \%$ aqueous $\mathrm{NH}_{3}$ to give $2 \mathrm{~d}$ as a white solid (13.0 $\mathrm{mg}, 60 \%$ yield, $60{ }^{\circ} \mathrm{C} ; 17.8 \mathrm{mg}, 82 \%$ yield, $80{ }^{\circ} \mathrm{C}$ ).

m.p. $129^{\circ} \mathrm{C}$

${ }^{1} \mathbf{H}$ NMR $\left(400 \mathrm{MHz}, \mathrm{CDCl}_{3}\right) \delta 8.33(\mathrm{~d}, J=11.1 \mathrm{~Hz}, 1 \mathrm{H}), 7.91(\mathrm{~s}, 1 \mathrm{H}), 7.56(\mathrm{~d}, J=7.7$ $\mathrm{Hz}, 2 \mathrm{H}), 7.45(\mathrm{t}, J=7.2 \mathrm{~Hz}, 1 \mathrm{H}), 7.36(\mathrm{t}, J=7.4 \mathrm{~Hz}, 2 \mathrm{H}), 7.12(\mathrm{t}, J=7.3 \mathrm{~Hz}, 1 \mathrm{H}), 6.91$ $(\mathrm{t}, J=8.1 \mathrm{~Hz}, 1 \mathrm{H}), 6.72(\mathrm{~s}, 1 \mathrm{H}), 3.90(\mathrm{~s}, 3 \mathrm{H}), 3.70(\mathrm{~d}, J=16.9 \mathrm{~Hz}, 1 \mathrm{H}), 3.55(\mathrm{~s}, 3 \mathrm{H})$, $3.34(\mathrm{~d}, J=16.9 \mathrm{~Hz}, 1 \mathrm{H}), 2.63$ (s, 2H), 1.68 (brs, 1H), 1.16 (s, 3H), 1.08 (s, 3H).

${ }^{13} \mathrm{C}$ NMR $\left(100 \mathrm{MHz}, \mathrm{CDCl}_{3}\right) \delta 165.2,162.6(\mathrm{~d}, J=243.8 \mathrm{~Hz}, 1 \mathrm{C}), 151.4,144.6,137.0$ (d, $J=11.7 \mathrm{~Hz}, 1 \mathrm{C}), 134.6,131.9$ (2C), 131.0 (d, $J=9.2 \mathrm{~Hz}, 1 \mathrm{C}), 128.84$ (2C), 128.3, 127.0 (2C), 125.9, 122.0 (d, $J=3.3 \mathrm{~Hz}, 1 \mathrm{C}), 113.9,111.0$ (d, $J=21.8 \mathrm{~Hz}, 1 \mathrm{C}), 108.8$ (d, $J=27.3 \mathrm{~Hz}, 1 \mathrm{C}), 61.2,56.0,48.3,42.8,42.0,28.3,27.0$.

IR (FT-ATR, $\mathrm{cm}^{-1}, \mathrm{CH}_{2} \mathrm{Cl}_{2}$ ) $v_{\max } 2952,2852,1673,1599,1527,1482,1467,1429$, 1395, 1380, 1361, 1331, 1293, 1257, 1200, 1179, 1134, 1053, 1028, 1010, 976, 834, 799, 720.

HRMS (ESI) $m / z$ : $[\mathrm{M}+\mathrm{H}]^{+}$Calcd for $\mathrm{C}_{26} \mathrm{H}_{27} \mathrm{FN}_{2} \mathrm{O}_{3} 435.2084$; found 435.2078.

Optical: $[a]^{20} \mathrm{D}=+11.1^{\circ}\left(c=0.57, \mathrm{CHCl}_{3}, 95 \%\right.$ e.e. $)$

HPLC (Chiralpak AD, ${ }^{i} \mathrm{PrOH} / n$-hexane $=20 / 80$ with $0.1 \% \mathrm{Et}_{2} \mathrm{NH}$, flow rate $=1.0$ $\mathrm{mL} / \mathrm{min}, \lambda=254 \mathrm{~nm}$ ) $t_{\mathrm{R}}=13.7 \mathrm{~min}$ (major), $16.5 \mathrm{~min}$ (minor)

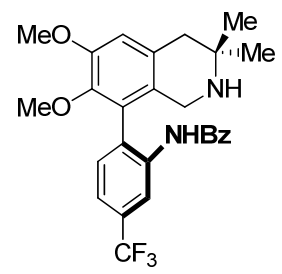

$N$-(2-(6,7-Dimethoxy-3,3-dimethyl-1,2,3,4-tetrahydroisoquinolin-8-yl)-5-

(trifluoromethyl)phenyl)benzamide (2e) was synthesized by following Procedure 19 from 1e. The crude material was purified by normal-phase column chromatography using an eluent of $10 \% \mathrm{MeOH} / \mathrm{EtOAc}$ with $1 \%$ aqueous $\mathrm{NH}_{3}$ to give $2 \mathrm{e}$ as a white solid (14.5 mg, $60 \%$ yield, $60{ }^{\circ} \mathrm{C} ; 18.9 \mathrm{mg}, 78 \%$ yield, $80{ }^{\circ} \mathrm{C}$ ).

m.p. $169^{\circ} \mathrm{C}$

${ }^{1} \mathbf{H}$ NMR $\left(400 \mathrm{MHz}, \mathrm{CDCl}_{3}\right) \delta 8.25(\mathrm{~d}, J=8.3 \mathrm{~Hz}, 1 \mathrm{H}), 7.87(\mathrm{~s}, 1 \mathrm{H}), 7.57(\mathrm{dt}, J=8.5$, $1.7 \mathrm{~Hz}, 2 \mathrm{H}), 7.43$ (ddd, $J=6.8,4.0,1.3 \mathrm{~Hz}, 1 \mathrm{H}), 7.37-7.32(\mathrm{~m}, 2 \mathrm{H}), 7.24$ (dd, $J=8.4$, $1.8 \mathrm{~Hz}, 1 \mathrm{H}), 6.99(\mathrm{~d}, J=1.9 \mathrm{~Hz}, 1 \mathrm{H}), 6.68(\mathrm{~s}, 1 \mathrm{H}), 3.89(\mathrm{~s}, 3 \mathrm{H}), 3.74(\mathrm{~d}, J=17.0 \mathrm{~Hz}$, $1 \mathrm{H}), 3.55(\mathrm{~s}, 3 \mathrm{H}), 3.37(\mathrm{~d}, J=17.0 \mathrm{~Hz}, 1 \mathrm{H}), 2.67-2.56(\mathrm{~m}, 2 \mathrm{H}), 2.36(\mathrm{~s}, 3 \mathrm{H}), 1.17(\mathrm{~s}$, 3H), $1.06(\mathrm{~s}, 3 \mathrm{H})$.

${ }^{13}$ C NMR (100 MHz, $\left.\mathrm{CDCl}_{3}\right) \delta 165.3,151.5,144.3,136.4,134.5,132.2,132.1,131.0$ (q, $J=32.0 \mathrm{~Hz}, 1 \mathrm{C}), 130.5,130.3,128.90$ (2C), 127.9, 127.03 (2C), 125.4, 120.7 (q, $J$ $=3.6 \mathrm{~Hz}, 1 \mathrm{C}), 118.5$ (q, $J=4.0 \mathrm{~Hz}, 1 \mathrm{C}), 114.3,61.3,56.1,48.4,42.8,42.1,28.2,27.1$. IR (FT-ATR, $\mathrm{cm}^{-1}, \mathrm{CH}_{2} \mathrm{Cl}_{2}$ ) $v_{\max } 3411,2962,1680,1580,1536,1467,1427,1380$, 1356, 1330, 1257, 1229, 1168, 1125, 1091, 1073, 1029, 993, 962, 921, 903, 825, 797, 754, 709, 668, 588, 479.

HRMS (ESI) m/z: [M+H] $]^{+}$Calcd for $\mathrm{C}_{27} \mathrm{H}_{27} \mathrm{~F}_{3} \mathrm{~N}_{2} \mathrm{O}_{3} 485.2052$; found 485.2047.

Optical: $[a]^{20}{ }_{\mathrm{D}}=+3.5^{\circ}\left(c=0.26, \mathrm{CHCl}_{3}, 97 \%\right.$ e.e. $)$

HPLC (Chiralpak AD, ${ }^{i} \mathrm{PrOH} / n$-hexane $=20 / 80$ with $0.1 \% \mathrm{Et}_{2} \mathrm{NH}$, flow rate $=1.0$ $\mathrm{mL} / \mathrm{min}, \lambda=254 \mathrm{~nm}$ ) $t_{\mathrm{R}}=6.8 \mathrm{~min}$ (major), $14.9 \mathrm{~min}$ (minor) 


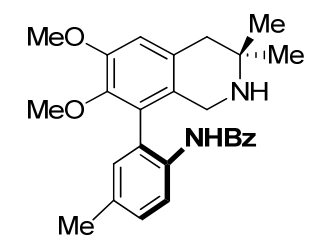

$N$-(2-(6,7-Dimethoxy-3,3-dimethyl-1,2,3,4-tetrahydroisoquinolin-8-yl)-4methylphenyl)benzamide (2f) was synthesized by following Procedure 19 from 1 f. The crude material was purified by normal-phase column chromatography using an eluent of $10 \% \mathrm{MeOH} / \mathrm{EtOAc}$ with $\%$ aqueous $\mathrm{NH}_{3}$ to give $\mathbf{2} \mathbf{f}$ as a white solid (11.6 $\mathrm{mg}$, $54 \%$ yield, $60{ }^{\circ} \mathrm{C} ; 18.7 \mathrm{mg}, 87 \%$ yield, $80{ }^{\circ} \mathrm{C}$ ).

m.p. $195^{\circ} \mathrm{C}$

${ }^{1} \mathbf{H}$ NMR $\left(500 \mathrm{MHz}, \mathrm{CDCl}_{3}\right) \delta 8.26(\mathrm{~d}, J=8.3 \mathrm{~Hz}, 1 \mathrm{H}), 7.88(\mathrm{~s}, 1 \mathrm{H}), 7.60-7.56(\mathrm{~m}$, $2 \mathrm{H}), 7.44$ (ddd, $J=6.8,4.0,1.3 \mathrm{~Hz}, 1 \mathrm{H}), 7.38-7.33(\mathrm{~m}, 2 \mathrm{H}), 7.27$ (s, 1H), $7.26(\mathrm{dd}, J$ $=8.4,1.8 \mathrm{~Hz}, 1 \mathrm{H}), 7.00(\mathrm{~d}, J=1.9 \mathrm{~Hz}, 1 \mathrm{H}), 6.70(\mathrm{~s}, 1 \mathrm{H}), 3.91(\mathrm{~s}, 3 \mathrm{H}), 3.75(\mathrm{~d}, J=17.0$ $\mathrm{Hz}, 1 \mathrm{H}), 3.57(\mathrm{~s}, 3 \mathrm{H}), 3.38(\mathrm{~d}, J=17.0 \mathrm{~Hz}, 1 \mathrm{H}), 2.69-2.58(\mathrm{~m}, 2 \mathrm{H}), 2.37(\mathrm{~s}, 3 \mathrm{H}), 1.18$ $(\mathrm{s}, 3 \mathrm{H}), 1.08(\mathrm{~s}, 3 \mathrm{H})$.

${ }^{13}$ C NMR $\left(125 \mathrm{MHz}, \mathrm{CDCl}_{3}\right) \delta 165.2,151.3,144.4,135.1,133.9,133.1,131.7,131.6$, 130.6, 129.6, 129.4, 128.7 (2C), 127.2, 127.0 (2C), 125.9, 122.2, 113.6, 61.2, 56.1, 48.3, 42.9, 42.1, 28.2, 27.2, 21.1 .

IR (FT-ATR, $\mathrm{cm}^{-1}, \mathrm{CH}_{2} \mathrm{Cl}_{2}$ ) $v_{\max } 3416,3309,2960,2926,1673,1591,1516,1476$, 1464, 1426, 1399, 1379, 1362, 1329, 1308, 1253, 1213, 1188, 1147, 1125, 1095, 1033, 1001, 962, 932, 893, 845, 821, 796, 760, 708, 675, 568, 542, 483.

HRMS (ESI) $m / z$ : $[\mathrm{M}+\mathrm{H}]^{+}$Calcd for $\mathrm{C}_{27} \mathrm{H}_{30} \mathrm{~N}_{2} \mathrm{O}_{3} 431.2335$; found 431.2329 .

Optical: $[a]^{20}{ }_{\mathrm{D}}=-6.0^{\circ}\left(c=0.68, \mathrm{CHCl}_{3}, 97 \%\right.$ e.e. $)$

HPLC (Chiralpak AD, ${ }^{i} \mathrm{PrOH} / n$-hexane $=20 / 80$ with $0.1 \% \mathrm{Et}_{2} \mathrm{NH}$, flow rate $=1.0$ $\mathrm{mL} / \mathrm{min}, \lambda=254 \mathrm{~nm}$ ) $t_{\mathrm{R}}=59.2 \mathrm{~min}$ (major), $16.5 \mathrm{~min}$ (minor)

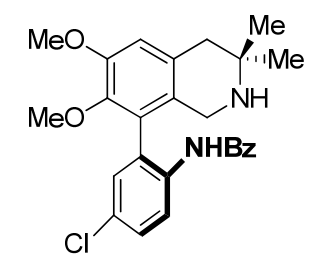

N-(4-Chloro-2-(6,7-dimethoxy-3,3-dimethyl-1,2,3,4-tetrahydroisoquinolin-8-

yl)phenyl)benzamide (2g) was synthesized by following Procedure 19 from 1 g. The crude material was purified by normal-phase column chromatography using an eluent of $10 \% \mathrm{MeOH} / \mathrm{EtOAc}$ with $0.5 \%$ aqueous $\mathrm{NH}_{3}$ to give $\mathbf{2 g}$ as a white solid (12.4 $\mathrm{mg}$, $55 \%$ yield, $60{ }^{\circ} \mathrm{C} ; 17.4 \mathrm{mg}, 77 \%$ yield, $80{ }^{\circ} \mathrm{C}$ ).

m.p. $66{ }^{\circ} \mathrm{C}$

${ }^{1} \mathbf{H}$ NMR $\left(400 \mathrm{MHz}, \mathrm{CDCl}_{3}\right) \delta 8.40(\mathrm{~d}, J=8.8 \mathrm{~Hz}, 1 \mathrm{H}), 7.87(\mathrm{~s}, 1 \mathrm{H}), 7.55(\mathrm{~d}, J=7.7$ $\mathrm{Hz}, 2 \mathrm{H}), 7.45(\mathrm{t}, J=7.1 \mathrm{~Hz}, 1 \mathrm{H}), 7.40(\mathrm{~d}, J=8.8 \mathrm{~Hz}, 1 \mathrm{H}), 7.35$ (t, $J=7.5 \mathrm{~Hz}, 2 \mathrm{H}), 7.17$ $(\mathrm{s}, 1 \mathrm{H}), 6.72(\mathrm{~s}, 1 \mathrm{H}), 3.90(\mathrm{~s}, 3 \mathrm{H}), 3.74(\mathrm{~d}, J=17.0 \mathrm{~Hz}, 1 \mathrm{H}), 3.58(\mathrm{~s}, 3 \mathrm{H}), 3.35(\mathrm{~d}, J=$ $16.9 \mathrm{~Hz}, 1 \mathrm{H}), 2.62$ (s, 2H), 1.57 (brs, 1H), 1.16 (s, 3H), 1.07 (s, 3H).

${ }^{13}$ C NMR $\left(100 \mathrm{MHz}, \mathrm{CDCl}_{3}\right) \delta 165.2,151.4,144.3,134.7,134.5,132.0,131.9,129.8$, $129.2,128.8$ (2C), 128.7, 128.7, 127.9, 127.0 (2C), 125.6, 123.2, 114.2, 61.3, 56.1, $48.3,42.8,42.0,28.3,27.0$. 
IR (FT-ATR, $\mathrm{cm}^{-1}, \mathrm{CH}_{2} \mathrm{Cl}_{2}$ ) $v_{\max } 3414,3309,2961,2934,1676,1592,1576,1510$, 1492, 1479, 1463, 1425, 1394, 1364, 1329, 1303, 1252, 1191, 1176, 1154, 1124, 1095, 1030, 998, 962, 903, 844, 823, 795, 707, 569, 541, 477, 422.

HRMS (ESI) $m / z$ : $[\mathrm{M}+\mathrm{H}]^{+}$Calcd for $\mathrm{C}_{26} \mathrm{H}_{27} \mathrm{ClN}_{2} \mathrm{O}_{3} 451.1789$; found 451.1783.

Optical: $[a]^{20} \mathrm{D}=+11.7^{\circ}\left(c=0.24, \mathrm{CHCl}_{3}, 99 \%\right.$ e.e. $)$

HPLC (Chiralpak AD, ${ }^{i} \mathrm{PrOH} / n$-hexane $=20 / 80$ with $0.1 \% \mathrm{Et}_{2} \mathrm{NH}$, flow rate $=1.0$ $\mathrm{mL} / \mathrm{min}, \lambda=254 \mathrm{~nm}$ ) $t_{\mathrm{R}}=27.9 \min$ (major), $21.8 \mathrm{~min}$ (minor)

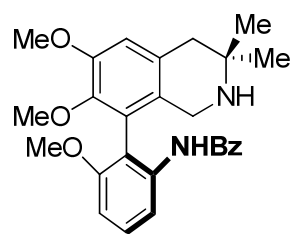

$N$-(2-(6,7-Dimethoxy-3,3-dimethyl-1,2,3,4-tetrahydroisoquinolin-8-yl)-3-

methoxyphenyl)benzamide (2h) was synthesized by following Procedure 19 from $\mathbf{1 h}$. The crude material was purified by normal-phase column chromatography using an eluent of $10 \% \mathrm{MeOH} / \mathrm{EtOAc}$ with $1 \%$ aqueous $\mathrm{NH}_{3}$ to give $\mathbf{2 h}$ as a white solid (12.3 $\mathrm{mg}, 55 \%$ yield, $60{ }^{\circ} \mathrm{C} ; 21.0 \mathrm{mg}, 94 \%$ yield, $80^{\circ} \mathrm{C}$ ).

m.p. $77^{\circ} \mathrm{C}$

${ }^{1} \mathbf{H}$ NMR $\left(400 \mathrm{MHz}, \mathrm{CDCl}_{3}\right) \delta 8.10(\mathrm{~d}, J=8.2 \mathrm{~Hz}, 1 \mathrm{H}), 7.78(\mathrm{~s}, 1 \mathrm{H}), 7.52(\mathrm{~d}, J=7.4$ $\mathrm{Hz}, 2 \mathrm{H}), 7.48-7.38(\mathrm{~m}, 2 \mathrm{H}), 7.34$ (t, $J=7.4 \mathrm{~Hz}, 2 \mathrm{H}), 6.80$ (d, $J=7.8 \mathrm{~Hz}, 1 \mathrm{H}), 6.70$ (s, $1 \mathrm{H}), 3.90(\mathrm{~s}, 3 \mathrm{H}), 3.76(\mathrm{~s}, 3 \mathrm{H}), 3.68-3.51(\mathrm{~m}, 4 \mathrm{H}), 3.36(\mathrm{~d}, J=16.7 \mathrm{~Hz}, 1 \mathrm{H}), 2.68-2.55$ (m, 2H), 1.52 (brs, $1 \mathrm{H}), 1.15(\mathrm{~s}, 3 \mathrm{H}), 1.07(\mathrm{~s}, 3 \mathrm{H})$.

${ }^{13}$ C NMR $\left(100 \mathrm{MHz}, \mathrm{CDCl}_{3}\right) \delta 165.2,156.9,151.3,144.6,136.6,135.0,131.7,131.4$, $129.5,128.7$ (2C), 127.0 (2C), 126.8, 125.4, 115.4, 114.1, 113.6, 106.8, 61.0, 56.0, 55.7, 48.4, 42.2, 42.1, 28.3, 26.8 .

IR (FT-ATR, $\mathrm{cm}^{-1}, \mathrm{CH}_{2} \mathrm{Cl}_{2}$ ) v $v_{\max } 3410,2960,2935,2836,1678,1588,1525,1438$, 1412, 1379, 1330, 1309, 12571191, 1146, 1128, 1095, 1071, 1029, 996, 962, 934, 845, 783, 735, 709, 624, 576.

HRMS (ESI) $m / z$ : $[\mathrm{M}+\mathrm{H}]^{+}$Calcd for $\mathrm{C}_{27} \mathrm{H}_{30} \mathrm{~N}_{2} \mathrm{O}_{4}$ 447.2284; found 447.2281 .

Optical: $[a]^{20}{ }_{\mathrm{D}}=-11.7^{\circ}\left(c=0.30, \mathrm{CHCl}_{3}, 98 \%\right.$ e.e. $)$

HPLC (Chiralpak AD, ${ }^{i} \mathrm{PrOH} / n$-hexane $=20 / 80$ with $0.1 \% \mathrm{Et}_{2} \mathrm{NH}$, flow rate $=1.0$ $\mathrm{mL} / \mathrm{min}, \lambda=254 \mathrm{~nm}$ ) $t_{\mathrm{R}}=10.1 \mathrm{~min}$ (major), $16.6 \mathrm{~min}$ (minor)

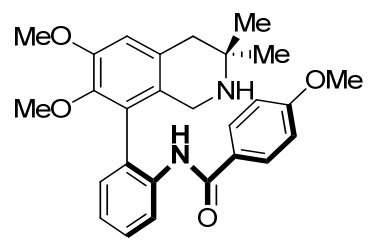

$N$-(2-(6,7-Dimethoxy-3,3-dimethyl-1,2,3,4-tetrahydroisoquinolin-8-yl)phenyl)-4methoxybenzamide (2i) was synthesized by following Procedure 19 from 1i. The crude material was purified by normal-phase column chromatography using an eluent of $10 \% \mathrm{MeOH} / \mathrm{EtOAc}$ with $1 \%$ aqueous $\mathrm{NH}_{3}$ to give $2 \mathbf{i}$ as a white solid $(16.5 \mathrm{mg}, 74 \%$ yield, $\left.60^{\circ} \mathrm{C}\right)$.

m.p. $111^{\circ} \mathrm{C}$ 
${ }^{1} \mathbf{H}$ NMR $\left(400 \mathrm{MHz}, \mathrm{CDCl}_{3}\right) \delta 8.25(\mathrm{~d}, J=8.1 \mathrm{~Hz}, 1 \mathrm{H}), 7.80(\mathrm{~s}, 1 \mathrm{H}), 7.51(\mathrm{~d}, J=8.4$ $\mathrm{Hz}, 2 \mathrm{H}), 7.43(\mathrm{t}, J=7.6 \mathrm{~Hz}, 1 \mathrm{H}), 7.21(\mathrm{t}, J=7.3 \mathrm{~Hz}, 1 \mathrm{H}), 7.11(\mathrm{~d}, J=7.4 \mathrm{~Hz}, 1 \mathrm{H}), 6.80$ $(\mathrm{d}, J=8.3 \mathrm{~Hz}, 2 \mathrm{H}), 6.68(\mathrm{~s}, 1 \mathrm{H}), 3.90-3.85(\mathrm{~m}, 4 \mathrm{H}), 3.79(\mathrm{~s}, 3 \mathrm{H}), 3.57-3.48(\mathrm{~m}, 4 \mathrm{H})$, $2.83(\mathrm{dd}, J=44.8,16.8 \mathrm{~Hz}, 2 \mathrm{H}), 1.30$ (s, 3H), 1.22 (s, 3H).

${ }^{13}$ C NMR $\left(100 \mathrm{MHz}, \mathrm{CDCl}_{3}\right) \delta 165.3,162.4,152.2,145.1,135.8,130.0,129.9,129.2$, 129.1, 128.9 (2C), 126.8, 126.8, 124.9, 123.3, 119.9, 113.9 (2C), 113.3, 61.1, 56.0, 55.5, 51.9, 40.6, 39.8, 25.8, 24.2.

IR (FT-ATR, $\mathrm{cm}^{-1}, \mathrm{CH}_{2} \mathrm{Cl}_{2}$ ) $v_{\max } 3415,3302,2939,2841,2629,1671,1606,1580$, $1528,1506,1482,1458,1394,1379,1360,1330,1303,1254,1199,1176,1153,1131$, 1056, 1028, 1010, 997, 965, 844, 799, 764, 720, 696, 640, 574, 527.

HRMS (ESI) $m / z$ : $[\mathrm{M}+\mathrm{H}]^{+}$Calcd for $\mathrm{C}_{2} 7 \mathrm{H}_{30} \mathrm{~N}_{2} \mathrm{O}_{4} 447.2284$; found 447.2278.

Optical: $[a]^{20} \mathrm{D}=+5.5^{\circ}\left(c=0.31, \mathrm{CHCl}_{3}, 95 \%\right.$ e.e. $)$

HPLC (Chiralpak AD, ${ }^{i} \mathrm{PrOH} / n$-hexane $=20 / 80$ with $0.1 \% \mathrm{Et}_{2} \mathrm{NH}$, flow rate $=1.0$ $\mathrm{mL} / \mathrm{min}, \lambda=254 \mathrm{~nm}$ ) $t_{\mathrm{R}}=39.1 \mathrm{~min}$ (major), $16.7 \mathrm{~min}$ (minor)

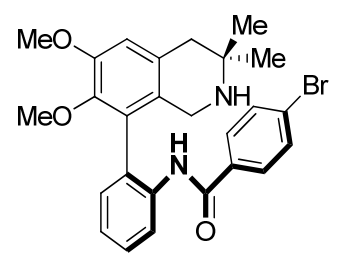

4-Bromo- $N$-(2-(6,7-dimethoxy-3,3-dimethyl-1,2,3,4-tetrahydroisoquinolin-8yl)phenyl)benzamide $(\mathbf{2 j})$ was synthesized by following Procedure 19 from $\mathbf{1 j}$. The crude material was purified by normal-phase column chromatography using an eluent of $10 \% \mathrm{MeOH} /$ EtOAc with $1 \%$ aqueous $\mathrm{NH}_{3}$ to give $2 \mathbf{j}$ as a white solid (19.6 mg, $79 \%$ yield, $60{ }^{\circ} \mathrm{C}$ ).

m.p. $117^{\circ} \mathrm{C}$

${ }^{1} \mathbf{H}$ NMR $\left(400 \mathrm{MHz}, \mathrm{CDCl}_{3}\right) \delta 8.33(\mathrm{~d}, J=8.1 \mathrm{~Hz}, 1 \mathrm{H}), 7.91(\mathrm{~s}, 1 \mathrm{H}), 7.55-7.40(\mathrm{~m}$, $5 \mathrm{H}), 7.25-7.14(\mathrm{~m}, 2 \mathrm{H}), 6.69(\mathrm{~s}, 1 \mathrm{H}), 3.89(\mathrm{~s}, 3 \mathrm{H}), 3.75(\mathrm{~d}, J=16.9 \mathrm{~Hz}, 1 \mathrm{H}), 3.53(\mathrm{~s}$, $3 \mathrm{H}), 3.34(\mathrm{~d}, J=16.9 \mathrm{~Hz}, 1 \mathrm{H}), 2.64(\mathrm{~s}, 2 \mathrm{H}), 1.17(\mathrm{~s}, 3 \mathrm{H}), 1.09(\mathrm{~s}, 3 \mathrm{H})$.

${ }^{13} \mathrm{C}$ NMR $\left(100 \mathrm{MHz}, \mathrm{CDCl}_{3}\right) \delta 164.4,151.4,144.3,135.4,133.9,132.0$ (2C), 131.7, $130.2,129.3,128.9,128.7$ (2C), 127.2, 126.4, 125.3, 124.6, 122.3, 113.6, 61.2, 56.0, 48.6, 42.7, 41.9, 28.3, 26.6.

IR (FT-ATR, $\mathrm{cm}^{-1}, \mathrm{CH}_{2} \mathrm{Cl}_{2}$ ) $v_{\max } 3413,3306,2960,2928,1678,1590,1524,1479$, 1464, 1454, 1428, 1380, 1363, 1331, 1314, 1258, 1192, 1151, 1085, 1070, 1030, 1012 , $962,895,841,799,757,712,639,568,529,469,421$.

HRMS (ESI) $m / z$ : $[\mathrm{M}+\mathrm{H}]^{+}$Calcd for $\mathrm{C}_{26} \mathrm{H}_{27} \mathrm{BrN}_{2} \mathrm{O}_{3} 495.1283$; found 495.1278 .

Optical: $[a]^{20} \mathrm{D}=+28.9^{\circ}\left(c=0.18, \mathrm{CHCl}_{3}, 99 \%\right.$ e.e. $)$

HPLC (Chiralpak AD, ${ }^{i} \mathrm{PrOH} / n$-hexane $=20 / 80$ with $0.1 \% \mathrm{Et}_{2} \mathrm{NH}$, flow rate $=1.0$ $\mathrm{mL} / \mathrm{min}, \lambda=254 \mathrm{~nm}$ ) $t_{\mathrm{R}}=53.6 \mathrm{~min}$ (major), $15.5 \mathrm{~min}$ (minor)

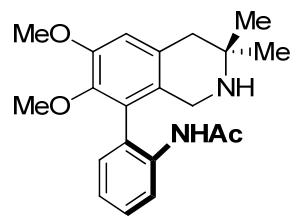

$\mathrm{N}$-(2-(6,7-Dimethoxy-3,3-dimethyl-1,2,3,4-tetrahydroisoquinolin-8-

yl)phenyl)acetamide (2k) was synthesized by following Procedure 19 from $\mathbf{1 k}$. The 
crude material was purified by normal-phase column chromatography using an eluent of $10 \% \mathrm{MeOH} / \mathrm{EtOAc}$ with $1 \%$ aqueous $\mathrm{NH}_{3}$ to give $2 \mathbf{k}$ as a yellow solid $(9.9 \mathrm{mg}, 56 \%$ yield, $60{ }^{\circ} \mathrm{C}$; $14.2 \mathrm{mg}, 80 \%$ yield, $80{ }^{\circ} \mathrm{C}$ ).

m.p. $150^{\circ} \mathrm{C}$

${ }^{1} \mathbf{H}$ NMR $\left(400 \mathrm{MHz}, \mathrm{CDCl}_{3}\right) \delta 8.19(\mathrm{~d}, J=7.5 \mathrm{~Hz}, 1 \mathrm{H}), 7.36(\mathrm{t}, J=7.6 \mathrm{~Hz}, 1 \mathrm{H}), 7.14$ $(\mathrm{t}, J=6.7 \mathrm{~Hz}, 1 \mathrm{H}), 7.11-7.02(\mathrm{~m}, 2 \mathrm{H}), 6.68(\mathrm{~s}, 1 \mathrm{H}), 3.88(\mathrm{~s}, 3 \mathrm{H}), 3.67(\mathrm{~d}, J=16.7 \mathrm{~Hz}$, $1 \mathrm{H}), 3.49(\mathrm{~d}, J=1.4 \mathrm{~Hz}, 3 \mathrm{H}), 3.33(\mathrm{~d}, J=16.8 \mathrm{~Hz}, 1 \mathrm{H}), 2.77-2.58(\mathrm{~m}, 2 \mathrm{H}), 2.35$ (brs, $1 \mathrm{H}), 1.93(\mathrm{~s}, 3 \mathrm{H}), 1.17(\mathrm{~d}, J=4.9 \mathrm{~Hz}, 6 \mathrm{H})$.

${ }^{13}$ C NMR $\left(100 \mathrm{MHz}, \mathrm{CDCl}_{3}\right) \delta 168.5,151.3,144.4,135.6,131.3,130.0,129.4,128.7$, 126.7, 125.1, 124.2, 122.3, 113.3, 61.0, 55.8, 48.6, 42.6, 41.9, 28.3, 26.6, 24.7.

IR (FT-ATR, $\mathrm{cm}^{-1}, \mathrm{CH}_{2} \mathrm{Cl}_{2}$ ) $v_{\max } 3411,3329,3300,2960,2927,2853,1685,1583$, 1521, 1480, 1453, 1428, 1367, 1331, 1316, 1298, 1258, 1192, 1175, 1152, 1090, 1031, $962,926,838,799,757,637,576,539,507,418$.

HRMS (ESI) $m / z$ : $[\mathrm{M}+\mathrm{H}]^{+}$Calcd for $\mathrm{C}_{21} \mathrm{H}_{26} \mathrm{~N}_{2} \mathrm{O}_{3}$ 355.2022; found 355.2016.

Optical: $[a]^{20}{ }_{\mathrm{D}}=-9.5^{\circ}\left(c=0.11, \mathrm{CHCl}_{3}, 81 \%\right.$ e.e. $)$

HPLC (Chiralpak AD, ${ }^{i} \mathrm{PrOH} / n$-hexane $=20 / 80$ with $0.1 \% \mathrm{Et}_{2} \mathrm{NH}$, flow rate $=1.0$ $\mathrm{mL} / \mathrm{min}, \lambda=254 \mathrm{~nm}$ ) $t_{\mathrm{R}}=28.5 \mathrm{~min}$ (major), $23.4 \mathrm{~min}$ (minor)

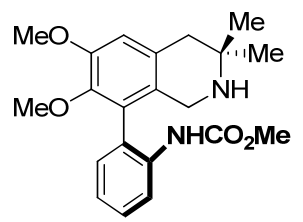

Methyl (2-(6,7-Dimethoxy-3,3-dimethyl-1,2,3,4-tetrahydroisoquinolin-8yl)phenyl)carbamate (2I) was synthesized by following Procedure 19 from 11. The crude material was purified by normal-phase column chromatography using an eluent of $10 \% \mathrm{MeOH} / \mathrm{EtOAc}$ with $0.5 \%$ aqueous $\mathrm{NH}_{3}$ to give $2 \mathrm{l}$ as a colorless gum (7.4 mg, $40 \%$ yield, $60{ }^{\circ} \mathrm{C}$; $6.5 \mathrm{mg}$, $35 \%$ yield, $80{ }^{\circ} \mathrm{C}$ ).

${ }^{1} \mathbf{H}$ NMR $\left(400 \mathrm{MHz}, \mathrm{CDCl}_{3}\right) \delta 8.11(\mathrm{~d}, J=6.2 \mathrm{~Hz}, 1 \mathrm{H}), 7.37(\mathrm{t}, J=7.7 \mathrm{~Hz}, 1 \mathrm{H}), 7.15-$ $7.03(\mathrm{~m}, 2 \mathrm{H}), 6.68(\mathrm{~s}, 1 \mathrm{H}), 6.40(\mathrm{~s}, 1 \mathrm{H}), 3.88(\mathrm{~s}, 3 \mathrm{H}), 3.67-3.58(\mathrm{~m}, 4 \mathrm{H}), 3.51(\mathrm{~s}, 3 \mathrm{H})$, $3.33(\mathrm{~d}, J=16.9 \mathrm{~Hz}, 1 \mathrm{H}), 2.65$ (s, 2H), 1.76 (brs, 1H), 1.17 (d, $J=3.8 \mathrm{~Hz}, 6 \mathrm{H})$.

${ }^{13}$ C NMR $\left(100 \mathrm{MHz}, \mathrm{CDCl}_{3}\right) \delta 154.1,151.4,144.8,135.7,131.3,130.0,129.1,128.8$, 125.7, 125.5, 123.3, 119.9, 113.5, 61.0, 55.9, 52.3, 48.6, 42.7, 42.0, 28.1, 27.1.

IR (FT-ATR, $\left.\mathrm{cm}^{-1}, \mathrm{CH}_{2} \mathrm{Cl}_{2}\right) v_{\max } 3414,3312,2959,1737,1585,1523,1478,1454$, 1428, 1380, 1332, 1315, 1302, 1214, 1193, 1152, 1118, 1094, 1065, 1031, 996, 961, 833, 767, 644, 619, 565.

HRMS (ESI) $m / z$ : $[\mathrm{M}+\mathrm{H}]^{+}$Calcd for $\mathrm{C}_{21} \mathrm{H}_{26} \mathrm{~N}_{2} \mathrm{O}_{4}$ 371.1971; found 371.1970.

Optical: $[a]^{20} \mathrm{D}=-3.6^{\mathrm{o}}\left(c=0.28, \mathrm{CHCl}_{3}, 28 \%\right.$ e.e. $)$

HPLC (Chiralpak AD, ${ }^{i} \mathrm{PrOH} / n$-hexane $=10 / 90$ with $0.1 \% \mathrm{Et}_{2} \mathrm{NH}$, flow rate $=1.0$ $\mathrm{mL} / \mathrm{min}, \lambda=254 \mathrm{~nm}$ ) $t_{\mathrm{R}}=23.1 \mathrm{~min}$ (major), $14.8 \mathrm{~min}$ (minor) 


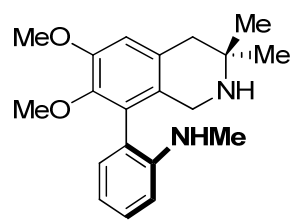

2-(6,7-Dimethoxy-3,3-dimethyl-1,2,3,4-tetrahydroisoquinolin-8-yl)- $\mathrm{N}$ -

methylaniline (2m) was synthesized by following Procedure 19 from $\mathbf{1 m}$. The crude material was purified by normal-phase column chromatography using an eluent of $10 \%$ $\mathrm{MeOH} / \mathrm{EtOAc}$ with $0.5 \%$ aqueous $\mathrm{NH}_{3}$ to give $\mathbf{2 m}$ as a white solid $(6.5 \mathrm{mg}, 40 \%$ yield, $60{ }^{\circ} \mathrm{C} ; 11.6 \mathrm{mg}, 71 \%$ yield, $\left.80{ }^{\circ} \mathrm{C}\right)$.

m.p. $60{ }^{\circ} \mathrm{C}$

${ }^{1} \mathbf{H}$ NMR $\left(400 \mathrm{MHz}, \mathrm{CDCl}_{3}\right) \delta 7.31-7.23(\mathrm{~m}, 1 \mathrm{H}), 6.92(\mathrm{~d}, J=7.3 \mathrm{~Hz}, 1 \mathrm{H}), 6.74(\mathrm{t}, J$ $=7.4 \mathrm{~Hz}, 1 \mathrm{H}), 6.69(\mathrm{~d}, J=8.2 \mathrm{~Hz}, 1 \mathrm{H}), 6.64(\mathrm{~s}, 1 \mathrm{H}), 3.87(\mathrm{~s}, 3 \mathrm{H}), 3.62(\mathrm{~d}, J=16.9 \mathrm{~Hz}$, $1 \mathrm{H}), 3.54$ (s, 3H), $3.42(\mathrm{~d}, J=16.8 \mathrm{~Hz}, 1 \mathrm{H}), 2.75(\mathrm{~s}, 3 \mathrm{H}), 2.64(\mathrm{q}, J=16.2 \mathrm{~Hz}, 2 \mathrm{H})$, $1.16(\mathrm{~d}, J=8.2 \mathrm{~Hz}, 6 \mathrm{H})$.

${ }^{13}$ C NMR $\left(100 \mathrm{MHz}, \mathrm{CDCl}_{3}\right) \delta 151.4,146.6,145.3,130.8,130.5,129.7,128.9,126.0$, 121.6, 116.7, 112.8, 109.9, 61.0, 55.9, 48.5, 42.5, 42.1, 30.9, 28.6, 26.8.

IR (FT-ATR, $\mathrm{cm}^{-1}, \mathrm{CH}_{2} \mathrm{Cl}_{2}$ ) $v_{\max } 3421,3314,2960,2930,2823,1603,1580,1513$, 1478, 1460, 1425, 1378, 1362, 1315, 1289, 1252, 1192, 1168, 1147, 1114, 1095, 1065, 1031, 996, 962, 929, 855, 834, 794, 749, 641, 607, 565, 476, 435, 422.

HRMS (ESI) $m / z$ : [M+H] $]^{+}$Calcd for $\mathrm{C}_{20} \mathrm{H}_{26} \mathrm{~N}_{2} \mathrm{O}_{2}$ 327.2073; found 327.2072.

Optical: $[a]^{20} \mathrm{D}=+17.6^{\circ}\left(c=0.26, \mathrm{CHCl}_{3}, 78 \%\right.$ e.e. $)$

HPLC (Chiralpak AD, ${ }^{i} \mathrm{PrOH} / n$-hexane $=2 / 98$ with $0.1 \% \mathrm{Et}_{2} \mathrm{NH}$, flow rate $=1.0$ $\mathrm{mL} / \mathrm{min}, \lambda=280 \mathrm{~nm}$ ) $t_{\mathrm{R}}=28.0 \mathrm{~min}$ (major), $22.6 \mathrm{~min}$ (minor)

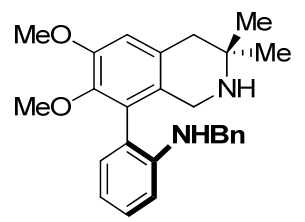

$N$-Benzyl-2-(6,7-dimethoxy-3,3-dimethyl-1,2,3,4-tetrahydroisoquinolin-8-

yl)aniline (2n) was synthesized by following Procedure 19 from $1 \mathbf{n}$. The crude material was purified by normal-phase column chromatography using an eluent of $10 \%$ $\mathrm{MeOH} / \mathrm{EtOAc}$ with $1 \%$ aqueous $\mathrm{NH}_{3}$ to give $\mathbf{2 n}$ as a colorless gum $(9.7 \mathrm{mg}, 48 \%$ yield, $60{ }^{\circ} \mathrm{C} ; 10.9 \mathrm{mg}, 54 \%$ yield, $80{ }^{\circ} \mathrm{C}$ ).

${ }^{1} \mathbf{H}$ NMR $\left(400 \mathrm{MHz}, \mathrm{CDCl}_{3}\right) \delta$ 7.33-7.24 (m, 4H), 7.23-7.14 (m, 2H), 6.95 (d, J= 7.2 $\mathrm{Hz}, 1 \mathrm{H}), 6.75(\mathrm{t}, J=7.3 \mathrm{~Hz}, 1 \mathrm{H}), 6.67-6.59(\mathrm{~m}, 2 \mathrm{H}), 4.31$ (d, $J=3.5 \mathrm{~Hz}, 2 \mathrm{H}), 3.95-$ $3.81(\mathrm{~m}, 4 \mathrm{H}), 3.67-3.49(\mathrm{~m}, 5 \mathrm{H}), 2.63(\mathrm{~s}, 2 \mathrm{H}), 1.16(\mathrm{~d}, J=11.3 \mathrm{~Hz}, 6 \mathrm{H})$.

${ }^{13}$ C NMR (100 MHz, $\left.\mathrm{CDCl}_{3}\right) \delta 151.4,145.3,139.8,130.9,130.4,129.9,128.8,128.6$ (2C), $127.2(2 \mathrm{C}), 127.0,126.2,126.2,121.8,117.0,112.9,110.8,61.1,55.8,48.5,48.0$, 42.6, 42.1, 27.8, 27.7.

IR (FT-ATR, $\mathrm{cm}^{-1}, \mathrm{CH}_{2} \mathrm{Cl}_{2}$ ) $v_{\max } 3423,3028,2959,2929,1725,1601,1579,1510$, 1477, 1452, 1425, 1378, 1361, 1327, 1314, 1288, 1250, 1191, 1174, 1162, 1148, 1122 , 1092, 1029, 995, 962, 928, 855, 829, 796, 739, 698, 642, 605, 566, 536, 457.

HRMS (ESI) $m / z$ : $[\mathrm{M}+\mathrm{H}]^{+}$Calcd for $\mathrm{C}_{26} \mathrm{H}_{30} \mathrm{~N}_{2} \mathrm{O}_{2}$ 403.2386; found 403.2384.

Optical: $[a]^{20} \mathrm{D}=+9.0^{\circ}\left(c=0.86, \mathrm{CHCl}_{3}, 61 \%\right.$ e.e. $)$ 
HPLC (Chiralpak IC, ${ }^{i} \mathrm{PrOH} / n$-hexane $=10 / 90$ with $0.1 \% \mathrm{Et}_{2} \mathrm{NH}$, flow rate $=1.0$ $\mathrm{mL} / \mathrm{min}, \lambda=254 \mathrm{~nm}$ ) $t_{\mathrm{R}}=40.5 \mathrm{~min}$ (major), $13.5 \mathrm{~min}$ (minor)

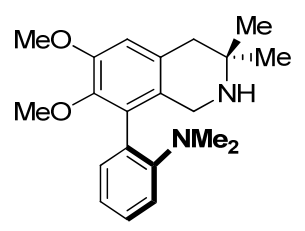

2-(6,7-Dimethoxy-3,3-dimethyl-1,2,3,4-tetrahydroisoquinolin-8-yl)- $N, N$ -

dimethylaniline (2o) was synthesized by following Procedure 19 from 1o. The crude material was purified by normal-phase column chromatography using an eluent of $10 \%$ $\mathrm{MeOH} / \mathrm{EtOAc}$ with $0.5 \%$ aqueous $\mathrm{NH}_{3}$ to give $2 \mathrm{o}$ as a colorless gum $(5.3 \mathrm{mg}, 31 \%$ yield, $60{ }^{\circ} \mathrm{C} ; 7.0 \mathrm{mg}, 41 \%$ yield, $80{ }^{\circ} \mathrm{C}$ ).

${ }^{1} \mathbf{H}$ NMR $\left(400 \mathrm{MHz}, \mathrm{CDCl}_{3}\right) \delta$ 7.33-7.24 (m, 1H), 7.06-6.93 (m, 3H), $6.60(\mathrm{~s}, 1 \mathrm{H})$, $3.87(\mathrm{~s}, 3 \mathrm{H}), 3.74-3.64(\mathrm{~m}, 4 \mathrm{H}), 3.39(\mathrm{~d}, J=17.0 \mathrm{~Hz}, 1 \mathrm{H}), 2.63(\mathrm{~s}, 2 \mathrm{H}), 2.54(\mathrm{~s}, 6 \mathrm{H})$, 1.72 (brs, $1 \mathrm{H}), 1.15(\mathrm{~d}, J=7.3 \mathrm{~Hz}, 6 \mathrm{H})$.

${ }^{13}$ C NMR $\left(100 \mathrm{MHz}, \mathrm{CDCl}_{3}\right) \delta 151.9,151.2,144.4,133.9,131.3,129.9,129.6,128.3$, 125.6, 121.1, 118.0, 112.1, 60.9, 55.7, 48.6, 43.5 (2C), 42.6, 42.2, 28.5, 26.7.

IR (FT-ATR, $\mathrm{cm}^{-1}, \mathrm{CH}_{2} \mathrm{Cl}_{2}$ ) $v_{\max } 2934,2827,2779,1678,1593,1494,1476,1463$, $1452,1426,1378,1361,1327,1314,1289,1249,1191,1174,1148,1114,1098,1048$, 1032, 996, 948, 863, 835, 798, 761, 746, 717, 639, 607, 581, 556, 540, 480, 440.

HRMS (ESI) $m / z$ : [M+H] $]^{+}$Calcd for $\mathrm{C}_{21} \mathrm{H}_{28} \mathrm{~N}_{2} \mathrm{O}_{3}$ 341.2229; found 341.2227.

Optical: $[a]^{20} \mathrm{D}=+11.7^{\circ}\left(c=0.24, \mathrm{CHCl}_{3}, 19 \%\right.$ e.e. $)$

HPLC (Chiralpak AD, ${ }^{i} \mathrm{PrOH} / n$-hexane $=3 / 97$ with $0.1 \% \mathrm{Et}_{2} \mathrm{NH}$, flow rate $=1.0$ $\mathrm{mL} / \mathrm{min}, \lambda=254 \mathrm{~nm}$ ) $t_{\mathrm{R}}=16.6 \mathrm{~min}$ (major), $7.3 \mathrm{~min}$ (minor)

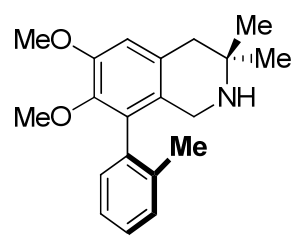

6,7-Dimethoxy-3,3-dimethyl-8-(o-tolyl)-1,2,3,4-tetrahydroisoquinoline (2q) was synthesized by following Procedure 19 from 1q. The crude material was purified by normal-phase column chromatography using an eluent of $10 \% \mathrm{MeOH} / \mathrm{EtOAc}$ with $0.5 \%$ aqueous $\mathrm{NH}_{3}$ to give $2 \mathrm{q}$ as a colorless oil $\left(7.5 \mathrm{mg}, 48 \%\right.$ yield, $\left.80{ }^{\circ} \mathrm{C}\right)$.

${ }^{1} \mathbf{H}$ NMR $\left(400 \mathrm{MHz}, \mathrm{CDCl}_{3}\right) \delta$ 7.29-7.23 (m, 2H), 7.23-7.18 (m, 1H), $7.06(\mathrm{~d}, J=7.2$ $\mathrm{Hz}, 1 \mathrm{H}), 6.62(\mathrm{~s}, 1 \mathrm{H}), 3.87(\mathrm{~s}, 3 \mathrm{H}), 3.54(\mathrm{~d}, J=17.0 \mathrm{~Hz}, 1 \mathrm{H}), 3.50(\mathrm{~s}, 3 \mathrm{H}), 3.29(\mathrm{~d}, J=$ $16.9 \mathrm{~Hz}, 1 \mathrm{H}), 2.64(\mathrm{~s}, 2 \mathrm{H}), 2.04(\mathrm{~s}, 3 \mathrm{H}), 1.15(\mathrm{~d}, J=9.6 \mathrm{~Hz}, 6 \mathrm{H})$.

${ }^{13}$ C NMR $\left(100 \mathrm{MHz}, \mathrm{CDCl}_{3}\right) \delta 151.1,144.4,136.4,136.1,133.6,130.3,123.0,129.3$, $127.5,125.6,124.9,112.2,60.7,55.8,48.4,43.0,42.1,27.9,27.5,19.8$.

IR (FT-ATR, $\mathrm{cm}^{-1}, \mathrm{CH}_{2} \mathrm{Cl}_{2}$ ) $v_{\max } 2958,2931,2395,2360,2339,1726,1593,1477$, $1427,1379,1360,1329,1250,1192,1151,1119,1092,1032,997,962,827,754,731$, 478, 453, 445, 436, 422, 417, 401, 480, 449, 434, 422, 415, 401, 411.

HRMS (EI) $m / z$ : [M] $]^{+}$Calcd for $\mathrm{C}_{20} \mathrm{H}_{25} \mathrm{NO}_{2} 311.1885$; found 311.1882 .

Optical: $[a]^{20}{ }_{\mathrm{D}}=-21.8^{\circ}(c=0.26, \mathrm{MeOH}, 60 \%$ e.e. $)$ 
HPLC (Chiralpak IC, ${ }^{i} \mathrm{PrOH} / n$-hexane $=10 / 90$ with $0.1 \% \mathrm{Et}_{2} \mathrm{NH}$, flow rate $=1.0$ $\mathrm{mL} / \mathrm{min}, \lambda=254 \mathrm{~nm}$ ) $t_{\mathrm{R}}=9.1 \mathrm{~min}$ (major), $20.0 \mathrm{~min}$ (minor)

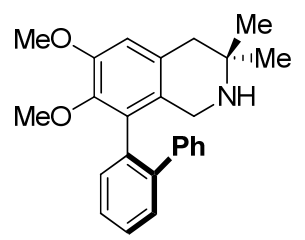

8-([1,1'-Biphenyl]-2-yl)-6,7-dimethoxy-3,3-dimethyl-1,2,3,4-

tetrahydroisoquinoline (2r) was synthesized by following Procedure 19 from 1r. The crude material was purified by normal-phase column chromatography using an eluent of $10 \% \mathrm{MeOH} / \mathrm{EtOAc}$ with $0.5 \%$ aqueous $\mathrm{NH}_{3}$ to give $2 \mathrm{r}$ as a colorless gum $(2.5 \mathrm{mg}$, $13 \%$ yield, $60{ }^{\circ} \mathrm{C} ; 4.9 \mathrm{mg}, 27 \%$ yield, $80{ }^{\circ} \mathrm{C}$ ).

${ }^{1} \mathbf{H}$ NMR $\left(400 \mathrm{MHz}, \mathrm{CDCl}_{3}\right) \delta$ 7.46-7.42 (m, 2H), 7.42-7.36 (m, 1H), 7.24-7.18 (m, $3 \mathrm{H}), 7.18-7.08(\mathrm{~m}, 3 \mathrm{H}), 6.52(\mathrm{~s}, 1 \mathrm{H}), 3.84(\mathrm{~s}, 3 \mathrm{H}), 3.67(\mathrm{~s}, 3 \mathrm{H}), 3.32(\mathrm{~d}, J=17.0 \mathrm{~Hz}$, $1 \mathrm{H}), 3.17(\mathrm{~d}, J=17.0 \mathrm{~Hz}, 1 \mathrm{H}), 2.56(\mathrm{~d}, J=16.3 \mathrm{~Hz}, 1 \mathrm{H}), 2.40(\mathrm{~d}, J=16.1 \mathrm{~Hz}, 1 \mathrm{H})$, $1.11(\mathrm{~s}, 3 \mathrm{H}), 0.65(\mathrm{~s}, 3 \mathrm{H})$.

${ }^{13}$ C NMR $\left(100 \mathrm{MHz}, \mathrm{CDCl}_{3}\right) \delta 151.0,145.1,141.5,141.3,134.9,133.6,130.1,130.1$, 129.6, 129.0 (2C), 128.9, 128.0, 127.8 (2C), 127.2, 126.7, 112.5, 61.0, 55.8, 42.8, 41.7, 29.3, 29.1, 24.7.

IR (FT-ATR, $\mathrm{cm}^{-1}, \mathrm{CH}_{2} \mathrm{Cl}_{2}$ ) $v_{\max } 3635,3057,2960,2933,2360,2339,1728,1595$, $1479,1427,1379,1331,1290,1255,1192,1151,1117,1093,1034,997,843,777,742$, 702, 617, 523, 488, 467, 447, 434, 417, 409, 401, 445, 434, 422, 411.

HRMS (EI) $m / z$ : [M] ${ }^{+}$Calcd for $\mathrm{C}_{25} \mathrm{H}_{27} \mathrm{NO}_{2} 373.2042$; found 373.2037.

Optical: $[a]^{20} \mathrm{D}=-6.3^{\circ}(c=0.076, \mathrm{MeOH}, 80 \%$ e.e. $)$

HPLC (Chiralpak AD-H, ${ }^{i} \mathrm{PrOH} / n$-hexane $=3 / 97$ with $0.1 \% \mathrm{Et}_{2} \mathrm{NH}$, flow rate $=1.0$ $\mathrm{mL} / \mathrm{min}, \lambda=254 \mathrm{~nm}$ ) $t_{\mathrm{R}}=15.9 \mathrm{~min}$ (major), $19.1 \mathrm{~min}$ (minor)

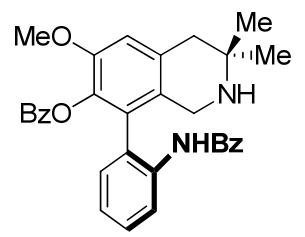

8-(2-Benzamidophenyl)-6-methoxy-3,3-dimethyl-1,2,3,4-tetrahydroisoquinolin-7yl benzoate (2s) was synthesized by following Procedure 19 from 1s. The crude material was purified by normal-phase column chromatography using an eluent of $10 \%$ $\mathrm{MeOH} / \mathrm{EtOAc}$ with $1 \%$ aqueous $\mathrm{NH}_{3}$ to give $2 \mathrm{~s}$ as a yellow gum $(11.9 \mathrm{mg}, 47 \%$ yield, $60{ }^{\circ} \mathrm{C} ; 11.7 \mathrm{mg}, 46 \%$ yield, $80{ }^{\circ} \mathrm{C}$ ).

${ }^{1} \mathbf{H}$ NMR $\left(400 \mathrm{MHz}, \mathrm{CDCl}_{3}\right) \delta 8.13(\mathrm{~d}, J=14.5 \mathrm{~Hz}, 1 \mathrm{H}), 7.92(\mathrm{~d}, J=6.5 \mathrm{~Hz}, 2 \mathrm{H}), 7.75$ $(\mathrm{d}, J=4.2 \mathrm{~Hz}, 2 \mathrm{H}), 7.52(\mathrm{t}, J=7.0 \mathrm{~Hz}, 1 \mathrm{H}), 7.49-7.43(\mathrm{~m}, 1 \mathrm{H}), 7.43-7.28(\mathrm{~m}, 6 \mathrm{H})$, $7.16(\mathrm{~s}, 2 \mathrm{H}), 6.76(\mathrm{~s}, 1 \mathrm{H}), 3.84(\mathrm{~s}, 3 \mathrm{H}), 3.51(\mathrm{~s}, 2 \mathrm{H}), 2.65(\mathrm{dd}, J=48.5,16.0 \mathrm{~Hz}, 2 \mathrm{H})$, $1.14(\mathrm{~s}, 3 \mathrm{H}), 0.89(\mathrm{~s}, 3 \mathrm{H})$.

${ }^{13}$ C NMR (100 MHz, $\left.\mathrm{CDCl}_{3}\right) \delta 165.3,149.9,136.2,135.7,134.6,133.9,133.7,131.9$, $131.7,130.6$ (2C), 130.2, 129.5, 129.3, 129.0, 128.8 (2C), 128.7, 128.5 (2C), 128.3, $128.0,127.3$ (2C), 126.0, 125.0, 113.2, 56.2, 48.4, 42.3, 42.2, 28.8, 25.8. 
IR (FT-ATR, $\mathrm{cm}^{-1}, \mathrm{CH}_{2} \mathrm{Cl}_{2}$ ) $v_{\max } 3401,3065,2960,2925,2853,1721,1675,1602$, $1582,1523,1494,1475,1452,1380,1362,1333,1306,1250,1239,1216,1176,1153$, 1081, 1063, 1026, 839, 799, 760, 709, 640, 583, 505.

HRMS (ESI) $m / z$ : $[\mathrm{M}+\mathrm{H}]+$ Calcd for $\mathrm{C}_{32} \mathrm{H}_{30} \mathrm{~N}_{2} \mathrm{O}_{4}$ 507.2284; found 507.2279.

Optical: $[a]^{20} \mathrm{D}=-94.7^{\circ}\left(c=0.34, \mathrm{CHCl}_{3}, 99 \%\right.$ e.e. $)$

HPLC (Chiralpak AD, ${ }^{i} \mathrm{PrOH} / n$-hexane $=30 / 70$ with $0.1 \% \mathrm{Et}_{2} \mathrm{NH}$, flow rate $=1.0$ $\mathrm{mL} / \mathrm{min}, \lambda=254 \mathrm{~nm}$ ) $t_{\mathrm{R}}=36.3 \mathrm{~min}$ (major), $29.5 \mathrm{~min}$ (minor)

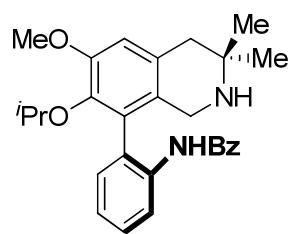

$\mathrm{N}$-(2-(7-Isopropoxy-6-methoxy-3,3-dimethyl-1,2,3,4-tetrahydroisoquinolin-8-

yl)phenyl)benzamide (2t) was synthesized by following Procedure 19 from 1t. The crude material was purified by normal-phase column chromatography using an eluent of $10 \% \mathrm{MeOH} / \mathrm{EtOAc}$ with $0.5 \%$ aqueous $\mathrm{NH}_{3}$ to give $\mathbf{2 t}$ as a colorless gum (10.9 $\mathrm{mg}$, $49 \%$ yield, $60{ }^{\circ} \mathrm{C} ; 16.9 \mathrm{mg}, 76 \%$ yield, $80{ }^{\circ} \mathrm{C}$ ).

${ }^{1}$ H NMR $\left(400 \mathrm{MHz}, \mathrm{CDCl}_{3}\right) \delta 8.31(\mathrm{~d}, J=8.1 \mathrm{~Hz}, 1 \mathrm{H}), 8.22(\mathrm{~s}, 1 \mathrm{H}), 7.64(\mathrm{~d}, J=8.1$ $\mathrm{Hz}, 2 \mathrm{H}), 7.47-7.33(\mathrm{~m}, 4 \mathrm{H}), 7.18(\mathrm{~d}, J=4.2 \mathrm{~Hz}, 2 \mathrm{H}), 6.66$ (s, 1H), 4.11 (p, $J=6.2 \mathrm{~Hz}$, $1 \mathrm{H}), 3.89-3.78(\mathrm{~m}, 4 \mathrm{H}), 3.33(\mathrm{~d}, J=17.0 \mathrm{~Hz}, 1 \mathrm{H}), 2.67-2.54(\mathrm{~m}, 2 \mathrm{H}), 1.38$ (brs, $1 \mathrm{H})$, $1.17(\mathrm{~s}, 3 \mathrm{H}), 1.11(\mathrm{~d}, J=6.1 \mathrm{~Hz}, 3 \mathrm{H}), 1.07(\mathrm{~s}, 3 \mathrm{H}), 0.80(\mathrm{~d}, J=6.1 \mathrm{~Hz}, 3 \mathrm{H})$.

${ }^{13} \mathrm{C}$ NMR $\left(100 \mathrm{MHz}, \mathrm{CDCl}_{3}\right) \delta 165.3,151.7,142.3,135.7,135.1,131.6,131.5,130.9$, $130.3,128.7$ (2C), 128.6, 128.2, 127.1 (2C), 126.0, 124.1, 122.6, 113.5, 76.4, 56.0, 48.3, 43.2, 42.1, 28.7, 26.9, 22.7, 22.3.

IR (FT-ATR, $\left.\mathrm{cm}^{-1}, \mathrm{CH}_{2} \mathrm{Cl}_{2}\right) v_{\max } 3415,3063,2968,2930,1673,1580,1520,1494$, 1444, 1379, 1328, 1306, 1248, 1190, 1175, 1154, 1139, 1104, 1013, 970, 937, 842, 795, 756, 708, 640, 585, 482 .

HRMS (ESI) $m / z$ : $[\mathrm{M}+\mathrm{H}]^{+}$Calcd for $\mathrm{C}_{28} \mathrm{H}_{32} \mathrm{~N}_{2} \mathrm{O}_{3} 445.2491$; found 445.2491 .

Optical: $[a]^{20} \mathrm{D}=-43.6^{\circ}\left(c=0.50, \mathrm{CHCl}_{3}, 89 \%\right.$ e.e. $)$

HPLC (Chiralpak IC, ${ }^{i} \mathrm{PrOH} / n$-hexane $=20 / 80$ with $0.1 \% \mathrm{Et}_{2} \mathrm{NH}$, flow rate $=1.0$ $\mathrm{mL} / \mathrm{min}, \lambda=254 \mathrm{~nm}$ ) $t_{\mathrm{R}}=11.5 \mathrm{~min}$ (major), $20.2 \mathrm{~min}$ (minor)

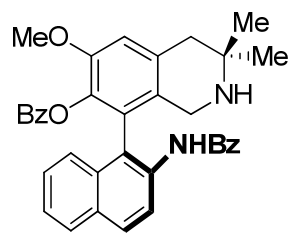

8-(2-Benzamidonaphthalen-1-yl)-6-methoxy-3,3-dimethyl-1,2,3,4-

tetrahydroisoquinolin-7-yl benzoate (2v) was synthesized by following Procedure 19 from 1v. The crude material was purified by normal-phase column chromatography using an eluent of $10 \% \mathrm{MeOH} / \mathrm{EtOAc}$ with $1 \%$ aqueous $\mathrm{NH}_{3}$ to give $2 \mathbf{v}$ as a white solid (9.2 mg, 33\% yield, $60{ }^{\circ} \mathrm{C} ; 12.0 \mathrm{mg}, 43 \%$ yield, $80{ }^{\circ} \mathrm{C}$ ).

m.p. $198^{\circ} \mathrm{C}$

${ }^{1} \mathbf{H}$ NMR $\left(400 \mathrm{MHz}, \mathrm{CDCl}_{3}\right) \delta 8.44(\mathrm{~s}, 1 \mathrm{H}), 8.13(\mathrm{~s}, 1 \mathrm{H}), 7.82(\mathrm{t}, J=7.6 \mathrm{~Hz}, 4 \mathrm{H}), 7.67$ $(\mathrm{d}, J=7.5 \mathrm{~Hz}, 2 \mathrm{H}), 7.57-7.34(\mathrm{~m}, 7 \mathrm{H}), 7.23(\mathrm{t}, J=7.7 \mathrm{~Hz}, 2 \mathrm{H}), 6.84(\mathrm{~s}, 1 \mathrm{H}), 3.88(\mathrm{~s}$, 
$3 \mathrm{H}), 3.54(\mathrm{~d}, J=17.0 \mathrm{~Hz}, 1 \mathrm{H}), 3.18(\mathrm{~d}, J=17.2 \mathrm{~Hz}, 1 \mathrm{H}), 2.75(\mathrm{~d}, J=16.0 \mathrm{~Hz}, 1 \mathrm{H})$, $2.60(\mathrm{~d}, J=16.2 \mathrm{~Hz}, 1 \mathrm{H}), 1.69(\mathrm{brs}, 1 \mathrm{H}), 1.11(\mathrm{~s}, 3 \mathrm{H}), 0.86(\mathrm{~s}, 3 \mathrm{H})$.

${ }^{13} \mathrm{C}$ NMR $\left(100 \mathrm{MHz}, \mathrm{CDCl}_{3}\right) \delta 165.5,150.0,136.9,134.5,134.4,133.9,133.6,132.0$, 131.8, 131.3, 130.1 (2C), 129.0, 128.7 (2C), 128.6, 128.4 (3C), 128.1 (2C), $127.4(2 \mathrm{C})$, $126.8,126.6,125.4,125.0,123.2,123.1,113.4,56.2,48.4,42.3,42.1,29.8,29.0$.

IR (FT-ATR, $\mathrm{cm}^{-1}, \mathrm{CH}_{2} \mathrm{Cl}_{2}$ ) $v_{\max } 3385,3062,2960,2923,2852,1720,1675,1598$, $1583,1523,1503,1488,1462,1427,1380,1328,1316,1273,1234,1214,1177,1152$, $1118,1084,1067,1025,1000,902,860,815,798,782,749,706,654,639,584,528$, 427.

HRMS (ESI) $m / z$ : $[\mathrm{M}+\mathrm{H}]^{+}$Calcd for $\mathrm{C}_{36} \mathrm{H}_{32} \mathrm{~N}_{2} \mathrm{O}_{4}$ 557.2440; found 557.2438.

Optical: $[a]^{20} \mathrm{D}=-6.61^{\circ}\left(c=0.62, \mathrm{CHCl}_{3}, 99 \%\right.$ e.e. $)$

HPLC (Chiralpak AD, ${ }^{i} \mathrm{PrOH} / n$-hexane $=50 / 50$ with $0.1 \% \mathrm{Et}_{2} \mathrm{NH}$, flow rate $=1.0$ $\mathrm{mL} / \mathrm{min}, \lambda=254 \mathrm{~nm}$ ) $t_{\mathrm{R}}=10.2 \mathrm{~min}$ (major), $23.3 \mathrm{~min}$ (minor)

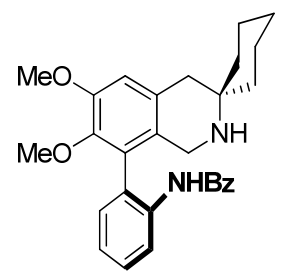

$N$-(2-(6',7'-Dimethoxy-2',4'-dihydro-1' $H$-spiro[cyclohexane-1,3'-isoquinolin]-8'yl)phenyl)benzamide (2w) was synthesized by following Procedure 19 from $\mathbf{1 w}$. The crude material was purified by normal-phase column chromatography using an eluent of $10 \% \mathrm{MeOH} / \mathrm{EtOAc}$ with $1 \%$ aqueous $\mathrm{NH}_{3}$ to give $2 \mathrm{w}$ as a white solid $(19.6 \mathrm{mg}, 86 \%$ yield, $\left.60^{\circ} \mathrm{C}\right)$.

m.p. $64{ }^{\circ} \mathrm{C}$

${ }^{1} \mathbf{H}$ NMR $\left(400 \mathrm{MHz}, \mathrm{CDCl}_{3}\right) \delta 8.42(\mathrm{~d}, J=8.0 \mathrm{~Hz}, 1 \mathrm{H}), 7.91(\mathrm{~s}, 1 \mathrm{H}), 7.57(\mathrm{~d}, J=7.3$ $\mathrm{Hz}, 2 \mathrm{H}), 7.44(\mathrm{t}, J=6.2 \mathrm{~Hz}, 2 \mathrm{H}), 7.35(\mathrm{t}, J=7.4 \mathrm{~Hz}, 2 \mathrm{H}), 7.23-7.12(\mathrm{~m}, 2 \mathrm{H}), 6.72(\mathrm{~s}$, $1 \mathrm{H}), 3.90(\mathrm{~s}, 3 \mathrm{H}), 3.67(\mathrm{~d}, J=16.8 \mathrm{~Hz}, 1 \mathrm{H}), 3.54(\mathrm{~s}, 3 \mathrm{H}), 3.31(\mathrm{~d}, J=16.8 \mathrm{~Hz}, 1 \mathrm{H})$, $2.63(\mathrm{q}, J=16.3 \mathrm{~Hz}, 2 \mathrm{H}), 7.61(\mathrm{brs}, 1 \mathrm{H}), 1.56-1.27(\mathrm{~m}, 10 \mathrm{H})$.

${ }^{13}$ C NMR $\left(100 \mathrm{MHz}, \mathrm{CDCl}_{3}\right) \delta 165.2,151.3,144.3,135.7,135.0,131.7,131.6,130.1$, $129.3,128.8,128.7$ (2C), 127.0 (2C), 126.9, 126.3, 124.2, 121.8, 113.8, 61.2, 56.0, 49.8, 41.8, 40.6, 36.3, 35.3, 26.3, 22.0, 21.9.

IR (FT-ATR, $\mathrm{cm}^{-1}, \mathrm{CH}_{2} \mathrm{Cl}_{2}$ ) v $v_{\max } 3413,3319,3064,2926,2852,1717,1677,1581$, 1521, 1494, 1478, 1451, 1425, 1348, 1309, 1258, 1191, 1154, 1101, 1079, 1028, 966, $918,895,841,797,756,735,706,641,584,528,491,449,437$.

HRMS (ESI) $m / z$ : $[\mathrm{M}+\mathrm{H}]^{+}$Calcd for $\mathrm{C}_{29} \mathrm{H}_{32} \mathrm{~N}_{2} \mathrm{O}_{3} 457.2491$; found 457.2491.

Optical: $[a]^{20}{ }_{\mathrm{D}}=+13.2^{\circ}\left(c=0.97, \mathrm{CHCl}_{3}, 96 \%\right.$ e.e. $)$

HPLC (Chiralpak AD, ${ }^{i} \mathrm{PrOH} / n$-hexane $=10 / 90$ with $0.1 \% \mathrm{Et}_{2} \mathrm{NH}$, flow rate $=1.0$ $\mathrm{mL} / \mathrm{min}, \lambda=254 \mathrm{~nm}$ ) $t_{\mathrm{R}}=61.7 \mathrm{~min}$ (major), $28.3 \mathrm{~min}$ (minor) 


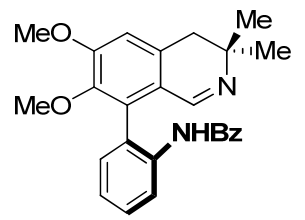

$N$-(2-(6,7-Dimethoxy-3,3-dimethyl-3,4-dihydroisoquinolin-8-

yl)phenyl)benzamide (3a) was synthesized by following Procedure 19 from 1a. The crude material was purified by normal-phase column chromatography using an eluent of $25 \% \mathrm{EtOAc} / \mathrm{Hx}$ to give $\mathbf{3 a}$ as a yellow gum.

${ }^{1} \mathbf{H}$ NMR $\left(400 \mathrm{MHz}, \mathrm{CDCl}_{3}\right) \delta 8.30(\mathrm{~d}, J=7.9 \mathrm{~Hz}, 1 \mathrm{H}), 8.24(\mathrm{~s}, 1 \mathrm{H}), 7.78(\mathrm{~s}, 1 \mathrm{H}), 7.66$ $(\mathrm{d}, J=7.3 \mathrm{~Hz}, 2 \mathrm{H}), 7.52-7.41(\mathrm{~m}, 2 \mathrm{H}), 7.37(\mathrm{t}, J=7.4 \mathrm{~Hz}, 2 \mathrm{H}), 7.23(\mathrm{t}, J=7.5 \mathrm{~Hz}$, $1 \mathrm{H}), 7.16(\mathrm{~d}, J=7.5 \mathrm{~Hz}, 1 \mathrm{H}), 6.75(\mathrm{~s}, 1 \mathrm{H}), 3.96(\mathrm{~s}, 3 \mathrm{H}), 3.56(\mathrm{~s}, 3 \mathrm{H}), 2.68(\mathrm{~s}, 2 \mathrm{H}), 1.21$ $(\mathrm{d}, J=12.3 \mathrm{~Hz}, 6 \mathrm{H})$.

${ }^{13}$ C NMR $\left(100 \mathrm{MHz}, \mathrm{CDCl}_{3}\right) \delta 165.4,155.1,155.0,144.4,136.1,135.0,134.4,131.9$, 131.7, 131.6, 129.4, 128.8 (2C), 127.1 (2C), 125.6, 124.4, 123.1, 119.5, 112.2, 61.3, $56.2,53.8,38.9,29.0,26.8$.

IR (FT-ATR, $\mathrm{cm}^{-1}, \mathrm{CH}_{2} \mathrm{Cl}_{2}$ ) $v_{\max } 3421,2962,2927,2856,1711,1673,1625,1587$, $1520,1465,1446,1431,1384,1343,1316,1304,1253,1193,1177,1156,1116,1073$, 1029, 997, 971, 881, 862, 836, 820, 796, 757, 709, 649, 625, 580, 474.

HRMS (ESI) $m / z$ : [M+H]+ Calcd for $\mathrm{C}_{26} \mathrm{H}_{26} \mathrm{~N}_{2} \mathrm{O}_{3}$ 415.2022; found 415.2016. 


\subsection{NMR Spectra of 2}

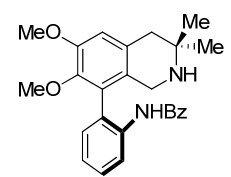

$2 \mathbf{a}$

$\left({ }^{1} \mathrm{H} \mathrm{NMR}, 400 \mathrm{MHz}, \mathrm{CDCl}_{3}\right)$

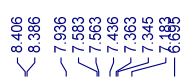

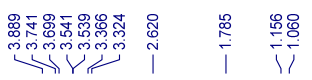
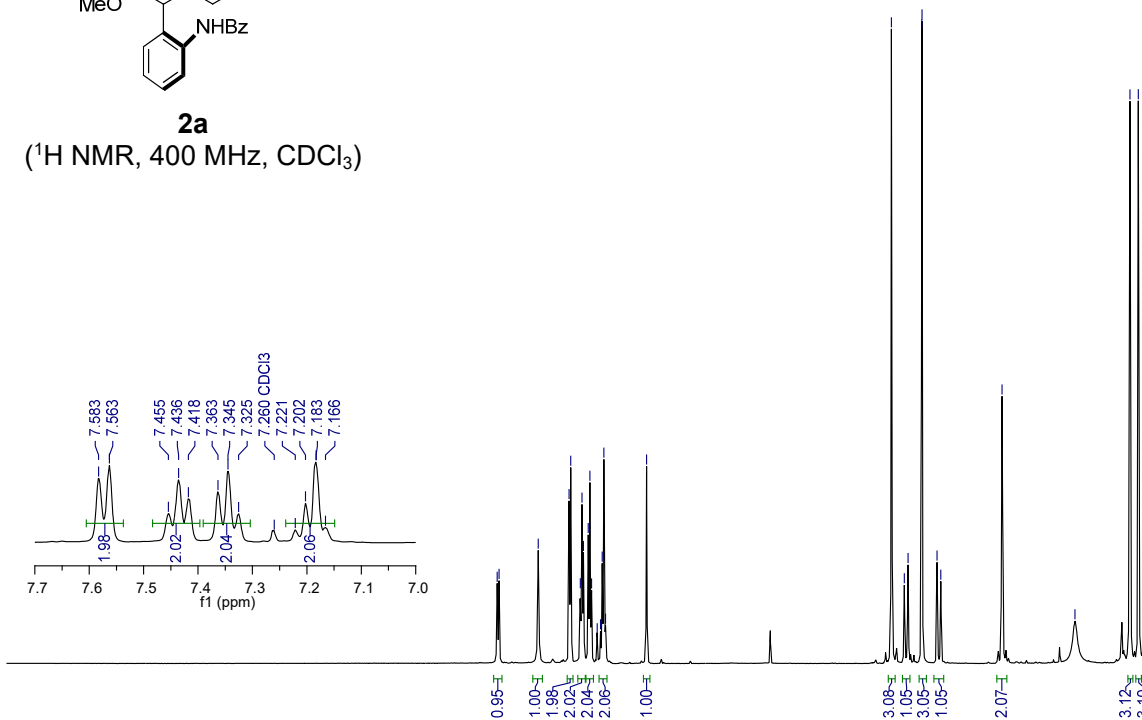

14

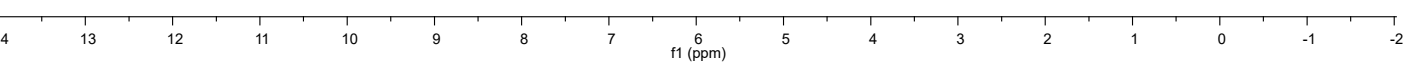

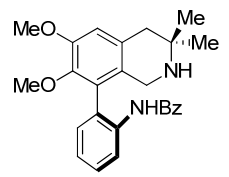

$2 a$

$\left({ }^{13} \mathrm{C}\right.$ NMR, $\left.100 \mathrm{MHz}, \mathrm{CDCl}_{3}\right)$

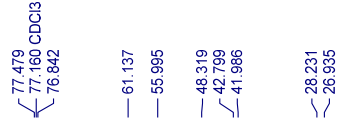

|

।

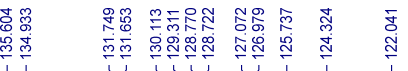

II Y I Y Y I I I I

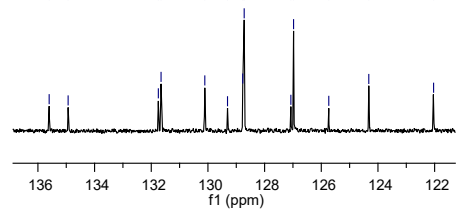




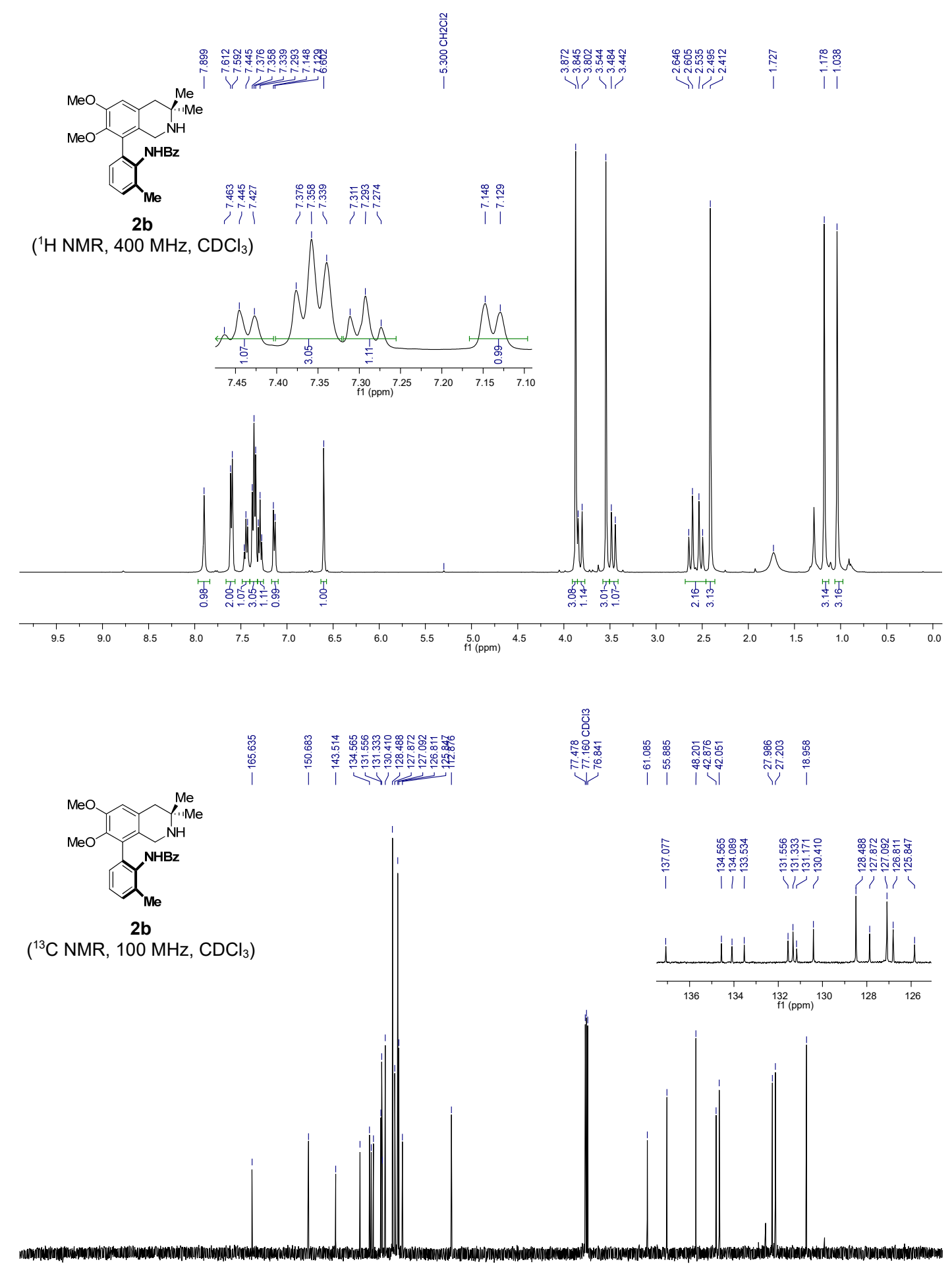

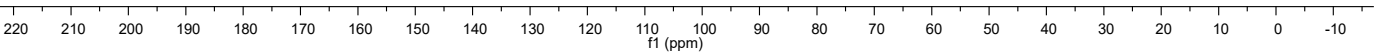




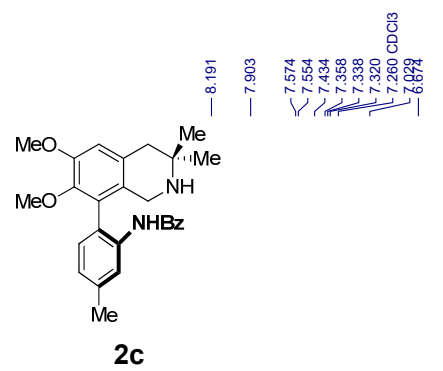

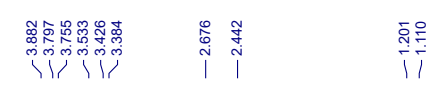

$\left({ }^{1} \mathrm{H} \mathrm{NMR}, 400 \mathrm{MHz}, \mathrm{CDCl}_{3}\right)$
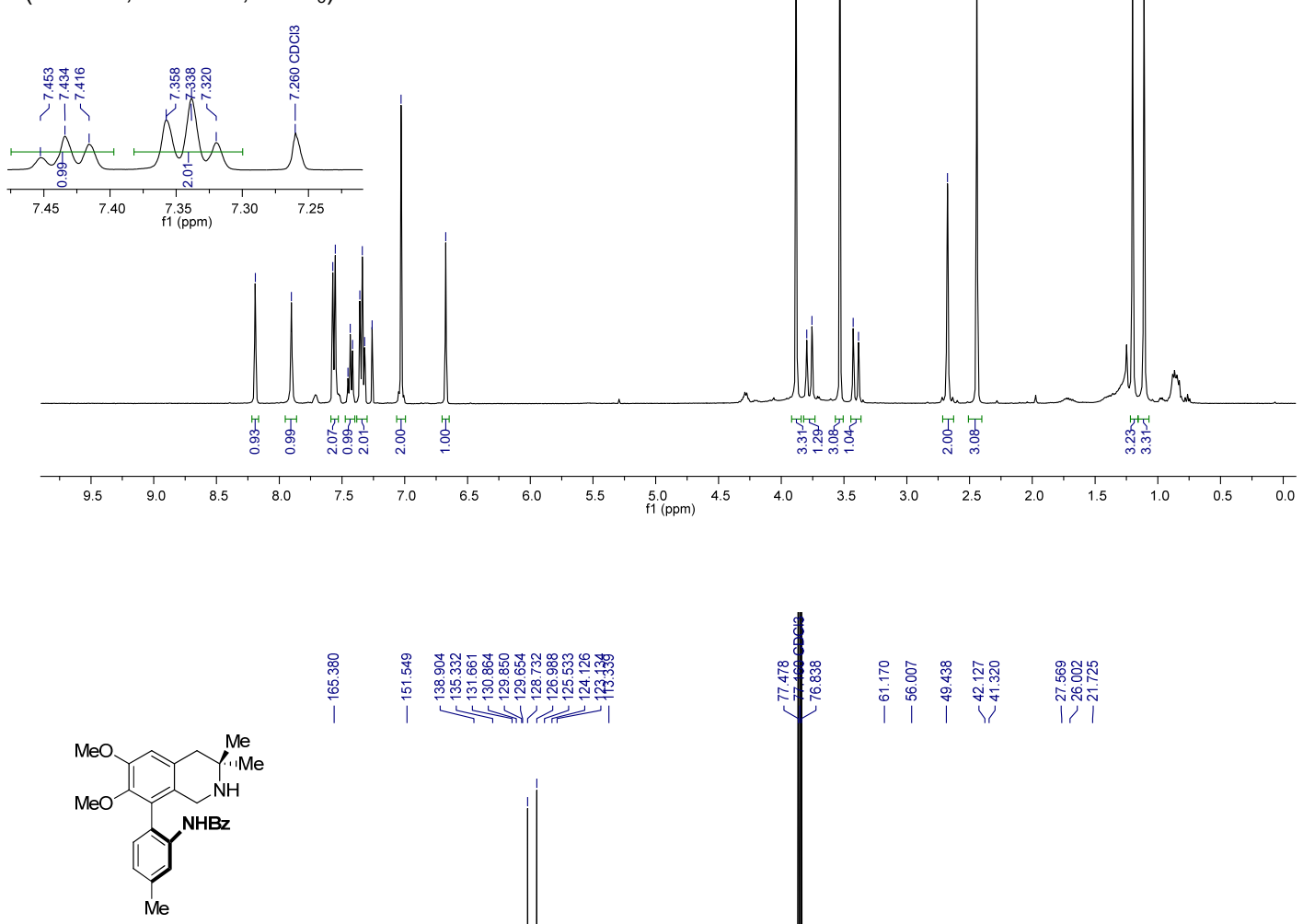

2c

$\left({ }^{13} \mathrm{C} \mathrm{NMR}, 100 \mathrm{MHz}, \mathrm{CDCl}_{3}\right)$

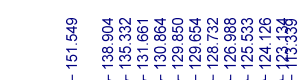

i

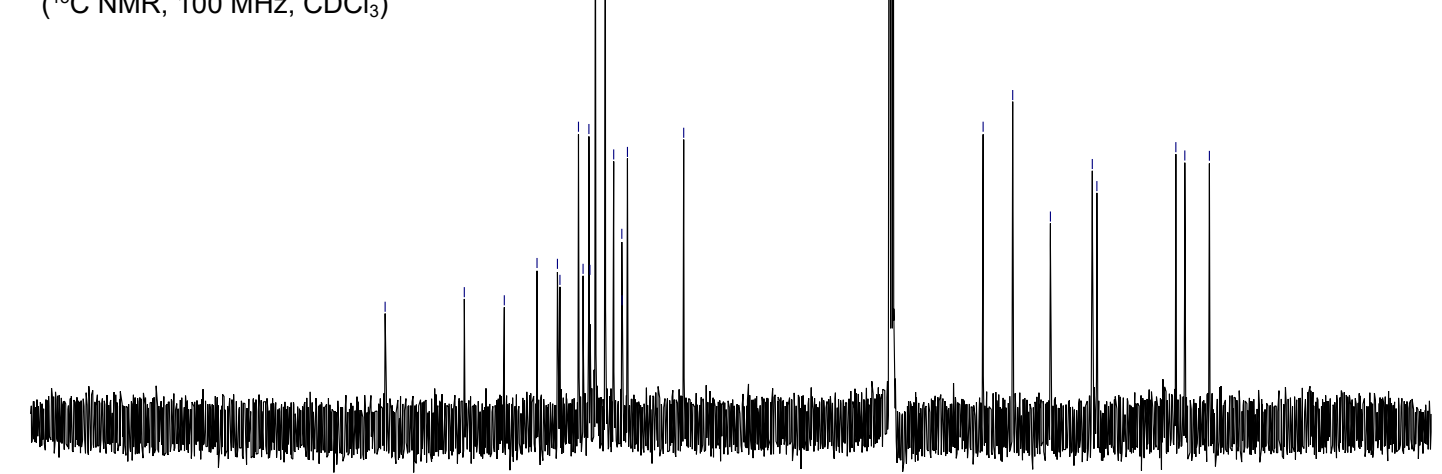

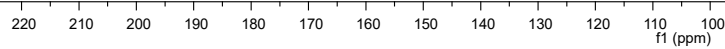



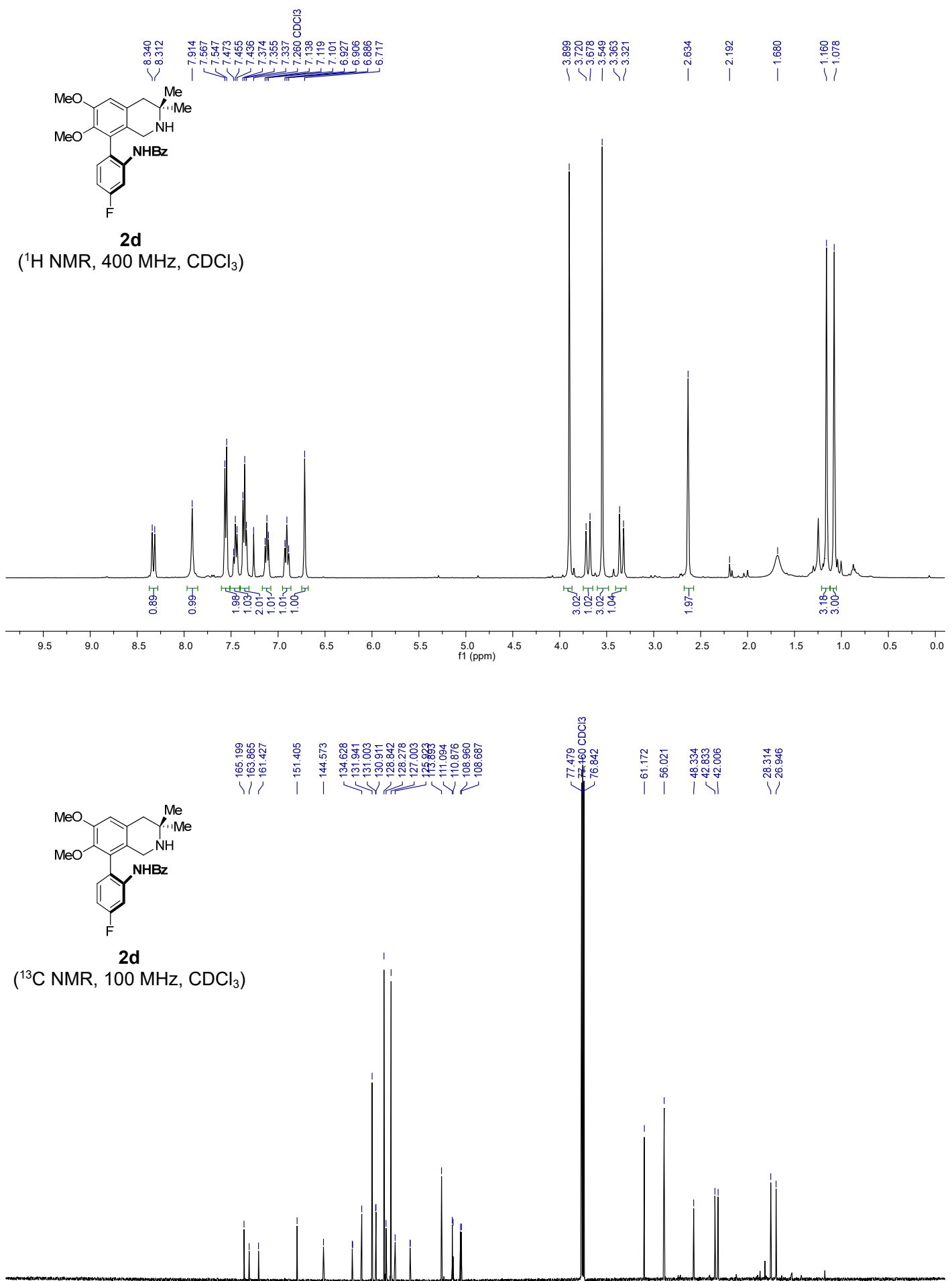

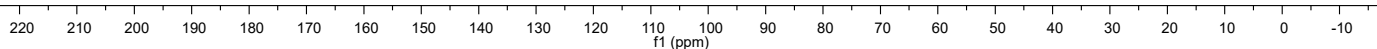



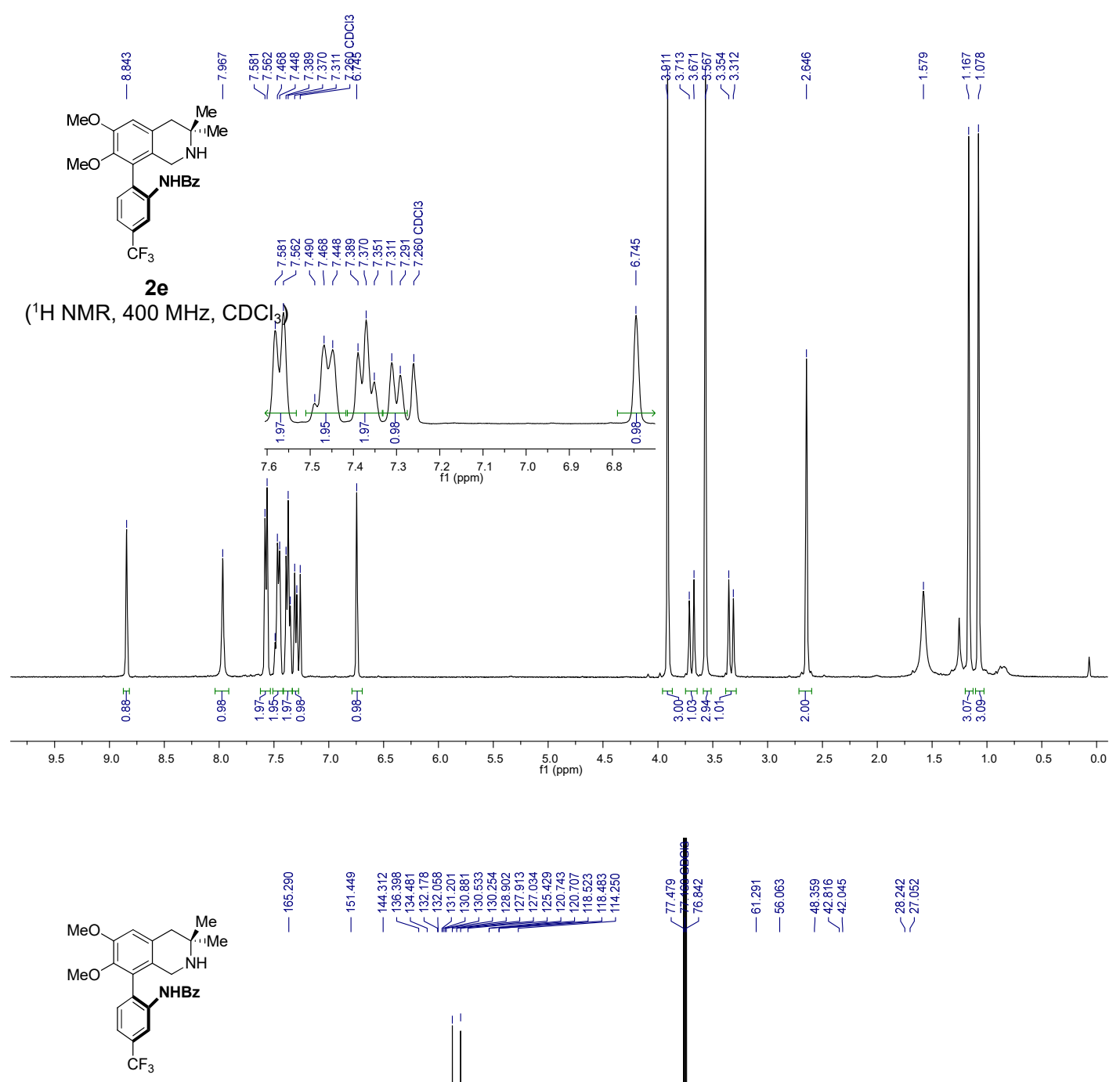

$\left({ }^{13} \mathrm{C} \mathrm{NMR,}, 100 \mathrm{MHz}, \mathrm{CDCl}_{3}\right)$

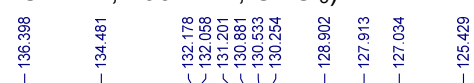

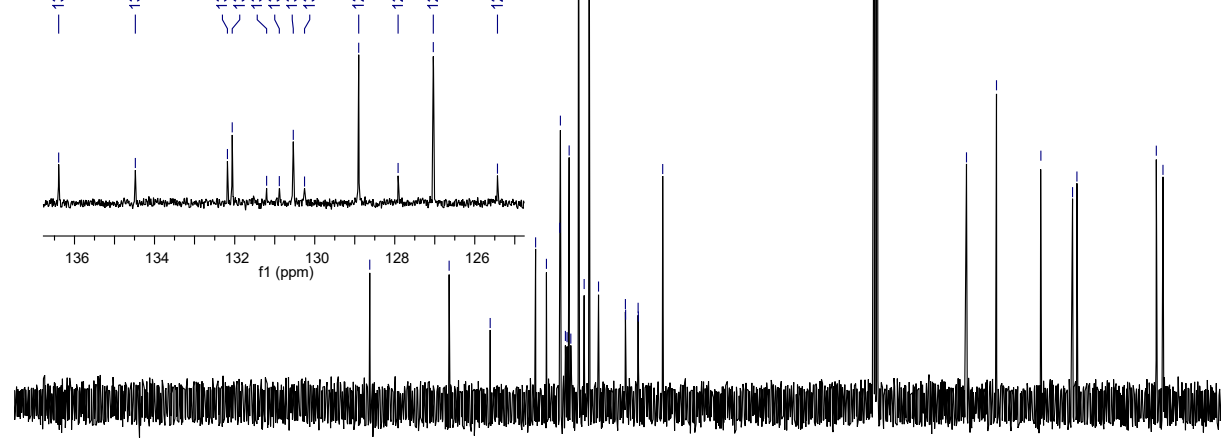

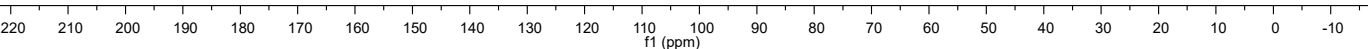



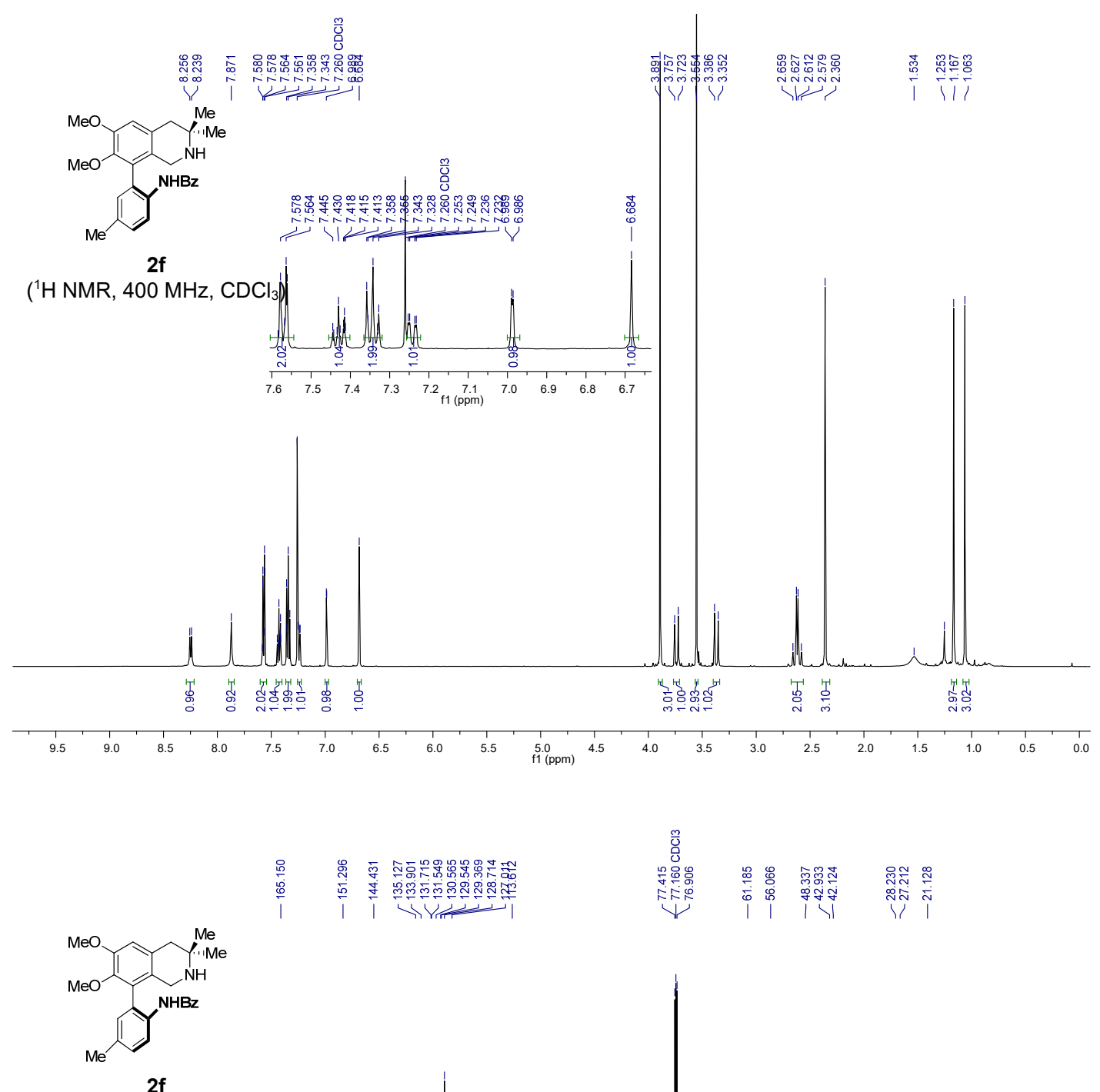

$\left({ }^{13} \mathrm{C}\right.$ NMR, $\left.100 \mathrm{MHz}, \mathrm{CDCl}_{3}\right)$

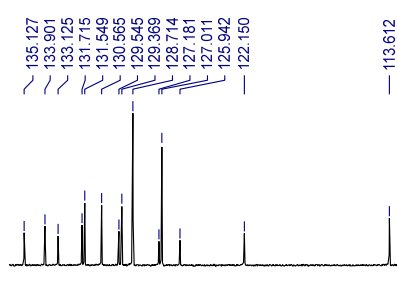

|

i j
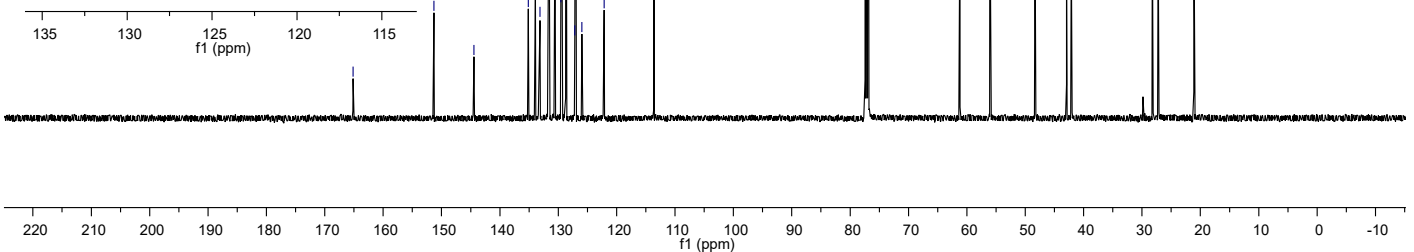


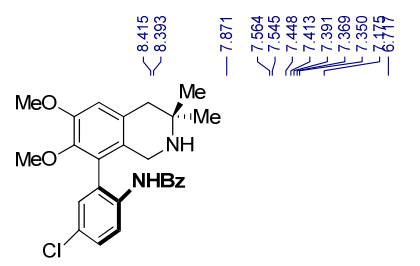

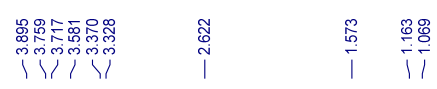

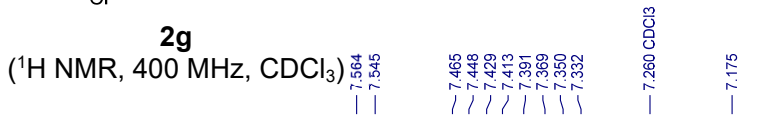
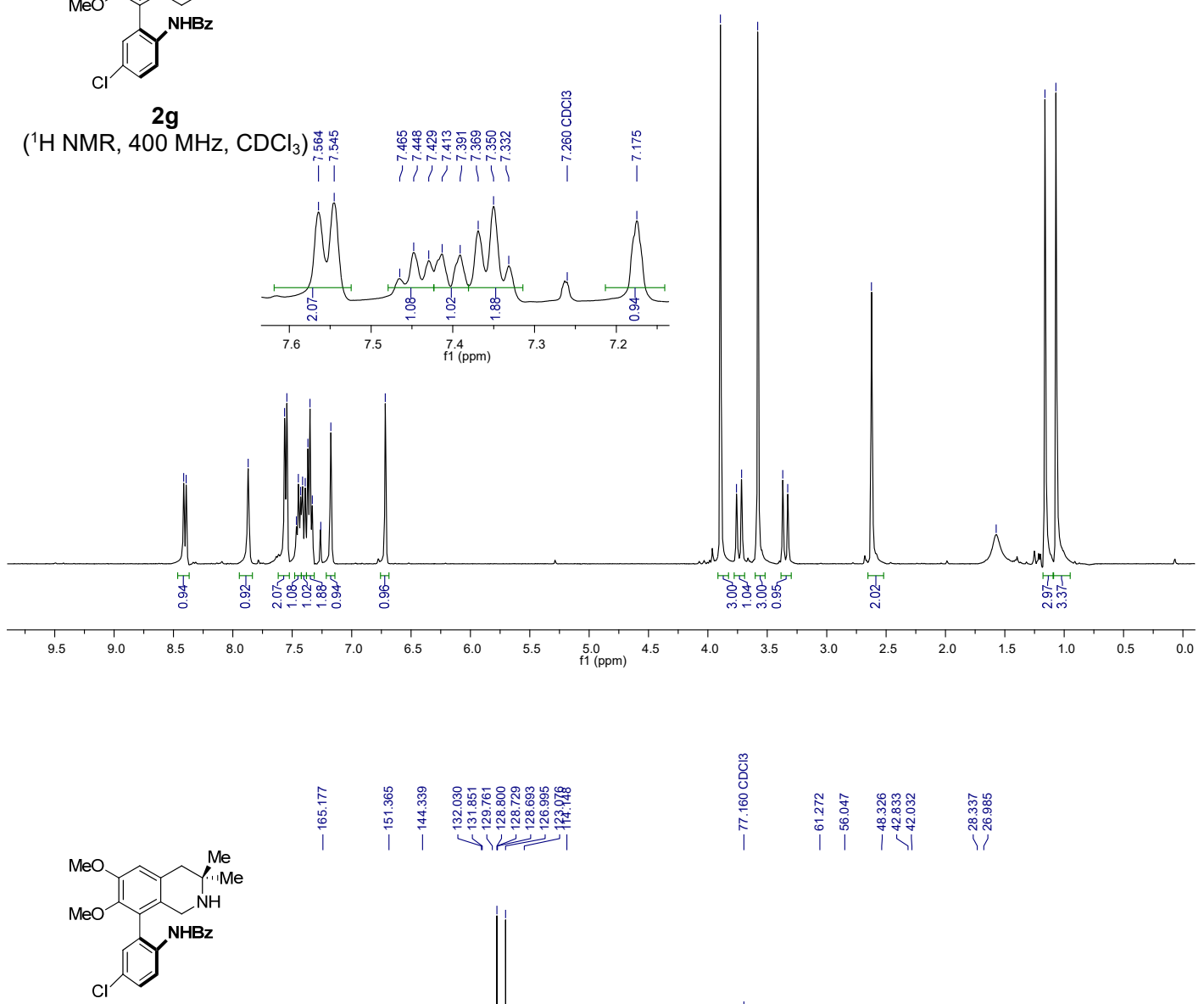

$2 \mathrm{~g}$

$\left({ }^{13} \mathrm{C} \mathrm{NMR}, 100 \mathrm{MHz}, \mathrm{CDCl}_{3}\right)$

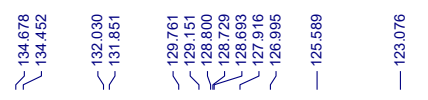

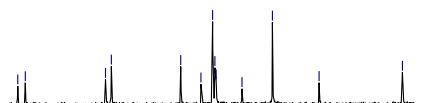

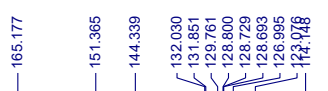

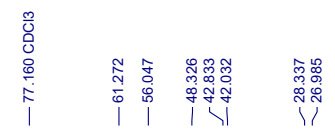

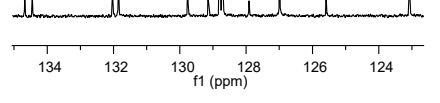

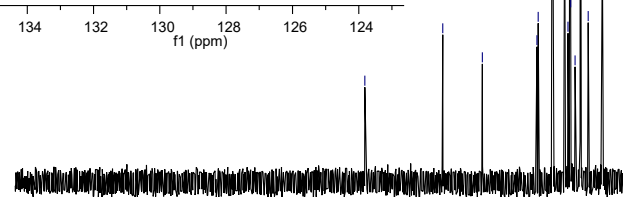

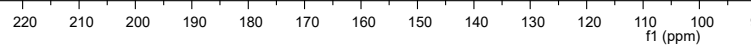




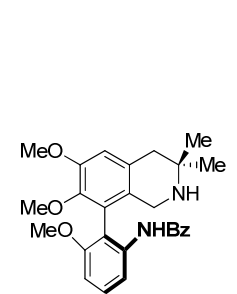

2h

( ${ }^{1} \mathrm{H} \mathrm{NMR}, 400 \mathrm{MHz}, \mathrm{CDCl}_{3}$ )

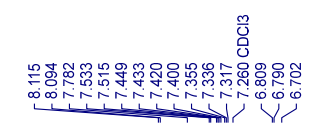

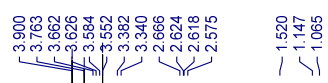

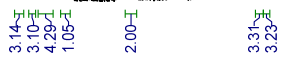

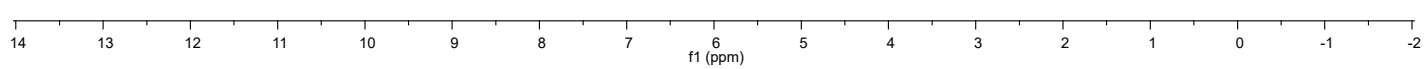

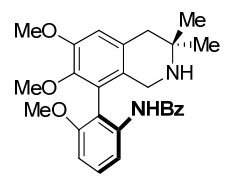

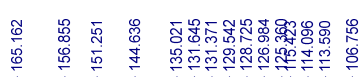

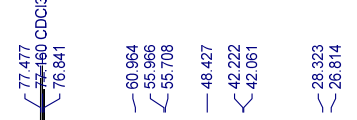

2h

$\left({ }^{13} \mathrm{C} \mathrm{NMR}, 100 \mathrm{MHz}, \mathrm{CDCl}_{3}\right)$

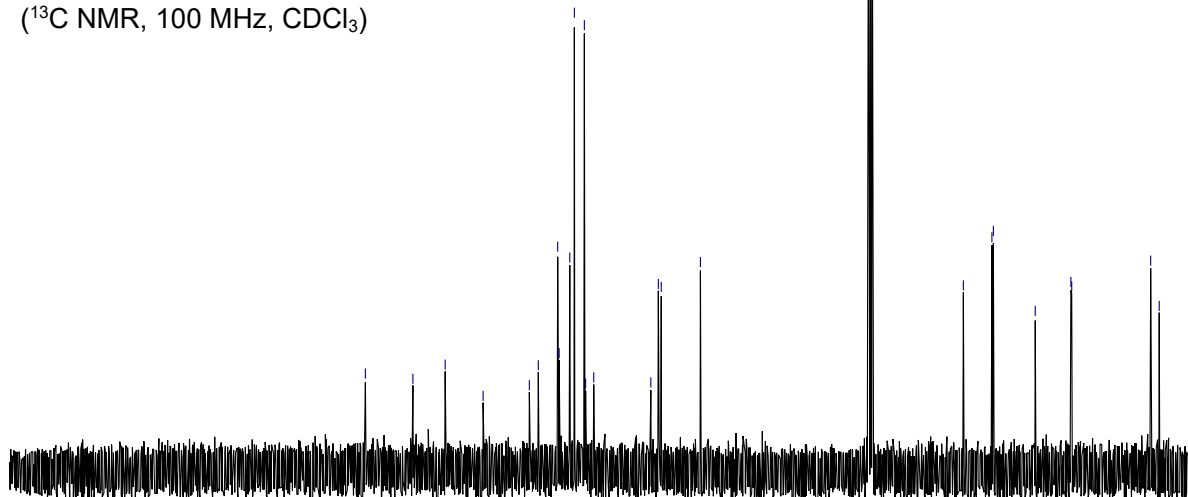

$\begin{array}{lllllllllllll}220 & 210 & 200 & 190 & 180 & 170 & 160 & 150 & 140 & 130 & 120 & \begin{array}{c}110 \\ \mathrm{f} 1(\mathrm{ppm})\end{array} \\ 100 & \end{array}$ 

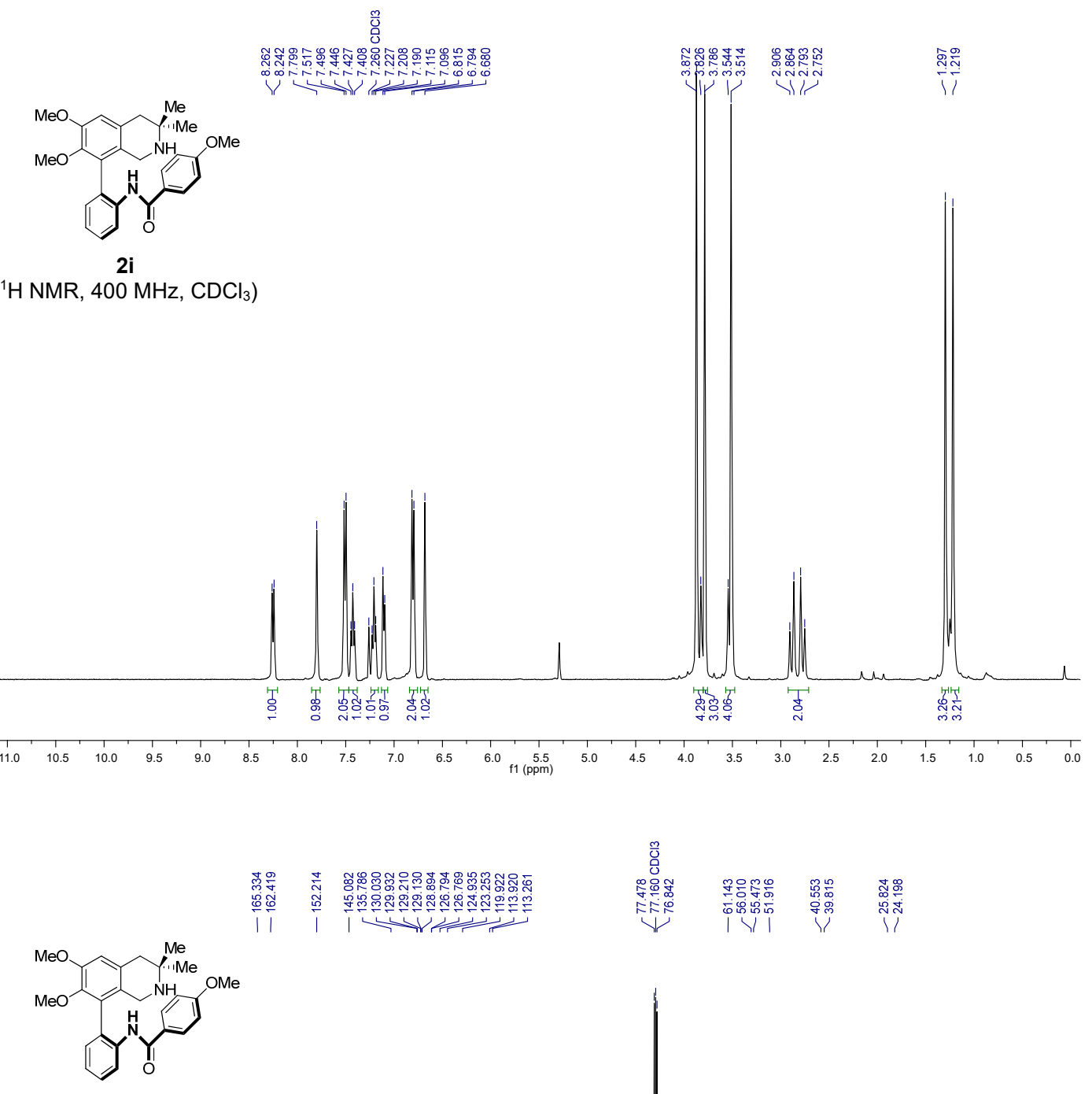

$\left({ }^{13} \mathrm{C} \mathrm{NMR}, 100 \mathrm{MHz}, \mathrm{CDCl}_{3}\right)$
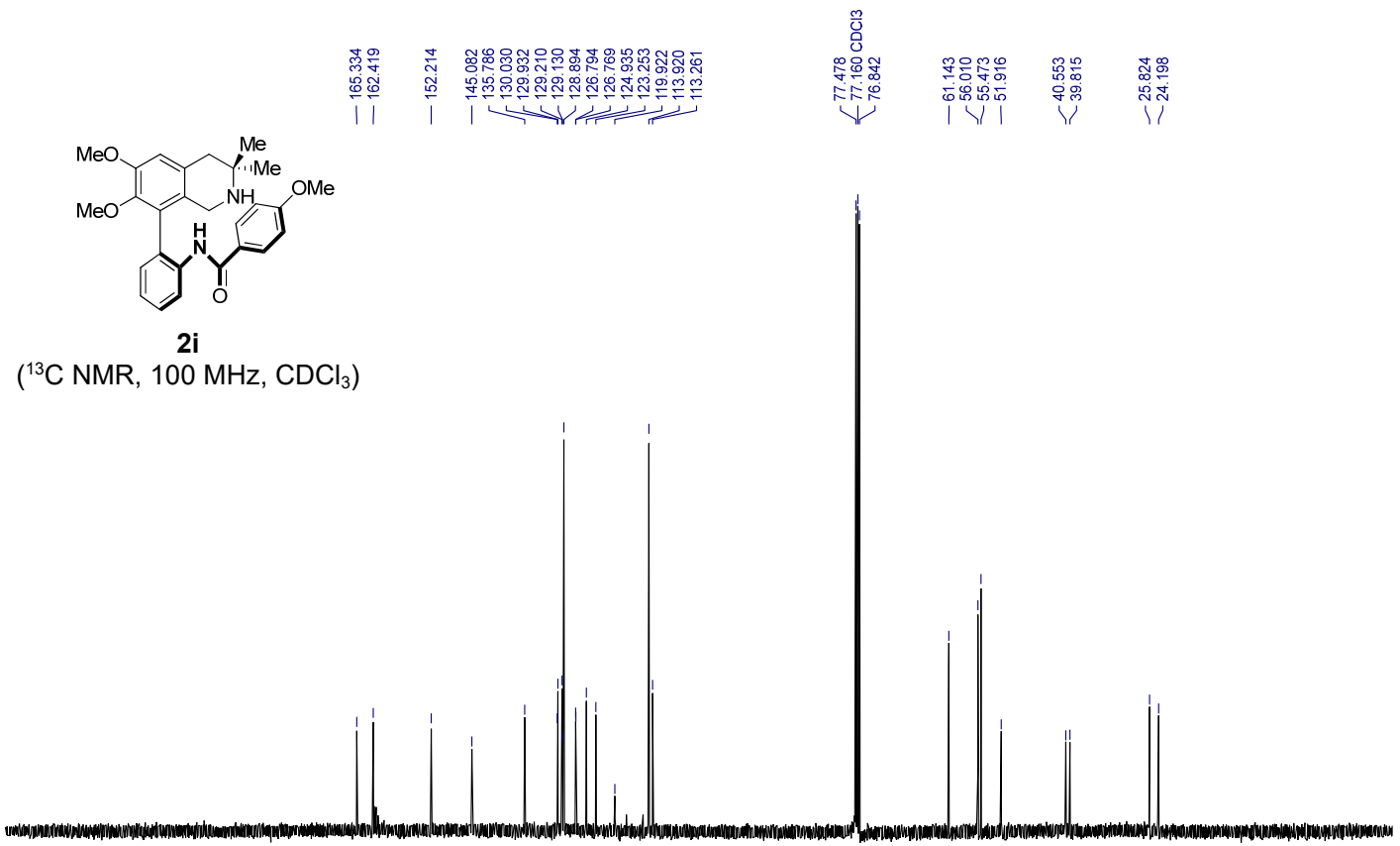

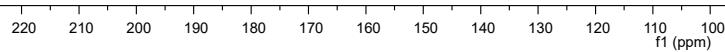



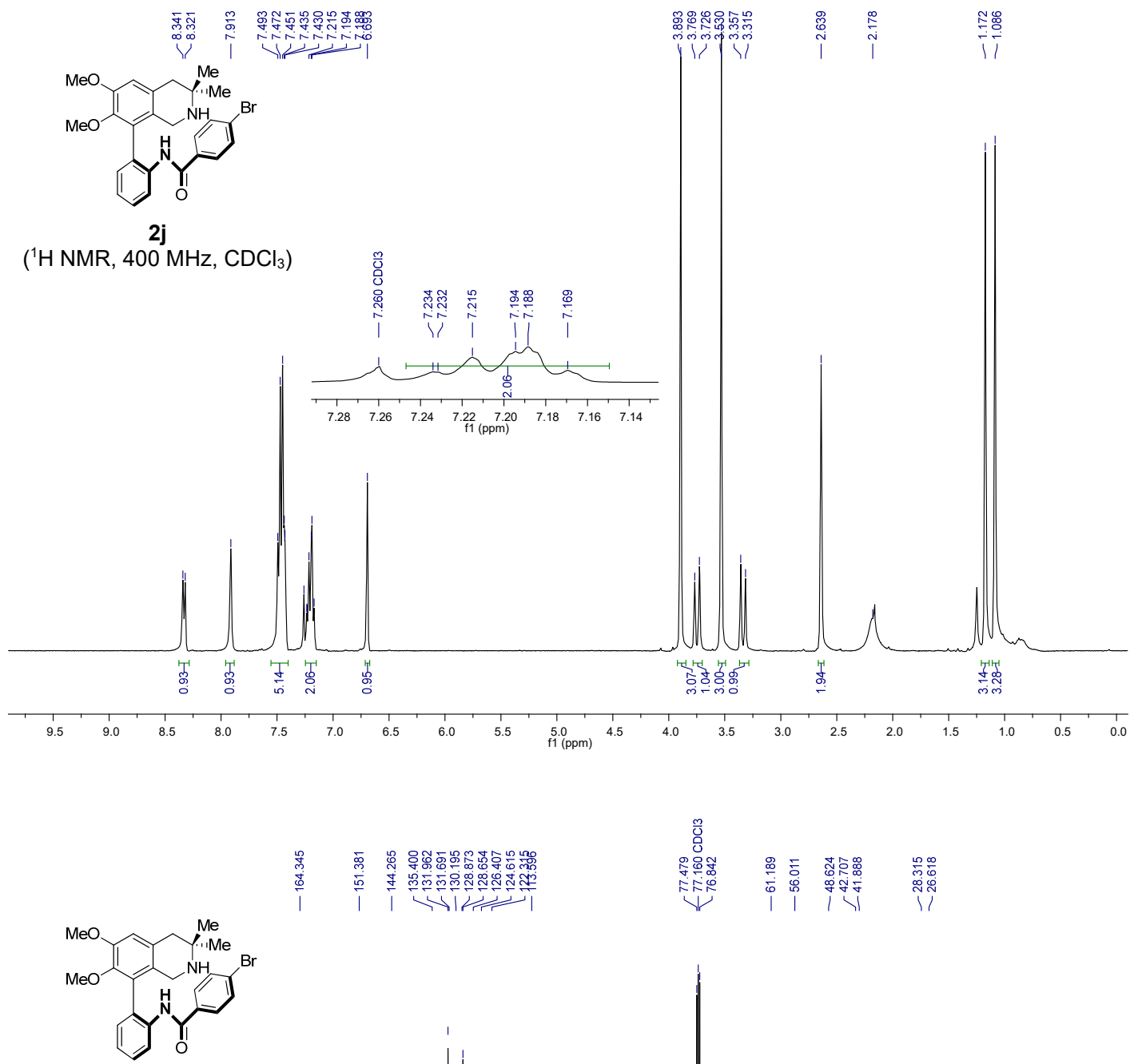

2j

$\left({ }^{13} \mathrm{C} \mathrm{NMR}, 100 \mathrm{MHz}, \mathrm{CDCl}_{3}\right)$
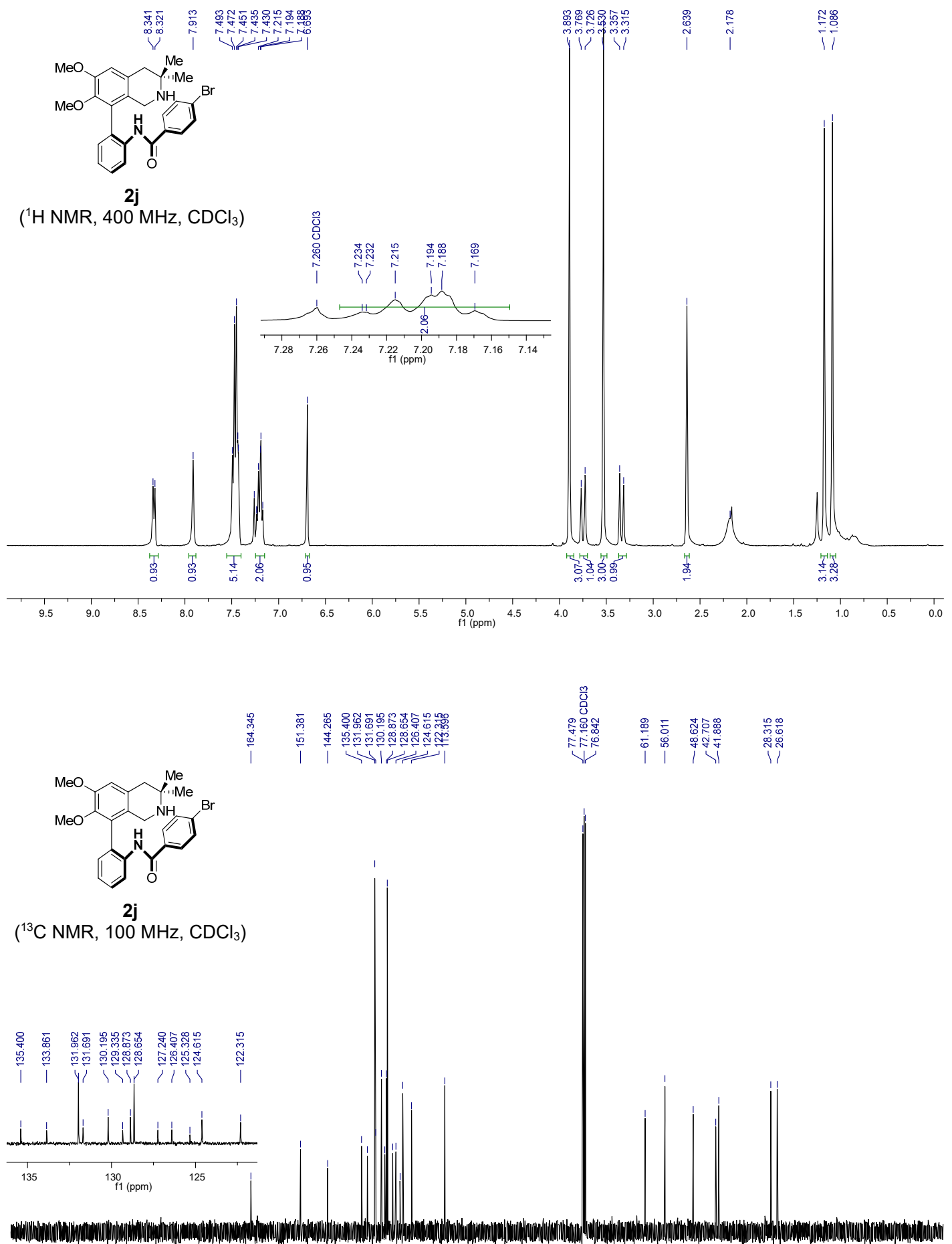

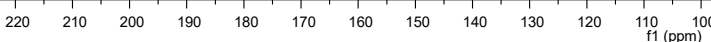




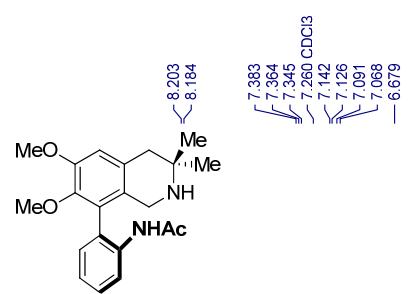

$2 \mathrm{k}$

( ${ }^{1} \mathrm{H}$ NMR, $400 \mathrm{MHz}, \mathrm{CDCl}_{3}$ )

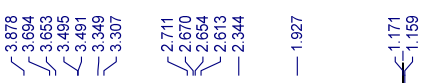

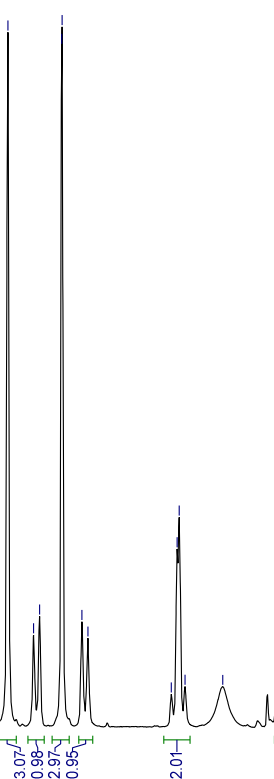

a
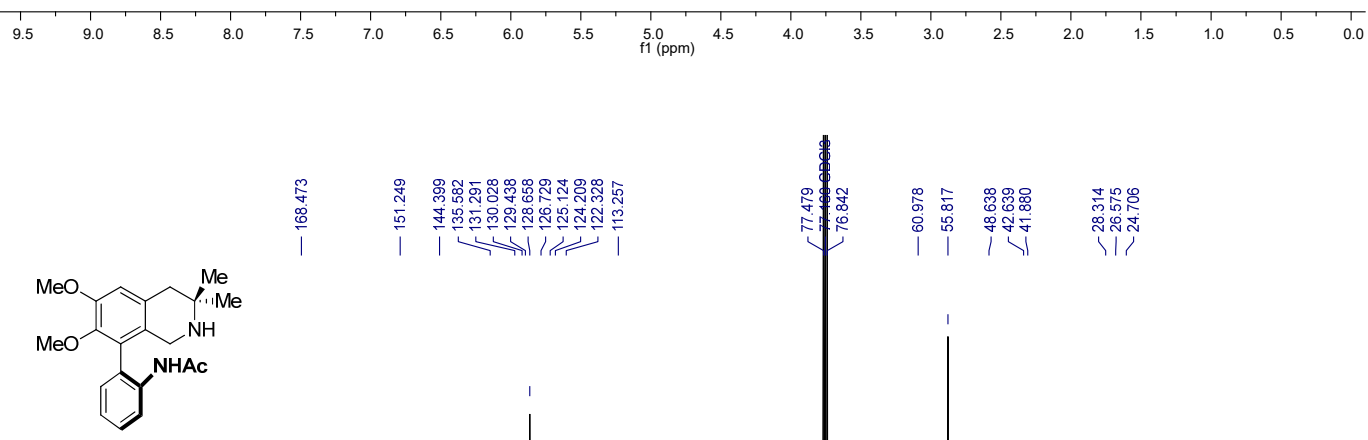

2k

$\left({ }^{13} \mathrm{C}\right.$ NMR, $\left.100 \mathrm{MHz}, \mathrm{CDCl}_{3}\right)$

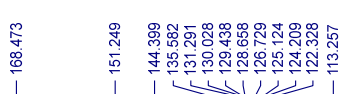

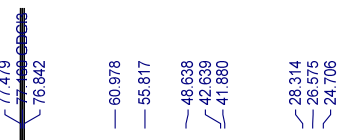

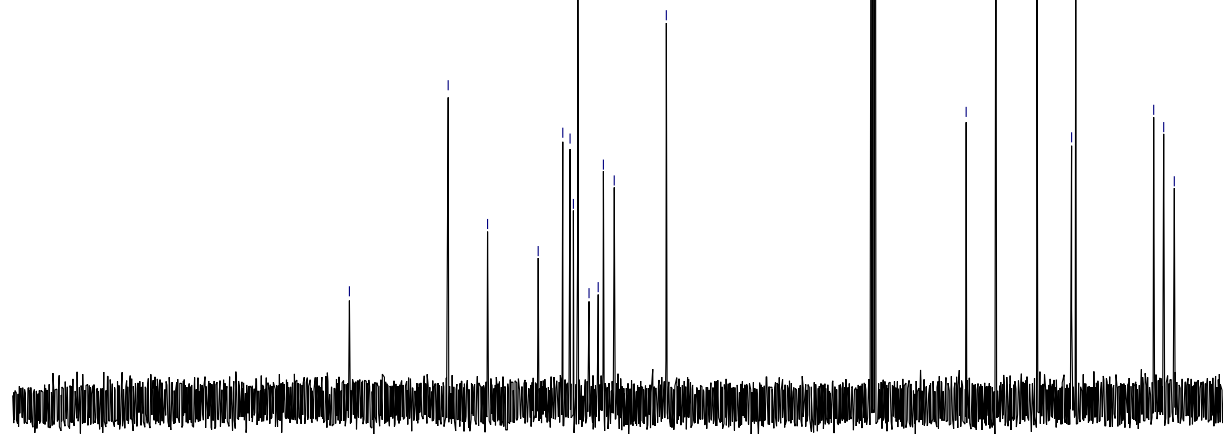

$\begin{array}{lllllllllllll}220 & 210 & 200 & 190 & 180 & 170 & 160 & 150 & 140 & 130 & 120 & \begin{array}{l}110 \\ \mathrm{f} 1(\mathrm{ppm})\end{array} \\ 100 & & \end{array}$ 


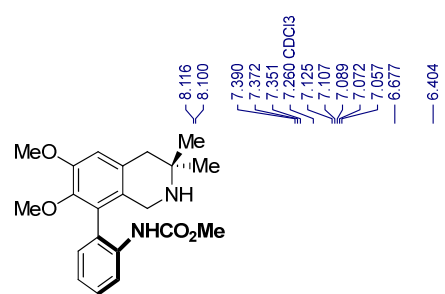

21

( ${ }^{1} \mathrm{H}$ NMR, $400 \mathrm{MHz}, \mathrm{CDCl}_{3}$ )
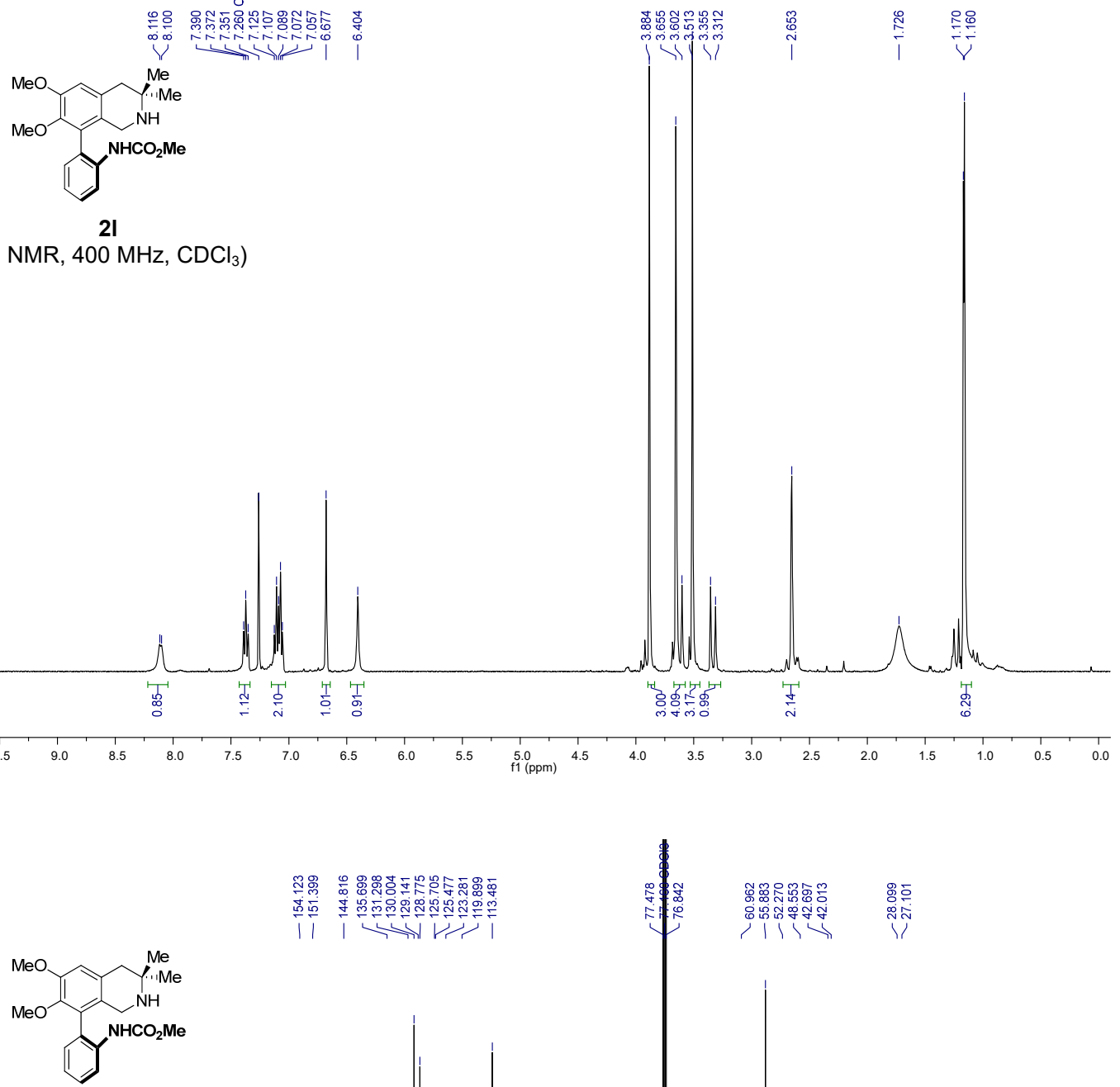

21

$\left({ }^{13} \mathrm{C} \mathrm{NMR}, 100 \mathrm{MHz}, \mathrm{CDCl}_{3}\right)$
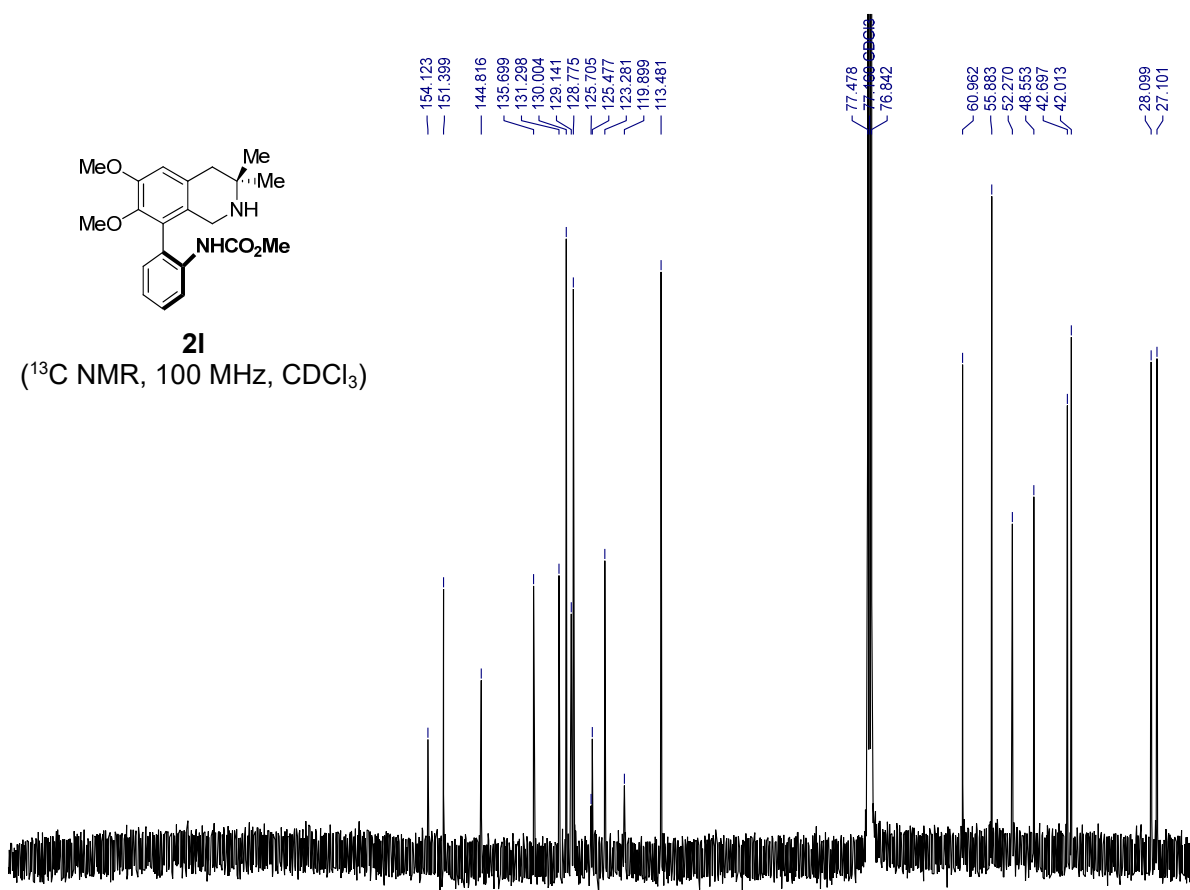

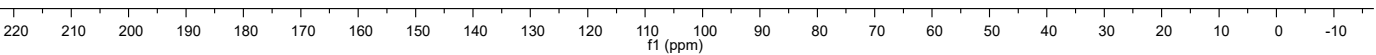



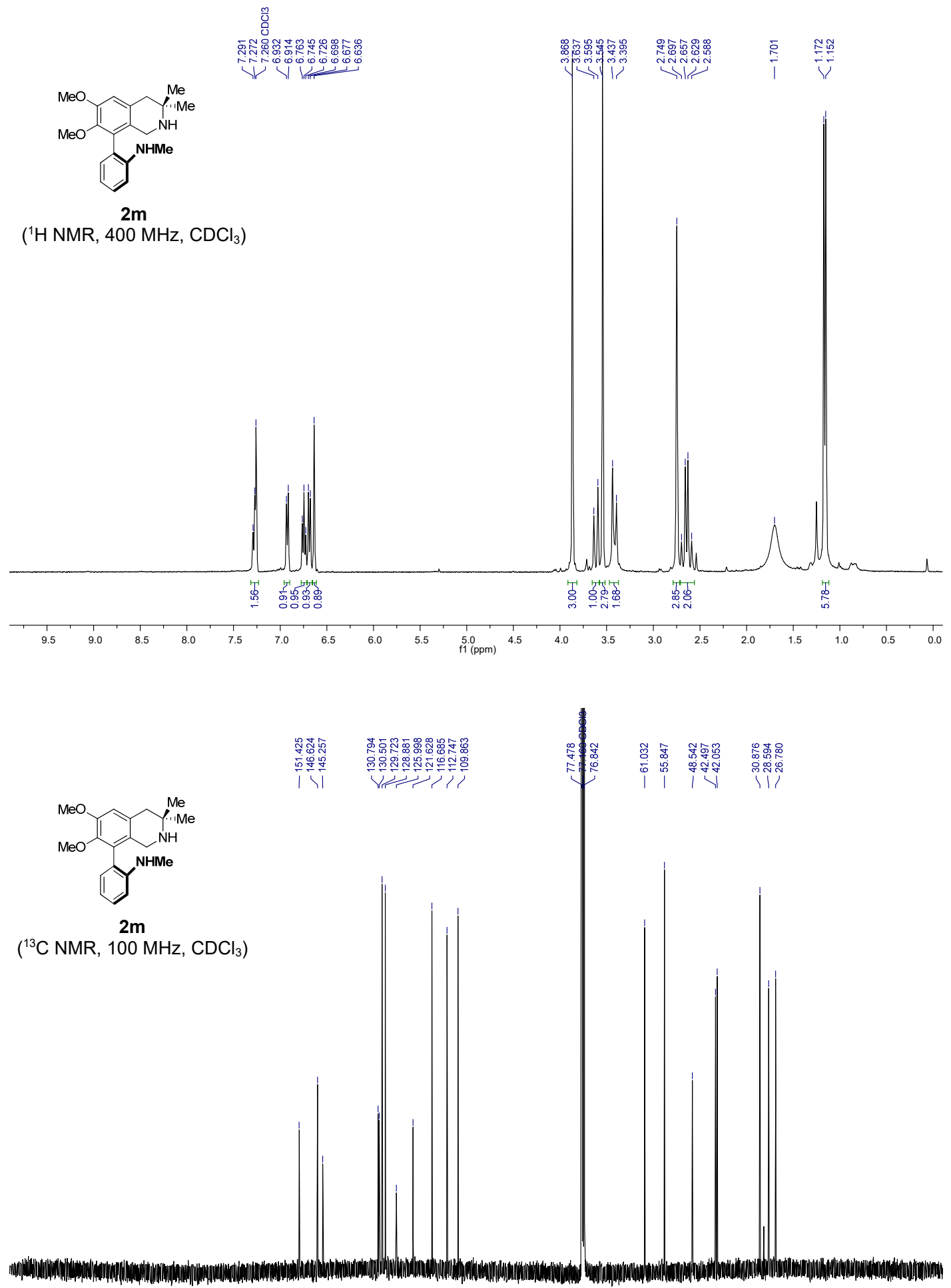

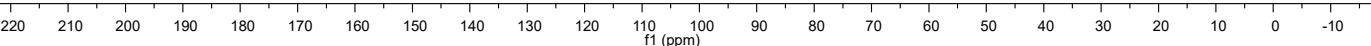



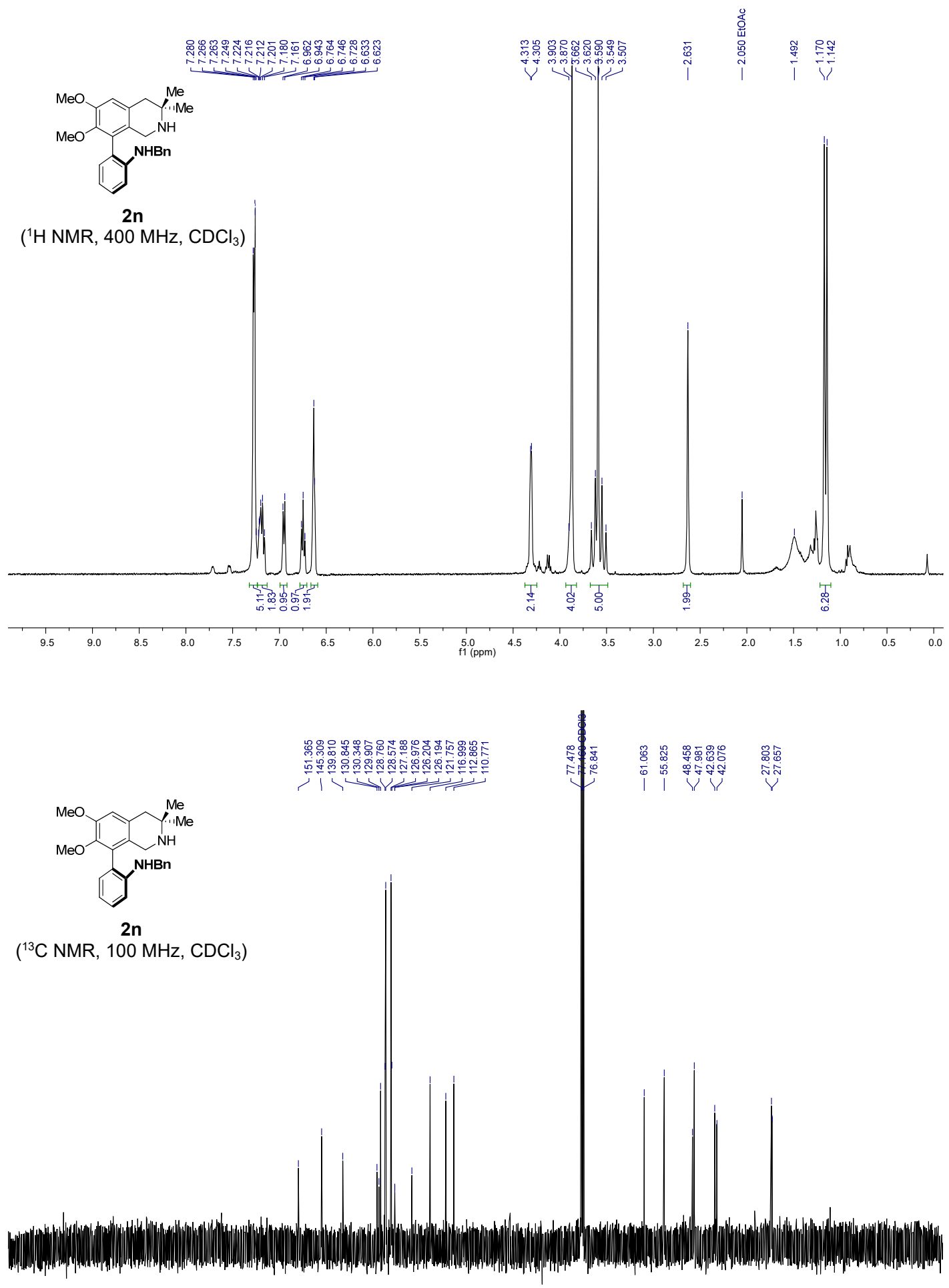

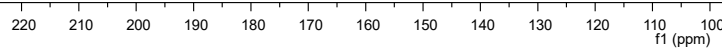




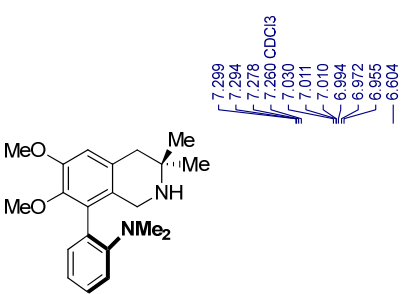

20

( ${ }^{1} \mathrm{H} \mathrm{NMR}, 400 \mathrm{MHz}, \mathrm{CDCl}_{3}$ )
111

害

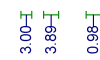

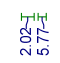

20

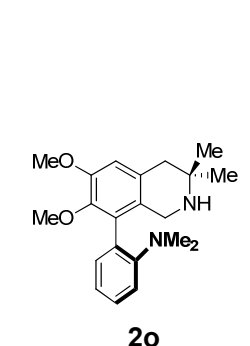

$\left({ }^{13} \mathrm{C}\right.$ NMR, $\left.100 \mathrm{MHz}, \mathrm{CDCl}_{3}\right)$

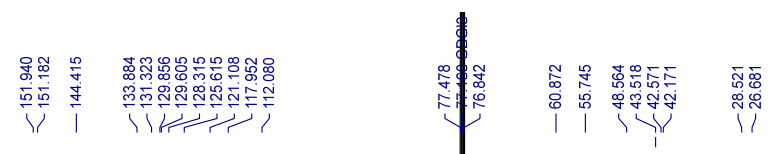

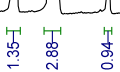

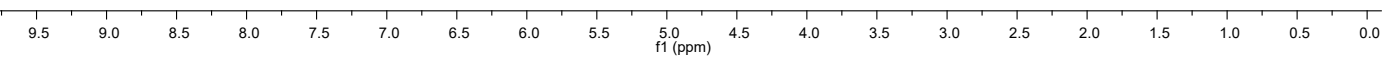

,

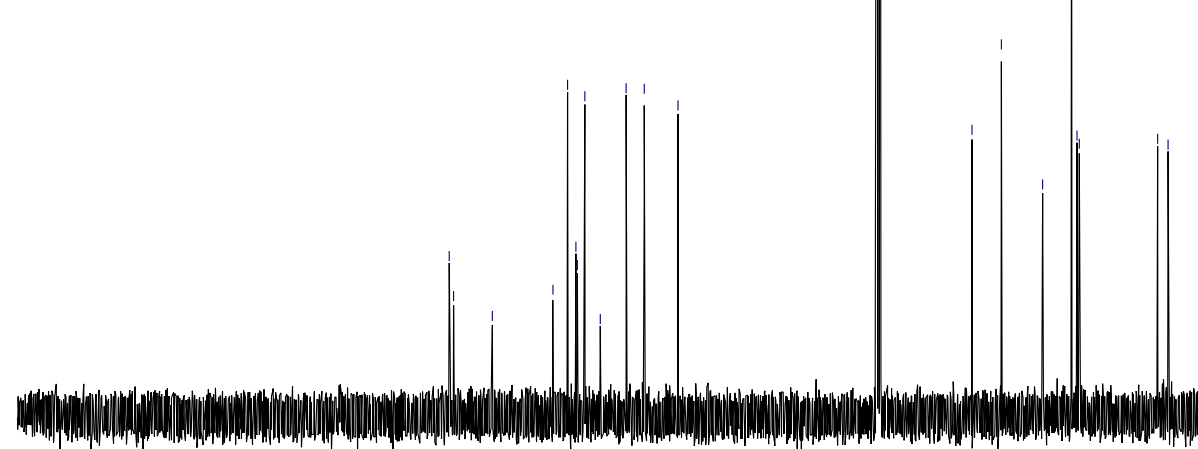

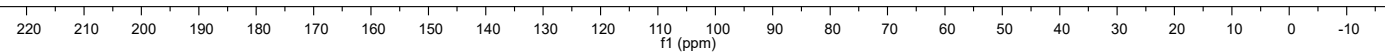



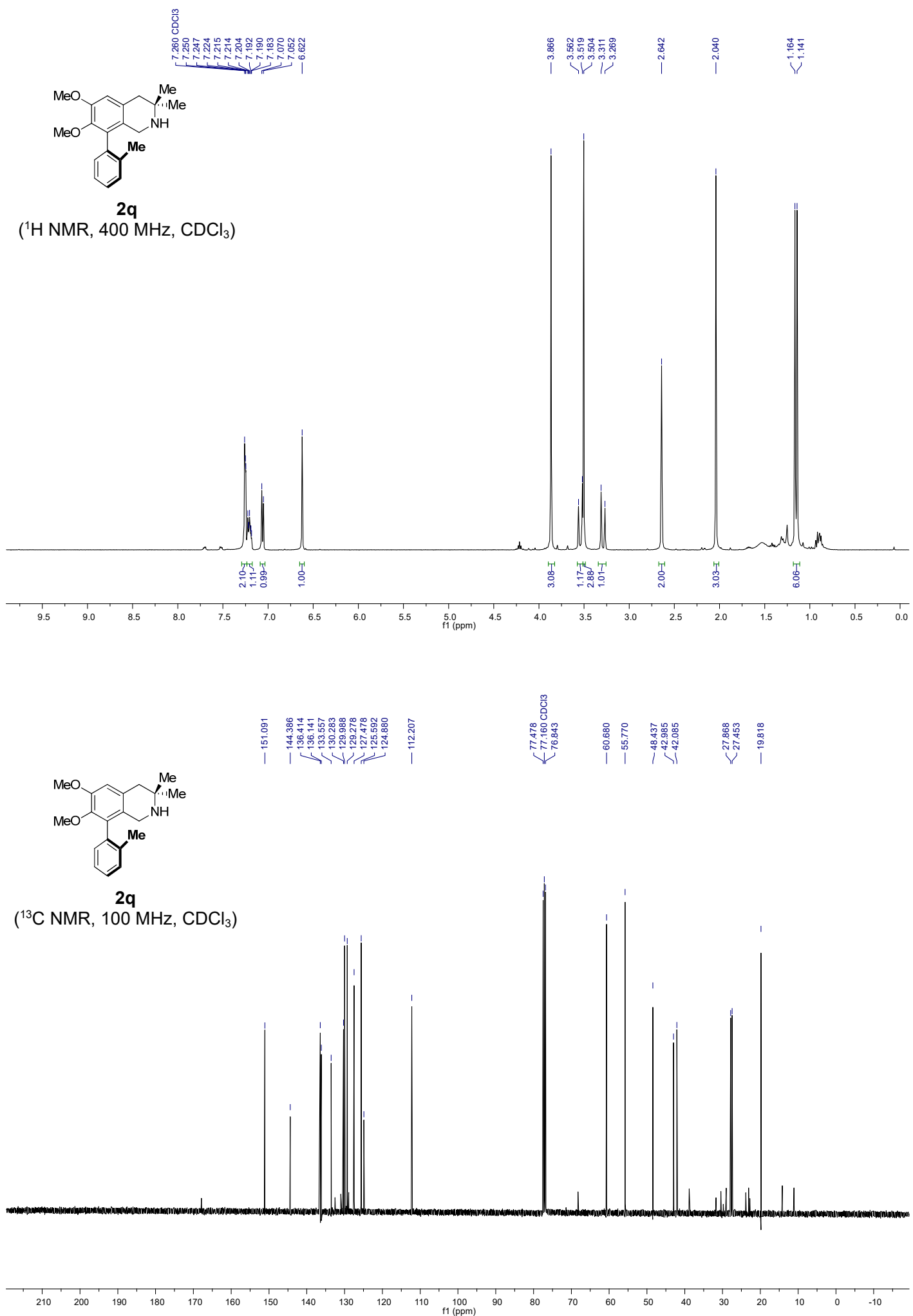


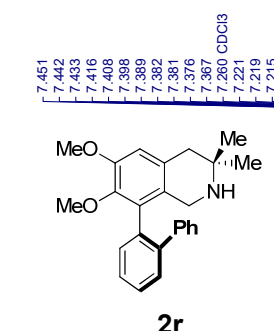

( ${ }^{1} \mathrm{H}$ NMR, $400 \mathrm{MHz}, \mathrm{CDCl}_{3}$ )

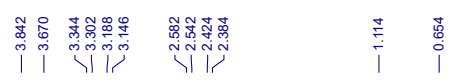

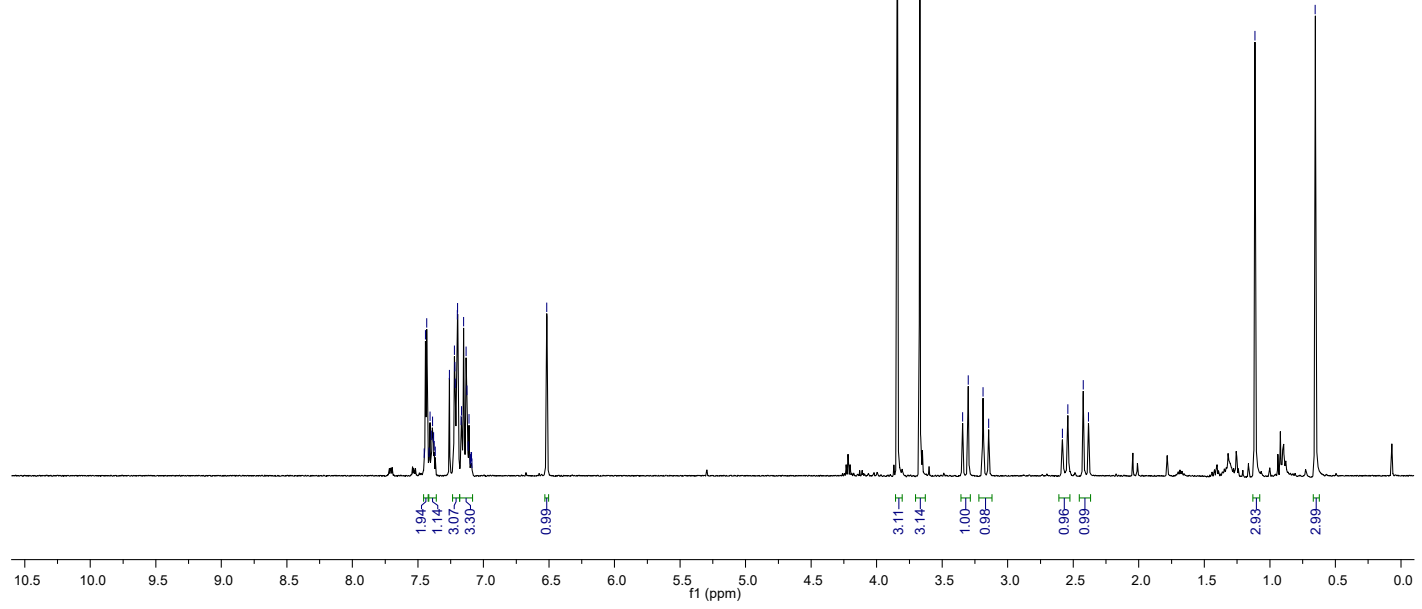

至

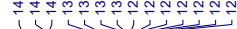

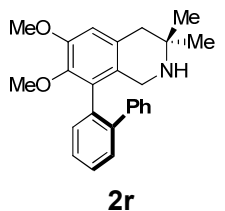

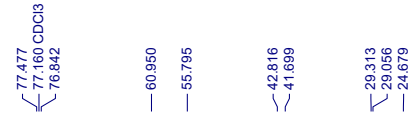

$\left({ }^{13} \mathrm{C}\right.$ NMR, $\left.100 \mathrm{MHz}, \mathrm{CDCl}_{3}\right)$

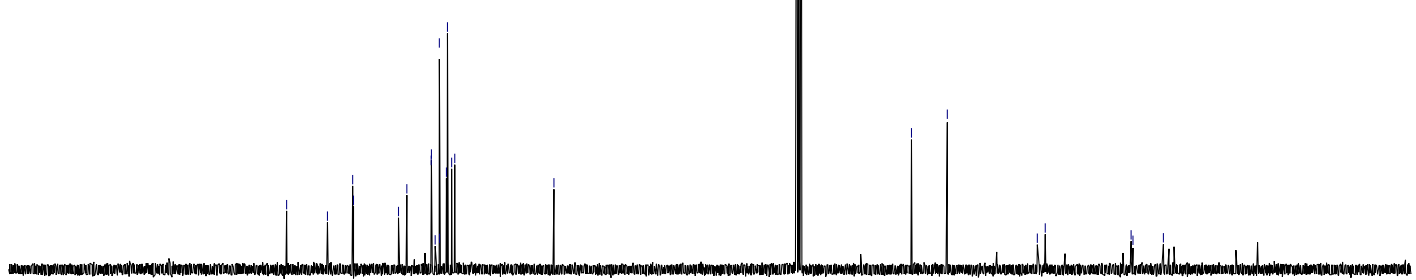


\%

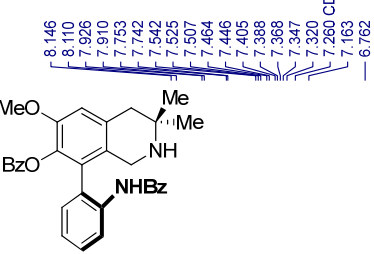

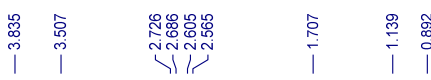

2s

( ${ }^{1} \mathrm{H}$ NMR, $400 \mathrm{MHz}, \mathrm{CDCl}_{3}$ )
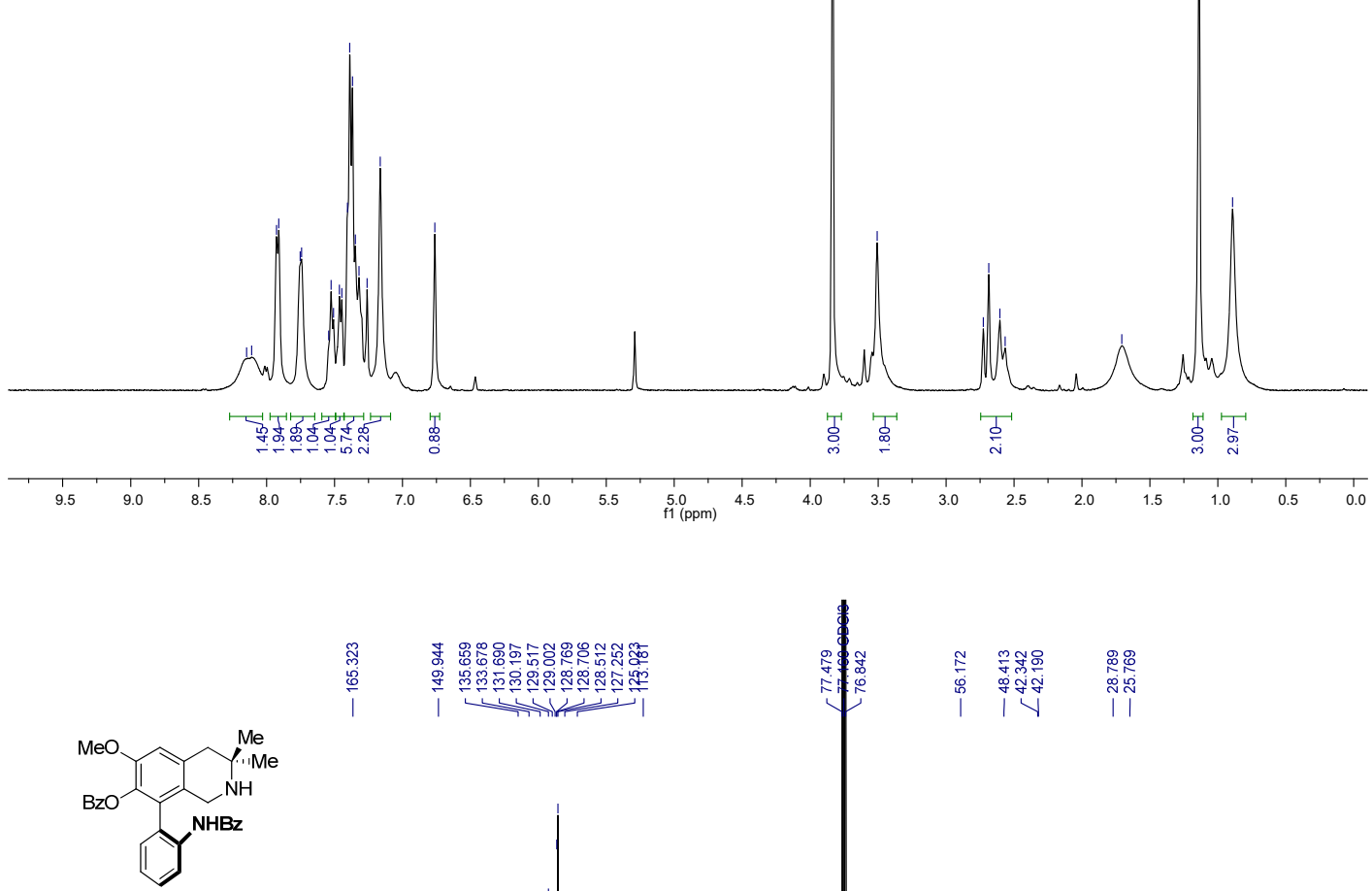

2s

$\left({ }^{13} \mathrm{C}\right.$ NMR, $\left.100 \mathrm{MHz}, \mathrm{CDCl}_{3}\right)$

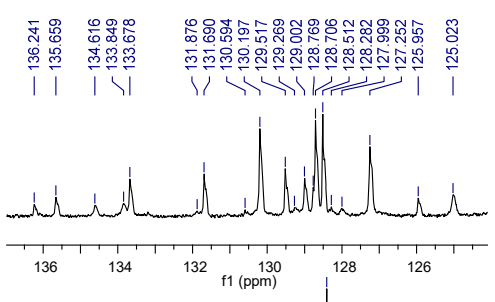

$\begin{array}{llllllllllll}220 & 210 & 200 & 190 & 180 & 170 & 160 & 150 & 140 & 130 & 120 & \begin{array}{l}110 \\ \mathrm{f} 1(\mathrm{ppm})\end{array}\end{array}$ 

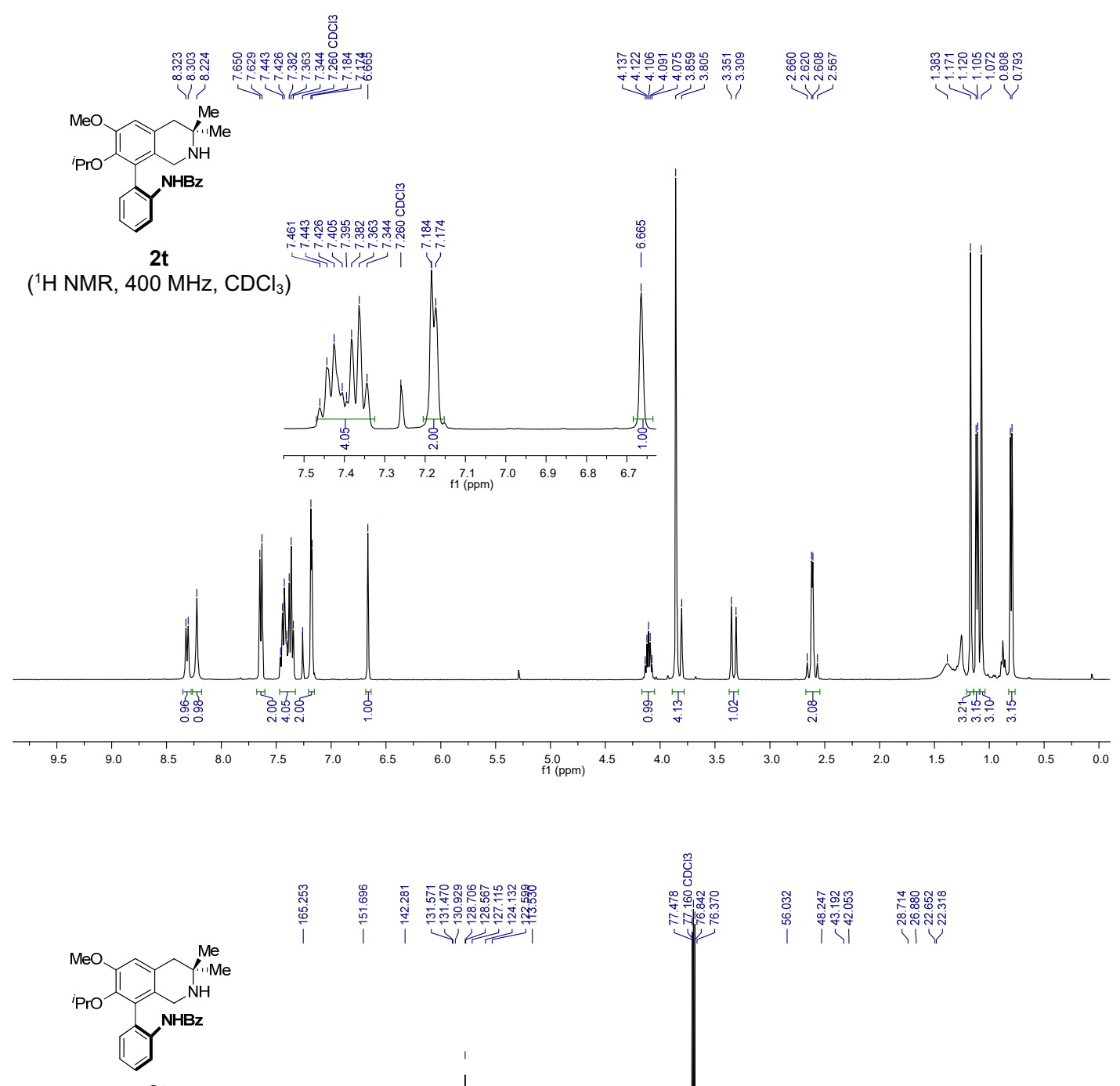

$\left({ }^{13} \mathrm{C} \mathrm{NMR}, 100 \mathrm{MHz}, \mathrm{CDCl}_{3}\right)$

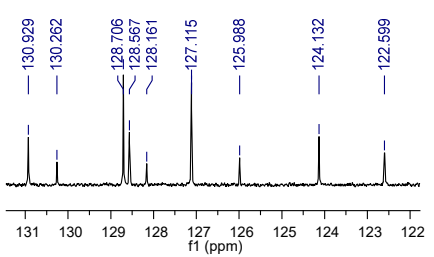

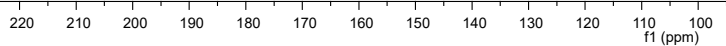




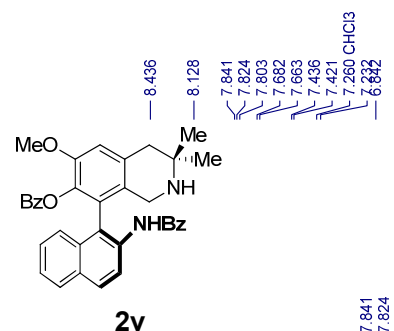

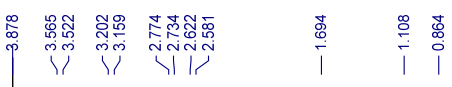

$\left({ }^{1} \mathrm{H} \mathrm{NMR}, 400 \mathrm{MHz}, \mathrm{CDCl}_{3}\right)$
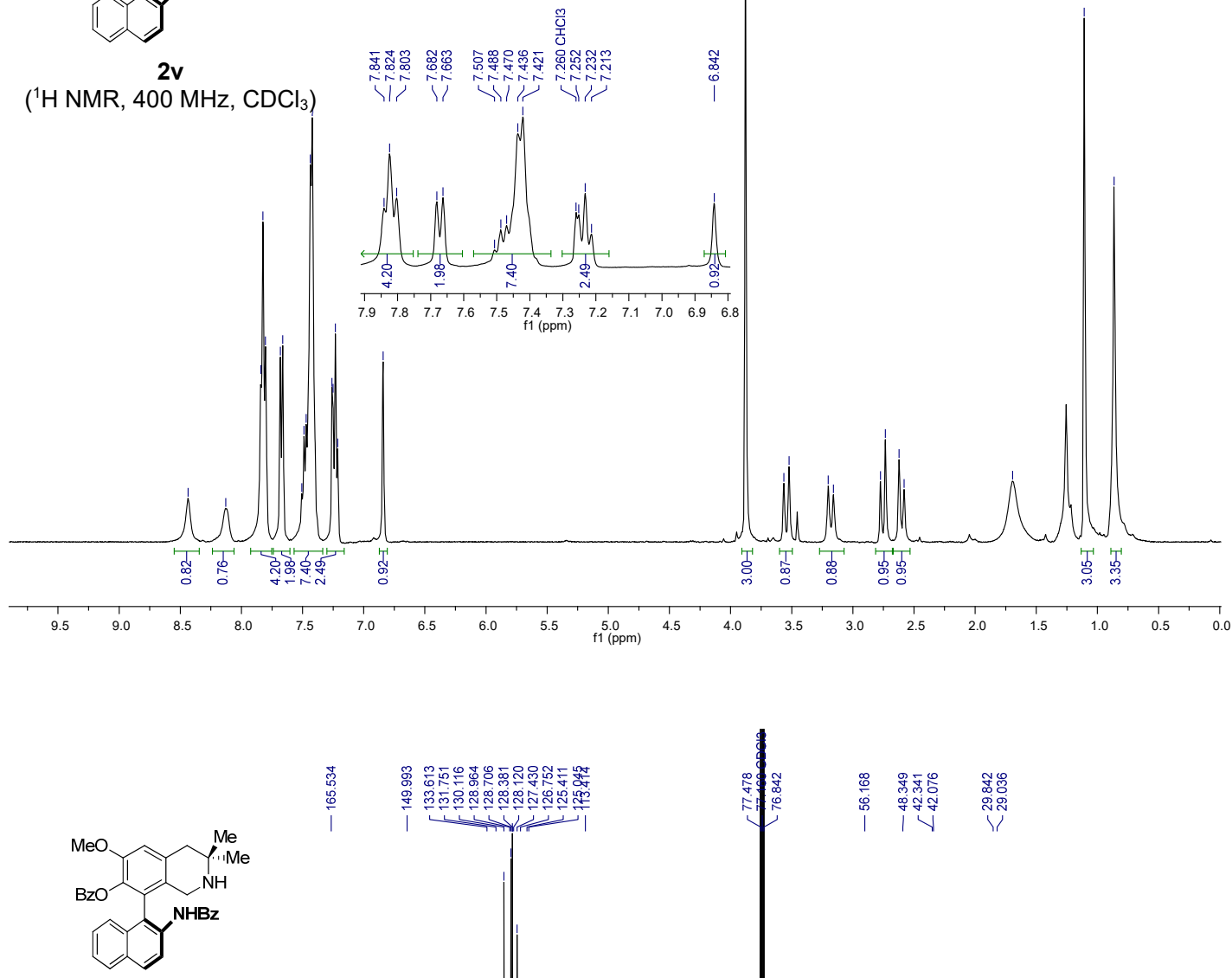

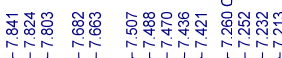

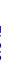

$\begin{array}{llll}9.5 & 9.0 & 8.5 & 8.0\end{array}$

$2 v$

$\left({ }^{13} \mathrm{C} \mathrm{NMR}, 100 \mathrm{MHz}, \mathrm{CDCl}_{3}\right)$

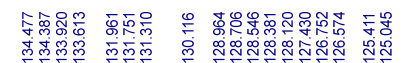

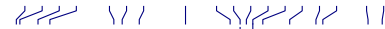

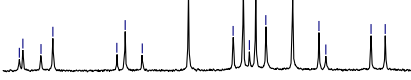

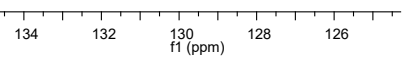

$\mid$

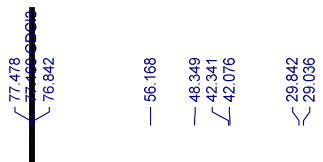

$\begin{array}{llllllllllll}220 & 210 & 200 & 190 & 180 & 170 & 160 & 150 & 140 & 130 & 120 & \begin{array}{c}110 \\ \mathrm{f} 1(\mathrm{ppm})\end{array} \\ 100 & \end{array}$ 


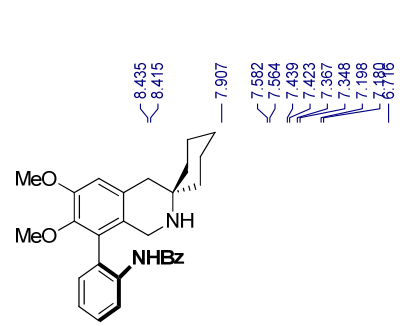

$\stackrel{2 \mathbf{w}}{\left({ }^{1} \mathrm{H} \mathrm{NMR}, 400 \mathrm{MHz}, \mathrm{CDCl}_{3}\right)}$
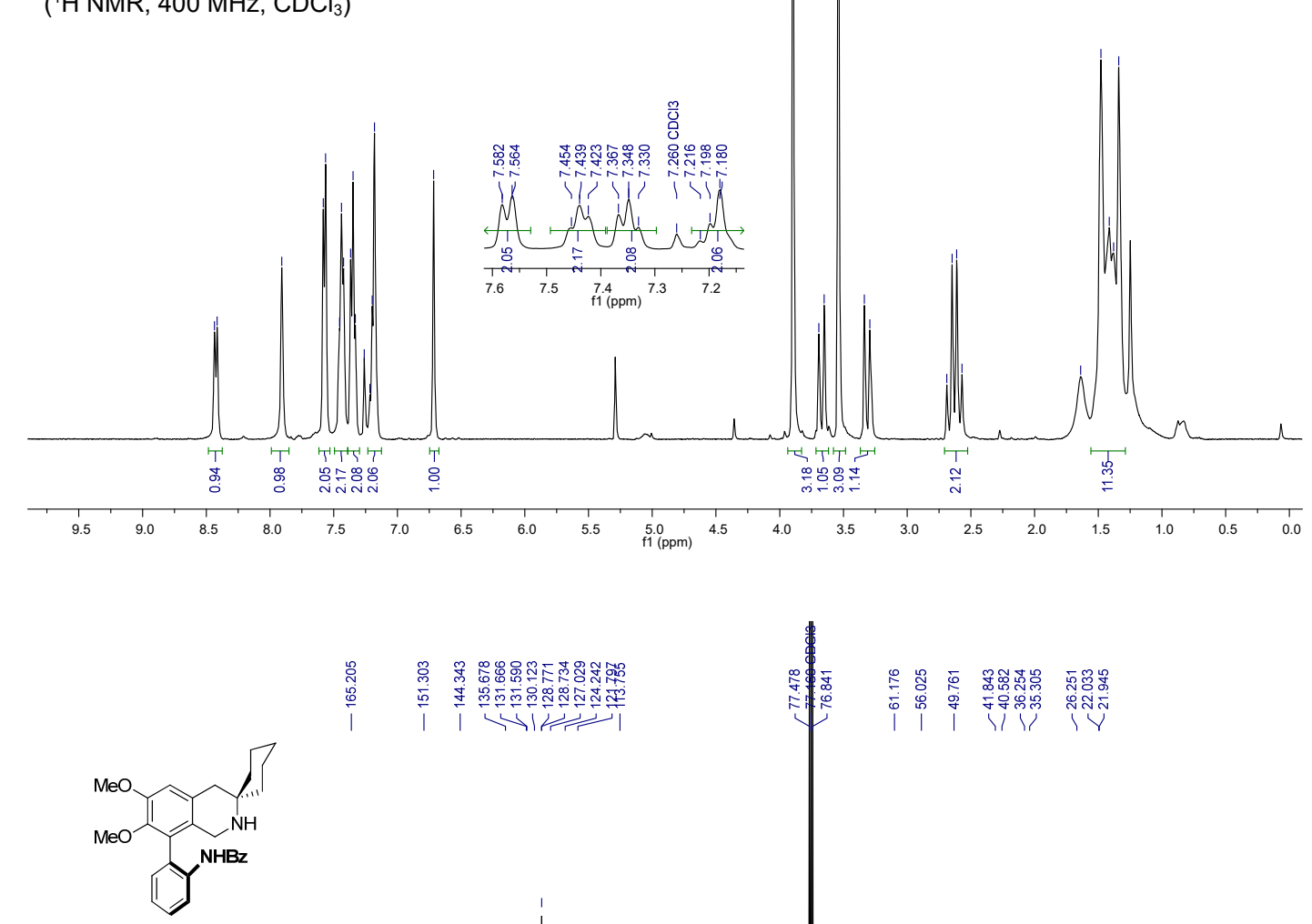

$\left({ }^{13} \mathrm{C} \mathrm{NMR,}, 100 \mathrm{MHz}, \mathrm{CDCl}_{3}\right)$

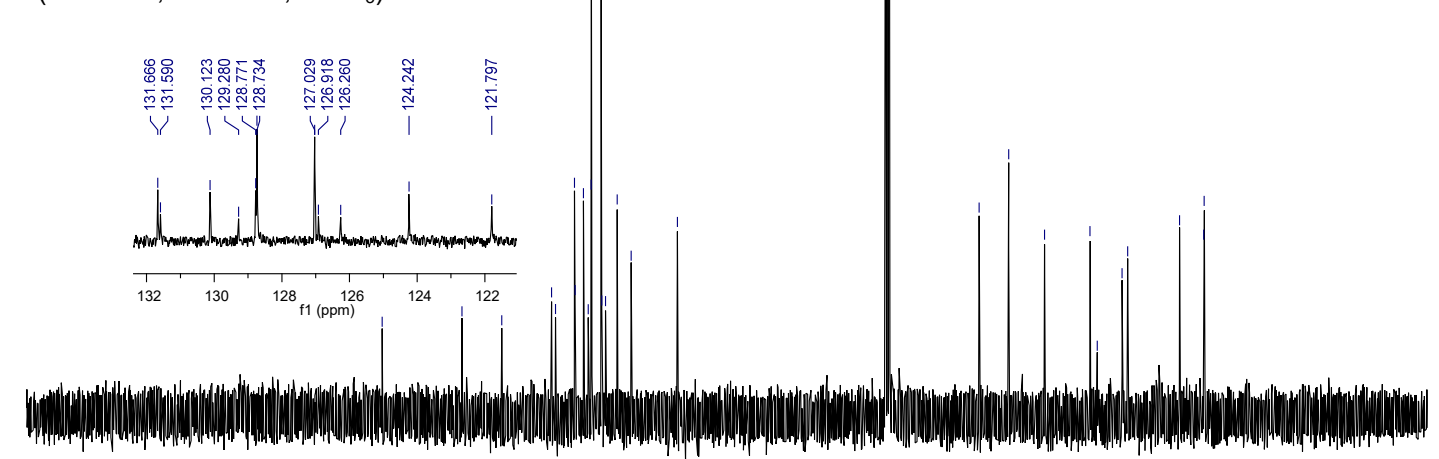

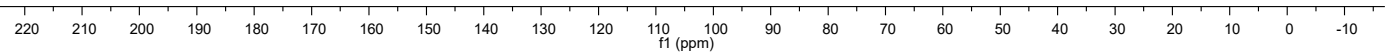




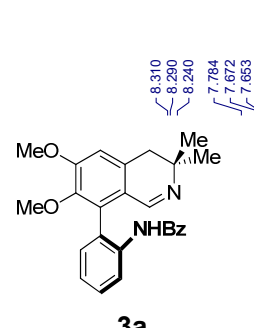

$\left({ }^{1} \mathrm{H} \mathrm{NMR}, 400 \mathrm{MHz}, \mathrm{CDCl}_{3}\right.$ )
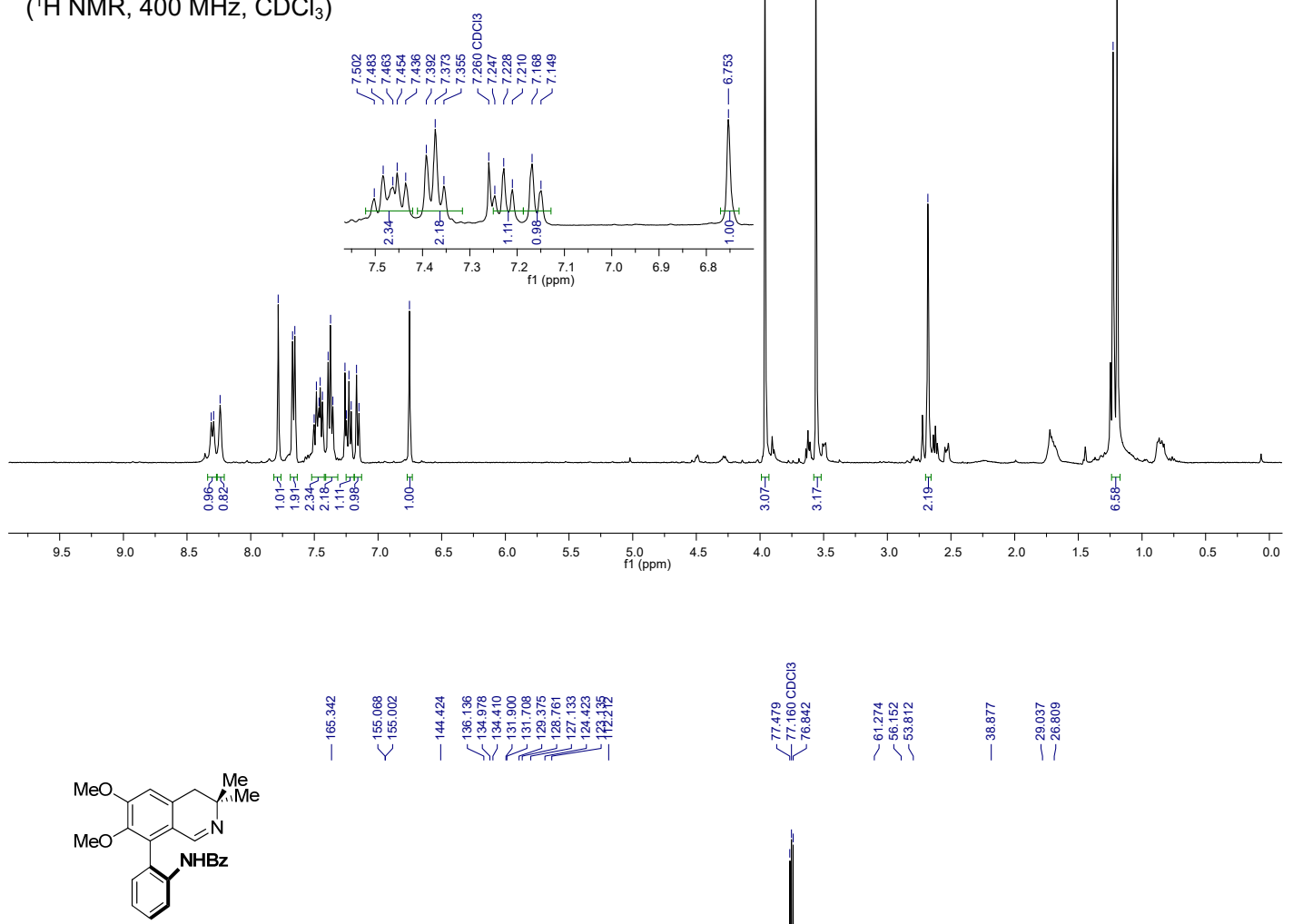

3a

$\left({ }^{13} \mathrm{C}\right.$ NMR, $\left.100 \mathrm{MHz}, \mathrm{CDCl}_{3}\right)$

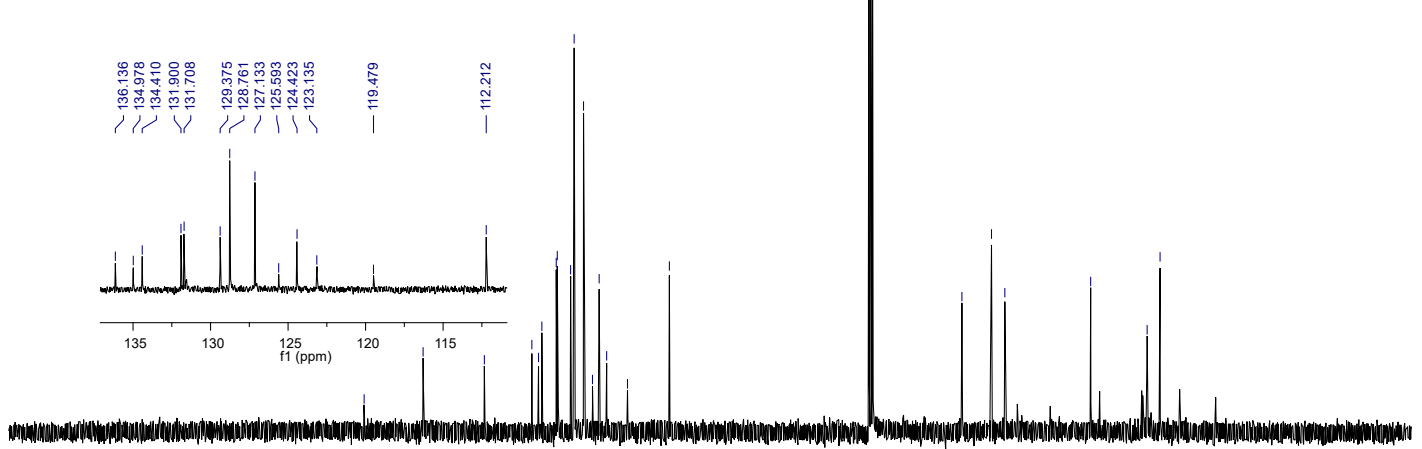


2D NOESY NMR Spectrum of $2 \mathrm{f}\left(500 \mathrm{MHz}, \mathrm{CDCl}_{3}\right)$

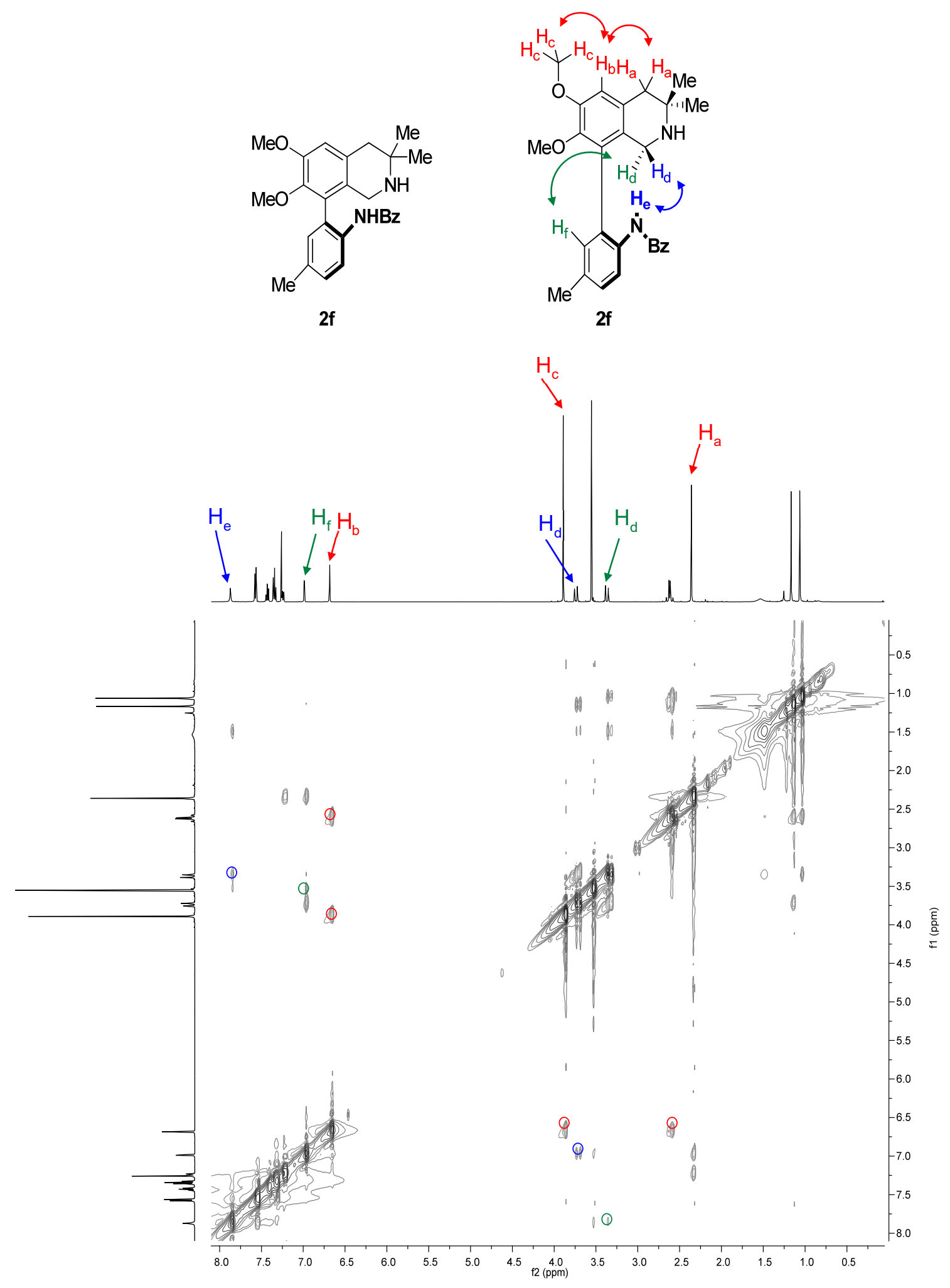


2D NOESY NMR Spectrum of $20\left(500 \mathrm{MHz}, \mathrm{CDCl}_{3}\right)$

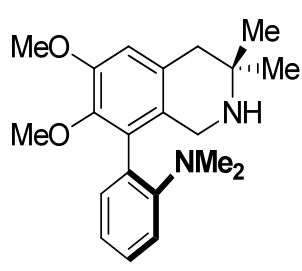

20

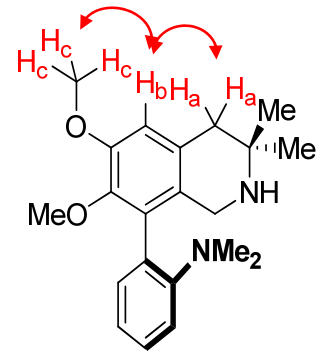

20

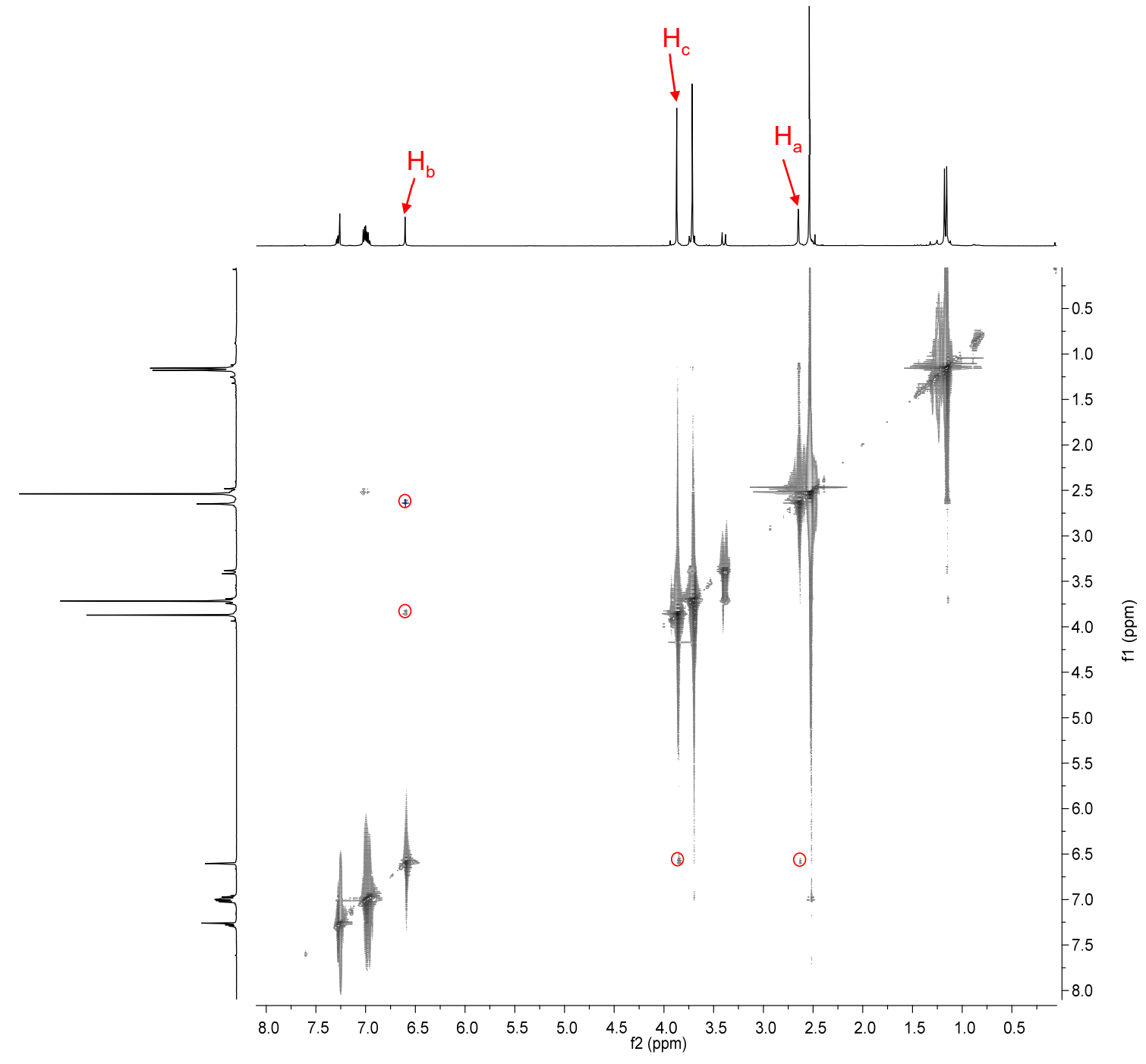


2D NOESY NMR Spectrum of regioisomeric mixture 20 and regio-2o (2o : regio-2o $\left.=1: 1.4,500 \mathrm{MHz}, \mathrm{CDCl}_{3}\right)$<smiles>COc1cc2c(c(-c3ccccc3[N+](=O)[O-])c1OC)CNC(C)(C)C2</smiles>

20<smiles>COc1ccccc1-c1cc2c(c(OC)c1OC)CNC(C)(C)C2</smiles>

regio-2o

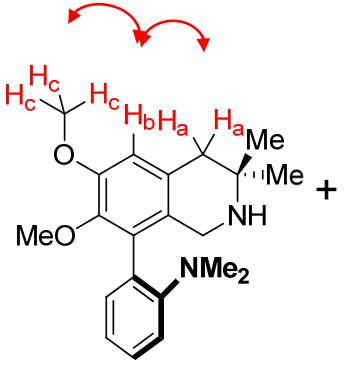

20

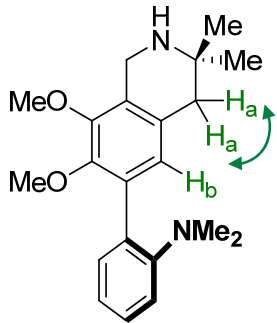

regio-2o

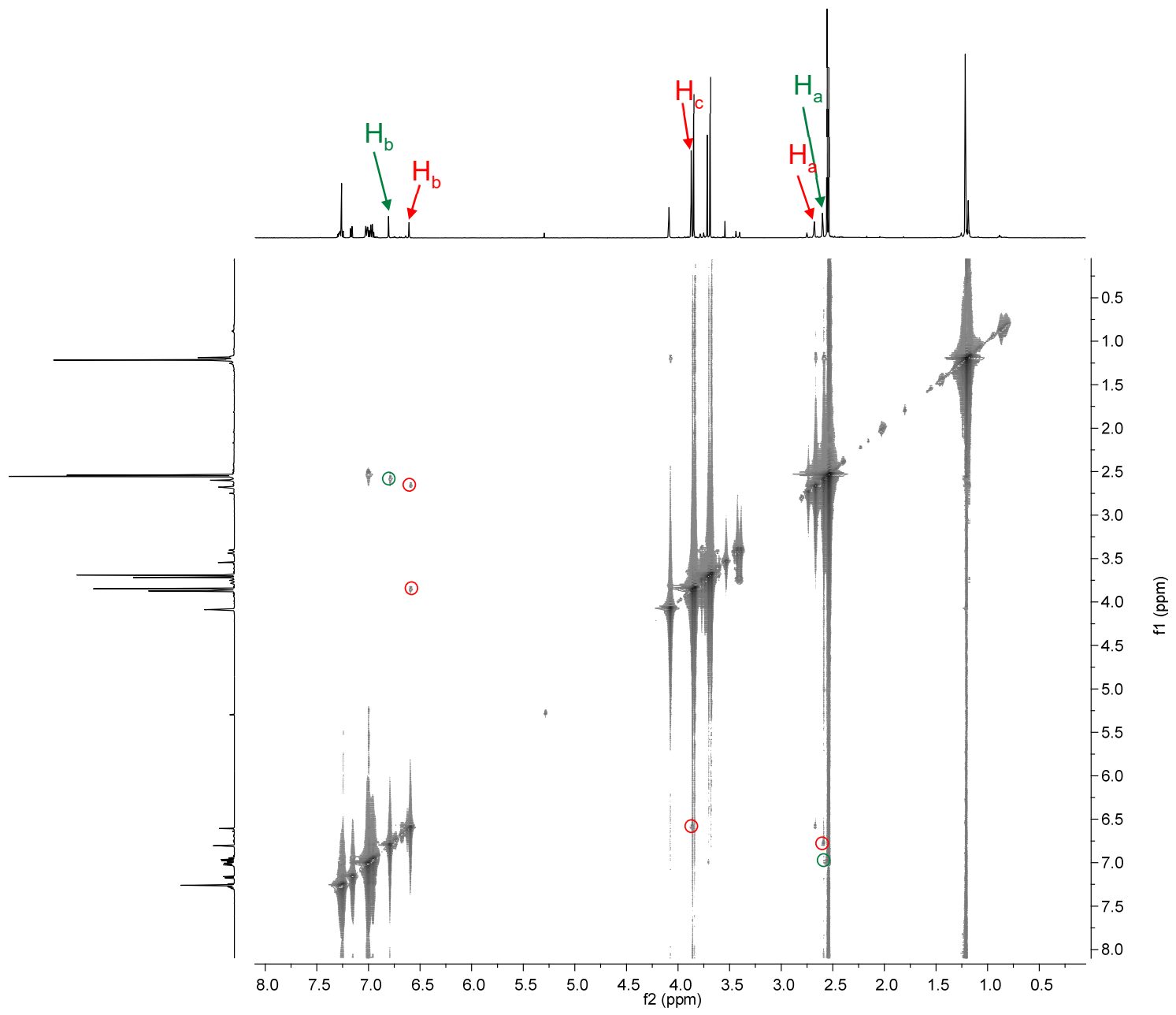




\subsection{HPLC Traces of 2}

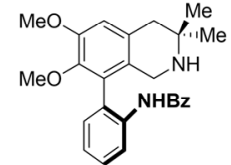

$2 a$

HPLC conditions:

Chiralpak AD, $10 \%$ ' $\mathrm{PrOH} / n$-Hexane with $0.1 \% \mathrm{Et}_{2} \mathrm{NH}$ eluent

$1.0 \mathrm{~mL} / \mathrm{min}, 254 \mathrm{~nm}$

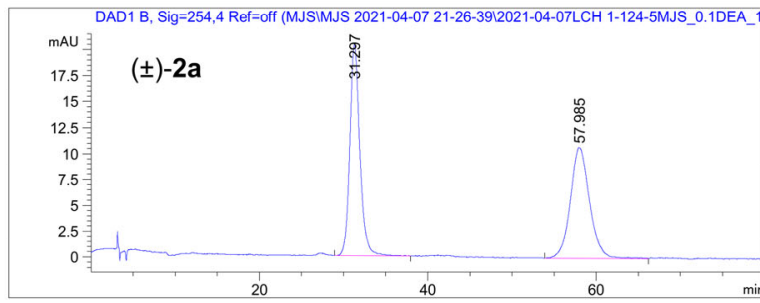

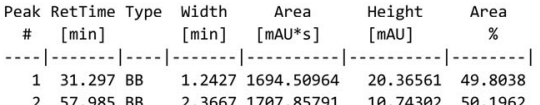
Totals

$3402.36755 \quad 31.10863$

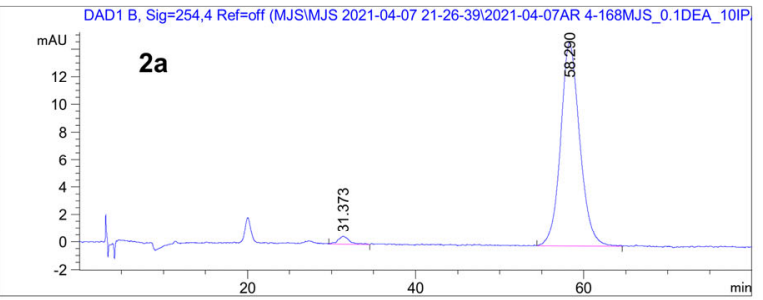

Peak RetT1me Type width Area He1ght Area \# $[\mathrm{min}] \quad[\mathrm{min}]\left[\mathrm{mAU}^{*} \mathrm{~s}\right][\mathrm{mAU}] \%$ $\begin{array}{llllll}31.373 \text { BV R } & 1.2859 & 52.77548 & 5.71289 \mathrm{e}-1 & 2.1999\end{array}$ $\begin{array}{llllll}2 & 58.290 \text { BV R } & 2.4472 & 2346.22656 & 14.75481 & 97.8001\end{array}$ Totals : $2399.00204 \quad 15.32601$ 


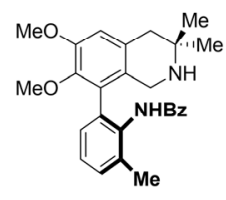

$2 \mathbf{b}$

HPLC conditions:

Chiralpak AD, 20\% 'PrOH $/ n$-Hexane with $0.1 \% \mathrm{Et}_{2} \mathrm{NH}$ eluent

$1.0 \mathrm{~mL} / \mathrm{min}, 254 \mathrm{~nm}$

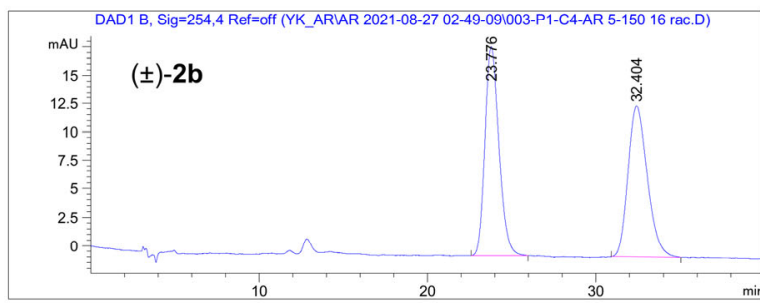

Peak RetTime Type width Area Height Area \# [min] $[\mathrm{min}]\left[\mathrm{mAU}^{*} \mathrm{~s}\right][\mathrm{mAU}] \quad \%$

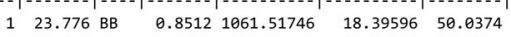
$\begin{array}{llllll}2 & 32.404 \text { BB } & 0.9894 & 1059.93091 & 13.27651 & 49.9626\end{array}$ Totals : $2121.44836 \quad 31.67247$

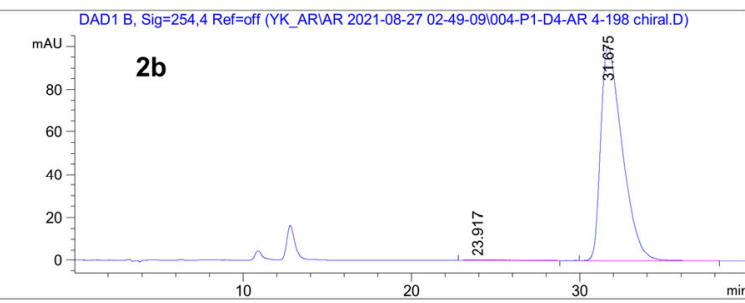

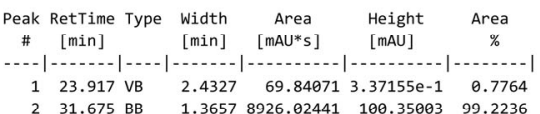
Totals :

$8995.86512 \quad 100.68718$

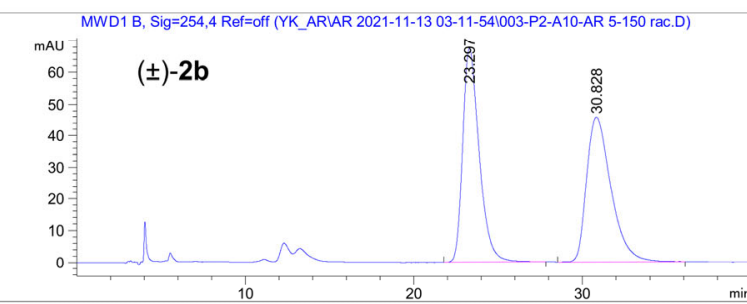

Peak RetTime Type width Area Height Area $\#[\mathrm{~min}]$
-

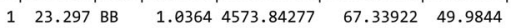
$\begin{array}{lllllll}2 & 30.828 \text { BB } & 1.5219 & 4576.69141 & 45.63918 & 50.0156\end{array}$ Totals : $9150.53418 \quad 112.97840$

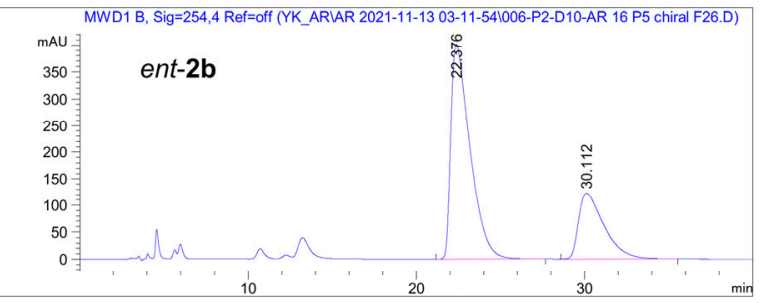

Peak RetTime Type width Area Height Area \# [min] [min] [mAU*s] [maU] \% $\begin{array}{lllllll}1 & 22.376 & \text { BV R } & 1.1481 & 3.17023 \mathrm{e} 4 & 401.96945 & 71.2475\end{array}$ $\begin{array}{lllll}30.112 \text { VV R } & 1.5188 & 1.27937 e 4 & 122.46703 & 28.7525\end{array}$ Totals : $4.44968 \mathrm{e} 4 \quad 524.43648$ 


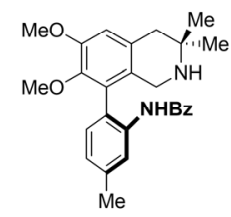

2c
HPLC conditions:

Chiralpak AD, $20 \%$ ' $\mathrm{PrOH} / n-\mathrm{H}$ exane with $0.1 \% \mathrm{Et}_{2} \mathrm{NH}$ eluent

$1.0 \mathrm{~mL} / \mathrm{min}, 254 \mathrm{~nm}$

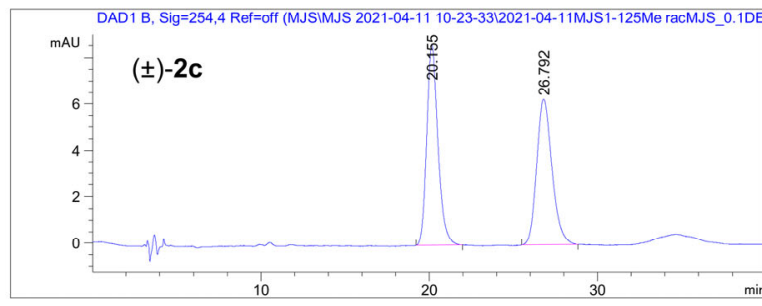

$$
\begin{aligned}
& \begin{array}{ccccc}
\begin{array}{c}
\text { Peak } \\
\#
\end{array} \text { RetTime Type } & \text { Width } & \text { Area } & \text { Height } & \text { Area } \\
{[\mathrm{min}]} & {[\mathrm{min}]} & {[\mathrm{mAU} * \mathrm{~s}]} & {[\mathrm{mAU}]} & \%
\end{array}
\end{aligned}
$$

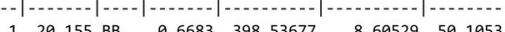

$$
\begin{aligned}
& \begin{array}{lllllll}
26.792 & \text { BB } & 0.8423 & 396.86203 & 6.27091 & 49.8947
\end{array} \\
& \text { Totals : }
\end{aligned}
$$

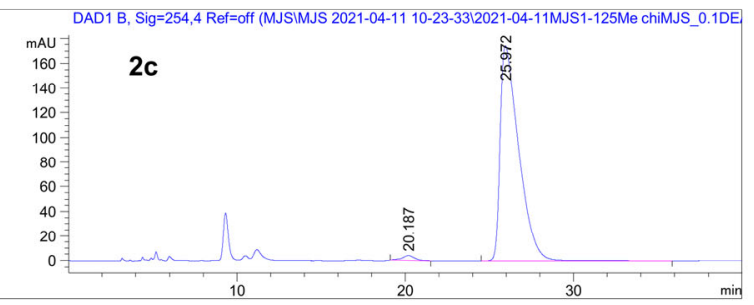

Peak RetTime Type Width Area
$\#$ [min] \# [min] $[\mathrm{min}]\left[\mathrm{mAU}^{*} \mathrm{~s}\right] \quad[\mathrm{mAU}] \quad \%$

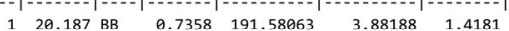

$\begin{array}{llllrr}1 & 29.187 \text { RB } & 0.7358 & 191.58963 & 3.88188 & 1.4181 \\ 2 & 25.972 \text { BB } & 1.1473 & 1.33176 \mathrm{e} 4 & 174.31396 & 98.5819\end{array}$

Totals :

$1.35092 e 4 \quad 178.19585$ 


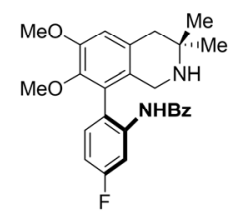

2d

HPLC conditions:

Chiralpak AD, $20 \% \mathrm{PrOH} / n$-Hexane with $0.1 \% \mathrm{Et}_{2} \mathrm{NH}$ eluent

$1.0 \mathrm{~mL} / \mathrm{min}, 254 \mathrm{~nm}$

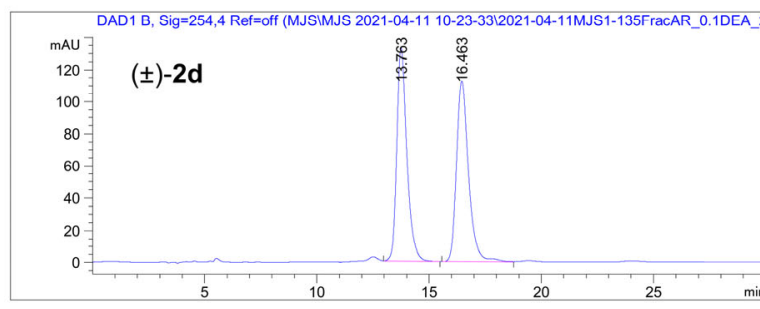

Peak RetTime Type width Area Height Area \# [min] [min] [mAU*s] [mAU] \%

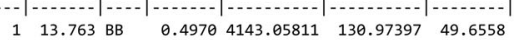

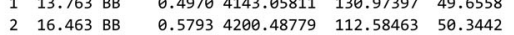
Totals :

$8343.54590 \quad 243.55860$
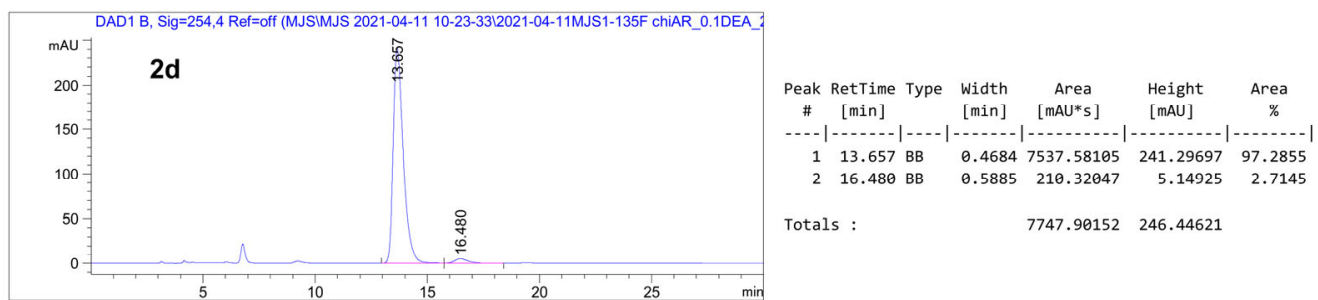

$\begin{array}{lllllll}1 & 13.657 & \text { BB } & 0.4684 & 7537.58105 & 241.29697 & 97.2855\end{array}$

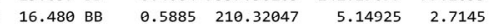
Totals :

$7747.90152 \quad 246.44621$

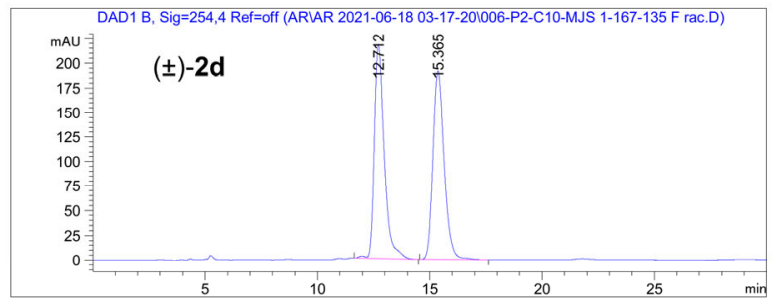

Peak RetTime Type Width Area Height Area \# [min] [min] [mAU*s] [mAU] $\%$ $\begin{array}{llllllll}1 & 12.712 & \text { VB R } & 0.4638 & 6673.65918 & 217.58028 & 49.9944\end{array}$ $\begin{array}{llllll}2 & 15.365 \text { вВ } & 0.5325 & 6675.16357 & 191.59926 & 50.0056\end{array}$ Totals :

$1.33488 \mathrm{e} 4 \quad 489.17953$

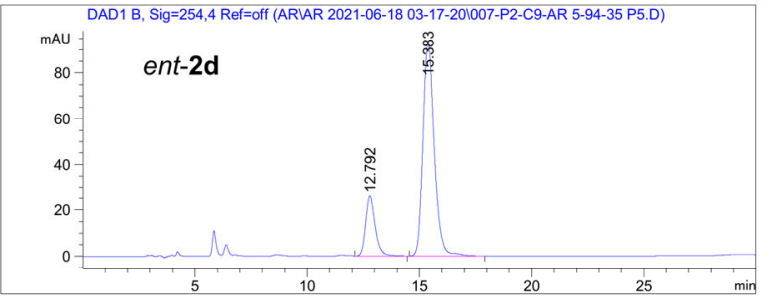

Peak RetTime Type width Area Height Area \# [min] [min] [maU*s] [maU] \% $\begin{array}{lllllll}1 & 12.792 & \text { BB } & 0.4489 & 759.93701 & 25.85584 & 18.9103\end{array}$

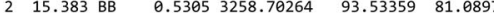
Totals : $4018.63965 \quad 119.38943$ 


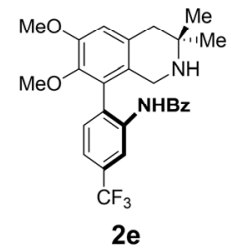

HPLC conditions:

Chiralpak AD, 20\% $\mathrm{PrOH} / n$-Hexane with $0.1 \% \mathrm{Et}_{2} \mathrm{NH}$ eluent

$1.0 \mathrm{~mL} / \mathrm{min}, 254 \mathrm{~nm}$

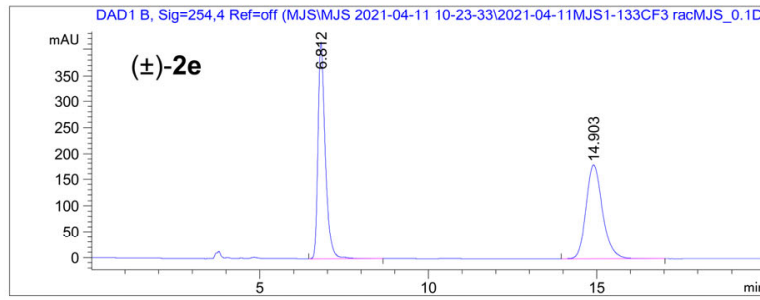

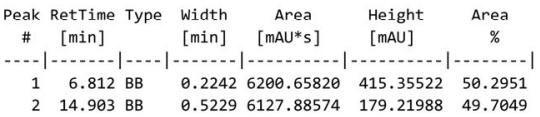
Totals :

$1.23285 \mathrm{e} \quad 594.57510$

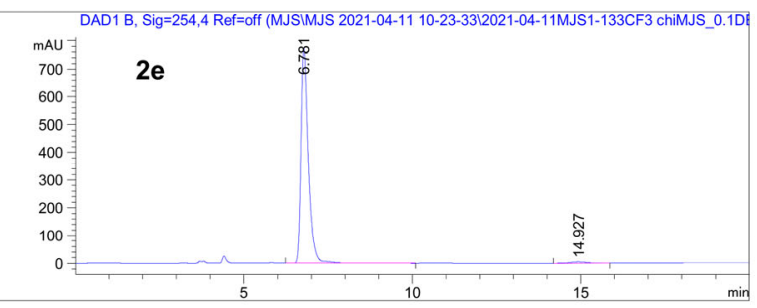

Peak RetTime Type width Area Height Area \# [min] [min] [mAU*s] [mAU] \%

$\begin{array}{llllll}1 & 6.781 W R & 0.2245 & 1.15796 \mathrm{e} 4 & 773.99963 & 98.6418\end{array}$ \begin{tabular}{ll}
$4.81897 \quad 1.3582$ \\
\hline
\end{tabular} Totals :

$1.17390 \mathrm{e} 4 \quad 778.81860$ 


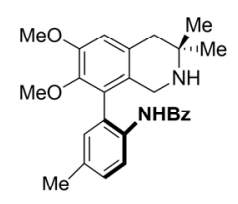

$2 f$

HPLC conditions:

Chiralpak AD, 20\% $\mathrm{PrOH} / n$-Hexane with $0.1 \% \mathrm{Et}_{2} \mathrm{NH}$ eluent

$1.0 \mathrm{~mL} / \mathrm{min}, 254 \mathrm{~nm}$

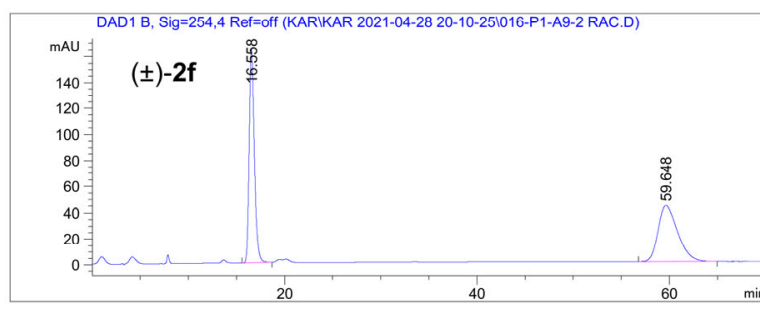

Peak RetTime Type width Area Height Area \# [min] $[\mathrm{min}]\left[\mathrm{mAU}^{*} \mathrm{~s}\right][\mathrm{mAU}] \quad \%$

$\begin{array}{llllll}1 & 16.558 \text { BV R } & 0.5733 & 6143.22119 & 163.89221 & 50.1477\end{array}$

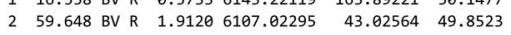
Totals :

$.22502 \mathrm{e} 4 \quad 206.91785$

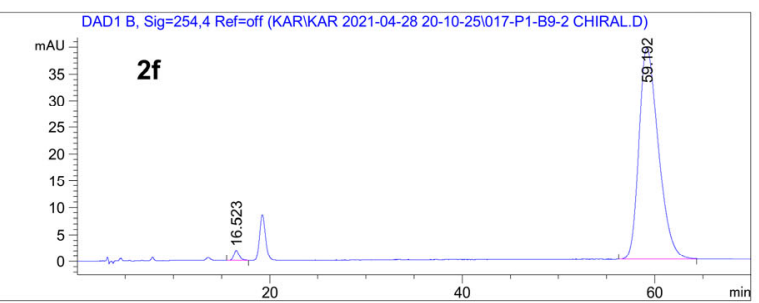

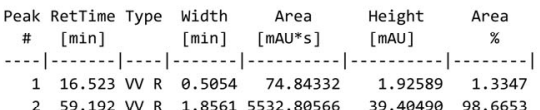

Totals :

$5607.64898 \quad 41.33079$

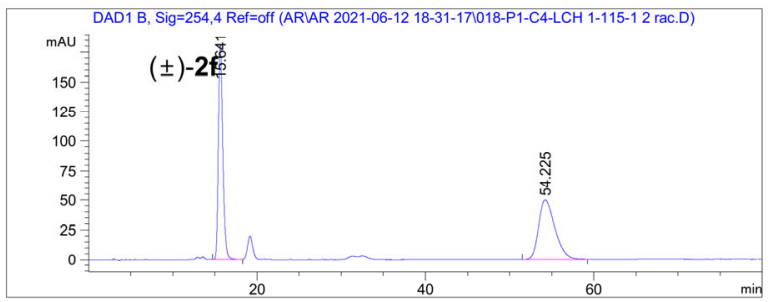

Peak RetTime Type Width Area Height Area \# [min] $[\mathrm{min}] \underset{[\mathrm{mAU} * \mathrm{~s}]}{\mathrm{m}] \mathrm{mAU}]} \begin{gathered}\text { Area } \\ \%\end{gathered}$ $\begin{array}{lllllll}1 & 15.641 \text { BV R } & 0.5604 & 6612.42529 & 180.92783 & 50.0900\end{array}$ $2 \quad 54.225$ BV R $1.8194 \quad 6588.65888 \quad 49.35549 \quad 49.9100$ Totals :

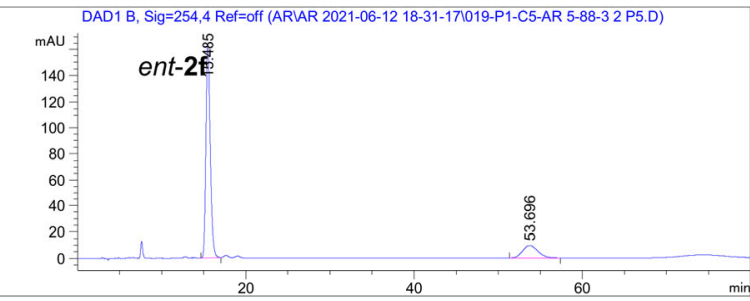

Peak RetTime Type width Area Height Area \# [min] [min] [mAU*s] [mAU] \%

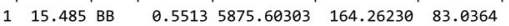
$\begin{array}{llllll}2 & 53.696 \mathrm{BB} & 1.4811 & 1200.32959 & 9.58649 & 16.9636\end{array}$ Totals : $7075.93262 \quad 173.76878$ 


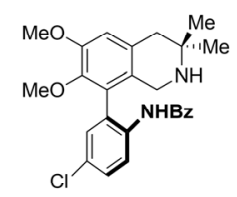

$2 \mathrm{~g}$
HPLC conditions:

Chiralpak AD, 20\% $\mathrm{PrOH} / n$-Hexane with $0.1 \% \mathrm{Et}_{2} \mathrm{NH}$ eluent

$1.0 \mathrm{~mL} / \mathrm{min}, 254 \mathrm{~nm}$

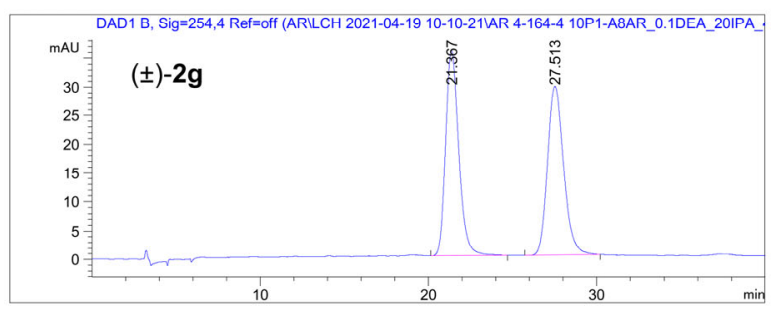

$$
\begin{aligned}
& \begin{array}{c}
\text { Peak RetTime Type Width Area Height Area } \\
{[\mathrm{min}]} \\
{\left[\mathrm{mAU} \mathrm{U}^{*} \mathrm{~s}\right]}
\end{array} \\
& \text { \# [min] }[\mathrm{min}]\left[\mathrm{mAU}^{*} \mathrm{~s}\right][\mathrm{mAU}] \quad \% \\
& \begin{array}{lllll}
21.367 \text { BV R } & 0.8340 & 1939.57886 & 35.69262 & 49.7912
\end{array} \\
& \begin{array}{llllll}
2 & 27.513 \text { BV R } & 1.0172 & 1955.84375 & 29.35800 & 50.2088
\end{array}
\end{aligned}
$$

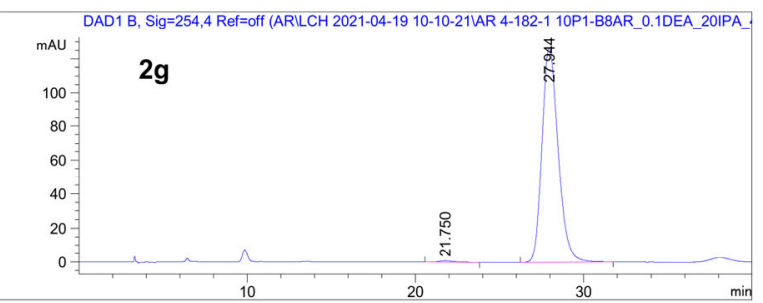

$$
\begin{aligned}
& \text { Peak RetTime Type Width Area Height Area } \\
& \text { \# [min] }[\mathrm{min}]\left[\mathrm{mAU}^{*} \mathrm{~s}\right][\mathrm{mAU}] \text { \% } \\
& \begin{array}{llllll}
1 & 21.750 \text { вв } & 0.7301 & 49.86015 & 8.35677 \mathrm{e}-1 & 0.5863
\end{array}
\end{aligned}
$$

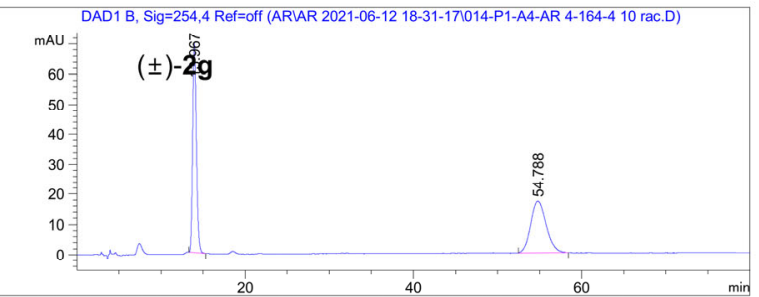

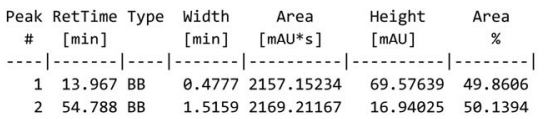

Totals :

$4326.36401 \quad 86.51664$

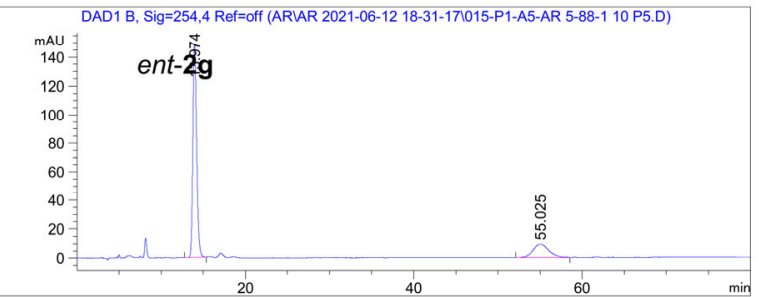

Peak RetTime Type Width Area Height Area

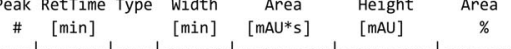
$\begin{array}{lllllll}1 & 13.974 \text { BB } & 0.4797 & 4648.71875 & 149.11816 & 79.5325\end{array}$ $\begin{array}{lllll}55.025 \text { WV R } & 1.4765 & 1196.33459 & 9.27729 & 20.4675\end{array}$

Totals : $\quad 5845.05334 \quad 158.39545$ 


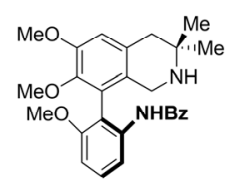

$2 h$
HPLC conditions:

Chiralpak AD, $20 \% \mathrm{PrOH} / n$-Hexane with $0.1 \% \mathrm{Et}_{2} \mathrm{NH}$ eluent

$1.0 \mathrm{~mL} / \mathrm{min}, 254 \mathrm{~nm}$

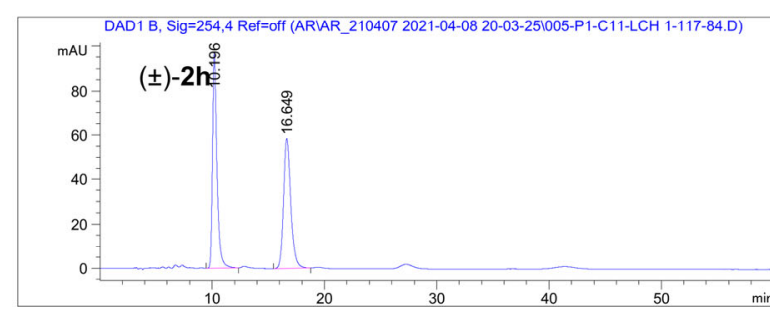

$$
\begin{aligned}
& \begin{array}{ccccc}
\text { Peak RetTime Type width } & \text { Area } & \text { Height Area } \\
\# \text { [min] } & {[\mathrm{min}]} & {[\mathrm{mAU} * \mathrm{~s}]} & {[\mathrm{mAU}]} &
\end{array} \\
& \text { \# [min] } \mid \text { [min] [mAU"s] [ [mAU] }
\end{aligned}
$$

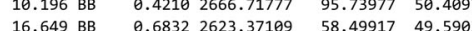

$$
\begin{aligned}
& \text { Totals : }
\end{aligned}
$$

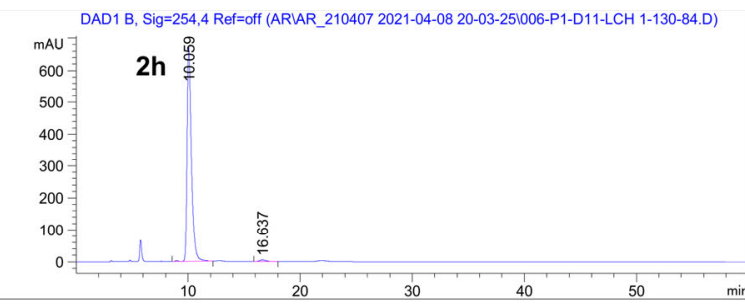

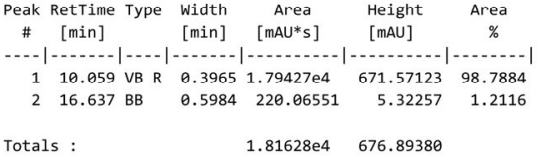




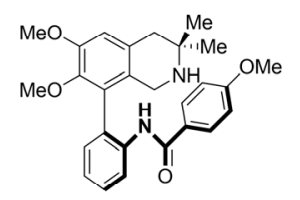

$2 \mathbf{i}$

HPLC conditions:

Chiralpak AD, $20 \% \mathrm{PrOH} / n$-Hexane with $0.1 \% \mathrm{Et}_{2} \mathrm{NH}$ eluent

$1.0 \mathrm{~mL} / \mathrm{min}, 254 \mathrm{~nm}$

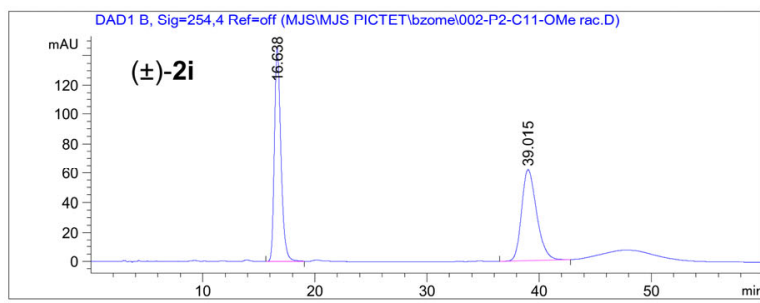

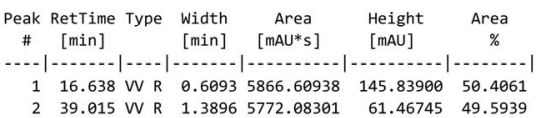

Totals :

$1.16387 \mathrm{e4} \quad 287.30645$

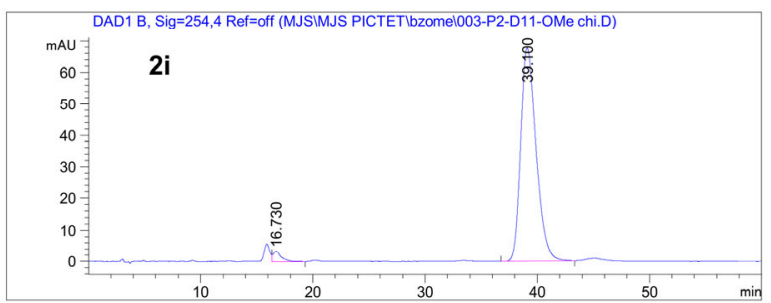

Peak RetTime Type width Area Height Area \# [min] [min] [mAU*s] [mAU] \% $\begin{array}{lllllll}1 & 16.730 \text { VB } & 0.7206 & 169.39801 & 3.33476 & 2.5529\end{array}$ Totals :

$6635.49664 \quad 70.98235$

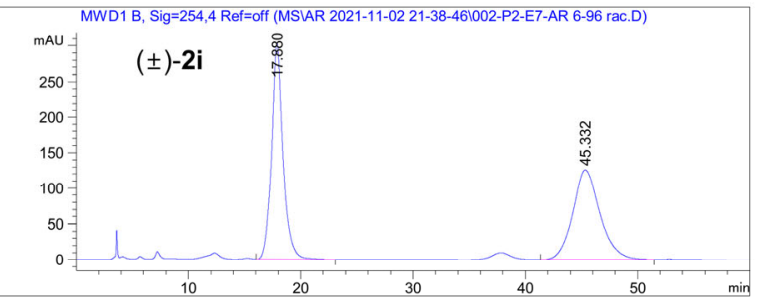

Peak RetTime Type width Area Height Area

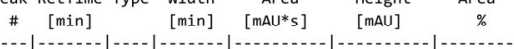
$\begin{array}{llllll}1 & 17.880 \text { BB } & 1.0058 & 2.13745 e 4 & 303.45477 & 50.3755\end{array}$ $\begin{array}{llll} & 3465 \quad 2.18558 \mathrm{e} & 125.81335 & 49.6245\end{array}$ Totals : $4.24303 \mathrm{e} 4 \quad 429.26812$

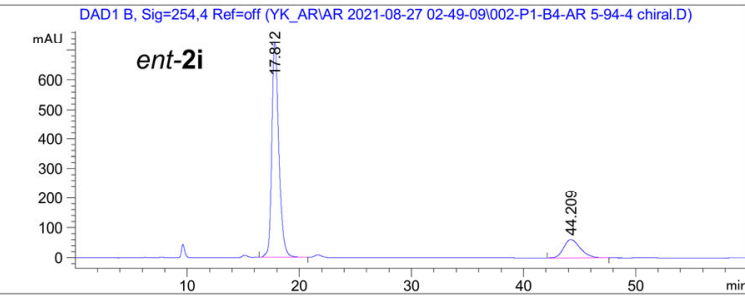

Peak RetTime Type width Area Height Area \# [min] [min] [mAU*s] [mAU] \% $\begin{array}{lllllll}1 & 17.812 & \text { BB } & 0.6571 & 3.16618 \mathrm{e} 4 & 728.50043 & 83.3999\end{array}$ $\begin{array}{lllllll}2 & 44.289 & \text { BB } & 1.5122 & 6302.05762 & 61.25736 & 16.6901\end{array}$ $\begin{array}{lll}\text { Totals : } & 3.79639 \mathrm{e} 4 & 789.75779\end{array}$ 


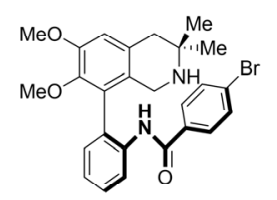

2j

HPLC conditions:

Chiralpak AD, 20\% $\mathrm{PrOH} / n$-Hexane with $0.1 \% \mathrm{Et}_{2} \mathrm{NH}$ eluent

$1.0 \mathrm{~mL} / \mathrm{min}, 254 \mathrm{~nm}$

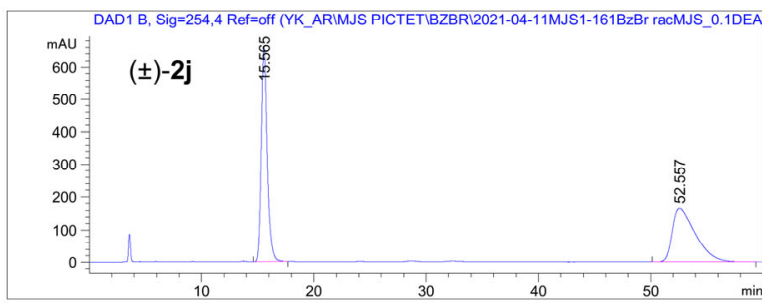

Peak RetTime Type width Area Height Area \# [min] $[\mathrm{min}]\left[\mathrm{MAU}^{*} \mathrm{~s}\right]{ }_{[\mathrm{mAU}]} \%$ $\begin{array}{lllllll}1 & 15.565 & \text { BB } & 0.5561 & 2.43672 \mathrm{e} 4 & 660.95966 & 50.4600\end{array}$

$4.8290204 \quad 826.96471$

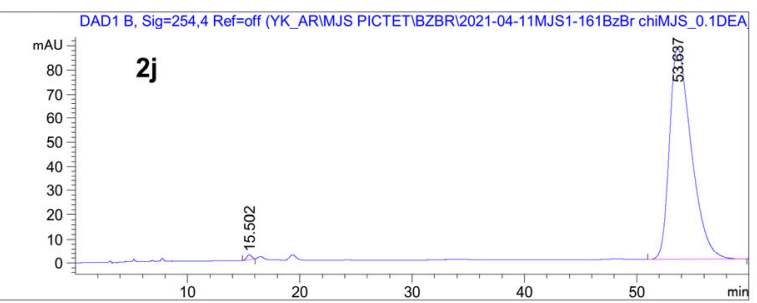

Peak RetTime Type width Area Height Area \# [min] [min] [mAU*s] [mAU] \% $\begin{array}{llllll}1 & 15.502 \mathrm{BB} & 0.4332 & 61.39697 & 2.05146 & 0.5146\end{array}$ Totals :

$1.19306 \mathrm{e} 4 \quad 89.71839$

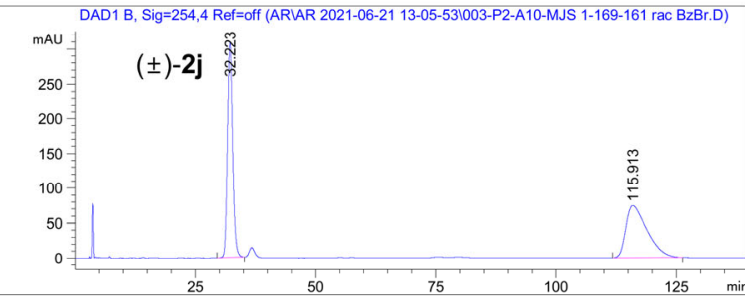

Peak RetTime Type Width Area Height Area \# [min] [min] [mAl*s] $\begin{gathered}\text { Height } \\ \text { [mAU] }\end{gathered}$

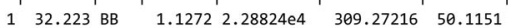
$\begin{array}{llll}3.5919 & 2.27773 e 4 \quad 74.39676 & 49.884\end{array}$ Totals : $4.56597 e 4 \quad 383.66891$

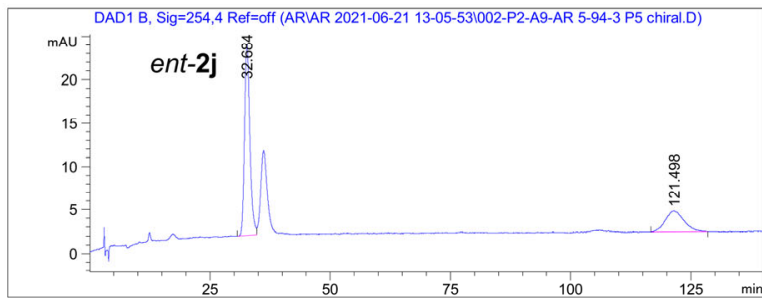

Peak RetTime Type width Area Height Area \# [min] [min] [mAU*s] [mAU] \% $\begin{array}{llllll}1 & 32.684 \mathrm{BV} & 1.1827 & 1701.73914 & 21.99244 & 72.3701\end{array}$

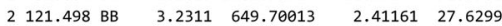
Totals : $2351.43927 \quad 24.40405$ 


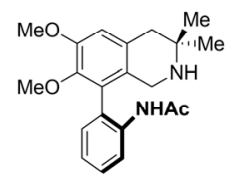

$2 k$
HPLC conditions:

Chiralpak AD, $20 \% \mathrm{PrOH} / n$-Hexane with $0.1 \% \mathrm{Et}_{2} \mathrm{NH}$ eluent

$1.0 \mathrm{~mL} / \mathrm{min}, 254 \mathrm{~nm}$

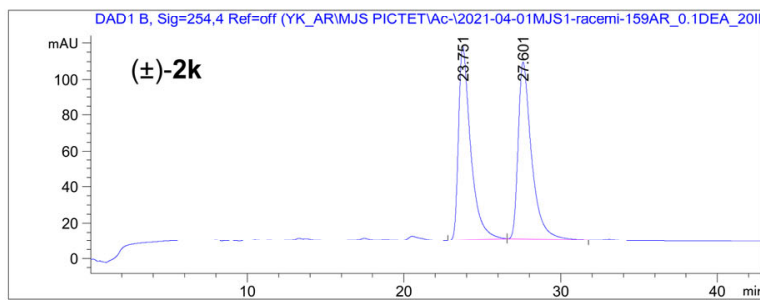

Peak RetTime Type width Area Height Area \# [min] ${ }^{[m i n]}\left[\right.$ [mAU*s] $_{\text {[mAU] }} \%$ $\begin{array}{lllllll}1 & 23.751 \text { вB } & 0.7960 & 5796.06836 & 107.66891 & 49.9857\end{array}$

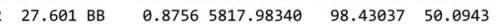
Totals :

$1.16141 \mathrm{e} \quad 206.09928$

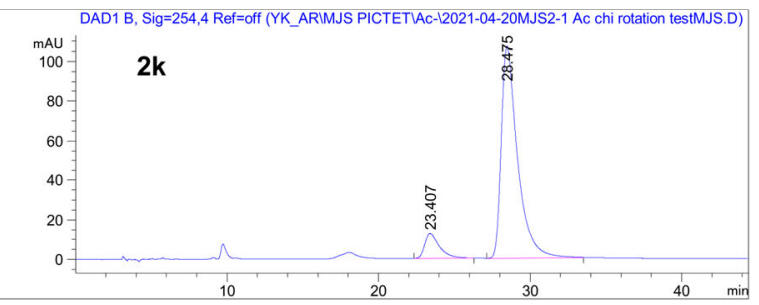

Peak RetTime Type Width Area
\# [min] \begin{tabular}{cccccc}
$\#$ & {$[\mathrm{~min}]$} & {$[\mathrm{min}]$} & {$[\mathrm{mAU} * \mathrm{~s}]$} & {$[\mathrm{mAU}]$} & $\%$ \\
\hdashline 1 & $23.497 \mathrm{BB}$ & 0.9347 & 826.77014 & 12.32292 & 9.3832
\end{tabular} $\begin{array}{rrrrrr}1 & 23.467 \text { BB } & 0.9347 & 826.77014 & 12.32292 & 9.3832 \\ 2 & 28.475 \text { BB } & 1.0958 & 7984.37646 & 106.85850 & 98.6168\end{array}$ Totals : 8811.14661119 .17342 


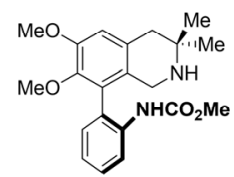

2I
HPLC conditions:

Chiralpak AD, $10 \% \mathrm{PrOH} / n$-Hexane with $0.1 \% \mathrm{Et}_{2} \mathrm{NH}$ eluent

$1.0 \mathrm{~mL} / \mathrm{min}, 254 \mathrm{~nm}$

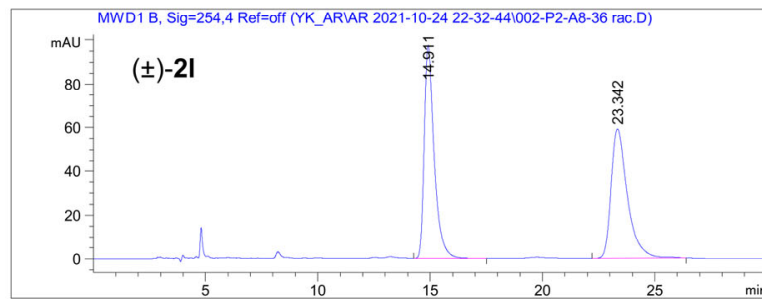

$\begin{array}{ccccc}\text { Peak RetTime Type Width Area } & \text { Height Area } \\ \text { \# [min] } & {[\mathrm{min}]} & {[\mathrm{mAU} * \mathrm{~s}]} & {[\mathrm{maU}]} & \end{array}$ \# [min]

$\begin{array}{lllllll}1 & 14.911 & \text { BV R } & 0.4587 & 2994.37744 & 97.38841 & 49.9368\end{array}$

$\begin{array}{llllll}2 & 23.342 \text { BV R } & 0.7504 & 3001.95996 & 59.32301 & 50.0632\end{array}$

Totals :

$5996.33740 \quad 156.71142$

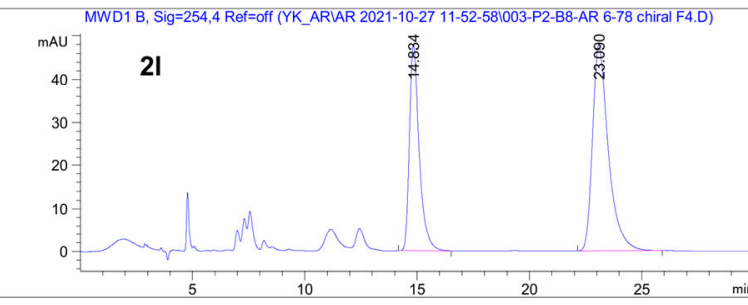

Peak RetTime Type Width Area Height Area \# [min] [min] [mAU*s] [mAU] \% $\begin{array}{llllll}1 & 14.834 \mathrm{BB} & 0.4534 & 1446.38379 & 47.75195 & 38.0447\end{array}$

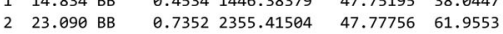
Totals : $3801.79883 \quad 95.52951$ 


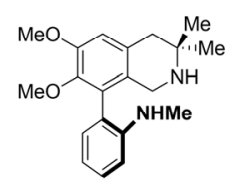

$2 m$
HPLC conditions:

Chiralpak AD, $2 \%$ PrOH $/ n$-Hexane with $0.1 \% \mathrm{Et}_{2} \mathrm{NH}$ eluent

$1.0 \mathrm{~mL} / \mathrm{min}, 280 \mathrm{~nm}$

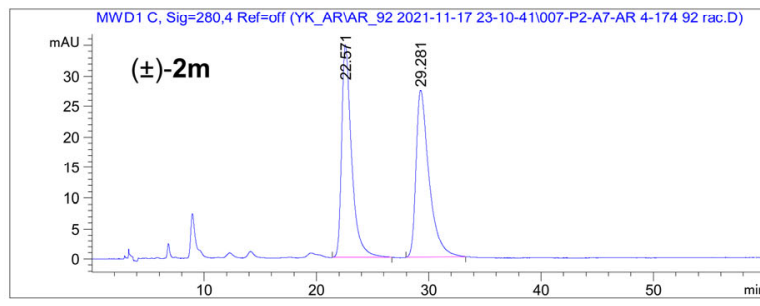

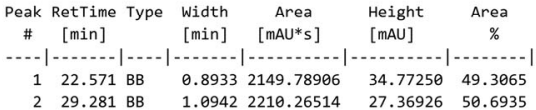

$\begin{array}{lll}\text { Totals : } & 4360.05420 \quad 62.14176\end{array}$

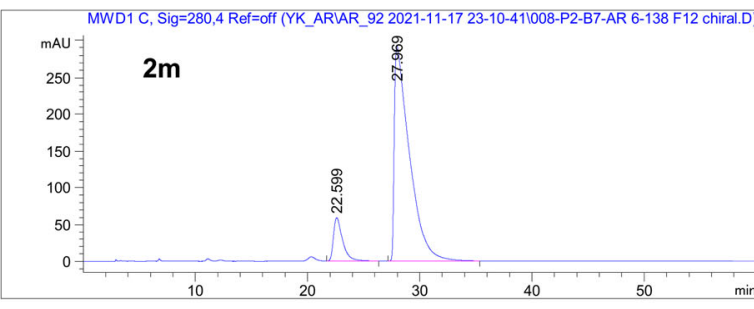

Peak RetTime Type width Area Height Area \# [min] [min] [mAU*s] [mAU] \% $\begin{array}{lllllll}1 & 22.599 \text { Bв } & 0.8264 & 3331.44263 & 59.39157 & 10.8544\end{array}$ $\begin{array}{llllll}2 & 27.969 \text { BB } & 1.3109 & 2.73606 \mathrm{e} & 291.53448 & 89.1456\end{array}$ Totals :

$3.06920 \mathrm{e} 4 \quad 350.92606$ 


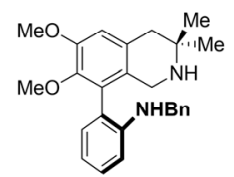

$2 n$
HPLC conditions:

Chiralpak IC, $10 \%$ PrOH$/ n$-Hexane with $0.1 \% \mathrm{Et}_{2} \mathrm{NH}$ eluent

$1.0 \mathrm{~mL} / \mathrm{min}, 254 \mathrm{~nm}$

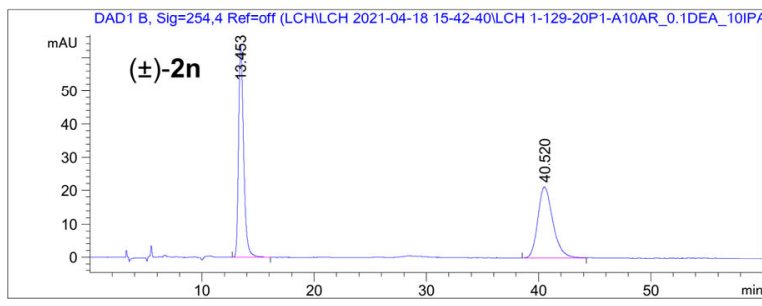

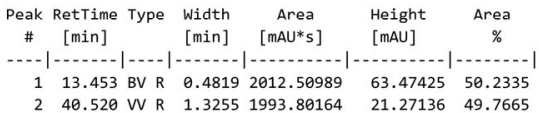
Totals : $4006.31152 \quad 84.74561$

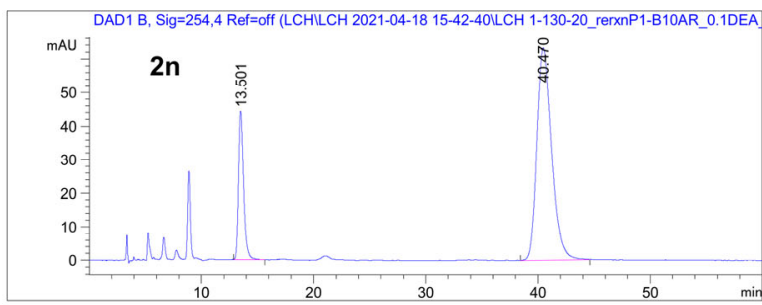

Peak RetT1me Type W1dth Area Height Area \# [min] $[\mathrm{min}][\mathrm{mAU} * \mathrm{~s}] \quad[\mathrm{mAU}] \quad \%$ $\begin{array}{llllll}1 & 13.501 \text { BB } & 0.1803 & 1397.21133 & 11.26066 & 19.5111 \\ 2 & 40.470 \text { BB } & 1.4006 & 5762.92383 & 63.15221 & 80.4859\end{array}$ Totals

$7160.16516 \quad 107.41287$ 


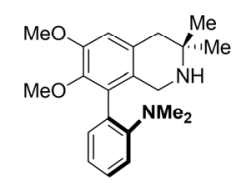

20
HPLC conditions:

Chiralpak AD, 3\% EtOH $/ n$-Hexane with $0.1 \% \mathrm{Et}_{2} \mathrm{NH}$ eluent

$1.0 \mathrm{~mL} / \mathrm{min}, 254 \mathrm{~nm}$

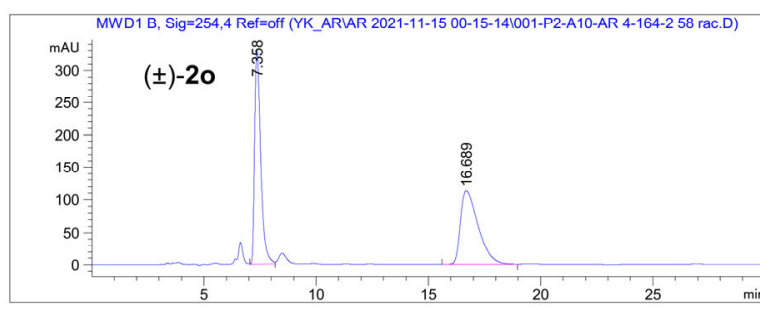

Peak RetTime Type width Area Height Area \# [min] [min] [mAU*s] [mAU] \% $\begin{array}{llllll}1 & 7.358 \mathrm{VF} & 0.2899 & 6278.79541 & 330.05215 & 50.1468\end{array}$ $\begin{array}{llllll}2 & 16.689 \text { BB } & 0.8317 & 6242.02490 & 113.13393 & 49.8532\end{array}$ Totals :

$1.25208 \mathrm{e} 4 \quad 443.18608$

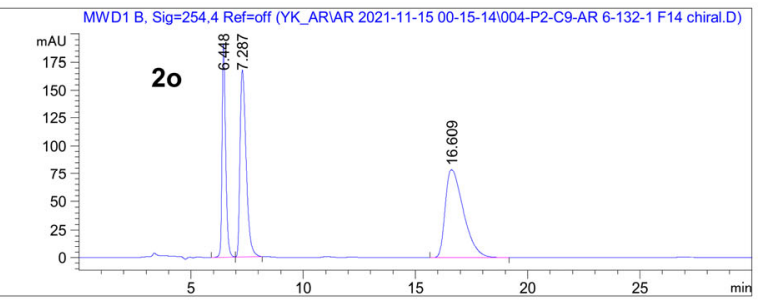

Peak RetTime Type Width Area Height Area H [min] [min] [mAU*s] [mAU] \%

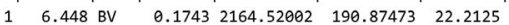
$\begin{array}{llllll}7.287 \mathrm{VF} & 0.2873 & 3147.80884 & 167.48235 & 32.3031\end{array}$ $\begin{array}{lllllll}3 & 16.609 \text { BB } & 0.8622 & 4432.26709 & 78.80264 & 45.4844\end{array}$ Totals : $9744.59595 \quad 437.15971$

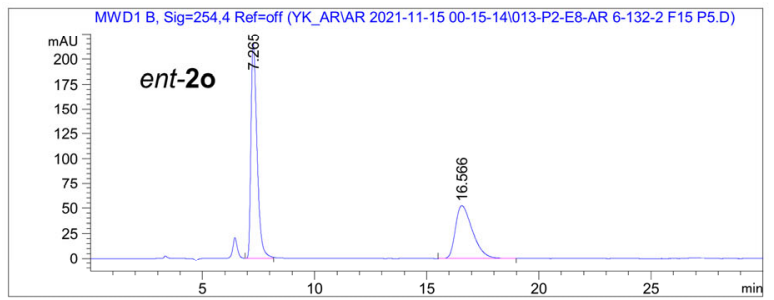
\begin{tabular}{cccccc}
$\begin{array}{c}\text { Peak RetTime Type } \\
* \\
{[\text { [min] }}\end{array}$ & $\begin{array}{c}\text { Width } \\
{[\text { min] }}\end{array}$ & $\begin{array}{c}\text { Area } \\
{[\text { [mAU*s] }}\end{array}$ & $\begin{array}{c}\text { Height } \\
{[\text { mAU] }}\end{array}$ & $\begin{array}{c}\text { Area } \\
\%\end{array}$ \\
\hdashline 1 & $7.265 \mathrm{BF}$ & 0.2898 & 4040.99609 & 214.52324 & 59.4952 \\
\hline 2 & $16.566 \mathrm{BB}$ & 0.7923 & 2751.14380 & 52.24555 & 40.5848
\end{tabular} Totals : $\quad 6792.13989 \quad 266.76879$

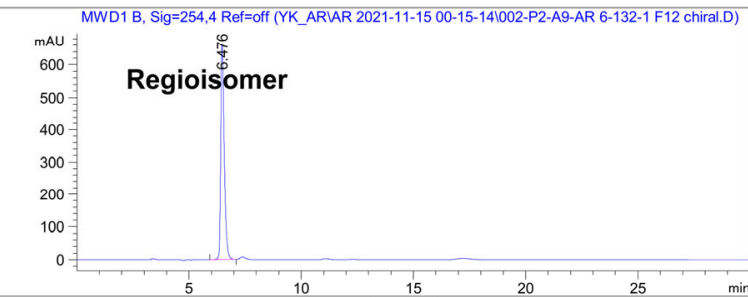

Peak RetTime Type width Area Height Area \# [min] [min] [mA**s] [mAU] \% 10.476 BB $\quad 0.16817294 .35596 \quad 664.29456 \quad 100.0000$ Totals : $7294.35596 \quad 664.29456$ 


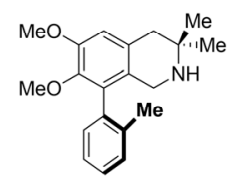

$2 q$
HPLC conditions:

Chiralpak IC, $10 \%$ 'PrOH$/ n$-Hexane with $0.1 \% \mathrm{Et}_{2} \mathrm{NH}$ eluent

$1.0 \mathrm{~mL} / \mathrm{min}, 254 \mathrm{~nm}$

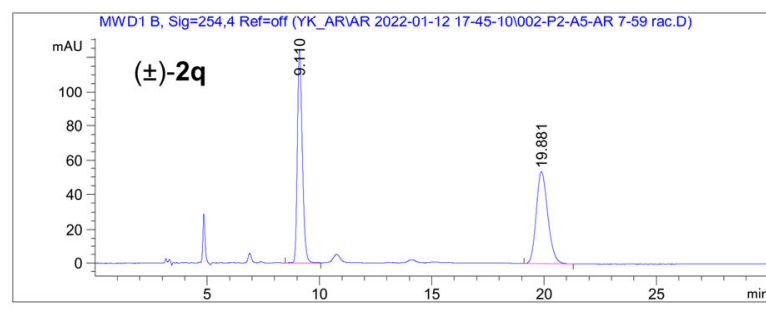

Peak RetTime Type width Area Height Area \# [min] $[$ [min] [mAU*s] [mAU] \% $\begin{array}{llllll}1 & 9.110 \text { BB } & 0.2360 & 1921.08081 & 124.48693 & 50.1178\end{array}$ $\begin{array}{llllll}19.881 & \text { BB } & 0.5460 & 1912.04736 & 53.37032 & 49.8822\end{array}$ Totals : $3833.12817 \quad 177.85725$

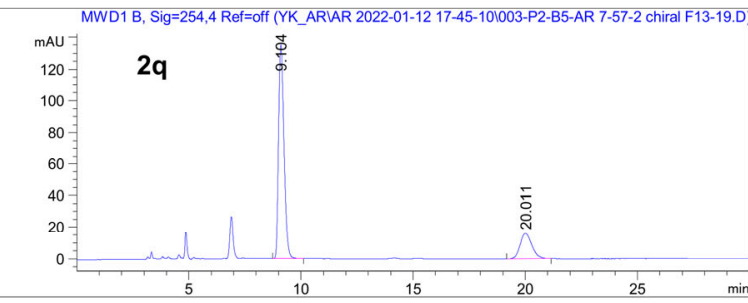

Peak RetTime Type Width Area Height Area
$\#$ [min] \# [min] [min] [mAU*s] [mAU] \%

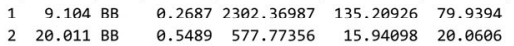
Totals :

$2880.14343 \quad 151.15024$ 


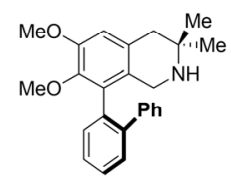

$2 r$
HPLC conditions:

Chiralpak AD-H, $3 \%{ }^{\mathrm{i}} \mathrm{PrOH} / n-\mathrm{Hexane}$ with $0.1 \% \mathrm{Et}_{2} \mathrm{NH}$ eluent

$1.0 \mathrm{~mL} / \mathrm{min}, 254 \mathrm{~nm}$

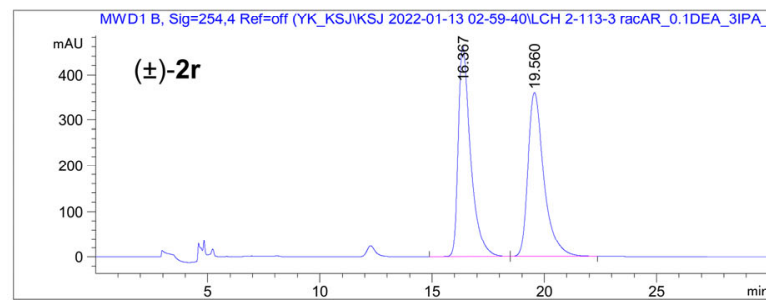

$$
\begin{aligned}
& \begin{array}{c}
\text { Peak RetTime Type Width Area } \\
\begin{array}{c}
\text { \# [min] } \\
{[\text { min] }}
\end{array} \text { [mAU*s] }
\end{array} \\
& \text { H [min] }[\mathrm{min}][\mathrm{mAU} * \mathrm{~s}][\mathrm{mAU}] \text { \% } \\
& \begin{array}{llllll}
1 & 16.367 \text { BB } & 0.5713 & 1.76411 \mathrm{e} 4 & 460.15869 & 49.8434
\end{array}
\end{aligned}
$$
Totals :

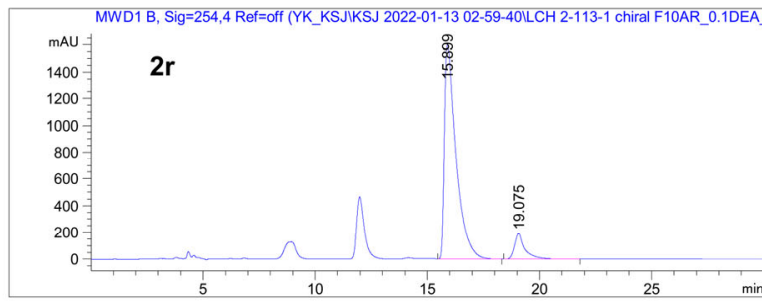

Peak RetTime Type Width Area lleight Area \# [min] $[$ min] [mAU*s] [mAU] \%

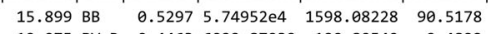
9.4822 Totals :

$6.35180 \mathrm{e} 4 \quad 1788.88768$ 


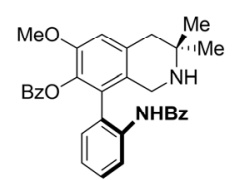

2s
HPLC conditions:

Chiralpak AD, 30\% $\mathrm{PrOH} / n-\mathrm{Hexane}$ with $0.1 \% \mathrm{Et}_{2} \mathrm{NH}$ eluent

$1.0 \mathrm{~mL} / \mathrm{min}, 254 \mathrm{~nm}$

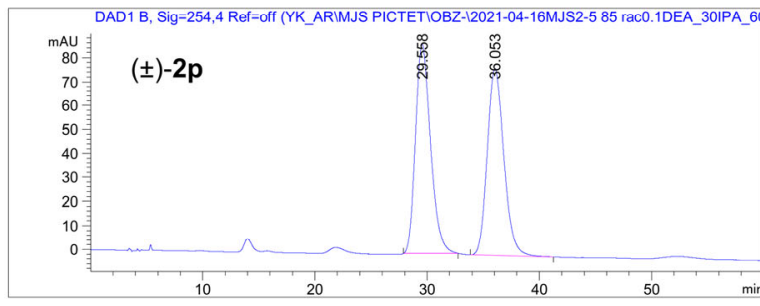

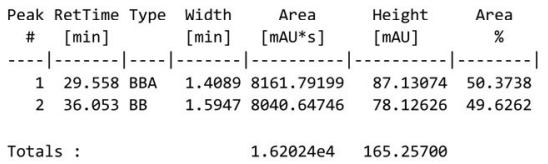

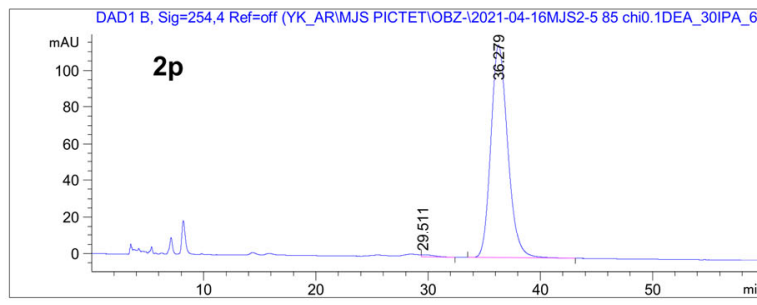

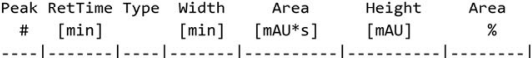

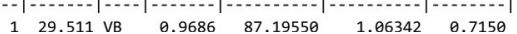
$\begin{array}{llllrr}1 & 29.511 \text { VB } & 0.9686 & 87.19550 & 1.06342 & 0.7150 \\ 2 & 36.279 \text { W R R } & 1.6401 & 1.21076 \mathrm{e} 4 & 115.57946 & 99.2850\end{array}$ Totals :

$1.21948 \mathrm{e} 4 \quad 116.64288$ 


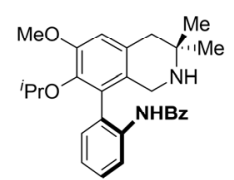

$2 t$

HPLC conditions:

Chiralpak IC, $20 \%$ PrOH$/ n$-Hexane with $0.1 \% \mathrm{Et}_{2} \mathrm{NH}$ eluent

$1.0 \mathrm{~mL} / \mathrm{min}, 254 \mathrm{~nm}$

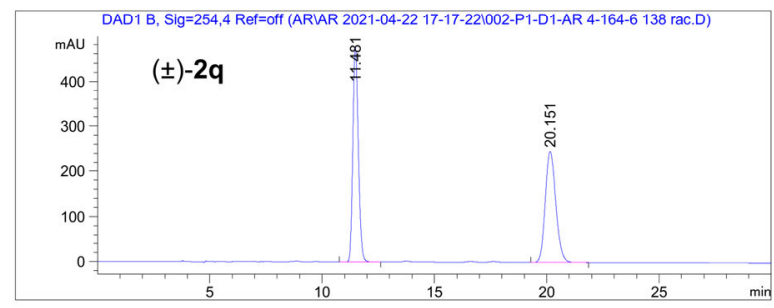

Peak RetTime Type Width Area Height Area
$\#$ [min] \# [min] $\left[\right.$ min] $\left[\mathrm{MAU}^{*}{ }^{2}\right][\mathrm{mAU}] \quad \%$

$0.5046 .13232 \quad 476.11194 \quad 50.0504$

Totals :

$1.60761 \mathrm{e} 4 \quad 722.91957$

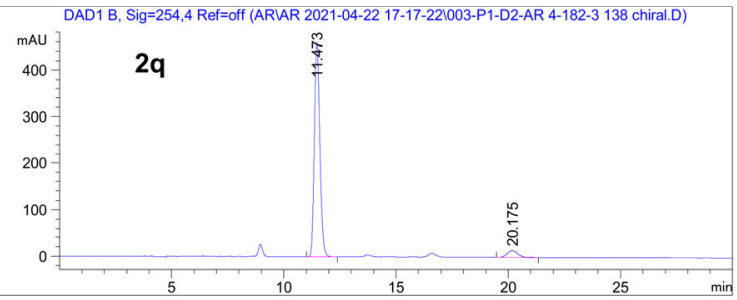

$\begin{array}{cccc}\begin{array}{c}\text { Peak RetTime Type Width Area } \\ \text { \# [min] }\end{array} & \text { Height } & \text { Area } \\ {[\mathrm{min}]} & {[\mathrm{mAU} * \mathrm{~s}]} & {[\mathrm{mAU}]} & \%\end{array}$ \# [min] [min] [mAU*s] [MAU] \%

$\begin{array}{lllllll}1 & 11.473 \text { RB } & 0.26117750 .18918 \quad 458.79071 & 94.3994\end{array}$

$\begin{array}{lrrrrr}1 & 11.473 \text { BB } & 0.2611 & 7756.18918 & 458.79071 & 94.3994 \\ 2 & 20.175 \text { BB } & 0.4946 & 460.59000 & 14.34329 & 5.6096\end{array}$

Totals :

$8210.77017 \quad 473.13400$ 


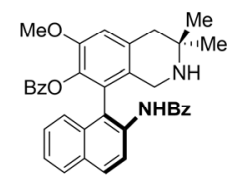

$2 v$
HPLC conditions:

Chiralpak AD, $50 \%$ $\mathrm{PrOH} / n$-Hexane with $0.1 \% \mathrm{Et}_{2} \mathrm{NH}$ eluent

$1.0 \mathrm{~mL} / \mathrm{min}, 254 \mathrm{~nm}$

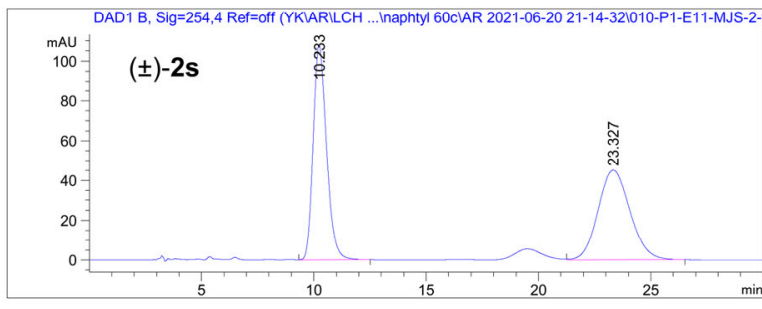

Peak RetTime Type Width Area Height Area

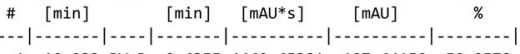

$\begin{array}{lllllll}10.233 & \text { BV R } & 0.6355 & 4460.65381 & 107.64150 & 50.0578\end{array}$

$\begin{array}{llllll}23.327 \text { W R } & 1.4056 & 4450.34863 & 44.85212 & 49.9422\end{array}$

Totals :

$8911.00244 \quad 152.49362$

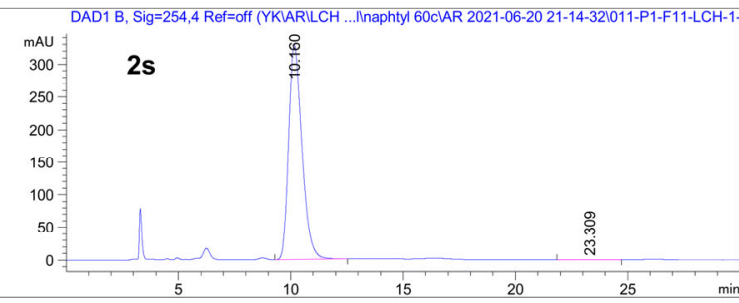

Peak RetTime Type Width Area Height Area \# [min] [min] [mAU*s] [mAU] \%

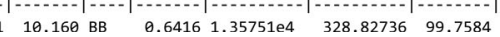
$\begin{array}{llllll}1 & 19.160 \text { BB } & 0.6416 & 1.35751 e 4 & 328.82736 & 99.7584 \\ 2 & 23.309 \text { BV } & 1.0879 & 32.87439 & 3.71687 \mathrm{e}-1 & 0.2416\end{array}$ Totals :

$1.36080 \mathrm{e} 4 \quad 329.19905$ 


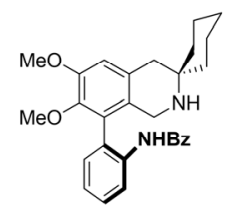

$2 w$
HPLC conditions:

Chiralpak AD, $10 \% \mathrm{PrOH} / n$-Hexane with $0.1 \% \mathrm{Et}_{2} \mathrm{NH}$ eluent

$1.0 \mathrm{~mL} / \mathrm{min}, 254 \mathrm{~nm}$

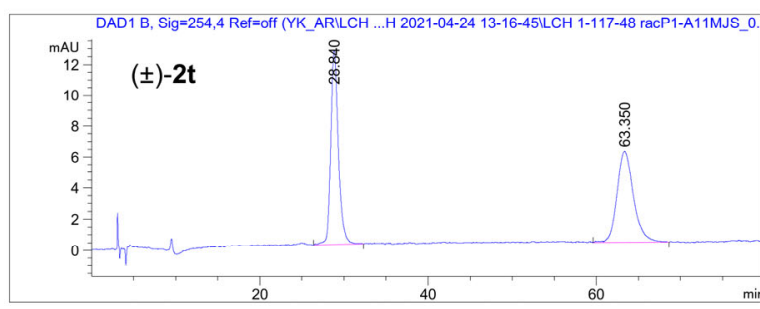

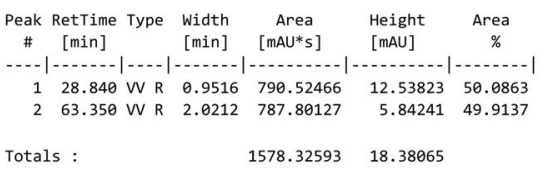

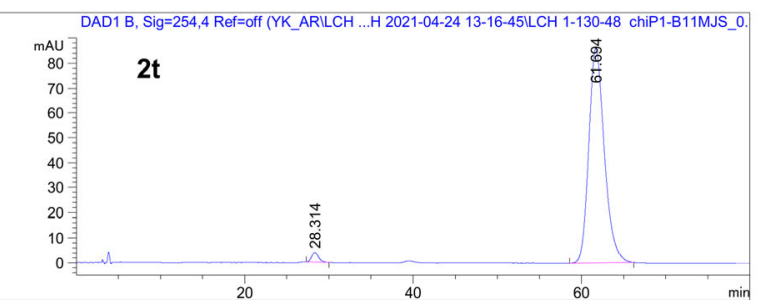

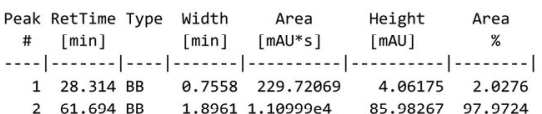

Totals :

$.13296 \mathrm{e} 4 \quad 90.04443$

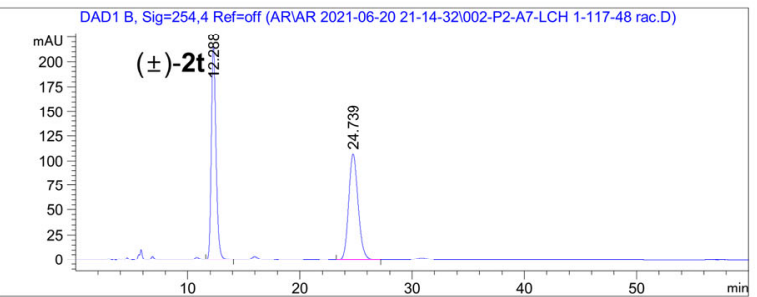

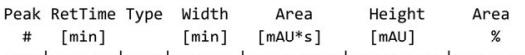
1. 12.288 B日 $0.440326279 .66357 \quad 217.84012 \quad 50.2008$ 0.4903 $6279.6357 \quad 107.8041159 .2008$ Totals :

$1.25091 \mathrm{e} 4 \quad 324.84423$

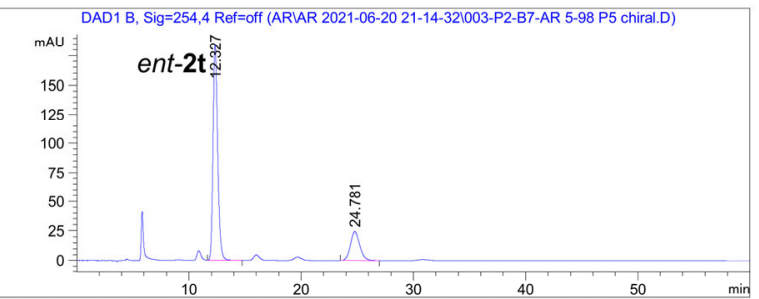

Peak RetTime Type Width Area Height Area \# [min] [min] [mAU*s] [mAU] \% $\begin{array}{llllll}1 & 12.327 \mathrm{BB} & 0.4364 & 5280.51807 & 184.23817 & 78.9477\end{array}$ $\begin{array}{llllll}24.781 \text { BB } & 0.8794 & 1408.11194 & 24.25200 & 21.0523\end{array}$ Totals : $6688.63000 \quad 208.49017$ 


\section{Experiment for X-ray Crystallograpy of $2 b$}

\section{Experimental details}

Single crystals of $\mathbf{2 b}$ were obtained from dichloromethane-hexane co-solvent system (17 $\mathrm{mg}$ of $\mathbf{2 b}$ and $0.6 \mathrm{~mL}$ of 1:5, dichloromethane-hexane) by slow evaporation for 12 h. A suitable crystal was selected and the single crystal X-ray diffraction data was collected on a SuperNova, Dual, $\mathrm{Cu}$ at home/near, AtlasS2 diffractometer. The crystal was kept at 294.3(9) K during data collection. Using Olex $2,{ }^{7}$ the structure was solved with the ShelXT ${ }^{8}$ structure solution program using Direct Methods and refined with the ShelXL ${ }^{9}$ refinement package using Least Squares minimization. The full numbering scheme of compound $\mathbf{2} \mathbf{b}$ can be found in the full details of the X-ray structure determination (CIF), which is included as Supporting Information. CCDC number 2122088 (2b) contains the supplementary crystallographic data for this paper. These data can be obtained free of charge from The Cambridge Crystallographic Data Center via www.ccdc.cam.ac.uk/data_request/cif.

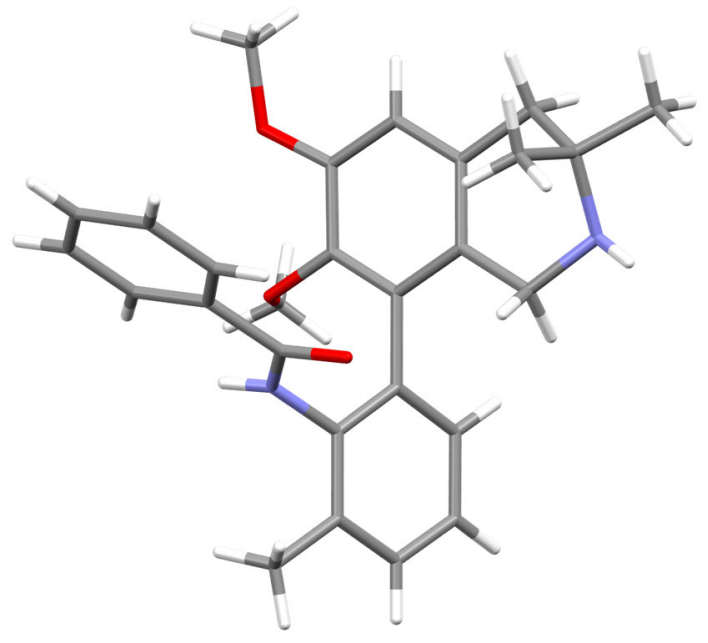

Figure S1. The single crystal X-ray structure of $\mathbf{2 b}$; ellipsoid contour at the $50 \%$ probability level. 
Table S6 Crystal data and structure refinement for $\mathbf{2 b}$.

\begin{tabular}{|c|c|}
\hline Identification code & $2 b$ \\
\hline Empirical formula & $\mathrm{C}_{27} \mathrm{H}_{30} \mathrm{~N}_{2} \mathrm{O}_{3}$ \\
\hline Formula weight & 430.53 \\
\hline Temperature/K & 294.3(9) \\
\hline Crystal system & orthorhombic \\
\hline Space group & $\mathrm{P} 212121$ \\
\hline $\mathrm{a} / \AA ̊$ & $9.8973(2)$ \\
\hline $\mathrm{b} / \AA ̊$ & $10.8332(3)$ \\
\hline $\mathrm{c} / \AA ̊$ & $22.4444(5)$ \\
\hline$\alpha /^{\circ}$ & 90 \\
\hline$\beta /{ }^{\circ}$ & 90 \\
\hline$\gamma /{ }^{\circ}$ & 90 \\
\hline Volume $/ \AA^{3}$ & $2406.46(10)$ \\
\hline $\mathrm{Z}$ & 4 \\
\hline$\rho_{\text {calcg }} / \mathrm{cm}^{3}$ & 1.188 \\
\hline$\mu / \mathrm{mm}^{-1}$ & 0.616 \\
\hline $\mathrm{F}(000)$ & 920.0 \\
\hline Crystal size $/ \mathrm{mm}^{3}$ & $0.2 \times 0.15 \times 0.1$ \\
\hline Radiation & $\mathrm{CuK} \alpha(\lambda=1.54184)$ \\
\hline \multicolumn{2}{|c|}{$2 \Theta$ range for data collection $/{ }^{\circ} 7.878$ to 147.664} \\
\hline Index ranges & $-11 \leq \mathrm{h} \leq 7,-7 \leq \mathrm{k} \leq 13,-25 \leq 1 \leq 27$ \\
\hline Reflections collected & 5979 \\
\hline Independent reflections & $3818\left[\mathrm{R}_{\text {int }}=0.0298, \mathrm{R}_{\text {sigma }}=0.0277\right]$ \\
\hline Data/restraints/parameters & $3818 / 0 / 294$ \\
\hline Goodness-of-fit on $\mathrm{F}^{2}$ & 1.065 \\
\hline Final $R$ indexes $[\mathrm{I}>=2 \sigma(\mathrm{I})]$ & $\mathrm{R}_{1}=0.0705, \mathrm{wR}_{2}=0.1981$ \\
\hline Final $\mathrm{R}$ indexes [all data] & $\mathrm{R}_{1}=0.0767, \mathrm{wR}_{2}=0.2078$ \\
\hline \multicolumn{2}{|c|}{ Largest diff. peak/hole / e $\AA^{-3} 0.23 /-0.29$} \\
\hline Flack parameter & $0.1(3)$ \\
\hline
\end{tabular}

Table S7 Fractional Atomic Coordinates $\left(\times 10^{4}\right)$ and Equivalent Isotropic Displacement Parameters $\left(\AA^{2} \times 10^{3}\right)$ for $2 \mathrm{~b}$. $U_{\text {eq }}$ is defined as $1 / 3$ of the trace of the orthogonalised $\mathrm{U}_{\mathrm{IJ}}$ tensor.

$\begin{array}{llll}\text { Atom } \boldsymbol{x} & \boldsymbol{y} & \boldsymbol{z} & \mathbf{U ( e q )} \\ \text { O001 5416(3) } & 2805(3) & 4630.3(15) & 80.0(8) \\ \text { O002 9837(3) } & 4394(3) & 4267.9(11) & 74.7(8) \\ \text { N003 7583(3) } & 3036(3) & 4904.0(12) & 57.8(7) \\ \text { O004 10159(3) } & 4108(5) & 3090.6(12) & 103.4(13) \\ \text { C005 7417(4) } & 4240(3) & 5161.8(13) & 54.3(7) \\ \text { C006 7470(4) } & 5273(3) & 4799.5(13) & 60.1(8) \\ \text { C007 6581(4) } & 2410(3) & 4640.3(14) & 58.4(8) \\ \text { C008 8798(4) } & 4727(4) & 3902.2(13) & 64.6(9) \\ \text { C009 6954(4) } & 1206(3) & 4358.9(13) & 58.0(8) \\ \text { C00A 7604(4) } & 5156(3) & 4132.5(13) & 60.7(9)\end{array}$




$\begin{array}{llll}\text { C00B 7274(4) } & 4338(4) & 5779.0(14) & 63.0(8) \\ \text { C00C 4202(5) } & 5703(4) & 2981.7(17) & 72.2(10) \\ \text { N00D 4213(5) } & 6334(4) & 3564.8(17) & 94.0(13) \\ \text { C00E 6536(5) } & 5488(3) & 3757.2(14) & 64.5(9) \\ \text { C00F 7237(5) } & 5501(4) & 6022.4(15) & 72.1(10) \\ \text { C00G 7304(5) } & 6541(4) & 5676.4(17) & 77.6(12) \\ \text { C00H 8949(4) } & 4605(5) & 3279.0(15) & 76.8(12) \\ \text { C00I 6714(5) } & 5407(4) & 3136.2(15) & 69.6(10) \\ \text { C00J 5921(5) } & 506(4) & 4116.4(19) & 77.8(11) \\ \text { C00K 7416(6) } & 6430(4) & 5063.7(16) & 76.4(12) \\ \text { C00L 8234(5) } & 712(4) & 4355(2) & 85.8(13) \\ \text { C00M 5166(5) } & 5852(4) & 3998.6(17) & 79.5(12) \\ \text { C00N 7460(6) } & -1086(4) & 3852.1(18) & 84.1(14) \\ \text { C00O 7915(5) } & 4962(5) & 2911.1(15) & 81.5(13) \\ \text { C00P 7177(7) } & 3204(4) & 6158.2(17) & 90.1(15) \\ \text { C00Q 6181(6) } & -629(5) & 3859(2) & 90.0(14) \\ \text { C00R 10858(6) } & 5307(7) & 4311(2) & 101.7(18) \\ \text { C00S 5607(6) } & 5816(5) & 2715.7(18) & 86.1(13) \\ \text { C00T 3794(6) } & 4382(5) & 3051(2) & 87.3(13) \\ \text { C00U 8482(6) } & -424(5) & 4100(3) & 96.0(15) \\ \text { C00V 3168(7) } & 6382(6) & 2595(3) & 102.2(16) \\ \text { C00W 10266(7) } & 3767(11) & 2499(3) & 151(4)\end{array}$

Table S8 Anisotropic Displacement Parameters $\left(\AA^{2} \times 10^{3}\right)$ for $2 \mathrm{~b}$. The Anisotropic displacement factor exponent takes the form: $-2 \pi^{2}\left[h^{2} a * 2 U_{11}+2 h k a * b * U_{12}+\ldots\right]$.

$\begin{array}{llllll}\text { Atom } \mathbf{U}_{\mathbf{1 1}} & \mathbf{U}_{\mathbf{2 2}} & \mathbf{U}_{\mathbf{3 3}} & \mathbf{U}_{\mathbf{2 3}} & \mathbf{U}_{\mathbf{1 3}} & \mathbf{U}_{\mathbf{1 2}} \\ \text { O001 59.8(15) } & 78.0(18) & 102(2) & -22.1(16) & -4.1(16) & 0.3(14) \\ \text { O002 70.6(16) } & 100.0(19) & 53.6(11) & 6.4(13) & -4.0(11) & -9.5(17) \\ \text { N003 59.6(17) } & 54.5(14) & 59.2(13) & -5.7(11) & 0.8(13) & 1.6(14) \\ \text { O004 70.3(18) } & 183(4) & 56.7(13) & -18(2) & 3.7(13) & 6(2) \\ \text { C005 59.6(18) } & 56.3(16) & 46.8(13) & -3.2(11) & -0.5(13) & -3.3(17) \\ \text { C006 75(2) } & 56.9(17) & 48.1(14) & -3.3(12) & -2.3(16) & -2.4(18) \\ \text { C007 65(2) } & 60.0(18) & 50.3(13) & -4.0(13) & 1.5(14) & -4.3(17) \\ \text { C008 71(2) } & 77(2) & 46.4(14) & 1.8(14) & -1.4(15) & -12(2) \\ \text { C009 65(2) } & 61.6(18) & 47.4(13) & -4.3(13) & 2.9(14) & -5.7(17) \\ \text { C00A 76(2) } & 58.5(17) & 47.4(14) & -1.1(12) & 1.7(16) & -8.8(18) \\ \text { C00B 67(2) } & 74(2) & 47.6(14) & 1.0(14) & 0.5(14) & 1(2) \\ \text { C00C 82(3) } & 69(2) & 65.7(18) & -2.6(17) & -4.5(19) & 8(2) \\ \text { N00D 104(3) } & 96(3) & 82(2) & -18(2) & -11(2) & 29(3) \\ \text { C00E 81(2) } & 62.3(19) & 50.6(14) & 1.9(13) & 1.1(16) & -5.7(19) \\ \text { C00F 85(3) } & 85(2) & 45.6(13) & -13.6(15) & -1.9(16) & 6(2) \\ \text { C00G 104(3) } & 64.1(19) & 64.5(18) & -18.7(16) & -17(2) & 8(2) \\ \text { C00H 65(2) } & 114(3) & 51.7(16) & -8.0(19) & 3.3(17) & -13(3) \\ \text { C00I 74(2) } & 85(2) & 50.1(15) & 3.7(16) & -2.0(16) & -12(2) \\ \text { C00J 74(2) } & 75(2) & 84(2) & -18(2) & -5(2) & -13(2)\end{array}$




$\begin{array}{llllll}\text { C00K 110(3) } & 58.6(18) & 60.8(17) & -3.4(15) & -9(2) & -3(3) \\ \text { C00L 75(3) } & 76(3) & 106(3) & -29(2) & 2(3) & -2(2) \\ \text { C00M 98(3) } & 84(3) & 56.7(16) & -3.4(18) & 6.3(19) & 13(3) \\ \text { C00N 117(4) } & 63(2) & 72(2) & -12.3(17) & 17(3) & -4(3) \\ \text { C00O 79(3) } & 124(3) & 41.8(13) & 0.5(19) & 3.5(16) & -18(3) \\ \text { C00P 118(4) } & 98(3) & 54.0(17) & 16.0(19) & 4(2) & -11(3) \\ \text { C00Q 102(4) } & 78(3) & 89(3) & -23(2) & 0(3) & -24(3) \\ \text { C00R 97(3) } & 138(4) & 71(2) & 11(3) & -19(2) & -36(4) \\ \text { C00S 97(3) } & 99(3) & 63.0(19) & 17(2) & -5(2) & -5(3) \\ \text { C00T 83(3) } & 82(3) & 96(3) & -8(2) & -2(3) & -2(3) \\ \text { C00U 86(3) } & 80(3) & 122(4) & -28(3) & 9(3) & 7(3) \\ \text { C00V 104(4) } & 105(3) & 97(3) & 4(3) & -16(3) & 21(4) \\ \text { C00W 83(4) } & 294(10) & 76(3) & -63(5) & 2(3) & 25(6)\end{array}$

Table S9 Bond Lengths for $\mathbf{2 b}$.

\begin{tabular}{|c|c|}
\hline Atom Atom Length/Å & Atom Atom Length/§̊ \\
\hline O001 C007 1.229(5) & C00B C00P 1.498(5) \\
\hline O002 C008 1.365(5) & C00C N00D 1.476(5) \\
\hline O002 C00R 1.417(6) & C00C C00S 1.519(7) \\
\hline N003 C005 $1.436(4)$ & C00C C00T $1.494(7)$ \\
\hline N003 C007 1.340(5) & C00C C00V 1.531(7) \\
\hline $\mathrm{O} 004 \mathrm{C} 00 \mathrm{H} 1.379(6)$ & N00DC00M 1.453(6) \\
\hline O004 C00W 1.383(6) & C00E C00I $1.407(4)$ \\
\hline C005 C006 1.384(4) & C00E C00M 1.513(6) \\
\hline C005 C00B 1.397(4) & C00F C00G 1.371(6) \\
\hline C006 C00A 1.508(4) & C00G C00K 1.385(5) \\
\hline C006 C00K 1.387(5) & $\mathrm{C} 00 \mathrm{H} \mathrm{C} 00 \mathrm{O} 1.371(7)$ \\
\hline C007 C009 1.496(5) & C00I C00O 1.379(7) \\
\hline C008 C00A 1.371(6) & C00I C00S 1.512(6) \\
\hline $\mathrm{C} 008 \mathrm{C} 00 \mathrm{H} 1.413(4)$ & C00J C00Q 1.383(6) \\
\hline C009 C00J 1.384(6) & C00L C00U 1.379(6) \\
\hline C009 C00L 1.375(7) & C00N C00Q 1.359(8) \\
\hline C00AC00E 1.399(6) & C00N C00U 1.360(7) \\
\hline
\end{tabular}

Table S10 Bond Angles for $\mathbf{2 b}$.

Atom Atom Atom Angle/ ${ }^{\circ}$

C008 O002 C00R 113.2(4)

C007 N003 C005 123.6(3)

$\mathrm{C} 00 \mathrm{H}$ O004 C00W 117.7(4)

C006 C005 N003 119.5(3)

C006 C005 C00B 121.7(3)

C00B C005 N003 118.7(3)

C005 C006 C00A 121.2(3)

C005 C006 C00K 118.5(3)
Atom Atom Atom Angle/
N00D C00C C00V 106.6(4)
C00S C00C C00V 110.5(4)
C00T C00C C00S 111.5(4)
C00T C00C C00V 109.8(5)
C00M N00D C00C 115.6(4)
C00A C00E C00I 119.1(4)
C00A C00E C00M 121.9(3)
C00I C00E C00M 118.9(4) 
C00K C006 C00A $120.2(3)$
O001 C007 N003 $121.8(3)$
O001 C007 C009 $121.8(3)$
N003 C007 C009 $116.4(3)$
O002 C008 C00A $120.8(3)$
O002 C008 C00H $119.4(4)$
C00A C008 C00H $119.7(4)$
C00J C009 C007 $117.4(4)$
C00L C009 C007 $124.7(3)$
C00L C009 C00J $117.7(4)$
C008 C00A C006 $118.6(3)$
C008 C00A C00E $120.8(3)$
C00E C00A C006 $120.6(4)$
C005 C00B C00P $120.5(3)$
C00F C00B C005 $117.8(3)$
C00F C00B C00P $121.6(3)$
N00D C00C C00S $107.7(4)$
N00D C00C C00T $110.6(4)$

Table S11 Torsion Angles for $\mathbf{2 b}$. A B C D Angle/ O001 C007 C009 C00J -3.9(5) O001 C007 C009 C00L -179.9(4) O002 C008 C00AC006 1.0(5) O002 C008 C00A C00E -179.5(3) O002 C008 C00HO004 1.9(7) O002 C008 C00HC00O -178.8(4) N003 C005 C006 C00A 4.2(6) N003 C005 C006 C00K -174.9(4) N003 C005 C00B C00F 174.3(4) N003 C005 C00B C00P -5.2(6) N003 C007 C009 C00J 176.1(3) N003 C007 C009 C00L 0.1(5) O004 C00HC00OC00I 177.4(5) C005 N003 C007 O001 -4.1(5) C005 N003 C007 C009 175.9(3) C005 C006 C00A C008 -69.1(5) C005 C006 C00A C00E 111.4(4) C005 C006 C00K C00G -0.3(8) C005 C00B C00F C00G 1.6(7) C006 C005 C00B C00F -2.4(6) C006 C005 C00B C00P 178.1(4) C006 C00A C00E C00I 177.8(3) C006 C00A C00E C00M-6.2(6) C007 N003 C005 C006 -82.5(4)

C00G C00F C00B 121.9(3)

C00F C00G C00K 119.6(3) $\mathrm{O} 004 \mathrm{C} 00 \mathrm{H} \mathrm{C008} \mathrm{115.6(4)}$ $\mathrm{C} 00 \mathrm{O} \mathrm{C} 00 \mathrm{H} \mathrm{O} 004$ 125.0(3) $\mathrm{C} 00 \mathrm{O} \mathrm{C} 00 \mathrm{H} \mathrm{C008}$ 119.4(4) C00E C00I C00S 120.6(4) C00O C00I C00E 119.5(4) C00O C00I C00S 119.9(3) C00Q C00J C009 120.9(5) C00G C00K C006 120.4(4) C009 C00L C00U 120.9(5) N00D C00MC00E 115.8(3) C00Q C00N C00U 119.7(4) $\mathrm{C} 00 \mathrm{H}$ C00O C00I 121.5(3) C00N C00Q C00J 120.1(5) C00I COOS C00C 113.2(3) C00N C00U C00L 120.5(5)

$\begin{array}{lllll}\text { A } & \text { B } & \text { C } & \text { D } & \text { Angle } \\ & \circ\end{array}$ C00A C00E CO0I C00O 2.3(6) C00A C00E C00I C00S -177.0(4) C00A C00E C00MN00D 171.9(4) C00B C005 C006 C00A -179.1(4) C00B C005 C006 C00K 1.8(6) C00B C00F C00G C00K -0.2(8) C00C N00D C00MC00E 40.2(6) N00D C00C C00S C00I 51.7(5) C00E COOI COOO C00H -0.6(8) C00E C00I C00S C00C -27.8(7) C00F C00G C00K C006 -0.4(8) C00H C008 C00A C006 179.8(4) $\mathrm{C} 00 \mathrm{H}$ C008 C00A C00E -0.7(6) C00I C00E C00MN00D -12.1(6) C00J C009 C00L C00U 2.1(7) C00K C006 C00A C008 110.0(5) C00K C006 C00A C00E -69.5(6) C00L C009 C00J C00Q -2.4(6) C00M C00E C00I C00O-173.7(4) C00M C00E C00I CO0S 6.9(6) C00O C00I C00S C00C 152.8(4) C00P C00B C00F C00G -178.9(5) C00Q C00N C00U C00L -0.5(8) C00R O002 C008 C00A -101.0(5) 
C007 N003 C005 C00B 100.7(4)

C007 C009 C00J C00Q -178.7(4)

C007 C009 C00L C00U 178.0(4)

C008 C00A C00E C00I -1.7(6)

C008 C00A C00E C00M 174.3(4)

$\mathrm{C} 008 \mathrm{C} 00 \mathrm{HC} 00 \mathrm{OC} 00 \mathrm{I}-1.8(8)$

C009 C00J C00QC00N 1.4(7)

C009 C00L C00UC00N -0.7(9)

C00A C006 C00K C00G -179.5(5)

$\mathrm{C} 00 \mathrm{AC} 008 \mathrm{C} 00 \mathrm{HO} 004-176.9(4)$

C00AC008 C00HC00O 2.5(7)
C00R O002 C008 C00H 80.3(5)

C00S C00C N00D C00M-59.7(6)

$\mathrm{C} 00 \mathrm{~S}$ C00I $\mathrm{C} 00 \mathrm{O} \mathrm{C} 00 \mathrm{H} 178.7(5)$

C00T C00C N00D C00M62.4(6)

C00T C00C C00S C00I -69.9(6)

C00U C00N C00Q C00J $0.1(8)$

C00V C00C N00D C00M-178.3(5)

C00V C00C C00S C00I 167.8(4)

C00W O004 C00H C008 168.8(7)

$\mathrm{C} 00 \mathrm{~W} \mathrm{O004} \mathrm{C} 00 \mathrm{H} \mathrm{C00O}-10.5(10)$

Table S12 Hydrogen Atom Coordinates $\left(\AA \times 10^{4}\right)$ and Isotropic Displacement Parameters $\left(\AA^{2} \times 10^{3}\right)$ for $2 b$.

$\begin{array}{llll}\text { Atom } \boldsymbol{x} & \boldsymbol{y} & \boldsymbol{z} & \mathbf{U}(\mathbf{e q}) \\ \text { H003 8369.3 } & 2699.14 & 4919.71 & 69 \\ \text { H00D 3412.05 } & 6392.53 & 3710.87 & 113 \\ \text { H00F 7165.64 } & 5584.25 & 6433.59 & 87 \\ \text { H00G 7273.73 } & 7318.45 & 5852.09 & 93 \\ \text { H00J 5039.66 } & 803.71 & 4126.75 & 93 \\ \text { H00K 7454.91 } & 7134.53 & 4827.79 & 92 \\ \text { H00L 8942.51 } & 1150.49 & 4526.1 & 103 \\ \text { H00A 4764.4 } & 5134.13 & 4186.98 & 95 \\ \text { H00B 5296.67 } & 6470.81 & 4305.43 & 95 \\ \text { H00N 7635.05 } & -1849.26 & 3678.49 & 101 \\ \text { H00O 8025.78 } & 4902.73 & 2500.68 & 98 \\ \text { H00C 6420.74 } & 2715.49 & 6031.21 & 135 \\ \text { H00E 7056.42 } & 3439.36 & 6567.12 & 135 \\ \text { H00H 7991.92 } & 2729.86 & 6119.45 & 135 \\ \text { H00Q 5479.6 } & -1081.26 & 3690.53 & 108 \\ \text { H00I 11136.63 } & 5549.69 & 3918.61 & 153 \\ \text { H00M 11618.33 } & 4977.02 & 4523.87 & 153 \\ \text { H00P 10510.81 } & 6011.55 & 4520.28 & 153 \\ \text { H00R 5652.16 } & 5321.87 & 2355.97 & 103 \\ \text { H00S 5763.57 } & 6669.79 & 2604.46 & 103 \\ \text { H00T 4452.65 } & 3957.5 & 3288.75 & 131 \\ \text { H00U 3738.07 } & 4001.32 & 2665.76 & 131 \\ \text { H00V 2928.64 } & 4340.44 & 3243.4 & 131 \\ \text { H00W 9356.3 } & -739.35 & 4097.7 & 115 \\ \text { H00X 2311.59 } & 6398.85 & 2795.39 & 153 \\ \text { H00Y 3073.32 } & 5960.81 & 2220.64 & 153 \\ \text { H 3470.47 } & 7211.36 & 2525.37 & 153 \\ \text { H00Z 10289.49 } & 4492.47 & 2253.41 & 226 \\ \text { HA 9500.91 } & 3269.88 & 2390.22 & 226 \\ \text { HB 11080.24 } & 3300.62 & 2441.4 & 226\end{array}$




\section{References}

1. G. R. Fulmer, A. J. M. Miller, N. H. Sherden, H. E. Gottlieb, A. Nudelman, B. M. Stoltz, J. E. Bercaw and K. I. Goldberg, Organometallics, 2010, 29, 2176-2179.

2. D. Ando and J. Ralph, ACS Sustain. Chem. Eng., 2019, 7, 18624-18629.

3. T. Deng, W. Mazumdar, R. L. Ford, N. Jana, R. Izar, D. J. Wink and T. G. Driver, J. Am. Chem. Soc., 2020, 142, 4456-4463.

4. $\quad$ Kettle, J. G.; Simpson, I.; Phillips, C.; Boyd, S.; Steward, O. R.; Bodnarchuk, M. S.; Cassar, D. J.; Pike, K. G. Fused Tricyclic Compounds Useful as Anticancer Agents, WO2020178282, September 10, 2020.

5. $\quad$ La, H. J.; Kim, G. Y.; Kim, Y. U.; Yang, J. H.; Choi, J. S.; Choi, D. H.; Eum, S. J.; Lee, J. D. Preparation of nitrogen-containing polycyclic compounds as organic light-emitting device materials. KR2016052399, May 12, 2016.

6. D. Qiu, L. Jin, Z. Zheng, H. Meng, F. Mo, X. Wang, Y. Zhang and J. Wang, J. Org. Chem., 2013, 78, 1923-1933.

$7 . \quad$ O. V. Dolomanov, L. J. Bourhis, R. J. Gildea, J. A. K. Howard and H. Puschmann, J. Appl. Crystallogr., 2009, 42, 339-341.

8. G. Sheldrick, Acta Crystallogr. A, 2015, 71, 3-8.

9. G. Sheldrick, Acta Crystallogr. A, 2008, 64, 112-122. 